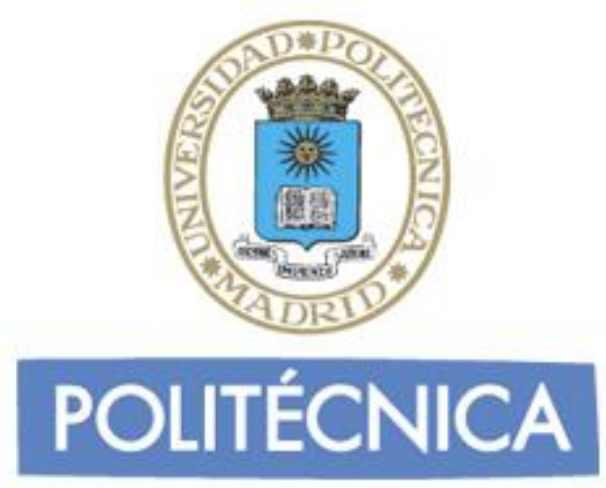

Universidad Politécnica de Madrid

Escuela Técnica Superior de Ingeniería y Sistemas de Telecomunicación

\title{
CONTRIBUTION TO THE DESIGN, IMPLEMENTATION AND STANDARDIZATION OF SEMANTIC MIDDLEWARE ARCHITECTURES FOR THE SMART GRID
}

\section{DOCTORAL THESIS}

\author{
Jesús Rodríguez Molina \\ Master in Systems and Services Engineering \\ for the Information Society
}



Centro de Investigación en Tecnologías de Software y

Sistemas Multimedia para la Sostenibilidad

Escuela Técnica Superior de Ingeniería y Sistemas de Telecomunicación

\title{
CONTRIBUTION TO THE DESIGN, IMPLEMENTATION AND STANDARDIZATION OF SEMANTIC MIDDLEWARE ARCHITECTURES FOR THE SMART GRID
}

\section{DOCTORAL THESIS}

\author{
Jesús Rodríguez Molina \\ Master in Systems and Services Engineering \\ for the Information Society
}

Supervisor:

Prof. PhD. José-Fernán Martínez Ortega

Universidad Politécnica de Madrid 



\section{DOCTORADO EN INGENIERÍA DE SISTEMAS Y SERVICIOS PARA LA SOCIEDAD DE LA INFORMACIÓN}

\begin{tabular}{|c|c|c|}
\hline \multicolumn{3}{|c|}{ Tesis Doctoral } \\
\hline Título & \multicolumn{2}{|c|}{$\begin{array}{c}\text { Contribution to the design, implementation and } \\
\text { standardization of semantic middleware architectures for the } \\
\text { Smart Grid }\end{array}$} \\
\hline Autor & \multicolumn{2}{|l|}{ Jesús Rodríguez Molina } \\
\hline Director & Dr. José-Fernán Martínez Ortega & $\mathrm{V}^{0} \mathrm{~B}^{\mathrm{o}}$ \\
\hline \multicolumn{3}{|c|}{ Tribunal } \\
\hline \multicolumn{3}{|l|}{ Presidente } \\
\hline \multicolumn{3}{|l|}{ Secretario } \\
\hline \multicolumn{3}{|l|}{ Vocal $1^{\circ}$} \\
\hline \multicolumn{3}{|l|}{ Vocal $2^{\circ}$} \\
\hline \multicolumn{3}{|l|}{ Vocal $3^{\circ}$} \\
\hline \multicolumn{3}{|l|}{ Suplente } \\
\hline \multicolumn{3}{|l|}{ Suplente } \\
\hline $\begin{array}{l}\text { Lugar y fecha } \\
\text { de lectura }\end{array}$ & \multicolumn{2}{|c|}{ E.T.S.I. y Sistemas de Telecomunicación (U.P.M.) } \\
\hline Calificación & & \\
\hline
\end{tabular}

El Presidente

El secretario

Los vocales

Tesis Doctoral para la obtención del título de Doctor por la Universidad Politécnica de Madrid 

A happy life is impossible; the best a man can attain is a heroic life, such as is lived by one who struggles against overwhelming odds in some way and some affair that will benefit the whole of mankind, and who in the end triumphs, although he obtains a poor reward or none at all

Arthur Schopenhauer

You promised me Mars colonies. Instead, I got Facebook

Buzz Aldrin

Now I must go to war. We must all believe we have a future. We must fight for those who aren't even born yet!

Terra Branford 



\section{Table of contents}

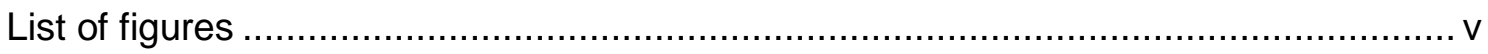

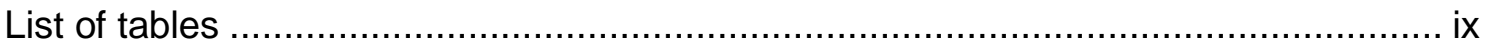

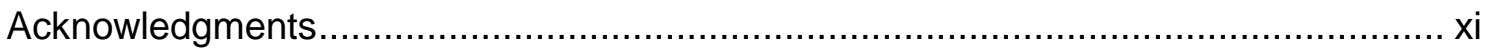

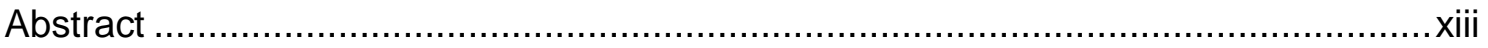

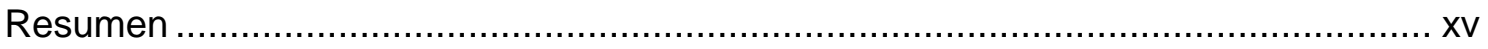

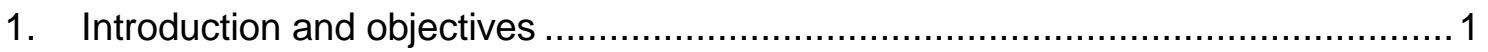

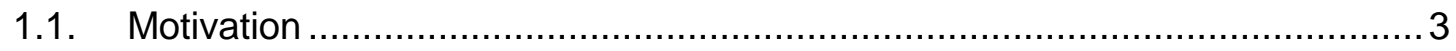

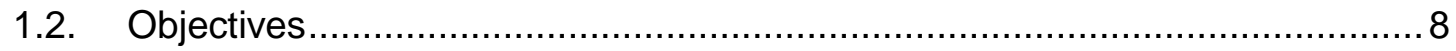

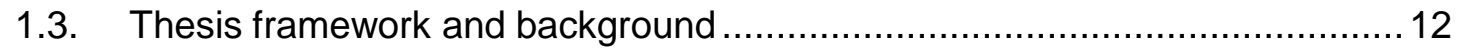

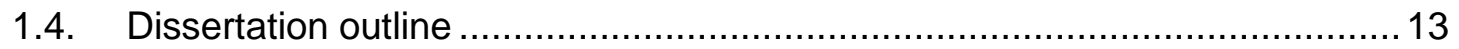

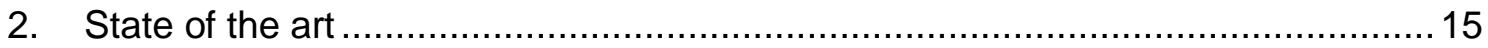

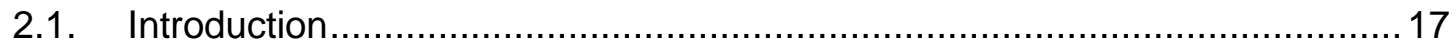

2.2. State of the art in middleware architectures for the Smart Grid .................... 18

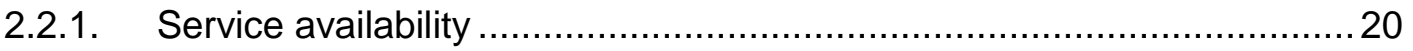

2.2.2. Computational capabilities of deployment hardware .............................2 21

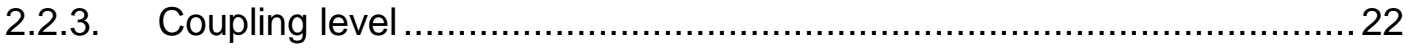

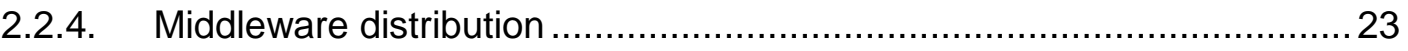

2.2.5. Taxonomy for middleware in distributed systems..............................2 24

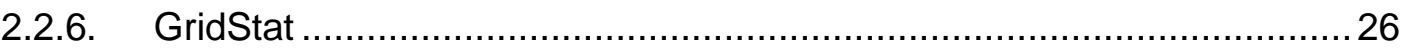

2.2.7. Service-Oriented Middleware for Smart Grid........................................28

2.2.8. Ubiquitous Sensor Network Middleware (USN) ..................................30

2.2.9. OSHNet (Object-Based Middleware for Smart Home Network) ..............32

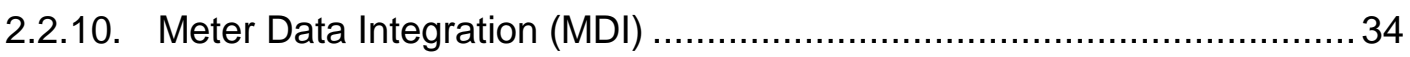

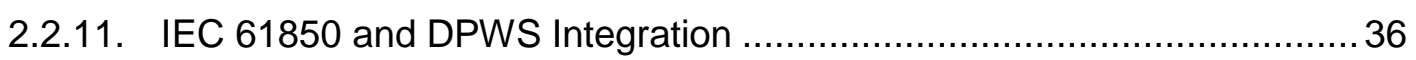

2.2.12. Intelligent Agents Platform ......................................................... 38

2.2.13. Self-Organizing Smart Grid Services ............................................... 40

2.2.14. Secure Decentralized Data-Centric Information Infrastructure ...............42 42

2.2.15. A cloud optimization perspective...................................................... 44

2.2.16. KT Smart Grid Architecture and Open Platform ..................................46 46

2.2.17. Smart microgrid monitoring with DDS ........................................... 48

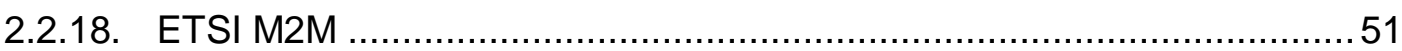

2.2.19. Smart Middleware Device for Smart Grid Integration ............................53

2.2.20. WAMPAC-based Smart Grid communications ...................................55 


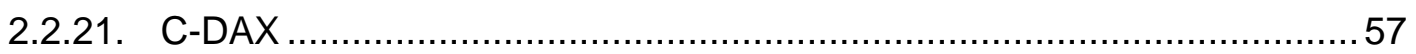

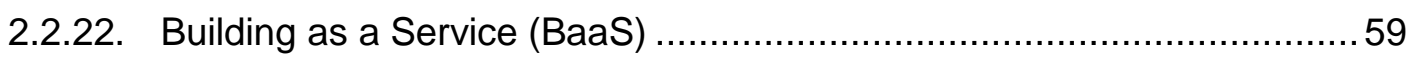

2.2.23. Middleware-based management for the Smart Grid...........................61 61

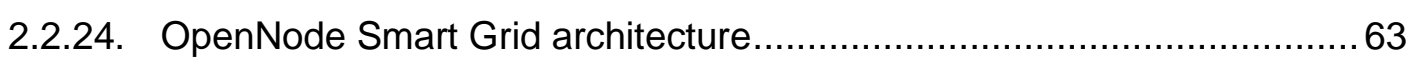

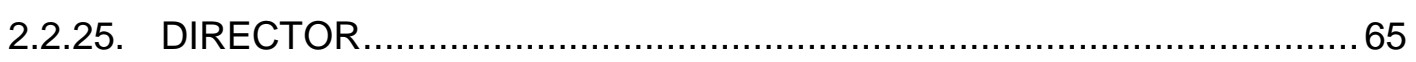

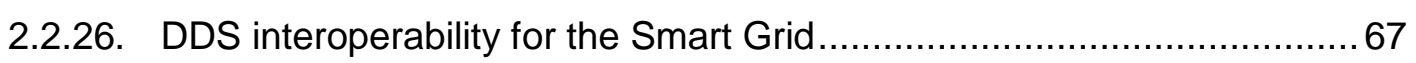

2.3. State of the art in Enterprise Service Bus solutions....................................70

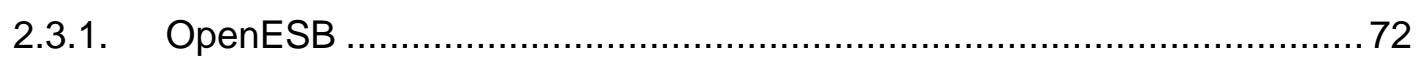

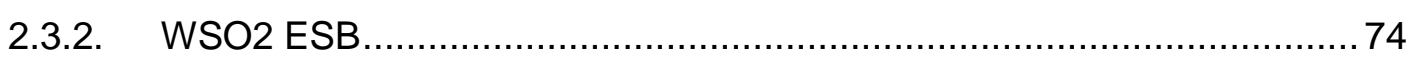

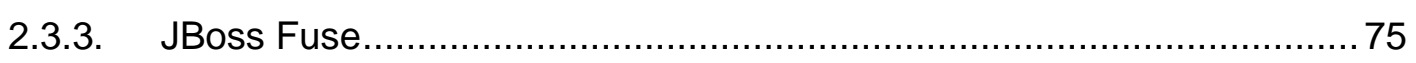

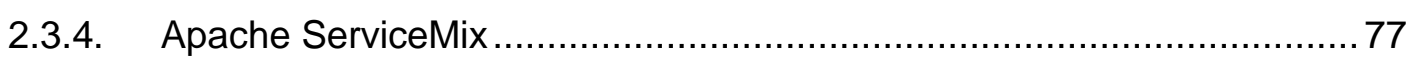

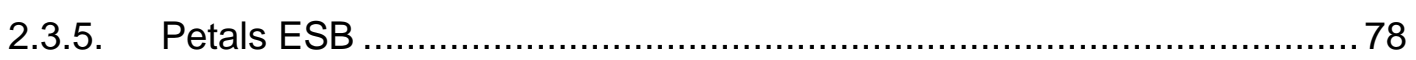

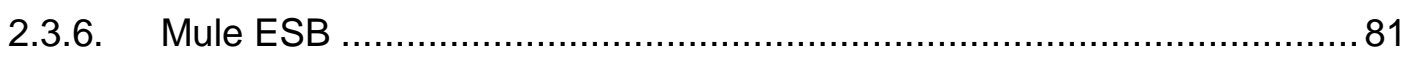

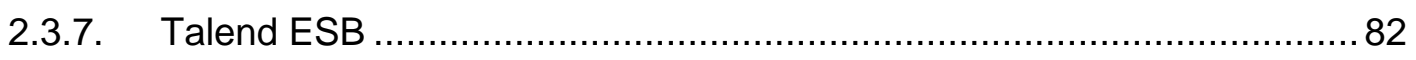

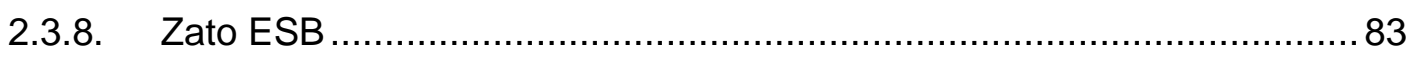

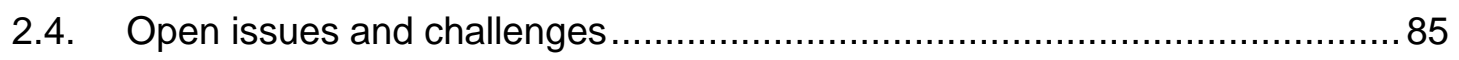

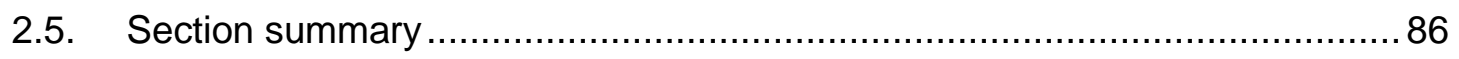

3. Proposal for a Common Middleware Architecture ............................................. 89

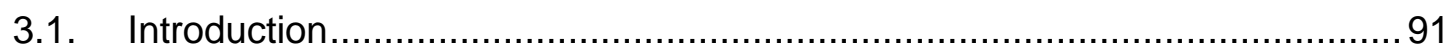

3.2. Common Middleware Architecture: purpose and scope .............................92

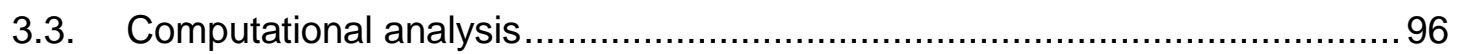

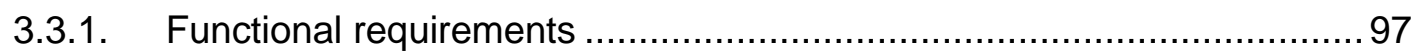

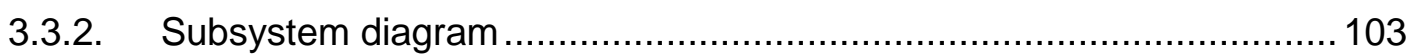

3.3.3. Component diagrams .......................................................... 104

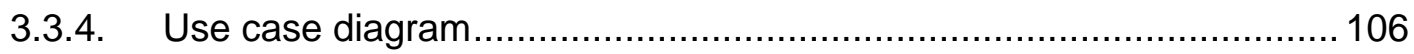

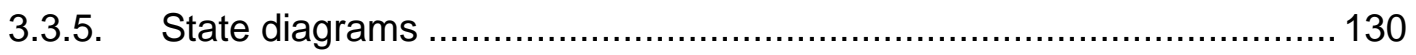

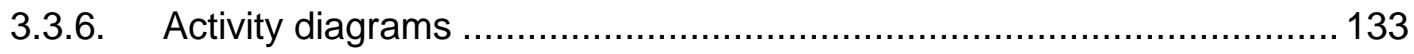

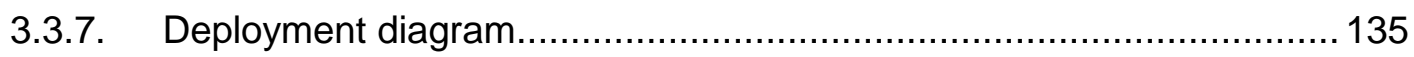

3.4. Architecture Description for ISO/IEC/IEEE 42010 ................................. 136

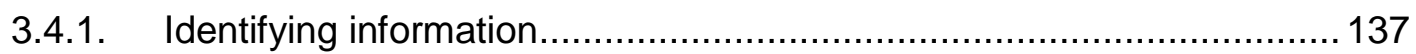

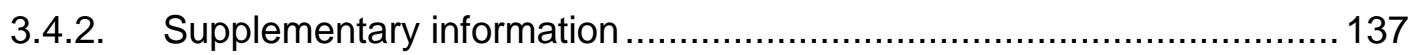

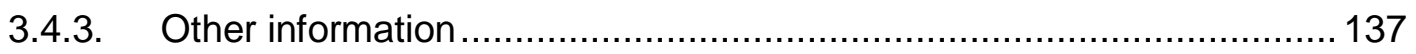

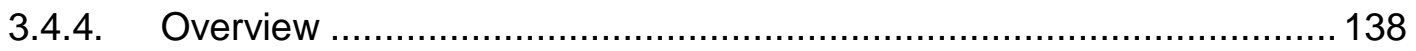

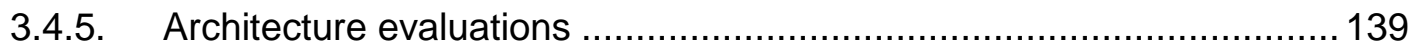

3.4.6. Rationale for key decisions .................................................. 139 
3.4.7. Stakeholders, concerns and concern-stakeholder traceability .............139

3.4.8. Viewpoint names ............................................................... 142

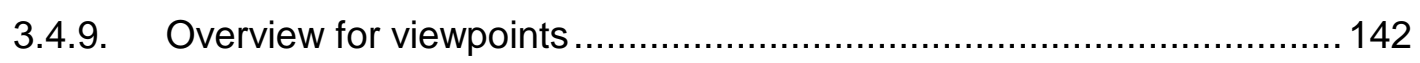

3.4.10. Stakeholders, concerns and "anti-concerns" for viewpoints ................ 143

3.4.11. Operations on views .............................................................. 144

3.4.12. Views and views name ......................................................... 145

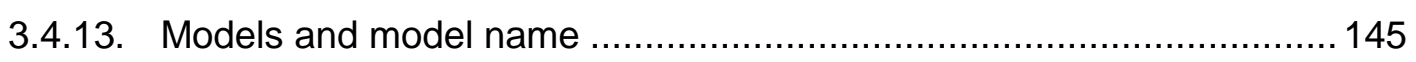

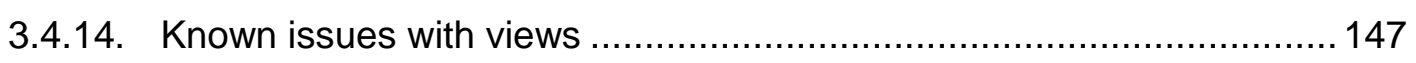

3.4.15. Consistency and correspondences ............................................ 147

3.4.16. Known inconsistencies ............................................................. 148

3.4.17. Correspondences in the Architecture Description ............................ 148

3.4.18. Correspondence rules .......................................................... 148

3.4.19. Architecture decisions and rationale ............................................ 149

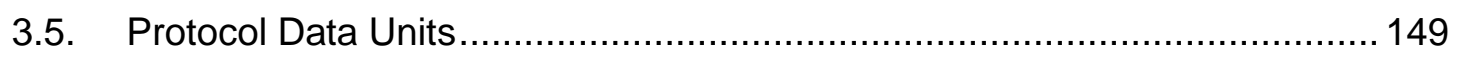

3.5.1. Generic PDU format ............................................................. 150

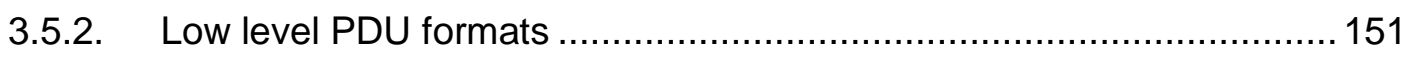

3.6. Business models and middleware exploitation ....................................... 152

3.6.1. Offline model without the Smart Grid ................................................. 153

3.6.2. Offline model within the Smart Grid .................................................. 154

3.6.3. Online model without the Smart Grid ................................................. 155

3.6.4. Online model within the Smart Grid ..................................................... 156

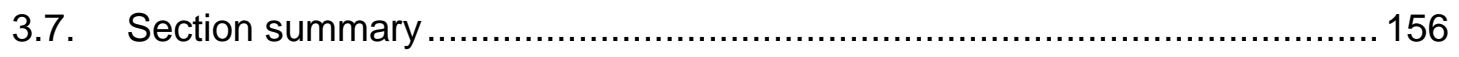

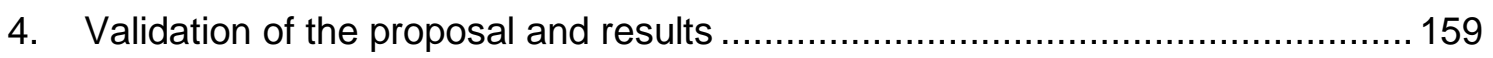

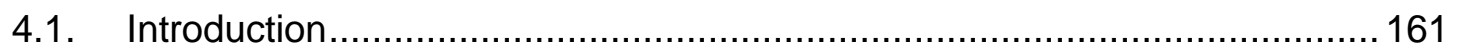

4.2. Framework for the Common Middleware Architecture ............................. 163

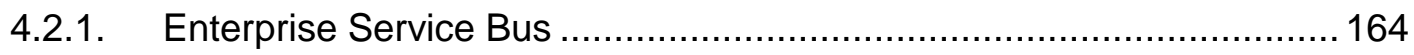

4.2.2. Programming language and Integrated Development Environment ..... 165

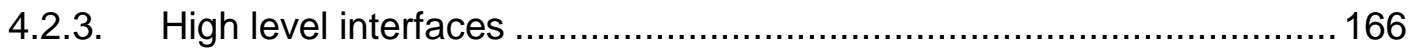

4.2.4. Low level interfaces ................................................................ 173

4.2.5. Interconnectivity of software components ...................................... 181

4.2.6. CMA ontology implementation .................................................. 184

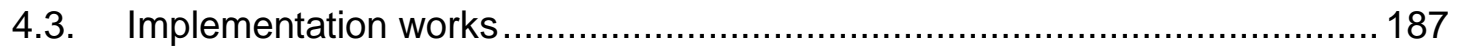

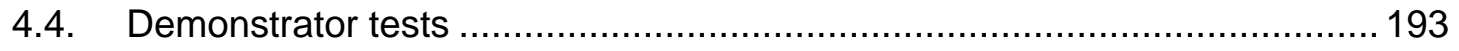

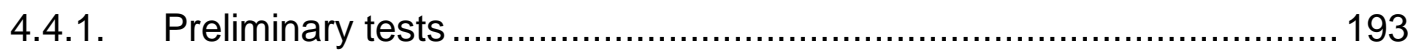

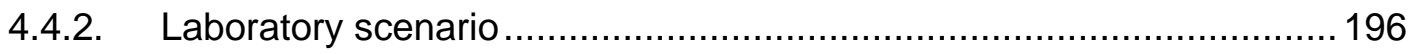


4.4.3. Ylivieska scenario ........................................................... 210

4.4.4. Steinkjer scenario ............................................................ 211

4.4.5. Smart Grid scenario test results............................................... 213

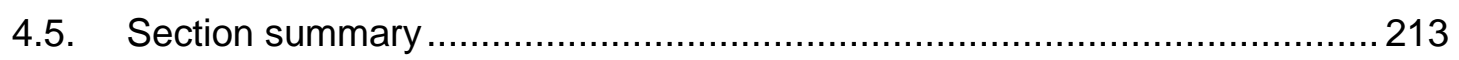

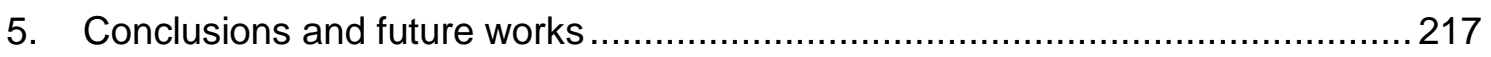

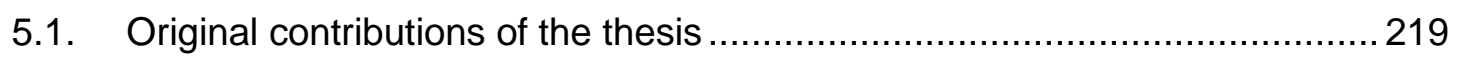

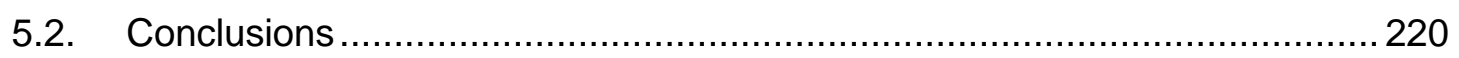

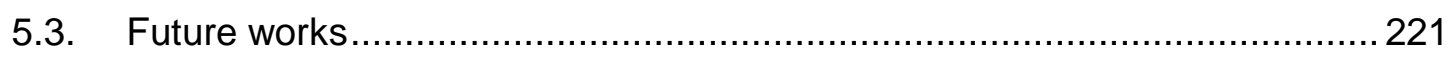

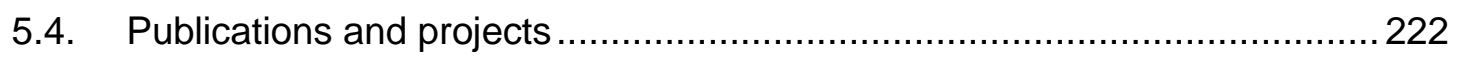

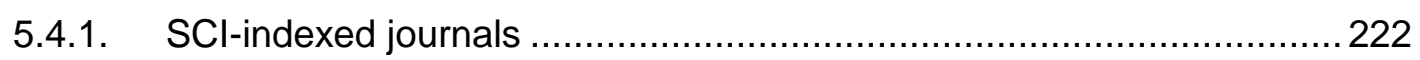

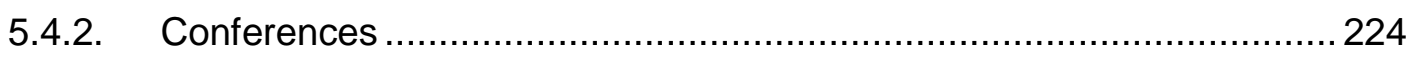

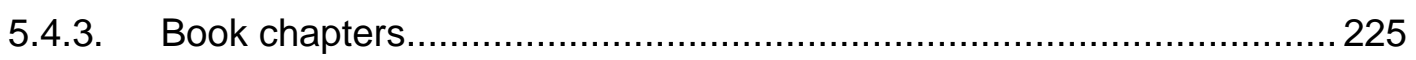

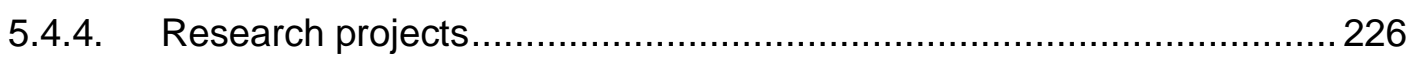

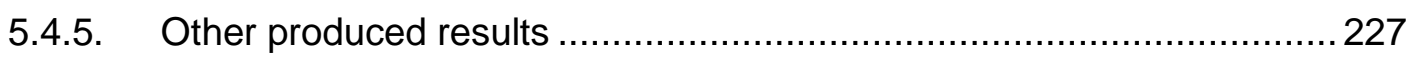

Appendix A: development procedures ........................................................ 231

Appendix B: Application Programming Interface ............................................... 245

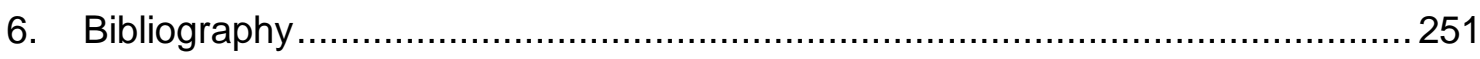

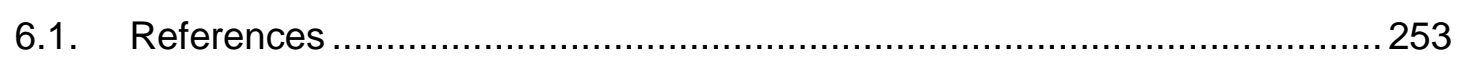

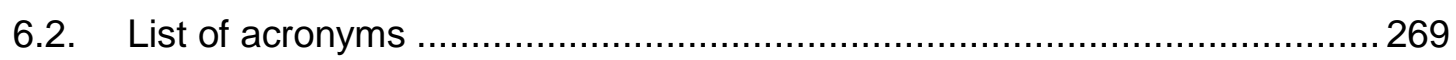




\section{List of figures}

Figure 1. Graphical comparison between the conventional power grid and the Smart Grid, inspired from [11]

Figure 2. Energy market value chain focused on the prosumer, as represented in [11]. 7

Figure 3. Middleware location in a distributed system ........................................... 8

Figure 4. Comparison between common elements in the loT and the Smart Grid .......11

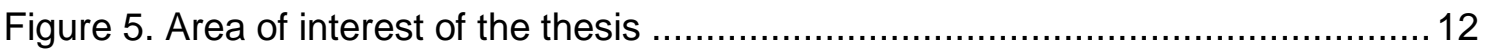

Figure 6. Project logos where middleware-related research activities have been carried

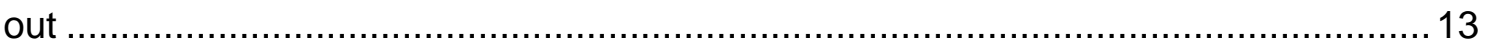

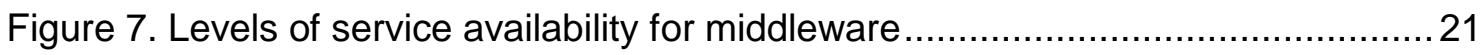

Figure 8. Levels of computational capabilities for middleware .....................................22

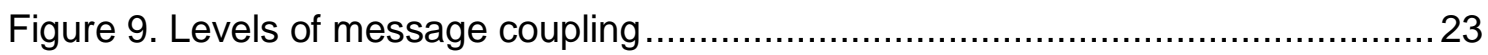

Figure 10. Levels of middleware distribution ..................................................... 23

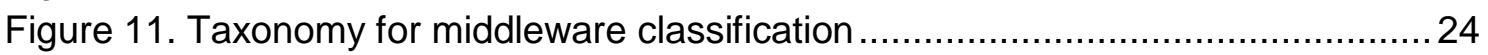

Figure 12. Matrix for Smart Grid middleware taxonomy ........................................... 25

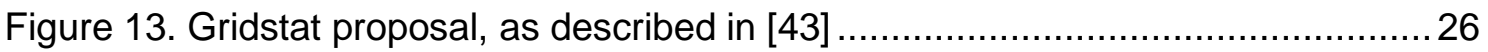

Figure 14. Service-Oriented Middleware proposal, as described in [47] .....................29

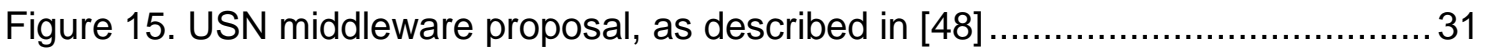

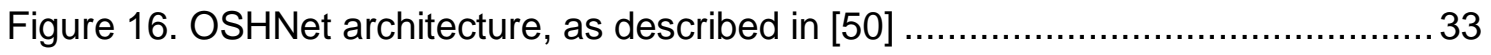

Figure 17. Meter Data Integration Proposal, as described in [51] .............................. 35

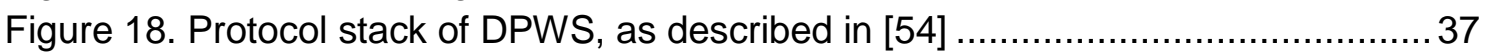

Figure 19. IAP-INMS appearance, as described in [56]........................................ 39

Figure 20. Solution structure (a) and main levels of the proposal (b), as described in

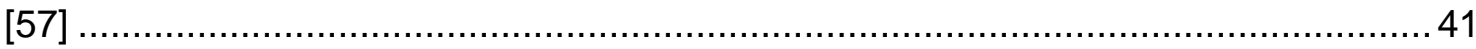

Figure 21. Data-Centric Information Infrastructure, as described in [58] ..................... 43

Figure 22. Main components of the cloud optimisation service, as shown in [59] ........45

Figure 23. Main components of the Open Service Platform, as described in [64] ........47

Figure 24. Main features of a DDS deployment, as described in [65] ........................50

Figure 25. Structure of the ETSI M2M proposal, as described in [76] ..........................52

Figure 26. Smart Middleware Device, as described in [78] .......................................5

Figure 27. WAMPAC controller location, as described in [79] .................................56

Figure 28. C-DAX middleware appearance, as described in [81] .............................58

Figure 29. High level architecture of the BaaS system part of the proposal, as

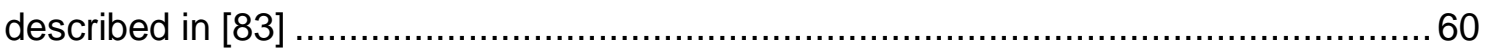

Figure 30. Block diagram of the EMD structure, as described in [85] .........................62

Figure 31. Middleware high level location in the proposal, as described in [88]..........64

Figure 32. Location of the DIRECTOR proposal in a Smart Grid deployment, as stated

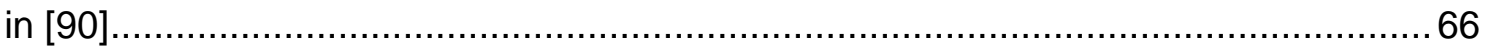

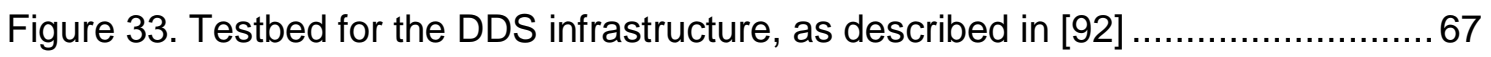

Figure 34. ESB location and most usual services in a Cyber-Physical System ...........71

Figure 35. Legacy and standalone required software components, as described in [99]

Figure 36. Facilities offered by WSO2 Enterprise Service Bus and their relationships, as described in [101]. 
Figure 37. JBoss Fuse layers and services as described in [105] ............................ 77

Figure 38. Petals ESB software components, as described in [126]......................... 80

Figure 39. Mule ESB main software components, as described in [128] ..................... 81

Figure 40. Main architectural functional blocks in Talen, as described in [131]............83

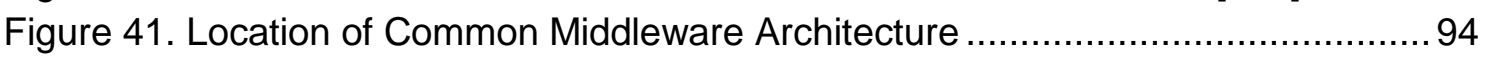

Figure 42. SGAM Framework, as depicted in [153] ........................................... 95

Figure 43. Comparison between the scope of SGAM and CMA..............................96

Figure 44. High level appearance of the Common Middleware Architecture .............. 101

Figure 45. Common Middleware Architecture location in Smart Grid devices ............ 103

Figure 46. CMA subsystem diagram with all the groups of services represented ...... 104

Figure 47. Components diagram from the High Level MW services subsystem ......... 105

Figure 48. Components diagram from the General Purpose Services subsystem ..... 105

Figure 49. Components diagram from the General Purpose Services subsystem ..... 106

Figure 50. Use case diagram for Common Middleware Architecture ......................... 107

Figure 51. Use case diagram for further Middleware Management Information

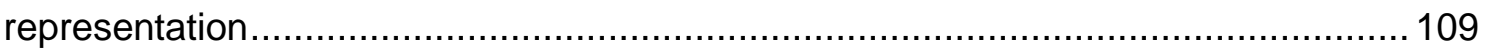

Figure 52. Sequence diagram for device registration started by a human operator ... 110

Figure 53. Sequence diagram for device registration started by a device...................111

Figure 54. Sequence diagram for device registration started by a middleware service

Figure 55. Use case diagram for further Central Controller Support representation .. 113 Figure 56. Data requests done in the Central Controller support (Middleware Access

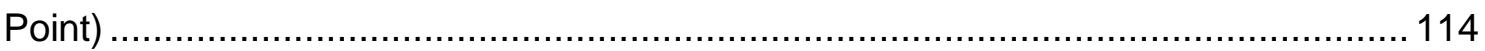

Figure 57. Data requests done in the Central Controller support (Middleware

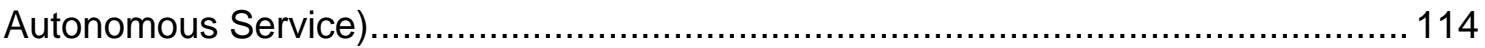

Figure 58. Use case diagram for further Local Controller Support representation ..... 116

Figure 59. Sequence of a data request done to the Local Controller support ............117

Figure 60. Use case diagram for service discovery representation ...........................118

Figure 61. Sequence of a data request done to the Local Controller support ............119

Figure 62. Use case diagram for service discovery representation ......................... 120

Figure 63. Sequence diagram with data collection and securitization........................ 122

Figure 64. Class diagram from the Middleware Access Point component .................. 123

Figure 65. Class diagram from a Middleware Autonomous Service component ........ 124

Figure 66. Class diagram of the Registration component ...................................... 125

Figure 67. Class diagram of the Context Awareness component ............................. 126

Figure 68. Class diagram of the semantic capabilities component ........................... 127

Figure 69. Class diagram of the Hardware Abstractor component ........................... 128

Figure 70. Class diagram of the Service Composition component ...........................129

Figure 71. Class diagram of the Securitization component ................................... 130

Figure 72. State diagram for service registration................................................. 131

Figure 73. State diagram for service request and answer .................................... 132

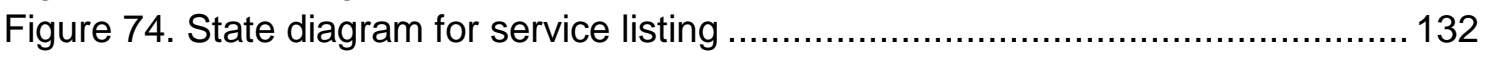

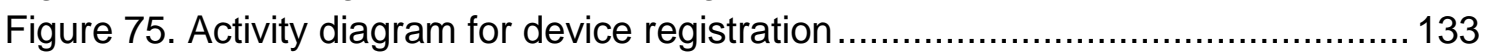

Figure 76. Activity diagram for service requests.................................................. 134

Figure 77. Activity diagram for service list request ............................................. 135

Figure 78. Deployment diagram of a system with the Common Middleware Architecture

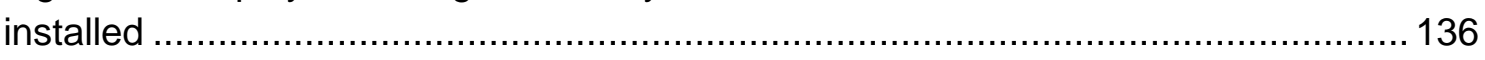


Figure 79. PDU format for a high level data request ............................................ 150

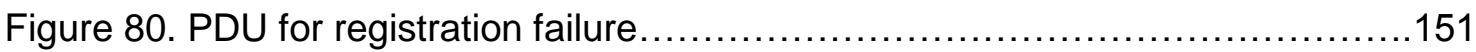

Figure 81. PDU for registration requests ....................................................... 151

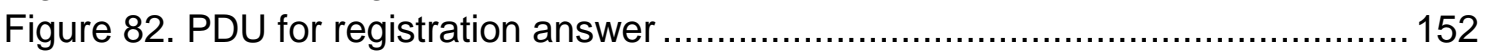

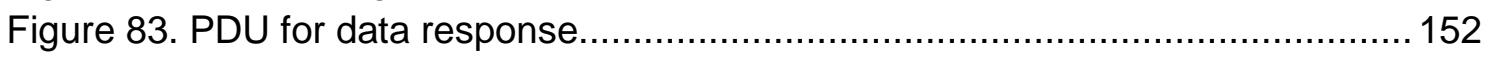

Figure 84. High-level view of component deployments ......................................... 157

Figure 85. Theoretical and actual implementations of the middleware and its adjacent

layers.

Figure 86. Waterfall model (enhanced with feedback and incremental prototyping) for

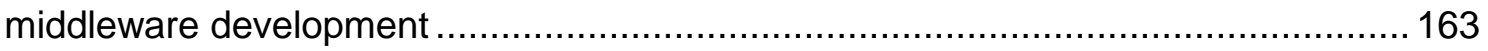

Figure 87. Connectivity among elements of an ESB, as depicted in [154]................ 165

Figure 88. Web service dependencies location ................................................... 167

Figure 89. URIs for available middleware high level components............................ 173

Figure 90. Device registration with the intervention of the ontology of the middleware,

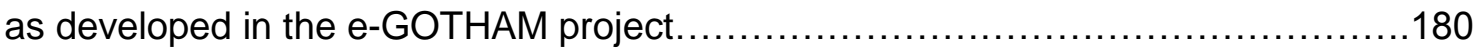

Figure 91. Conceived CMA ontology........................................................... 186

Figure 92. Running JBoss Fuse with the list of active bundles ................................ 188

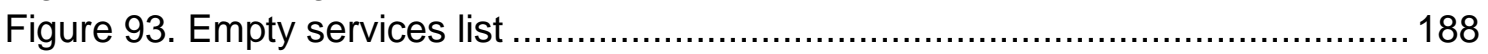

Figure 94: registration message with the identifier. ............................................ 189

Figure 95. Middleware bundles and the new one created during registration ............ 189

Figure 96. Features of the device that has become registered................................. 190

Figure 97. Security sequence of steps, as shown in [154] .................................... 192

Figure 98. Time used to answer temperature requests done to nodes, as described in

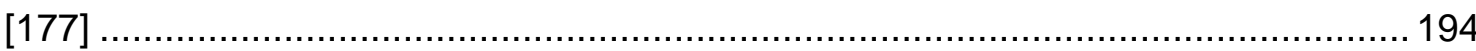

Figure 99. Time used to answer to obtain data from a composed service, as described

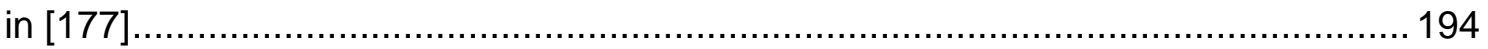

Figure 100. Performance results for simple (high graph) and composed (low graph)

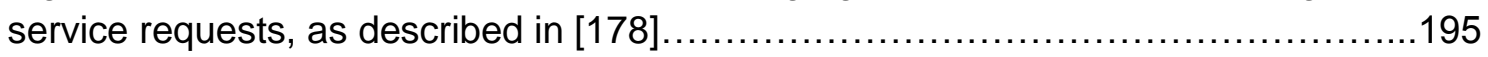

Figure 101. Deployment made in Ylivieska. Middleware is at the centre of the

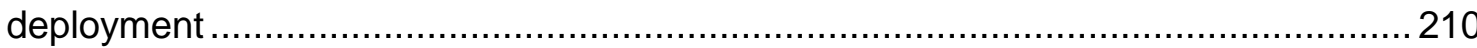

Figure 102. Steinjker deployment with the middleware solution, as depicted in [34]..211

Figure 103. Middleware tests results, as shown in [34] .........................................212

Figure 104. Different areas of knowledge and projects used for the conception of CMA

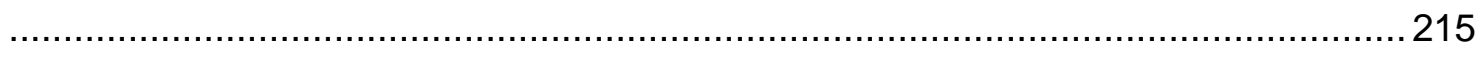

Figure 105. Cash prize certificate for Middlegrid (Spanish) ..................................228

Figure 106. Chronological summarization of all the outputs resulting in the thesis ....229

Figure 107. Maven project access in Eclipse IDE for Ubuntu ....................................233

Figure 108. Creation of a maven project for Eclipse in Ubuntu...............................233

Figure 109. Location of the activemq broker and deploy directories..........................234

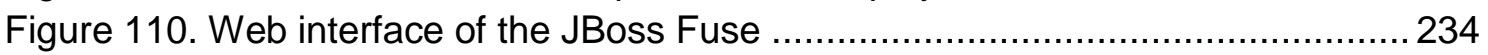

Figure 111. User.properties file in JBoss Fuse .................................................... 235

Figure 112. Software bundles from the middleware ............................................ 236

Figure 113. Virtual Private Network settings for remote connections to the Vitrociset

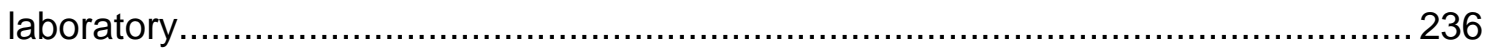

Figure 114. Example of ESB and components deployed in Vitrociset facilities..........2237

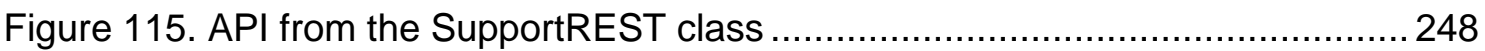

Figure 116. API from the Support class ......................................................... 248

vii 
Figure 117. API of the ContextAwarenessController class ......................................249

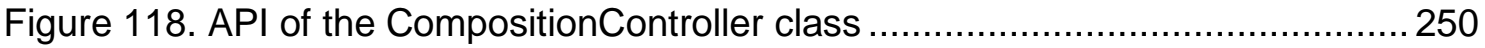

Figure 119. API of the HALController class ....................................................... 250 


\section{List of tables}

Table 1. Main characteristics of the studied middleware solutions .............................68

Table 2. Main characteristics of the studied open source ESB solutions ...................... 84

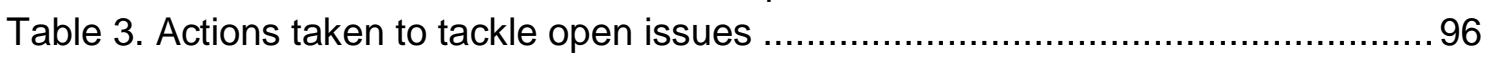

Table 4. Functional and Non-functional requirements for middleware ..........................99

Table 5. Software components defined from the requirements .................................99

Table 6. Actions taken to satisfy non-functional requirements and their justification.. 100

Table 7. Description of Middleware Management Information.................................. 107

Table 8. Description of Central Controller support use case .....................................112

Table 9. Description of Local Controller support use case ..................................... 115

Table 10. Description of Service Discovery use case ............................................... 117

Table 11. Description of Information Security use case ........................................... 119

Table 12. Traceability for the concerns of the stakeholders ................................... 141

Table 13. Viewpoints and their associated views and architecture models................ 147

Table 14. Main concepts for ISO/IEC/IEEE 42010 standardization ........................... 158

Table 15. Device information table filled by e-GOTHAM partners ............................177

Table 16. Support middleware component testing ............................................... 197

Table 17. Device registration testing activities.................................................... 207 


\section{Acknowledgments}

This thesis is the culmination of a collection of works and efforts shared with many people in the Next Generation Networks and Services Group (GRyS) who have been true comrades in arms during the good and the bad times that I have gone through there. It is when trouble and stress happen that the true nature of a person is revealed, and I am grateful to have found workmates that have helped me through thick and thin, regardless of my opinions or comments.

I would like to start by thanking the supervisor of my thesis, Professor José-Fernán Martínez Ortega. He has given me lots of motivation and encouragement during all the stages of my education, from the Bachelor thesis to my doctoral studies.

I would also like to thank Pedro Castillejo for all the support that he has provided me during all these years. It has been a pleasure and a privilege working with him all this time, and it is great being able to count on such a knowledgeable and patient person. I also want to thank David Gómez for his positivity and good spirit. He has been a firm source of tranquillity in moments of utter chaos.

I would also like to thank Alexandra Cuerva, Carlos Estévez, Esther Moreno and José Antonio Sánchez for the times we have spent together, either working, writing papers or just sharing a laugh in the cafeteria of the university. I am sure that whatever is ahead for them in their lives will be great. I also want to mention the strong inspiration that the hard work that my Chinese colleagues Xin Li, Ning Li, and Xin Yuan give me. As for the newest additions to the group, I am sure that Yuwen Chen and Zhaoyu Zhai are on their way to become great researchers.

Thanks to the combined efforts of my supervisor, Professor José-Fernán Martínez Ortega, and Doctor Geir Mathisen I did a three month stay in the Information and Communication Technologies department of SINTEF, a large research institution located in Norway, where I made part of my thesis and learnt a bit about how things are done in that country. I got to meet brilliant researchers with very impressive backgrounds, and I am very grateful for that too.

I want to thank my girlfriend Corianne for her unconditional love during all this time, no matter how harsh circumstances have been for us sometimes. Also, I would like to use these lines to thank my parents for their positivity, regardless of how bad things looked.

Last but not least, I want to mention all my friends and people that are part of my life outside work: my friend Javiii, my university friends César, Félix, Adriana, Álvaro, Esther, Carlos, Diego, Guille, Randa, Fran, my little nieces Alejandra and Olivia, my brother-in-law José Carlos and my sister Mónica.

Thank you all and let us hope we have a bright future. Tomorrow never knows. 


\section{Abstract}

The Smart Grid, conceived as the power grid enhanced with Information and Communication Technologies aimed to optimizing electricity consumption, enabling a bidirectional participation in the energy provided and improving the power grid with features like Demand Response, Demand Side Management or Optimal Power Flow, is becoming one of the most compelling Cyber-Physical Systems that is being currently developed. Its capacity to increase usable energy in a sustainable manner, along with collect more information about its actual usefulness, makes possible the improvement of living standards for many people all around the world in a more transparent and open way. What is more, since it is able to integrate the comparatively small power supplied by the Renewable Energy Sources provided by the end users that participate in the Smart Grid (which become "prosumers", as they both produce and consume electricity), it democratizes access to energy and enables a higher degree of competitiveness between traditional actors in the energy markets and newcomers, thus granting the overall improvement of the services that can be provided by the utility companies.

However, there are still many open issues that must be solved before completely using the Smart Grid to our advantage. Among these open issues, interoperability of its installed devices is a major one. The equipment that is used in deployments of this kind (Advanced Metering Infrastructure, Phasor Measurement Units, Remote Terminal Units, etc.) is manufactured by different companies with different backgrounds and interests. Therefore, the implementation of their products has usually different ways to transmit information or even proprietary solutions with a low degree of compatibility with other pieces of hardware. In a way, the status quo is similar to the situation of computer networks before the first standards were released: $a$ plethora of manufacturers offer their own solutions to provide services and connectivity, but they struggle to work cooperatively with developments of other equipment vendors that may have different perspectives on the technologies that can be used for data transmission.

In addition to that, the services that should be available for either a Smart Grid or one of its smaller scale counterparts (microgrids, nanogrids) are not made clear, neither in terms of what services they should be or where they should be located. While there are some high level functionalities that are usually regarded as almost mandatory (the aforementioned Demand Side Management, device registration, Demand Response, Optimal Power Flow), other more data-centric facilities are often portrayed in a way vaguer manner. Security specifications, the existence of semantic capabilities, how to access the capabilities in a specific deployment or even how the hardware devices become integrated is not described with enough detail. That issue jeopardizes the main purpose of installing and developing components in this area of knowledge, because it makes difficult a further integration of both legacy systems that may have been used by large utility companies for a long time and new developments done by smaller companies that want to play a role in the Smart Grid.

Fortunately, many of these challenges can be solved by implementing a software layer located between the applications that can be included for the benefit of the end users and the xiii 
hardware and network infrastructure installed for package and binary data interchange. This software layer, commonly referred as middleware, has as its main purpose abstracting the heterogeneity and complexity of the underlying distributed hardware components, so that it will offer to the high, more application-based layer a collection of facilities of homogeneous, centralized appearance, usually shaped as an Application Programming Interface that can be accessed by the application developers. Middleware is a very useful software tool for CyberPhysical Systems and distributed solutions because it grants the integration of almost any kind of device, either by adding software components in the device itself or in another part of the system, which must be open enough to have the components installed or have the required computational capabilities to have those components installed.

The main original contribution to knowledge of this doctoral thesis is offering a proposal for a model of a semantic middleware architecture for the Smart Grid, based on software components for distributed solutions. This model is aimed to be used in any kind of deployment related to the Smart Grid, as well as providing a common set of components and interfaces to be observed in future implementations. This architecture has been called Common Middleware Architecture (CMA), as it aims to provide the necessary software components for middleware development under any imaginable use case within this application domain. It has been designed based on the experience accumulated from several research projects where the implementation of a middleware layer was one of the main achievements. CMA has been designed with the main needs of a middleware solution in mind, such as hardware abstraction, context awareness, device registration, interfaces for the upper level, securitization and device integration. While the main domain of CMA is the Smart Grid, and demonstrators based on the Smart Grid have been used to validate it, CMA can also be adapted to other environments.

All in all, the main objective of this thesis is creating a reliable framework for the development of middleware solutions for the Smart Grid, which can be used in other application domains where there are requirements of hardware abstraction and service availability resembling the ones that can be found in this area of knowledge. Another major objective of this thesis is making contributions to the standardization of middleware development for the Smart Grid, so that there will be a specific set of services to be developed in order to comply with the most important functionalities of middleware (hardware abstraction, homogeneous set of services for applications, encasing services based on semantic capabilities and security). These two objectives have been achieved with the contributions done in the study of the State of the Art, the inference of open issues and challenges, the establishment of a list of functional and nonfunctional requirements and the validation of the proposal put forward in this manuscript. The solutions developed can be regarded as the background of the architecture described here, and therefore its performance should be good enough for the functionalities carried out for this kind of software layer, which should be present in any distributed or Cyber-Physical System that uses a collection of deployed pieces of equipment with different capabilities. Besides, since this is a middleware solution solves problems for issues present in other distributed and/or Cyber-Physical Systems (the Internet of Things, underwater robotics) it can be ported to other domains with ease, as services as high level interface access or device registration are used in those situations as well.

xiv 


\section{Resumen}

La Red Eléctrica Inteligente, concebida como el tendido eléctrico mejorado con las Tecnologías de la Información y las Comunicaciones dirigidas a optimizar el consumo de electricidad, permitir una participación bidireccional en la energía suministrada y mejorar la red eléctrica con características como la Respuesta ante la Demanda, Gestión de la Demanda o el Flujo de Potencia Óptimo, se está convirtiendo en uno de los sistemas ciberfísicos más convincentes desarrollado actualmente. Su capacidad para aumentar la energía utilizable de manera sostenible, así como obtener más información sobre su utilidad real, hace posible la mejora del nivel de vida de muchas personas en todo el mundo de una manera más transparente y abierta. Además, al poder integrar la relativamente pequeña potencia suministrada por fuentes de energía renovable aportadas por los usuarios finales que participan en la Red Eléctrica Inteligente (que se convierten en "prosumidores", ya que ambos producen y consumen electricidad), democratiza el acceso a la energía y permite un mayor grado de competitividad entre los actores tradicionales de los mercados energéticos y los recién llegados, garantizando así la mejora general de los servicios que pueden aportar las empresas de utilidades.

Sin embargo, todavía hay muchas cuestiones abiertas que deben ser resueltas antes de utilizar completamente el potencial de la Red Eléctrica Inteligente en nuestro beneficio. Entre estas cuestiones, la interoperabilidad de los dispositivos instalados es una de los principales. El equipo que se utiliza en los despliegues de este tipo (Infraestructura de Medición Avanzada, Unidades de Medida de Fasores, Unidades Terminales Remotas, etc.) es fabricado por diferentes compañías con diferentes conocimientos e intereses. Por tanto, la implementación de sus productos tiene a menudo diferentes formas de transmitir información o incluso soluciones propietarias con un bajo grado de compatibilidad con otros dispositivos. De alguna manera, el statu quo es similar a la situación de las redes de ordenadores antes de que se publicaran los primeros estándares: un conjunto de fabricantes ofrecen sus propias soluciones para proporcionar servicios y conectividad, pero tienen problemas al cooperar con los desarrolladores de otros fabricantes de equipos que pueden tener diferentes perspectivas sobre las tecnologías que se pueden utilizar para la transmisión de datos.

Aparte de eso, los servicios que deberían estar disponibles para una Red Eléctrica Inteligente o una de sus contrapartes de menor escala (microrred, nanorred) no están claros, ni en términos de qué servicios deben ser o dónde deben estar ubicados. Si bien hay algunas funcionalidades de alto nivel que generalmente se consideran casi obligatorias (las anteriormente mencionadas Gestión de Lado de la Demanda, registro de dispositivos, Respuesta a la Demanda, Flujo Óptimo de Potencia), otras facilidades centradas en datos se describen de una manera más vaga. Las especificaciones de seguridad, la existencia de capacidades semánticas, cómo acceder a las capacidades en un despliegue específico o incluso cómo los dispositivos de hardware se integran no se describen con suficiente detalle. Esta cuestión pone en peligro el objetivo principal de instalar y desarrollar componentes en esta área de conocimiento, ya que dificulta una mayor integración de tanto sistemas heredados que pueden haber sido utilizados 
por las grandes empresas de utilidades durante mucho tiempo como nuevos desarrollos realizados por empresas más pequeñas que quieren desempeñar un papel en la Smart Grid.

Afortunadamente, muchos de estos retos pueden resolverse mediante la implementación de una capa de software ubicada entre las aplicaciones que se pueden incluir para el beneficio de los usuarios finales y el hardware y la infraestructura de red instalada para el intercambio de datos binarios y de paquetes. Esta capa de software, comúnmente referida como middleware, tiene como objetivo principal abstraer la heterogeneidad y complejidad de los componentes de hardware distribuidos subyacentes, de manera que ofrecerá a la capa alta y más basada en aplicaciones una colección de facilidades de aspecto homogéneo y centralizado, comúnmente conformada como una Interfaz de Programación de Aplicaciones a la que pueden acceder los desarrolladores de aplicaciones. El middleware es una herramienta de software muy útil para sistemas distribuidos y ciberfísicos porque permite la integración de casi cualquier tipo de dispositivo, ya sea añadiendo componentes de software en el propio dispositivo o en otra parte del sistema, la cual debe ser lo suficientemente abierta para tener los componentes instalados, o bien debe tener las capacidades de computación necesarias para tener esos componentes instalados.

La principal contribución original al conocimiento de esta tesis doctoral es ofrecer una propuesta para un modelo de arquitectura de middleware semántico para la Red Eléctrica Inteligente, basado en componentes software para soluciones distribuidas. Este modelo está destinado a ser utilizado en cualquier tipo de despliegue relacionado con la Red Eléctrica Inteligente, así como a proporcionar un conjunto común de componentes e interfaces a ser tenidos en cuenta en futuras implementaciones. Esta arquitectura se ha denominado Common Middleware Architecture (CMA), ya que tiene como objetivo proporcionar los componentes de software necesarios para el desarrollo de middleware en cualquier caso de uso imaginable dentro de este dominio de aplicación. Se ha diseñado contando con la experiencia acumulada en varios proyectos de investigación en los que la implementación de una capa de middleware fue uno de los principales logros. La CMA ha sido diseñada teniendo en cuenta las principales necesidades de una solución middleware, como abstracción de hardware, conocimiento del contexto, registro de dispositivos, interfaces para el nivel superior, securización e integración de dispositivos. Aunque el dominio principal de la CMA es la Red Eléctrica Inteligente, y se han utilizado demostradores basados en la Red Eléctrica Inteligente para validarla, la CMA también puede adaptarse a otros entornos.

En general, el objetivo general de esta tesis es crear un marco fiable para el desarrollo de soluciones de middleware para la Red Eléctrica Inteligente que pueda ser utilizado en otros dominios de aplicaciones donde existen requisitos de abstracción de hardware y disponibilidad de servicio similares a los que se pueden encontrar en esta área del conocimiento. Otro objetivo principal de esta tesis es contribuir a la estandarización del desarrollo de middleware para la Red Eléctrica Inteligente, de modo que habrá un conjunto específico de servicios desarrollados para poder cumplir con las funcionalidades más importantes del middleware (abstracción del hardware, conjunto homogéneo de servicios para aplicaciones, encapsulación de servicios basados en capacidades semánticas y seguridad). Estos dos objetivos se han logrado con las aportaciones realizadas en el estudio del estado del arte, la inferencia de 
cuestiones y temas abiertos, el establecimiento de una lista de requisitos funcionales y no funcionales y la validación de la propuesta presentada en este manuscrito. Las soluciones desarrolladas pueden ser consideradas como los antecedentes de la arquitectura aquí descrita, por lo que su rendimiento debe ser lo suficientemente bueno para las funcionalidades realizadas para este tipo de capa de software, la cual debería estar presente en cualquier sistema ciberfísico o distribuido que use un grupo de equipos de diferentes capacidades que haya sido desplegado. Además, puesto que se trata de una solución de middleware que resuelve problemas para cuestiones presentes en otros sistemas distribuidos y / o ciberfísicos (Internet de las Cosas, robótica subacuática), puede ser portado a otros dominios con facilidad, ya que servicios como interfaces de acceso a alto nivel o registro de dispositivos también se utilizan en esas situaciones. 
1. Introduction and
objectives 
This chapter offers information about the historical context where the Smart Grid and middleware started off and were developed. In addition to that, a description of the objectives that were established at the start of the thesis is present in this section as well. Finally, the background used for research activities and the outlines of the dissertation have been included as well.

\subsection{Motivation}

Electricity is pivotal in the development of all the goods and services that are manufactured, developed and traded since the Second Industrial Revolution, which happened around more than one hundred years ago [1]. It was during that time when fossil fuels began to be burned in an intensive manner to obtain electricity as an output, rather than using the generated heat to move mechanical parts of steam-powered machinery. While electricity generation was being crafted, the development of infrastructures that made possible its supply to the production centres where it was being used was also required. Later on, as standards of living improved, electricity became a service demanded in dwells and households. Consequently, a power grid was built to transport electricity from its production facilities to the clients and end users. Typically, a power grid will work by transferring high voltage electricity originated in a power plant (usually of an order of magnitude ranging in the hundreds of thousand Volts; High Voltage Direct Current or HVDC is the most common way to transport electricity at this stage [2]), which is better suited for travelling long distances, and it will be progressively converted to lower voltages until it is delivered to its consumption location, where voltage gets scaled down to a range from 125 to 240 Volts, depending on factors as the country or the age of electric infrastructure owned by the end user. Power lines will have different characteristics depending on the voltage they are expected to work with. In this kind of system, there are several actors that, while presenting some differences depending on the country and their legislation, play a role in the development and exploitation of such a system. These actors are:

- Distribution System Operator (DSO): it is responsible for electricity generation in the system. Commonly, DSOs own the power plants required for electricity generation, that may use renewable (hydropower, photovoltaic or thermal solar power, etc.) or fossil fuels (coal, oil, natural gas). Thus, a DSO plays a more important role in electricity generation rather than distribution.

- Transmission System Operator (TSO): it provides the required infrastructure to transfer electricity from the power plant to the consumption points. Power lines used for that purpose are usually property of the TSO.

- Aggregator/Retailer: it is the entity that purchases electricity from the DSO and sells it to the end users. Depending on how "smart" the power grid is, it will either carry out functionalities related to aggregation or it will be solely focused on electricity trade.

- End user/Client: it is the entity that purchases the electricity offered by the former actor and consumes it. As far as the conventional power grid is concerned, this is where the electricity flow ends, as the energy it carries is often transformed into other kinds (luminous, mechanical, thermal, etc.). 
Overall, these actors tend to be the same in most of the world, even though there might be some major differences depending on the countries (in Spain, for example, it is common that the Distribution System Operator or DSO will also play an active role in the commercialization of electricity to end users via second party companies, so the DSO is also present as a retailer). The principles of the currently used power grid are based on the theoretical and practical work performed by researchers such as Nikola Tesla [3] [4] and its implementation and installation makes use of techniques that date back to at least the 1960s [5] [6]. As a direct consequence of the latter fact, the regular power grid has become outdated in many ways, revealing itself incapable of keeping pace with several technological innovations that have taken place during the last decades. To begin with, renewable energies are increasingly used to produce electricity as an alternative to non-renewable, fossil-based energy sources. Despite some issues such as its intermittent availability or aesthetic impact [7] [8], its friendliness towards the environment and availability for comparatively small users forecast a growing utilization of these resources in the immediate and distant future, even in the worst case scenarios that have been devised [9]. Secondly, the availability of Renewable Energy Sources (RESs) for the small users as Distributed Energy Resources (DERs) has the potential of significantly changing the traditional, one-way flow of the electricity that has been traditionally implemented. Last but not least, by means of using means of energy storage (such as home batteries), electric energy does not need to be consumed as it is received and can be turned into other kinds of energy (for example, chemical energy), stored and used during a more suitable time, which may be when it is actually needed (in case of blackouts or power failures) or when trade opportunities appear in the electricity market, if the user has technology capable of interacting with them. Consequently, the end user can be not just a consumer of energy, but an actor capable of producing its own electricity, thus becoming enabled to either use it for themselves or obtaining a profit from its trade; hence the term prosumer, which is heavily linked to the Smart Grid and is referred to an end user capable of producing and consuming energy at the same time (producer and consumer are merged as prosumer).

It is due to these facts that the conventional power grid can be turned into a Smart Grid. The Smart Grid can be defined as the power grid after becoming enhanced with Information and Communication Technology (ICT) with the aim of providing a rationalized usage of energy, more oriented to the supplied resources (thus attempting to make use of what is available at a particular moment) rather than solely taking demand into account (which can be satisfied by adding more power plants and energy resources, but not at a sustainable pace in a finite world). As stated in [10], "Smart Grids increase the connectivity, automation and coordination between suppliers, consumers and network by modernizing grid features like demand side management, generation, real-time pricing, and automated meter activation and reading". Figure 1 shows the changes that are introduced by adding Smart Grid capabilities to the overall system. As it can be seen, while many of the actors and roles participating in the Smart Grid are still the same, there are major changes in the functionalities performed by end users (or more accurately said, prosumers). 


\section{Conventional power grid}
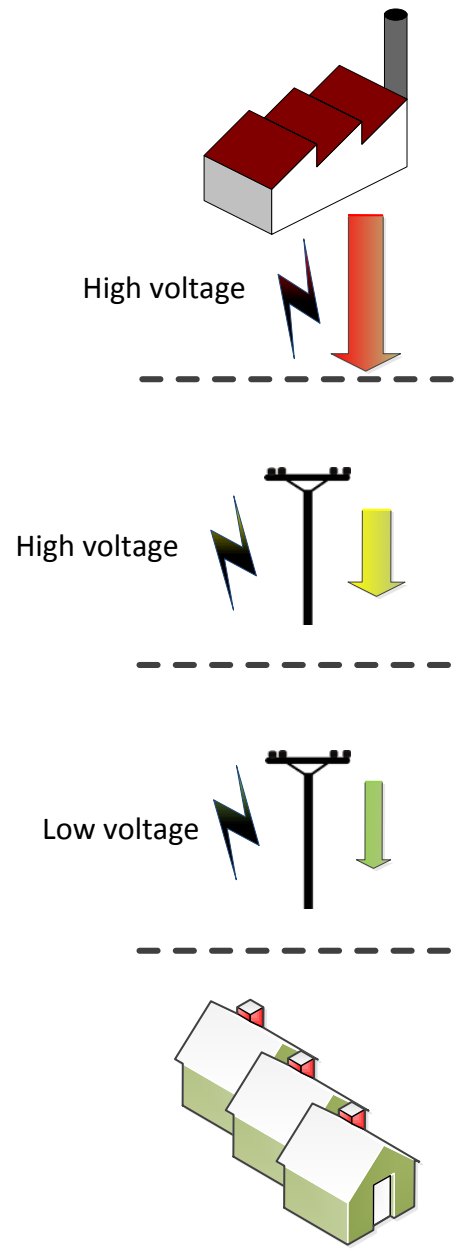

Smart Grid

DSO

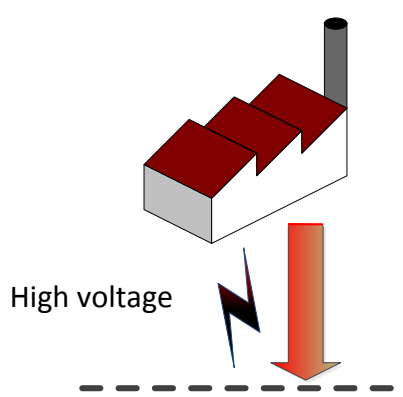

TSO

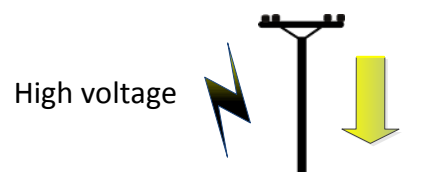

Aggregator/

Retailer

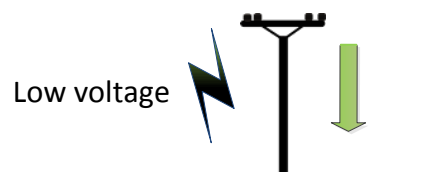

End users/Clients

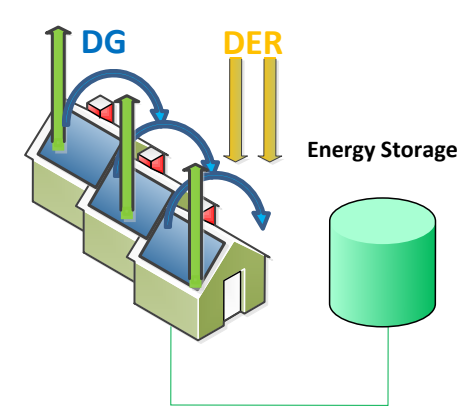

Figure 1. Graphical comparison between the conventional power grid and the Smart Grid, inspired from [11]

The decentralization of electricity production introduces some more significant changes in the whole behaviour of the Smart Grid. As it has already been mentioned, it adds a collection of small energy producers that require that the power flow will become bidirectional, as there is no longer a single major electricity supplier with the energy production centralized in one or a group of facilities supplying the electricity to peripheral consumers. Furthermore, the transition to a supply-based way to consume energy rather than a demand-based one is also encouraged by the adoption of the Smart Grid, where prosumers can trade their electricity surplus, taking into account how much and where it is needed or at what price it can be supplied. Prosumers as a new actor in the way electricity is produced, traded and consumed are a major innovation of the Smart Grid, with a great impact in the whole system due to several reasons:

1. Energy access democratization. Prosumers own means for energy production, which are commonly based on Renewable Energy Sources (RES). Consequently, they are able 
to meet their power demands to an extent, depending on the extension of their available RESs and the energy they consume, and do not depend on DSOs as heavily as regular consumers. In this way, energy becomes available in a way that was not possible before.

2. Energy consumption patterns. Prosumers integrated in the power grid alter power consumption patterns dramatically; depending on the time of the day, month of the year and their consumption history, they will either inject power to the grid or will demand less than a regular consumer. Those two actions will greatly change how energy is consumed in a specific area, regardless of its geographical limits (nanogrid, microgrid or larger).

3. Trade for small users. Prosumers could be willing to trade their produced electricity as if it was any other commodity. Depending on their willingness or knowledge, trading algorithms could be used in the development of business models based on energy storage and delivery according to the demands of other prosumers, regular consumers or even the DSOs.

4. Environment-friendliness. Since the overwhelming majority of the prosumers use to produce electricity infrastructures based on renewable energies, more energy is available in an environment-friendly manner, with all the positive consequences that it carries (lower amount of carbon dioxide released to the atmosphere, less pollution, etc.).

5. Impact in other partners involved in the power grid. The integration of this new actor with the power grid will force a redefinition of the roles of other entities involved in the production, transfer and consumption of electricity, as they will require to adapt their offer to what prosumers can provide (DSOs) or gather information about consumption patterns (aggregators).

Overall, and as stated in [11], prosumers will be fully involved in the economic and technological parts of energy consumption, as well as creating new value for the enhanced power grid. A mathematical analysis that models the potential profitability of their activities has been included in the next chapter. The integration of prosumers in the Smart Grid is one of the benefits of enabling middleware in this application domain, for it will enable the necessary mechanisms for data transmission among the different hardware elements of a deployment where prosumers are contributing to the power flow with their own Renewable Energy Sources.

Figure 2 summarizes that new paradigm in the Smart Grid once prosumers have been fully integrated, along with the possible cooperation between entities that they offer: they are capable of receiving and providing assets (either electricity or information) to the Energy Services Company (ESCO) or to other prosumers, which can be organized as a cluster in a Virtual Power Plant (VPP) style. At the same time, DSOs can trade electricity with prosumers (either as a way to have energy or have it as a complementary resource) and with the aggregator in order to offer its resources and information. All these relations are included in the generation of electricity rather than its mere consumption (as regular end users do) and its transmission by means of the infrastructure owned by the Transmission System Operator. 


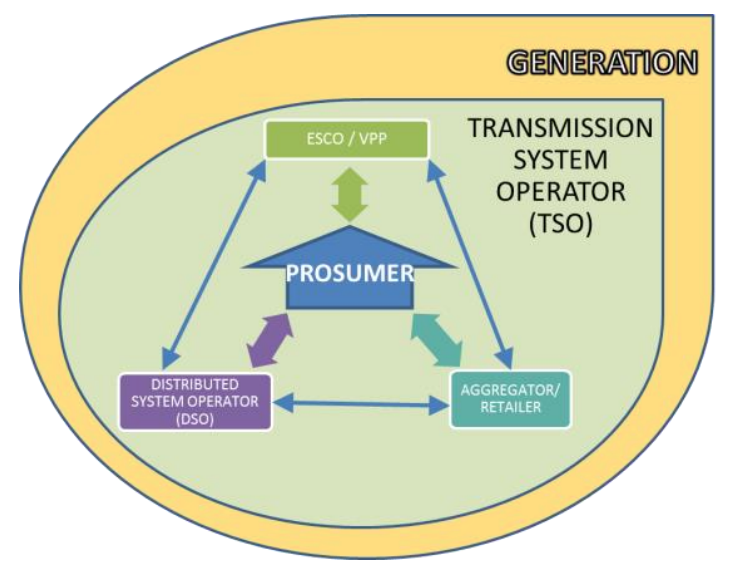

Figure 2. Energy market value chain focused on the prosumer, as represented in [11]

The integration of prosumers and their small scale facilities used to generate electricity is a critical task for the Smart Grid, especially if all the changes that have to be made to guarantee it are taken into account. These latter can be defined by two different categories:

1. Technical: there are several activities that must be undertaken to offer integration at several layers, such as the physical one (providing all the necessary connections to the power grid and the information transferred from one piece of equipment to the other at the bit level), the network (so as to interchange packages between the existing prosumers, which will effectively behave as a distributed system) and the data-based ones (in order to exchange information about the services and features of the system that will be mutually understood by all the ends involved in the communication).

2. Legislative: prosumers are different from the entities traditionally involved in the generation of energy in the sense that their infrastructure is small-sized and is scattered in a certain area rather than centralized in a position. That would require the rearrangement of the legislative framework used to include the prosumers into the energy markets. For example, the European Energy Exchange includes, among other preconditions for admission, liable equity of at least 50,000 $€$ [12]. This and other requirements might be challenging to be met by individual prosumers. Nevertheless, they could be offered an alternative, such as federating themselves into a larger cluster of energy providers making use of an aggregator.

Information and Communication Technologies must also be described. The Smart Grid makes use of very different appliances with a huge disparity regarding their features, manufacturers or data formats used to transfer data throughout the system. The lack of standardized procedures specific to the software in the Smart Grid or software solutions to interoperate these devices at the data level is one of the major challenges that are faced in this application domain, as interoperability of the devices used cannot be guaranteed and there is a chance for them to become isolated from the other devices used for data transmissions. Fortunately, this issue can be solved by means of middleware. Whereas middleware is of more recent conception than the power grid, it was firstly referenced in a NATO document that dates back to 1968 [13], so it is still almost 50 years old. Popularity of middleware grew at a slowly pace during a time where distributed systems were underdeveloped and mainframes were the most common location of most of the computational and logical resources in the firstly available 
computer networks (thus having a central hardware component providing most of the services, instead of a collection of computers). By the 1980s, though, middleware was being increasingly used as a way to interconnect legacy systems with new ones. As it will be described in subsequent chapters of this thesis, this functionality is still satisfied by middleware for distributed systems. Its usability and utility became greatly expanded during the late 1990s and early 2000s with the uprising of Internet usage, the first developments dealing with the Internet of Things (IOT) and mobile computing, as those are systems that are by definition distributed and heterogeneous [14] [15]. It was during that time that middleware for distributed systems was definitely established as the software entity capable of abstracting heterogeneity from an underlying collection of hardware devices in order to offer a set of homogenous, centralized-looking facilities (typically, an Application Programming Interface or API) to the application layer [16] [17]. Further developments, such as Cloud Computing, have made middleware a software entity of critical importance in the development of distributed systems [18] [19]. Figure 2 illustrates the typical location of middleware in the latter; due to this and how it is accessed by the application layer it effectively withholds the different (and quite often, opposite) hardware components present in a deployment, as well as their local firmware and operating systems. Even though these systems usually interconnect with each other by interchanging messages at the network layer, they still are lacking data formats that can be apprehended by a human being, as middleware does when sending information upwards.

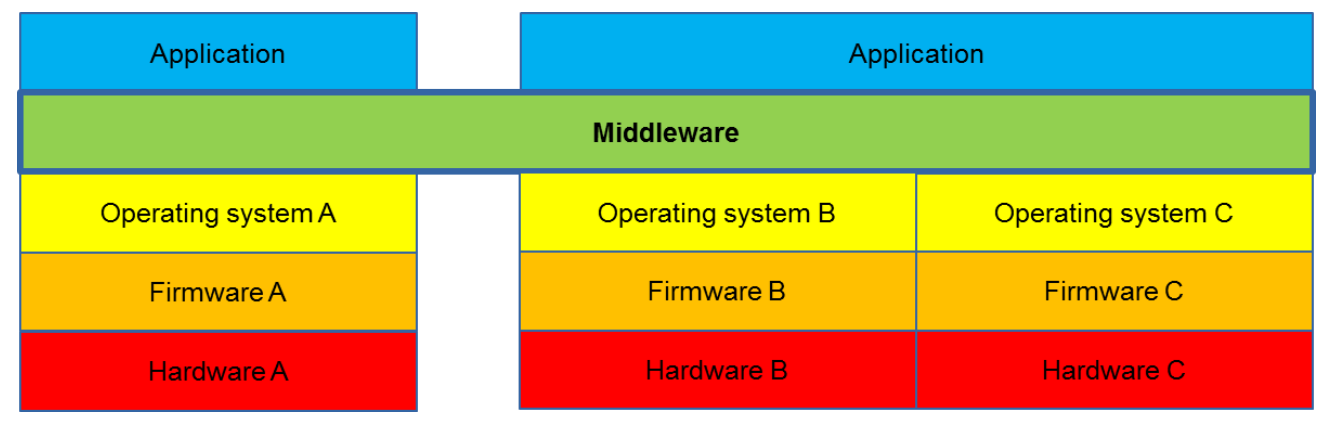

Figure 3. Middleware location in a distributed system

Since the Smart Grid can be regarded as a distributed system that must cope with most of its defined issues, the importance of having a middleware architecture that a) abstracts the heterogeneity of the appliances present in the Smart Grid, b) provides a collection of services that can be used by every entity making use of them, c) offers a way to access those services from the application layer in a homogeneous way and d) allows interoperability and scalability for all the parties involved in a deployment, becomes capital for the reasonable development and deployment of hardware, services and applications.

\subsection{Objectives}

There are several objectives that have been set for this thesis; all of them are related to a great extent to middleware as a software component of the Smart Grid. They have been listed as follows: 
1. Providing a study on the state of the art with regards to existing middleware solutions for the Smart Grid where their strengths and weaknesses are acknowledged and assessed. It will be made clear during this thesis that the existence of a semantic middleware in this application domain makes possible not only the abstraction of hardware heterogeneity (thus easing the integration of Renewable Energy Sources referred to as RESs- in the Smart Grid) but also adding other services (semantic capabilities, security, context awareness, service composition) that result in the improvement of the information and applications that can be included at higher levels. However, an evaluation of the existing middleware solutions for the Smart Grid must be carried out in order to know how optimal the current solutions are. This step is required so as to know how the current status quo with regards to the scope of the thesis is looking like, and contributions can be focused in the areas where they are most needed, which have been identified as open issues to be dealt with.

2. Contributing to the standardization of design and implementation of semantic middleware architectures for the Smart Grid. A proposal for a standard in semantic middleware architectures for the Smart Grid is put forward in this thesis; it has been called Common Middleware Architecture (CMA). The contributions that are made by CMA are mostly focused on a) the establishment of a collection of functional and non-functional requirements that can be regarded as mandatory for a good design of a semantic middleware architecture for the Smart Grid, b) listing a set of software components that must be present in any semantic middleware implementation that is planned to be used in this application domain, c) how these components must interact with each other in order to guarantee the expected services that a middleware for the Smart grid should provide and d) the steps to be carried out during implementation activities where software components are codified. In a more detailed way, contributions have been made in order to establish a collection of characteristics, software modules, interfaces and technologies that can be applied for efficient middleware implementation activities. These contributions are based on the developed middleware works that have been made in different European projects. While these works have been done following the waterfall software development cycle (analysis, design, implementation, testing and maintenance), it has been combined with incremental prototyping for the development works that have been carried out in the middleware architecture implementation. Although CMA could be used as a standard for the design and implementation of middleware architectures for the Smart Grid, its contributions can also be incorporated to any other standard that resembles the functionalities that have been put forward here and enjoys a higher level of popularity. Furthermore, the addition of a software layer that is performing several complex actions when dealing with the data requested that has to be transferred among the different components of a system could suppose that the pieces of equipment where it is installed would be hindered by its inclusion and demand of computational resources. Nevertheless, performance results show that including middleware in architectures providing service registration or composition does not affect the performance of a deployment, with the added befits of including services that can be utilized by an end user or a prosumer. 
3. Proving that a semantic middleware architecture is a key element to create business models where new actors (especially prosumers) can join a new scenario where energy access and trade are democratized and more distributed than before. Since middleware is expected to be a major agent to use in order to integrate distributed, small-scale RESs, it must be proved that its inclusions adds to the trend of including prosumers in this application domain that will make energy access an easier procedure that will enhance energy availability and competitiveness in the energy markets.

This thesis describes the latest progress made regarding middleware architecture designs that become implemented as middleware solutions for the Smart Grid. Again, it has to be noted that from the software engineering point of view, the Smart Grid can be regarded as a distributed system that will be enhanced by the usage of middleware, so it is very convenient to have an implementation like this. It is due to those presented progress works that a proposal for an intermediation architecture for the Smart Grid application domain can be formulated with a high level of detail. Taking software development into account, along with the environments where the resulting work is deployed, the following core procedures were used for the implementation:

1. Describing the actions used to formalize, design, implement and validate a middleware architecture that has been used with success in a Smart Grid-like development. This is an area of knowledge where inclusion of ICTs (let alone middleware) is often implemented in a very poor way, or ignored altogether. Consequently, advantages that can be offered by ICT (a variety of services, applications for end users, hardware seamless integration, etc.) are not fully developed for power grids. The work that is going to be presented here shows that it is not only feasible, but also desirable, including a middleware layer within a Smart Grid, as it offers several facilities that greatly improve its usability. To name but a few, heterogeneity abstraction, interoperability among pieces of equipment, software and hardware scalability, security or context awareness can all be offered by middleware. In addition to that, Smart Grid-specific functionalities such as demand Response or Demand Side Management can be provided in an easier, more efficient way.

2. Describing the common combined features with regards to the efforts done in this area of industry, so that the model previous referred to as Common Middleware Architecture (CMA) can be put forward as a way to offer fixed interfaces, services and data units to be implemented. This proposal describes a) the software modules that can be regarded as mandatory for a functional middleware architecture, b) where those modules are located, c) how they should be interfaced and d) how they should be implemented into a middleware solution. In addition to that, the model presented here might be usable for some other application domains with technical similarities, such as the Internet of Things (IoT), and generally speaking, any kind of Cyber-Physical System that implies several pieces of heterogeneous hardware cooperating with each other by sharing information. In this sense, the ideas and principles described in this thesis have been ported to another European project named SWARMs (Smart and Networking UnderWAter Robots in Cooperation Meshes, [20]) that has similar challenges regarding interoperability of Autonomous Underwater Vehicles (AUVs). 
Figure 4 offers an illustrated example of this idea for an loT-based development: despite having a small collection of features specific for each of the environments where middleware is deployed (such as the loT), a significant amount of them is likely to keep reappearing. It is only natural for this to happen, as there are a group of functionalities that must be implemented on a constant basis.

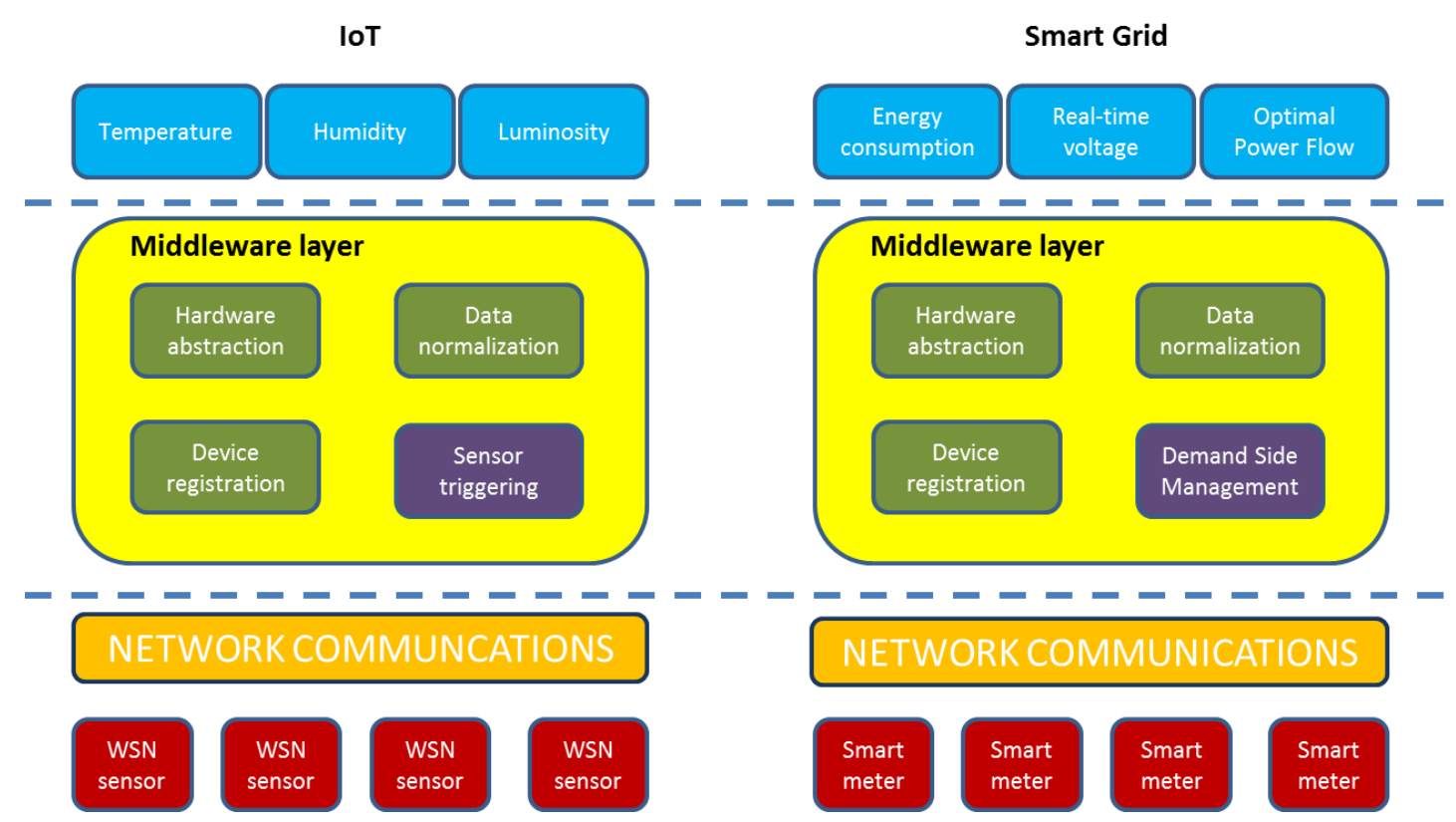

Figure 4. Comparison between common elements in the loT and the Smart Grid

As a starting ground to fulfil these goals, there are several activities that have been conducted in this thesis to have an accurate grasp of the current state of the art in several characteristics where middleware architectures are implemented:

1. The state of the art in middleware solutions for the Smart Grid has been studied and a taxonomy has been applied to classify the possible middleware solutions that can be found as a way to locate the main topic of the thesis (that is, semantic middleware architectures for the Smart Grid) within the possible software developments related to middleware and distributed systems.

2. A state of the art regarding the tools that have been used for the middleware implementation works used to validate the proposal for a Common Middleware Architecture. Since the most prominent of them is the open source Enterprise Service Bus architecture that was used in the validation scenarios, the most profound study for the software tools has been carried out here.

3. Last but not least, the potential for business models in middleware architectures has been studied as well. This is a matter of major importance because the commercial point of view is a pivotal element in the diffusion and acknowledgment of the proposal for a Common Middleware Architecture. Currently, business models for middleware exploitation involve a reduced set of companies with proprietary solutions that extend the closeness of their development works to the systems where they are used. Additionally (or sometimes as a consequence), middleware is often regarded as a nuisance rather than an opportunity to provide profitable 
services. Effective exploitation of middleware under different perspectives so that it will be a decisive agent for business models where prosumers can play a prominent role is described as part of the thesis.

The most prominent pieces of information described have been presented to the research community in varied forms, such as contributions for European research projects, scientific papers or software implementations, as they will be shown in a more extensive manner in the following sections.

\subsection{Thesis framework and background}

The concepts displayed are done according to two major parameters that must be taken into account: on the one hand, the location, from a software and layered architecture point of view, of the research that has been done during the period of time required to complete the assignments related to middleware. On the other hand, the experience acquired in research European projects from different call (ITEA-3, ARTEMIS, FP7, ECSEL).

1. If the different layers that have been previously described are taken into account, the research activities will be located in the middleware layer and slightly extended beyond the boundaries of this layer both upwards (to the application layer) and downwards (to the network layer interconnecting the hardware components). The reason to include these parts is due to the fact that interfacing the middleware layer, along with the technologies used to develop those interfaces, has to be taken into account when designing the different software modules to be programmed in the middleware solution. In a more accurate manner, the background of the area of the research done is in Figure 5.

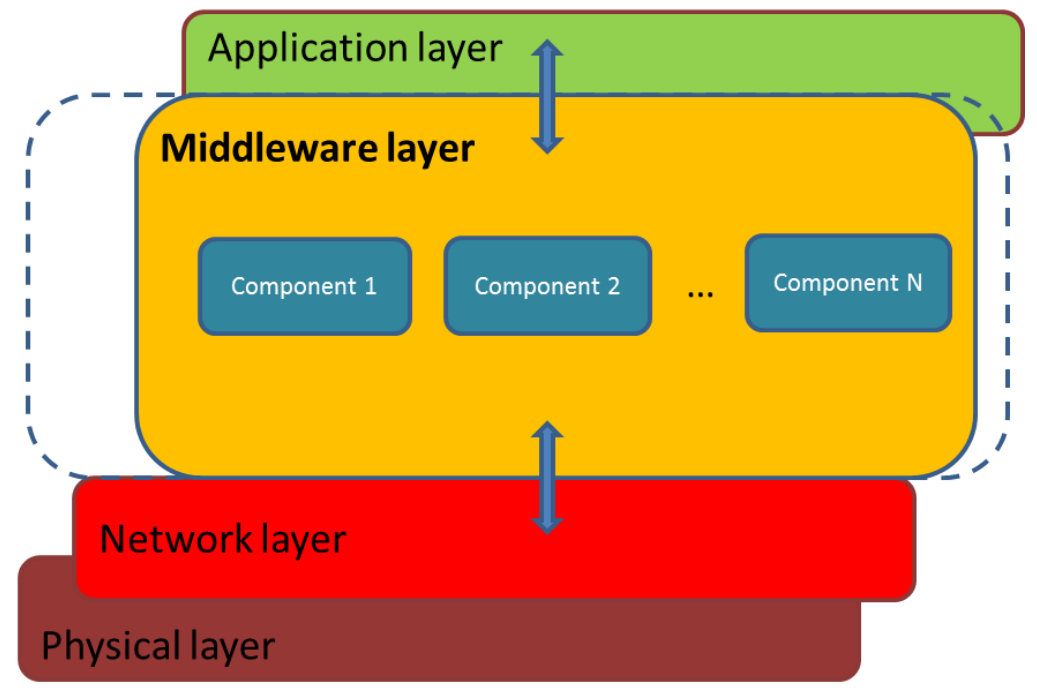

Figure 5. Area of interest of the thesis

2. At the same time, the implementation works done on this thesis have been done within the framework of European research projects. This is a pivotal feature of the presented research, as it has been validated by means of the scenarios that have been used in those 
projects. The most prominent contributions have been done in the ARTEMIS-funded eGOTHAM project (Sustainable-Smart Grid Open System for the Aggregated Control, Monitoring and Management of Energy [21]) and in the FP7-funded I3RES project (ICTbased Intelligent management of Integrated RES for the smart grid optimal operation [22]), which have obtained overall satisfactory results with acknowledged positive contributions regarding research and development in many different areas, including middleware solutions. Some of the ideas of middleware for distributed systems (hardware abstraction, information distribution, generic API for the application layer) were also implemented in the ITEA-3 project called LifeWear (LifeWear: Mobilized Lifestyle With Wearables [23]). Logos of these projects can be seen in Figure 6. In addition to that, contributions regarding middleware have also been done in ITEA-3 Web of Objects project [24] and in the currently undergoing ECSEL SWARMs project (Smart and Networking UnderWAter Robots in Cooperation Meshes [20]). e-GOTHAM and I3RES are projects strongly focused on the Smart Grid, whereas LifeWear and WoO deal with the Internet of Things and SWARMs with underwater robotics.

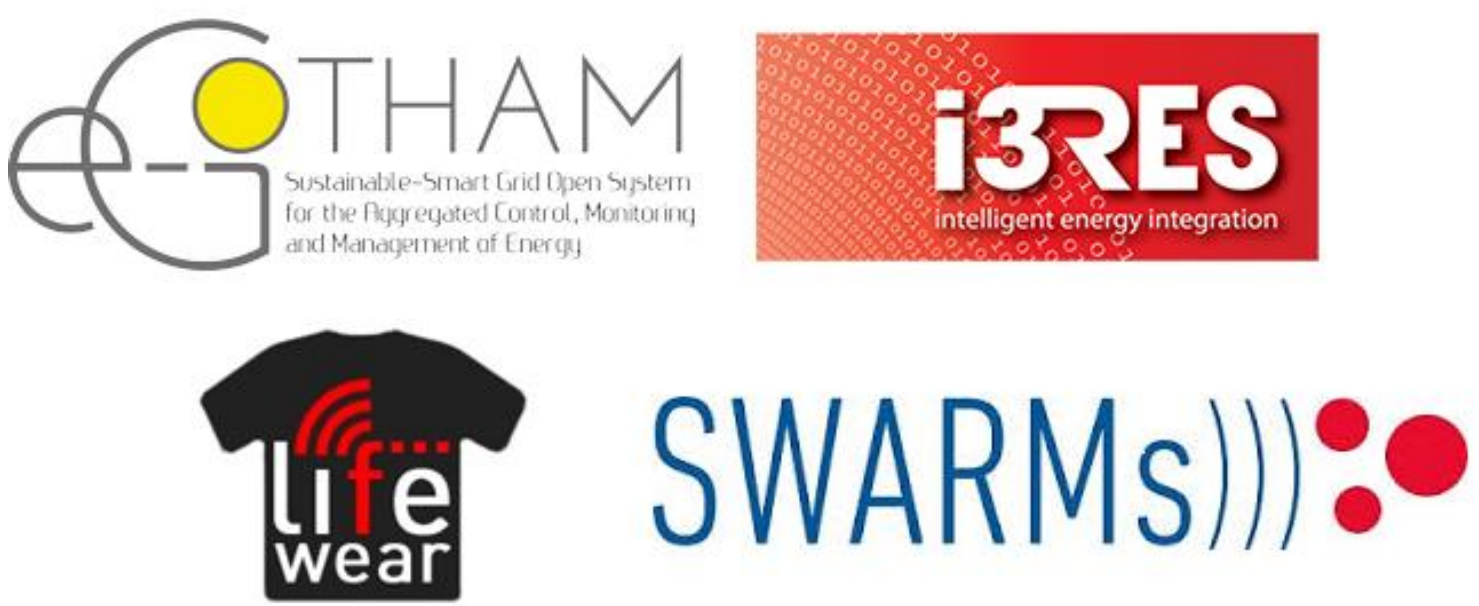

Figure 6. Project logos where middleware-related research activities have been carried out

The experience acquired with these European projects also provides a good starting point for future works that will be done regarding middleware architectures in the future.

\subsection{Dissertation outline}

This thesis has been structured in six different sections, each of them dealing with different characteristics:

1. Chapter one introduces the most high-level features of the thesis, such as the background where it is developed, the main issues that are tackled and a first approach to the development works that have been carried out.

2. Chapter two displays a study on the state of the art done on the major features that imply the research works done, that is to say, middleware architectures for the Smart Grid, tools 
for their implementation and business models for their exploitation. Open issues and challenges to be tackled are inferred as a result.

3. Chapter three includes the dissertation and a complete description about the main contributions done by this thesis: an outline of the Common Middleware Architecture for the Smart Grid is put forward, the lower and upper layer technologies used so that hardware and software components will be interfaced with ease, the description of the features that are used for implementation, and the functional and non-functional requirements that resulted in this proposal.

4. Chapter four deals with the validation scenarios used to prove that the proposal is capable of, showing good results in terms of performance both in the intermediate efforts and the final ones.

5. Chapter five describes the conclusions reached with the works presented in this thesis and puts forward some future works that can be carried out in the future. In addition to that, it shows the publications done in indexed journals (using the Journal Citation Report, JCR, as the chosen index), international conferences and other dissemination works done. Awards received are also mentioned.

6. Chapter six shows a list of all the references that have been used to complete this thesis.

7. Two appendixes have been added afterwards; one is used to offer a short description of some of the implementation works that have been carried out and the next one shows part of the API that was generated during the e-GOTAHM project, as it is directly related to the capabilities expected from CMA.

8. A table of acronyms closes the manuscript. 
2. State of the art 


\subsection{Introduction}

The main motivation for having a state of the art is that it is mandatory to have a good grasp on the status of the technologies and solutions that are being studied, as it can be considered as the starting ground for a researcher to become aware of the open issues and challenges that can be found in the corresponding application domain. Taking into account the existing middleware architectures for the Smart Grid, an accurate idea can be obtained regarding the available solutions in terms of research and marketable solutions. Middleware architectures for the Smart Grid usually involve their deployment in pieces of equipment of different features and capabilities, along with the set of services that can be offered to the prototype, demonstrator or permanent location where the Smart Grid is located. It is likely that the middleware will be added to a specific smaller structure, called microgrid, which basically represents a lower-scale Smart Grid (often covering a small, yet still sizable area, such as an industrial complex or a village [25]) that can be connected or disconnected to another larger entity (thus behaving in an island mode if required).

Other major aspect to be considered is the kind of software architecture that is used in the distributed model. Taking into account the existing solutions, one of the most suitable ones to be used is an Enterprise Service Bus or ESB. An ESB can be regarded as a model for a software architecture capable of transmitting messages throughout the entities connected to the bus used for data interchange, or as mentioned in [26] "a service computing platform based on event driven mechanism. Its task is to offer an integration management platform to the Service Providers (SP) and the Service Consumers (SC), which decreases the coupling degree of SP and $S C$ and eliminates the mess relationship between SP and SC". Considering that an ESB is mainly used as a software abstraction layer that withholds the differences among software applications, it can be easily used as a way to locate all the middleware services and components. While any other software architecture capable of embedding software components is still suitable, there are several more reasons to have come to the conclusion that an ESB is one of the best solutions for middleware implementation. The most prominent ones are as follows:

1. Service storage: when an Enterprise Service Bus is installed and run, locations are created as a way to store in a non-volatile way the different software packages that are created. Those software packages (which are often referred to in ESB terminology as bundles) can afterwards be transferred to another machine -which might even be of the same family of ESBs solutions but not the specific same one-, thus enabling system interoperability (since the software packages can be used regardless of the underlying hardware, as long as it is capable of having the ESB working without performance issues). It must be noted that a system that uses an Enterprise Service Bus will therefore be capable of containing a certain amount of services, so it will be used as more than a mere mean to transfer messages among the devices participating in a distributed system.

2. Design: an Enterprise Service Bus has been conceived as a software system that justifies its usage by allowing interoperability among different software-based 
applications that can be programmed in different languages [27]. This can be proven by the fact that ESBs have been ported to a variety of systems with different applications, such as healthcare [28] or C4I (Command, Control, Communications, Computers, and Intelligence) systems [29].

3. Middleware concept: middleware architectures' most prominent functionality (device heterogeneity abstraction) matches very accurately the purpose of having a software tool used to contain all the services that can be collected from the system, regardless of the hardware that is used to obtain them. When installed, ESBs will usually be turned into the embodiment of the middleware that has been designed for the system.

4. Distribution: ESBs make use of a bus to transfer information from one entity to the other. Consequently, there are no centralized locations that contain most of the information when an ESB solution is deployed in a system. This has several advantages, such as not having critical points that would leave the system seriously damaged in terms of performance whenever there is a flaw in the software or hardware use to run it in a specific location, or easing the addition of new peers in an already existing system, such as prosumers equipped with technology focused on obtaining electricity in a small scale (Distributed Energy Resources or DERs).

Overall, an Enterprise Service Bus solution will be therefore used as an intermediation level between applications (that in the scope of this thesis will be based on what can be obtained from the Smart Grid, like Demand Response, Demand Side Management, Optimal Power Flow, etc.) and information resources, such as pieces of information or databases. The justification of this statement will be shown during this chapter of the thesis.

Last but not least, it must also be taken into account that middleware architectures can be used as a way to either support or provide business models that will result in the profitable exploitation of either software solutions or devices that encased middleware. It has been stated that sometimes middleware is often deemed as a lesser evil that has to be dealt with [30], yet the potential for services and applications that middleware can offer in different application domains it is usually not acknowledged. This thesis proves that middleware can be used as a backbone for business related to the Smart Grid, even though distributed and CyberPhysical Systems will largely benefit from the implementation of a middleware layer that enhances the usability of most of the entities participating in a system of those features (the Internet of Things, Autonomous Underwater Vehicles, etc.).

\subsection{State of the art in middleware architectures for the Smart Grid}

When surveying middleware architectures for the Smart Grid, it has to be taken into account that they share a significant amount of features with the middleware architectures for other distributed or Cyber Physical Systems, such as the fact that they have software components often included in hardware devices working in a cooperative manner, or how they often use messages to transmit information from one element of a deployment to another different one. 
Therefore, there are several features that can be ported to other application domains as well. On the other hand, the variety on different middleware solutions applied to the area of knowledge of the Smart Grid is wide, with different backgrounds and legacy works that have been included, so there are several criteria that have to be taken into account to grasp an accurate idea of how the main challenges addressed by middleware are solved in each of the proposals. Consequently, in order to classify the different middleware proposals that have been used for distributed systems, a hierarchy or a way to identify common features among will have to be established, as the scope of this manuscript attempts to cover different proposals of differing areas of knowledge. Therefore, some characteristics have been established as a way to organize the knowledge that has been included. Although sometimes proposals will be sharing features from each of the features used in the same context, in the end there will be a more prominent one characterizing the proposal. These features are:

1. Service availability. This is used to describe how there are some distributed systems that rely on middleware just as a way to abstract hardware heterogeneity when compared to other proposals that define a more complete set of functionalities for middleware (high level interfaces, inner services, etc.). From a layered software point of view, this feature defines how "thick" a middleware proposal is, depending on the number of services that have been implemented.

2. Computational capabilities of deployed hardware. The hardware capabilities of the systems running middleware must be taken into account when the solutions are being evaluated, as they will have to work with a higher level of optimisation if they are installed in low capability devices that will impose strict non-functional requirements for its usage. It can often happen that middleware, being a software development capable of abstracting hardware features, will be deployed in devices of heterogeneous software capabilities. This particular case will also be taken into account, as using one kind of device over another one should be dictated by the actual needs of the middleware architecture.

3. Message coupling level. Depending on the specific needs of the platform where middleware solutions are deployed, it could happen that different degrees of message coupling are required. When describing message coupling, it must be understood as the amount of time that typically takes place when sending a message and receiving it at the other end of the communication. Requiring a longer time to do the transmissions does not necessarily imply that the middleware solution is worse, as it could happen that it has been designed with a high decoupling level.

4. Middleware distribution. While middleware is usually conceived for its usage in distributed systems, there are different degrees of distribution that imply the existence (or absence) of centres of information in the system. The level of relevance when they are present will be considered by means of this feature.

Overall, these features will be used as the backbone of the classification used to characterize the most prominent middleware proposals for the Smart Grid that have been researched. Each of them can be conceived as an axis that will showcase "minimum" and "maximum" values that, depending on the performance and the overall features of the proposal, will result more or less optimized for the usual functionalities expected from middleware. Thus, the chosen criterion will be further divided in intermediate levels that define their partial status and are 
encased within two values that represent the extreme, end values. Another way to conceive this is as a matrix where all the features will be categorized as a collection of rows and each of the intermediate states of the features will be located as part of the columns of the matrix. Both depictions will be further described in the next subsection.

\subsubsection{Service availability}

The quantity and extension of the services that have been included in the architecture will determine the capabilities of the middleware designed and the level of responsibilities assigned to it. It is not uncommon that a distributed system using low capability devices as a way to extract information will rely on services located in the middleware to perform operations that would otherwise be made at the applications or in the hardware components. On the contrary, sometimes most of the services will be offered outside the middleware and it will be deployed just for message interchanges or as an access layer for other parts of the deployed system. Four different levels of service availability have been defined to be included in the developed middleware taxonomy:

- Abstraction middleware. This kind of middleware offers the required set of operations to perform hardware abstraction, so that all the data will be presented to the applicationbased, upper levels in a homogenous-looking way that will make it easy to be utilized by end users and application developers.

- Intermediation middleware. Aside from hardware abstraction, this type of middleware offers an additional sublayer used to provide access points to the middleware for the applications that are located right above it. This is done so due to the fact that the applications may be too light or have too few software resources to run several procedures related to special characteristics (machine learning, big data) that will require them to locate part of their functionalities in the underlying middleware.

- Message-Oriented Middleware (MOM). In addition to the former capabilities, these middleware solutions are capable of interchanging messages with relevant content regarding the information that is transferred throughout the system, thus adding some knowledge to it. The main concept behind MOM is that messages will be interchanged among the participants in the distributed system, regardless of their location or the application iteration that are running in a particular machine [31].

- Middleware architecture. This middleware solution is characterized by offering a wide set of services that are of common usability for the system, ranging from security to semantic capabilities. It is the most complex and complete possibility available according to the studied middleware designs and implementations, and relies on a structure to encase the services and features of a software-based system. One example of this concept is Enterprise Service Bus architectures [32].

According to the description previously offered, the axial representation of this feature is as shown in Figure 7. Note that the subjacent feature that establishes the existing levels is the service availability in the middleware proposal. 


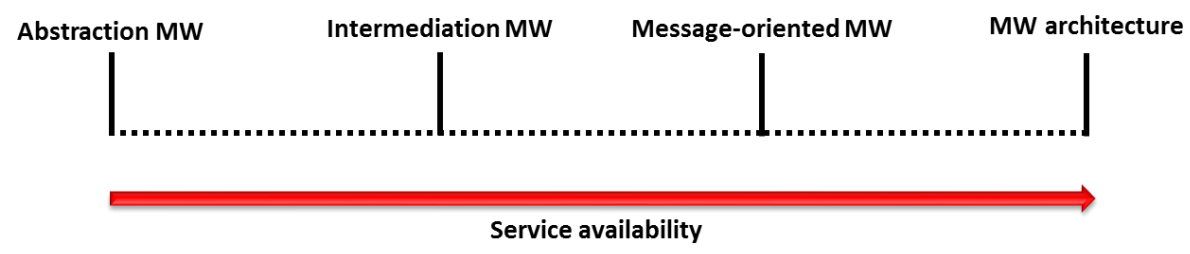

Figure 7. Levels of service availability for middleware

\subsubsection{Computational capabilities of deployment hardware}

Even though hardware will be an underlying element that middleware will abstract in order to withhold its complexity for end users and clients alike instead of part of the middleware itself, it is something that must be taken into account when designing it, for it will determine the kind of services that can be run in the system. When all is said and done, computational capabilities must be born in mind when designing the complexity of the services to be used, as the processing power provided by the hardware will determine if a service can be executed locally, in a distributed manner, or cannot be offered to higher levels at all due to its excessive workload demand. The levels that have been defined for computational capabilities are:

- End user domain devices. These are pieces of hardware that are usually included in the Smart Grid as equipment mounted in an end user's dwell or facility. The most common ones are Advanced Metering Infrastructure (AMI), which sometimes can be used and deemed as if it was part of an Internet of Things development [33] or home batteries for energy storage, which are becoming increasingly popular because of the energy they can provide when buying it from the electricity markets at a particular moment is disadvantageous [34]. The capability of these end user devices to have software components installed in them is usually acceptable, but issues related to Intellectual Property Rights (IPRs) or security may happen when proprietary solutions are used.

- Aggregator domain devices. These are the devices that will be used by the aggregator/retailer present in a deployment to, depending on how smart the power grid is, either trade with electricity among different clusters of users (in case the aggregator has the capabilities to do so) or just offer it to be sold to the end users. Commonly, these devices will imply some hardware with a database in them to store information about end user profiles or energy scheduling algorithms used to determine when energy should be purchased [35].

- TSO/DSO domain devices. These are devices that are used in a Smart Grid, but are not accessed by end users or the aggregator, as they are usually owned by the DSO or the TSO; if something, they will be accessed by the technicians and engineers participating in their deployment. Two examples of this kind of equipment are Remote Terminal Units (RTUs), used for functionalities such as executing demand response programs between the end users and the DSO [36], and Phasor Measurement Units (PMUs) focused on synchronizing the measurements on an electrical grid for monitoring and control purposes through the 
power grid [37]. As it happened in previous cases, issues related with Intellectual Property rights are prone to happen as well.

- Power plant domain. This term encases the different hardware devices used in the power plant management, which may or may not have middleware software components installed in them, depending on how services have been distributed in all the other hardware locations. The pieces of equipment present here usually require large computational capabilities or generate large amounts of data for power grid management (big data, [38]) or execute complex algorithms for overall knowledge inference (machine learning, [39]).

Considering the description of the different available levels shown before, Figure 8 shows them in a more graphical manner.

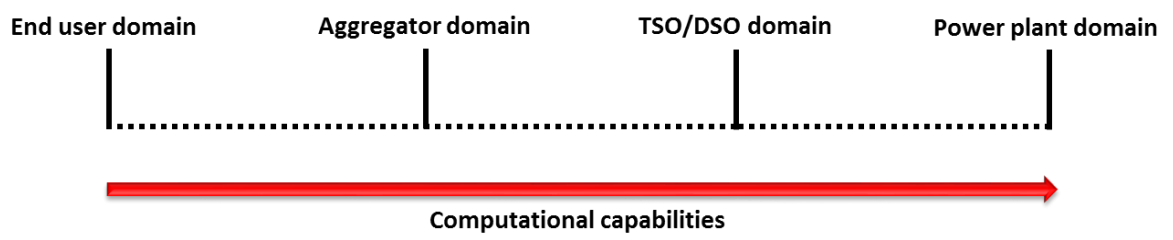

Figure 8. Levels of computational capabilities for middleware

\subsubsection{Coupling level}

This feature must be understood in the context of this manuscript as the level of interdependence between the element that produces data when a communication is established and the element that, at the other end, consumes the data that has been served to the system for any kind of purpose. Coupling level is closely linked to the need of providing the data as soon as it has been produced in the system. The four levels that have been defined for this feature are as follows:

- Real-time. In this case, data are served to the consumer as immediately as possible, to the point that it is virtually assumed that no delays are acceptable. Real-time systems are often prone to severe constrains in the requirements that are regarded as such [40].

- Client/Server. In this case, the system follows a pattern where data are located in one end of the communication (the server) and are retrieved by another end making requests to the server (the client) in the typical fashion used in distributed systems [41]. This model used for interchanging information is the one used in widespread systems such as the Internet and is followed by many middleware proposals as well.

- Polling. Under this paradigm, data are provided to a storage location where it is kept until it is retrieved by the data client that is expected to consume it [42]. As it can be inferred, the time required for the user to retrieve the data is less important, as long as the information is still available.

- Publish/Subscribe. Under this paradigm, several entities subscribe to a collection of services that can be offered by the system, so that whenever data is available for those 
services they will be retrieved by the subscribed elements. The main difference between polling and publish/subscribe is that when an entity subscribes to a service, it will receive the data without any further action, whereas under a polling paradigm the entity that needs the data will have to actively demand it to the entity that stores it.

As it happened with the other two criteria, there is a progression between the lowest message coupling level and the highest one, as shown in Figure 9.

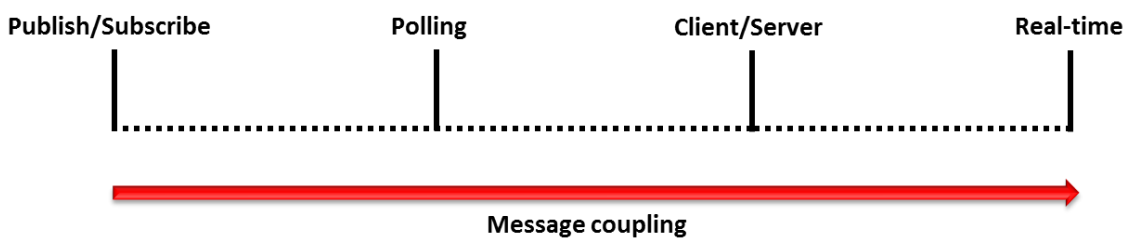

Figure 9. Levels of message coupling

\subsubsection{Middleware distribution}

This feature assesses the level of distribution that has been designed for each of the solutions. Four different levels have been defined by bearing in mind how many devices will have significant parts of the middleware installed:

- Fully centralized. The middleware solution has been installed in a single device (which implies that it will be powerful enough to run it). Although there might be other devices connected to the one with the middleware installed, the solution is still installed in a single one.

- Mostly centralized. The middleware has been distributed among several devices, but there is still a more prominent centre where most of the services or the system intelligence runs.

- Mostly decentralized. Most of the logic and the prominent features are located on the periphery of the system, despite the existence of a central node used to transfer information from one part of the middleware solution to the other.

- Peer-to-peer. No central elements are available in the solution; all the interchanged data is done so in a fully distributed manner. This is the paradigm that, for example, has been used the most for file sharing from a historical point of view [43].

Figure 10 shows the different levels that have been set for middleware distribution, ranging from next to no distribution to a peer-to-peer paradigm.

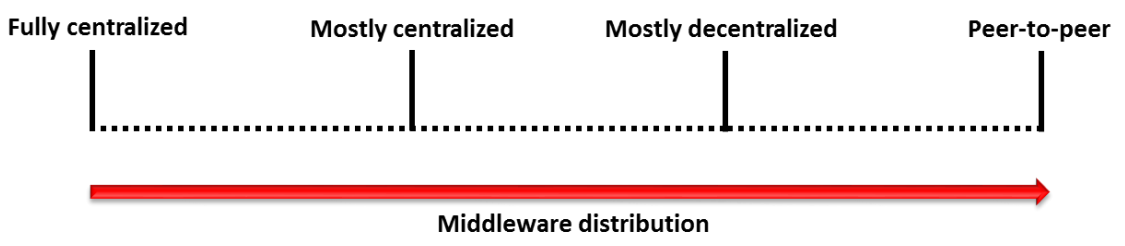

Figure 10. Levels of middleware distribution 


\subsubsection{Taxonomy for middleware in distributed systems}

The previous assessment of the criteria put to a use to classify and have a holistic perspective of the middleware solutions can also be taken into account from different perspectives. A direct one, where all the internal categories for each of the criterion become represented, has been used in Figure 11. It effectively implies that a taxonomy has been introduced as a result of the study on the state of the art of middleware architectures for the Smart Grid.

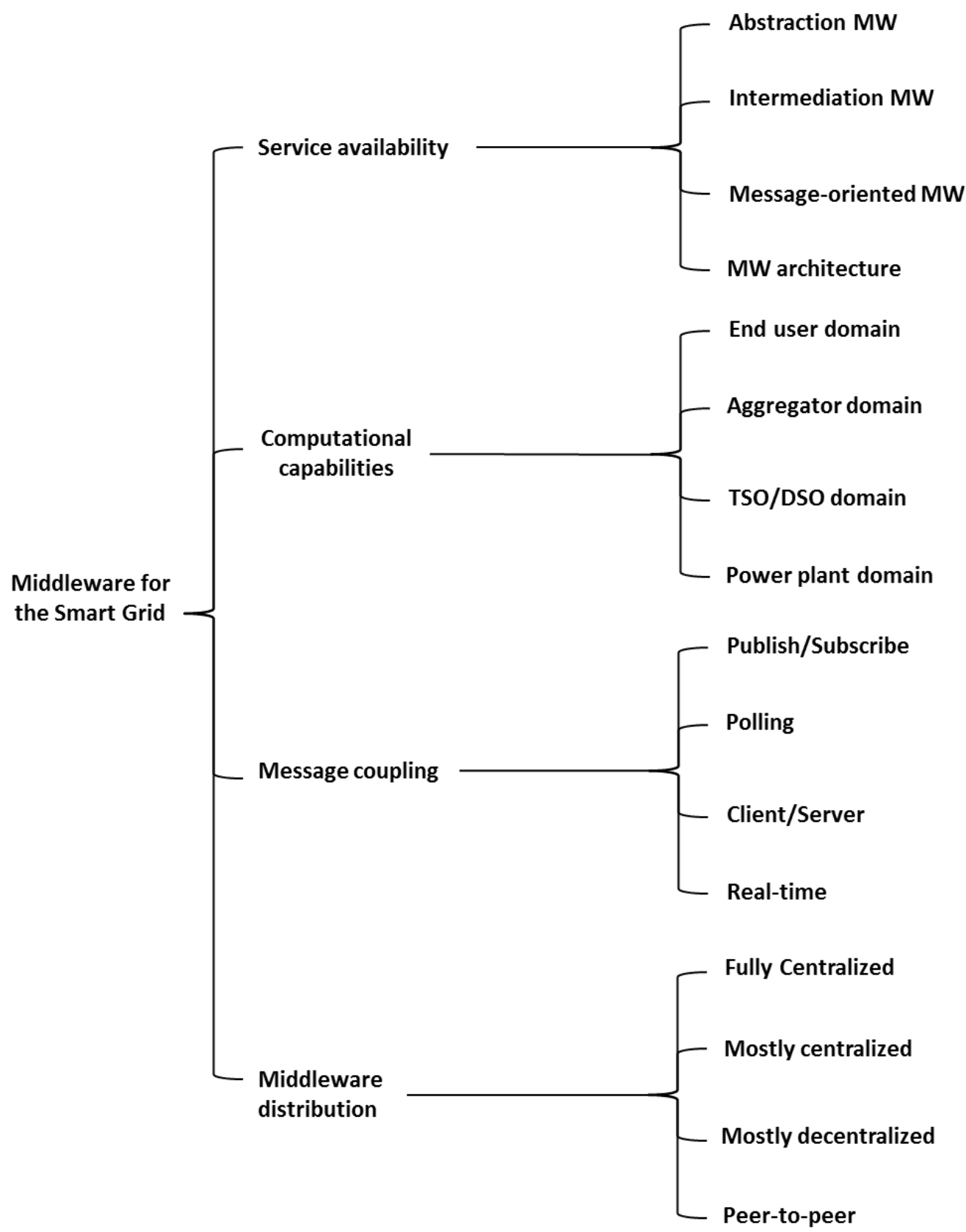

Figure 11. Taxonomy for middleware classification

In addition to that, there is another way that can be used to represent the taxonomy; rather than having a separated branch for each of the features, they can be placed altogether in a 
matrix where each of the rows will be occupied by the feature and each of the columns will be made up by the intermediate levels of every characteristic of the ones that are represented. This latter representation is more appealing from the mathematical point of view, as each of the features can be located as one element matching a row and column position, as depicted in Figure 12.

$\begin{array}{cccc}\text { Abstraction MW } & \text { Intermediation MW } & \text { Message-oriented MW } & \text { MW architecture } \\ \begin{array}{c}\text { Middleware } \\ \text { Smart Grid }= \\ \text { End user domain }\end{array} & \text { Aggregator domain } & \text { TSO/DSO domain } & \text { Power plant domain } \\ \text { Publish/Subscribe } & \text { Polling } & \text { Client/Server } & \text { Real-time } \\ \text { Fully centralized } & \text { Mostly centralized } & \text { Mostly decentralized } & \text { Peer-to-peer }\end{array}$

Figure 12. Matrix for Smart Grid middleware taxonomy

If the taxonomy is born in mind the way it has been depicted in the previous figure, the accurate definition of the features of each of the middleware architectures could be regarded a matter of choosing features that will match a collection of four positions in the given matrix, one for each row (feature established as a way to define the kind of middleware), so that the optimal middleware solution will be defined as the result of a mathematical equation. If, for example, a solution where a) a middleware architecture for b) end user devices based on c) a Publish/Subscribe paradigm that d) requires a peripheral information processing procedure but still has a centre to perform certain functionalities, then the solution could be described as follows:

Smart Grid Middleware $=$ Service Availability (element no.3) + Computational Capabilities (element no. 0) + Message Coupling (element no. 0) + Middleware Distribution (element no. 2).

Therefore, it can be represented as:

$$
S G M=S A(3)+C C(0)+M C(0)+M D(2)
$$

In this way, a precise idea can be obtained of the most prominent aspects of a middleware solution that has been conceived for the Smart Grid. In addition to that, once the characteristics of a particular architecture have become clear, an assessment of its advantages and disadvantages can also be done by considering how middleware functionalities are performed in each of the presented proposals. 


\subsubsection{GridStat}

The proposal elaborated by Harald Gjermundrød, et al. [44] focuses on several aspects inherent to middleware and distributed systems: it has been conceived to be used by scattered pieces of hardware involving Smart Grid deployments, Quality of Service (QoS) capabilities and a collection of functionalities within its very architecture. It was firstly presented as a way to distribute timestamped, time-synchronous data from Phasor Measurement Units [45] and further developed into a way to support RPC (Remote Procedure Call) mechanisms to use its own QoS semantic features [46]. The main purpose of this middleware proposal is creating a framework where data can be interchanged in a distributed manner under a Publish/Subscribe paradigm. In this way, messages will be transferred from one entity to another by mean of intermediate hardware appliances and software components. The following information can be inferred for each of the previously formulated criterion:

Service availability: while GridStat is regarded as a middleware framework by its authors and is referred to as an architecture, its functionalities are aimed at transferring information among different locations rather than providing any extra service, so it fits better a Message Oriented Middleware type of service availability. The authors claim that it offers secure, robust and flexible data communications that provides Quality of Service features, such as QoS-managed multicast. One of the most notorious features of the architecture is that it is divided in two different levels that are referred to as planes: the upper plane is the management plane; its main purpose is establishing the forwarding rules of the information sent. The other plane is called the data plane; it transfers data from the publishers to the subscribers, regardless of their location. Management plane uses QoS brokers to determine where information is sent in the data plane. QoS brokers in touch with the management plane (called leaf QoS brokers) directly manage the data plane clouds (a set of routers with the same resource management and cyber security policies) where the Status Routers are located; the latter have a flat organization within the clouds and forward the incoming data to the suitable communication link. Figure 13 depicts the appearance of the architecture.

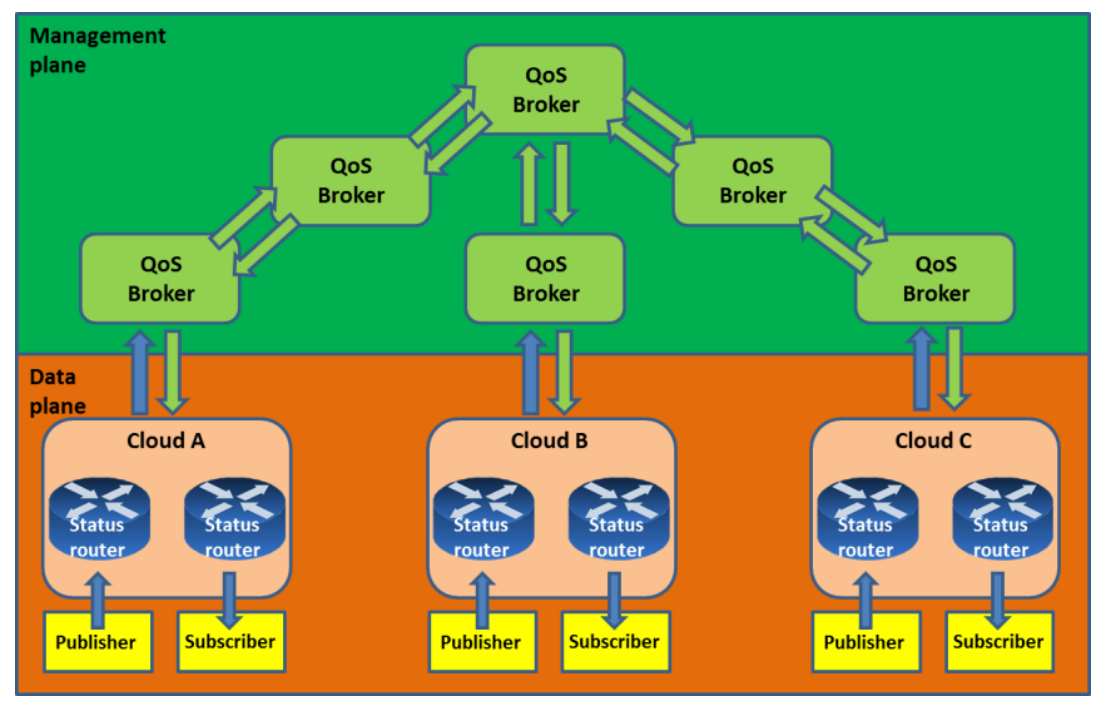

Figure 13. Gridstat proposal, as described in [43] 
Computational capabilities: The proposal is primarily aimed to provide substation automation (which implies that the software components can be located in the TSO/DSO infrastructures, as both entities have substations in the power grid). However, it is not explicitly mentioned that it cannot be equipped elsewhere, as the software elements that are described, such as QoS brokers or publishers and subscribers, are not necessarily located in one specific kind of hardware. The implementation that was used to provide experimental results uses Java as programming language (even though a C-written version, which should offer a higher performance, were under development at the time the proposal was introduced to the research community), whereas the hardware used implied Dell Power Edge 1750s servers for data transmission, Fedora 2 Linux as the operating system and a Java 2 Platform standard edition, so the requirements were more than acceptable to run the middleware architecture.

Message coupling: GridStat relies heavily on a Publish/Subscribe model in the data plane to send and receive information among the different entities where it is installed. This is clear from what has been shown of the data plane, which implies groups of routers receiving information from publishers and forwarding it to subscribers that will be dependent on either the same cloud or a different one (thus requiring forwarding commands from the management plane). Along with Publish/Subscribe, some other paradigms have been used as well: it is mentioned that Common Object Request Broker Architecture (CORBA), Client/Server communications are used for command interactions interchanged among the entities of the management plane.

Middleware distribution: The proposal offers a prominent degree of decentralization, but rather than following a peer-to-peer model, it has a hierarchy that keeps high level management decisions in an upper location. It is mentioned in the proposal that the management plane must readapt the network to changing conditions, like power system configurations or network failures, so a degree of control is given to some entities that others do not have.

If the matrix for the middleware in the Smart Grid is considered, the proposal can be defined as:

$$
S G M=S A(2)+C C(2)+M C(0)+M D(2)
$$

Advantages of the proposal: GridStat offers a detailed framework not only from the theoretical but also from the practical point of view, as there are implementation works to prove the design that has been conceived by the authors and the performance results show the feasibility of the proposal. The fact that it does not require many computational resources to have it running and its significant distribution degree are also appealing, as it can be used in different low capability devices the Smart Grid usually involves, such as smart meters.

Disadvantages of the proposal: The proposal does not have a collection of services that can be used by applications; instead of that, GridStat seems to have been conceived for the mere transmission of data one level above network capabilities. Even though security is mentioned several times in the proposal, not enough detail is provided (such as how access is securitized or whether cryptography is used). Lastly, important features used for middleware and 
information enhancement (ontologies, information models) are not mentioned in the proposal.

\subsubsection{Service-Oriented Middleware for Smart Grid}

L. Zhou and J. Rodrigues put forward a proposal that puts its stress on services for the Smart Grid and how they get to be applied in a deployment [47]. The authors claim that they have developed an efficient and integrated middleware solution for heterogeneous services of the Smart Grid, aiming to obtain a high level of software sustainability and stability. The idea of offering efficient services in the proposal is a major one for the authors, as they consider that serving for power allocation and consumption is closely linked to the idea of service applied to a middleware solution for the enhancement of the power grid. The proposal deals with one usual problem of middleware solutions, that is to say, the difficulty to be reused from one development to another, due to the different nature of the services that are requested in each application domain. Consequently, the service-oriented middleware has been decoupled according to several application-based functionalities. Its most prominent characteristics are:

Service availability: As it usually happens with middleware, this proposal is divided into several layers that split the functionalities in different levels, so that they will be accessed and used in different ways. As it has been displayed in Figure 14 there are three levels: a user part, a control part and a transmission layer. The user part is responsible for providing both QoS and Quality of Experience (QoE) for the end user (which usually determines bandwidth, delay, reliability or jitter parameters); it also takes care of scheduling flexibility to offer the best available QoS possible. Secondly, the control part connects the user part with the transmission layer; its functionalities are the ones more typical of a middleware solution, as it is expected to handle the different devices interoperating in the system and transferring information via transmission layer while maintaining an acceptable level of QoS and QoE in the user part. Lastly, the transmission layer is regarded as the foundation of the whole system and uses its services in the Advanced Metering Infrastructure of any deployment where the proposal is used. Data interchanges are carried out both among contiguous levels and between the user part and the transmission layer. Due to the separation of the proposal in different layers, and its differentiated services, it can be regarded as a middleware architecture. 


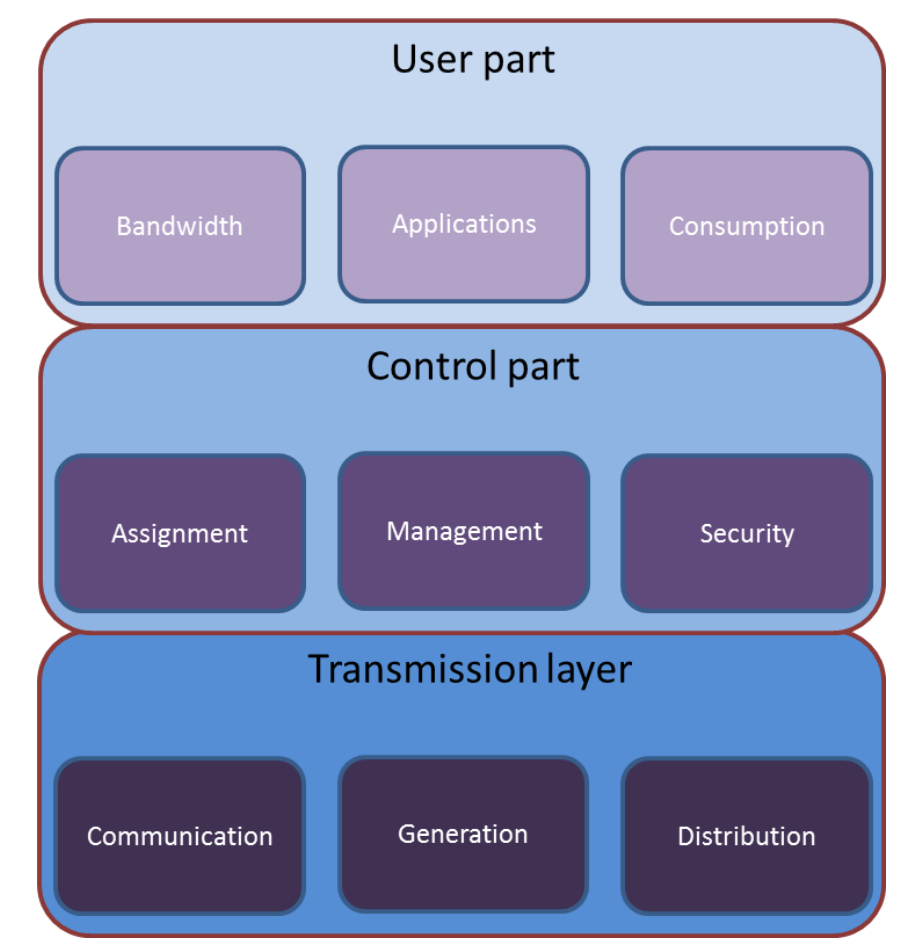

Figure 14. Service-Oriented Middleware proposal, as described in [47]

Computational capabilities: according to the results that have been obtained from the tests, the middleware solution offers a high Mean Option Score (MOS) in four different situations (access control, message transmission, power allocation and service quality) without compromising its performance. As for the tools that have been used, the network simulator ns-2 was used to test the proposal. The collecting node has been modeled with a regular personal computer. Each of the smart meters has been modeled as a node with an ARM processor. It can be estimated that end user pieces of equipment and aggregator infrastructure would be the most likely to receive the developed middleware.

Message coupling: the proposal mentions that they have developed an application access control principle; hence, there is a homogeneous way to access the middleware. Additionally, it is also said that message transmission was tested, so the proposal can be regarded as a Message Oriented Middleware.

Middleware distribution: due to the tests that have been done and the fact that the proposal is expected to be mounted in devices such as smart meters, it can be argued that it is a mostly decentralized proposal, as it will be used in many devices of comparatively low computational resources but still follow the hierarchy to be expected from the Smart Grid. Specific information about this criterion is scarce in the proposal, as simulation tests are provided rather than having the software running in actual machines.

If the matrix for the middleware in the Smart Grid is considered, the proposal can be defined as:

$$
S G M=S A(3)+C C(0|| 1)+M C(2)+M D(2)
$$


Advantages of the proposal: there is a collection of services provided clearly stated by the authors. Apart from that, performance tests have been made in order to offer actual data about the behavior of the middleware solution. Even they have been done in the controlled environment of a simulator, they offer an idea of the capabilities of the middleware solution. Finally, it is also mentioned that symmetric algorithms have been considered for security implementation.

Disadvantages of the proposal: overall, the services that are provided are not that prominent or complex. In addition to that, it is not mentioned how distributed it can be or the devices that would be able to have installed the software packages it is made of. In addition to that, the monitoring system that is referred to in the proposal is mainly composed by elements that, although are used for communication purposes, can also be regarded as part of levels usually assigned to physical and network-based ones (such as Wi-Fi, satellite networks, the Internet, etc.). Overall, few data are provided with regards of the nature of each of the services present in the three layers of the proposal; information about the functionalities of each of them is missing too.

\subsubsection{Ubiquitous Sensor Network Middleware (USN)}

Zaballos et al. offer their own interpretation about what a middleware architecture for the Smart Grid should look like [48]. It is mainly focused on adapting the framework of the ITU ubiquitous sensor architecture to the application domain of the Smart Grid, which is conceived by the authors of the proposal as a network of Intelligent Electronic Devices, sensors, distributed generators, dispersed loads and smart meters requiring a heterogeneous communication paradigm. It is also mentioned how by using the framework referred to as the Ubiquitous Sensor Network architecture at defining interoperability, along with a Next Generation Network (NGN) as the backbone where the proposal is deployed, end-to-end integration of devices can be achieved. The proposal relies heavily on Internet of Things-like features, such as lightweight transmission protocols (such as IEEE 802.15.4, used for bit transmission among constrained devices like nodes from a Wireless Sensor Network, even when adding security capabilities to it [49]). In addition to that, it also makes use of technologies like Power Line Communication (PLC) or Worldwide Interoperability for Microwave Access (WiMAX).

Service availability: there are several kinds of services that have been conceived, as it can be seen in Figure 15, so the proposal can be regarded as a middleware architecture. There are three different levels closely related to the kind of level they are closest to. The higher level has a directory containing information about the sensor network. Furthermore, it has an open API that can be accessed by the application developers in order to access the middleware architecture. The middle level is used to process either events related to the deployment or the sensing data that can be mined. Finally, the lower level is devoted to interface and monitor the sensor network that is present in the lower levels. Interestingly enough, there is a security manager module that is used in every level of the architecture. 


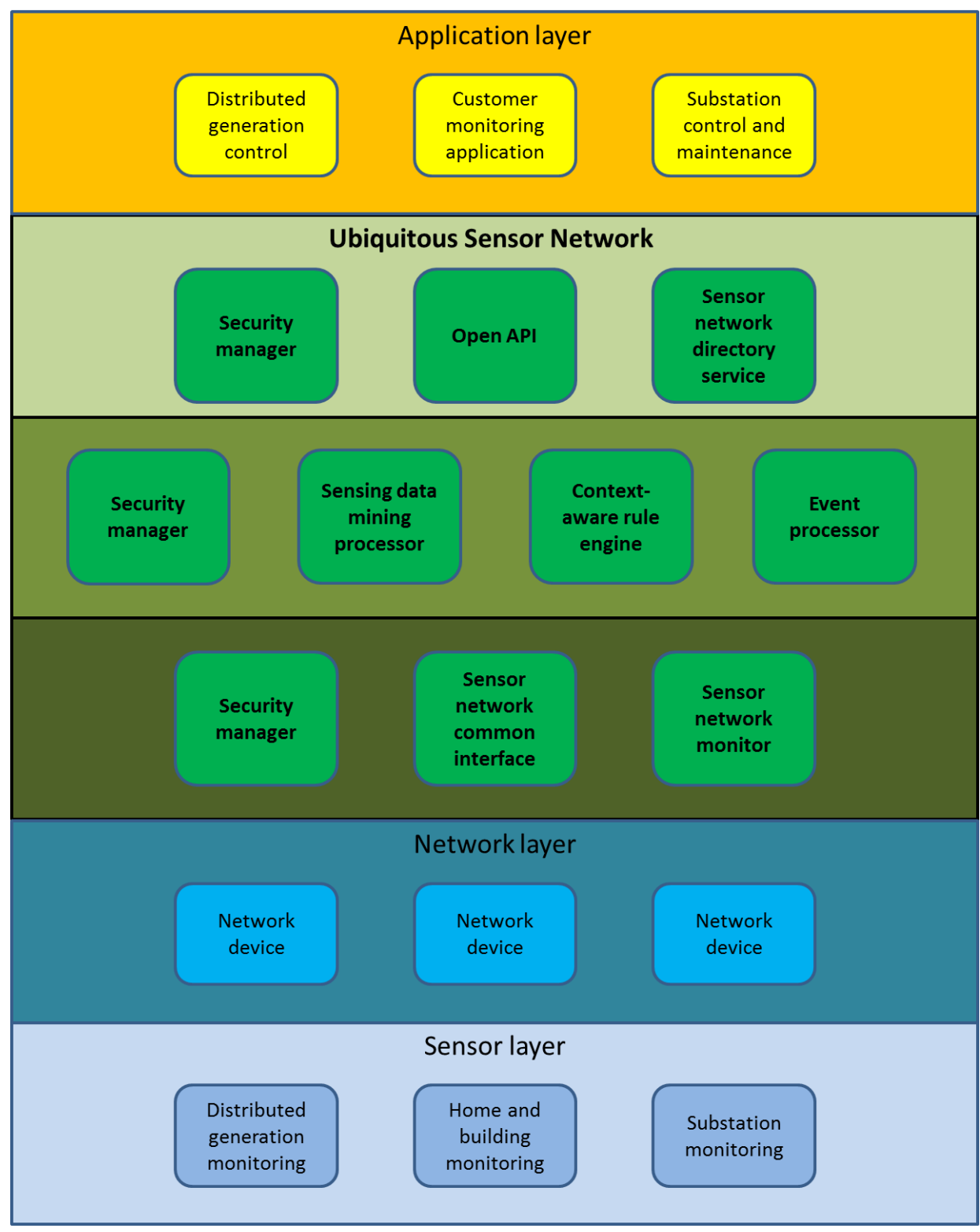

Figure 15. USN middleware proposal, as described in [48]

Computational capabilities: while the proposal uses sensor-based networks for information transfer, little is said about what kind of devices in the Smart Grid would equip the software components it is made of. It can be presumed that they would be equipped by entities located one level higher than the network one, so virtually any computer, aggregator or TSO/DSO domain equipment would be able to have it installed.

Message coupling: the proposal mentions that the USN application level is a technology platform that can be used effectively in real-time and control functions. Besides, there is an example showing how connection and authentication procedures would be performed that implies a Client/Server communication. It seems that Client/Server requests could be addressed in real-time as a best case scenario, but there is no accurate information about it.

Middleware distribution: as it happened before, there is little information about this topic. Since a network layer is supposed to be installed below the middleware proposal, it is 
supposed to be either mostly centralized or a mostly decentralized proposal that is able to run in several devices simultaneously.

This proposal can be described as:

$$
S G M=S A(3)+C C(0|| 1|| 2)+M C(3)+M D(1|| 2)
$$

Advantages of the proposal: the proposal makes use, or at least is compatible with, several different proved technologies used in other levels of the system, such as IEEE 802.15.4 or WiMAX. It aims at being reasonable decentralized.

Disadvantages of the proposal: the proposal tends to mix several different concepts that, due to their inherent features and locations in a system architecture, are not part of middleware (such as applications or Wireless Sensor Networks). What is more, there are no explicit mentions on what kind of equipment would be used to encase the proposal in a Smart Grid or a microgrid. Performance tests, either simulated or in actual appliances, are not shown to get a grasp on how the proposal gets needs solved, such as consumption data or data transport. Overall, the proposal feels more like an Internet of Things development that was ported to the Smart Grid rather than something that was made from the scratch for the latter application domain.

\subsubsection{OSHNet (Object-Based Middleware for Smart Home Network)}

Sang Oh Park et al. describe a proposal based on interoperability between home devices and Smart Grid-related ones [50]. The proposal is structured in three different layers: the Application layer (which offers the home users the proposal is aimed to five different Application Programming Interfaces), the Library layer (responsible for providing the necessary information about the present home devices) and the Network layer (which provides device interoperability among devices using different kinds of protocols). Thus, it expands its borders further than what is strictly located as the domain of a middleware architecture, which usually relies on data received from the network to be provided to an application; taking into account this idea, the library layer of OSHNet would be the one that is fitting an actual middleware solution, even though it is the network layer the one that is more closely aimed to the usual functionalities of middleware. Home devices characteristics are included in the Library layer as four different kinds of objects: Control object (applied to controlling neighbouring home appliances), Streaming object (focused on managing input and output data), Status object (used to maintain current status of the home devices included in the deployment) and Function object (utilized to execute functionalities in home appliances, like powering on or switching TV channels).

Service availability: the proposal can be regarded as a middleware architecture, as it is composed by several levels (referred to in the proposal as layers) with different services in each of them. As it was mentioned before, the highest one has the suitable API used to access the application level, whereas the library layer has the aforementioned four kinds of objects (Control, Streaming, Function and Status) used to categorize each of the devices in the home 
deployment, as well as three modules that are used to invoke services from the proposal: the Object Management Module (in order to control the functionalities that are offered by the devices where the solution is present), the Object Discovery Module (to gather information about other home peer devices) and the Connection Management Module (used to establish, maintain and terminate connections among devices). One lower level, but still within the middleware architecture, the Network layer presents the Virtual Network Adapter (VNA), which is the software entity interconnecting the different devices that are used outside the middleware proposal. Figure 16 displays the most prominent software components of the proposal.

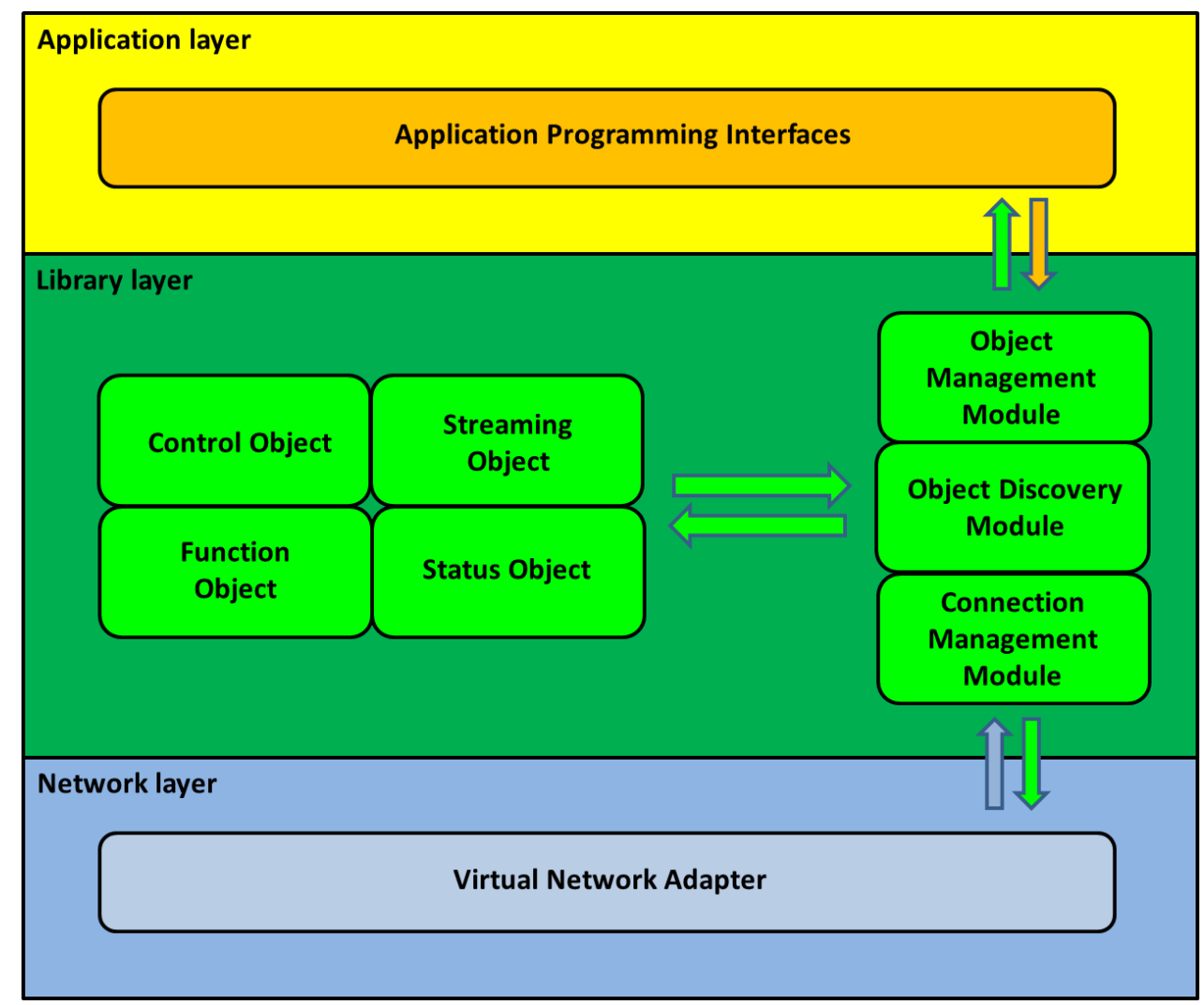

Figure 16. OSHNet architecture, as described in [50]

Computational capabilities: the proposal often refers to home systems as the devices that will equip the middleware solution. In addition to that, the proposal documentation offers screenshots of mobile devices as smartphones, so it can be assumed that End User equipment of the Smart Grid, such as Advanced Metering Infrastructure, would suffice to have the software components installed. The tests that have been carried out involve a set of virtual devices, such as a humidifier, a wind-powered generator, a smart meter, a smartphone, three laptops or an external information center.

Message coupling: since there are user interfaces shown in the proposal, it can be assumed that it is capable of handling Client/Server requests. Information is fed onto those interfaces, so at least they should be able to support some kind of polling, even though the actual way to interact with the simulated environment is not completely clear. 
Middleware distribution: it is mentioned that the proposal is expected to be installed in Distributed Energy Resources, as well as in other devices of somewhat lower computational capabilities. In addition to that, there are other pieces of equipment as laptops that are expected to have higher ruling capabilities over the system, so it can be said that the proposal deals with a mostly distributed middleware solution. The devices that are included are supposed to be simulated, though.

According to the matrix that was previously defined, this proposal can be described as:

$$
S G M=S A(3)+C C(0)+M C(2)+M D(2)
$$

Advantages of the proposal: the middleware architecture that is put forward here contains a collection of services where a group of them are specifically designed to tackle hardware interoperability. In addition to that, simulated devices and user interfaces have been created to have a realistic idea of what can be expected from the proposal.

Disadvantages of the proposal: The proposal also uses confusing names in the sense that there are several levels referred to as "layers" still within the boundaries of the middleware architecture, as the network layer or the application layer. In addition to that, while some services are added to the architecture, those are meant for the internal performance of the proposal, rather than offering a more compelling functionality for end users.

\subsubsection{Meter Data Integration (MDI)}

Zhao Li et al. offer in this proposal their own conception of what a middleware solution should be [51]. While the proposal is referred to in the proposal as a Meter Data Integration (MDI) solution, it is clear from the text that is supposed to integrate different kinds of AMI in a common deployment, so it is actually performing middleware functionalities to the point that is described as a middleware located between the Advanced Metering Infrastructure and the Distributed Management System (DMS). Some other elements are defined as well: a Meter Data Management System (MDMS) is used as a certain kind of data server and a Meter Data Collector gathers all the information obtained from the deployed Smart Meters. This proposal takes into account implementation details about the smart meters that are either used or manufactured by several large companies, such as Pacific Gas \& Electricity or Siemens, so a more realistic approach can be taken when functionalities are designed.

Service availability: the proposal described here makes used of three different levels of information to separate and determine the specific functionalities that will be carried out. Consequently, and taking into account that each of the layers contains different services, it can be regarded as a middleware architecture. The first level contains the services used to adapt the information utilized by the DMSs to the possible transport interfaces used in the deployment. The intermediate layer is the more complex one: there is a temporal database used to verify and translate the collected smart meter data, the Loosely Couple Event (LCE) infrastructure used for message publication and subscription and a MDI monitor used to track the status of the functional components existing in the overall MDI layer. Lastly, the lower 
level is used to contain all the adaptors used for connectivity with the AMI network. The overall appearance of the services of this middleware architecture is shown in Figure 17.

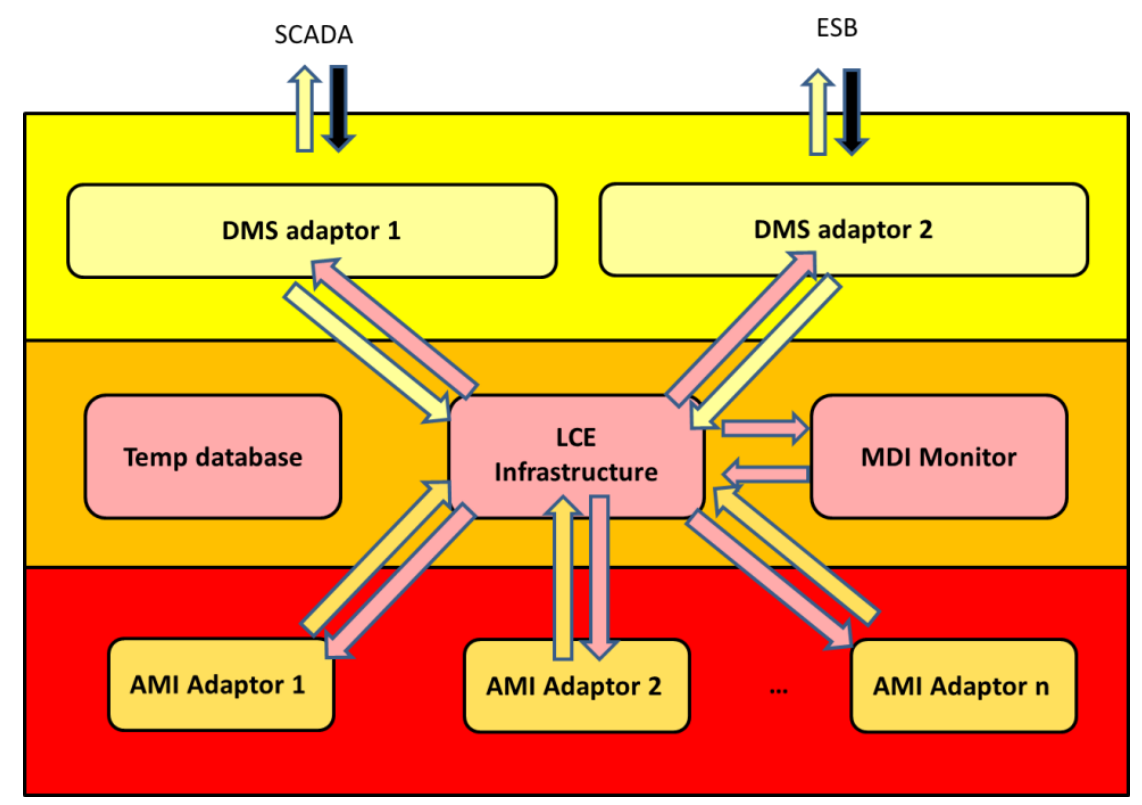

Figure 17. Meter Data Integration Proposal, as described in [51]

Computational capabilities: according to the tests that have been performed on the proposal, the devices used are two simulated smart meters (which use separated pieces of hardware and Windows Server 2003 and 2008 as the operating systems) and another one used as a server containing the MDI layer (it uses a Windows 2008 Server operating system). The proposal is mainly focused on managing Advanced Metering Infrastructure, although taking into account that the MDI components have been located in a different machine and the fact that there are data obtained from the smart meters, arguably it can be expected to be installed in aggregator-located equipment.

Message coupling: the proposal is explicitly described as using a Publish/Subscribe paradigm, as it has been designed to work publishing and subscribing the messages that are transferred by means of the LCE infrastructure. According to the information on this middleware architecture, it is loosely coupled in the sense that message senders and receivers run in different spaces and if for any reason one of them stops working, the other side of the communications will still be operational.

Middleware distribution: the proposal has been installed in a single computer in the tests that have been carried out. On the other hand, it is commanding several devices that have been portrayed as part of an Advanced Metering Infrastructure. Due to these reasons, it has been considered a mostly centralized middleware architecture.

This proposal can be described as:

$$
S G M=S A(3)+C C(0|| 1)+M C(0)+M D(1)
$$


Advantages of the proposal: the middleware architecture aims to provide interoperability of different devices that are used as real smart meters. In addition to that, it is clearly justified why the proposal is conceived as one using a publisher/subscriber and the computational resources that are suitable to run it with good enough performance.

Disadvantages of the proposal: as it happened with several previous proposals, there is very little information about how the services can be implemented or any detail about their performance on different programming languages. What is more, services provide not so many functionalities beyond interoperability and interconnectivity; security or semantics, for example, seem not to be supported by the proposal. In addition to that, the information about the tests that have been carried out is referred to as a small group of devices that are simulating actual ones; having more devices that were located in an application domain that involved a microgrid or something alike would have been welcomed, especially when actual smart meters have been studied for the proposal.

\subsubsection{IEC 61850 and DPWS Integration}

Stjepan Sucic et al. combine in their proposal the usage of DPWS (Device Profile for Web Services, a standard put forward to promote interoperability among constrained implementations of Web services, along with higher interoperability with more flexible client implementations [52]) and an electric standard (IEC 61850, a communication model used for functionalities like setting requirements for device models or the description of the language for communication among substations [53]) typical of the power grid needs that the authors present [54]. Their main idea is that, since the principles of IEC 61850 are defined under a nonPlatform Specific or software specific point of view, and in fact, they use a description known as Abstract Communication Service Interface (ACSI) that is not linked to any specific middleware solution, Web services can be used to complement one effective middleware mapping enabling IEC 61850 communications. This mapping enabling is known as Manufacturing Message Specification or MMS, and is also used for distributed transmission of power control [55]. Rather than replacing this technology, the proposal seeks to improve some of its weaknesses; the authors cite its complexity and involving a full OSI stack that makes integration of any distributed solution more expensive.

Service availability: there are several levels that are defined in this middleware proposal as an architecture. One important fact is that, despite their different features, they are all focused on providing facilities to the Web services that are present in it. DPWS is used here, as it is claimed by the authors that it is a technology aimed to provide interoperability for heterogeneous and constrained devices that may have limited and low capability resources. The three different levels that are present are located as it is usual in middleware solutions, that is to say, between the application layer and the network one. The uppermost of them is used for Web service discovery (which provides an addressing mechanism for Web services), Web service Metadata Exchange (defines operations to retrieve device and service metadata, along with datatypes) and Web service eventing (for management of the publish/subscribe mechanism used in the proposal. These capabilities are linked to the intention of using the 
functionalities that a Service Oriented Architecture (SOA) could use in a distributed system. The middle layer contains all the functionalities related to security (specification on how confidentiality and several security token formats should be used), Web service policy (describes constrains and capabilities of available policies) and addressing (addressing mechanisms for the Web services). Finally, the lower layer contains Simple Object Access Protocol (SOAP) functionalities and metadata facilities like eXtensible Markup Language (XML) schemas or Web Services Description Language (WSDL)-formatted information. The authors claim that the proposal can deal with the requirements imposed by both ACSI (real-time, distributed applications, integration at the device level etc.) and other new control models (Virtual Power Plants or VPPs). These features have been depicted in Figure 18.

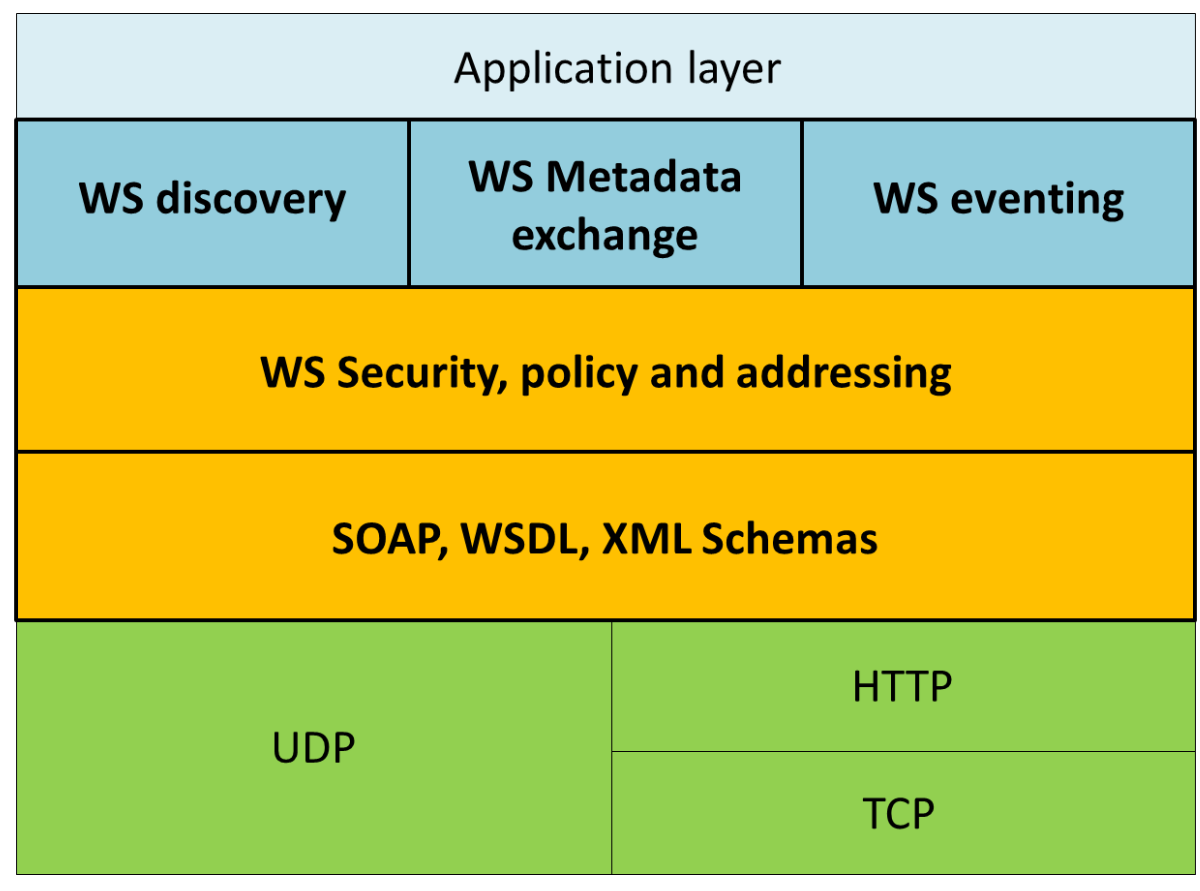

Figure 18. Protocol stack of DPWS, as described in [54]

Computational capabilities: this proposal covers a wide range of Smart Grid devices, as the electric protocol that is enabled is usually implemented in substations, and more recently, in wind power plants or in Distributed Energy Resources. Consequently, it can be said that the proposal covers the area of TSO/DSO domain, as well as the power plant one. Two more aspects of the proposal have to be taken into account too: on the one hand, the Web services can be installed in a myriad of different devices, so hardware to be used can be very flexible. On the other hand, the proposal makes use of the concepts present in DPWS about the devices holding the services (hosting devices), along with the devices they are keeping (hosted services). It is mentioned in the proposal that it can be used for integration of Virtual Power Plants.

Message coupling: the proposal is mentioned to work with a Publish/Subscribe paradigm in several cases, being the most prominent one any interaction that involves the Web service Eventing component. For instance, a subscriber can establish a link with an event source, and if an event is triggered, an event sink will be used to publish a message containing data about it. 
Additionally, it is mentioned in the proposal that ACSI runs with a Report Control Block that require a Publish/Subscribe model for its correct performance.

Middleware distribution: while still making use of the hierarchy of the actors involved in the Smart Grid (the infrastructure that the proposal is most aimed at are power plants, either physical or virtual), the fact that it can be used for the integration of the distributed resources of a Virtual Power Plant provides an idea of how distributed the proposal has been conceived. Therefore, it can be argued that, as far as middleware distribution is concerned, it is a mostly distributed one.

This proposal for middleware in the Smart Grid can be characterized as:

$$
S G M=S A(3)+C C(2|| 3)+M C(0)+M D(2)
$$

Advantages of the proposal: unlike many of the other proposals that have been studied, this one openly mentions how semantic resources are used for interoperability among devices, and provides information about the technologies used to offer the application layer an homogeneous set of services. What is more, wind power plants and DERs available in Virtual Power Plants are considered in a level that other proposals hardly ever do. Lastly, the proposal presents services genuinely useful for the whole deployment such as security.

Disadvantages of the proposal: the middleware architecture presented here lacks any relevant information about tests that may have been carried out, so it is hard to get an idea of the actual performance of the proposal. Furthermore, the proposal is lacking information about how to abstract hardware heterogeneity, as DPWS is more focused on higher levels an ACSI mechanisms are not detailed.

\subsubsection{Intelligent Agents Platform}

Álvaro Paricio et al. offer a middleware solution that tackles the heterogeneity existing among devices linked to both the Smart Grid (AMI) and other areas as Home Area Network devices [56]. The proposal refers to an Intelligent Agents Platform (IAP) used as the framework where message interchange is done. The devices present in a Smart Grid where this proposal is deployed (smart meters, sensors, etc.) are controlled by IAP Mediation Devices. The required management among the elements that are using the Smart grid framework is guaranteed by Integrated Network Management (INMS) functions, which rely on several functional blocks participating of actions like fault handling, integration capabilities or configuration management. The proposal makes use of Enterprise Service Buses for internal data transfer among its distributed components, so it will be regarded as an architecture.

Service availability: the proposal consists of three differentiated elements: two management layers that use internal buses for data transfer (the Network Mediation Layer and the Management Application Layer), and an intermediate one connecting the other two (Middleware Communication Services) that may or may not appear according to the specific needs of operational models. The Network Mediation Layer processes in a bi-directional fashion the usually large amount of information transferred through the deployment. There 
are pieces of equipment called IAP Mediation Device (MD) that use this layer for control purposes. Any management traffic type is controlled with these MDs, regardless of its nature (status monitoring, security, configuration, operation flows, etc.). The Management Applications Layer is responsible for using a collection of functional application blocks that are closely tied to an end user functionality as a reporting engine, alarm handling or a task scheduler. Lastly, the middleware communication services basically interconnect one layer with the immediately other one in case data have to be transported from the applications backend to the mediation system. All these features have been represented in Figure 19.
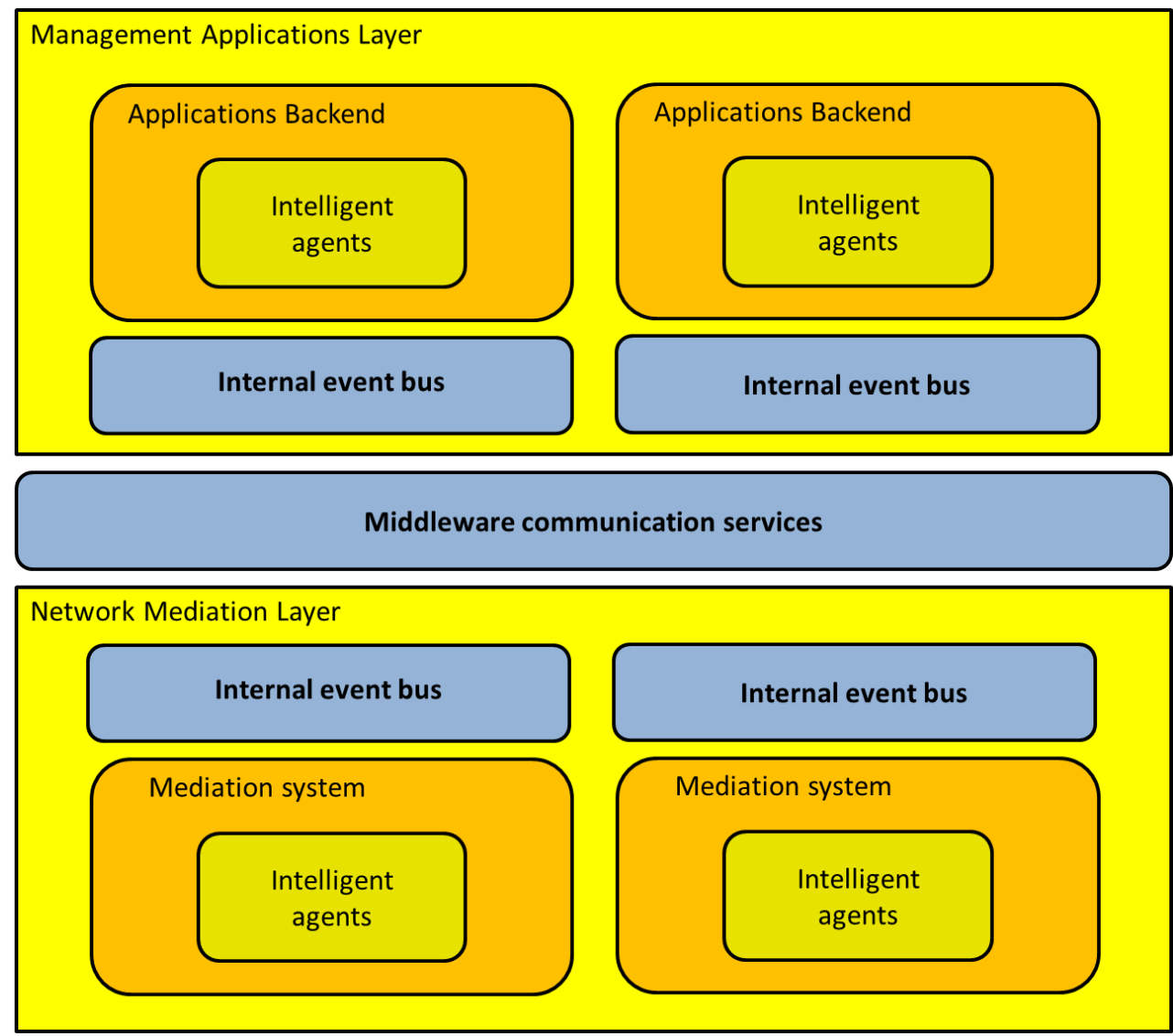

Figure 19. IAP-INMS appearance, as described in [56]

Computational capabilities: it is mentioned that the proposal has been tested repeatedly in different application domains. Furthermore, Customer Premises Equipment has been used as a way to employ a Smart Grid-like deployment where information could be transmitted via IP network. Unfortunately, there are scarce data of the equipment that was used for the text, or even if it was simulated or not (it is mentioned, for instance, that each Mediation Device will control one hundred concentrators, so a total of ten thousand AMIs will be managed).

Message coupling: there are two different kinds of paradigms that are used in this proposal. The first on is real-time for event collection, with no message decoupling whatsoever. On the other hand, a Publish/Subscribe mechanism is also utilized for the Intelligent Agents Platform used for the implementation and testing activities. There are even other kinds of paradigms, such as agents using peer-to-peer communications, or polling done on the concentrators used for testing activities. 
Middleware distribution: as it happened before, not many data are available regarding what kind of distribution is expected to use the proposal. Since Mediation Devices, as well as the IAP platform are mentioned in the tests, it can be argued that the equipment will not be located as part of the power plant premises. Also, since the AMI seems to be controlled by the software components of the proposal, it can be said that this is a mostly decentralized proposal.

This proposal can be described as follows:

$$
S G M=S A(3)+C C(0|| 1|| 2|| 3)+M C(0)+M D(2)
$$

Advantages of the proposal: it is implied by the information provided that this middleware architecture is capable of handling data of very different characteristics. The proposal also makes use of an Enterprise Service Bus, which is considered to be a very suitable option for distributed and Cyber Physical Systems with the features of the Smart Grid. Behavioral rules provide some degree of a semantics level. Some tests have been carried out in order to assess how the proposal would work in a distributed system.

Disadvantages of the proposal: the amount of services offered by the proposal is lower than in the other ones, despite using an ESB (which could be filled with software packages that will contain the services that can be provided). What is more, information about the performance results obtained in the tests, or what the devices used for them looked like, is missing. Finally, even though the middleware architecture can transfer many different kinds of data, it is not explained where services of major importance, like security or hardware abstraction, can be obtained.

\subsubsection{Self-Organizing Smart Grid Services}

Abdalkarim Awad and Reinhard German offer their own concepts for Smart Grid services capable of be aware of the context where they are deployed upon [57]. The authors introduce several metrics, referred to as degrees, to be used in order to define what features a development should have and to what extent. These degrees are: degree of scalability (among other facts, it assesses whether information can be created using just local messages), robustness (which evaluates the adaptability of the self-organizing services), target orientation (how any node creates its own data from an initial state), emergence (a phenomenon that can be witnessed at a macro level), flexibility (providing redundancy so that there will not be single points of failure in the deployment), reliability (ability of the self-organizing service to find alternative solutions in case any issue appears, like route unavailability) and parallelism (ability of the service to join or leave the system from different sides simultaneously).

Service availability: this proposal focuses on the services that can be provided in a Smart Grid and treats the middleware as a software component located in one of the two levels that are shown in Figure 20 (a). Here, it can be seen that the two levels of the proposal include a decision level (where the data is received and design, supervision or control are performed) and an infrastructure level that provides feedback to take decisions. Those two levels mimic the solution structure that is provided in Figure 20 (b), where decision support is done at the 
decision level and data communication combined with physical devices roughly matches the infrastructure level. It is expected from middleware that it will cope with several processes: data routing, aggregation, filtering and replication are mentioned by the authors. Middleware as a software participant in the system is less relevant here than in other proposals, as it is used in the infrastructure side of the communications that take place throughout the system. Therefore, it can be regarded as an abstraction middleware.

Computational capabilities: the proposal makes no explicit mention about the devices that are expected to be used to install the proposal. The physical level seems to correspond with Advanced Metering Infrastructure, whereas the decision support centers could be located in the aggregator side, as they are controlling the devices present on the very end of the deployment and they are able to send commands.

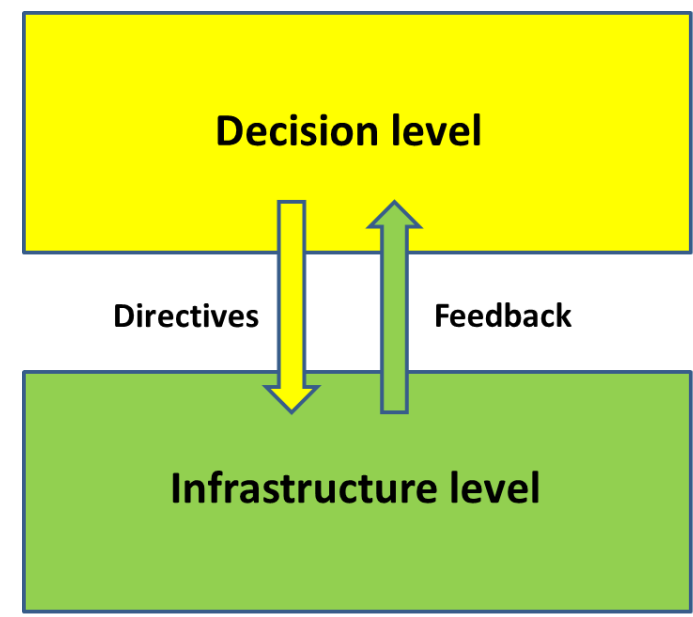

a)

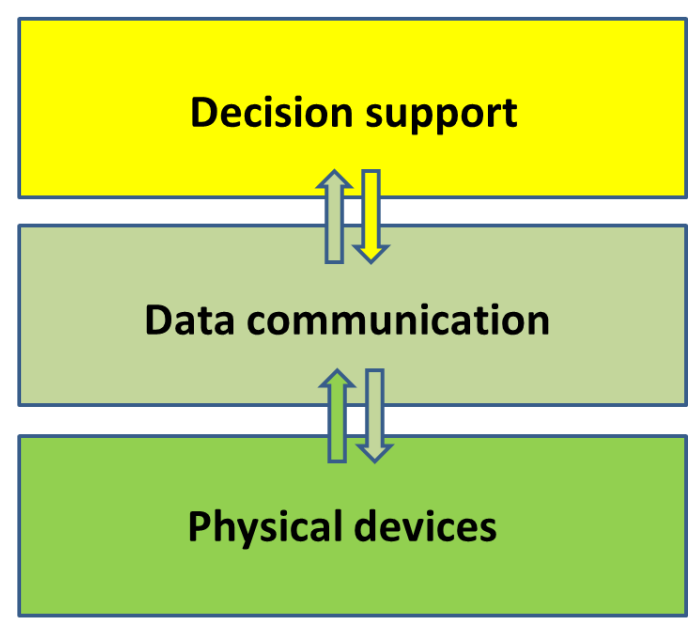

b)

Figure 20. Solution structure (a) and main levels of the proposal (b), as described in [57]

Message coupling: real-time communications are mentioned the most in the proposal as one of the most critical ways to interchange data among the elements of the Smart Grid. Aside from that, not many details are providing any other form of message coupling (publish/subscribe, polling or Client/Server communications are not mentioned).

Middleware distribution: the proposal openly criticizes centralized middleware solutions, and it is also mentioned that all nodes have the same importance here. However, while it can be inferred from the proposal that there is some degree of distribution (which could mean that the proposal is a mostly decentralized one), it is not clear where the solution is expected to be installed. Peer-to-peer is mentioned in the proposal as the most preferred used for networks in the proposal.

Considering all these facts, the proposal can be described as:

$$
S G M=S A(0)+C C(0|| 1)+M C(3)+M D(3)
$$

Advantages of the proposal: the middleware architecture put forward here offers a distributed way to interchange data among several software entities. 
Disadvantages of the proposal: there is very few information on how the proposal can be used in an actual deployment. Besides, not much information is provided on what tests have been carried out to validate the middleware part. Additionally, not prominent services are offered as part of the middleware (semantic features, security). The overall impression of this work is that middleware is a secondary element that is required to have the self-organizing services working fine.

\subsubsection{Secure Decentralized Data-Centric Information Infrastructure}

The proposal presented here by Young-Jin Kim et al. puts forward how a framework for a decentralized and distributed system can be applied to the Smart Grid [58]. The authors claim that their proposal tackles challenges related to security, latency, real-time events and distributed data sources. The Information and Communication technologies infrastructure makes use of the Internet Protocol as the underlying way to transmit information at the network level. In addition to that, it offers securitization for the services that have been included in the proposal. It also makes use of the Common Information Model as a way to interchange data between Energy and Distributed Management Systems (EMSs, DMSs) in a seamless way. It is assumed that each of the devices present in the grid is able to handle symmetric-key operations to establish secure channels, as public-key operations are way more costly in terms of time and performance.

Service availability: taking into account the layered approach that has been taken in this solution, it can be said that this is an example of a middleware architecture. There are several layers in the whole proposal to take into account: the highest level one deals with what the authors refer to as power applications, which is above the proper middleware and has been conceived as the applications that can be accessed by the end users. The Middleware Application Programming Interface is offered just one level below; it offers a description of how middleware is accessed from the application layer and the services it can provide to it. More importantly, it provides a way to access to the services provided for event management services (Non-time critical and time critical data/event) and control commands. At the same time, these services rely on other software components based on networking and distributed information transfers, such as the network cache for information storage (used for pulled data) and a publish/subscribe dissemination (responsible for pushed data). A Secured grid overlay network is used for the data transmitted in unicast, multicast and broadcast communications. Lastly, low-latency transport protocols have also been included in the proposal. The proposal has been portrayed in Figure 21. 


\begin{tabular}{|c|c|c|}
\hline \multicolumn{3}{|c|}{ Middleware Application Programming Interface } \\
\hline $\begin{array}{c}\text { Non-time critical } \\
\text { data/event }\end{array}$ & $\begin{array}{c}\text { Time critical } \\
\text { data/event }\end{array}$ & Control commands \\
\hline Network cache & $\begin{array}{c}\text { Publisher/Subscriber } \\
\text { dissemination }\end{array}$ \\
\hline \multicolumn{3}{|c|}{ Secured grid overlay network } \\
\hline Reliable, low-latency, lightweight transport
\end{tabular}

Figure 21. Data-Centric Information Infrastructure, as described in [58]

Computational capabilities: there are few data on what kind of equipment has been conceived to have the middleware components installed. The authors stress the idea that their proposal is data-centric, as opposed to host-centric, so hardware is expected just to have the components installed without worrying too much about the nature of the hardware and its location.

Message coupling: there is a component in the proposal that is stated as responsible for publisher and subscriber dissemination, so the messages are expected to at least follow this pattern when they have to be transmitted. Real-time communications are also taken into account here: the software components that manage the events do so taking into account their real-time nature; it is also mentioned in the proposal as something that can be provided by means of a Real Time Protocol (RTP).

Middleware distribution: it can be inferred from the proposal that this is a mostly decentralized one, since it relies on technologies like IP or security for a whole network. Still, an application layer has been conceived to be accessed by just a group of machines, so there will be some centralization left, even if it is held by several pieces of equipment.

Therefore, the proposal can be described in the following manner:

$$
S G M=S A(3)+C C(0|| 1|| 2|| 3)+M C(0|| 3)+M D(2)
$$

Advantages of the proposal: this middleware architecture is strongly based on the capabilities of any other distributed system, which makes the proposal easily portable to any other environment where CPSs are used. The proposal makes use of common technologies for networking and securitization, so its implementation and deployment should be easy enough. Alas, by having an API it is possible to access the middleware in a very accurate manner and have a good grasp on what it is capable of providing. Security is a concept of major importance in this proposal, as it is clearly stated by the presence of a layer used for securitization as its main role. 
Disadvantages of the proposal: there are some major services as the ones used to provide semantic capabilities that are missing in this middleware architecture. The proposal has also included a level used for low-latency transport layer that, while a welcome addition, falls out of the traditional scope of middleware and may represent an extra work to have it working with the most usual transport layer protocols like UDP or TCP. Finally, the implementation works that have been carried out are conceived as something extra done for the benefit of the IP network, rather than to create a separate distributed software layer for hardware interoperability.

\subsubsection{A cloud optimization perspective}

Xi Fang et al. put forward their own proposal for a Smart Grid middleware-like architecture that uses cloud computing as a way to provide services in a distributed manner [59]. In this context, it can be said that cloud computing is a large-scale computational paradigm where the resources required to provide a service (storage, network utilities, etc.) are done in distributed infrastructures and/or platforms that make use of tools as the Internet or web services [60]. Cloud computing platforms are used in a widespread manner; companies such as Amazon or Microsoft offer their own solutions for commercial and research purposes, like Amazon Web Services (AWS, [61]) or Microsoft Azure [62]. According to the authors, cloud computing is a paradigm that should be used as a way to offer services in the application domain of the Smart Grid due to four reasons: a) it fits the high information processing requirements of the Smart grid, where changing prices during short periods of time demand high computation capabilities, b) cloud computing can improve information integration by avoiding having what they call "islands of information", c) it can be used to outsource some of the tasks related to information management, thus having a less complex system and d) it eases the duties of Distributed Energy Generation parties willing to enter the electricity marketplace, who are usually of smaller scale that traditional participants.

Service availability: the proposal relies on several domains that encircle the core functionalities that have been conceived by the authors, namely, the Smart Grid domain (composed of seven subdomains that tackle different functionalities related to the application domain of this thesis: service providers, operations, markets, bulk generation, transmission, distribution and the customers), the cloud domain (used to offer storage or computing services), the broker domain (a mediation party between the cloud domain and the smart grid domain that collects the needs of the main actors in the Smart grid and finding the best cloud services available to satisfy them) and the network domain (which provides the network and the communications infrastructure). According to the subdomains that are offered as part of the Smart Grid domain, the proposal can be regarded as a middleware architecture, as it has a collection of functionalities that are matching a collection of services offered in a distributed manner. Figure 22 displays the location of the main components that have been included in the proposal. Interestingly enough, middleware as such is not mentioned in the proposal, even though it is clearly used to interconnect heterogeneous entities belonging to different domains by means of a broker. 


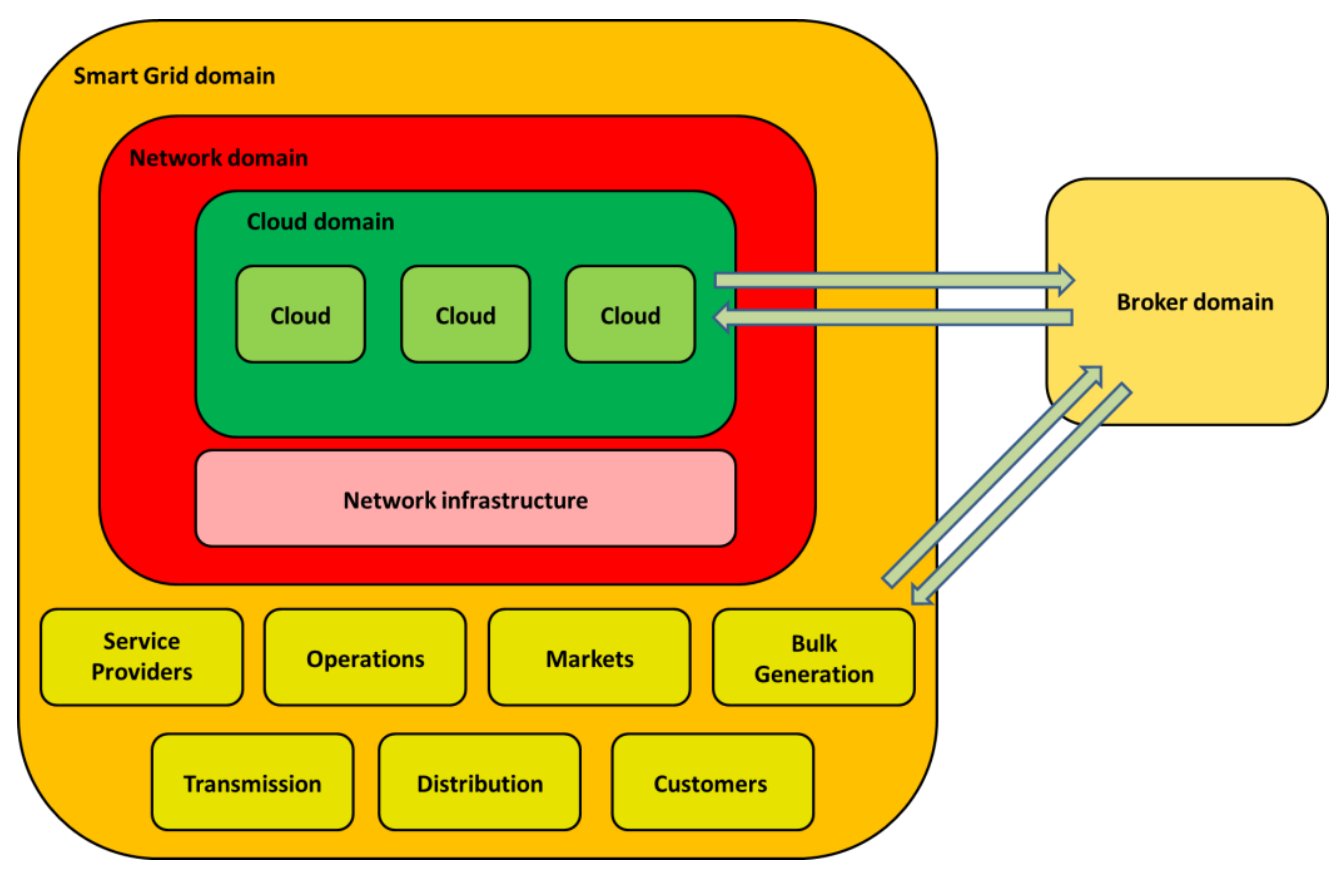

Figure 22. Main components of the cloud optimisation service, as shown in [59]

Computational capabilities: these are strongly linked to the paradigm of cloud computing. Since the underlying idea of the proposal is that the cloud will separate the ICT-related functionalities of the Smart Grid, any kind of device capable of running the algorithms and tools required for optimization (CPLEX Studio from IBM is mentioned as one of them) will be able to hold the middleware components. By "middleware components" it has to be assumed that they will be the main software subsystems associated to the subdomains present in the Smart Grid. The clouds that belong to the cloud domain might be regarded as part of the middleware as well, but due to the fact that they do not show what software components include, it can only be estimated what kind of facilities could be used. Overall, regular equipment (servers, Personal Computer-like appliances) falling within the facilities of the Aggregator or the TSO and DSO can be considered as the optimal choice to install the required software.

Message coupling: it is not possible to know what the main way to interchange messages in the proposal is. It can be assumed that a cloud computing-based infrastructure should be able to, at least, interchange data both in real-time (as mentioned in the related works that are described by the authors) or under a Publish/Subscribe paradigm (where data obtained from the Smart Grid domain can be stored until a subscriber interested in those data is registered).

Middleware distribution: since a cloud computing infrastructure is strongly implied in the proposal, it can be regarded as a mostly distributed one where even though most of the elements are distributed, there are also some more centralized ones (such as a the broker domain described by the authors) also included.

Therefore, the proposal can be described as:

$$
S G M=S A(3)+C C(0|| 1|| 2|| 3)+M C(0|| 3)+M D(2)
$$


Advantages of the proposal: the proposal is crystal clear in describing how it is a distributed one (by providing cloud computing services, any proposal becomes distributed almost by default). In addition to that, the idea of distinguishing the Information and Communication infrastructure from the power grid one is appealing in the sense that it makes easier to work in parallel in both areas, provided that detailed interfaces are provided to cooperate among them. Services with a major importance as security have also been included in the proposal, as well as some tests to assess its likely performance.

Disadvantages of the proposal: it would have been welcomed if the authors had provided some kind of API to reflect how interactions are done between the cloud, the network and the Smart Grid domain. While it can be assumed that commercial solutions have been used (Amazon Simple Storage Service, known for its ability to work with other cloud platforms [63], is mentioned in the proposal, as well as its pricing options) there is no more specific information. Finally, next to no information is given on how messages are interchanged among the software elements present in the proposal.

\subsubsection{KT Smart Grid Architecture and Open Platform}

Jisun Lee et al. have also described the approach that has been taken by KT (former Korea Telecom) to offer a commercial solution based on an energy management platform [64]. It is claimed to be based on the functionalities offered by a SOA, along with intelligent agents and business process management. The new functionalities that have to be dealt with in order to integrate the new facilities that become part of the Smart Grid (Demand Response, Distributed Energy Resources, Electric Vehicles, grid performance optimization or energy management for end users) are taken into account. One remarkable fact of this proposal is that despite being offered by a private company, it is regarded as an open service platform, so the scalability that it can offer a far as service availability is concerned should be guaranteed.

Service availability: while middleware as such is mentioned in the proposal, there are three different levels, as it usually is the case among middleware architectures. The first, uppermost level is the Customer Energy Management Systems (CEMS), which integrates, among others, the management capabilities required for home dwellers (Home Energy Management System, HEMS). The second one consists of a database that contains information about customers, energy usage or even metadata obtained from the system. The third level, referred to as KOC Platform, is used for management of functionalities as demand response (Demand Response Management System, DRMS), renewable energies (Renewable Energy Management System, REMS), smart metering information (Metering Data Management System) and for businessrelated operations (Business Supporting System, BSS). Apart from these levels, there is a low level interface used to connect hardware devices typical of the Smart Grid (Supervisory Control And Data Acquisition systems or SCADAs, AMI, power panels, etc.). The service platform that has been depicted in Figure 23 also enables the participation of third party providers and customers that will use the platform by means of service interfaces. 


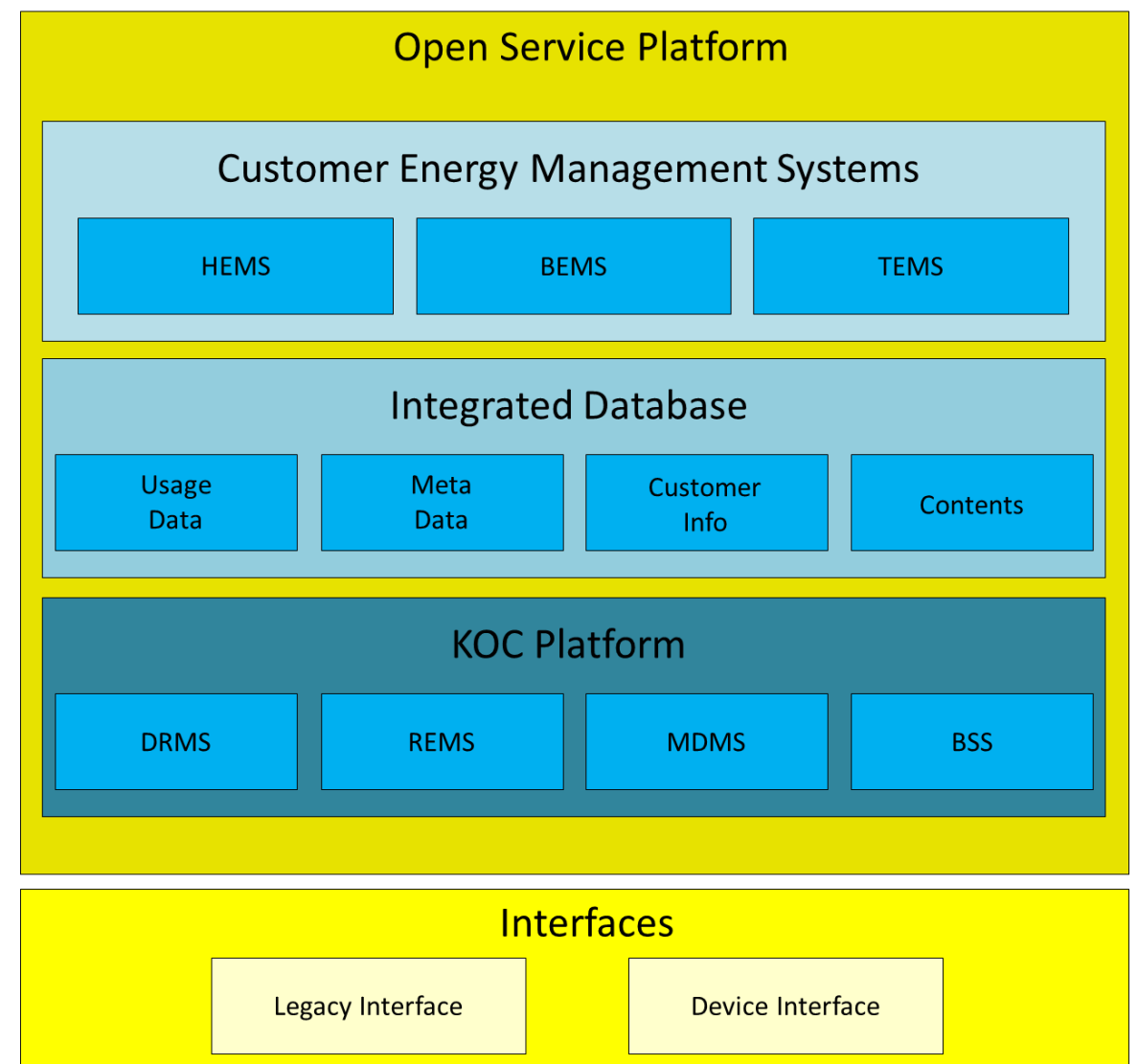

Figure 23. Main components of the Open Service Platform, as described in [64]

Computational capabilities: despite being clear that the proposal is accessing a plethora of devices, it is harder to tell where it is expected to be installed. Arguably, since information is going to be collected from AMIs or SCADAs, it could be located in a device that is outside those, so it would belong to either the aggregator or other participants like the TSO or the DSO. In that case, hardware as servers or mainframes should be powerful enough to have the components installed.

Message coupling: it is explicitly mentioned in the proposal that the data is analyzed and displayed in a real-time fashion. Apart from that, few data are provided on how the information is transferred throughout the architecture.

Middleware distribution: it is implied in the proposal that the platform will be installed throughout all the locations where its services are required. Yet there are several specific devices (specifically, the ones installed in the end users dwells) that are expected to feed data to the system, so it can be said that it will be a mostly decentralized proposal.

This middleware for the Smart Grid can be also described as:

$$
S G M=S A(3)+C C(1|| 2|| 3)+M C(0)+M D(2)
$$

Advantages of the proposal: the proposal has also been tested in an actual scenario where information about energy consumption or energy flow is offered to end users. Perhaps as a consequence of having a realistic deployment done, concerns about electricity usage and 
information about the users of the platform is widely available. The idea of having the platform as an open development is positive in the sense that it allows the improvement of the architecture according to the needs of the end users that may become participants of it.

Disadvantages of the proposal: there are some concepts and terms of the proposal are not correctly explained, such as how data is sent from one side of the architecture to the opposite one. Likewise, end users are regarded just as consumers, instead of taking into account that they could also be in the position of injecting electricity into the power grid. Furthermore, there are several aspects of the deployment, like securitization of services, that are not mentioned and it is not known how they would be implemented, or even if they would be. Finally, information about how to access the application layer or what kind of Application Programming Interfaces are offered is not available.

\subsubsection{Smart microgrid monitoring with DDS}

The authors of this proposal [65] rely on an already developed middleware solution in the form of Data Distributed Service (DDS) to offer interoperability among the devices present in a microgrid. DDS is a standard defined by the Object Management Group (OMG) that defines a middleware protocol and an Application Programming Interface for interconnectivity in a datacentric fashion [66]. It describes how to interchange information at the data level (as opposed to bits, frames, packages or frames) by means of a Publish/Subscribe system, among other functionalities offered. The DDS specification has been conceived as a Data-Centric Publish/Subscribe (DCPS) model that describes all the features required to understand the procedures associated to DDS, to the point that a Platform Independent and a Platform Specific Model (PIM and PSM) are thoroughly depicted [67]. The DCPS model also makes use of a lower level, real-time oriented architecture referred to as Real Time Publish Subscriber (RTPS) [68]. This architecture is used to interconnect different DCPS-based, upper level developments. In order to understand how DDS works, there are several terms that have to be known. A topic, for example, is a definition for an association of participants in a communication specified and distinguished from others by several features (the topic name, the topic type and the topic domain ID). A domain, on the other hand, is a data space that comprehends a logical network of participations $[69,70]$ where several entities referred to as domain participants publish data and become subscribed to other pieces of information. Lastly, the Publish/Subscribe paradigm is pivotal in the development of a DDS solution, as it is the most prominent communication paradigm used whenever data are going to be transferred by means of this solution. In an environment like this, the publisher will implement a data writer, whereas the subscriber will have a data reader to collect the data that was published by the other entity of the communication. Figure 24 shows the main participants of a DDS communication where data is published and transferred to the subscribers.

Since the OMG only provides a description of the standard, it is left to companies, universities and research institutions the actual implementation of DDS solutions. There are solutions that have been developed under the idea of providing an open source solution to a greater or a lower extent (OpenDDS [71] or the OpenSpliceDDS developed by PrismTech [72]) whereas 
other solutions utilize a more commercial approach (eProsima [73] or the CoreDX solution developed by Twin Oaks [74]). Commonly, commercial solutions require licensing agreements for their usage in any kind of middleware development; open source-based solutions hardly ever require the same, even though the kind of license they are using must be taken into account so as it will not backfire and creates issues when exploiting the developed software.

As for the proposal itself, the authors take advantage of the Quality of Service features provided by DDS as a way to enhance the power grid. The RTI distribution of DDS is explicitly mentioned as being capable of providing the required efficiency and reliability for data communications. They stress the importance of having a data-centric model due to the fact that they are able to avoid having to worry about other lower level tasks (network locations, etc.). As far as the implementation of the proposal is concerned, it uses the Interface Description Language (IDL) as a way to define the data that are going to be transferred (that is to say), since IDL files are the ones used by DDS implementations to include the data that is relevant for a deployment. IDL is oblivious to the programming language that is used, so it enables the implementation of a DDS-based solution regardless of how it is programmed. A microgrid monitoring system is also offered in the proposal, where a DDS-enabled data acquisition system is used for data publishing.

Service availability: this proposal solely conceives middleware to transfer messages that will collect information from microgrid devices while offering this information via DCPS (as it offers an API that can be used by applications to access the data). Therefore, it can be regarded as a Message-Oriented Middleware with no further services (such as security or semantics) encased in it.

Computational capabilities: little is said about what kind of devices is expected to have the DDS components installed. Since the middleware is collecting data from devices as wind turbines or IP cameras, it can be inferred that it will be installed in other appliances that receive those data. Therefore, the middleware proposal will be installed in pieces of equipment that would correspond to the aggregator or the TDO/DSO infrastructure. They might even be installed in power plants, since it can be used to track real-time events that force the electricity production to change in one way or another. 


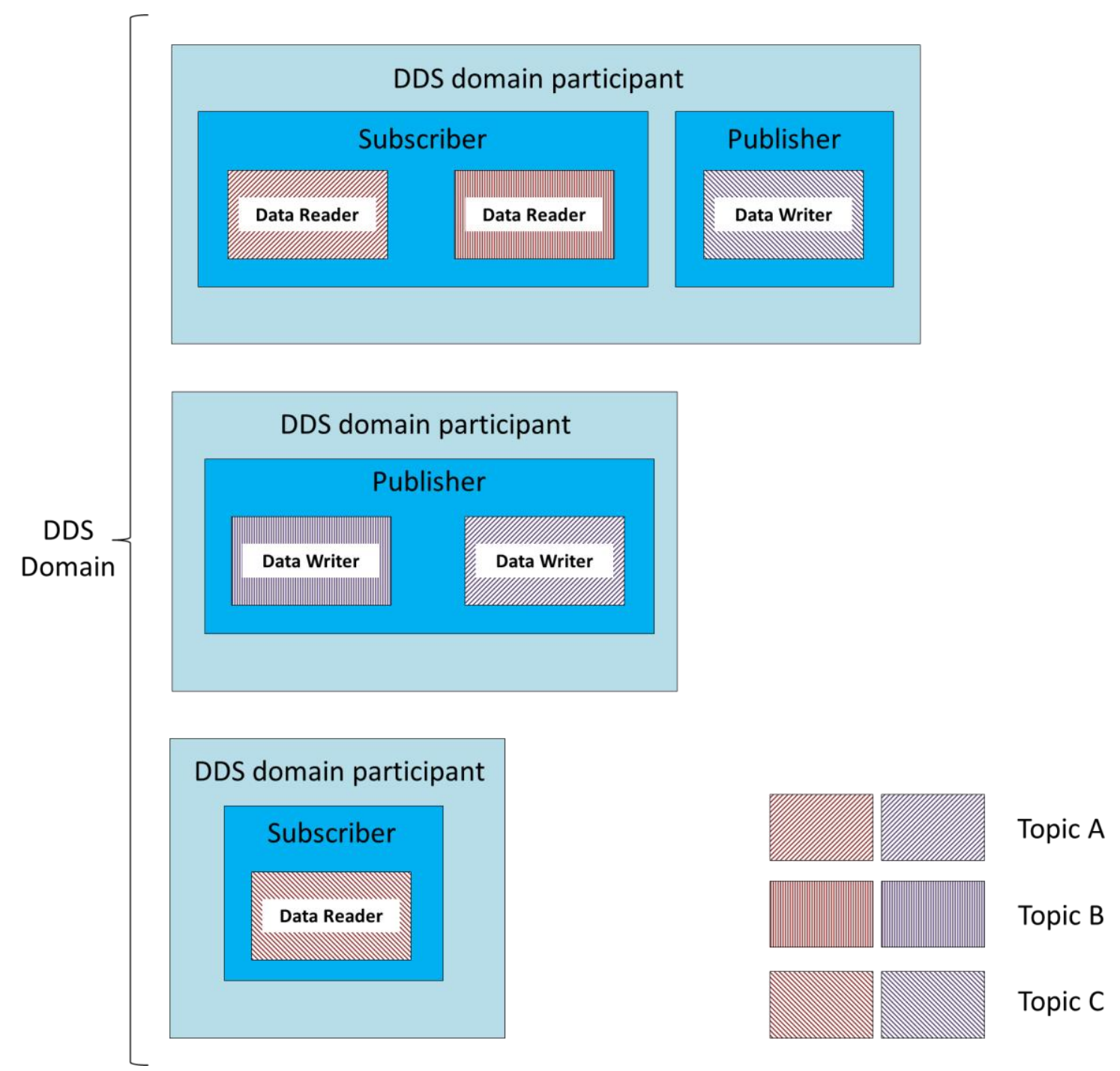

Figure 24. Main features of a DDS deployment, as described in [65]

Message coupling: the proposal is clearly oriented to two ways to transfer data, namely, Publish/Subscribe and real-time paradigms. On the one hand, DDS is heavily reliant on a Publish/Subscribe model for communications. On the other hand, a database has been conceived in the proposal where real-time data generated from the devices and the non-end user equipment. These data end up stored in the historical database for data playback, in case it is needed for information analysis or system performance.

Middleware distribution: DDS is consistent with the idea of having a distributed system sharing data among different elements. Consequently, it is a mostly distributed architecture that still has some elements more likely to have a higher level of computational capabilities (for example, a DB server will usually be more powerful than a mobile device) and hence have more functionalities installed.

The middleware solution that has been presented here should be regarded as:

$$
S G M=S A(2)+C C(1|| 2|| 3)+M C(0|| 3)+M D(2)
$$


Advantages of the proposal: DDS is a portable middleware solution that has already been proven as efficient in several application domains. It is flexible enough to support different kinds of communications (Publish/Subscribe, real-time) and is able to provide an API that can be used to the advantage of the application developers. In addition to that, its distributed nature makes possible the addition of an ever-increasing number of infrastructures used in Distributed Energy Resources.

Disadvantages of the proposal: While DDS results convincing in many ways as a middleware solution for the Smart Grid, it presents trickier challenges related to Intellectual Property Rights and licensing: OpenSpliceDDS is licensed under the third version of the GNU Lesser General Public License (LGPLV3) for its usage that, unlike the ordinary GPL, does not automatically imply that the developed software will also be of free nature [75]. Depending on the kind of development that is going to be carried out, this might be desirable or counterproductive. On the other hand and in case of CoreDX, a license might be provided for research and runtime or for commercial exploitation, which could imply a payment to Twin Oaks. In the end, many of the advantages and disadvantages are associated to the fact that a solution already developed by a third party is going to be ported to a microgrid, which may result in the need of getting commercial agreements on the libraries and the content that can be used. Regarding the aspects of the proposal that are not related to DDS, it can be said that, for the good and the bad, it merely ports the middleware standard to a microgrid, offering a reliable solution easy to port to other environments but no additional services that would enhance it are included. Besides, there is little information on what kind of devices is expected to have the middleware.

\subsubsection{ETSI M2M}

Guang Lu et al. put forward a proposal [76] where it is proven how a set of standards for Machine-to-Machine communications designed by European Telecommunications Standards Institute (ETSI) [77] can be ported to the Smart Grid. ETSI is an institution known for its efforts in Information and Communication Technologies in general, with significant contributions in the Internet of Things. It is stated by the authors of the proposal that the application domain involving the Smart Grid presents a series of challenges that are common to the ones that have to be tackled by middleware solutions, like interoperability (conceived in the proposal as the capacity of two or more systems to exchange information based on a common set of communication specifications) and scalability. Some other features such as security (key management, authentication, cryptography) and device management (configuration, performance, firmware/software, etc.) are considered in the proposal, at the same time as service specific of the Smart Grid (demand response). The architecture design that, according to the authors, is offered by ETSI M2M, offers the idea of Service Capabilities (SCs) as a way to provide the functionalities to be shared by the applications located in the upper layer. Some of the most prominent SCs are: Application Enablement, Remote Entity Management, Telco Operator Exposure or Interworking Proxy. 
Service availability: the information provided by the authors of the proposal mentions how the Service Capabilities in the ETSI M2M architecture can be installed in a quite distributed manner. The proposal is also claimed not to require a specific underlying access technology. Furthermore, an open API is mentioned as a way for the applications to obtain access to the middleware. Taking into account that security and device management are also implemented in the proposal, it can be mentioned that this is a middleware architecture. When applied to the Smart Grid, there will be a collection of control centres that will gather as particular domains of a M2M Core that communicates via network with a group of Smart Grid Systems that include the components that deal with the main facilities of a deployment (generation, transmission, distribution). Its overall appearance has been displayed in Figure 25.

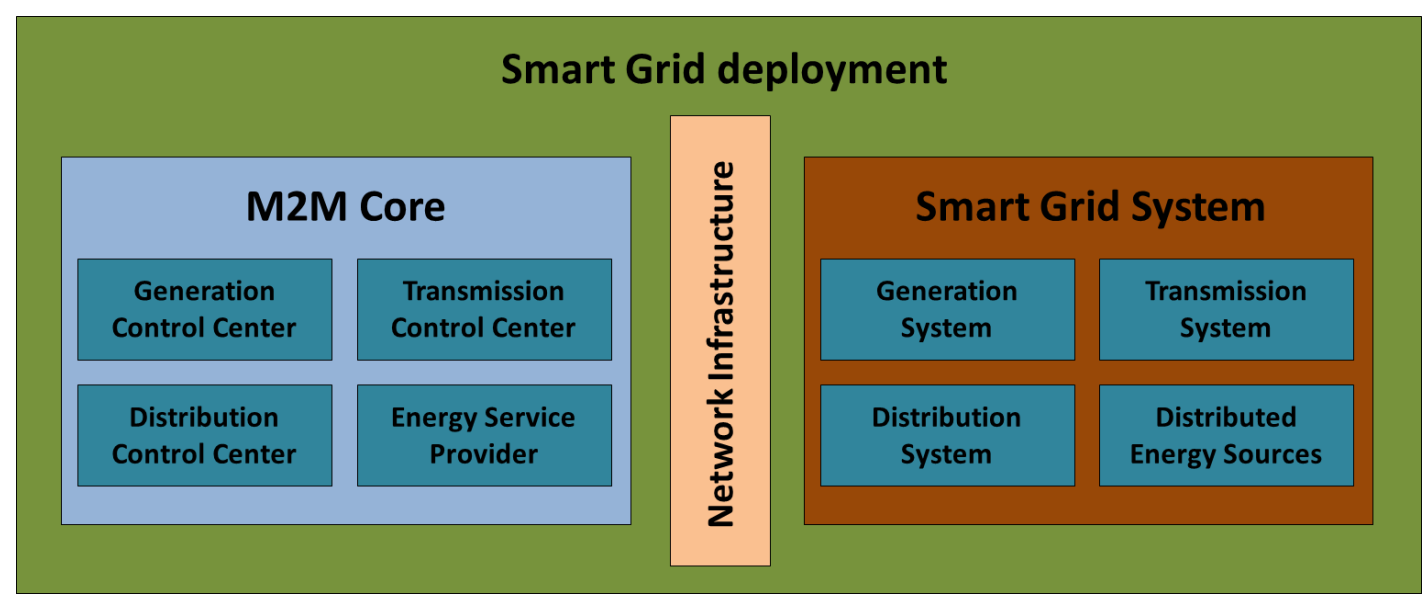

Figure 25. Structure of the ETSI M2M proposal, as described in [76]

Computational capabilities: it is clearly stated in the proposal that Service Capabilities are installed in devices as gateways and Machine-to-Machine communication cores, which can be estimated as regular PC-like or server-like hardware components in a deployment. In addition to that, since ETSI M2M is based on the Internet of Things, it has been conceived to be installed in almost any location. Therefore, the proposal could be installed almost anywhere in a deployment, such as the Advanced Metering Infrastructure of a home dweller, Aggregator, TSO or DSO equipment or even monitoring the facilities of a power plant.

Message coupling: there is little data about how messages are expected to be transferred. While it is mentioned once that a real-time automated response is to be expected, the most predominant idea that has been considered is that there are appliances like servers for information on power resources or how M2M servers have been included in the proposal, so it can be said that a Client/Server architecture is the one that is coming closer to how messages are interchanged. No information available about the existence of a broker is present either, so it can be assumed that a publish/subscribe paradigm is unlikely to be used in this case.

Middleware distribution: as it usually is the case with loT-like proposals, this is a mostly decentralized architecture that is able to run in devices of very different nature, even those that have limited computational resources. In addition to that, a peer-to-peer paradigm could be implemented as well, as three are several machines that will communicate with each other 
(which can be deduced from the preeminence of the concept of M2M in the proposal) without requiring the intervention of any end user or application with a Graphical User Interface (GUI).

This proposal can be represented by:

$$
S G M=S A(3)+C C(0|| 1|| 2|| 3)+M C(2)+M D(2)
$$

Advantages of the proposal: this middleware architecture covers most of the prominent issues that have to be tackled in a middleware for the Smart Grid, such as service and API availability, security or interoperability. Details about how prototyping is performed and the kind of tools that have been used are profuse and focused on each of the characteristics that have been deemed as important by the authors (interoperability and scalability, security, device management, demand response). The fact that this proposal is ported from an loT application domain also points out the fact that CPSs share multiple challenges that can be addressed with a similar kind of solution.

Disadvantages of the proposal: information offered about the challenges and the activities that have to be carried out to port the set of ETSI M2M standards from the loT to the Smart Grid is somehow confusing. While it is mentioned a wide plethora or services that can be added, sometimes it is not clear how they are implemented, if either a set of protocols cooperating with each other or as software components that are executed in different pieces of hardware. Finally, how data messages are transferred from one part of the deployment to the other is described with too scarce information.

\subsubsection{Smart Middleware Device for Smart Grid Integration}

Oliveira et al. [78] show in their work regarding middleware how it can be included as another software component in a single hardware device tailored for Smart Grid integration. One of the main issues that according to them is yet to be fully dealt with is the integration between devices and protocols such as Modbus (considered as an standard for the industry application domain), which would require special gateways in a deployment. This proposal puts forward a design and implementation for a gateway of this sort, which has been named Smart Middleware Device (DMI in the Portuguese acronym used by the authors). The appliance is capable of managing the software components to be used for protocol translations, along with data flow characteristics. This device is expected to be used as a connector between the power grid and the ICT domains, as it will be linked to other power stations present in the grid, as well as to routers included in the network infrastructure.

Service availability: DMI has been conceived as a middleware architecture because it explicitly mentions a plethora of services that have been developed. According to the authors, though, the middleware solution will be included just as a part of the device that has been implemented for that purpose, so it will be present only in one device. As for the services, they are part of two different groups: the translators and the core components. Translators will be used as a way to transfer information from the protocols deemed as standards to be considered by the proposal (Modbus, IEC 61850 and Distributed Network Protocol, version 3; it 
is hinted that other protocols might be integrated too). At the same time, the core components are used for the usual requirements faced by information transferring in the Smart Grid: queries done between its different elements and units, a repository to store those answers and a management component to control operations as queries (or queries pushed to a SCADA) or answers that need translations. The appearance of the internal components of the proposal and how they interact with external elements has been summarized in Figure 26.

Computational capabilities: rather than considering what kind of device could have the proposal installed, the middleware is expected to run in a specific one. According to the specifications offered by the authors of the proposal, a DMI has been assembled and runs as a service under a Linux, Berkeley Software Distribution (BSD)-like operating system. Considering those features, it could be included as part of the pieces of equipment installed in almost any location, even though the fact that it is used for translating protocols used in power substations points out that it could be located as part of the TSO or the DSO infrastructure.

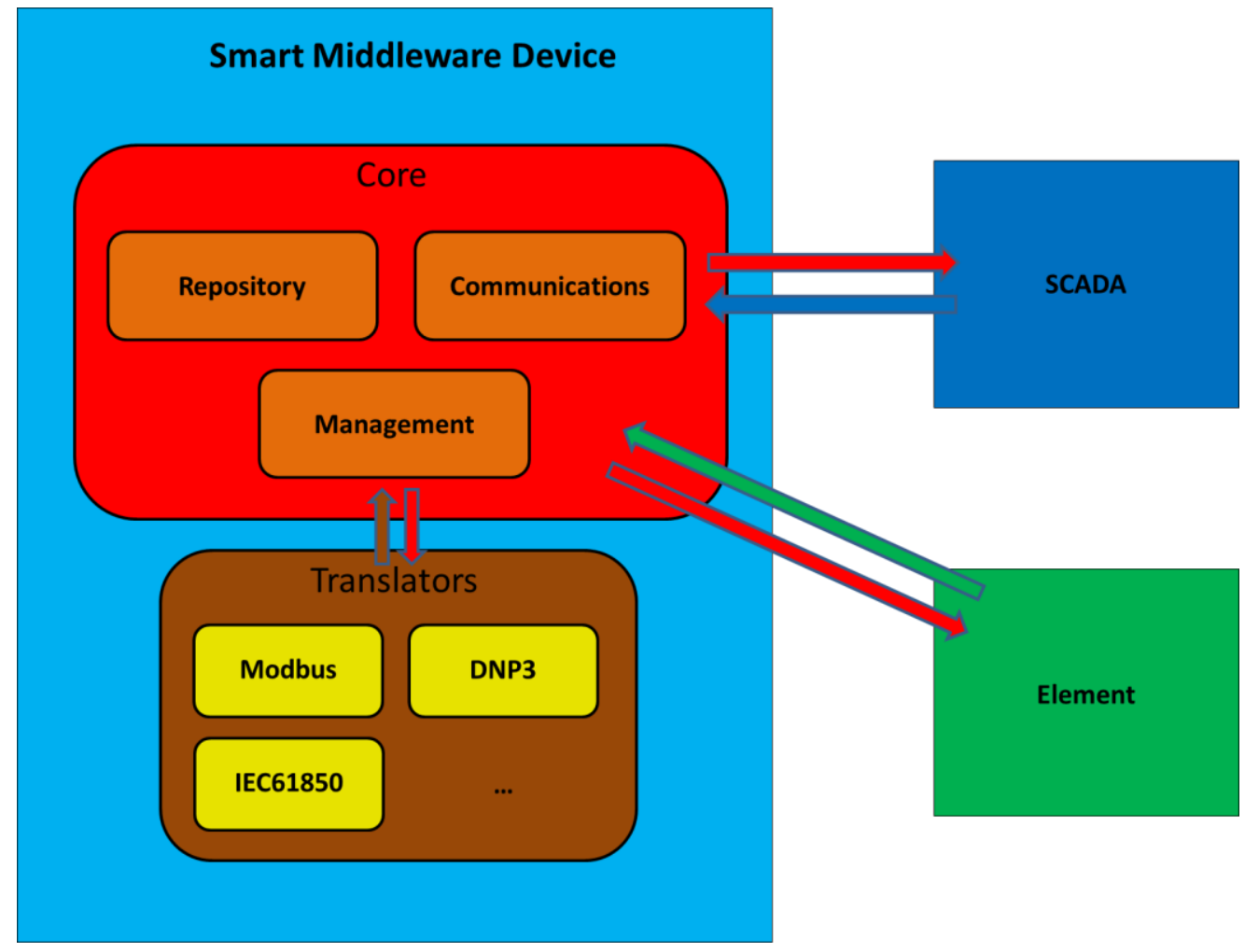

Figure 26. Smart Middleware Device, as described in [78]

Message coupling: it is mentioned by the authors that when tests were carried out with the device, it was able to receive real-time data from a power grid, which was used to configure Quality of Service indicators that would trigger alarms or actions to be taken. In addition to that, it is also explicitly stated that it has been defined within a client / server architecture.

Middleware distribution: this proposal is fully centralized in one single device (the Smart Middleware Device), which is the one used to include all the software components required for data transmission and protocol translation. 
According to the matrix that was defined before, this proposal can be defined as follows:

$$
S G M=S A(3)+C C(2|| 3)+M C(3)+M D(0)
$$

Advantages of the proposal: the authors have included information about actual tests that have been carried out by integrating the DMI with a Smart Meter, and a General Packet Radio Service (GPRS)-enabled device used by the device of the proposal to send commands and receive readings. Integration of different kinds of protocols by the same kind of entity is also addressed by the proposal; translating data representations from one format to the other is also to be expected considering how middleware is used.

Disadvantages of the proposal: one of the most important decisions taken by the authors of the proposal is confining the middleware in a single device, which will require having that device whenever middleware is required, so middleware is not actually providing hardware abstraction in this case. Overall, there are not many data on how the put forward solutions are going to be implemented for a distributed environment, and the fact that the middleware will be located in one single device makes it more vulnerable for the deployment, as middleware may completely fail in case there is a malfunction on the hardware that has it installed. What is more, the idea of having a device embodying the concept of middleware can be disorienting, as it looks as inconsistent with the intention of having middleware as a software layer installed in several devices and appliances that will negate the differences between them to offer a homogeneous set of facilities to an upper layer. Lastly, there are some major features like security, semantics or an API for the application layer, which are not mentioned in the proposal.

\subsubsection{WAMPAC-based Smart Grid communications}

Aditya Ashok et al. stress the importance of security in CPSs in their work [79]. Specifically, they address a Wide-Area Monitoring, Protection and Control subsystem that is included in a Smart Grid. It is claimed in the proposal that PMUs are the main components of the power grid that take advantage of the existence of a WAMPAC subsystem, as it leverages the PMUs to obtain real-time knowledge from the operations that are being carried out in the power grid where it is installed. According to the authors, WAMPAC can be divided into several different subdomains: Wide-Area Monitoring Systems (WAMS), Wide-Area Protection Systems (WAP) and Wide-Area Control (WAC). As it happened with former proposals, the authors rely on a SCADA system to collect information from outside the middleware. They also provide a classification on cyber security attacks (timing attacks, data integrity attacks, replay attacks) that can be performed onto a system. According to the proposal, deploying the different WAMPAC subsystems can have several challenges: for example, WAMS must be able to provide high availability, integrity and some degree of confidentiality in utility data. Furthermore, WAPAC schemes, based to an extent on power-related protocols such as IEC 61850, must guarantee that messages that are transferred ensuring that they have been authenticated to separate malicious information or commands. Finally, the idea of a real-time WAC using information from a PMU is planned to be further researched. The proposal makes 
use of a game theoretic framework in order to model features from both cyber and physical points of view.

Service availability: the authors' proposal is an architecture built on the three subdomains that WAMPAC is made of. WAP, for example, relies on large amounts of information collected from the whole deployed system, which will be used to enable decisions that will counter disturbances across the system. Furthermore, WAMS is claimed to be responsible for distributing information in a reliable way (which involves an infrastructure that must offer integrity and high availability regarding the PMU data) and needing a high-speed networking infrastructure. Lastly, WAC is mentioned as a potential way to offer applications as secondary voltage control, static control or inter-area oscillation damping. The location of the WAMPAC controller in a deployment is shown in Figure 27. Note that this WAMPAC component that has been included is regarded as part of a wider Smart Grid deployment bent on solving security issues, so it is not conceived to be a whole middleware solution.

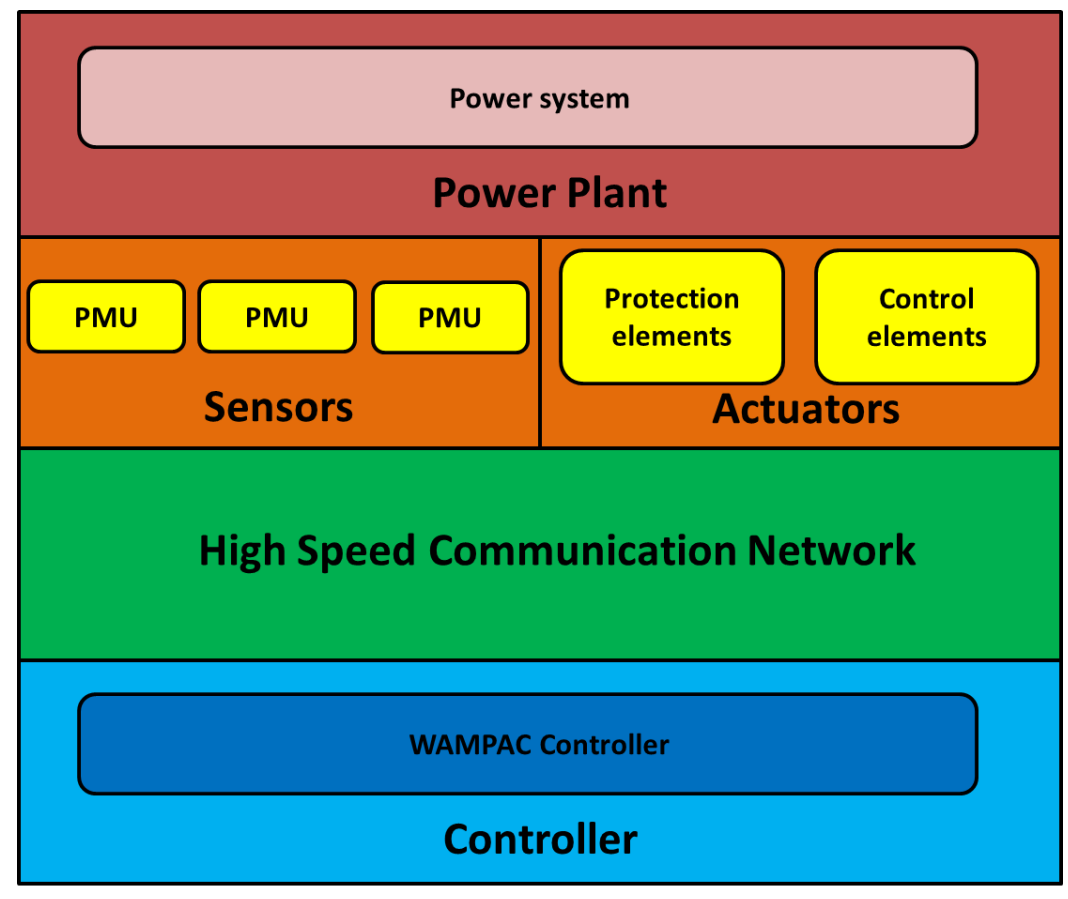

Figure 27. WAMPAC controller location, as described in [79]

Computational capabilities: even though not very much information is provided about this feature, it can be said that the WAMPC controller involves networking, security and data management solutions, so it is supposed to be part of the facilities used for communications and data interchange between the end users' equipment and the infrastructure used to generate electricity, that is to say, the TSO and DSO pieces of equipment.

Message coupling: as it happened with the former case, there are few data to get a grasp about how messages are transferred, except for the idea that securitization must be provided. Nevertheless, real-time communications are mentioned as the most usual ones that take place in the proposal scenario. Furthermore, the approach taken by the North American SynchroPhasor Initiative (NASPI) regarding secure synchronized data measurement infrastructure 
(NASPInet, [80]), where a Publisher/Subscribe component has been implemented, is mentioned in the proposal as a known solution.

Middleware distribution: while the proposal makes use of a distributed domain for information interchange, and it is included in a distributed system as the Smart Grid is, it is not made clear how devices are expected to be deployed where the proposal can be installed. Considering the kind of infrastructure mentioned in the proposal, it can be claimed that it will be a mostly decentralized solution, as the WAMS component requires a network for data delivery.

This proposal can be further described as:

$$
S G M=S A(3)+C C(2|| 3)+M C(0|| 3)+M D(2)
$$

Advantages of the proposal: security is strongly tackled in the proposal and it is made clear how the Smart Grid can be securitized against the most damaging attacks. Also, the concept of using a game theory framework as a tool for security in Cyber-Physical Systems is interesting and offers a different point of view on how to address it. The authors of the proposal have also provided a justification of how a testbed can be implemented to test security in a Smart Gridlike deployment when game formulations are involved.

Disadvantages of the proposal: while the focus of the proposal on security is welcomed, as it is a functionality of major importance in a distributed system, there is less information about all the other services expected to be part of a middleware architecture (semantics, API, etc.). Clearly, the main idea that the authors want to transmit is that they have created a system capable to provide secure communications, rather than a middleware solution with several services encased.

\subsubsection{C-DAX}

Michael Hoefling et al. [81] provide their own vision of what a secure middleware should be according to what has been designed in the research project called Cyber-secure Data and Control Cloud for power grids (C-DAX, [82]). As it was presented in other proposals, their solution is focused on solving security issues found in data exchanges for the Smart Grid (the authors refer to Active Distribution Networks or ADNs). Facilities that are likely to make use of this proposal according to the criteria of the authors are: Phasor Measurement Units (PMUs), the ones related to Real-Time State Estimation (RTSE) and Phasor Data Concentrators (PDCs). RTSE applications are described as receiving aggregated information from the PDCS and feeding it in a mathematical model used to estimate the current conditions of the grid. PDCs are used for the reception of data that have been timestamped (refreshed at a frequency of 50 $\mathrm{Hz}$ ), time-aligned and aggregated from different PMUs. Information is sent throughout a deployment with this solution according to topics established to separate one kind of content from the others. These topics are defined as representations of abstract nature used for unidirectional information channels with a certain storage capacity. PMU measurement is made by using NASPInet (North American Synchro-Phasor Initiative Network) as the protocol. 
Service availability: the middleware that has been prepared for this project can be regarded as a Message-Oriented Middleware, considering that the main efforts of the proposal are centred in creating a secure procedure for the interchange of messages in the deployment, instead of offering a collection of services that will be contained in the software infrastructure chosen for the middleware. Two planes of information have been defined as a way to organize the data: control plane and data plane; the latter is utilized in combination with Designated Nodes (DNs) that grant access to the cloud used to implement the middleware proposal for the C-DAX project. Data Brokers (DBs) are used as a way to either store or send forward information. Finally, there is a Monitoring and Management System devoted to monitor and control the deployed devices. The overall appearance of the proposal has been displayed in Figure 28.

Computational capabilities: the proposal is expected to use pieces of equipment that are to be expected according to a regular Information and Communication Technologies deployment. It is also mentioned in the proposal that in order to perform the tests, a $100 \mathrm{Mbit} / \mathrm{s}$ link has been used. Therefore, and taking into account that the proposal deals mostly with data transmission and securitization, it can be inferred that it is expected that TSO or DSO infrastructure will be the main beneficiaries of the security measures that are described by the authors. In addition to that, pieces of equipment present in power stations may also welcome having the proposal installed, as it will add security to information of critical importance for their performance, as it will depend on the feedback obtained from a Smart Grid.

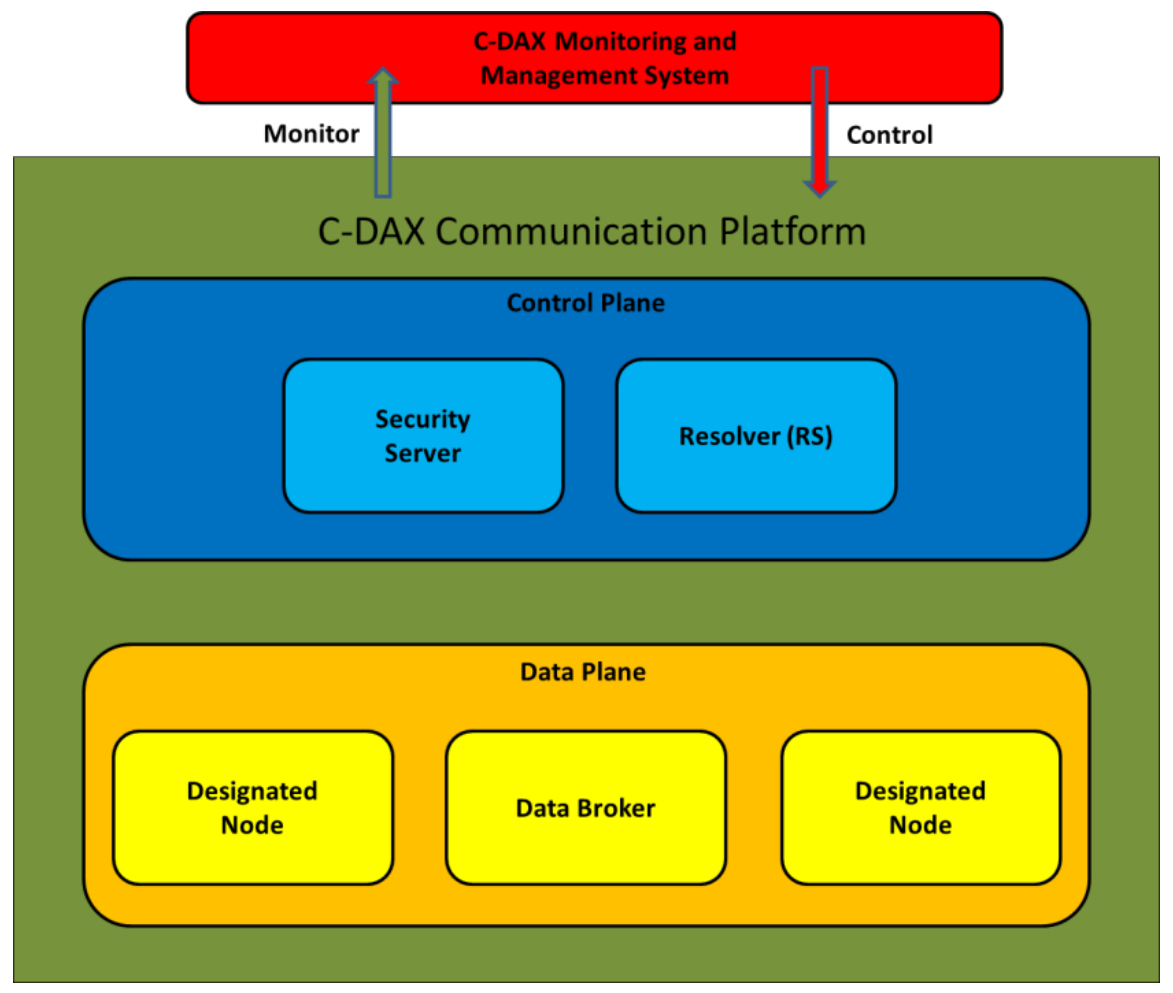

Figure 28. C-DAX middleware appearance, as described in [81]

Message coupling: the proposal makes possible several different ways to establish communications. The authors distinguish three of them: streaming communication mode (where subscribers receive topic data after becoming part of one of them in a continuous, noninterrupted way), query communication mode (subscribers send explicit data queries) and 
point-to-point communications (without using Designated Nodes or Data Brokers). However, it can be said that there are two different paradigms in the proposal that are heavily mentioned and used. On the one hand, real-time data are required to be taken into account, as they usually imply information obtained from the smart meters deployed in the Smart Grid deployment in an almost instantaneous fashion. On the other hand, information is published throughout the data plane (and by means of the designated nodes that are at both ends) using a Publish/Subscribe paradigm, where application data is published in one end of the communication and is consumed by the subscribed located at the other end. It is explicitly stated by the authors that one of the objectives of the project is to adapt the Publish/Subscribe paradigm to the needs of power grids. Finally, Client/Server communications are discouraged by the authors of the proposal, claiming that they demand servers to be configured properly and clients have to be re-configured when they have to establish connections with backup servers.

Middleware distribution: the proposal is a mostly decentralized middleware solution due to the fact that it is distributed in several different pieces of equipment that are part of the ICT infrastructure of the Smart Grid (subscribers, publishers, databases) and a communication infrastructure has been used in order to perform tests on the proposal. Since there are elements that manage data reception and delivery (such as the Data Brokers) there will be still some more prominence of several software elements over others, thus proving that the solution is not entirely peer-to-peer, with similar features in each of the nodes where the proposal has been installed.

This proposal can be described as:

$$
S G M=S A(2)+C C(2|| 3)+M C(0)+M D(2)
$$

Advantages of the proposal: the authors offer a compelling proposal that has been tested in a realistic scenario (a laboratory belonging to one of the project partners). There are several scenarios that have been consider for testing activities, being failing DBs one of them.

Disadvantages of the proposal: The number of services included in the proposal is more modest than in other ones, due to its condition of being a Message-Oriented Middleware. No information is provided regarding an API to be offered to the higher levels as a specific way to interact with the middleware shown here.

\subsubsection{Building as a Service (BaaS)}

The main concept that has been implemented in the proposal led by Susana Martin et al. is the usefulness of the Smart Grid for energy efficiency in buildings [83]. According to the authors, buildings can be used to obtain services from them (hence the term Building as a Service) by developing a middleware solution used to link a collection of entities of a CPS that stresses the importance of energy consumption. The Open Services Gateway initiative (OSGi, [84]), which is claimed to offer openness, interoperability and transparency, offers interfaces in the implementation works required for the solution. Several other ideas common to this kind of 
application domain (Event-Driven Architecture, Service-Oriented Architecture) have also been included in the proposal. Interoperability among several interlinked entities is provided by integrating Building Information Models (BIMs), Data Warehouses (so as to store information as a whole) and Building Management Systems (BMSs) and the legacy ICT facilities as a single system. Furthermore, services based on optimal control, prediction and assessment have been included too.

Service availability: The proposal is considered to be a middleware architecture due to the fact that it has included several devices that are distributed in different layers. However, the only software component regarded as middleware by the authors of the proposal is the Communication Logic Layer or CLL. It is encased with two more levels as part of the intermediation architecture defined for this proposal, that is to say, the Application Layer and the Data Layer. The Data Layer has included information dealing with the services related to the building used for the BaaS concept (for example, the ICT infrastructure for weather and access control), the Data Warehouse infrastructure, the Building Information Modelling Server and the external services responsible for collection information. The Communication Logic Layer is composed by two sub levels: the Core Communication and the Data Access Object sublayer. The first one consists of the Domain Controllers or DCs and the Data Acquisition Control Manager or DACM. The second one adds the Data Access Objects for the DC and the DACM. Figure 29 shows the overall appearance of the proposal.

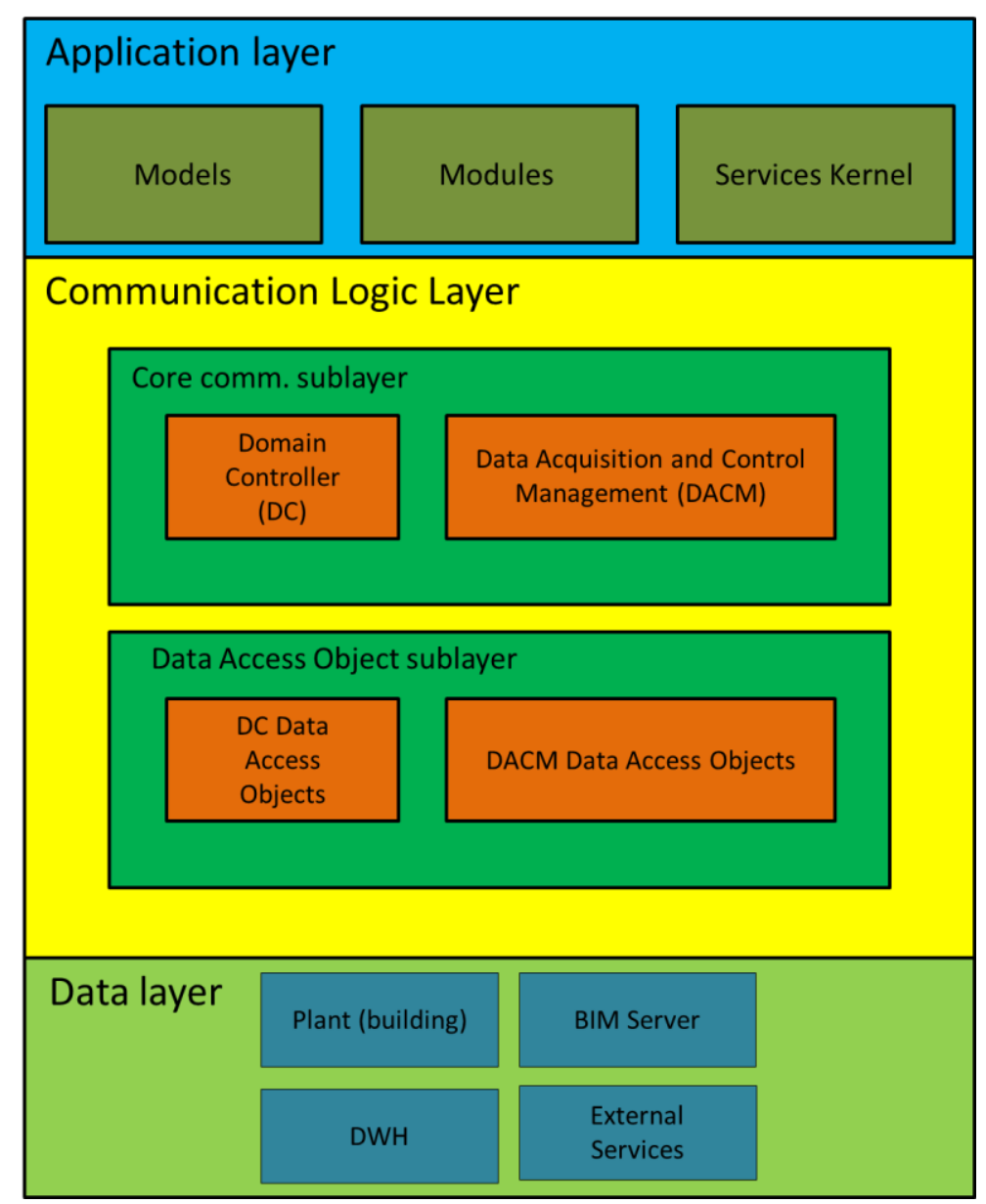

Figure 29. High level architecture of the BaaS system part of the proposal, as described in [83] 
Computational capabilities: the software components that the middleware architecture is made of are implemented as OSGi-bundles that are usually on the kilobyte or the megabyte scale, so it is easy to install the software in any kind of piece of hardware with normal and even low capabilities. Considering that data is being obtained from sensors it can be argued that the middleware solution can be installed in any intermediate piece of equipment belonging to the Aggregator, the TSO or the DSO.

Message coupling: it is explicitly said in the proposal that this middleware has been conceived for applications that follow a Client/Server architecture. Additionally, it is mentioned that the proposal has been designed taking SOA and EDA concepts into account and a modular eventdriven design has been created. On the other hand, it is added that components can be subscribed to events in an EDA architecture, so it is subtly implied that there could some kind of Publish/Subscribe system. However, this point is never mentioned explicitly, so a Client/Server approach should still be considered as the one that is used here.

Middleware distribution: the proposal is a mostly decentralized one due to the fact that while it is distributed in different elements that are independent from each other (the buildings) there are still some elements that have a higher degree of responsibility than others, such as the Data Acquisition and Control Manager.

Considering all these features, this middleware proposal can be described like this:

$$
S G M=S A(3)+C C(1|| 2)+M C(2)+M D(2)
$$

Advantages of the proposal: the proposal makes use of an API for data interchange that makes it easier for application developers to access the middleware solution, and how levels are interfaced with each other is made clear. Moreover, the usage of OSGi technologies is consistent with the idea of having open source software technologies for the middleware, which is a desirable feature for further scalability and improvement of the implementation works done. Finally, the proposal makes use of a collection of standardized technologies that ease the replication and implementation of software solutions. The proposal makes use of software technologies of widespread usage that have proven to be useful, such as Java Database Connectivity (JDBC) in the Data Warehouse component, JavaScript Object Notation (JSON) for the Building Information Model or Simple Object Access Protocol (SOAP) in the Building Management System.

Disadvantages of the proposal: Services of major importance to be encased by a middleware solution that are related to security or the semantic enhanced of information are not present in this related work. Furthermore, it is very centered on the idea of using it only for energy consumption in buildings, which may make challenging to port the middleware architecture to other applications related to the Smart Grid, or even other Cyber-Physical System.

\subsubsection{Middleware-based management for the Smart Grid}

Villa et al. put forward in their proposal [85] how a hardware platform can be used to manage, control and integrate electrical installations that are large scale and heterogeneous. 
Specifically, they refer to it as Embedded Metering Devices (EMDs), capable of controlling pieces of equipment that require no parameters to be changed. Among the features that are claimed to be enabled by the authors, adaptability (EMDs for devices that require no changes), scalability, availability (segments of the network can continue working in an autonomous way in case of failure) and hierarchical design are the most prominent ones. Other advantages related to the hardware and its components are: its small size and cost, low energy consumption, flexible access, access transparency and conventional tools. The proposal aims to have a generic platform capable of develop power management services for any kind of environment. It should be noted that the main interest of the authors is regarding the device as Advanced Metering Infrastructure, rather than software components to be installed in one piece of hardware in a microgrid.

Service availability: while there is very scarce information about the services that can be provided, it can be said that the software used for device interconnectivity is solely employed for that purpose. Consequently, it is a hardware abstraction kind of middleware where no other services are offered as part of the deployed hardware. What is more, there are no mentions on how the middleware is accessed by any other higher layer (in case it is a possibility), so the existence of an API is unlikely. The main hardware elements that are to be mounted on a node that is using the device (Bluetooth interfaces, the microcontroller or the very Embedded Metering Device) are present in Figure 30.

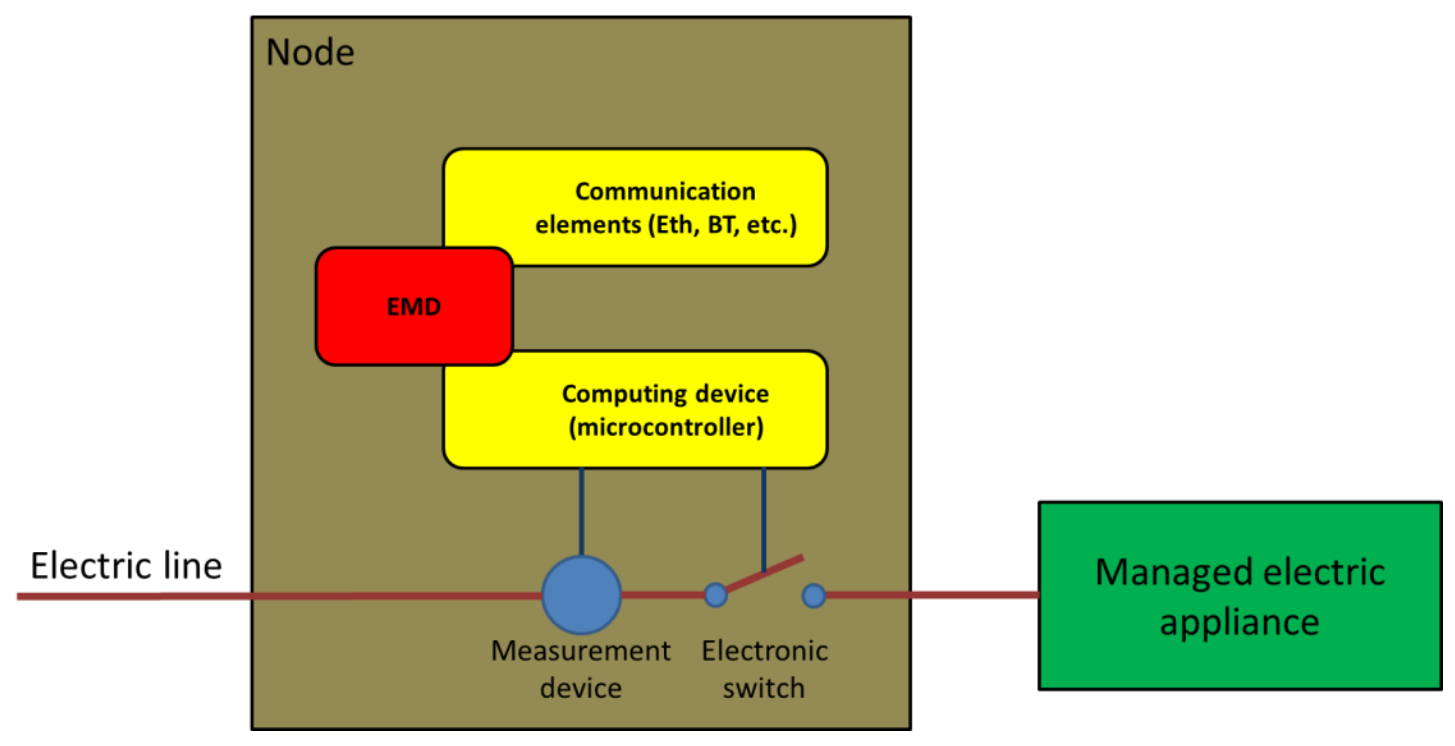

Figure 30. Block diagram of the EMD structure, as described in [85]

Computational capabilities: the proposal aims very specifically to be a scalable piece of Advanced Metering Infrastructure, so it has been aimed to be part of the AMI infrastructure that an end consumer/prosumer would have in their dwell or store. No other location of a Smart Grid deployment (TSO, DSO, Aggregator or power plant) is expected to be used to have the software components related to the middleware installed.

Message coupling: it is stated by the authors that their prototypes were working under CORBA [86] and the Internet Communications Engine (Ice, which provides a Remote Procedure Call 
protocol using standards from the transport layer [87]), so it essentially works under a Client/Server paradigm.

Middleware distribution: since the middleware solution is strongly linked to the hardware where it has been installed, it has been considered as a mostly centralized proposal that is running in a single kind of element (AMI) that is likely to be present in the facilities of many end users.

Bearing all these features in mind, the proposal can be described as:

$$
S G M=S A(0)+C C(0)+M C(2)+M D(1)
$$

Advantages of the proposal: the authors have made a great effort in creating a realistic way to have a piece or AMI capable of establishing connections with other parts of any possible deployed infrastructure of this sort by using middleware in it. Besides, they take into account aspects like the cost or the size that are usually not considered for prototyping purposes in other pieces of work.

Disadvantages of the proposal: the proposal is too focused on the hardware components that have been mounted in order to have EMDs that can be considered as a scalable AMI, so the limitations of the proposal come from that fact. Moreover, the EMD shown here basically makes use of CORBA and Ice as the actual middleware implemented within it, so it is not possible to use any other standard or a more novel solution that what is being provided here. Therefore, in order to port the software elements to other part of a Smart Grid, a significant recoding work is likely to be needed. In addition to that, software-related features such as security, semantics for information enhancement, or offering a specific API to access the services that may be provided by the device, are not stated in the proposal.

\subsubsection{OpenNode Smart Grid architecture}

Florent Leménager et al. offer their own novel concepts for a middleware solution focused on the Smart Grid [88]. They show the implementation works that have been made as part of the OpenNode project [89]. In the proposal, it is shown that there is a collection of key concepts (modularity, extensibility, distribution of intelligence, an open common reference architecture, usage of open standards and cost effectiveness) of major importance by the authors. The efforts carried out in this proposal have been directed to creating an open development for Secondary Substation Nodes (SSNs) located within secondary substations of the power grid, and a middleware solution used to interconnected the SSN with the utility systems related to the electrical facilities and infrastructures. It is mentioned by the authors that the middleware, always regarded as something separated from the SSNs and running above them, is used to deal with the stakeholder diversification and the flexibility needed to interoperate among the power grid, the network and the management methods.

Service availability: judging from the information available in the proposal, the middleware is solely focused on hardware abstraction, as there is no information about the kind of services that could be encased in the middleware proposal or in any message format interchanged 
between the data-based levels and the lower ones. There are two separated roles for the new software components developed both at the middleware and the SSN level: the Secondary Substation Node is used for interacting with any smart meter or local Intelligent Electronic Device (IED), whereas the middleware is conceived as a way to interchange metering data, along with grid automation information. Thus, it can be argued that some of the SSN functionalities resemble the ones that are expected from middleware from the point of view of hardware interoperability. The open nature of the content that is provided by the project is of major importance for the project partners, as it is claimed that the project aims to provide a reference architecture publicly available to the community for the integration of Distributed Energy Resources. At the same time, middleware transfers information to an Enterprise Service Bus in case it might come in handy to be transfer to other utility management systems. The locations of the middleware and the SSN in the project have been displayed in Figure 31.

Computational capabilities: according to the tests that were carried out, SSN prototypes were developed by using industrial PCs and embedded Linux CPUs. Smart Meters from five different manufacturers are also used in the testbed. Since the middleware is going to be interacting with software resources of the Secondary Substations deployed in the power grid, it is expected that it will be deployed in the aggregator, TSO or DSO facilities (in fact, it is explicitly mentioned in the proposal that the time constrains based on operational DSOs have been defined in the testbed).

Message coupling: no information is provided about how data is expected to be transferred from one side to other one in the proposal. It can be expected that, due to its location, realtime information will be exchanged in the system.

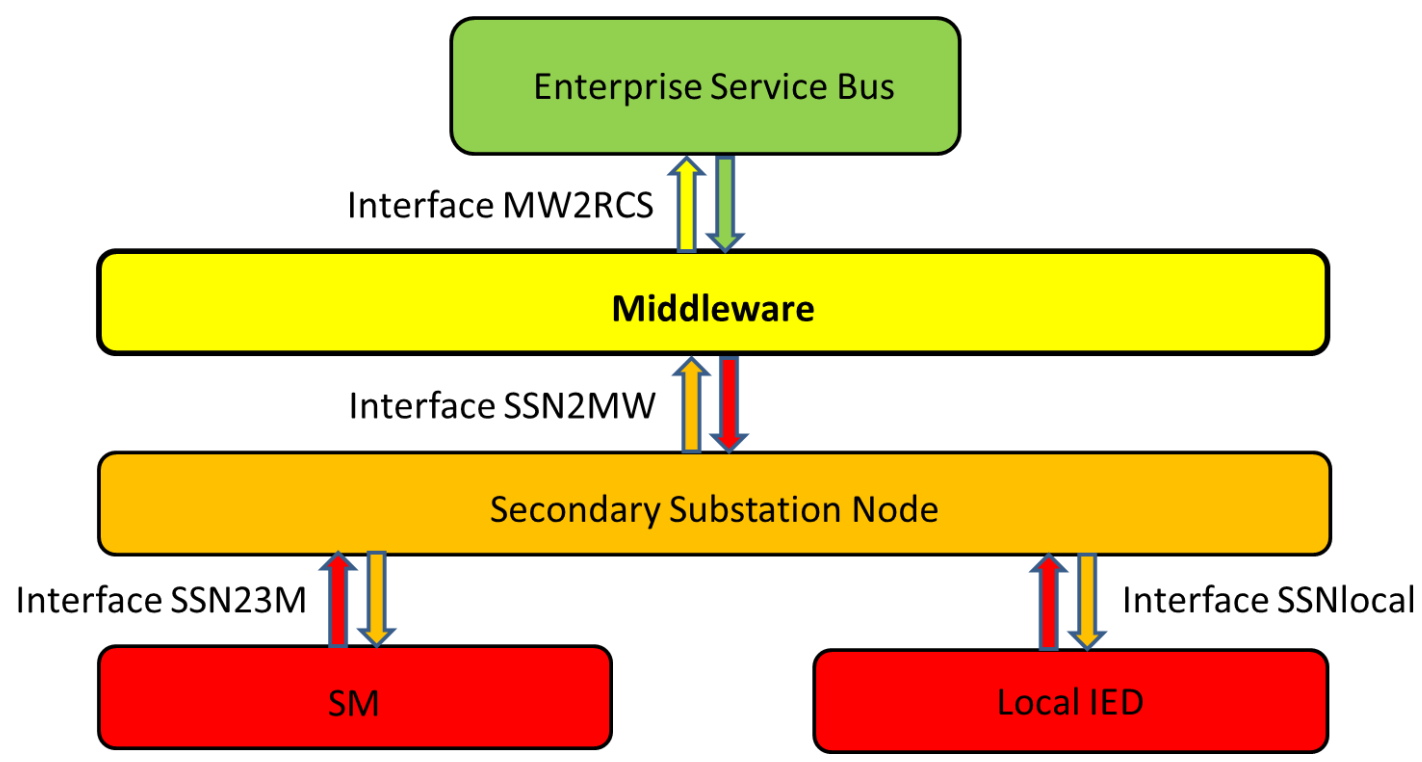

Figure 31. Middleware high level location in the proposal, as described in [88]

Middleware distribution: the proposal is a fully decentralized one, as there is not a prominent middleware location that will rule over other entities with middleware components installed and it is supposed to be interacting with SSNs scattered in a Smart Grid. 
Consequently, this proposal can also be described like:

$$
S G M=S A(0)+C C(1|| 2)+M C(3)+M D(3)
$$

Advantages of the proposal: the authors ensure that what has been developed will have a high degree of applicability to the power grid in order to enhance its performance. Furthermore, the proposal has been tested in several realistic scenarios where two physical and one virtual tests were carried out.

Disadvantages of the proposal: the proposal relies in middleware only to transfer information from the Secondary Substation Node to the Enterprise Service Bus that is used to interchange information with other utility deployments. Besides, data about major characteristics of the middleware are absent, such as how data messages are transferred from one area to the other or how to enable semantic capabilities or security as components of the middleware solution.

\subsubsection{DIRECTOR}

James Wilcox and Mahesh Sooriyabandara present what they refer to as a distributed communication transport manager for the Smart Grid [90]. It is expected from DIRECTOR that it will be able to manage the requirements of application communications throughout a Smart Grid. It has been placed by the authors between the application layer and the socket Application Programming Interfaces located right below the DIRECTOR middleware layer. It is claimed to instruct those sockets to generate an optimised interface that supports application needs to its best possible. Messages in the middleware domain contain the work payload, a list of destinations and the priority of the message.

Service availability: among what the proposal is capable of providing, hardware abstraction is the most prominent and obvious service. However, message orientation is also strong, stating that the socket configuration can be changed when sending them and different levels of bandwidth efficiency are required, so it has been regarded as an intermediation middleware. According to the authors, the DIRECTOR middleware is made up by several functionalities: an application interface (conceived as an inter-process communication transport socket), network health (offered by monitoring data exchanges over an Ethernet bridge), a custom transport layer (generated after considering the inputs provided both by the application interface and the network health information) and a software component used for custom socket (generated with negotiated transport features). The location of the middleware layer has been displayed in Figure 32. 


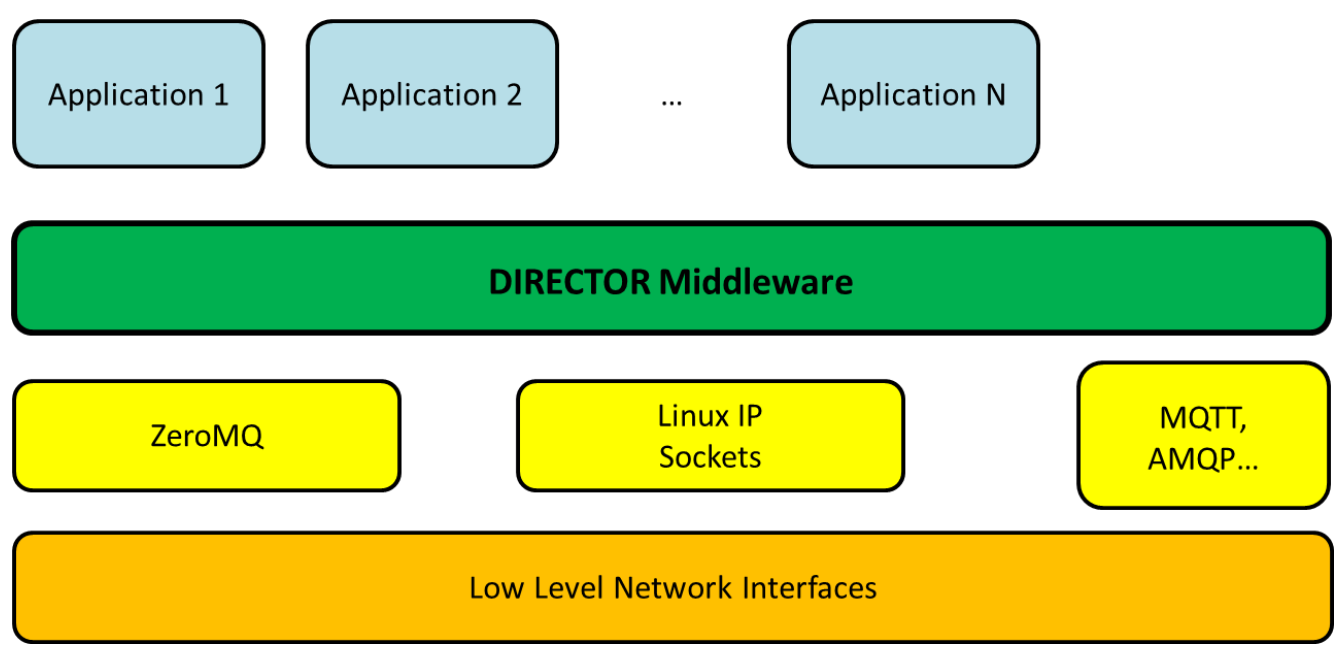

Figure 32. Location of the DIRECTOR proposal in a Smart Grid deployment, as stated in [90]

Computational capabilities: the proposal can be installed in almost any piece of equipment present in the Smart Grid, as tests were carried out in five Raspberry Pi platforms that, according to the authors and the specifications of the Raspberry Pi model B [91] have a 700 $\mathrm{MHz}$ ARM processor and $512 \mathrm{MB}$ of Random Access Memory (RAM). Therefore, and considering that a smart meter can be implemented out of a Raspberry Pi, even hardware located at the end user's facilities could handle the computational requirements of the middleware solution, let alone any other piece of equipment in the aggregator, TSO, DSO or power plant.

Message coupling: the proposal mentions that it has been conceived for distributed and realtime embedded systems, so it must be capable of transferring that kind of information. Furthermore, a virtualized Demand Response Automation Server has been used to simulate an actual Demand Response scenario.

Middleware distribution: it can be regarded as a fully decentralized middleware architecture that works under a peer-to-peer level, due to the fact that there is not a single component that adds an extra prominence when compared to all the others. However, this is due to the fact that no Publish/Subscribe (and thus, centralizing elements such as brokers) or any other message interchange paradigm is ever used.

Therefore, this architecture can be described as follows:

$$
S G M=S A(1)+C C(0|| 1|| 2|| 3)+M C(3)+M D(3)
$$

Advantages of the proposal: DIRECTOR has been tested under a deployment using hardware easy to find in a more realistic scenario (such as a microgrid, a power plant, etc.). The solution makes use of a data model to exemplify how applications would work. Lastly, the location of the proposal in a distributed system is made very clear, as it is aimed to be placed between the application layer and the sockets that are used for transport layer communications.

Disadvantages of the proposal: there are no mentions about how security or semantic capabilities can be added to the proposal, or any service that may go beyond interoperability 
among different devices. Besides, the proposal seems to be just involved in the messages that are sent through the transport layer but shows less information about how the information is transferred to the application layer, or the kind of APIs that are available for that level. As far as message coupling is concerned, it is not easy to figure out the kind of criteria that has been used in the proposal to use one paradigm or another.

\subsubsection{DDS interoperability for the Smart Grid}

As it happened with a former one, the proposal of Tarek A. Youssef et al. makes use of Data Distribution Service as a way to provide middleware to the Smart Grid [92]. DDS is utilized combined with data structures and standard interfaces in order to have a scalable Smart Grid infrastructure that can be used in the testbed utilized to assess the feasibility of the hypotheses formulated. It is claimed that it makes possible integration with other deployments, experimentation, data gathering and algorithm testing. At the same time, DDS enables the system to establish a global Data Space where data structures are used to generate information that can be interchanged under a Publish/Subscribe paradigm. Interoperability is guaranteed by means of Real Time Publish Subscribe Protocol (RTPS), the DDS lower level layer used for this purpose, whereas an API is provided for the higher levels to access the middleware services.

Service availability: the proposal is bent on providing connectivity between the devices present in the testbed and the applications that would be requested by the end users. The authors focus on the idea of providing a gateway for each of the applications that are estimated to be required in a Smart Grid deployment. These gateways will be used as a way to provide an Application Programming Interface for the topmost level of the architecture to access the middleware facilities. Therefore, it can be said that this is an example of Message-Oriented Middleware rather than an architecture with several distinguished devices. The most foreseeable services to be included have been depicted in Figure 33 .

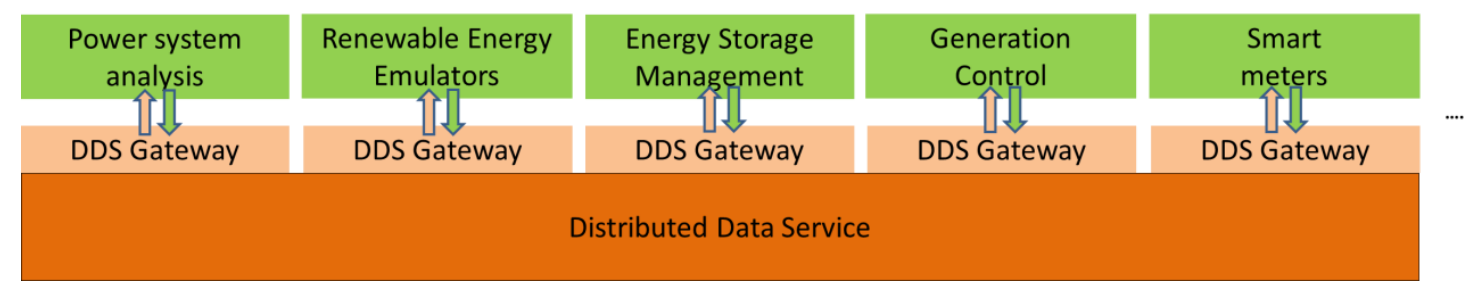

Figure 33. Testbed for the DDS infrastructure, as described in [92]

Computational capabilities: the proposal is expected to be installed in devices capable of handling DDS, so a regular computer should be able to handle it. Regarding the pieces of equipment expected to be included here, PC- or server-like appliances belonging to the aggregator and the TSO/DSO are likely to encase the software components of the proposal.

Message coupling: DDS strongly relies on a Publish/Subscribe paradigm to deliver data; consequently, it is used in this proposal as well, in order to deliver information related to the 
applications typical of the Smart Grid. In addition to that, real-time data is cited to be added as one kind of information that can be included in the deployment.

Middleware distribution: although there is not a clear mention about it, if the testbed that has been used by the authors is taken into account, the proposal can be expected to include and transfer data from end user devices. Thus, it can be located in any facility that is handling them, such as the aggregator infrastructure or the pieces of equipment installed as part of the TSO and the DSO.

This proposal can be further displayed as:

$$
S G M=S A(2)+C C(1|| 2)+M C(0|| 3)+M D(1)
$$

Advantages of the proposal: as it happened before, many of the advantages and disadvantages of the proposal are linked to the idea of using DDS as the provider of the middleware architecture. DDS has been proven to be effective in several environments using CPSs and so it demonstrates that it can seamless interconnect the devices and applications of the deployment put forward by the authors of the proposal. In addition to that, a compelling testbed is provided where experiments can be run to test the feasibility of the ideas related to the proposal. In addition to that, an Application Programming Interface is available for any DDS development compliant with the standard.

Disadvantages of the proposal: the main focus provided by the authors is solely on interconnectivity of high and low level capabilities, so additional services to be included in the middleware (semantic capabilities, security) are not considered. Furthermore, due to licensing in the used proposals, it might be a problem to exploit the developed works done under a DDS implementation.

All the information that has been collected from the proposals is shown in a more summarized manner in Table 1. It is here where the advantages and disadvantages offered by the state of the art are immediately present, and an accurate idea of the developments done can be conceived.

Table 1. Main characteristics of the studied middleware solutions

\begin{tabular}{|c|c|c|}
\hline Proposal name & Advantages & Disadvantages \\
\hline GridStat & $\begin{array}{l}\text { Detailed framework. } \\
\text { Implementation works. } \\
\text { Requires low computational } \\
\text { capabilities. }\end{array}$ & $\begin{array}{l}\text { No remarkable services } \\
\text { available. No details } \\
\text { regarding security } \\
\text { implementations. No } \\
\text { semantics. }\end{array}$ \\
\hline $\begin{array}{l}\text { Service-Oriented Middleware } \\
\text { for Smart Grid }\end{array}$ & $\begin{array}{l}\text { Collection of services. } \\
\text { Performance tests. Security } \\
\text { solutions conceived. }\end{array}$ & $\begin{array}{l}\text { No remarkable services } \\
\text { available. Scarce information } \\
\text { about distribution. Vague } \\
\text { boundaries defined. }\end{array}$ \\
\hline $\begin{array}{l}\text { Ubiquitous Sensor Network } \\
\text { Middleware }\end{array}$ & $\begin{array}{l}\text { Decentralization is enabled. } \\
\text { Compatibility with a plethora } \\
\text { of technologies. }\end{array}$ & $\begin{array}{l}\text { Vague boundaries defined. } \\
\text { Scarce information about the } \\
\text { pieces of equipment where } \\
\text { the proposal would be }\end{array}$ \\
\hline
\end{tabular}




\begin{tabular}{|c|c|c|}
\hline Proposal name & Advantages & Disadvantages \\
\hline & & $\begin{array}{l}\text { included. No performance } \\
\text { tests are included. }\end{array}$ \\
\hline OHSNet & $\begin{array}{l}\text { Significant collection of } \\
\text { services for hardware } \\
\text { interoperability. }\end{array}$ & $\begin{array}{l}\text { Vague boundaries defined. } \\
\text { Services do not provide value } \\
\text { for end users. }\end{array}$ \\
\hline MDI & $\begin{array}{l}\text { Precise and abundant } \\
\text { information about devices to } \\
\text { have the proposal installed } \\
\text { and computational resources. }\end{array}$ & $\begin{array}{l}\text { Very few data regarding } \\
\text { implementation. Security or } \\
\text { semantics have not been } \\
\text { enabled. }\end{array}$ \\
\hline IEC 61850 and DPWS & $\begin{array}{l}\text { Information about semantic } \\
\text { capabilities and } \\
\text { implementation is provided. } \\
\text { Security is provided. }\end{array}$ & $\begin{array}{l}\text { No information about tests or } \\
\text { hardware abstraction carried } \\
\text { out. }\end{array}$ \\
\hline IAP-INMS & $\begin{array}{l}\text { Data heterogeneity is taken } \\
\text { care of. An ESB is used. Tests } \\
\text { have been made on the } \\
\text { proposal. }\end{array}$ & $\begin{array}{l}\text { No data about hardware } \\
\text { abstraction or security. }\end{array}$ \\
\hline $\begin{array}{l}\text { Self-Organizing Smart Grid } \\
\text { Services }\end{array}$ & High degree of distribution. & $\begin{array}{l}\text { No data about how to use the } \\
\text { proposal in a deployment. } \\
\text { Scarce information about } \\
\text { tests. No prominent services } \\
\text { offered. }\end{array}$ \\
\hline $\begin{array}{l}\text { Secure Decentralized Data- } \\
\text { Centric Information } \\
\text { Infrastructure }\end{array}$ & $\begin{array}{l}\text { Easily portable proposal. } \\
\text { Networking and securitization } \\
\text { capabilities. API is provided. } \\
\text { Security is a major service in } \\
\text { the proposal. }\end{array}$ & $\begin{array}{l}\text { No semantic capabilities } \\
\text { provided. The proposal is too } \\
\text { much focused on transport } \\
\text { and network layers. }\end{array}$ \\
\hline $\begin{array}{l}\text { A cloud optimization } \\
\text { perspective }\end{array}$ & $\begin{array}{l}\text { High degree of distribution. } \\
\text { Parallel works can be done. } \\
\text { Security can be included. }\end{array}$ & $\begin{array}{l}\text { No API is provided. No data is } \\
\text { offered about software } \\
\text { elements of the proposal. }\end{array}$ \\
\hline $\begin{array}{l}\text { KT's Smart Grid Architecture } \\
\text { and Open Platform }\end{array}$ & $\begin{array}{l}\text { Platform as an open } \\
\text { development. Realistic } \\
\text { deployment done. }\end{array}$ & $\begin{array}{l}\text { End users are regarded as just } \\
\text { consumers in the proposal. } \\
\text { Service securitization } \\
\text { information is missing. No } \\
\text { data about the API. }\end{array}$ \\
\hline $\begin{array}{l}\text { Smart microgrid monitoring } \\
\text { with DDS }\end{array}$ & $\begin{array}{l}\text { DDS is very suitable for } \\
\text { middleware solutions. } \\
\text { Computational capabilities } \\
\text { are suitable. }\end{array}$ & $\begin{array}{l}\text { Licensing can be a challenge } \\
\text { depending on the purpose of } \\
\text { the deployment. No } \\
\text { additional, Smart Grid-related } \\
\text { services are offered. }\end{array}$ \\
\hline ETSI M2M & $\begin{array}{l}\text { API available. Profuse } \\
\text { information about } \\
\text { prototyping. Successful port } \\
\text { of another distributed } \\
\text { systems solution. }\end{array}$ & $\begin{array}{l}\text { Confusing exposition of the } \\
\text { implemented services. Not } \\
\text { many data about message } \\
\text { transmission. }\end{array}$ \\
\hline $\begin{array}{l}\text { Smart Middleware Device for } \\
\text { Smart Grid Integration }\end{array}$ & $\begin{array}{l}\text { Tests carried out on Smart } \\
\text { meter and devices with real } \\
\text { technologies. }\end{array}$ & $\begin{array}{l}\text { Main focus on encasing one } \\
\text { piece of hardware with } \\
\text { specific characteristics. No } \\
\text { information about how data }\end{array}$ \\
\hline
\end{tabular}




\begin{tabular}{|c|c|c|}
\hline Proposal name & Advantages & Disadvantages \\
\hline & & $\begin{array}{l}\text { becomes distributed in the } \\
\text { system is given. Security, API } \\
\text { or semantics are missing. }\end{array}$ \\
\hline $\begin{array}{l}\text { WAMPAC-based Smart Grid } \\
\text { communications }\end{array}$ & $\begin{array}{l}\text { Security is described very } \\
\text { accurately. Information about } \\
\text { how to build a testbed is } \\
\text { present. }\end{array}$ & $\begin{array}{l}\text { No description provided } \\
\text { about other services } \\
\text { unrelated to security } \\
\text { (semantics). No API is } \\
\text { described. }\end{array}$ \\
\hline C-DAX & $\begin{array}{l}\text { Tests carried out in a real } \\
\text { scenario regarding } \\
\text { components and use cases. } \\
\text { Security is enabled. }\end{array}$ & $\begin{array}{l}\text { Other services aside from } \\
\text { security are not described. }\end{array}$ \\
\hline Building as a Service & $\begin{array}{l}\text { An API and popular software } \\
\text { technologies (JSON, SOAP, } \\
\text { JDBC) are used. }\end{array}$ & $\begin{array}{l}\text { No information about data } \\
\text { semantics or securitization. A } \\
\text { too specific scenario (energy } \\
\text { consumption for buildings) } \\
\text { may be challenging if the } \\
\text { solution is going to be ported. }\end{array}$ \\
\hline $\begin{array}{l}\text { Middleware-based } \\
\text { management for the Smart } \\
\text { Grid }\end{array}$ & $\begin{array}{l}\text { Great effort in improving } \\
\text { Advanced Metering } \\
\text { Infrastructure by means of } \\
\text { middleware. }\end{array}$ & $\begin{array}{l}\text { Solution extremely } \\
\text { dependent on a single kind of } \\
\text { hardware. Solution only } \\
\text { proved with a small set of } \\
\text { specific technologies (CORBA, } \\
\text { Ice). }\end{array}$ \\
\hline $\begin{array}{l}\text { OpenNode Smart Grid } \\
\text { architecture }\end{array}$ & $\begin{array}{l}\text { High applicability to the } \\
\text { power grid. Tests carried out } \\
\text { in realistic scenarios. }\end{array}$ & $\begin{array}{l}\text { Middleware designed for very } \\
\text { specific functionalities. } \\
\text { Information missing about } \\
\text { important middleware } \\
\text { services. }\end{array}$ \\
\hline DIRECTOR & $\begin{array}{l}\text { Tests done in realistic pieces } \\
\text { of equipment. Clearly } \\
\text { described distributed } \\
\text { features. }\end{array}$ & $\begin{array}{l}\text { Important services } \\
\text { (semantics, securitization) are } \\
\text { not mentioned. No API is } \\
\text { available. It is difficult to } \\
\text { know what kind of message } \\
\text { coupling is used. }\end{array}$ \\
\hline $\begin{array}{l}\text { DDS interoperability for the } \\
\text { Smart Grid }\end{array}$ & $\begin{array}{l}\text { DDS is a suitable solution for } \\
\text { middleware. Compelling } \\
\text { testbed provided. }\end{array}$ & $\begin{array}{l}\text { Only focused on } \\
\text { interconnectivity of high and } \\
\text { low level capabilities. }\end{array}$ \\
\hline
\end{tabular}

A further analysis on open source ESB solutions will be provided, as they have been found to be the most adequate solution to use in middleware for the Smart Grid.

\subsection{State of the art in Enterprise Service Bus solutions}

Among the several ways possible to find a way to embody middleware in an actual implementation, using an Enterprise Service Bus (ESB) platform is a suitable solution for the 
challenges that may be found in the Internet of Things. Typically, an ESB will contain several features to guarantee that interoperability can be provided:

1. Rather than having a massive number of point-to-point mesh-based communications between the integrated applications, which would be impractical and a source of delays, a bus is used to connect them all and transfer the data. This enables the good usage of the software entity used for data transfer.

2. An ESB hides away the heterogeneity and complexity of the services and applications that is interconnecting, so it fits in accurately with the purpose of middleware.

3. An ESB allows the usage of software packages (bundles) that make possible their port from one deployment to a different one, thus saving time and resources in development works.

The position of an ESB (and of middleware when it is embedded in an ESB) in a CPS-based deployment (with similar features that can be found in a Smart Grid from the software point of view) has been depicted in Figure 34. Note that apart from the services that it is interconnecting, the ESB is capable of containing some more if required (device registration, securitization), so it effectively becomes a way to implement a middleware architecture.

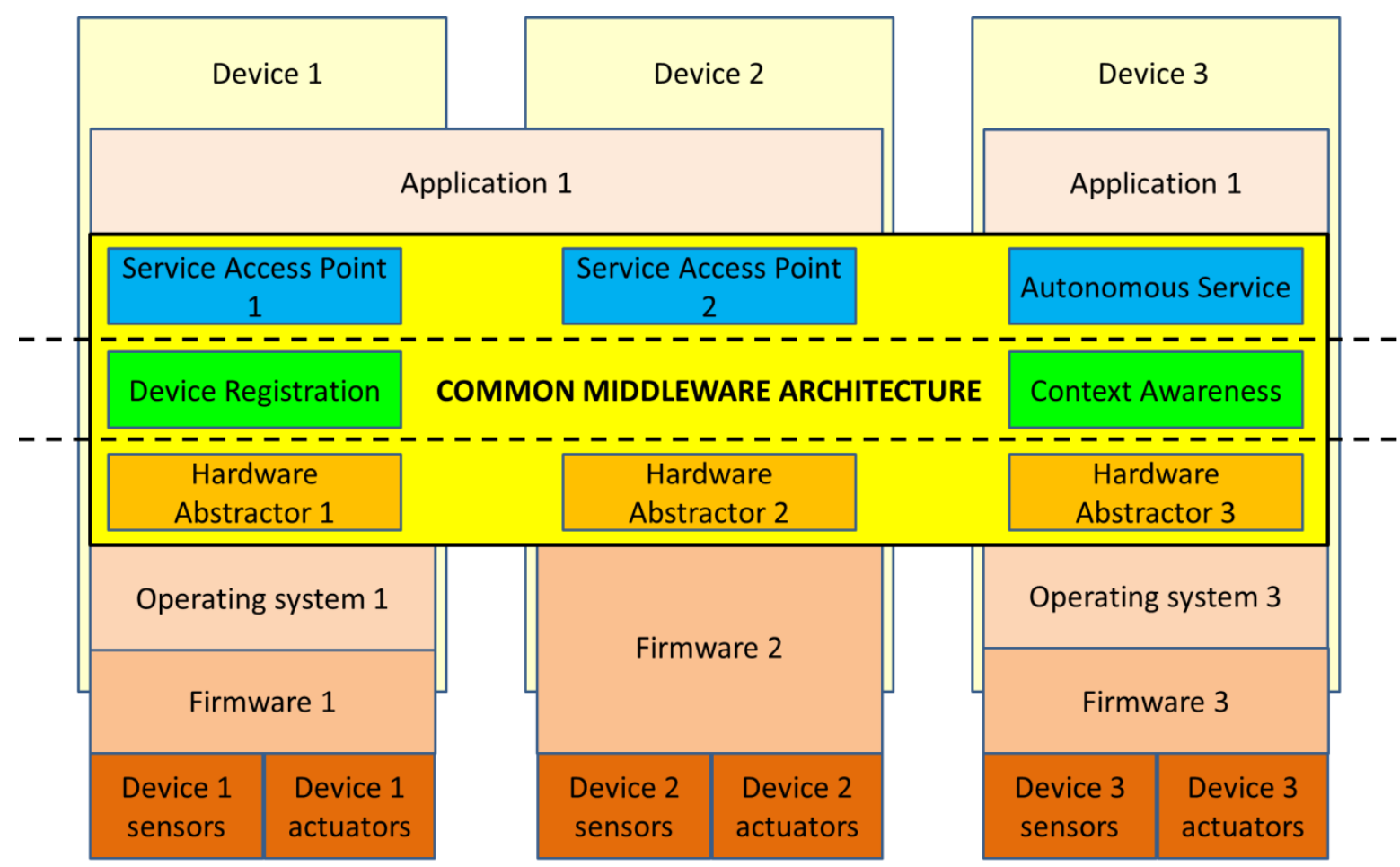

Figure 34. ESB location and most usual services in a Cyber-Physical System

The study that has been done about the state of the art in Enterprise Service Bus solutions focuses only on the ones that are offered under open source agreements. The motivation to do it like this is because two reasons:

1. From a purely economic point of view, it is much more convenient to use an open source solution, as they usually offer a number of services and interfaces enough for the idea of providing a middleware architecture for the Smart Grid. While open source 
solutions usually offer a weaker support than commercial ones, the development and integration issues that may be found are usually likely to have taken place before, so there is usually a way to solve them. However, having a strong community behind an ESB solution is more important here than in other solutions were support is a paid service.

2. As far as the research projects where implementation works were done are concerned, a non-open source ESB was never considered as an eligible solution due to the European Commission policies, which favour the usage of open source solutions, and the utilization of inexpensive tools that will not challenge budget requests.

The most interesting options that have been found matching the previous requirements have been listed in the following subsections.

\subsubsection{OpenESB}

According to their developers, OpenESB is an open-source ESB written in Java and expected to be used as a platform with a dual purpose: enterprise application integration (the original purpose that ESBs were designed for) and a service-oriented architecture (much closer to what a middleware architecture represents), as mentioned in [93]. It is claimed by its creators that "OpenESB is the unique open source ESB relying on standards that provides you with Simplicity, Efficiency, Long term durability, save on your present and future investments with a very low TCO (Total Cost of Ownership)" [94]. OpenESB makes use of Java Business Integration (JBI) as a way to provide an architecture that will encase components for service production and consumption [95]. It is maintained by the OpenESB community that was created after Sun Microsystems was purchased by Oracle. This ESB has five different parts organized as follows:

1. Framework: it is an implementation of JBI claimed to be lightweight [96]. Even though it works in close cooperation with the used container, the framework remains container-agnostic, so one can be chosen among a plethora of them. The framework is encased in a Java Virtual Machine (JVM) that makes use of binding components to communicate with other frameworks. In addition to that, it is claimed to be fully manageable by Java Management Extensions (JMX)-related tools. Lastly, the framework is the part that contains the virtual bus (named Normalised Message Router) to be utilized in this ESB.

2. Container: the container can be regarded as the application server used to store the facilities that might be needed by the applications. OpenESB is claimed to be capable of processing more than 10 million complete messages per day [10] that use this container to be transferred. The current version of OpenESB has six different containers available: GlassFish Version 2 [97] (the one that is included by default), JBoss [98] Version 4, Version 5 and Version 7 and the vey used JVM.

3. Components: these are the software entities able to deliver the ESB the facilities used to transfer information. There are two different kinds of them: one is the Services Engine and the other one is the Binding Component. The former are used for receiving and sending messages to the bus, without dealing with any messages that have to be 
sent outside it, whereas the latter interacts with messages outside the defined bus, but can generate either messages that will be sent inside the bus or messages transferred within the bus that leave it afterwards.

4. Integrated Development Environment and development plugins: it refers to the facility that is used for programming, debugging and testing the code that is produced. They usually include any plugging that is required for code generation. XML Schema Definition (XSD), WSDL, Business Process Execution Language (BPEL), Data Mapper and Composite Application editors are available to build, deploy, un-deploy, run, test and perform debugging tasks.

It is also noteworthy to mention that an OpenESB instance is able to work with both legacy solutions, which are dealt with by Domains and Node Agents working together with a Glassfish application server, and standalone stacks (that rely solely on an OpenESB instance making use of a Java Virtual Machine) [99]. A comparison of what is required for each of the iterations can be seen in Figure 35.
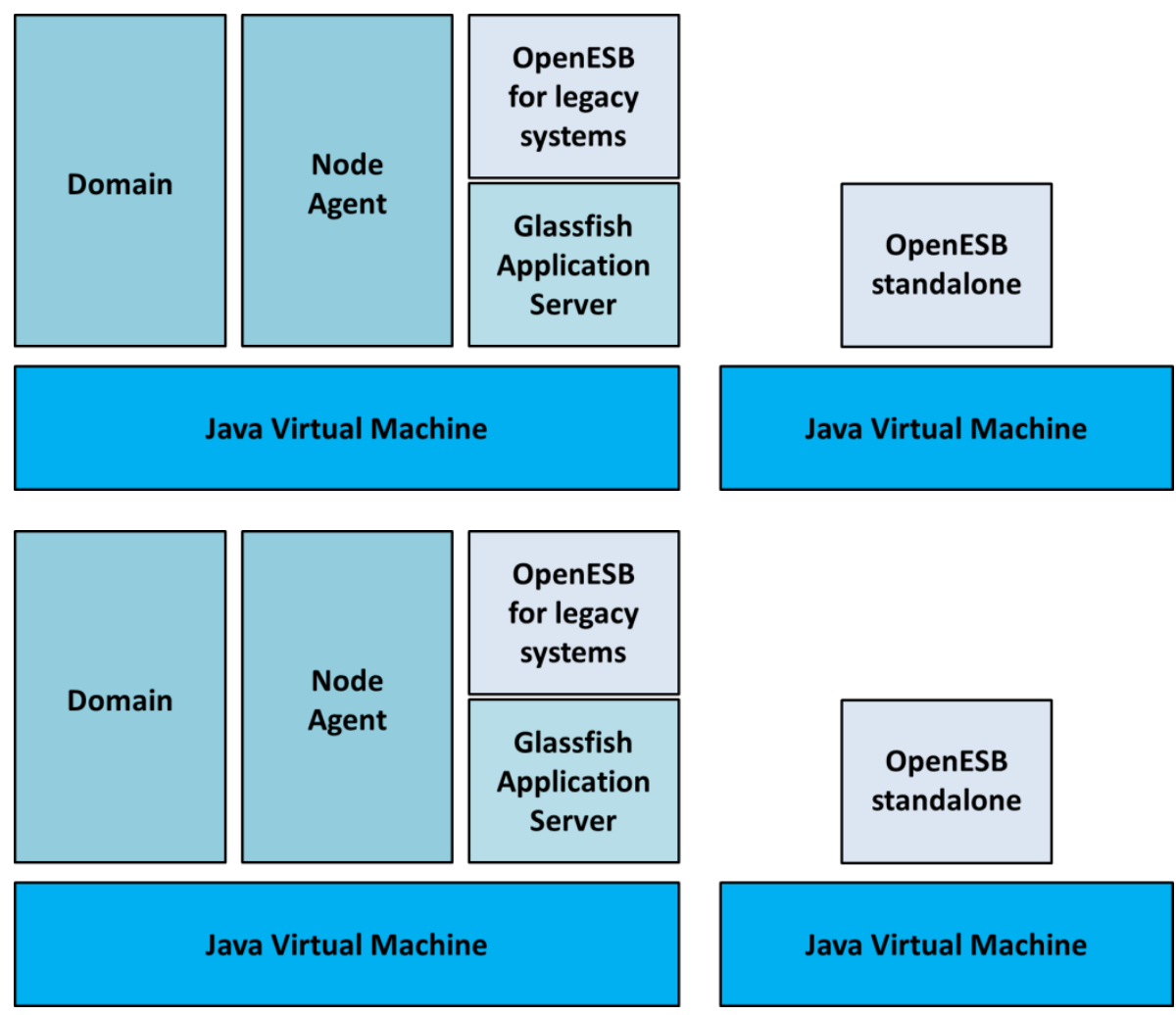

Figure 35. Legacy and standalone required software components, as described in [99]

As it can be inferred, OpenESB works in a way that implies that messages are sent from and to the applications accessing the bus where the legacy and the standalone stack are used. These messages will be sent via Binding Components and Services engines when they are required to travel inside the bus. All these components will be included in a selected container provided by a framework, and they will be worked upon by means of the Integrated Development Environment and the development plugins added to the distribution. One key feature of OpenESB is its openness; it is provided under a Common Development and Distribution License (CDDL) that makes possible providing a world-wide, royalty-free, non-exclusive license that 
enables the owner of the licensed software to use, reproduce, modify, display, perform, sublicense and distribute the original software [100].

OpenESB provides a good framework for services and delivers messages to the applications that require them. It has a foreseeable behaviour as it uses a bus to transport all the information required to interconnect the applications that have been distributed. However, the fact that is focused on one single programming language might be an issue for developers that are not proficient with that programming language.

\subsubsection{WSO2 ESB}

As it happened with the previous proposal, this iteration of the Enterprise Service Bus is claimed to be lightweight. In addition to that, it also has an open source development that explicitly regards itself as middleware conceptually relying on a SOA approach. Among the main features that are supported [101] the most important ones are:

1. WSO2 ESB makes use of WSO2 Carbon as the core of the platform, which provides a componentized architecture able to adapt business activity automatically in response to market events.

2. This proposal is expected to offer seamless adaptation among public, private and hybrid clouds, along with on premise solutions.

3. The developers of this proposal say WSO2 is optimized for the Internet of Things. The architecture offers a server-side and a cloud-based Reference Architecture so that the developers working on the loT can use it as a starting point.

4. Flexibility is also a stressed feature: all the products linked to the proposal are said to be completely based on open source solutions and open standards. Legacy and package apps are integrated in WSO2, and the developers are able to extend the platform and customize it if required.

5. The possibility of having an Original Equipment Manufacturer (OEM) is also offered in order to offer subsystems for an end product provided by a third party.

Figure 36 shows the most important services that the WSO2 architecture is capable of providing; security for the deployed components, facilities for portals and stores (namely, asset storage), device management focused on mobile devices and the loT, app development and management (working closely with what can be offered to portals and stores), API management (interweaved with the integration functionalities and the facilitates offered to portals and stores, as they require the services of an API to provide their own functionalities), integration by means of connectors with legacy systems and other facilities (along with service orchestration and composition) and analytics services. Note that several of them make use of other functionalities in order to offer their own, whereas there are some other services delivered in all the involved levels of the communication (such as security or analytics). 


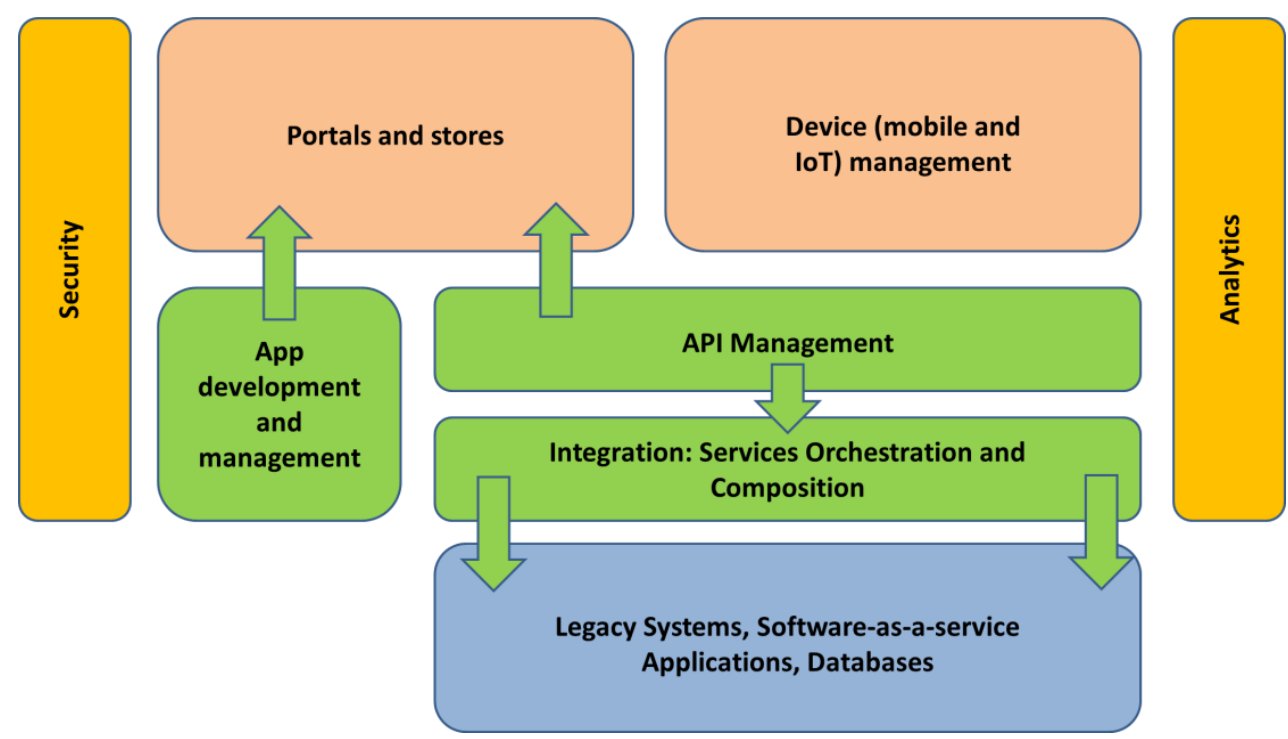

Figure 36. Facilities offered by WSO2 Enterprise Service Bus and their relationships, as described in [101]

WSO2 works sending and receiving messages by means of the several components of the architecture, which has been prepared to transfer information by using the following procedure [102]: when an application sends a message to the ESB it is received by a "transport", that is to say, a software component capable of receiving and sending messages over a collection of transport and application level protocols to guarantee communications among a distributed systems [103]. This message is sent by the transport through a message pipe that will handle it according to two different models, either a mediating message (where a single pipe is used) or proxy services (where separate pipes connect the transport to different proxies). Quality of Service is taken into account in this stage. The message routing and the transformation operations required to deal with it are considered as one (mediation framework in WSO2). When the message is injected to the pipes responsible for its delivery in the correct destinations Quality of Service is determined again. Lastly, the transport layer responsible for lower level communications will handle the message according to the required transport protocol transformations.

This WSO2 provides a complete set of facilities to build a middleware layer for a distributed system able to communicate applications in remote locations. However, the platform configuration still has to be managed, which also implies configuring the configuration repository, implement platform management tools or standardize and maintain platform change processes [104].

\subsubsection{JBoss Fuse}

This proposal has been implemented as a work done by Red Hat, which claims JBoss Fuse to be "an open, standards-based integration platform. It reduces the complexity of integrating disparate applications by leveraging the principles of Service Oriented Architecture (SOA) and standardized packaging frameworks" [105]. This solution shares with the former ones its open source nature and free availability of use. In fact, it is provided under a GNU General Public License which enables the owners of this ESB proposal to copy and distribute the received 
product, as long as an appropriate copyright notice and disclaimer of warranty is provided in a conspicuous and appropriated manner [106]. Furthermore, it is noted that any modification must be notified, along with providing those modifications or derived works free of charge [107] so that they will have the same status as the other parts of the original solution. JBoss Fuse has been conceived as a three-layered architecture divided by different functionalities depending on the layer that is taken into account:

1. Kernel layer: this layer is responsible for providing functionalities to the other upper layers. Consequently, most of its services have been conceived as part of a lightweight runtime offering management features and extending OSGi (former Open Services Gateway initiative, a worldwide consortium bent on creating open specifications and providing technology to ease the componentization of software modules and applications [108]) interfaces for handling and managing OSGi-based software packages (bundles) [109]. The most prominent of the services provided are: a console, logging capabilities, provisioning, configuration or security.

2. Services layer: this level embeds a collection of services consisting of interfaces and service implementations required to develop an instance of a specific service. The services that have been included in this layer are the ones used for Transactions, Messaging, Routing and Integration, Web and RESTful services and a JBI component, as the one that was used in OpenESB.

3. Application layer: this is the level that is closer to the application layer; therefore most of the contained services are focused on data format representation (Java-related services, Java API for XML Web Services -JAX-WS-, Java API for RESTful Web Services JAX-RS-). In addition to that, this is the level that provides the API used by developers to interact with JBoss Fuse to connect their application developments.

The appearance of the services that have been included in each of the layers has been depicted in Figure 37. Each of the services is able to communicate from one layer to the one above or underneath the one it is located in. As it can be inferred from previous information, this proposal works by deploying bundles in a repository and are activated when they have to be used. As explained in [110], when a software bundle is developed first and deployed afterwards in the application layer (the one that is immediately accessible for the developer), the kernel layer will read the Manifest.mf file that has been generated by the bundle to set up the application execution environment. Afterwards, the kernel will read the Blueprint.xml file in order to set up the resources needed for the route that defines the location of the bundle. Finally, the RouteBuilder class will access the routing and integration service via OSGi interface to define the routing runtime. JBoss Fuse also allows the addition of new bundles while it is operating, thus enhancing the capabilities of a deployment (for example, using it for adding interaction services between a regular computer and a Wireless Sensor Network [111]). It must be taken into account, though, that bundles to be deployed must be described by following the format of a Project Object Model file, or pom.xml. JBoss offers a satisfactory framework to deploy solutions for high-level communications in distributed systems, albeit the multiple options for configuration and debugging might create a learning curve that will result more or less challenging depending on the skills or experience of the developer. 


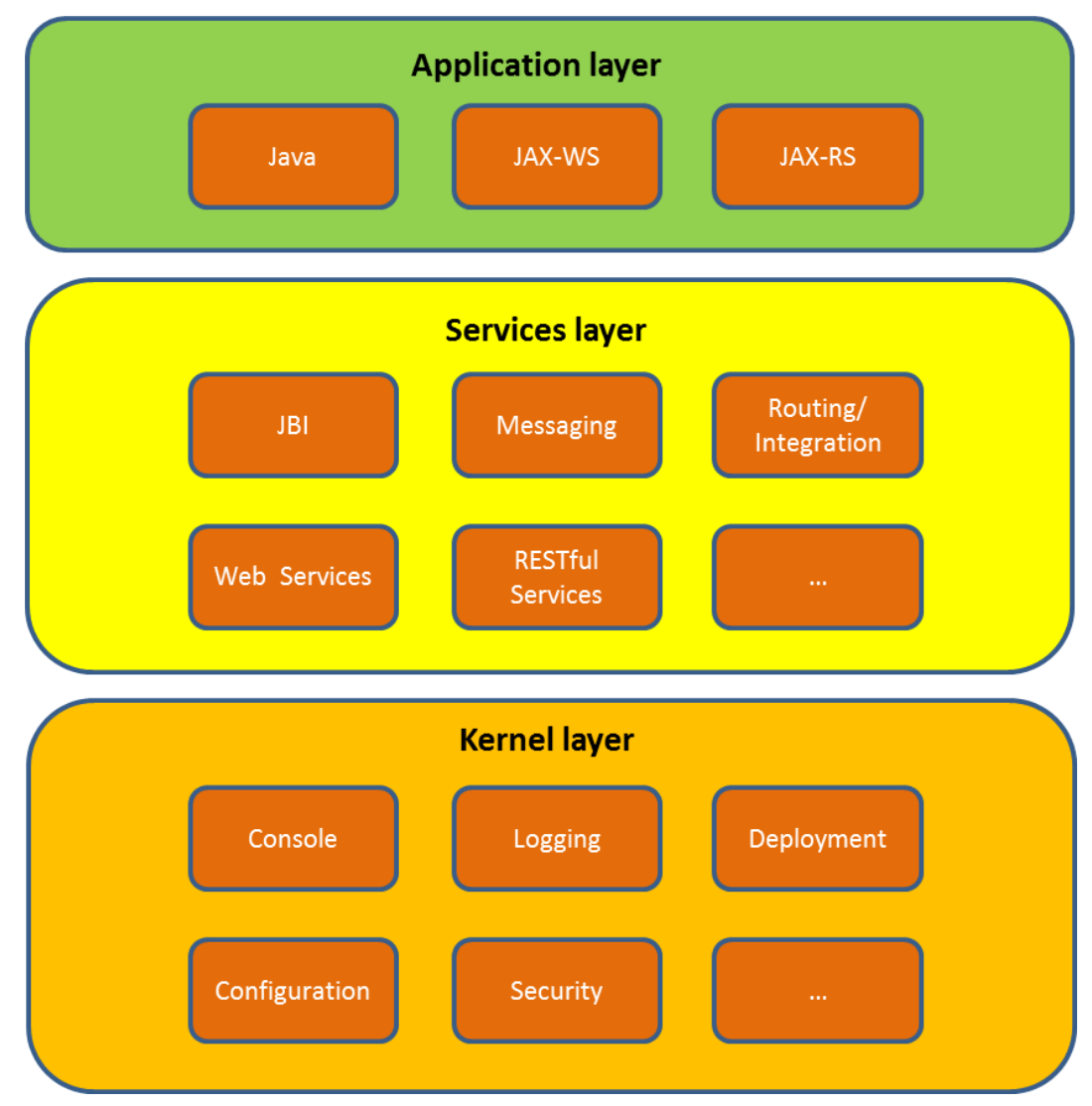

Figure 37. JBoss Fuse layers and services as described in [105]

\subsubsection{Apache ServiceMix}

This proposal is related to JBoss Fuse that has been previously presented in the sense that Apache ServiceMix is one of the components present in JBoss. Consequently, many of the features that were used by the former will appear in the latter. Apache ServiceMix is another open source proposal that claims to unify the features presented by several products, such as ActiveMQ, Camel, CXF and Karaf. In addition to that, it also makes use of OSGi interfaces in order to communicate some software packages with each other [112]. Additionally, this proposal is able to handle a Business Process Management engine (used as a systematic approach to optimize the workflow performed in a company [113]), support JPA by means of Apache OpenJPA and provide legacy support for the JBI standard, which was formerly deprecated in former versions of this architecture. Due to the capabilities offered by Apache ServiceMix, applications can be developed utilizing OSGi blueprints (such as the ones used by JBoss), declarative services (an OSGi functionality that makes possible defining and consuming services without dependencies in the source code to the OSGi framework [114]) and Spring Dynamic Modules (in order to allow functionalities like a better separation of application logic, deployment of several different versions of a module or library, etc. [115]) as a legacy solution. The functionalities that Apache ServiceMix is capable of offering rely on the integration of the former products, which make use of them as follows: 
1. Apache ActiveMQ: it is used as the messaging system and integration patterns server [116]. As the ESB proposal itself, it is also offered as an open source product. Among the features that it offers it is able to support a collection of cross language clients and protocols (Java, C, C++, C\#, Python, etc.), as well as Java Message Service 1.1, J2EE 1.4 and Enterprise Integration Patterns. Furthermore, it also offers support to Spring to embed ActiveMQ and fast persistence by means of Java Database Connectivity (JDBC, a Java-based industry standard for database-agnostic connectivity between Java and a set of SQL databases [117]).

2. Apache Camel: its main functionality is the definition of routing and mediation rules in domain-specific languages (Java-based Fluent API, Spring or Blueprint XML configuration files, as they were offered in JBoss Fuse). It makes use of Uniform Resource Identifiers to work with transport or messaging underlying standards [118]. It is claimed to provide seamless integration with the most popular frameworks, such as Spring, Blueprint and Guice.

3. Apache CXF: it can be regarded as an open source services framework used to develop services by using frontend programming APIs, such as the already mentioned JAX-WS and JAX-RS. As it has been described for other proposals, SOAP, XML/HTTP or RESTfulbased facilities are understood by this framework [119]. It therefore supports a collection of Web Services standards along with several frontend programming models.

4. Apache Karaf: it is used as the way to have a runtime server. Apache Karaf is actually capable of running itself as a container in a not dissimilar way than what would be used by a regular ESB architecture [120], albeit it would not provide as many components as an actual Enterprise Service Bus architecture would provide.

Apache ServiceMix works in a similar way to the one that was presented in JBoss, that is to say, when a bundle is developed, it will be done so as a regular project, but when it has to be deployed it will rely on two files, the blueprint for dependencies and another one for a description of the bundle (in this case, it will be a Spring file rather than a Project Object Model one). The deployed bundles will be stored in a deploy folder where they will be checked in order to know their status (Active, Failure status in case there has been any problem in their deployment, etc.) and, since it provides URIs, the services of the deployed bundles will be accessible remotely, as it happened with JBoss. Apache ServiceMix offers all the services and facilities that are expected from an ESB, but it lacks some features that the JBoss Fuse has, such as the "Fuse Fabric" technology that allows a location managing the installation and status of the ESB in a distributed system [121], so its advantages become reduced.

\subsubsection{Petals ESB}

This is another open source proposal for an Enterprise Service Bus primarily used for the implementation of a SOA that comes with a collection of heterogeneous services in its last distribution [122]. It has been developed by the OW2 consortium (an open source software community that claims to be independent, global and promote the development of software facilities as open-source middleware, generic business applications or cloud computing 
platforms [123]). Petals ESB is has eight different kinds of features that can be described as follows:

1. The Petals server: the latest distribution of Petals comes with a standalone Java server composed by several modules that supports a distributed mode, which implies that a group of instances can constitute a single virtual ESB.

2. JBI Components: they are used to bridge services included in Petals with external systems or applications (binding components) and provide internal logic-like service orchestration, routing or transformation (service engines).

3. Administration: it has two different components: the Petals ANT tasks (a collection of ANT tasks used to execute administration programs) and the Petals Command Line Interface (CLI, utilized to administrate a group of Petals servers).

4. Platform monitoring: a Java Management Extensions (JMX)-based server has been included. It has a JMX API conceived to work Cacti (a monitoring tool with graphical options used to display historical data gathered from monitoring probes [124]) and Nagios (another monitoring tool for mission-critical infrastructure components such as applications, network protocols or operating systems [125]).

5. Flow monitoring: in order to provide good enough monitoring capabilities, log services must be provided. Therefore, a log level called MONIT is included in the ESB. Furthermore, specific log handlers have also been added as an extra service.

6. Development: there are two software facilities that are offered by the Petals ESB: one is petals studio (described by the developers of this ESB as a custom Eclipse distribution) and the petals Maven plugin (a regular Maven plugin that offers additional support for Petals ESB).

7. Packaging: there are two ways offered to install this ESB: either by means of a compressed ZIP archive or by means of Devian packages offered for the Petals Command Line Interface, server and their extensions.

8. Experimental features: there some other facilities included that have been regarded as experimental by the creators of this ESB. One is the Petals web console (that is, a web application used to administrate a set of Petals servers), a Simple Flow Viewer (another web application used to visualize Petals flows) and a log handler to store information in a Mongo DB.

As for the Petals ESB itself, the authors claim that it has been developed according to the software component paradigm [126]. There are four different kinds of those that can be found:

1. Configuration: the software components present here are used for configuration duties: topology is utilized to acknowledge and update the topology information of the Petals ESB network, configuration has data about properties of the local Petals ESB container and the System State stores states and keeps consistency whenever a Java Business Integration (JBI) artefact state gets an update.

2. Distribution: these are the software components related to the distributed nature of the ESB: network provides information that is complementary to the one provided by 
the topology, whereas repository handles JBI artefacts resources needed at runtime by the ESB container.

3. Messaging is used for the external communications of Petals ESB. There are three software components to be mentioned in this case: delivery channel behaves as an interface with the $\mathrm{JBI}$ consumer/provider, router plays the role of choosing the endpoint to send any JBI message and uses and the transporter mediates between the router component and the communication channel.

4. Management is used to rule the capabilities of Petals ESB. This is the kind of software with a highest number or components. Deployment manages the deployment of service assemblies, whereas the installation service deals with installing the JBI components and their shared libraries. The Java Management Extensions (JMX) service is used to manage JMX connections to either a remote or a local petals ESB container. Lastly, there are other software components used for LifeCycle management purposes.

Their overall appearance has been displayed in Figure 38. Note that in order to send messages to the JBI consumer, the only kind of software components that is involved in this interchange is the Messaging one.

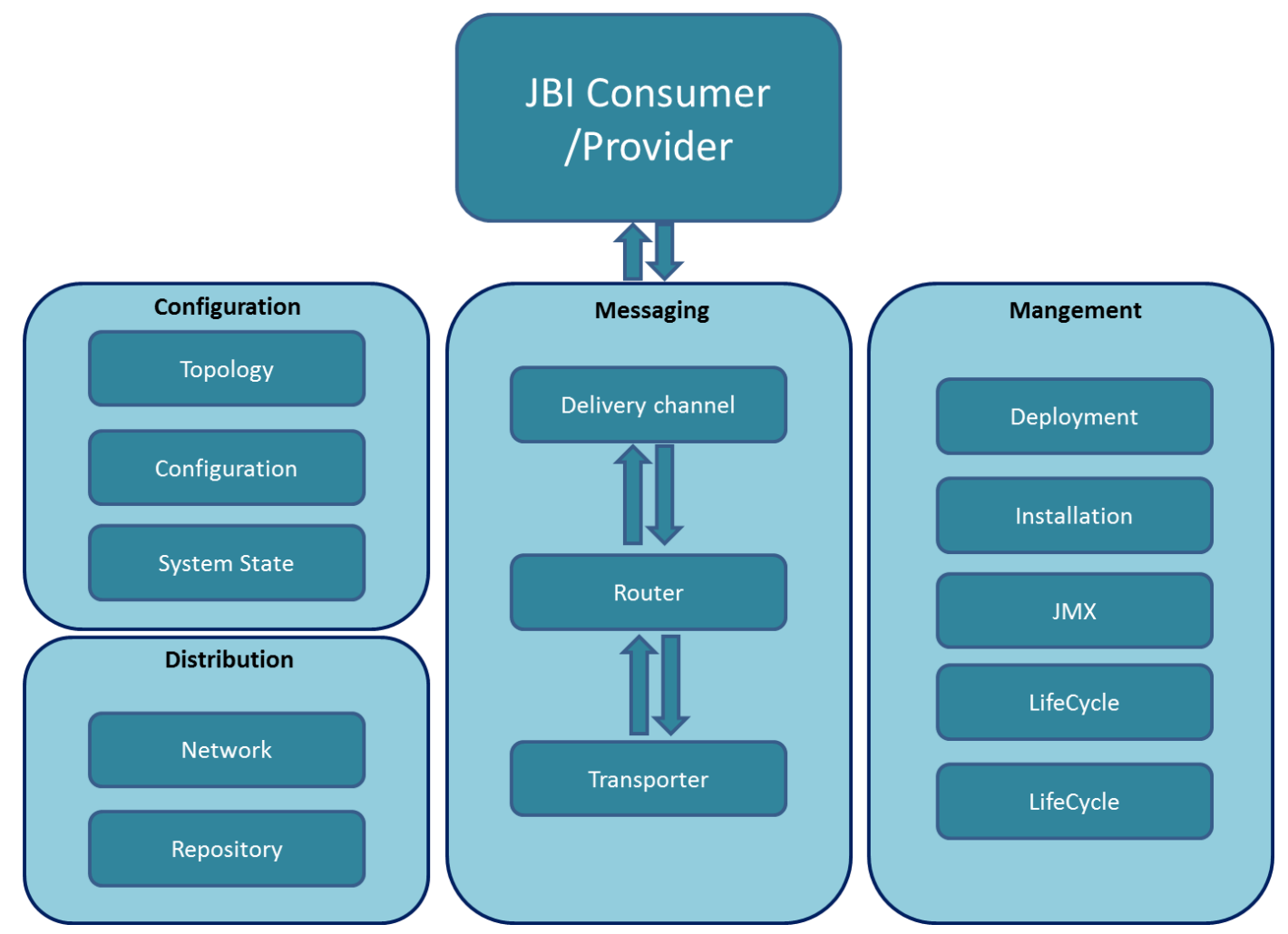

Figure 38. Petals ESB software components, as described in [126]

Petals ESB has a large number of services available to be immediately installed wherever it is required, such as a personal computer or a server. The main inconvenience that can be found in Petals is that it is not as popular or a widely used as other open source solutions, so it might be more challenging than wanted (fixing bugs, getting feedback from the community, etc.) during implementation duties. 


\subsubsection{Mule ESB}

According to its developers, this proposal for a source Enterprise Service Bus [127] is a lightweight one. There are several features to be taken into account in this. A description of all the services that are encased within Mule ESB can be found in [128]. This Enterprise Service Bus is offered in two different implementations called Mule ESB Community and Mule ESB Enterprise. The latter has a greater number of features, such as operational control facilities (web-based console, deployment and performance management, etc.) and additional security characteristics. Also, it is offered with additional support (for example, Service Level Agreements are provided). However, some of these functionalities are provided either by subscription or as separated products [129]. It is mentioned that the capabilities of Mule ESB include message routing (used for aggregating, filtering, routing and re-sequencing messages considering assigned rules and content), service mediation (negates differences among message protocols and services, separates business logic from messaging and makes possible location-independent service calls), service creation and hosting (hosts and exposes reusable services using the ESB as the container) and data transformation (utilized for exchanging information through several different formats and transport protocols). Figure 39 is displaying them as they have been described.

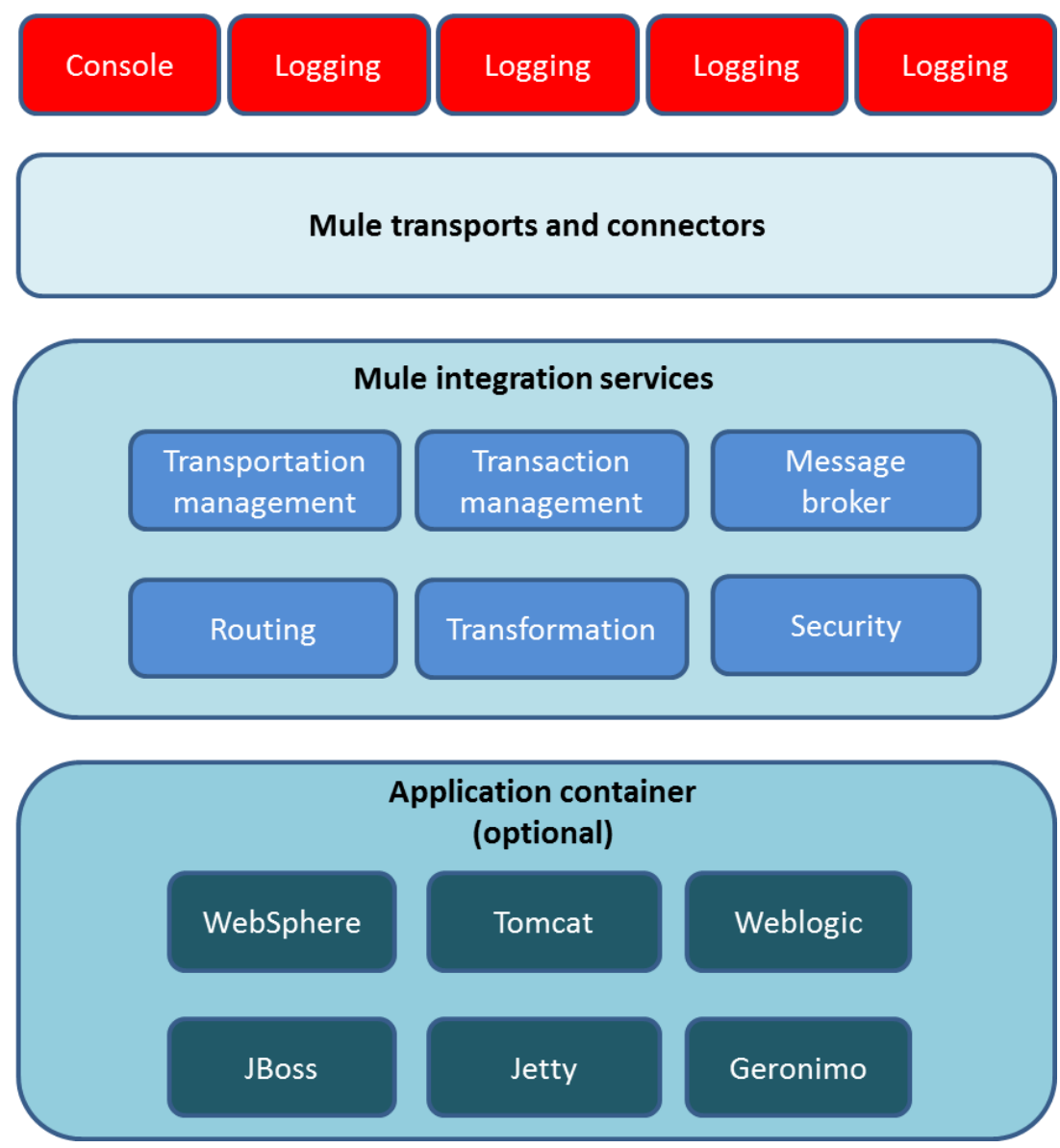

Figure 39. Mule ESB main software components, as described in [128] 
According to the performance metrics described in the MuleSoft white paper on MULE ESB Enterprise Performance [130], a customer should be able to process almost a million messages every day with less than 200 milliseconds latency and a peak rate of 600 messages per second. Testing activities run in a machine with 24 cores showed that an average latency of less than 1 second was measured for tests of increasing concurrency of up to 800 threads. A stable throughput with up to 4000 threads could be offered.

Among the advantages that the developers of Mule ESB claim their solution has, the following ones must be mentioned: a) the components that are encased by Mule ESB can be of very different kinds (ranging from plain Java projects to software components from other frameworks), b) this Enterprise Service Bus accepts many different formats for the messages that are transferred in a deployment (for example, SOAP or binary image files), c) components are conceived to be reusable without any need for specific code or a programmatic API, d) the kind of event-driven architecture that has been conceived (Stage Event-Driven Architecture or SEDA) by the developers allows a high degree of scalability and e) the core of the functionalities can be deployed without the need of an ESB. Overall, this ESB offers a wide set of interconnectivity capabilities for its users. However, some of the most advanced services have to be obtained via subscription (therefore, partially undermining the open source nature of the development).

\subsubsection{Talend ESB}

This Enterprise Service Bus is part of a larger software infrastructure based on what the developers refer to as Talend Open Studio [131] which comprises free tools for Big Data, Data Integration, Data Quality, the ESB and Master Data Management. The authors of this open source ESB implementation mention that it has been built taking into account former open source, Apache ESB-based technologies like Apache CXF, Apache camel and Apache ActiveMQ [132]. In addition to that, this Enterprise Service Bus solution is claimed to be lightweight and have a modular design that comes in handy for incremental deployment in distributed systems projects. Standards as SOAP or REST are also taken into consideration for service development and integration.

Talend ESB developers describe the proposal as made up by three autonomous architectural functional blocks [133], which have been represented in Figure 39. The names of the three functional blocks are: Client (which includes a Talend Studio used to enable data integration or data service processes, services or mediation routes), Talend Execution Servers (which describes one or more Talend Runtimes that make possible deploying and executing the services, jobs and routes previously created in the Studio located in the client part) and the Monitoring Databases (representing the Service Activity Monitoring database that monitors service calls). 


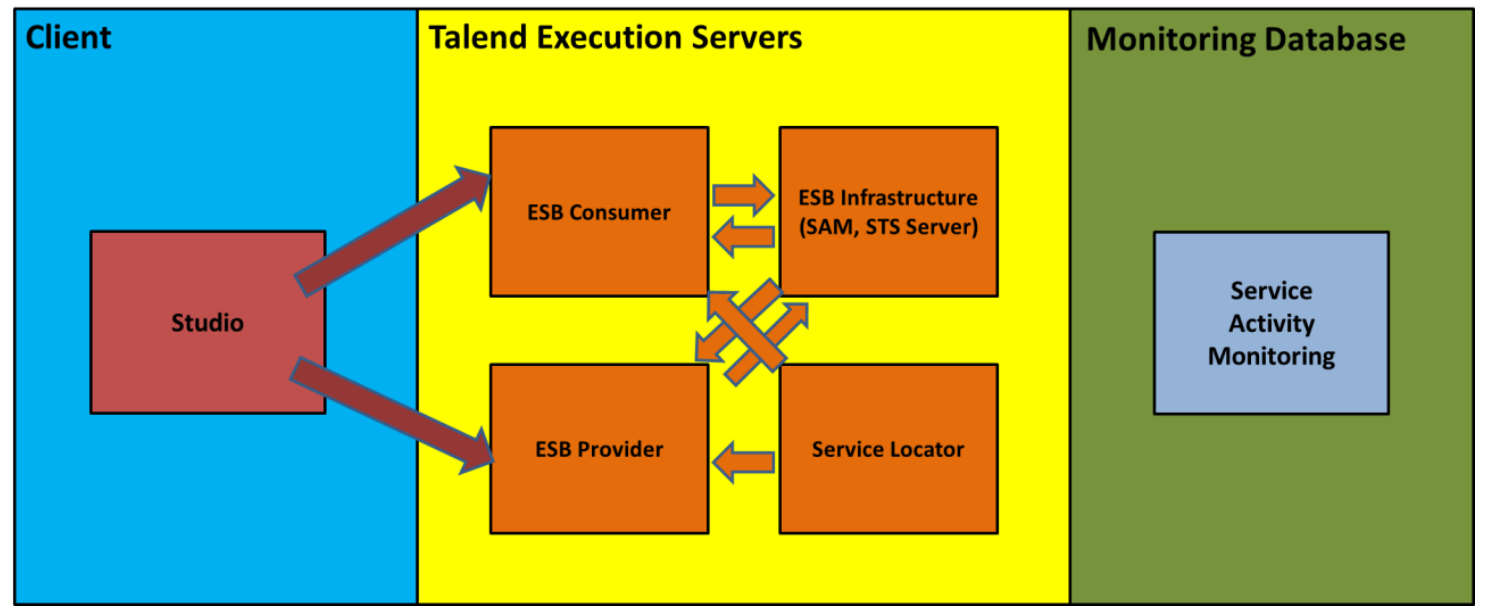

Figure 40. Main architectural functional blocks in Talen, as described in [131]

This solution offers services used to transfer data beyond what is expected from an ESN architecture (client facilities and a monitoring database have also been included in the proposal). At the time of writing this manuscript, though, it was not possible to obtain a white paper or a documented batch of tests regarding the performance of this solution, as the link supposed to provide that documentation seemed non-operational [134]. The boundaries of what is offered as an Enterprise Service Bus solution are not clear either, as the information that is shown tends to be mixed with all the other solutions of the company (for instance, it often looks as if it is part of an Open Studio broader solution).

\subsubsection{Zato ESB}

This is a proposal [135] that is described by its authors as capable of providing open source ESB, SOA, REST, APIs and cloud integrations in Python [136]. Orchestration of integration services, as well as exposing already existing or new APIs, are some of the capabilities that the developers of this proposal mention that Zato ESB has. In addition to that, this Enterprise Service Bus is unique in the sense that enables a very specific collection of features that are referenced in the information available from this ESB proposal:

1. Open source tools that have been codified in Python (APIs, REST interfaces, a ServiceOriented Architecture and the very open source ESB). According to the authors, the usage of Python optimizes the productivity that this ESB can offer.

2. Load balancer capabilities combined with hot deployment and hot reconfiguration make server restart unnecessary.

3. Web- and graphical-based tools that are offered for configuration purposes, easing this kind of activities where XML may be needed in other ESB solutions.

4. A wide collection of protocols and standards from industry are used. The most important ones are: Hypertext Transfer Protocol (HTTP), REST, SOAP, JSON, Advanced Message Queuing Protocol (AMQP), Structured Query Language (SQL), Simple Mail Transfer Protocol (SMTP) and File Transfer Protocol (FTP). Publish/subscribe communications are also possible. 
5. The community around the project is said to be growing, and training or commercial support are available.

The developers of this ESB describe how an ESB is suited to run different applications transmitting information among them when compared to one-to-one communications among a plethora of applications [137]. What is more, it offers a complete and clear set of documents that can be used to get a quick grasp of how this ESB works. However, in order to have secure APIs, and external tool called Vault must be installed separately [138]. Also, while the collection of features provided by the creators of Zato ESB is appealing, some of them are not that unique: for instance, hot deployment is also possible for JBoss Fuse, where software bundles can be deployed right away without having to stop the ESB. This latter ESB solution can also be managed by means of a web console where logs and deployed services are displayed. Furthermore, despite Python is a very simple and efficient programming language, it is not clear that is the optimal solution for productivity, due to the fact that it is a characteristic extremely subjective and dependent on the abilities of human developers. Plus, other programming languages like $\mathrm{C}++$ may offer better performance results that Python. Finally, in order to have Zato installed in machines with Windows or OS X as the operating systems, a virtual machine running a Long Term Support Ubuntu operating system is used in each of the cases [139].

All in all, the main features of the Enterprise Service Bus architectures presented here can be summarized as shown in Table 2:

Table 2. Main characteristics of the studied open source ESB solutions

\begin{tabular}{|c|c|c|}
\hline Proposal name & Advantages & Disadvantages \\
\hline Open ESB & $\begin{array}{l}\text { Variety of services. Clear } \\
\text { legacy system integration. }\end{array}$ & $\begin{array}{l}\text { Only one programming } \\
\text { language implementation } \\
\text { (Java). }\end{array}$ \\
\hline WSO2 ESB & $\begin{array}{l}\text { Service orchestration and } \\
\text { composition are offered. }\end{array}$ & $\begin{array}{l}\text { Multiple configuration } \\
\text { operations have to be done. }\end{array}$ \\
\hline JBoss Fuse & $\begin{array}{l}\text { Wide variety of services. } \\
\text { Easily portable components. } \\
\text { High scalability. Strong } \\
\text { community. }\end{array}$ & $\begin{array}{l}\text { Extra security resources } \\
\text { required. }\end{array}$ \\
\hline Apache ServiceMix & Simplicity of use. & Lack of available services. \\
\hline Petals ESB & Wide variety of services. & $\begin{array}{l}\text { Community not a strong as } \\
\text { in other ESBs. }\end{array}$ \\
\hline Mule ESB & $\begin{array}{l}\text { Strong community. Plethora } \\
\text { of available solutions. }\end{array}$ & $\begin{array}{l}\text { Some services are only } \\
\text { available via subscription. }\end{array}$ \\
\hline Talend ESB & $\begin{array}{l}\text { Capabilities out of the typical } \\
\text { ESB boundaries. }\end{array}$ & $\begin{array}{l}\text { Lack of performance } \\
\text { information. It is not clear } \\
\text { the scope of the ESB in the } \\
\text { Open Studio solution. }\end{array}$ \\
\hline Zato ESB & $\begin{array}{l}\text { User-friendliness (web and } \\
\text { graphical tools). Clarity of } \\
\text { documentation. }\end{array}$ & $\begin{array}{l}\text { Security requires another } \\
\text { tool. Features present in } \\
\text { other ESB solutions. } \\
\text { Dependent on Python. }\end{array}$ \\
\hline
\end{tabular}




\subsection{Open issues and challenges}

Taking into account the previous study done on both middleware solutions for the Smart Grid and Enterprise Service Bus architectures, it can be inferred that the proposed solutions tend to use a common approach to solve the challenges expected in terms of interoperability and service availability. Typically, they will include a way to interconnect devices at the data level (by means of message interchange, hardware abstraction layers or any other resource) and offer interfaces to the application layer. In a network model, these interfaces could be regarded as Service Access Points. In addition to that, the ESBs tend to encase a collection of different services capable of carrying out tasks like message brokering or legacy system integration. The disadvantage to this common way of solving issues is that there are also several common challenges that essentially remain unsolved. Overall, taking into account what has been researched in the presented solutions, the issues that have been found in middleware solutions for the Smart Grid usually resemble the ones found for the ESB architectures, as an ESB is used in this context as the embodiment of middleware. The ones that that could be listed as most significant are:

1. Lack of consistency in service availability. The services that are encased in middleware solutions vary greatly from one proposal to another; there is no list of criteria or fixed rules to define what and what not should be included in a middleware solution. That presents several problems that are difficult to solve. To begin with, the development of new middleware or using a new iteration of an already existing solution is more troublesome than for other kinds of software products, since it is harder to completely reuse the already available code due to the heterogeneity of the services middleware is developed. As a consequence, sometimes it looks more appealing starting from scratch when implementing middleware rather than porting an already existing solution, resulting in a significant loss of resources and an underuse of the already available code, which jeopardizes the scalability of the development works.

2. No common solutions to access services. While there are several ways to offer interfaces to the application layer, there is not a common API agreed to be used in terms of methods, parameters or services to be accessed (as a matter of fact, many solutions do not even have a defined API and solely rely on message interchange to connect remote hardware devices at the data level). This compromises the adaptability of the system to different application domains where a list of facilities to access middleware would have been welcome. In addition to that, it makes likely the update of the middleware solution every single time a new device has to be integrated, thus challenging middleware scalability and making the establishment of Long Term Support versions harder, as it is common in other software developments like operating systems.

3. Ambiguity regarding middleware design. Although a clear definition of middleware and what is expected from it has been accepted for decades, the design of a solution that will offer that kind of services is still unclear. Some solutions make use of services that are part of an architecture deployed in a distributed way (this is the most typical approach of system making use of an ESB, otherwise their service encasing capabilities would not be 
utilized at all), whereas some other solutions use little more than message interchange and prefer focusing on the higher or lower layers. The lack of a middleware design that is used in a generalized manner further complicates interoperability of hardware devices or among subsystems included as part of a larger system (for example, several microgrids included in a large Smart Grid [140] that, depending on their operating conditions, may work either connected to the macrogrid or operate in island mode, isolated from the larger infrastructure).

4. Ambiguity regarding middleware solution. As a direct consequence of the previous open issue, middleware implementation remains a convoluted process where local developments may not guarantee remote interoperability when those implementations have to be combined in something larger. If an open source Enterprise Service Bus is used, though, development can be eased, as in the end the software packages will be mutually understandable in different parts of the distributed systems, as long as the same kind of ESB is used (sometimes, using a slightly different kind of ESB in different sides of the system will still be possible if they have a common ground. For instance, it is shown in [141] that an constrained Apache ServiceMix ESB can work in a seamless way with a JBosslike ESB).

5. No specific methodology for middleware development. Unlike other works that have very accurate procedures for software implementation (for example, software design via UML [142]) or have standards that define languages for software architecture description [143], there is not a clearly defined approach or standardized manner to develop middleware solutions. Again, this is a source of challenges, as a team that has to implement this kind of software solution has to rely on their instincts or the experience of their senior members (if any), thus making the whole procedure less predictable.

In a nutshell, lack of standardized, specific software solutions that clearly describe what is required from middleware are a critical hinder for the development works that result in losing time and money in codification of software components hard to accommodate to any further development that might be carried out. These are the open issues that the proposal included in this doctoral thesis deals with, based on the requirement analysis, design, implementation and testing experience that has been gain through the participation in research projects where middleware development is required.

\subsection{Section summary}

It has been said at the start of this section that middleware is a software development desirable for the Smart Grid, as it makes possible several functionalities that can be regarded as mandatory for the satisfactory performance of a system where hardware devices (AMI, PMUs, RTUs, etc.) and software components have to be used. In addition to that, since this kind of software development can be found in different kinds of systems and fulfilling their functionalities in a diverse manner, its location in a matrix representing the different possible features of middleware for the Smart Grid has also been included. 
As the main objective of this thesis is the design and development of a complete middleware architecture for the application domain of the Smart Grid, a study on the state of the art in this topic has been carried out. In order to have a fixed of features to evaluate how each of the proposals solves the challenges that have to be faced by middleware, four different characteristics have been defined, with different degrees of depth that vary from one solution to the next one. Due to the nature of the middleware, decentralization and software architecture-like proposals are the most frequent ones, albeit they do not form a homogenous majority and have in fact deep differences regarding the services that have been included, how they are accessed or the way they tackled the underlying hardware present in a deployment.

Furthermore, a state of the art in open source Enterprise Service Bus solutions has been included too. Since the middleware solutions tend to include a number of layers bent on solving different kinds of issues, along with integrating services that have been previously implemented as software packages, the usage of an ESB has been justified. In addition to that, it is also stated why an open source solution is a more appealing option for the needs that research projects have had, as they are usually costless and have a strong community behind them willing to provide information in case any issue springs up. The most common problems found in the developed Enterprise Service Buses mirror to an extent the ones found in middleware solutions: either the services that they provide are scarce, the most important ones are not available, or it is not clearly stated how they are accessed by both the application and the hardware-based layers. The size of the community using them has been regarded as a major characteristic to take into account, as a professional customer service or completely reliable support cannot be guaranteed for an open source development most of the times and bugs and unforeseen issues are prone to appear in distributed systems that imply development activities made by several people.

Overall, the open issues and limitations that have been found are closely related to the lack of a standard (or at least, strongly advisable) solution that defines with clarity a collection of key features of a middleware development: the services that are going to be included, how they are going to be accessed, the requirements that should be taken into account when designing the solution, how to easily integrate local developments in a distributed system and the necessary steps to successfully implement middleware in the Smart Grid.

What the next section presents is a way to deal with all these open issues so that they can be either completely erased or mitigated to a great extent. The specific problems that are going to be solved are described as objectives that will be fixed in the proposed middleware solution. Considerations in the requirement analysis that is mandatory for almost any software development have been included. From those requirements, a design of software components will result. These software components will satisfy functionalities to be expected from a Smart Grid that has a significant amount of data being transmitted from one side to the other of a distributed system. 
3. Proposal for a Common Middleware Architecture 


\subsection{Introduction}

This section of the thesis has been included in order to have an accurate perspective of the proposal that is done for (as mentioned in the objectives at the introduction) the development of a standardized middleware architecture to guarantee interoperability among the devices present in a Smart Grid. Acknowledging this objective, the proposal has been named Common Middleware Architecture (CMA), as it aims at contributing to a common way to design and implement a semantic middleware solution for the Smart Grid that might be extensive to other CPSs where similar standardization issues are taking place. When reading through this section, it must be taken into account that several points of view have been considered with the purpose of providing a perspective on the proposal as holistic and detailed as possible. UML come here as a useful tool, since they make possible the representation of the software components required for the middleware. More specifically, the majority of the description that has been included in this section has been done so according the ideas put forward by the principles of the Model Driven Architecture (MDA), an initiative commanded by the Object Management Group, which aims to create software architectures that guarantee portability, interoperability, platform independence, domain specificity and productivity [144]. MDA offers a framework where there are two points of view with critical importance to understand the perspectives that can be provided for the CMA:

1. Computational Independent Model. Although it can be defined as a vocabulary of a problem domain useful to a variety of stakeholders [145], for other authors there are actually two different kinds of CIMs: ontology-based (made up by a formalized conceptual domain model) and UML-based ones [146]. As it can be inferred from its name, no rules are defined for computer-related implementation activities. This model is the most likely to be obtained when the requirement analysis is carried out (since it makes use of functional requirements, non-functional requirements, among other features, as explained in [147]), so it is the first one that is obtained when specifying a software system, according to the criteria established by MDA. According to [147], the UML diagrams that can be used to define software at this stage are sequence, activity and use case. In a way, a UML-based Computational Independent Model resembles a Platform Independent Model. The most important characteristic of this latter model is that it is obtained regardless of the platform where the software system is expected to run. A Platform Independent Model is of critical importance for the Model Driven Architecture, to the point that the OMG claims that "The MDA is OMG's way of developing applications and writing specifications, based on a platform-independent model (PIM) of the application or specification's business functionality and behavior" or "A complete MDA application consists of a definitive PIM, plus one or more PSMs and complete implementations, one on each platform that the application developer decides to support" [148]. If defined with UML, class, object, timing, interaction or communication diagrams are the most useful ones.

2. Validation of the platform. It is the most specific of the three models, as it is tailored to the pieces of equipment where it is going to be installed and must take into account 
information about specific, already existing solutions, such as network protocols [67]. According to [147], component, package and deployment diagrams are the ones most useful for its representation. Operational, system, support and user documentations are the ones regarded as most useful at this stage.

In addition to MDA, an iteration of the ISO/IEC/IEEE 42010 standard [143] has also been included as another way to describe CMS; it has been considered that viewpoints and standards of different nature enrich the proposal by incorporating other features that would be otherwise ignored. Unlike MDA, ISO/IEC/IEEE 42010 makes use of Architecture and/or Viewpoint Descriptions to define the characteristics of a software development. In any case, one vision is not incompatible with the other one, as there are ways to have an Architecture Description as stated by the ISO/IEC/IEEE standard that will make heavy use of UML, as MDA does.

Lastly, the structures used for the documentation in standards that have been settled under OASIS have also influenced this part of the manuscript, such as the documents that described MQTT [149] or AMQP [150]. They have been of great usefulness to state the different subsections that should be used for the Protocol Data Units when interchanging information at the low levels, as a detailed description is required for the different PDUs that are used in the proposal for the middleware architecture.

\subsection{Common Middleware Architecture: purpose and scope}

If CMA is assessed according to the same criteria that have been presented before (hardware abstraction for devices, high level interfaces for the application layer, UML description, etc.), it will result in the following features:

1. CMA has been conceived as a middleware architecture. Since it was deemed as the best possible solution because it is able to have the largest amount possible of services, an architecture with the three different levels that are most common in the studied proposals (one for hardware abstraction, another for the core services and a third one for interaction with the highest, application-based level) has been defined.

2. CMA can be used under a wide range of computational capabilities. Since CMA aims to provide a high level of decentralization, it has been conceived to being lightweight enough to be installed in almost every device imaginable that is present in the Smart Grid. It can be integrated as part of the software components of a smart meter or as part of the pieces of equipment located in any of the most prominent actors that were defined in the first chapter, regardless of the underlying software solution that is used during the implementation stages (ESB or any other are fine as long as they include the software components).

3. CMA has been designed as a Client/Server architecture capable of using other kinds of message coupling. The main idea behind the proposal is that it will be able to satisfy requests when they are started from the client side of the communication with the knowledge of the specific device among a plethora that should receive it (the server). 
However, as it will be described in chapter 4, there is no problem in using technologies (AMQP) that are mostly involved in a Publish/Subscribe paradigm.

4. Common Middleware Architecture is a mostly decentralized solution. While there will be a piece (or maybe even several pieces) of equipment that will have more software components installed that some others, many of the most prominent functionalities (data collection, device registration, etc.) will be done either in the periphery of the system or with the cooperation of it. As mentioned before, even though the validation of the proposal has been made with an ESB, there are no preconditions to have it implemented and installed with other architectural styles.

As it has been depicted in Figure 41, the CMA is located as any other middleware solution would be. As for the legacy systems that might be either too poor in computational resources to have middleware components running (regardless of how unlikely that is) or proprietary solutions that cannot be accessed because that would require unauthorized reprogramming of its software, they can still be integrated via gateways that will interface those legacy systems and adapt their information formats to the one used at the other parts of the deployment. 


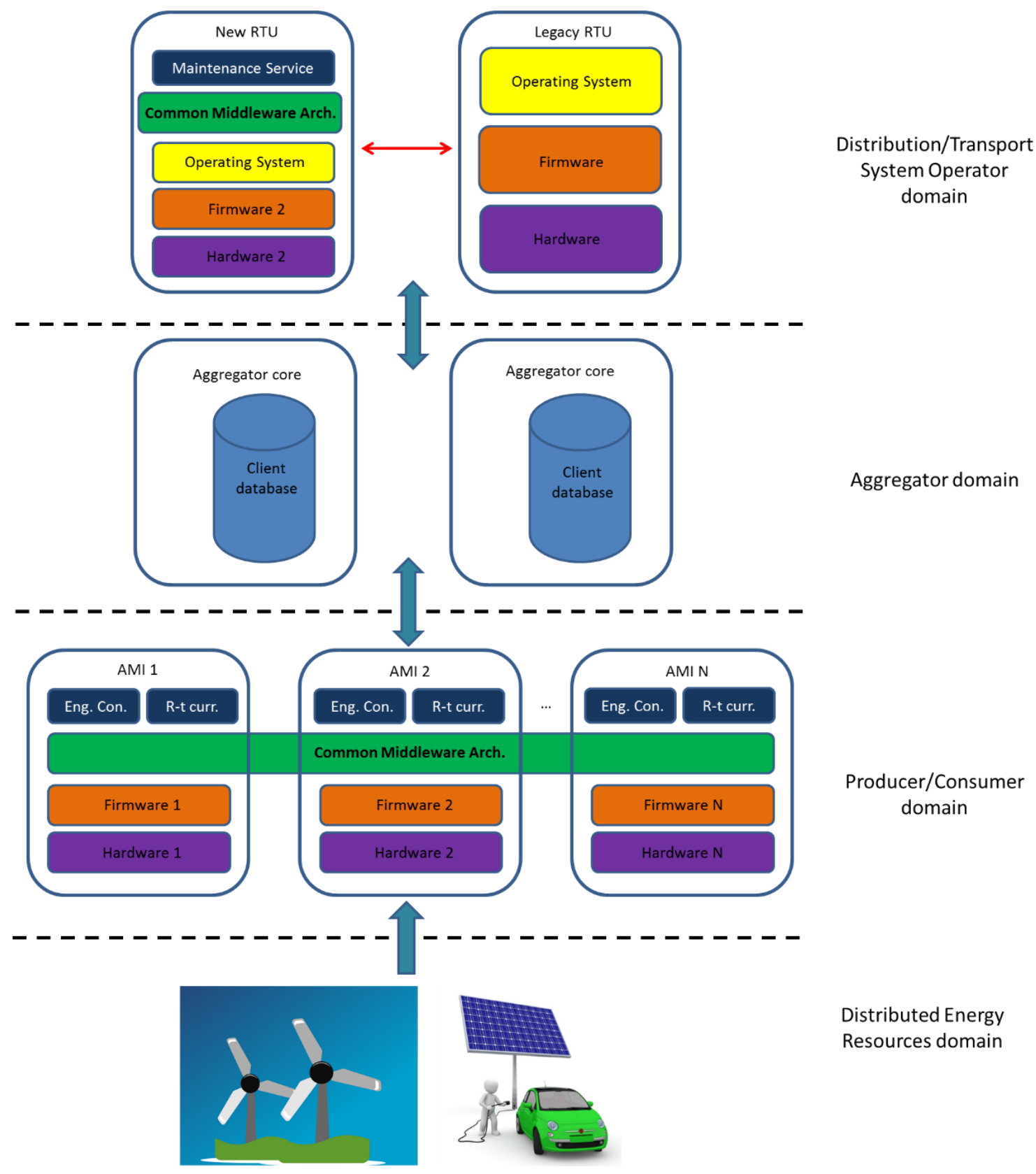

Figure 41. Location of Common Middleware Architecture

Therefore, it can be said that the purpose of this proposal of semantic middleware for the Smart Grid is tackling to the greatest extent possible the open issues found before, so that it will imply a major step forward the usability of the advantages that middleware can offer. Another purpose of this thesis is using CMA as a way to establish a reliable way to implement a middleware solution of the Smart Grid will also be able as a cornerstone for the standardization of middleware in this application domain. As explained before, the development of middleware implies several different services at different levels, so it is a complex procedure that can become convoluted and complex, especially if many different parties are involved in the development. By using CMA-based implementations, it becomes easy to know which are the required steps and building blocks to develop middleware; they 
can even be extended to other Cyber-Physical Systems with similar challenges to the ones present in this area of knowledge.

As far as the scope of the proposal is concerned, CMA is located at the data layer level that makes possible information transfers from heterogeneous devices and characteristics, as any other intermediation architecture like the ones that have been studied. Another perspective to look at it is comparing CMA with the model for the Smart Grid put forward by the Smart Grid Coordination Group (SG-CG), a collaboration of the Comité Européen de Normalisation (CEN, European Committee for Standardization [151]), the Comité Européen de Normalisation Electrotechnique (CENELEC, European Committee for Electrotechnical Standardization, [152]) and the European Telecommunications Standard Institute (ETSI, [77]). This group of institutions refers to it as the Smart Grid Architecture Model (SGAM) Framework [153]. SGAM takes into account several aspects that go beyond data exchange, such as business objectives, the infrastructures required to produce electricity or the data models and protocols required for data interchange. SGAM takes a three dimensional perspective composed by interoperability layers (business layer, function layer, information layer, communication layer, component layer), domains (generation, transmission, distribution, Distributed Energy Resources and customer premises) and zones (process, field, station, operation, enterprise, market). Figure 42 shows the appearance of SGAM.

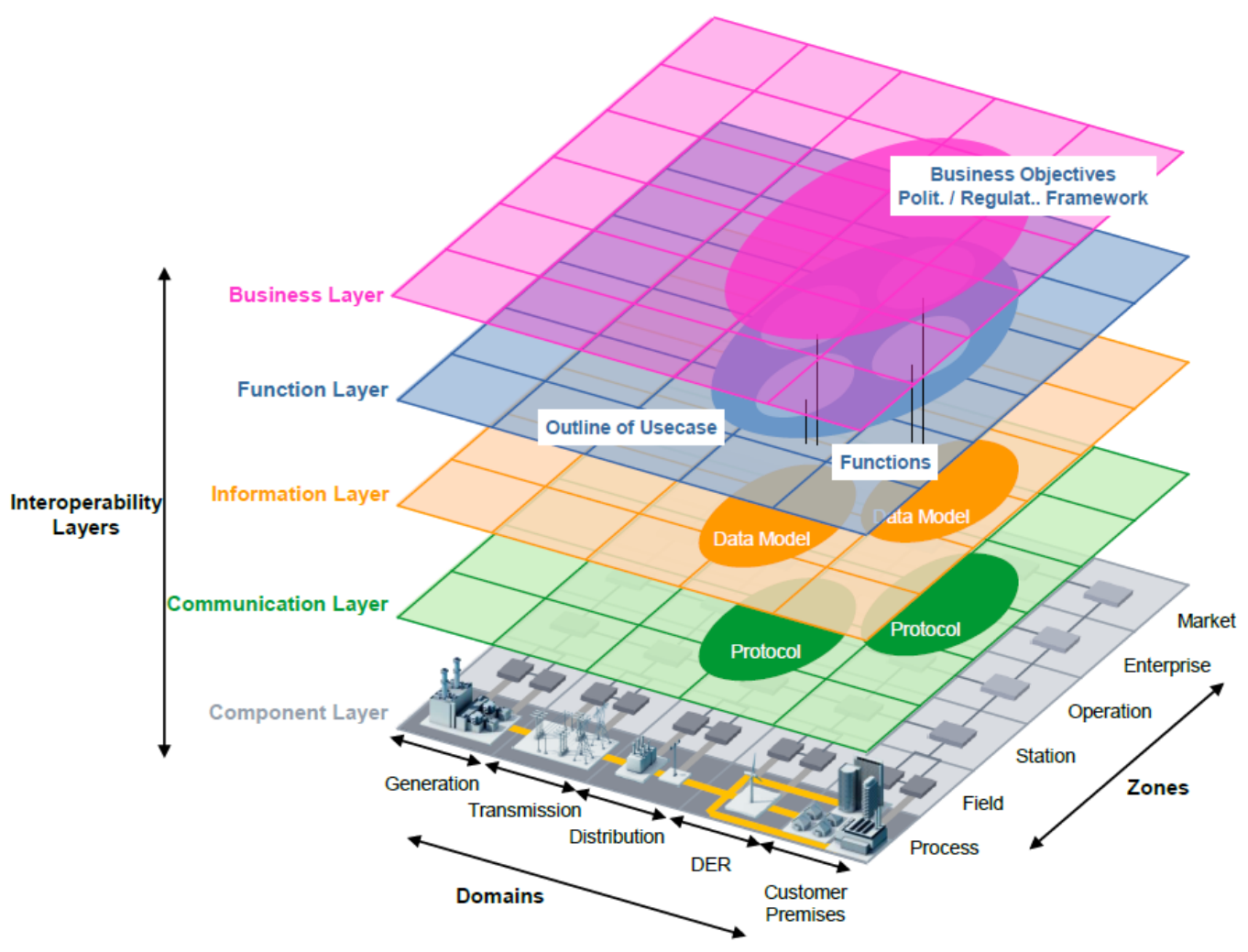

Figure 42. SGAM Framework, as depicted in [153]

This standardization proposal is more of a framework that defines every possible facility present in a Smart Grid with its different features (hardware components, facilities, regulation, business models, etc.) than a proper middleware layer. Nevertheless, its comparison with a 
middleware solution comes in handy due to the fact that some of the levels that are present in SGAM are also within the scope of CMA, to a greater or lesser degree. Specifically, CMA is almost entirely focused on the equivalent Information Layer of SGAM, due to the fact that the functionalities that it uses are placed almost exclusively at the information layer (as middleware operates at the data level). Figure 43 compares the two different scopes assumed by each of the proposals: whereas SGAM offers a holistic view of all the hardware, networking and software components involved in the Smart Grid, CMA takes information layer at a deeper level while takes business or components as already for granted. While CMA is located at the information layer and nowhere else, interfaces are offered to the communications layer represented by SGAM, due to the fact that it is a distributed system where aspects like networking have to be born in mind when designing the solution.
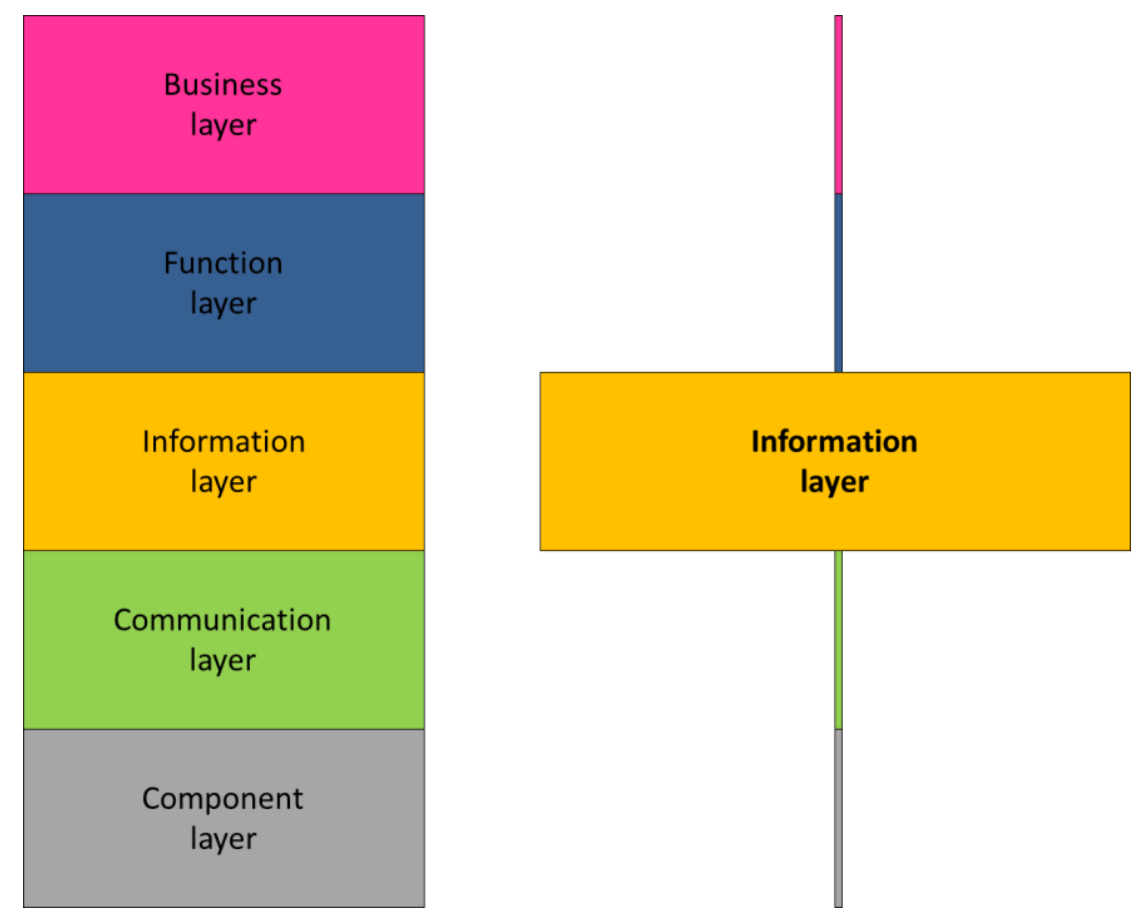

Figure 43. Comparison between the scope of SGAM and CMA.

\subsection{Computational analysis}

When compared to the traditional waterfall model for software development, this stage would be similar as the requirement analysis done prior to the design of a software system. Therefore, all the imaginable requirements of the system must be conceived at this point, so that they will be captured as software components performing the required functionalities.

Table 3. Actions taken to tackle open issues

\begin{tabular}{|l|l|l|}
\hline Open Issue/Challenge & Requirement & Action \\
\hline $\begin{array}{l}\text { Lack of consistency in service } \\
\text { availability. }\end{array}$ & $\begin{array}{l}\text { Information managed by the } \\
\text { middleware architecture. }\end{array}$ & $\begin{array}{l}\text { Design of a fixed collection } \\
\text { of services to be included in } \\
\text { the middleware architecture. }\end{array}$ \\
\hline No common solutions to & Elaboration of an Application & Inclusion of Middleware \\
\hline
\end{tabular}




\begin{tabular}{|l|l|l|}
\hline Open Issue/Challenge & Requirement & Action \\
\hline access services. & $\begin{array}{l}\text { Programming Interface to } \\
\text { access the semantic } \\
\text { middleware solution. }\end{array}$ & $\begin{array}{l}\text { Access Points to use its } \\
\text { services. }\end{array}$ \\
\hline $\begin{array}{l}\text { Ambiguity regarding } \\
\text { middleware design. }\end{array}$ & $\begin{array}{l}\text { Standardization procedures } \\
\text { for design in semantic } \\
\text { middleware for the Smart } \\
\text { Grid. }\end{array}$ & $\begin{array}{l}\text { Creation of a list of } \\
\text { functional and non- } \\
\text { functional requirements to } \\
\text { be included in middleware } \\
\text { design. }\end{array}$ \\
\hline $\begin{array}{l}\text { Ambiguity regarding } \\
\text { middleware solution. }\end{array}$ & $\begin{array}{l}\text { Standardization procedures } \\
\text { for implementation in } \\
\text { semantic middleware for the } \\
\text { Smart Grid. }\end{array}$ & $\begin{array}{l}\text { Development of middleware } \\
\text { as a collection of executable } \\
\text { components. }\end{array}$ \\
\hline $\begin{array}{l}\text { No specific methodology for } \\
\text { middleware development. }\end{array}$ & $\begin{array}{l}\text { Development of a } \\
\text { methodology for conception } \\
\text { and development of } \\
\text { semantic middleware. }\end{array}$ & $\begin{array}{l}\text { Appendix describing the } \\
\text { methodology of middleware } \\
\text { development. }\end{array}$ \\
\hline
\end{tabular}

\subsubsection{Functional requirements}

CMA has been defined the way it is due to the set of requirements that were used in the research projects related to the Smart Grid were middleware was required to be added as a way to guarantee device interoperability, interconnectivity at the data level and access to devices from applications. The functional requirements were described taking into account the three scenarios that had been set for the demonstrators where middleware was expected to be deployed: one of them was based on a residential scenario (where several home dwellings were equipped with Raspberry Pi-based Advanced Metering Infrastructure), another one for a factory used to manufacture olive oil (where all the processes were automated and the facility relied on SCADA systems to gather information) and a third one for a tertiary environment (which implied power infrastructure for a set of buildings). Consequently, the high level appearance of the functional requirements for the middleware architecture was as follows:

1. Information managed by the middleware architecture: considering the kind of application domain where the research projects took place, it was decided that middleware was to be involved in the transfer of information regarding prices, weather forecasting, energy consumption forecasting, energy generation forecasting, energy storage forecasting and historical data. By the term "managing", it was meant that data received by the middleware about the previously formulated topics would have to be sent to the corresponding higher level entities where it was supposed to be utilized in a significant way, as the services themselves were part of the applications that could be used in the demonstrator.

2. Support of Central and Local Controllers: as the implementation activities of the research projects went by, it was decided that the most suitable way to deploy the hardware components would be having a Central Controller than managed other smaller controllers located in different facilities; these latter were called Local Controllers. Therefore, the 
middleware components should be included in those hardware devices too, along with the ones dealing with the data transferred at the data level among them.

3. Service Discovery: the availability of services should be made clear for the application layer (as well as the devices present in the deployment as well. However, the middleware architecture described here is way more service- than device-oriented), as well as a registration mechanisms for them.

4. Information security: securitization of the interfaces that were used to access information had to be offered so that the whole system would guarantee some security dealing with access to the system.

As for the non-functional requirements that were established (as a way to strictly define what the system should be), the participants of the projects where middleware for the Smart Grid was to be developed decided that those requirements would be the following ones:

1. Usage of open source licenses. In order to maximize the usability and usefulness of the provided budget, it was decided that open source tools were the most useful for the development of the works done. As it will be explained in the next section, an open source Enterprise Service Bus was chosen to embody the middleware works done in the research projects that were carried out under the idea of having a common framework for middleware in the Smart Grid, as represented by CMA.

2. Usage of an ESB. As explained in [154], it has been deemed that the most suitable option for middleware in the Smart Grid is using an ESB, for it is able to interconnect any distributed system with ease for the developers using it (as all the complexity of the underlying software connection facilities are hidden from them) and is capable of encasing services and facilities at a level that other architectures do not do, which would imply reducing middleware to just a set of PDUs been transmitted among several devices.

3. Usage of semantic capabilities. A semantic middleware architecture designed to contain features for information inference from the data transferred among the deployment was expected to be implemented, as it was a common flaw found in the existing literature.

4. Middleware as a distributed system. There was no way that middleware could be implemented as a centralized effort in a single device, as it would not fit the distributed nature of the Smart Grid, where information is shared among many different elements scattered in a wide location, so it was decided that middleware would be present in different devices.

5. Middleware as a Service-Oriented Architecture. Instead of stressing the technical characteristics of the devices present in a deployment, it was considered that middleware should focus on the services that could be provided, as it was going to be embodied by a collection of software components capable of providing functionalities.

All these requirements were common for all the three demonstrators that were conceived to be undertaken during the testing and deployment of hardware and software activities. They were further defined in a more specific way as the design activities went by. When implementation works had to be done, all these requirements were given identifiers as a way to have more specific information about them. Once they had them assigned, it became easier 
to figure out which services should be implemented so that they could be included. The have been included in Table 4.

Table 4. Functional and Non-functional requirements for middleware

\begin{tabular}{|c|c|c|}
\hline Requirement identifier & Name and description & $\begin{array}{l}\text { Functional/ } \\
\text { Non-functional }\end{array}$ \\
\hline EGO-MDW-FUN-0010 & $\begin{array}{l}\text { Middleware managed information. Prices, } \\
\text { generation and storage forecast, weather } \\
\text { forecast, energy generation and storage } \\
\text { forecast, energy consumption forecast, } \\
\text { historical data. }\end{array}$ & Functional \\
\hline EGO-MDW-OPR-0020 & $\begin{array}{l}\text { Central Controller support. A piece of } \\
\text { hardware in the deployment will have } \\
\text { more middleware components. }\end{array}$ & Functional \\
\hline EGO-MDW-OPR-0030 & $\begin{array}{l}\text { Local Controller support. The less } \\
\text { significant pieces of hardware will still have } \\
\text { some middleware components. }\end{array}$ & Functional \\
\hline EGO-MDW-FUN-0040 & $\begin{array}{l}\text { Middleware service discovery. Middleware } \\
\text { will be able to register devices and offer } \\
\text { services to the higher layers. }\end{array}$ & Functional \\
\hline EGO-MDW-SEC-0050 & $\begin{array}{l}\text { Middleware information security. } \\
\text { Information will be access in a secure way. }\end{array}$ & Functional \\
\hline EGO-MDW-NFR-0010 & $\begin{array}{l}\text { Open source licenses. Middleware is } \\
\text { developed using open source licensed } \\
\text { tools. }\end{array}$ & Non-functional \\
\hline EGO-MDW-NFR-0020 & $\begin{array}{l}\text { Semantic capabilities. Middleware } \\
\text { architecture makes use of semantics in one } \\
\text { component used for device registration. }\end{array}$ & Non-functional \\
\hline EGO-MDW-NFR-0030 & $\begin{array}{l}\text { Enterprise Service Bus. An ESB is used to } \\
\text { guarantee message interchange and } \\
\text { services encased. }\end{array}$ & Non-functional \\
\hline EGO-MDW-NFR-0040 & $\begin{array}{l}\text { Distributed system. Middleware must be } \\
\text { deployed as a distributed system. }\end{array}$ & Non-functional \\
\hline EGO-MDW-NFR-0050 & $\begin{array}{l}\text { Service-Oriented Architecture. The } \\
\text { middleware architecture must be focused } \\
\text { on offering services to end users rather } \\
\text { than on device technical features. }\end{array}$ & Non-functional \\
\hline
\end{tabular}

Considering these requirements, it was made clear that a) there were several services that had to be designed to fulfil those requirements and b) they would end up modelled as software components. These procedures have been carried out as it has been depicted in Table 5:

Table 5. Software components defined from the requirements

\section{Functional requirement $\quad$ Software component designed $\quad$ Justification} identifier

\begin{tabular}{|l|l|l} 
EGO-MDW-FUN-0010 & High Level services related to & They are needed to either
\end{tabular}




\begin{tabular}{|c|c|c|}
\hline Functional requirement & Software component designed & Justification \\
\hline & energy. & $\begin{array}{l}\text { provide access to middleware or } \\
\text { perform functionalities by } \\
\text { themselves. }\end{array}$ \\
\hline EGO-MDW-OPR-0020 & $\begin{array}{l}\text { Three levels of the middleware } \\
\text { architecture present in the } \\
\text { Central Controller. }\end{array}$ & $\begin{array}{l}\text { They are needed to provide high } \\
\text { level access, perform hardware } \\
\text { abstraction and core } \\
\text { functionalities such as } \\
\text { registration and context } \\
\text { awareness. }\end{array}$ \\
\hline EGO-MDW-OPR-0030 & $\begin{array}{l}\text { Some of the mandatory services } \\
\text { for interoperability and device } \\
\text { registration have been included. }\end{array}$ & $\begin{array}{l}\text { Major operations cannot be } \\
\text { located in devices that might be } \\
\text { used in minor roles in the Smart } \\
\text { grid (for example, as a smart } \\
\text { meter). }\end{array}$ \\
\hline EGO-MDW-FUN-0040 & $\begin{array}{l}\text { Device Registration, Service } \\
\text { discovery components }\end{array}$ & $\begin{array}{l}\text { Required software for the } \\
\text { recognition of all the available } \\
\text { devices }\end{array}$ \\
\hline EGO-MDW-SEC-0050 & Interface securitization & $\begin{array}{l}\text { Required to implement security } \\
\text { in the system }\end{array}$ \\
\hline
\end{tabular}

At the same time, non-functional requirements may have different implications in the middleware, in the sense that they might either impose specific software components or other design features to the software architecture. Table 6 summarizes the actions to cover them and why they were taken.

Table 6. Actions taken to satisfy non-functional requirements and their justification

\begin{tabular}{|l|l|l|}
\hline $\begin{array}{l}\text { Functional requirement } \\
\text { identifier }\end{array}$ & Action taken & Justification \\
\hline EGO-MDW-NFR-0010 & $\begin{array}{l}\text { Open source tools have been } \\
\text { used for middleware } \\
\text { development. }\end{array}$ & $\begin{array}{l}\text { Strong community behind that } \\
\text { can be used to obtain support. } \\
\text { Most cost-efficient solution. }\end{array}$ \\
\hline EGO-MDW-NFR-0020 & $\begin{array}{l}\text { Semantics (and more } \\
\text { specifically, ontologies for data } \\
\text { representation formats) have } \\
\text { been used for device } \\
\text { registration. }\end{array}$ & $\begin{array}{l}\text { Needed for information } \\
\text { enhancement and better } \\
\text { knowledge gathering from the } \\
\text { devices. }\end{array}$ \\
\hline EGO-MDW-NFR-0030 & $\begin{array}{l}\text { Enterprise Service Bus is used as } \\
\text { a software architecture for the } \\
\text { middleware. }\end{array}$ & $\begin{array}{l}\text { Suitable architecture to encase } \\
\text { software components that will } \\
\text { be used as services. }\end{array}$ \\
\hline EGO-MDW-NFR-0040 & $\begin{array}{l}\text { Middleware is present in several } \\
\text { hardware devices in a system. }\end{array}$ & $\begin{array}{l}\text { Middleware must be distributed } \\
\text { for information gathering. }\end{array}$ \\
\hline EGO-MDW-NFR-0050 & $\begin{array}{l}\text { Middleware is conceived as a } \\
\text { Service-Oriented Architecture. }\end{array}$ & $\begin{array}{l}\text { Services to be provided are } \\
\text { more important than hardware. }\end{array}$ \\
\hline
\end{tabular}


When all is said and done, the appearance of the Common Middleware Architecture, from a high level point of view, will be as represented in Figure 44 .

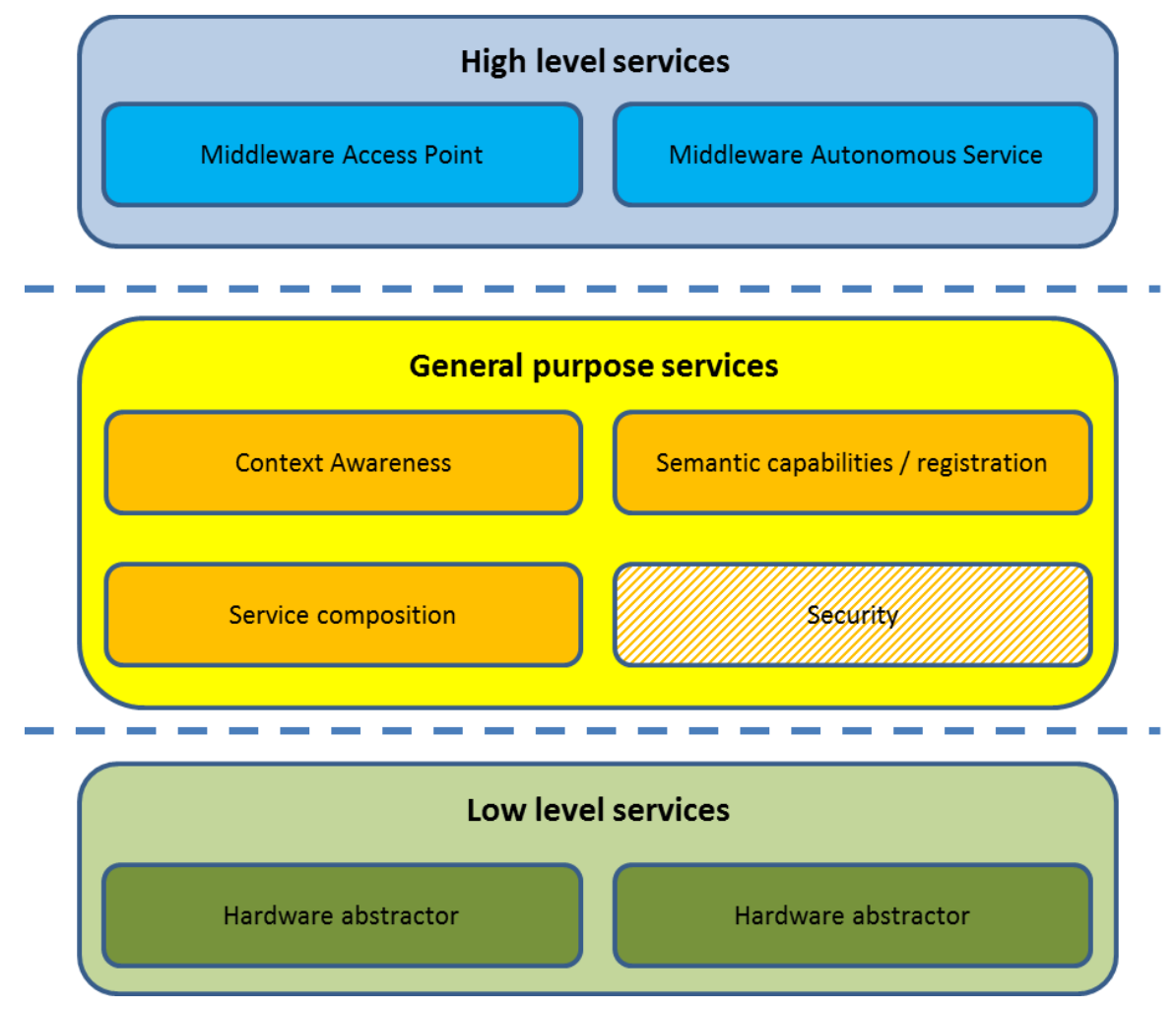

Figure 44. High level appearance of the Common Middleware Architecture

As it can be seen, the features that were imposed by the functional and non-functional requirements have been included in the architecture:

1. There are three levels for the services that have been included in the architecture: the High level contains the services that have been deemed most useful to interact with the application layer, that is to say, the ones that offer interfaces between the application layer and middleware (referred in Figure 44 as Middleware Access Point) and services that are capable of performing their functionalities without requiring further intervention from the other middleware elements (Middleware Autonomous Service).

2. The intermediate level contains the general purpose services; they have been called like that because they contain software components that are more typical of a software engineering product than the Smart Grid, as they do not take into account the nature of the hardware where they are deployed (that is a task that is performed by the high level and low level services, which must be aware of the components they are interfacing). The services that can be included here are more variable than in other developments, but there is still a core of services that can be considered as almost mandatory: context awareness must be offered as a way to be aware of the availability of services (and by proxy, devices, as they are the ones collecting the information required for services), semantic capabilities are implemented as a way to enhance the information provided by the devices and offer registration of services that will provide a higher degree of information, and service composition will be used as a way to obtain new services for the 
whole system based on simple services that are already present (for example, building a fire risk alarm based on a) temperature and b) power readings in a cable). Note that, among other components depending on the needs of the deployment, securitization can also be offered at this level if there are explicit implementation works done regarding information cyphering. While securitization was carried out in the research projects associated to this thesis by means of several operations regarding certificates for web browsers rather than actual implementation of a General purpose service component (as it will be explained in Chapter 4), it can be provided to a software component via libraries (for example, by means of Bouncy Castle ones [155]).

3. Low level services are responsible for the hardware abstraction of the underlying devices in a deployment. Not only they are used to collect the information that is received from the hardware, but also they rely on a specific information format to isolate the hardware particularities. That format is based on a collection of Protocol Data Units that has been included in section 3.6.

Note that despite the application domain where it has been implemented, this representation of the architecture has no specific features related to the Smart Grid, so it can be ported with ease to other environments that rely on CPSs to perform their functionalities. The previous picture shows CMA from a purely software point of view. However, if the hardware devices that are to contain middleware were to be considered, the overall appearance would be as shown in Figure 45. Basically, CMA has been conceived to be installed in different machines and appliances present in the Smart Grid, so that it will be a distributed enough development. There will be two exceptions to the deployment of middleware in this kind of devices: a) when the device will have too little power to have any extra software component installed (unlikely but still possible for some kind of Advanced Metering Infrastructure) or b) it is proprietary system that cannot be programmed by any third party (in that case, a specific interface with the remaining parts of the system will have to be carried out). 


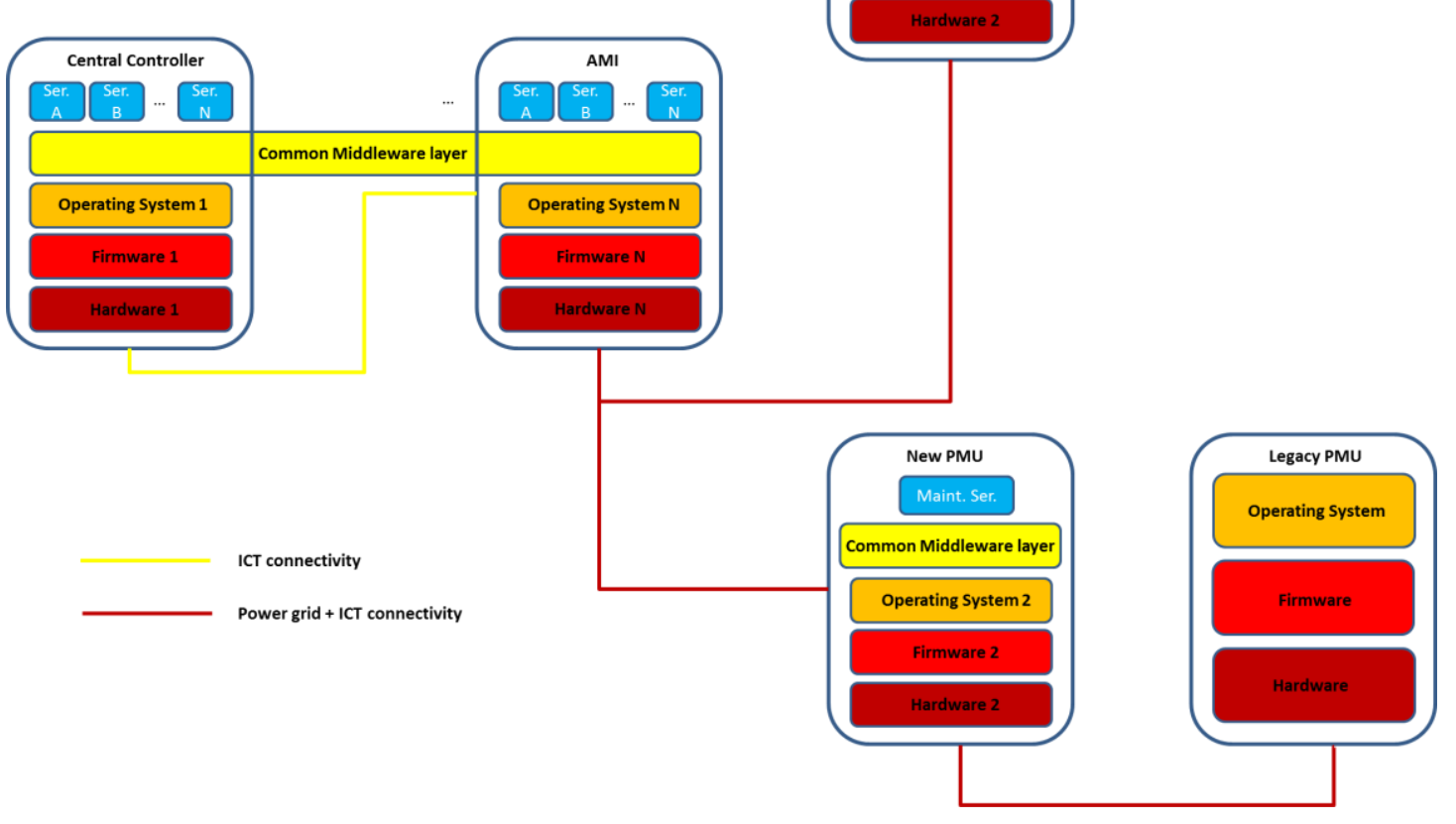

Figure 45. Common Middleware Architecture location in Smart Grid devices

So far, CMA has been depicted just as a representation of software components and levels. A more in-depth depiction based on UML will be done in the next subsections.

\subsubsection{Subsystem diagram}

The first UML diagram that has been included is referred to the highest level possible point of view, which is displaying the three different levels that Common Middleware Architecture is made of. Overall, each of the levels has been gathered in a subsystem that covers all those components. In this way, the High Level Services use a subsystem and interact with the applications and the General Purpose Services, whereas the latter ones form another subsystem that has the low layer services below them. These Low Level Services are also gathered in a subsystem that contains the software components that were defined previously, and will interact with the network infrastructure that has been used to deploy the middleware solution. All these subsystems have been represented in Figure 46. 


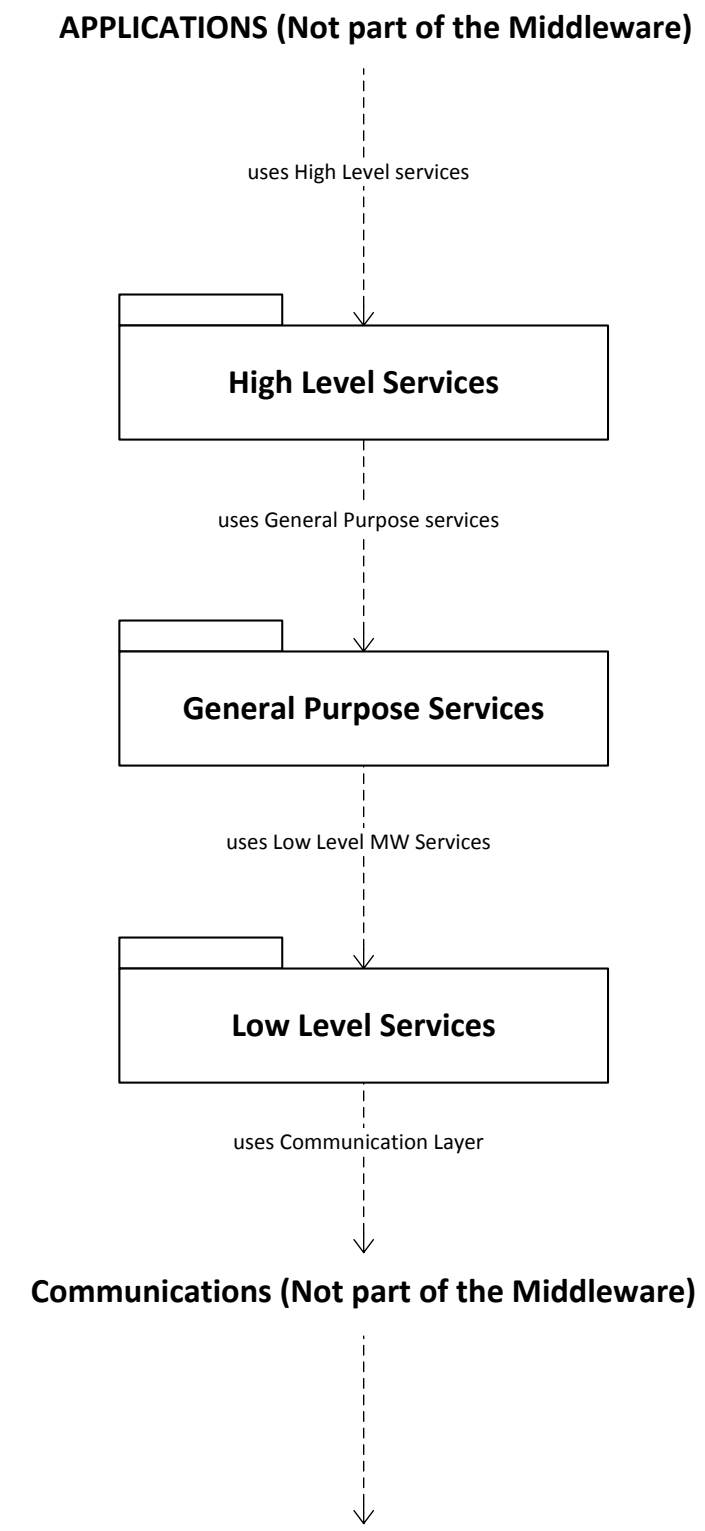

HARDWARE DEVICES (Not part of the Middleware)

Figure 46. CMA subsystem diagram with all the groups of services represented

Each of the subsystems that have been presented can be further divided into several components that interact with each other in the middleware solution.

\subsubsection{Component diagrams}

In the case of High Level Services, there are two different kinds of components that differ with each other in the way that has already been explained: there is a type of service merely used to access the middleware and collect the request done from the application layer (Middleware Access Point), whereas the other type (Middleware Autonomous Service) is used to satisfy the request that was sent, as this kind of service is capable of performing the operations by itself. This latter kind of service has to be implemented whenever it is required that a functionality can be accessed in a distributed manner but does not require any other component from the 
architecture for its performance. For the former type of middleware service, communications will be performed with the General Purpose Services. For Middleware Autonomous Services, on the contrary, only interactions with the application layer will be required, as it has been depicted in Figure 47.

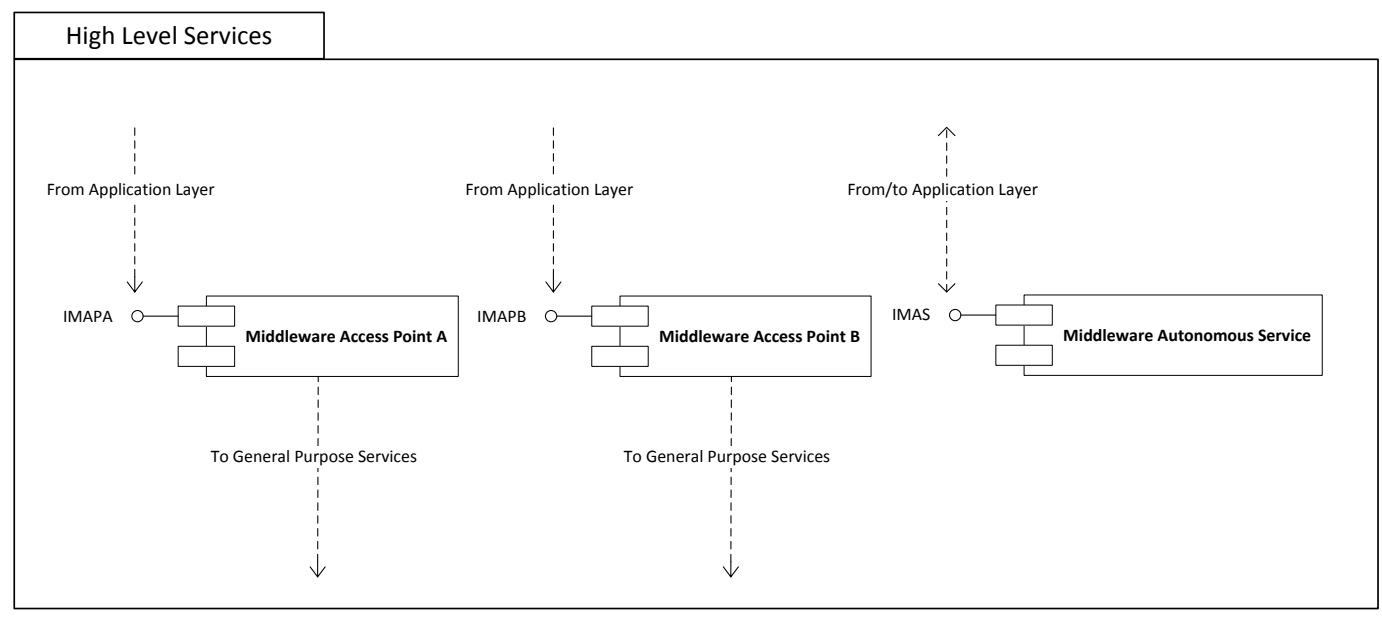

Figure 47. Components diagram from the High Level MW services subsystem

The General Purpose Services combine all the software components that are required for the normal performance of the middleware embedded in the devices that are part of the Smart Grid. The components that are present in this subsystem have a one-to-one correspondence to the software components described in the previous subsection of the thesis: a) a Context Awareness component is used to check on the surroundings of the middleware, along with its different elements, b) a Device Registration component is further used with the suitable semantic capabilities and contains the ontology designed for the system (which will be further used for the semantic enhancement of the information stored in the middleware architecture), c) a Service Composition component is used to merge simple services in new ones and d) a Security component (or at least, a procedure that will enable security capabilities) has been added. These components are represented in Figure 48.

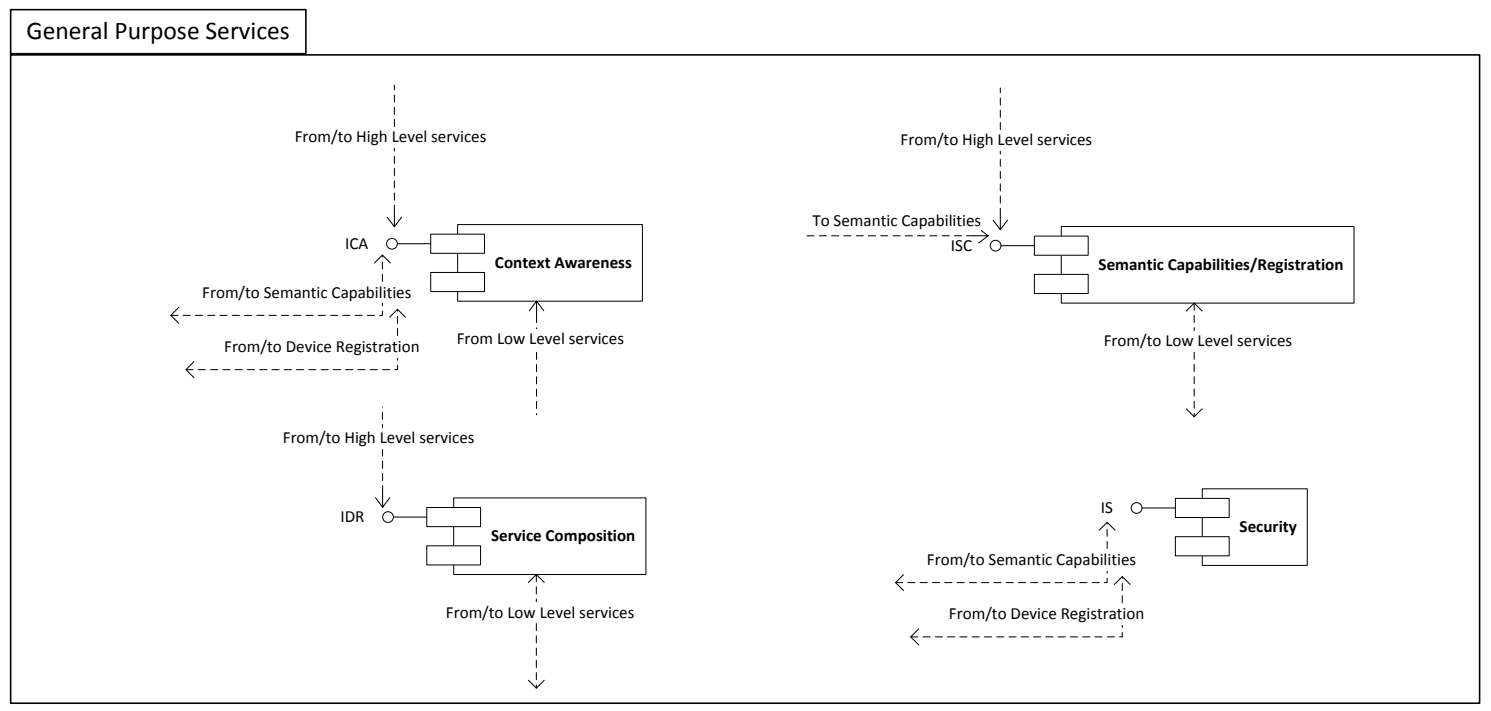

Figure 48. Components diagram from the General Purpose Services subsystem 
Low level services are represented by the hardware adaptors included in the subsystem. If a format based on a data level PDU is respected, one hardware adaptor will be enough, as its main functionality will be receiving the information according to the foreseen format. If for any other reason more components are needed, an additional hardware abstractor can be added for each service or group of services that is required. This idea is further represented in Figure 49, where different hardware abstractors are encased in the Low Level Services subsystem.

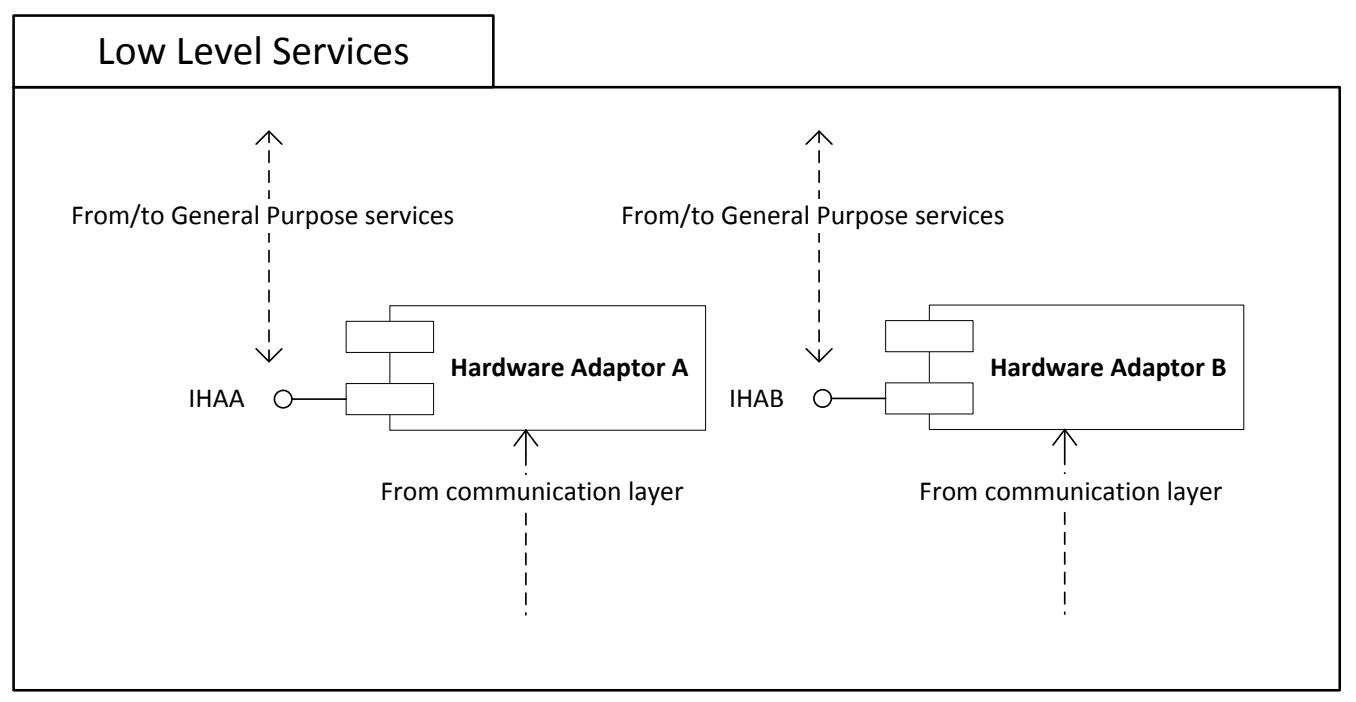

Figure 49. Components diagram from the General Purpose Services subsystem

\subsubsection{Use case diagram}

Use case diagrams have been used to describe with further accuracy the functionalities expected from a middleware architecture. There are two different levels that have been used in order to have a more accurate representation: on the one hand, a top, more generic-looking top level is used to address the most typical use cases that can be found in a development that requires a middleware solution. On the other hand, each of the uses cases (represented in the next diagram as ovals within the boundaries of the system represented by the Common Middleware Architecture) has been further split into other use case diagrams that show the relationships between the functionalities included in each of the use cases that were first formulated. To begin with, Figure 50 displays the main use cases that have been conceived for the middleware architecture. It has to be noted that the actors that have been included are parts of the overall system that is used along with middleware deployment (and therefore is surrounding the location where it is supposed to be) rather than human operators. Also, the boundaries of the system are provided by the CMA itself. 


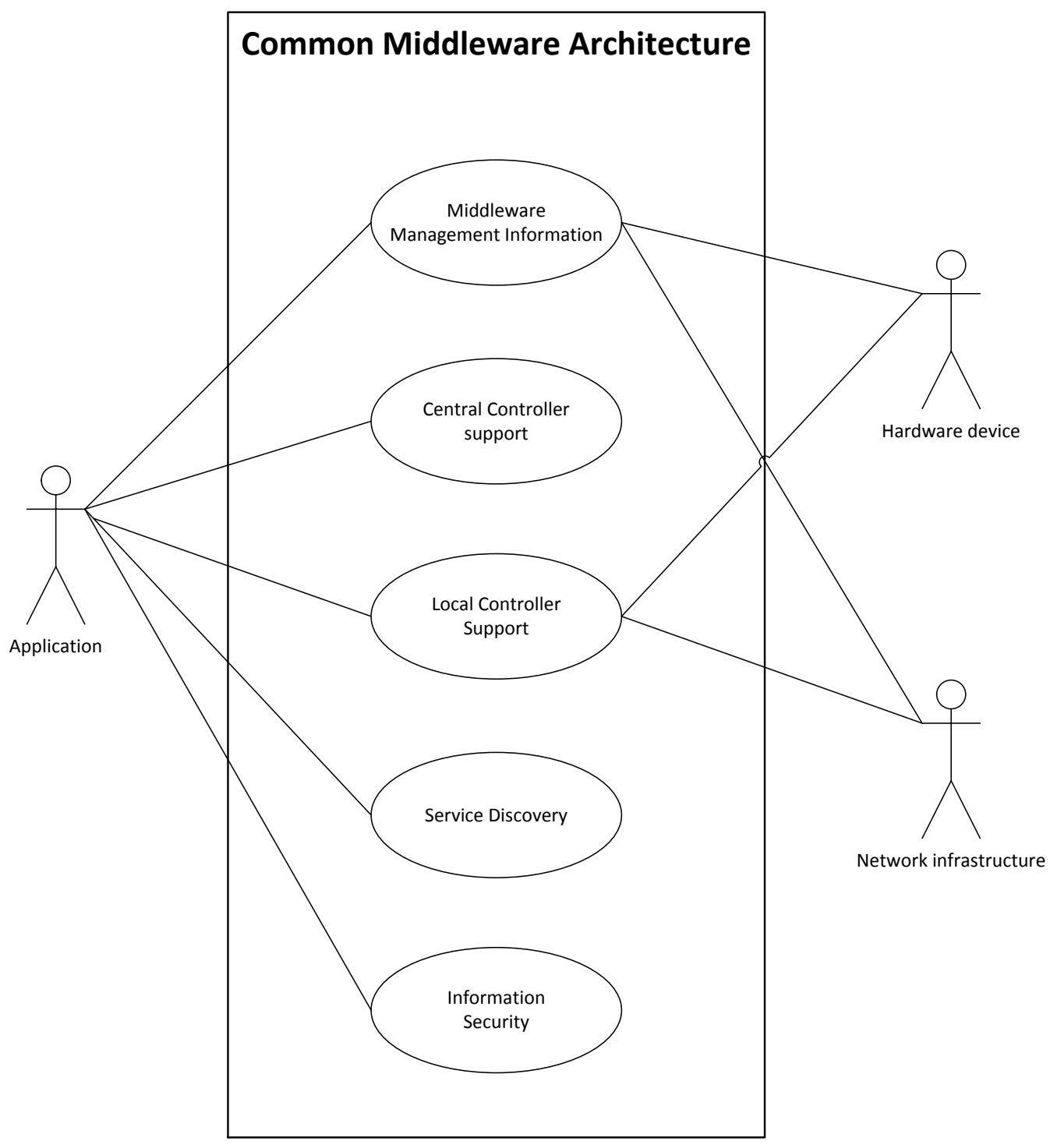

Figure 50. Use case diagram for Common Middleware Architecture

These use cases encase several functionalities with a collection of parameters that must be taken into account, such as the objectives of each of them, the actors implied in them and how they are executed. This information has been included in Table 7.

Table 7. Description of Middleware Management Information

\begin{tabular}{|l|l|}
\hline Name & EGO-MDW-FUN-0010 (Middleware Management Information) \\
\hline Scope and & $\begin{array}{l}\text { Deals with all aspects of information changes in the CMA. } \\
\text { Therefore, it must include how new data are registered in the } \\
\text { system, as well as take into account all the details regarding data } \\
\text { information in the architecture. }\end{array}$ \\
\hline Actors & Application, network infrastructure, hardware device \\
\hline Preconditions & $\begin{array}{l}\text { Middleware deployment in a Smart Grid (or a Cyber-Physical } \\
\text { System with Smart grid-like features). }\end{array}$ \\
\hline Postconditions & $\begin{array}{l}\text { Information regarding devices and services present in the system } \\
\text { is updated. }\end{array}$ \\
\hline Sequence & 1. Device requests to be registered with a message based on a \\
\hline
\end{tabular}




\begin{tabular}{|c|c|}
\hline description & $\begin{array}{l}\text { specific Protocol Data Unit that contains information regarding } \\
\text { its capabilities and services. } \\
\text { 2. Alternatively, a service within the middleware architecture } \\
\text { sends its own information about the functionalities that is } \\
\text { capable of providing. } \\
\text { 3. The information is formatted according to the data format } \\
\text { defined in the middleware. This will usually consist of an } \\
\text { ontology tailored for Smart Grid purposes. } \\
\text { 4. The information is included in a registry where devices and } \\
\text { services are present. In order to store this kind of information } \\
\text { a database will be required; a Triples Database (TDB) can be } \\
\text { used as the most suitable database to store semantically } \\
\text { enhanced information. TDB can be described as a Jena } \\
\text { (describe as a Java-based software framework used to build } \\
\text { Semantic Web developments, [156]) component used for } \\
\text { information storage and queries based on Resource } \\
\text { Description Framework (RDF, [157]). It can also be accessed } \\
\text { via SPARQL Protocol and RDF Query Language (SPARQL, a } \\
\text { language used to compose queries done to RDF-based } \\
\text { developments, [158]) queries in order to list services or } \\
\text { provide service discovery, which will come in handy for next } \\
\text { use cases. }\end{array}$ \\
\hline Exceptions & $\begin{array}{l}\text { No information is added from the applications. This is done so } \\
\text { because it is expected from this higher level that it will adapt to } \\
\text { the API provided, rather than having to tailor a solution for each of } \\
\text { the applications that might be included. }\end{array}$ \\
\hline
\end{tabular}

The sequence can be further described with the aid of UML. In Figure 51, it can be appreciated how the different procedures that give shape Middleware Management Information interact with each other. All the functionalities related to registration, regardless of the nature of what is registered, will be undertaken by including the Smart Grid ontology that has been defined for the Common Middleware Architecture, as well as by any activity required to obtain the data from either outside the middleware or any other software component present in the middleware architecture. The data format used for information representation must also be considered, as it will have to be adapted to the one that is used by the ontology developed for the deployment where the solution is used. As it was mentioned before, the components of this ontology and how it is developed is out of the scope of this thesis, but it will have to be able to include information regarding the services present in the devices to be expected from a Smart Grid. During the implementation works done in the research projects e-GOTHAM and I3RES it was decided that it was more practical to perform the format conversion within the middleware rather than providing it as the format to be used when data are transferred, as this had the consequence of having to deal with verbose messages in the network. 


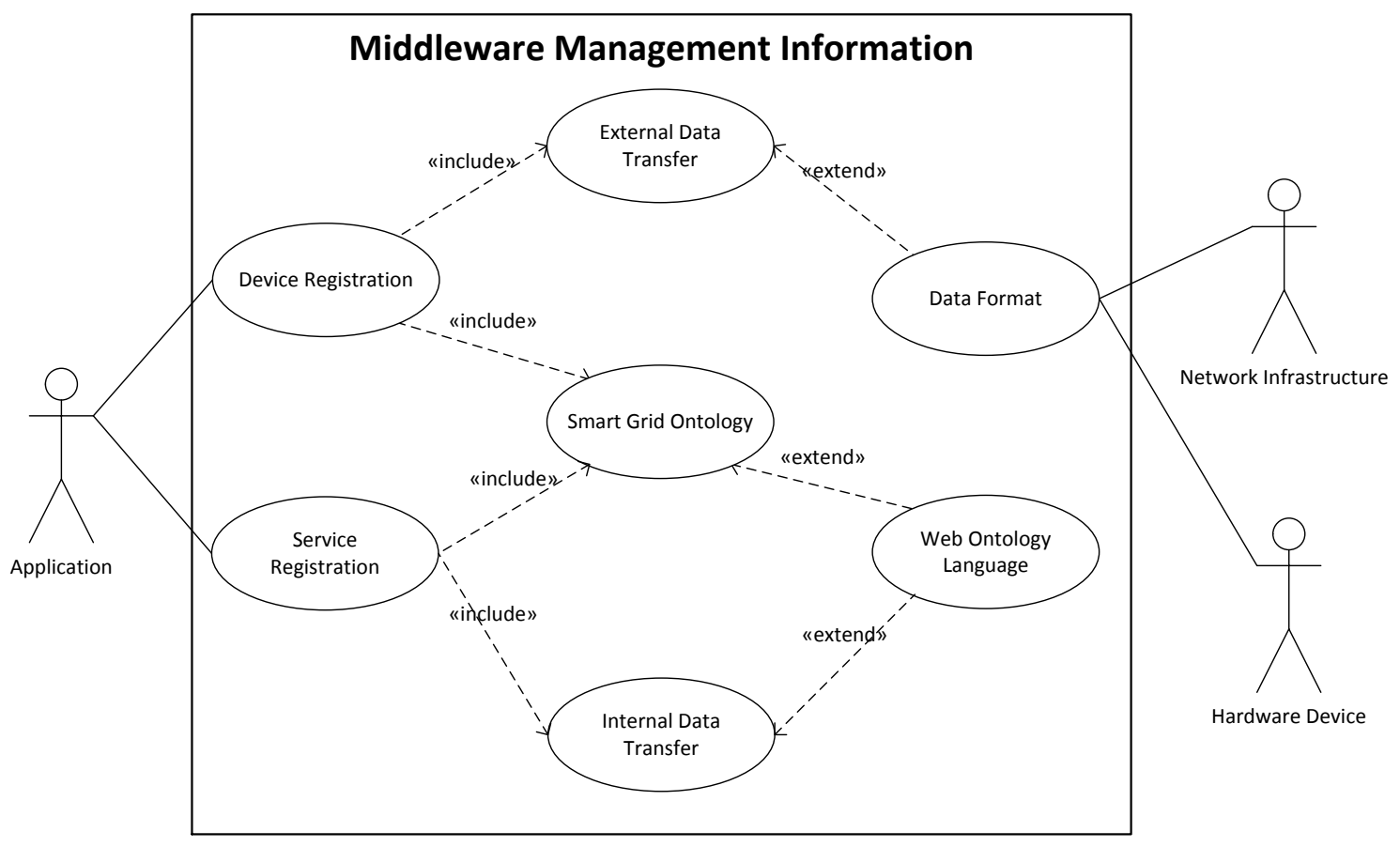

Figure 51. Use case diagram for further Middleware Management Information representation

The procedure that was explained previously can also be further defined with sequence diagrams. For example, registration can be carried out with three different actions, depending on the origin of the registration request. These procedures can be triggered either by a human operator, a device or a service within the middleware architecture. The first case has been depicted in the sequence diagram that is shown in Figure 52. Firstly, the human operator will request the registration of a specific device which will know previously. This request will be usually done via an Application layer facility (an app, a Graphical User Interface or even a web browser) that is capable of accessing the middleware solution via REST interface (1). Middleware is accessed via a Middleware Access Point that is used for merely transferring information to the other software components of the middleware architecture (as opposed to Middleware Autonomous Services). This registration request is further transferred to the software component responsible for device registration (2). According to the procedures defined during the e-GOTHAM and I3RES projects, it was made mandatory to check with the context awareness component whether that service or device was already registered in order to avoid redundancies that would result in listing duplicated or non-existing services, so the registration request message is sent to this Context Awareness component (3). Once an answer is sent back to the registration component stating that the service or device has not been registered yet (4), the registration request will involve the usage of semantic capabilities to adapt the request to the format that has been defined by the ontology of the middleware solution, so the message will be sent to the software component in charge for semantic capabilities in case they are not included locally (5), and will be formatted in terms of textfields and information according to the middleware ontology and sent back (6). It has to be noted that registration and semantic capabilities have been placed separately because due to the complexity of these procedures, they look clearer in a sequence diagram if they are defined as different instances of a class, even if they are implemented in the same software component. 
At this point, the request will look like an XML message with semantically annotated information about the features of the services, the location of the hardware device they belong to, the outputs that are expected from them and, all in all, the features that make the device unique. After this latter step, the device will be finally registered (7), and confirmation messages will be sent back the middleware high level (8) and the application (9). During the development works carried out in the e-GOTHAM project, an XML message was defined as the output for Middleware Access Point as a way to confirm that the registration had taken place. However, it could happen that the device that is about to be registered was already present. During the testing activities carried out the previously mentioned projects, it was considered as a situation unlikely but not impossible to happen, in case a device became unattached from the deployment and was connected again during a period of time that was shorter than the one that could be handled by the Context Awareness component. Consequently, a message was defined where the fact that a device has already been registered has been created and can be sent when required to the registration component (10), which will sent it back to the Middleware Access point (11) and be published in the application that is interacted by a human operator (12).

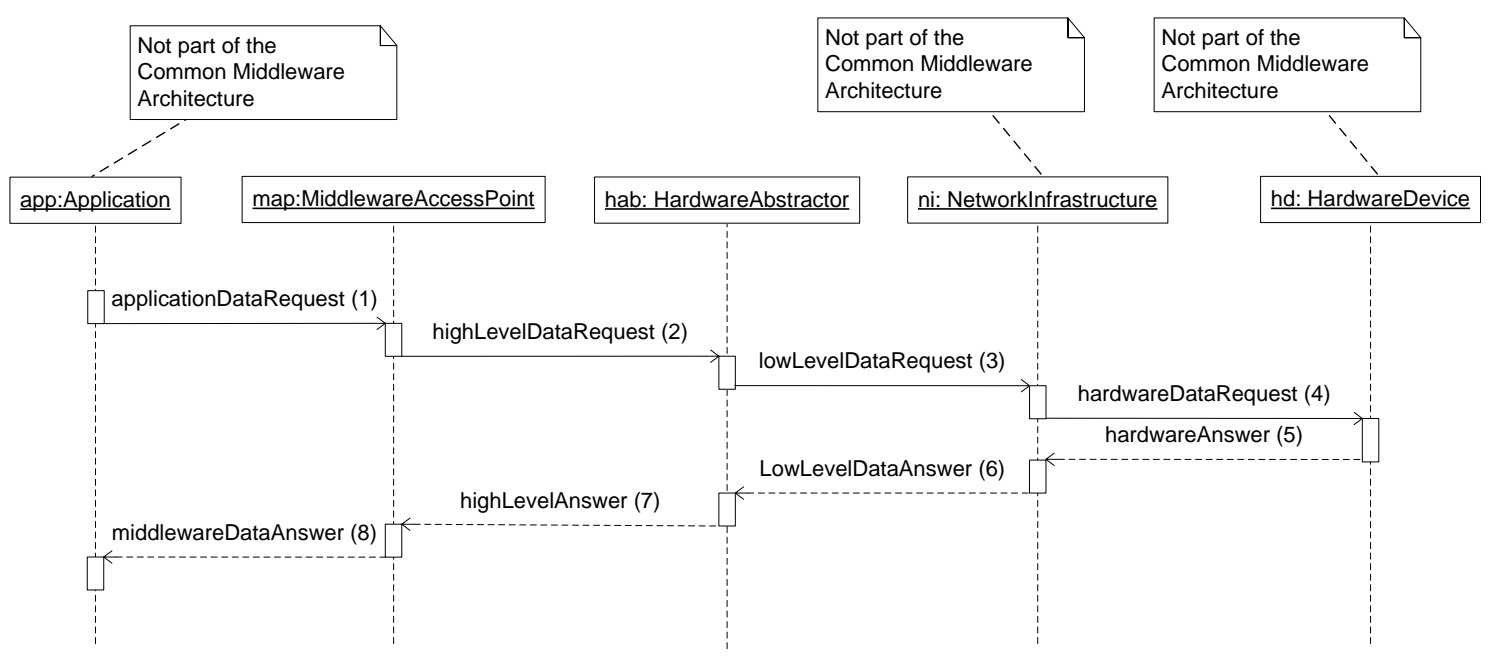

Figure 52. Sequence diagram for device registration started by a human operator

If the registration process is started by a device, the procedure is different in the first stages but similar in the latest ones: the device will send a registration request message according to a pre-established message format that involves the main data of the services and features that device is capable of providing (1). This message will be transferred throughout all the network elements present in the deployment (which are not part of the middleware, as this is conceived as software components between the network and the application levels) until it is reached by the registration component within the solution and send to the context awareness one (2) in order to check whether there is an already registered device with the exact same features that the one that is making the request. If the device has not been registered yet (3), the message will be sent to the middleware component that contains the ontology that is being used in the system (4) in order to format it according to its own rules (5). Once all these procedures are completed, the device will be registered in the database that is connected to the registration component (6). A confirmation will be sent to the device that is registered, 
consisting of a short message that will be using the same functionality as an acknowledgment (7). As it happened before, the device could be already registered; in that case, a message mentioning that the device has already has been registered will be sent from the context awareness component to the registration one (8), and a registration refusal will finally be sent to the device throughout all the network (9). All these messages that are interchanged have been portrayed in Figure 53 .

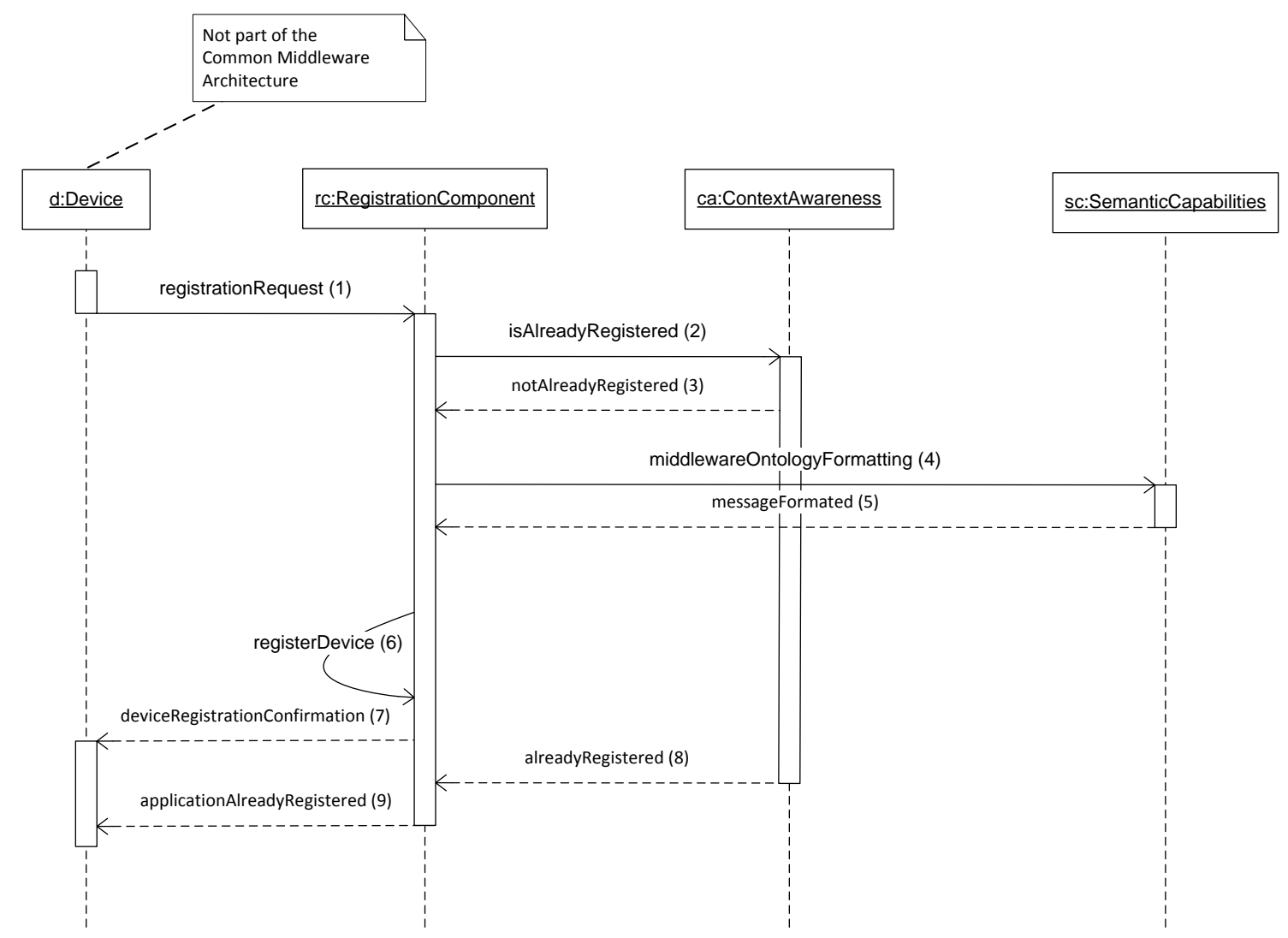

Figure 53. Sequence diagram for device registration started by a device

Lastly, the software components present in the middleware architecture will also be registered as a way to have a collection of them when they have to be discovered or listed. The procedure will be shorter than before due to several facts. To begin with, no communications outside the middleware solution will be required, so registration will be done in shorter periods of time. Secondly, services present in the middleware are not accessed from the outside, so there is no need to format them according to the ontology that is developed for the middleware solution (and, by proxy, no communications with the semantic capabilities component are required). Even though it seems unlikely to happen, the context awareness functionality that has been used before is still present here. Therefore, services from the middleware will be registered by sending a registration request message (1) to the registration component, which will check whether they have already been registered or not (2). If a confirmation is received about not having the service registered before (3), then it will be included in the TDB-based database (4). If it was already included, the registration message will be ignored in order to minimize the transfer within the middleware architecture (since neither a device or a human operator take place in the registration, they do not need to be 
informed about the presence of the device). When all is said and done, this third possible option to register a service will be done as shown in Figure 54.

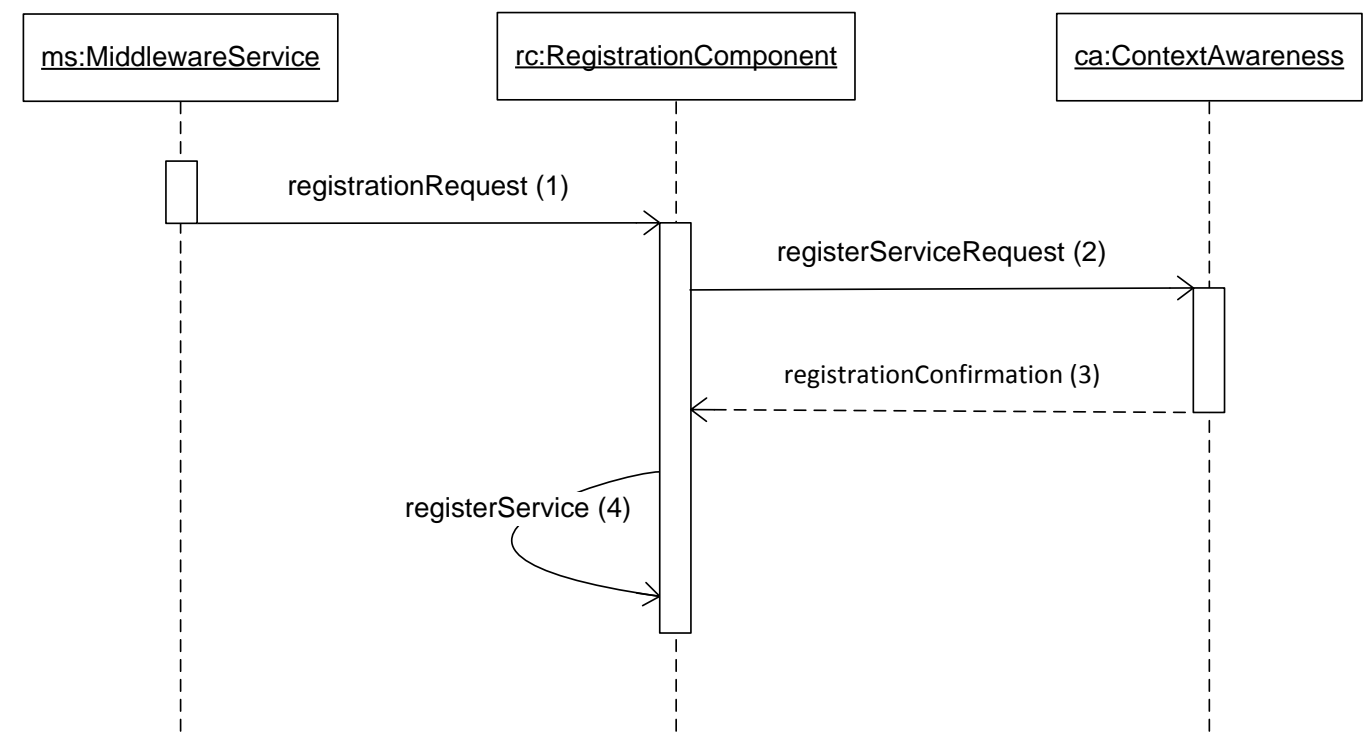

Figure 54. Sequence diagram for device registration started by a middleware service

The next general use case studied is the Central Controller support. This one involves all the activities required to request information to the central Controller where the most significant software components are located. In order to deliver the required data, data transfers will be required to at least interact with the hardware device that represents the Central Controller in the deployment. Therefore, data representation capabilities (represented by the middleware ontology composed by means of the Web Ontology Language) will be the ones to be used in the information transfers. The main characteristics of this use case have been further described in Table 8.

Table 8. Description of Central Controller support use case

\begin{tabular}{|c|c|}
\hline Name & EGO-MDW-OPR-0020 (Central Controller support) \\
\hline $\begin{array}{l}\text { Scope and } \\
\text { Objectives }\end{array}$ & $\begin{array}{l}\text { It is used to retrieve information that can be provided by } \\
\text { interacting with the Central Controller that has been deployed in } \\
\text { the system. The Central Controller is expected to be equipped with } \\
\text { most of the software services defined for the Common } \\
\text { Middleware Architecture. }\end{array}$ \\
\hline Actors & Application. \\
\hline Preconditions & $\begin{array}{l}\text { Common Middleware Architecture has been installed in a } \\
\text { hardware device (Central Controller). }\end{array}$ \\
\hline Postconditions & $\begin{array}{l}\text { Information is retrieved from the services present in the Central } \\
\text { Controller. }\end{array}$ \\
\hline $\begin{array}{l}\text { Sequence } \\
\text { description }\end{array}$ & $\begin{array}{l}\text { 1. Data is requested to the Central Controller about any of the } \\
\text { functionalities that can be provided by it (service listing or } \\
\text { service discovery). } \\
\text { 2. The request is sent to the Common Middleware Architecture. } \\
\text { 3. The request is attended by the services located at the central } \\
\text { Controller and an answer is sent back. }\end{array}$ \\
\hline
\end{tabular}




\begin{tabular}{|l|l|}
\hline & $\begin{array}{l}\text { 4. Alternatively, the request can be attended by a single service } \\
\text { located in the middleware solution (Middleware Autonomous } \\
\text { Service) and it can send the answer back. }\end{array}$ \\
\hline Exceptions & $\begin{array}{l}\text { No exceptions are present for this use case (the applications will } \\
\text { require to interact with the middleware or otherwise there will } \\
\text { not be data retrieval at all). }\end{array}$ \\
\hline
\end{tabular}

The relationships between the facilities that are needed for this use case to be successful are further explained in Figure 55. Data delivery will require internal data transfer throughout the components installed in the Central Controller, which at the same time require the information that has been formatted according to the Smart Grid ontology. The latter, as it happened before, relies on the Web Ontology Language for its composition.

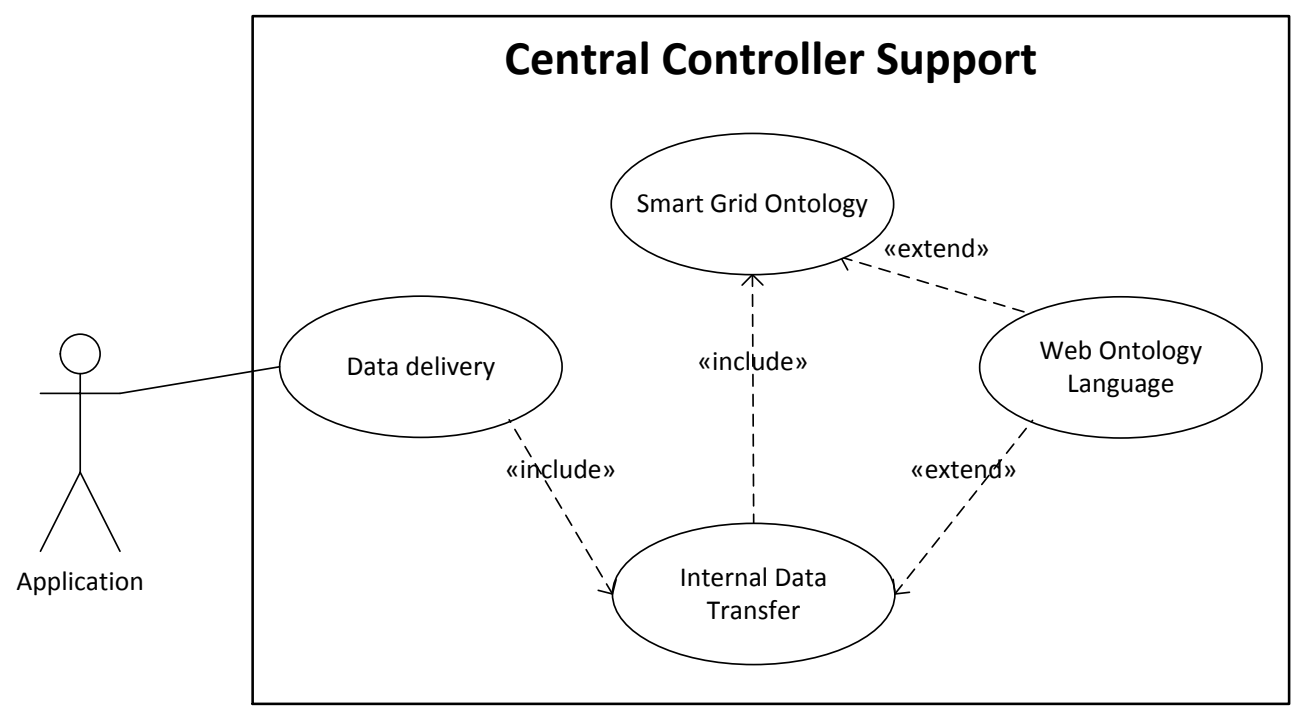

Figure 55. Use case diagram for further Central Controller Support representation

When the components of CMA are interacted in search of a service, they will usually imply showing the services that are already present in the deployment. Therefore, a request done against the Central Controller will imply reaching the list of services that have been included in the database that included the information about them formatted according to the ontology that was defined in the middleware solution. The procedure will start from a human operator making a data request though the application (1) to the Middleware Access Point. This CMA component will redirect the request to the registration component (2) which is the one connected to the database that has all the information stored. This component will answer by means of retrieving the information from the database (3) and sending it back to the middleware component that sent the request (4). Lastly, the data will be sent back to the application so that it can be used by the human operator that requested it in the first place (5). These steps have been represented with a sequence diagram in Figure 56. 


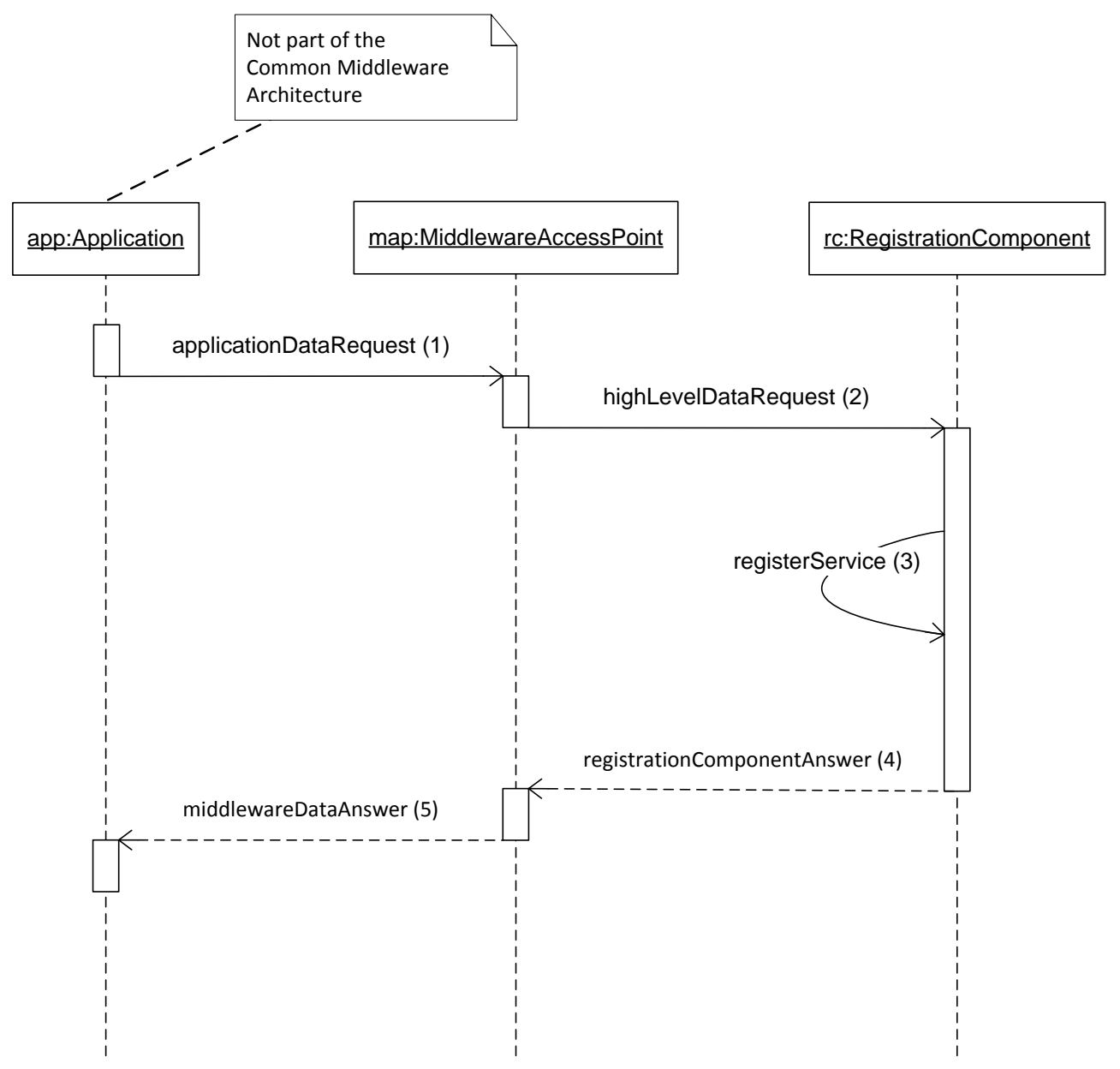

Figure 56. Data requests done in the Central Controller support (Middleware Access Point)

The other possible option is that the request done against the middleware components of the Central Controller can be satisfied by a service at the high layer. Therefore, a Middleware Autonomous Service will be used in order to retrieve the information. The sequence of events that should be used has been included in Figure 57.

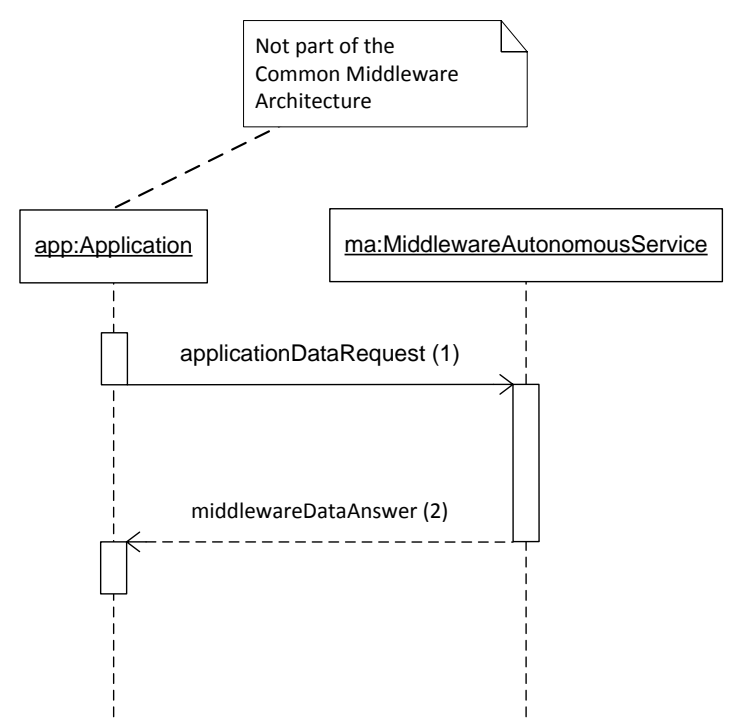

Figure 57. Data requests done in the Central Controller support (Middleware Autonomous Service) 
It can be seen how the procedure to obtain the data is simpler now than before, as it only implies sending the message from the application (1) to the suitable middleware component, which will send an answer back with the required data (2). The procedure used to distinguish the Middleware Autonomous Services from the Middleware Access Points, as well as how to justify their presence in the middleware solution have been described in Section 4.

Requests done to Local Controllers, on the other hand, require mode steps, as it has been represented in Table 9. Additionally, the application request will have to leave the Central Controller and be transferred through the network until the suitable Local Controller is reached.

Table 9. Description of Local Controller support use case

\begin{tabular}{|l|l|}
\hline Name & EGO-MDW-OPR-0030 (Local Controller support) \\
Objectives & $\begin{array}{l}\text { It is used to retrieve information that can be provided by one of } \\
\text { the Local Controllers that have been deployed in the system. } \\
\text { These Local Controllers will have some components from the } \\
\text { Common Middleware Architecture in order to provide information } \\
\text { through the distributed middleware, namely the ones required to } \\
\text { interact with all the other parts of the middleware architecture. }\end{array}$ \\
\hline Actors & $\begin{array}{l}\text { Common Middleware Architecture has been installed in the Local } \\
\text { Controller for the most prominent functionalities to be expected } \\
\text { from the devices present in the deployment (interaction with } \\
\text { Central Controller, etc.). }\end{array}$ \\
\hline Preconditions & $\begin{array}{l}\text { Information is retrieved from the hardware capabilities present in } \\
\text { the Local Controller (sensors, for example, can provide } \\
\text { information about electricity parameters, environment, etc.). }\end{array}$ \\
\hline Postconditions & $\begin{array}{l}\text { 1. A request is done for information via the application layer. } \\
\text { 2. The request is transferred throughout the middleware } \\
\text { components to the network that is located underneath. }\end{array}$ \\
\hline Sequence & $\begin{array}{l}\text { 3. The information request arrives to the device that has it and is } \\
\text { collected. }\end{array}$ \\
4escription & $\begin{array}{l}\text { The information is sent back to the middleware in a } \\
\text { standardized message format. } \\
\text { The middleware sends the information to the application } \\
\text { layer, where it is displayed. }\end{array}$ \\
\hline Exceptions & $\begin{array}{l}\text { No exceptions are present for this use case (the applications will } \\
\text { require to interact with the middleware or otherwise there will } \\
\text { not be data retrieval at all). }\end{array}$ \\
\hline
\end{tabular}

These functionalities have been further explained in the following use case diagram that deals with the specific functionalities needed for Local Controller support. As it happened in the previous use case, there will be some functionalities linked to the central Controller, since the communications will have to be done through it. However, external data transfers will be performed that will required a specific data format. Therefore, that functionality has also been included in Figure 58. 


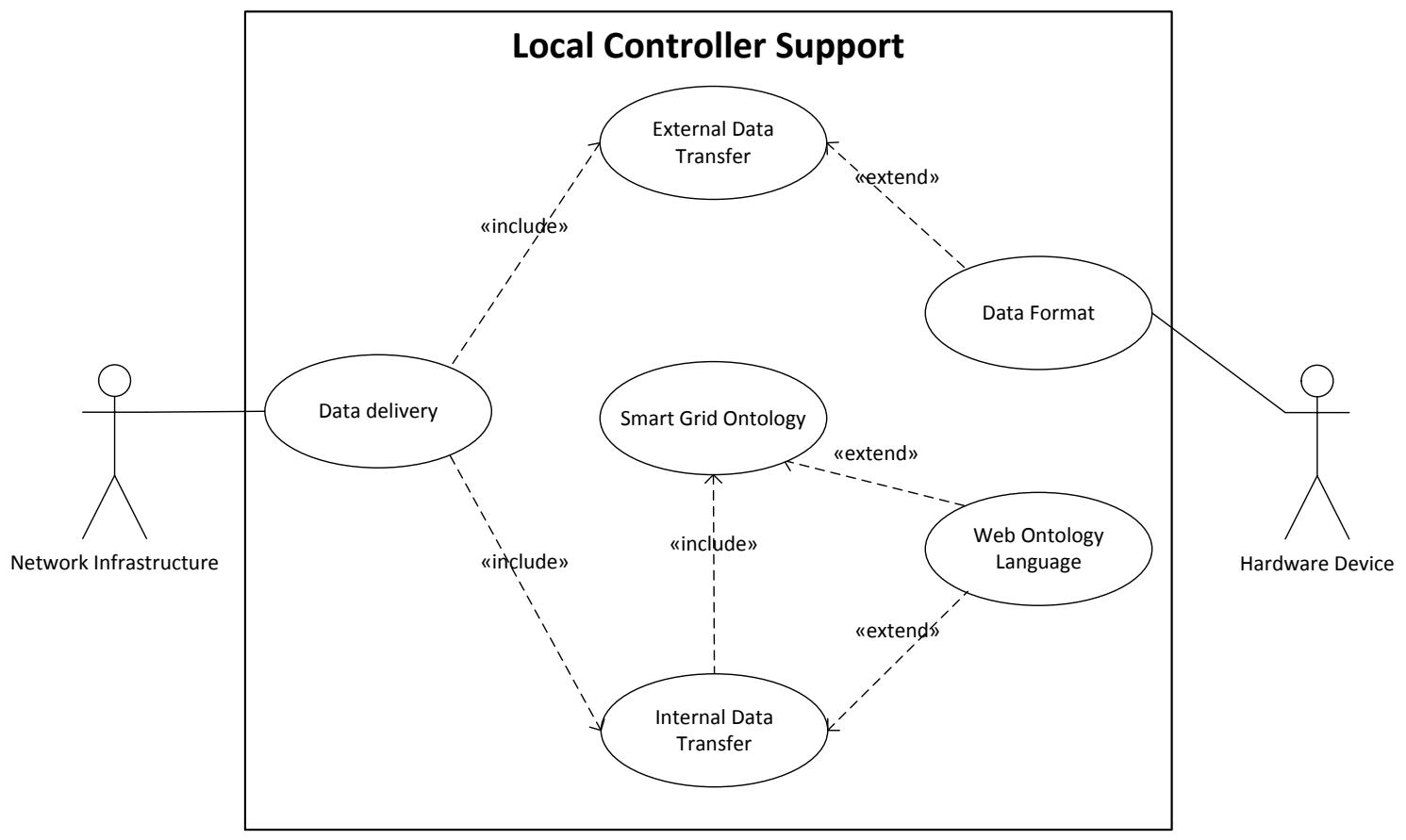

Figure 58. Use case diagram for further Local Controller Support representation

The procedure to obtain data from a device requires some more steps than previously, as there will be communications between the Central and Local Controller, thus requiring the intervention of the components used in the middleware to transfer data from one device to the other. According to the procedure that has been described in Figure 59, when an application requests data from a device it will send a request message to the Middleware Access Point (1). This request will be further transmitted down to the Hardware abstractor (2) and the network infrastructure that is located at a lower level than the middleware itself (3), to finally reach the device that has been requested (4). The answer will be sent back in the same fashion that was received by the hardware device: once the data is collected, it will be placed in the Protocol Data Unit used for data transmissions outside the middleware in order to transmit it throughout the network (5) back to the hardware abstractor (6) and the middleware. Lastly, the Middleware Access Point that was used to send the request will collect the answer (7) and send it to the application (8), so that it will be shown by the corresponding interface. The entities that have been defined as Local Controllers will be further identified in the next chapter. They can be regarded as the devices that have been installed in the end user facilities and are performing several significant functionalities for the system. Regardless of their functionality, a Local Controller will always have some middleware components that will be included. This is the particularity that will separate the Local Controller from any other device present in the deployment where CMA is installed. 


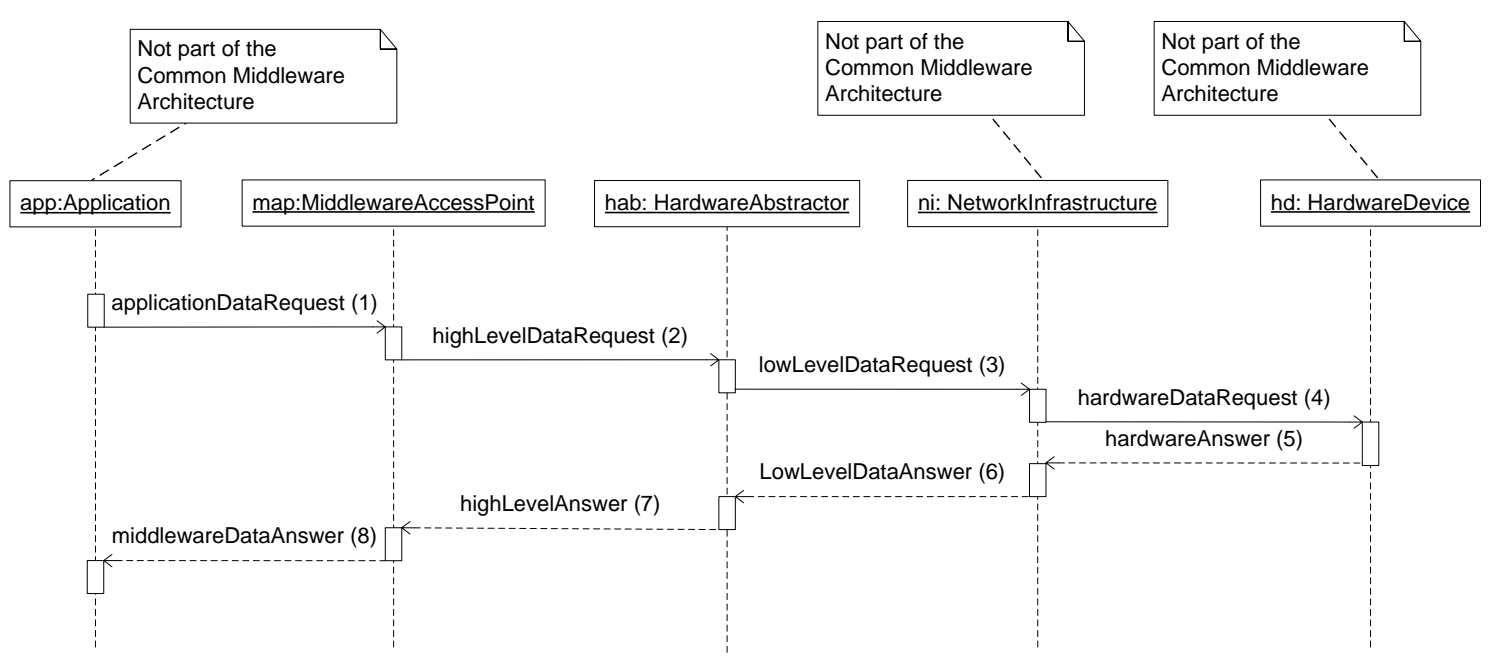

Figure 59. Sequence of a data request done to the Local Controller support

The next use case to be described is the one regarding Service Discovery. It is expected that whenever this service has to be used, it will be related to all the services present in the deployment that are unknown for the operator using the middleware architecture. Therefore, semantic capabilities will be required to provide complete descriptions of all the services that have been deployed. This service has been further described in Table 10.

Table 10. Description of Service Discovery use case

\begin{tabular}{|c|c|}
\hline Name & EGO-MDW-FUN-0040 (Middleware service discovery) \\
\hline $\begin{array}{l}\text { Scope and } \\
\text { Objectives }\end{array}$ & $\begin{array}{l}\text { Displaying the different services that are present in the system } \\
\text { when it is requested by an operation through an application. }\end{array}$ \\
\hline Actors & Application \\
\hline Preconditions & $\begin{array}{l}\text { All the services of interest have already been registered and can } \\
\text { be displayed. }\end{array}$ \\
\hline Postconditions & $\begin{array}{l}\text { Services that where unknown for a human operator become } \\
\text { known after information is requested about them. }\end{array}$ \\
\hline $\begin{array}{l}\text { Sequence } \\
\text { description }\end{array}$ & $\begin{array}{l}\text { 1. Services are request by a human operator via the application } \\
\text { layer. } \\
\text { 2. A semantically annotated list of available services is provided } \\
\text { with the most prominent information about them. }\end{array}$ \\
\hline Exceptions & $\begin{array}{l}\text { Aggregator customer does not belong to the DSO network. } \\
\text { Information received does not belong to the Aggregator portfolio. }\end{array}$ \\
\hline
\end{tabular}

These procedures have been split into several parts by means of a use case diagram represented in Figure 60 that shows the dependencies among the different elements used. 


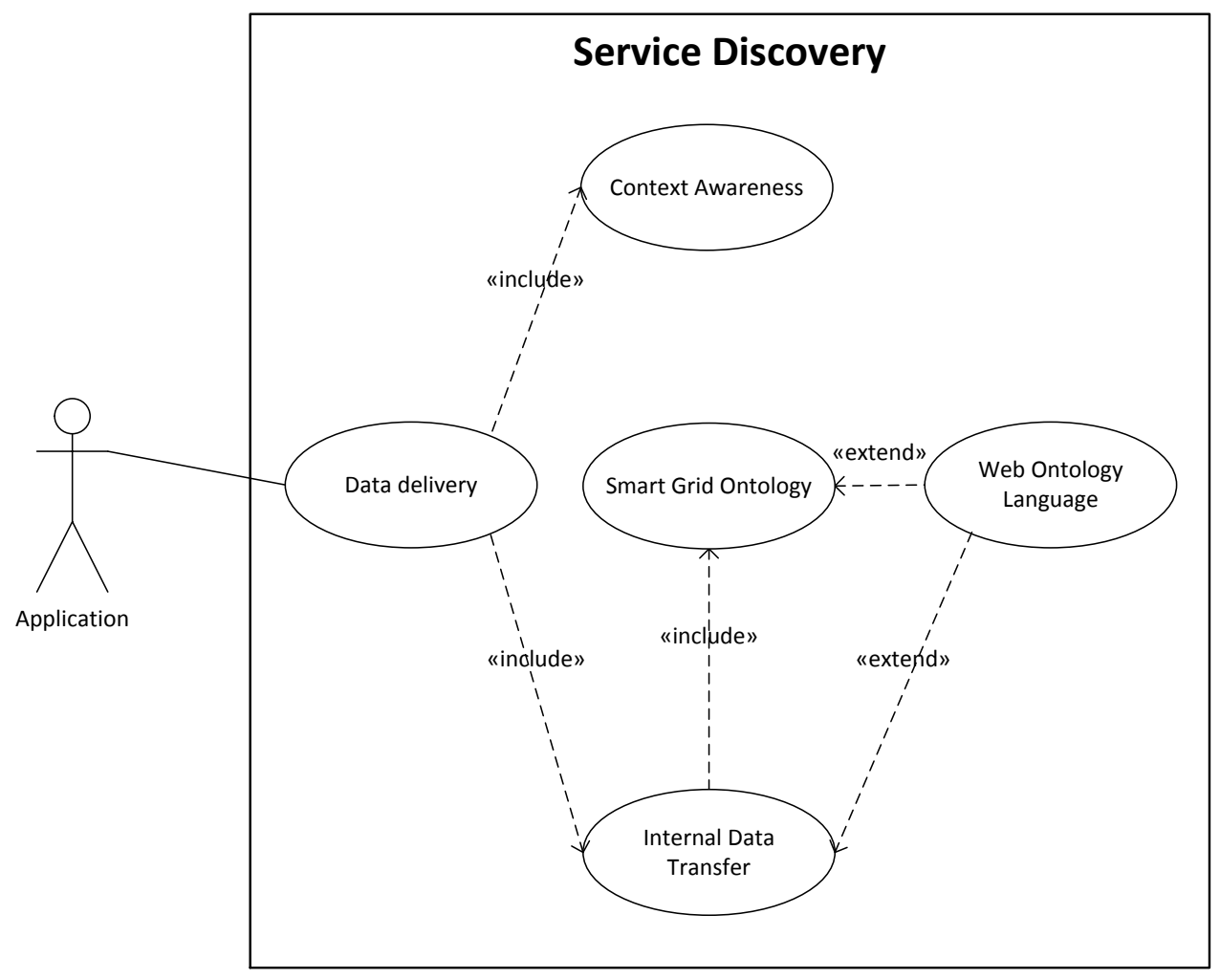

Figure 60. Use case diagram for service discovery representation

In this diagram it can be seen how the data delivery performed in this case relies on the context awareness facilities that have to be used in order to have an accurate idea of what services are present. Aside from that, the information present in the deployment is based on the format representation that has been chosen for the data. As it has been done before, the data format will be based on the ontology that has been implemented in the middleware solution. In addition to that, internal data transfer will also be required, as the semantic capabilities present in the middleware solution must be requested. This latter process can be further defined by means of a sequence diagram, such as the one portrayed in Figure 61. In this case, when a human operator requests the service discovery functionality from an application, it will be done so via Middleware Access Point (since a Middleware Autonomous Service would only access). When the request is sent from the application (1) to the high level middleware component, it will be resent to the registration one (2), as it is the one that has the data regarding the services that have been registered in the deployment. However, rather than getting a list of the services present in the middleware solution, the information will be updated with the semantic information that is used in the annotation of the service descriptions. Therefore, the request will be further sent to the component responsible for those semantic capabilities (3). Once the data is obtained, it will be sent back to the registration component (4) and the Middleware Access Point that collected the request in the first place will receive it (5) and send it back to the application layer (6). 


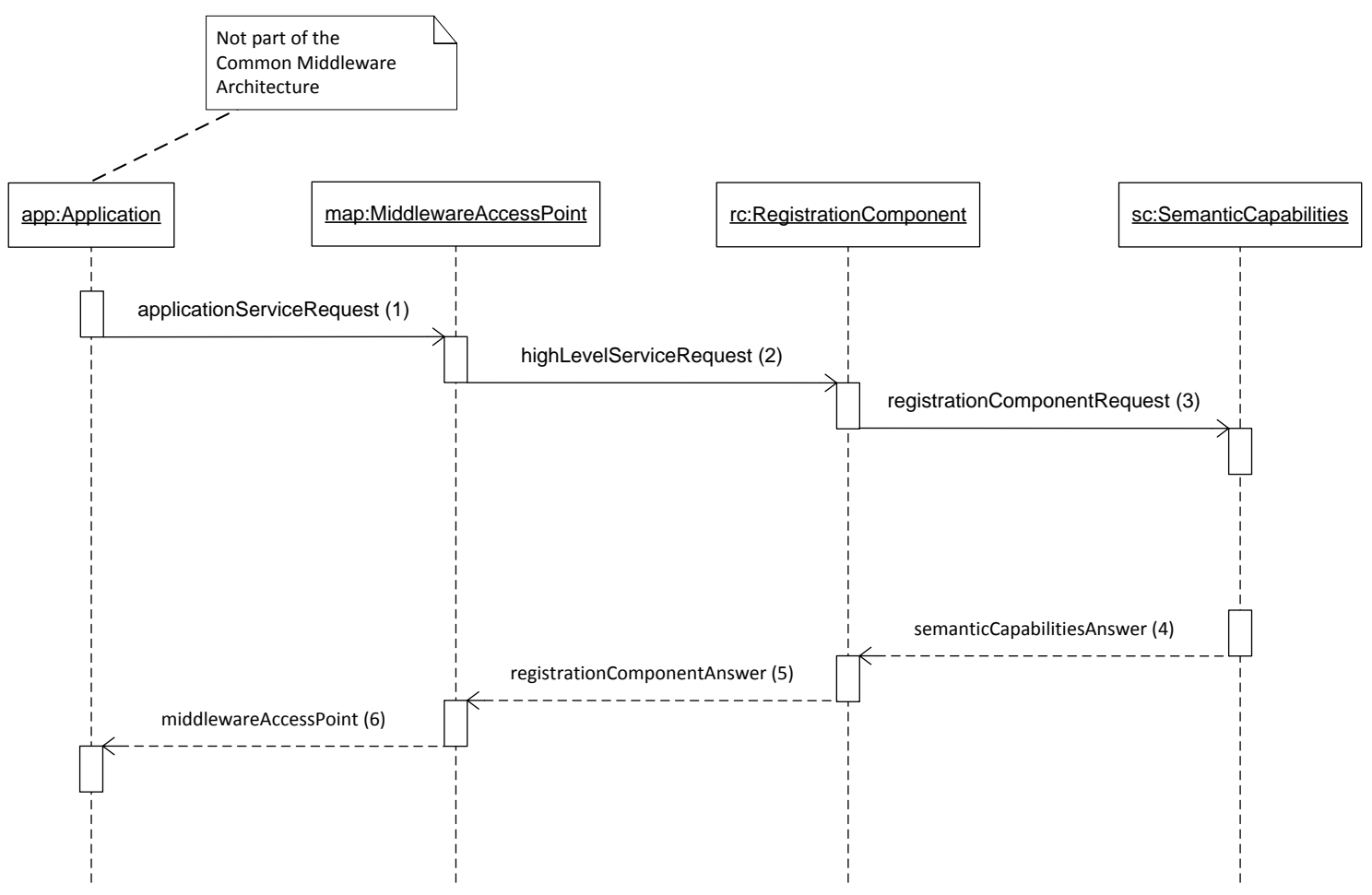

Figure 61. Sequence of a data request done to the Local Controller support

Lastly, securitization of the transactions that are going to take place must also be dealt with. There were two procedures that were deemed possible for this activity. The one dealing with certifications for the web browser whenever a user was connecting was the one finally used. It has been included in the section 4 of this thesis. As it has been done with the previous use cases, Table 11 is presented with the most important features of this use case.

Table 11. Description of Information Security use case

\begin{tabular}{|l|l|}
\hline Name & EGO-MDW-FUN-0050 (Information security) \\
Objectives & $\begin{array}{l}\text { Securitization of the transactions that are carried out among the } \\
\text { parties involved in the communications. It will guarantee some } \\
\text { security functionalities like confidentiality, integrity and } \\
\text { authentication, as long as a web browser is used in the data } \\
\text { transmissions. }\end{array}$ \\
\hline Actors & Application \\
\hline Preconditions & $\begin{array}{l}\text { The security software infrastructure (certification, etc.) has been } \\
\text { installed both in the remote application utilized by the end user } \\
\text { and the Central Controller that is used as an intermediary between } \\
\text { the user and the services that are requested. }\end{array}$ \\
\hline Postconditions & $\begin{array}{l}\text { Communications are carried out in a regular way. The end user } \\
\text { does not notice any change when security is used, but the } \\
\text { exchanged information cannot be accessed by any unwanted } \\
\text { party. }\end{array}$ \\
\hline $\begin{array}{l}\text { Sequence } \\
\text { description }\end{array}$ & $\begin{array}{l}\text { 1. Security certification is established previously by the parties } \\
\text { that are going to participate in the communication. } \\
\text { 2. Data interchange is carried out as usual. }\end{array}$ \\
\hline Exceptions & No exceptions should be allowed in the system; not using security \\
\hline
\end{tabular}


The operations that have been displayed in Table 11 can be further explained by means of the use case diagram that has been depicted in Figure 62.

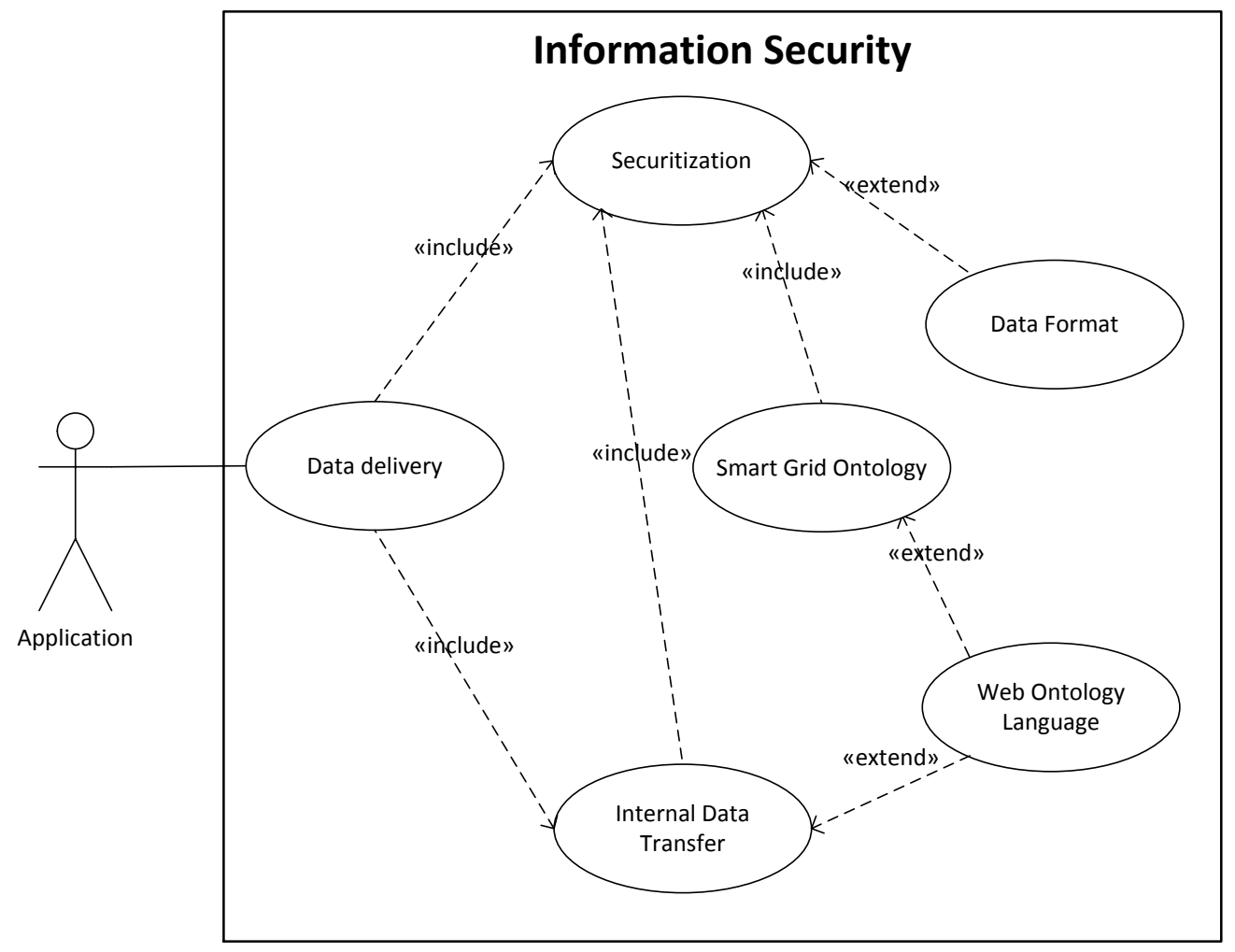

Figure 62. Use case diagram for service discovery representation

In the previous figure, it can be appreciated how the resources used for securitization are almost the same as the ones defined for other use cases, such as Central or Local Controller support and Service Discovery. The only significant difference, though, is that security must be taken into account for this case, as it will rule the data exchange that takes place among the parties that have been secured. Therefore, any data transfer and the very ontology that is used for the transmission of information must be involved in the security procedures that are developed.

Another way to explain these features is by means of a sequence diagram as the one that has been depicted in Figure 63 . The securitization operations that have been carried out in the sequence diagram correspond to the ones that can be performed when requesting information from a device, as it was depicted in the Local Controller support use case. In this way, when a human operator requests information from an application, the latter will send a request message (1) with the data that is required. The request will be sent to the Middleware Access Point used to transmit that piece of information (2). Afterwards, the securitization component will be used to cypher communications and transmit the data to the hardware abstractor component (3). Once this procedure has been completed, all the other data transmissions are undertaken as any other regular data request to a device: the information 
request will be transferred as packets at the network layer level (4) until it finally is delivered to the hardware device responsible for providing the needed information (5). The request will be deciphered in the hardware device so that it becomes clear the kind of data that is required (6) and the answered will be cyphered again in order to guarantee its secure delivery (7). Once an answer is obtained from the hardware (8), it will travel back to the middleware (9) until it reaches the hardware abstractor and is sent back to the securitization component (10). The latter will be responsible for deciphering the message received in the middleware solution and sending it back to the high level of the software architecture (11) and delivering it to the application that started the request (12).

It has to be highlighted that, according to what has been represented here, ciphering and deciphering operations can be done via symmetric or asymmetric data encryption. The only difference is that asymmetric encryption operations will take a longer time to be completed, which depending on the hardware of the end user device might take seconds to complete. Another different approach is the aforementioned usage of certifications to guarantee an acceptable level of security in the system. This is the solution that was implemented in the eGOTHAM and I3RES research projects. 


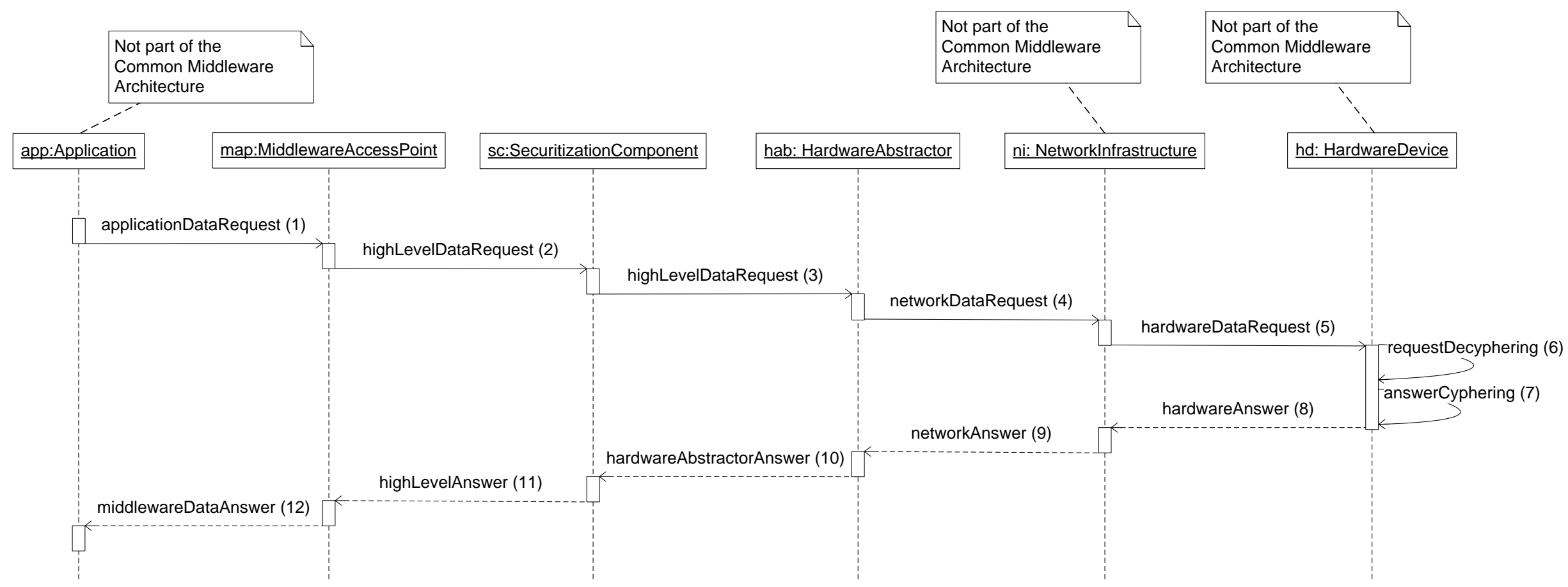

Figure 63. Sequence diagram with data collection and securitization 
As far as components are concerned, class diagrams for each of them must also be considered, due to the fact that they can be used as a decisive starting point for the implementation works that have to be carried out in order to have a realistic implementation of CMA. Although a Java implementation has been performed, these diagrams are neutral from the programming language point of view, thus providing a high degree of flexibility for implementation works depending on the abilities of developers. To begin with, the class diagram of the Middleware Access Point component is provided in Figure 64.

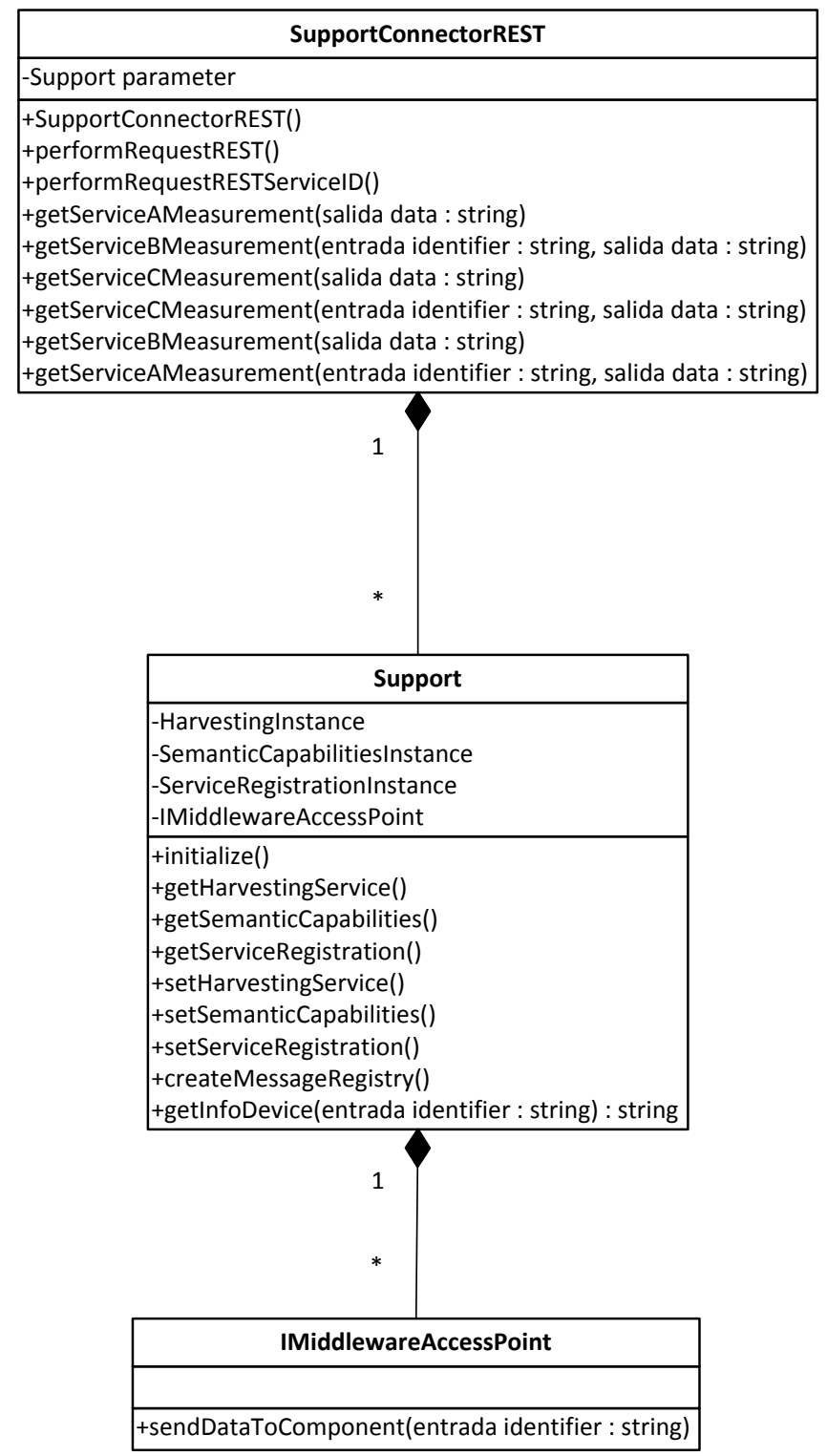

Figure 64. Class diagram from the Middleware Access Point component

There are several prominent aspects to take into account from this class diagram. The idea of having a REST-based interface to interact with the application layer can be clearly seen here as the Connector class. Not only it is used to receive all the requests that can be carried out from the application layer, but also takes of the REST-based connectivity among the different levels of a deployed system. Aside from that, two more facilities are required: an interface to connect this component with the lower layers of the middleware architecture (represented by IMiddlewareAccessPoint) and the variables needed to pick the information from these lower 
level components (made possible by using the methods in the support class). Note that IMiddlewareAccessPoint is not a regular Object Oriented Programming language interface but a class used to connect with the lower levels of the middleware, otherwise the UML relation would be of implementation rather than composition.

A Middleware Autonomous Service will require fewer features (due to the fact that they do not need to connect to any component of the middleware architecture) but these will be more complex (since they have to solve request that will not be done to other components of the middleware architecture). Therefore, the class required to interact with the application layer will still be present (SupportConnectorREST), but the other class will be specifically used to solve requests performed by the applications (such as service registration or perform the ancillary operations demanded by them). All these aspects have been portrayed in Figure 65 .

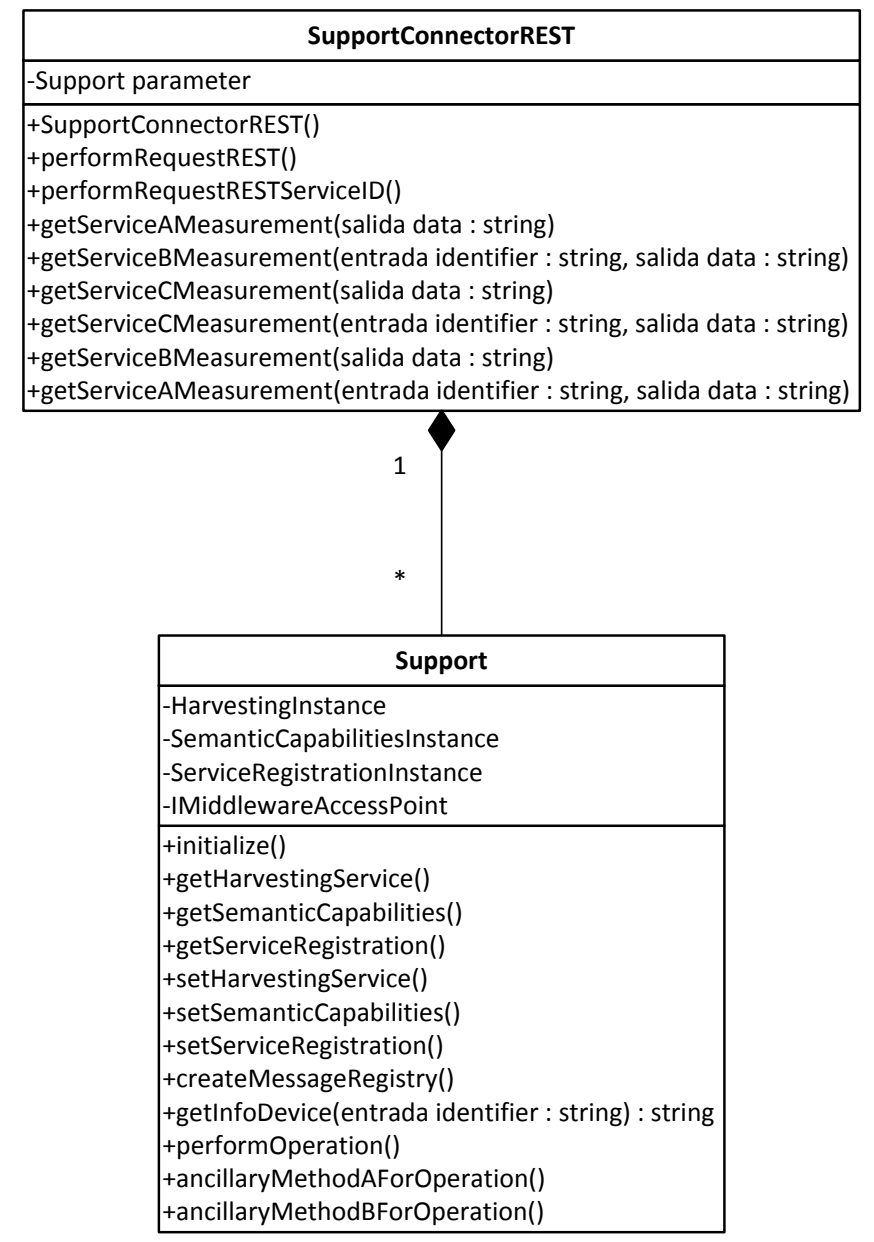

Figure 65. Class diagram from a Middleware Autonomous Service component

As far as registration is concerned, there are some facilities that must be guaranteed. Firstly, there must be a way to interact with the messages that are collected from the devices that have to be registered in the middleware solution. Secondly, the registration itself will have to be carried out in this component. Thirdly, the information that is transferred in the messages must be processed in order to know the specific features of the device that has become present in the system. Lastly, an entity capable of coordinating or managing these procedures will come in handy. All these aspects have been integrated in the class diagram that is 
portrayed in Figure 66. Depending on the complexity of the developments, the ontology can also be included in this component, but due to its potential complexity it is considered as located in a different one.

The relationships among the most prominent classes have also been included. Basically, the class responsible for providing the management facilities (MessageFormatManager) will control how the services belonging to devices will be registered, which will be collected from the interface that is offered to interact with the lower level components.

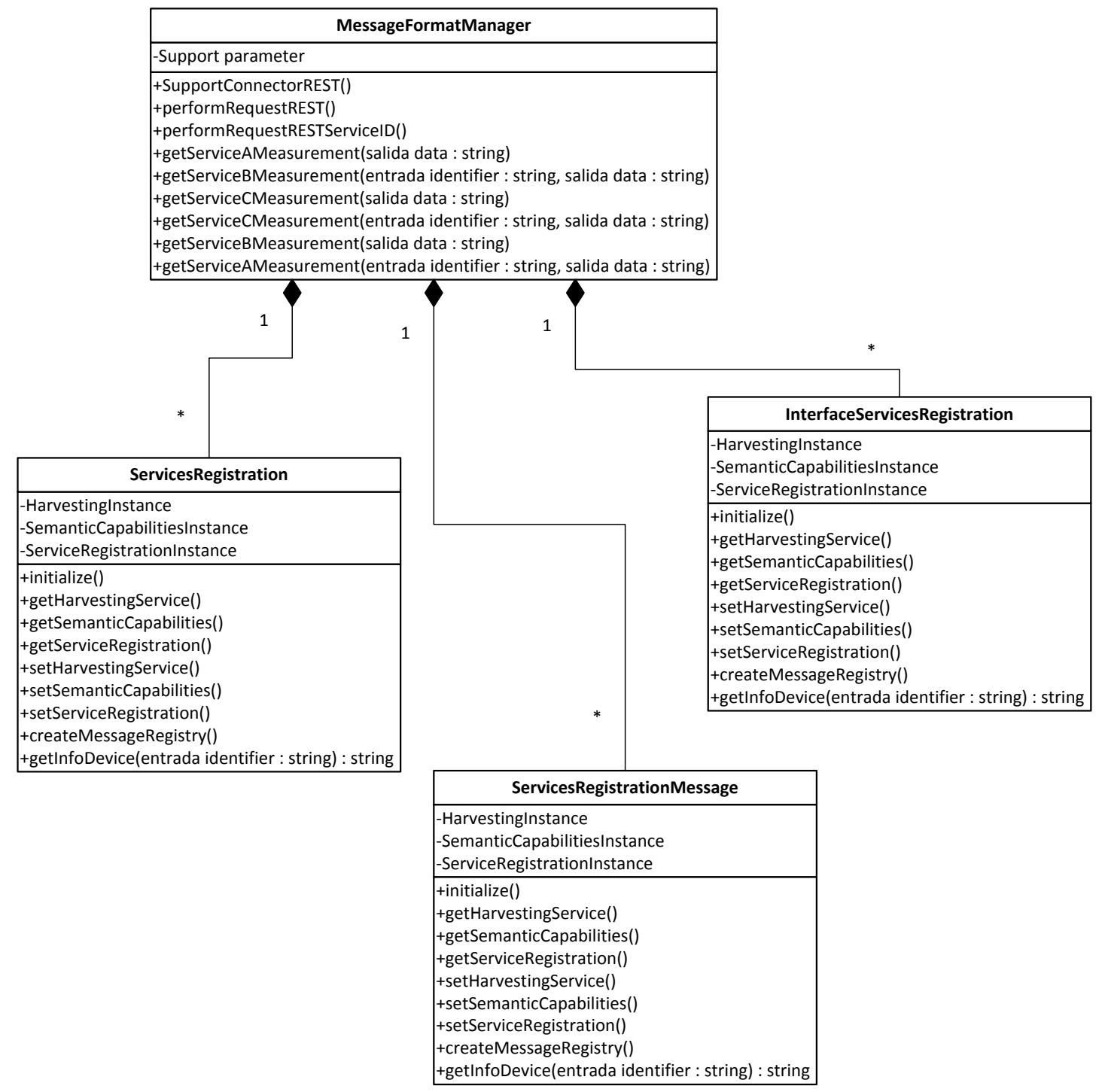

Figure 66. Class diagram of the Registration component

Additionally, the Context Awareness component will have several facilities that can be expected to obtain information from the environment and trigger any action that might be considered as necessary. One of the tasks to deal with it is processing the messages that will be received from the registered devices that are present in the system, so a class used for that purposed has been added (MessageManager). At the same time, an interface is required to obtain those messages both from outside the middleware architecture and other software components of the architecture, specifically the registration component that is used during 
service registration (hence the IContextAwarenessService interface and ContextAwarenessService class). As it was done for the previous component, a manager class will be used for management purposes when interchanging information with the ontology of the system or the service registration. Finally, a timestamp will be generated whenever a new message regarding the presence of devices is received by the component. These features have been included in Figure 67.

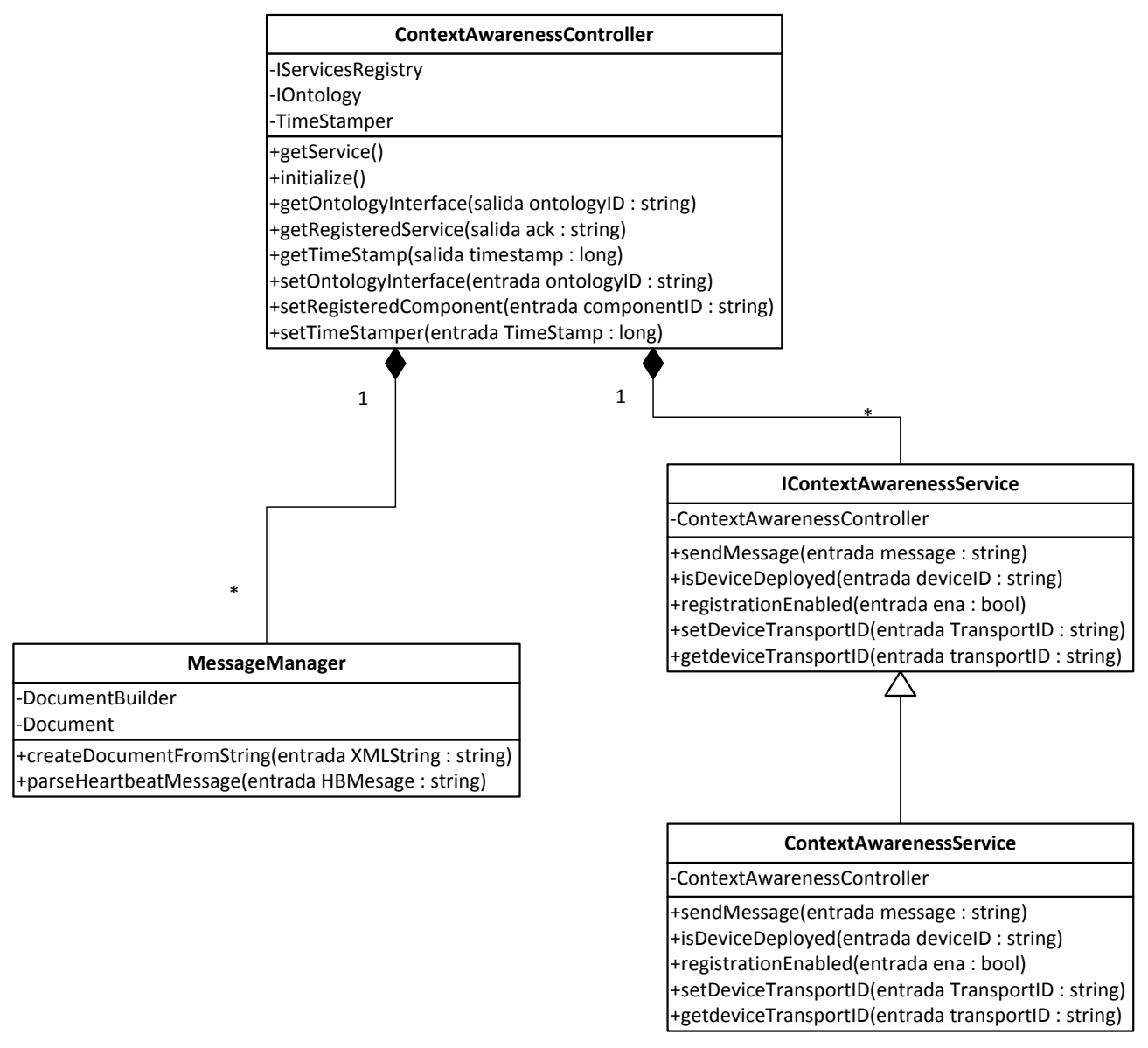

Figure 67. Class diagram of the Context Awareness component

The ontology itself has also been defined at a high level, due to the fact that its functionalities are of major importance for a system of these features. Apart from the managing class (OntologyConnector) and its interface (IOntologyConnector), there are two main classes where most of the functionalities of the ontology are located. The first one is used to contain all the templates of the devices established as the ones to be included in the deployment. This class has been represented as Templates, since it contains all those features to be expected from hardware (kind of device, geographical location, model, manufacturer, serial number) and software (service type, functionality or state). Note that even though hardware information was included in the ontology, the most relevant data that will be requested by the users communicating with the middleware will be related to the services. In addition to that, there is another class called QueryLanguageFacilities that has been used in order to define all the possible outcomes of the requests that are taken to be attended by the ontology (typically, they will be about alternative services to provide to the Context Awareness component, types 
of devices, hardware compliant with certain features, input and output parameters or listing devices and services). This has been designed like this due to the fact that it became evident during the design and development stages that SPARQL was the most suitable language to compose queries against the ontology that was defined for the system. The classes have been represented in Figure 68.

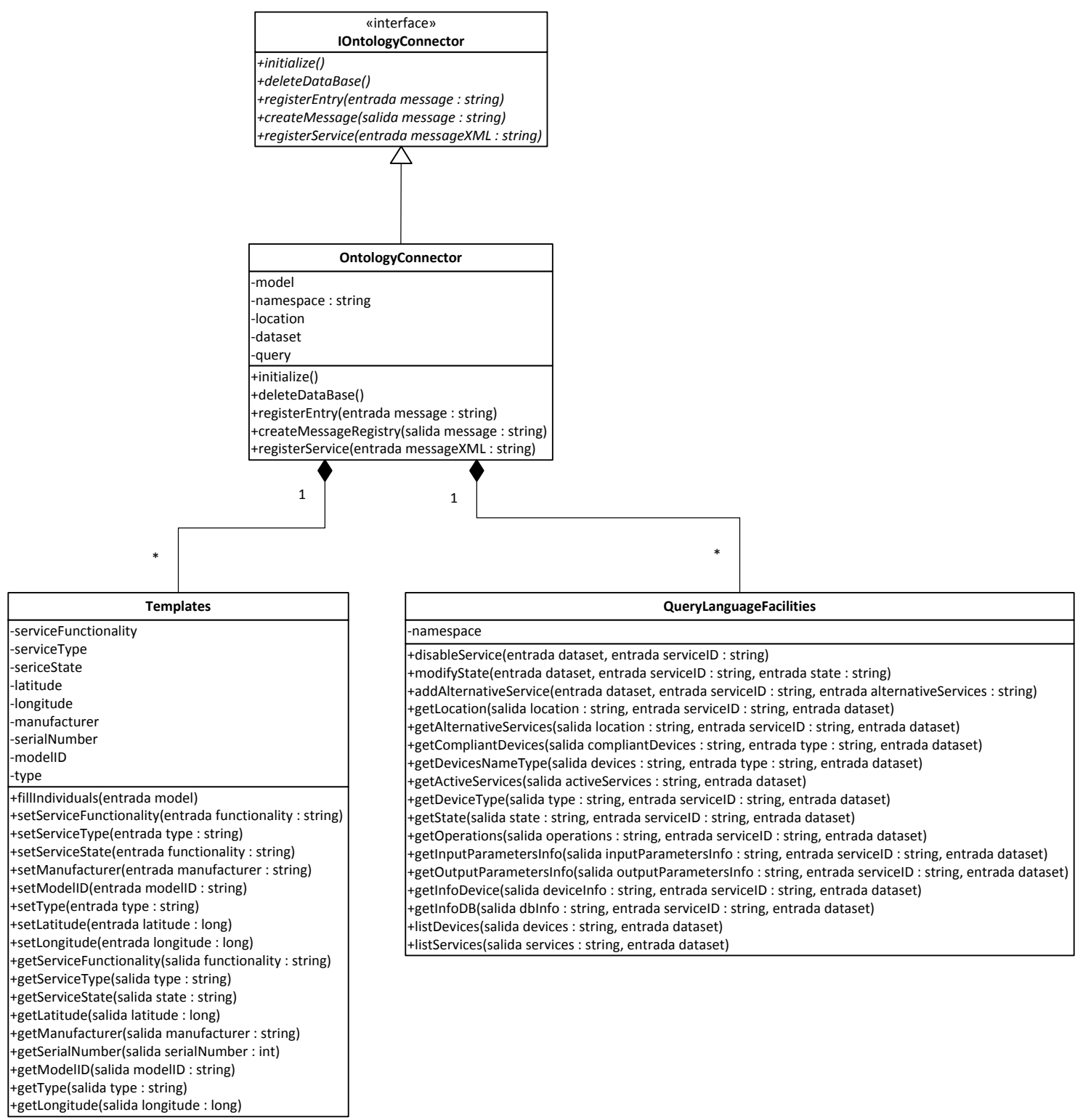

Figure 68. Class diagram of the semantic capabilities component

The CMA component used to abstract the hardware heterogeneity has also been portrayed as a set of classes in a diagram. The main particularity of this component is that it will have certain capabilities that will ensure that it is ready to receive information from the PDUs received from the network. Consequently, it implements a listener used to retrieve all the information regarding messages sent by the hardware devices deployed (HabServicelmplementation) that is implemented from an interface (IHabService). Furthermore, a managing class (called HardwareAbstractorManager) has also been included for sending and receiving messages, containing their most prominent data. This class makes 
use of a data listener that has been included in another one (DataListener) that is in permanent waiting mode so that it will immediately transfer the messages that are collected from the network. All these characteristics have been included in Figure 69. If the middleware architecture is deactivated due to any reason (hardware migration, etc.), the listening capabilities will remain disabled until they are either activated again or replaced with a different component.

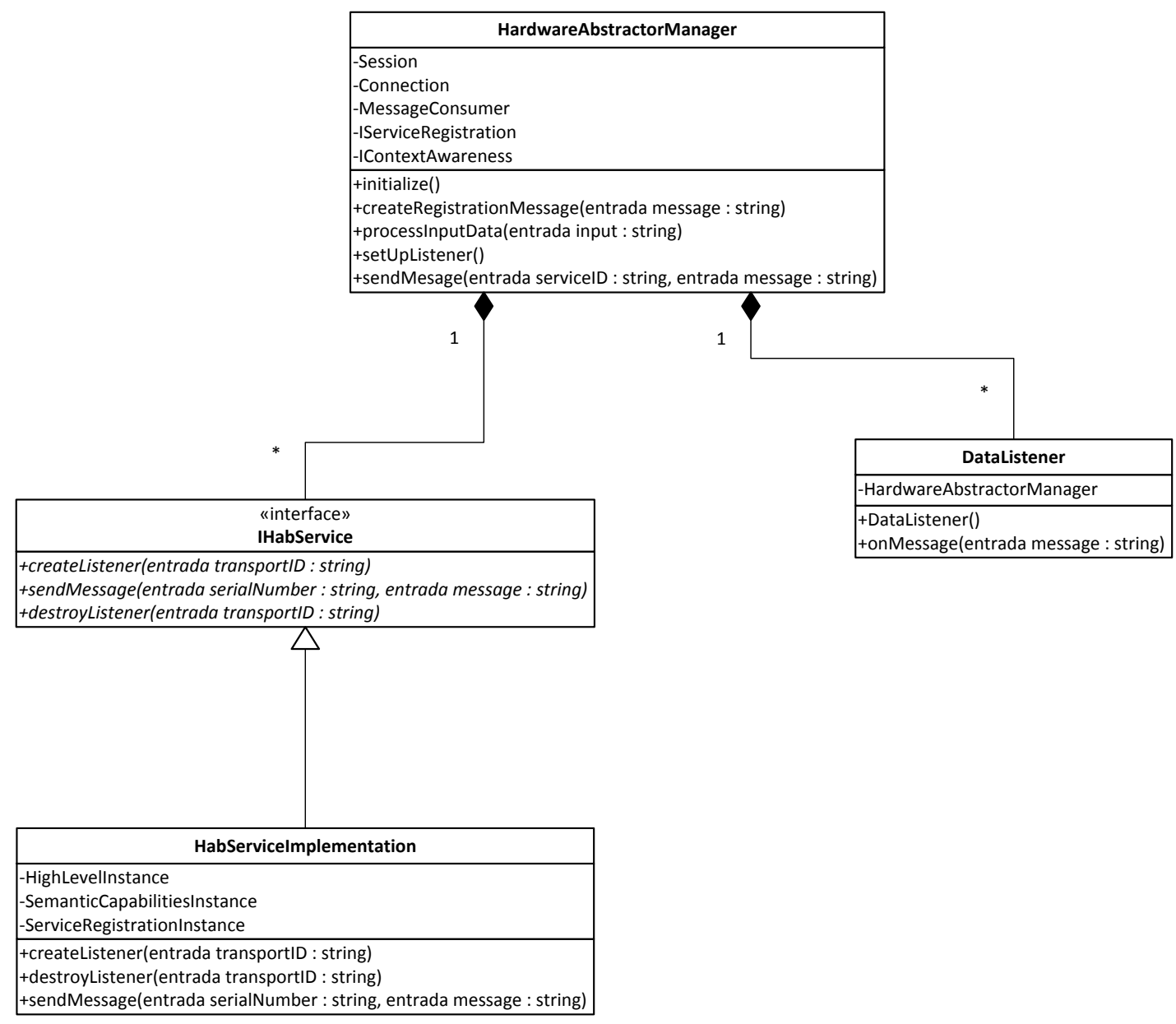

Figure 69. Class diagram of the Hardware Abstractor component

The component used for Service Composition can also be described in a similar manner. The elements that can be expected here are related to one class used to collect all the information from simple service requests and send it back to the high level middleware components. An agent is used as the Object Oriented Programming language interface because there are services that can be implemented and easily ported to other object instances. The class that implements the service composition functionalities receives the data from the other components, enables the composition and returns the result to its origins. The overall appearance of the class diagram that has been designed is present in Figure 70. 


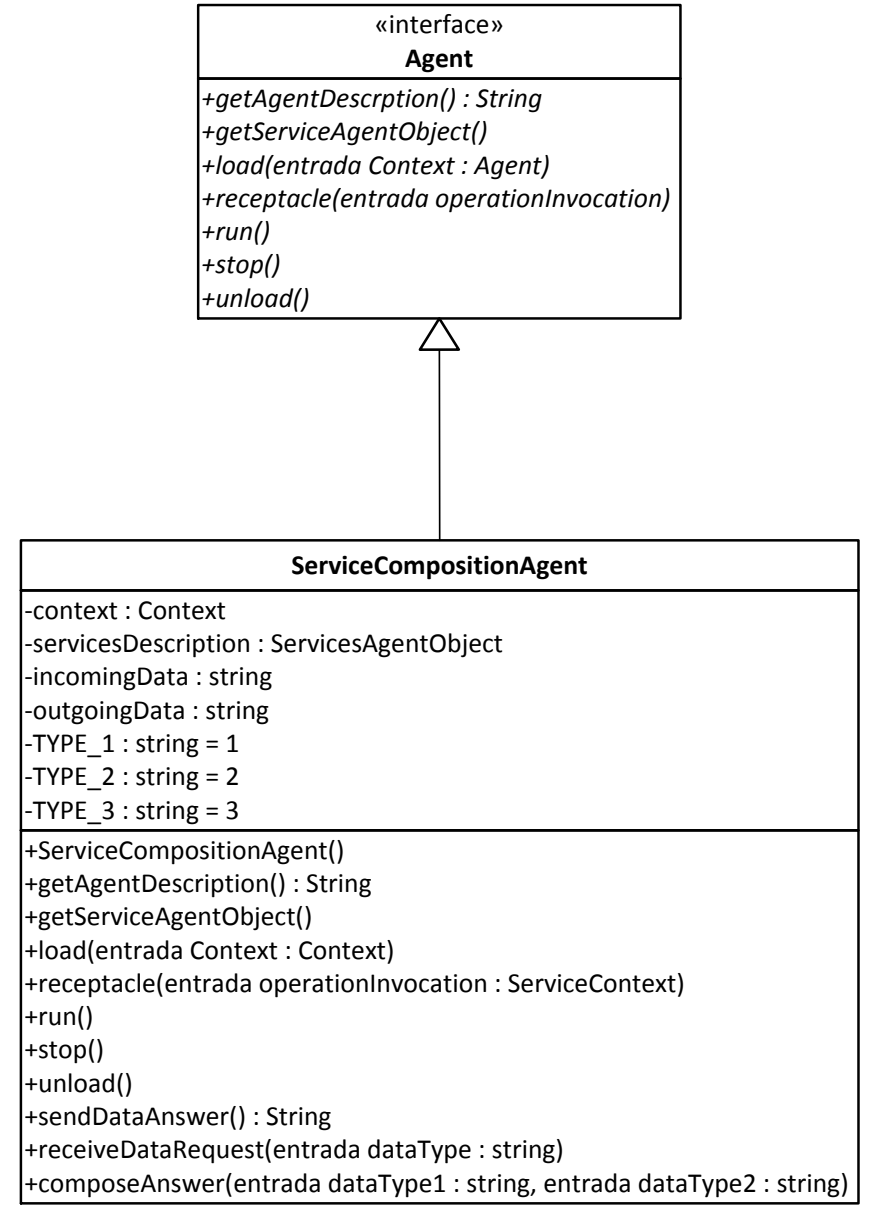

Figure 70. Class diagram of the Service Composition component

Lastly, the securitization component includes the functions used to encrypt and decrypt transferred information. They are very clearly separated between asymmetric (AsymmetricCryptography) and symmetric encryption (SymmetricCryptography), as it has been deemed useful that, should security be implemented by means of a software component, both options are available. Furthermore, a class used for ancillary purposes (AncillaryFunctionalities), such as choosing one kind of encryption over the other one, has been added to the software component. Figure 71 contains a representation of all these functionalities. 


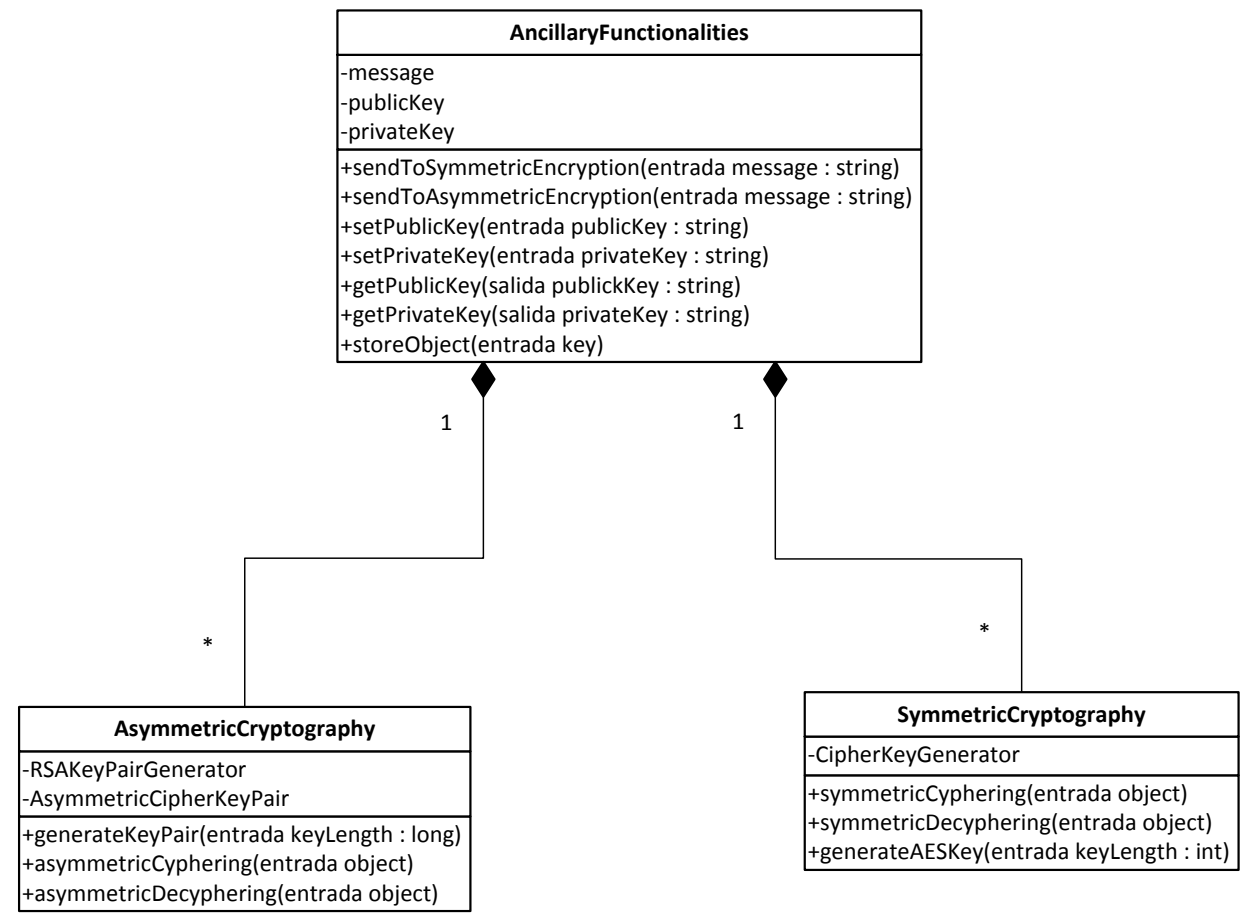

Figure 71. Class diagram of the Securitization component

\subsubsection{State diagrams}

There are two other ways that can be used to characterize from a Platform Independent Model point of view. One of them is by means state diagrams that show the different statuses where the system will work whenever it receives a specific input. Since they provide an interesting point of view regarding how a system changes its behaviour depending on the external stimuli it receives, they have been included in this thesis.

To begin with, the activity diagram for registration has been placed as Figure 72. From the point of view of a state diagram, the system stays in a "Waiting for registration request" status until it receives a device registration request, which will trigger a registration message that will be sent from the application to the middleware for its semantic treatment (1). The presence of the already registered device, along with all its associated services, will be checked by the Context Awareness component, since it will receive the request to do so (2). If the data corresponds to a non-registered device (3), the request will be further transferred (4) through the middleware architecture. It is interesting noting that during the message transmission, the system will move from one state to the next one without any other option. This is due to the fact that, as tests result will show in the next section, any other event happening once the request has started being transmitted through the middleware is extremely unlikely. As soon as the registration process is finished after the last transfer (5), the system will revert to its waiting status for further component registration (6). 


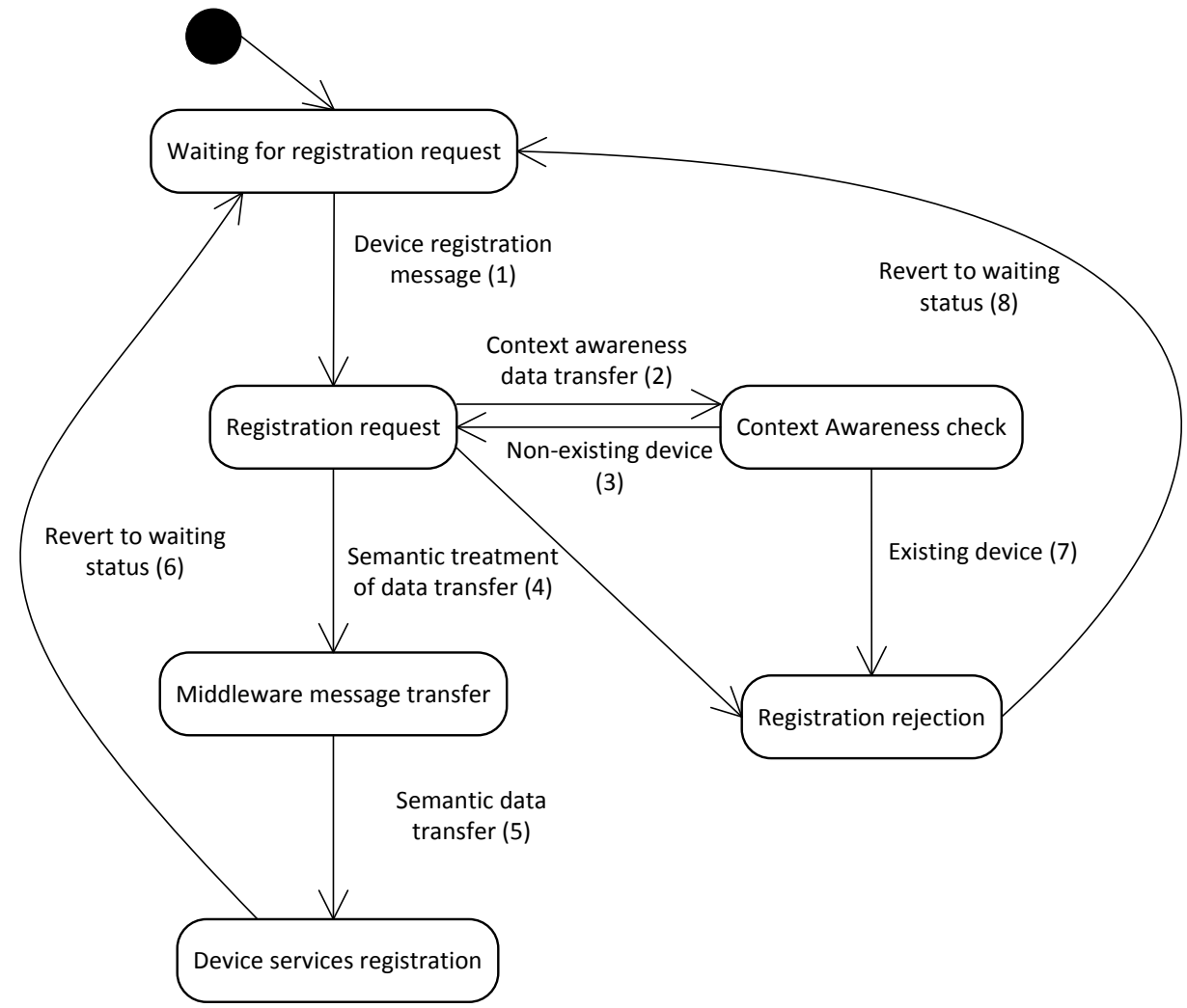

Figure 72. State diagram for service registration

Another situation that may happen, though, is that the device is already registered (7). Consequently, the Context Awareness component will refuse its registration, as it would be redundant. In any case, after this last change of status, the middleware architecture will go back to its initial status (8) and wait for further devices to be registered.

In case a request is received to obtain some kind of information from a service the sequence of states is as depicted in Figure 73. In this case, when the system is idle (it may be very well doing something else, but it is considered idle from the requests point of view) and a service request is sent to the middleware architecture (1) the service request will be checked by the Context Awareness component, which will look for an already registered device in order to satisfy it. If it is found, it will send back the confirmation of an existing device to the other part of the middleware (4) and the message will be further transferred throughout the architecture until it leaves the hardware device where it has been installed and the underlying network capabilities transfer the data request to the device itself (6). After some processing is done in the device (7), the information will be retrieved and send back to the hardware component that contained the Middleware Access Point that collected it (8). Once it has been delivered in the main part of the middleware architecture (9). The system will then change a status where the answer has already been received (10) and delivered to the middleware component that started it (11), to finally get back to its original idle state (12). As it happened before, other possible situation is that a request has been sent to a device that is not available. Consequently, the Context Awareness component will refuse its further processing (13) and the middleware architecture will revert to its original idle state. 


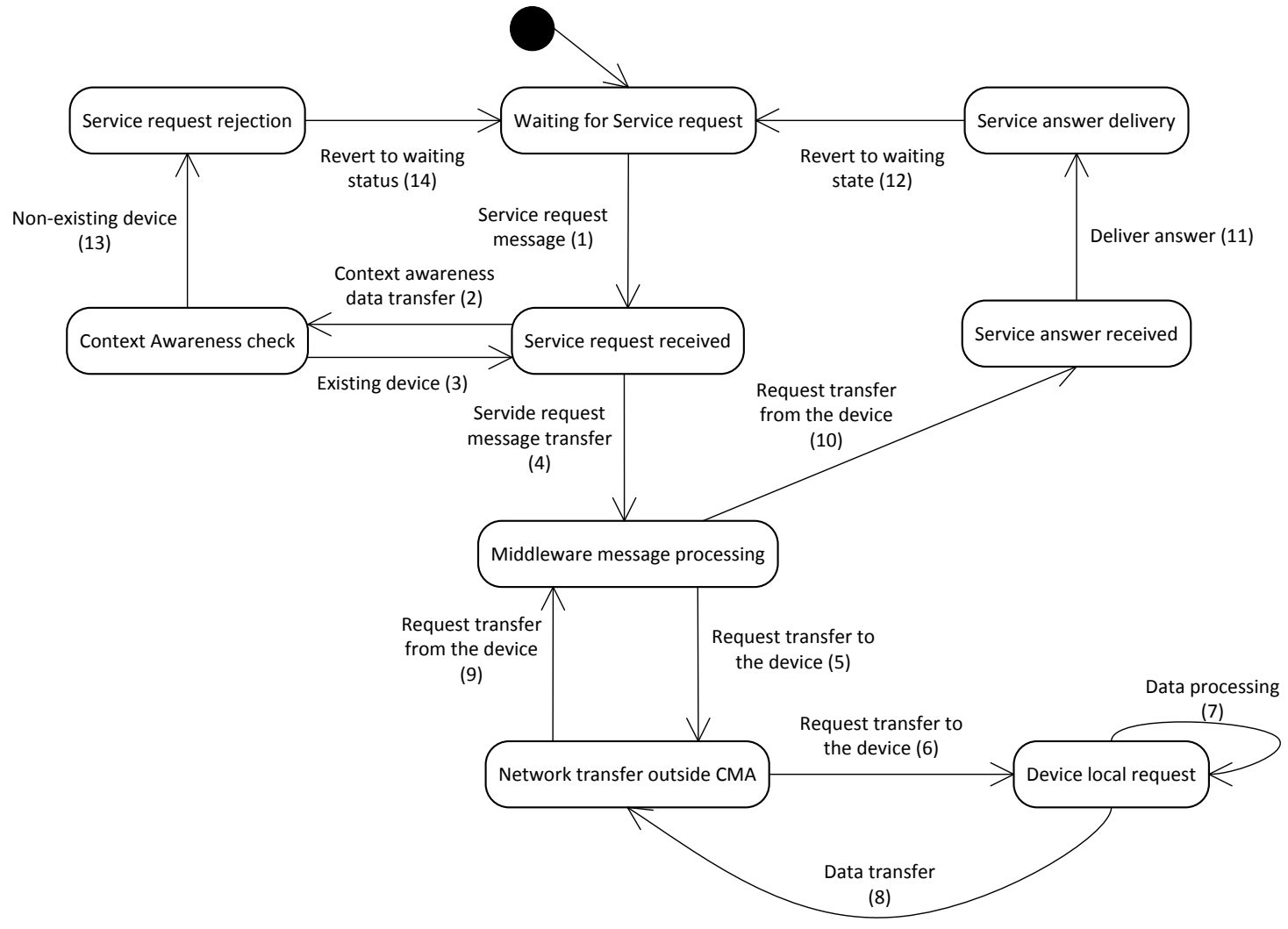

Figure 73. State diagram for service request and answer

The last prominent situation that is described by means of a state diagram is the request of listing the available services of the system. As portrayed in Figure 74, when the system is no taking any action with regards to listing services, and a new request is delivered to the system, it will transfer it (1) until it reaches the registration component capable of satisfying the query (2). The list will be provided as a set of data (3) and once it is received will be delivered to the application (4) and the system will return to its initial status (5).

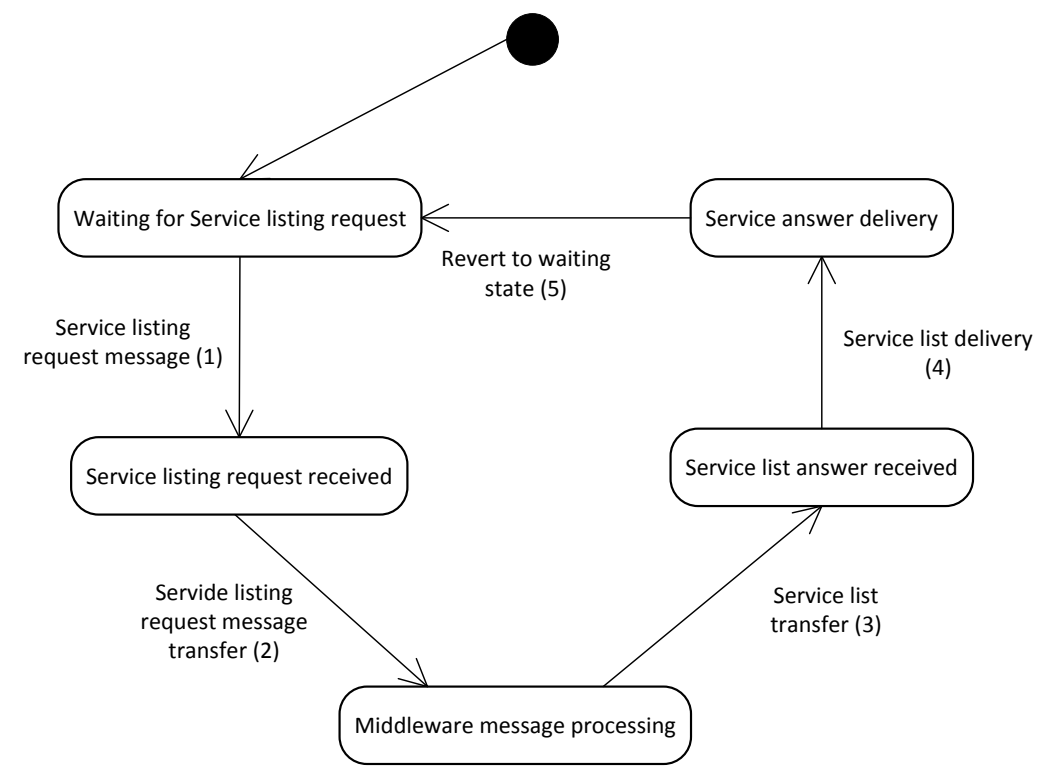

Figure 74. State diagram for service listing 


\subsubsection{Activity diagrams}

Activity diagrams show the different actions that can be taken by the parties implied in communications where the middleware solution is deployed. In the case of Common Middleware Architecture, they can be used to define the actions that have to be taken in order to complete a certain operation of interest for the system. They can be especially useful when determining the differences regarding a specific choice or option in the system behaviour that will result in a different set of actions. The same likely situations that were depicted in the previous section have been included here as well: device registration, service request and service list request.

When a device registration has to be done in the system, and according to the point of view that is provided by an activity diagram, the first action following the start of all the procedure will be sending forward the registration request until it reaches a point where either the device will be already registered or not. Should it be registered, any further step will become thwarted and the flow of actions will come to an end. In case it is not registered yet, it will have to be considered whether the query was started either from the device or the application layer. If the device has started the request, then it will imply that a network transfer has been performed and therefore CMA functionalities have been used. If the request was started from the application layer, the request will be attended by the suitable middleware component and the registration process will be finished, as shown in Figure 75.

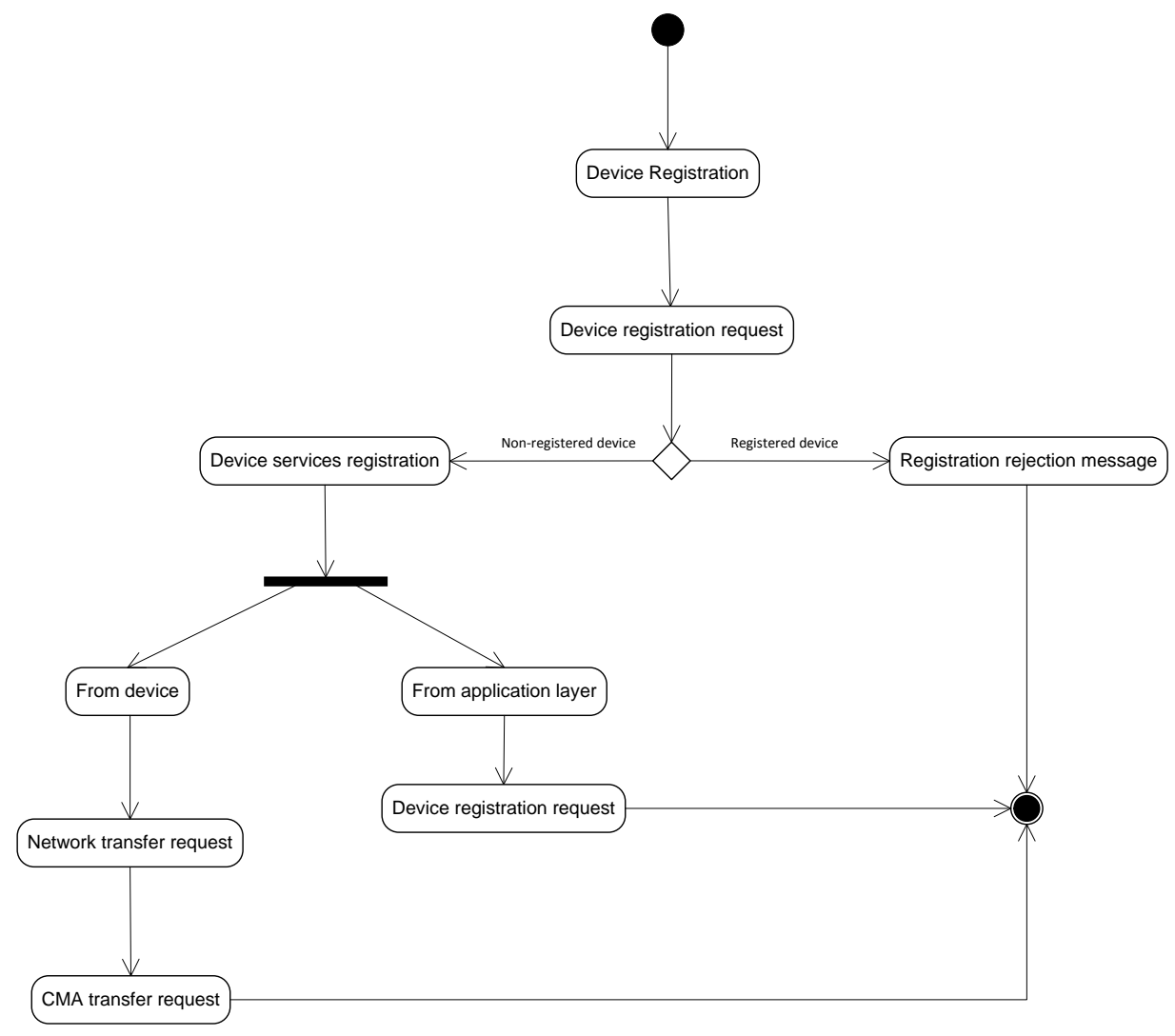

Figure 75. Activity diagram for device registration 
When a service is requested, it will be checked whether the device that is expected to provide it is present in the system or not. If it is not present, then the flow of information will be finished. If the device or service is present, then the service capable of providing an answer for the request will be invoked. This will be either one of the middleware high level components or a device present in the overall deployment. In the first case, the Middleware Autonomous Service will receive the request via transfer to the Common Middleware Architecture, process it and provide the result back to the application, thus finishing the information flow. If the request has to be answered by a device, it will be transferred via network to it and when the information is collected, embedded in a standardized Protocol Data Unit of the data level and send back to the middleware component that received the request, which will finally deliver the information to the application that originated it. All this sequence of actions has been depicted in Figure 76.

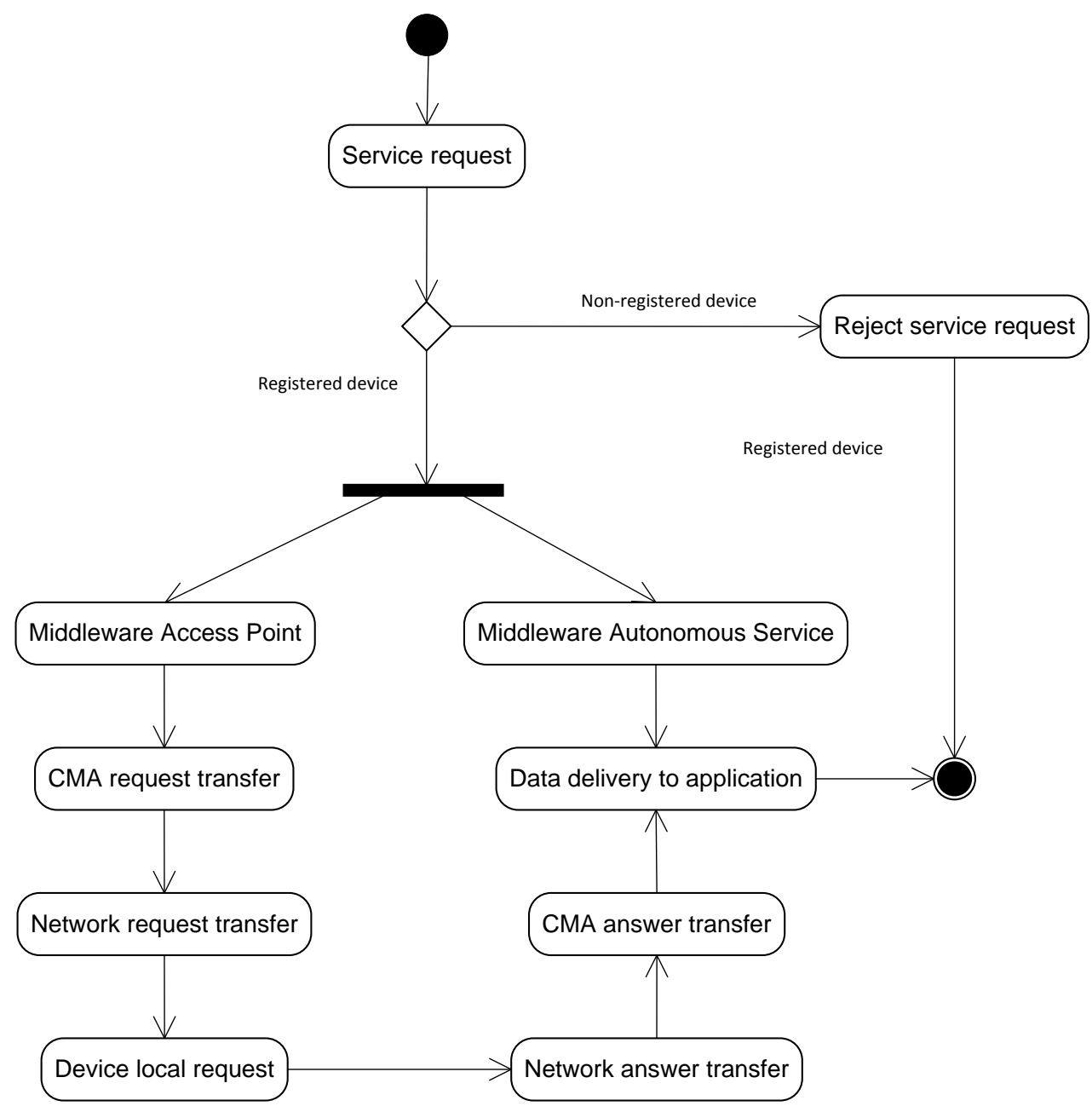

Figure 76. Activity diagram for service requests

Lastly, when a list of service is requested, the procedure will be less convoluted than in the previous examples. Figure 77 summarizes the steps taken in order to deliver the list to the application layer. From the point of view of an activity diagram, all this steps imply sending the listing request to $\mathrm{CMA}$, retrieving it in the fashion that has been explained previously with the sequence diagrams and provide it to the application that asked for it. Therefore, there will only 
be three different intermediate steps: the service list will be requested from the application layer, it will be transferred to the Common Middleware Architecture components (which will be, as shown before, the registration component and the semantic capabilities component) and will be transferred back to the application that requested the information to the middleware.

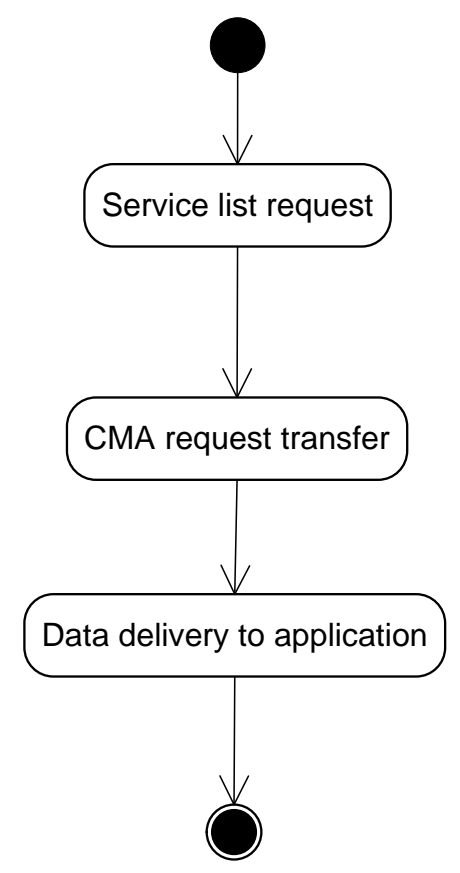

Figure 77. Activity diagram for service list request

\subsubsection{Deployment diagram}

The last UML diagram that is going to be used to represent the features and behaviour of the Common Middleware Architecture is a deployment one. As it has been mentioned before, the middleware architecture will be distributed among several different hardware devices present in a system with different hardware components belonging to a Smart Grid-like system (which has been referred to as "deployment"). Deployment diagrams show where the different parts of the middleware solution have been installed. By "where", it is meant the pieces of hardware that contain the middleware solution. In addition to that, the interconnections among all of them and the resources used to obtain them are also shown in this kind of diagram. All these ideas have been tied together in Figure 78. There, the elements that are used in a demonstrator or an actual working system can be better appreciated: the device referred to as the Central Controller connects itself with the device that is using the TCP/IP stack. At the same time, REST interfaces are used at the uppermost level of the stack (application) between the Central Controller and the device that is expected to use the application. This one can be based in a web browser or any other software technology, but in the end will have to count with some sort of Graphical User Interface. As far as the other devices are concerned, they will be connected with each other with the same kind of TCP/IP stack, as they have to be reached 
remotely, as it was done with the previous device that had the GUI installed in it. The number of local controllers that has been installed in the system will differ depending on the services that are demanded. In any case, they will be a collection that will at least focused on retrieving information either from the environment (as if they were part of an Internet of Things system) or from the power facilities installed in a certain area.

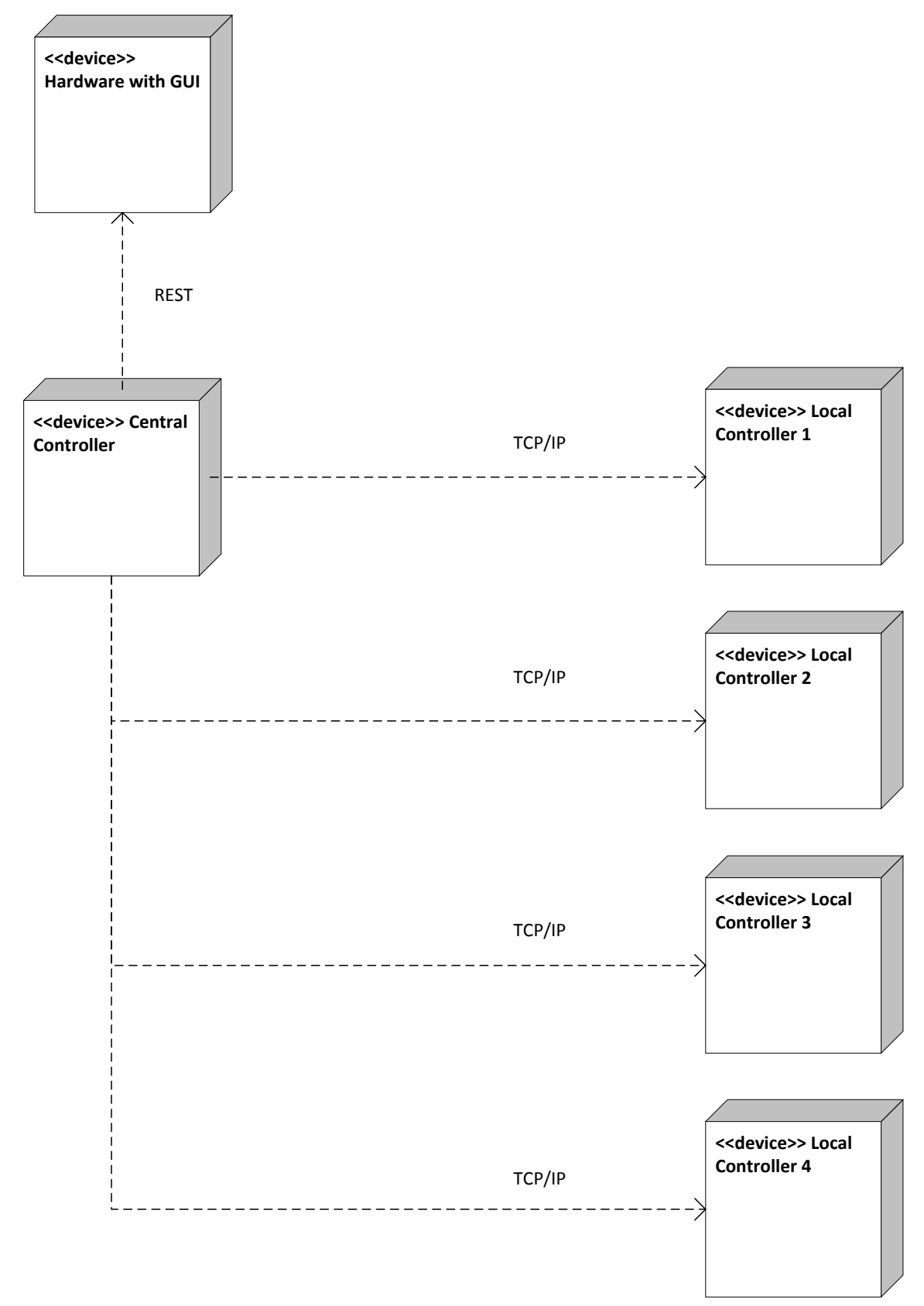

Figure 78. Deployment diagram of a system with the Common Middleware Architecture installed

\subsection{Architecture Description for ISO/IEC/IEEE 42010}

The description that has been placed in this section deals with the idea of describing CMA according to the models and principles formulated according to the ISO/IEC/IEEE 42010 
standard. The different subsections that have been included in this document have been done so according to the template defined by the ISO member Rich Hilliard for the description of software architectures [159], whom the author of this thesis established contact in order to clarify and obtain the suitable resources for this description. Rather than constantly referencing the standard, the next sections have been included as if having the architecture described according to this standard had been previously agreed upon. There are some aspects from this standard (as stated in [160]) that deal with the concept of model kinds and their features. Model kind names are a concept very specific to the ISO/IEC/IEEE 42010 standard, in the sense that according to it, architecture viewpoints are constituted by means of several architecture models which are ruled by a model kind that fixes conventions, notations and rules for the models of that type. The same idea is located in Figure 2 of [160], where it is represented that each Architecture Viewpoint is an aggregation of one or several model kinds that, at the same time, govern models for the architecture. It is also mentioned that "Examples of model kinds include data flow diagrams, class diagrams, Petri nets, balance sheets, organization charts and state transition models". Therefore, considering that these elements have already been included, and considering the scope of the thesis, model kinds and their features are not referenced in the description that has been included in this section. In order to make crystal clear the terms and parameters that have been included in this subsection, ,the ones that play a major role in it have been surrounded by <> symbols.

\subsubsection{Identifying information}

The $<$ Architecture Name $>$ that is described here is the $<$ Common Middleware Architecture $>$ or CMA. It aims to provide a significant contribution to a standard for the development of middleware for the <System of Interest> represented by the <Smart Grid>, although it can be extended with a minimal amount of modifications to other Cyber-Physical Systems, such as underwater robotics of the Internet of Things.

\subsubsection{Supplementary information}

This architecture has been defined as a result of the experience acquired during the collaboration of the author of this manuscript in several Smart Grid-themed projects, such as e-GOTHAM [21] and I3RES [22]. The specific needs for those projects were slightly different, in the sense that e-GOTHAM aimed to provide an ICT-based platform for the interoperability of hardware devices and energy-related applications, whereas I3RES was focused on the management of energy from a more electrical point of view, enabling new actors like aggregators or prosumers, and management of electricity from the end user side by means of services like Demand Response (DR) and Demand Side Management (DSM).

\subsubsection{Other information}

There are other important pieces of information that can be included from the architecture that is described here. Specifically, features related to the Purpose of $<$ Common Middleware 
Architecture>, its scope and the context of this architecture have been described in the following section.

\subsubsection{Overview}

CMA is a software middleware solution that has as its purpose guaranteeing interoperability of hardware devices interconnected via networked systems and the applications that can be accessed from the higher level endpoints that have been enabled in <Common Middleware Architecture>. In the same way, it establishes a number of processes, Processing Data Units and format details that target interconnectivity at the data level, so that data can be seamlessly transmitted from one part of a distributed system to the other.

The scope of <Common Middleware Architecture> strictly covers the middleware layer, as its main objectives are consistent with what is expected from this level (hardware abstraction, interoperability, Application Programming Interfaces for applications). Due to the nature of middleware, other levels such as applications networking, operating systems or hardware are not covered due to the following reasons:

1. Applications are supposed to be connected via access points to lower levels rather than perform the interconnection by themselves, due to the fact that applications are commonly unaware of all the other devices present in a distributed system, and if they had to be so, their complexity would grow exponentially and their scalability would be very limited.

2. Network layer perform interconnectivity functionalities, but these are limited to the PDUs typical of the network layer (packages) and its contents and format cannot be usually understood in an immediate manner by an application (let alone its human users).

3. Hardware devices are produced by different manufactures and vendors and, by themselves, their components at this level are not supposed to be aware of other devices. While middleware components can be added to hardware (as it will be shown in the next chapter), they are still part of the middleware layer from a distributed, CPS point of view. Alas, they are software-based, rather than actual hardware assembled in a production line.

As far as the context is concerned, the features presented in <Common Middleware Architecture $>$ have been conceived for the $<$ Smart Grid $>$ and any other CPS that results similar enough to it. Usually, any distributed system that presents hardware devices that receive and transfer information regarding application domain -or even contextual- information and can be acted upon via software will be likely to receive a port. In the end, by following the definition of what a Cyber-Physical System is - "CPSs refer to the next generation of engineered systems that require tight integration of computing, communication and control technologies to achieve stability, performance, reliability, robustness, and efficiency in dealing with physical systems of many application domains" [161] or the kind of deployment where computing devices and physical components are integrated [162]-, it can be determined how <Common Middleware Architecture> can be ported to those systems. 


\subsubsection{Architecture evaluations}

Two different scenarios have been used to assess the feasibility and performance of the semantic middleware proposals that have contributed to the creation of <Common Middleware Architecture>. In addition to that, [141] also includes a description and testing activities related to what was done in a laboratory-based environment using a Raspberry Pi as a Local Controller. For the sake of the manuscript that contains this description, they have been included in Chapter 4.

\subsubsection{Rationale for key decisions}

There are several decisions of major importance that have been taken in the context of the design and development of the <Common Middleware Architecture>. They have been taken considering some underlying aspects that are common of middleware architectures, such as the following ones:

1. Encapsulation of services was mandatory. Since the services that were going to be used by higher level entities (such as the application layer) have an immediate connection with the middleware, they had to be included in this latter layer.

2. Accessibility to the services present in the middleware had to be granted. Due to the same reason described before, several access points had to be created for the applications to access middleware.

3. The system presented had to be distributed. It was required that the facilities that can be offered by the middleware could be accessed from every device where it was supposed that middleware should be installed (such as the end user pieces of equipment like Advanced Metering Infrastructure)

4. Performance had to be acceptable. In the end, the system should work in a way that it was able to provide the data retrieved from the services at a reasonable speed, otherwise it would be useful for end users.

All in all, decisions match the criteria established for the non-functional requirements that were formulated in the projects were the middleware architectures were carried out.

\subsubsection{Stakeholders, concerns and concern-stakeholder traceability}

The main group of stakeholders can be roughly described as the group of people with an interest in information transmission in the <Smart Grid>. To put it more accurately, the following stakeholders have to be considered, according to what is defined in the ISO/IEC/IEEE 42010 standard for Architecture Description:

1. Users: the users of the middleware solution that is described in this thesis are the consumers at the very end of the $<$ Smart Grid $>$ that make use of the electricity that they have purchased to turn it into other different kinds of energy, depending on their needs 
(mechanical, chemical, etc.). It must be taken into account, though, that prosumers can also be included in this group, and they will be able to provide power to the system, in a bi-directional way that can be regarded as one of the most prominent contributions of the application domain that is being studied.

2. Operators: these are the staff involve in the correct performance of the systems in the $<$ Smart Grid>. Typically, they will involve ICT- and power-related personnel, as these are the two main implied areas of knowledge. Nevertheless, their functions will be rather similar: supervising the hardware and software equipment that is used in the system and controlling that the electricity is transferred according to their instructions.

3. Acquirers: the acquirers of the middleware solution that is being studied are the main parties involved in the ICT part of the <Smart Grid>: Distributed System Operator, Transmission System Operator and the aggregator all participate from the system that is being developed as part of the work performed in this thesis. Depending on the kind of devices used by the consumers/prosumers, middleware may also be extended to the local devices used in their dwellings, as shown in [141].

4. Owners: they will be the same entities that the acquirers are, as no transfer is planned to be done from one entity to another one out from the <Smart Grid $>$.

5. Suppliers: the entity expected to offer the middleware architecture will fall within the same category as the ones providing ICT-related services. Software developers, telecommunication companies or even start-ups related to the business of the power grid can supply the required.

6. Developers: the developers of a middleware solution will usually be part of the staff that is working on the ICT part of the <Smart Grid>. They might be either from the third party software company responsible for the development and installation of the middleware solution or from the power grid actors (DSO, TSO or the aggregator) in case they want to extend their areas of interest to $<$ Smart Grid $>$ software.

7. Builders: since what is presented here is a middleware architecture, there is no hardware to build except for the one that will have all the required software installed. Commonly, the computational capabilities of this solution will not be very demanding, so the hardware does not require being expensive.

8. Maintainers: there will be part of the staff implied in the middleware to check its correct performance, even though maintenance is likely to be minimal, as most of the services implied take care of the needs of the system. Replacement of devices or their upgrade (with new sensors and services) may require minor updates in the deployed middleware solution.

Concerns involving <Common Middleware Architecture> involve interoperability and interconnectivity at the data level of hardware devices typical of a power grid. Specifically, and as a way to answer the concerns addressed by the template of the ISO/IEC/IEEE 42010 standard for Architecture Description:

1. The purpose of the system-of-interest: allow the interoperability of the hardware devices that are present in the distributed, Cyber-Physical System represented by the <Smart Grid>. That is to say, the integration of different equipment regardless of their features and complexity in a deployment where all of the will be working in a cooperative manner. 
2. Suitability of the architecture: the suitability is very high, due to the fact that middleware is being used since the 1980s to guarantee interoperability between legacy and new systems. While the amount of services that is included here are way vaster than during that era, and they are applied to an application domain that was not present back then, middleware is proven to be a suitable solution for that kind of challenges.

3. Feasibility of the construction and deployment of the system-of-interest: Platform Specific Models of the system that has been included have been installed and tested in two different locations, so the system is capable of being successfully developed and deployed in a realistic manner.

4. Potential risks and impacts: there are two different kinds of risks and impacts. The first one involves technical complications, namely: issues related to the information transferred among the parties (especially regarding data privacy), complexity of the required software services (context awareness, device registration, etc.). The other one is more related to legislative aspects. For example, legal regulations may prevent prosumers to enter electricity markets by themselves, or a minimum level of security in data transactions must be guaranteed not to compromise privacy and integrity of the information transferred in the $<$ Smart Grid $>$.

5. Maintenance and evolution of the system of interest. It will be done as in any other software system that requires periodic updates: whenever there is a new device or service that has to be included, it will be done so that it will become accessible for the applications and their users.

Last but not least, the traceability of the solution that is put forward here has been defined by means of Table 12, where the concerns that have to be faced by each of the stakeholders have been depicted.

Table 12. Traceability for the concerns of the stakeholders

\begin{tabular}{|l|l|l|l|l|}
\hline Concerns & DSO & TSO & Aggregator & User/Prosumer \\
\hline Purpose & $\begin{array}{l}\text { Relevant } \\
\text { (information } \\
\text { transmission) }\end{array}$ & $\begin{array}{l}\text { Relevant } \\
\text { (information } \\
\text { transmission) }\end{array}$ & $\begin{array}{l}\text { Relevant } \\
\text { (information, } \\
\text { profitability) }\end{array}$ & $\begin{array}{l}\text { Very relevant } \\
\text { (new actor, } \\
\text { profitability) }\end{array}$ \\
\hline Suitability & Relevant & $\begin{array}{l}\text { Relevant } \\
\text { (integration) }\end{array}$ & $\begin{array}{l}\text { Relevant } \\
\text { (integration) }\end{array}$ & $\begin{array}{l}\text { Relevant } \\
\text { (integration) }\end{array}$ \\
\hline Feasibility & $\begin{array}{l}\text { Relevant (game } \\
\text { (inanger) }\end{array}$ & $\begin{array}{l}\text { Relevant } \\
\text { (information } \\
\text { transmission) }\end{array}$ & $\begin{array}{l}\text { Relevant } \\
\text { (information } \\
\text { transmission) }\end{array}$ & $\begin{array}{l}\text { Relevant } \\
\text { (profitability) }\end{array}$ \\
\hline Potential risks & Relevant & Relevant & Relevant & Irrelevant \\
\hline $\begin{array}{l}\text { Maintenance } \\
\text { and evolution }\end{array}$ & Relevant (new & $\begin{array}{l}\text { Relevant (new } \\
\text { facilities) }\end{array}$ & $\begin{array}{l}\text { Relevant (new } \\
\text { users/prosumers) }\end{array}$ & Irrelevant \\
\hline
\end{tabular}

As it can be seen, the overwhelming majority of the provided concerns affect to all of the entities participating in any deployment done implying <Smart Grid>-based hardware and software. The only concerns that are irrelevant are the ones concerning the potential risk of the installed software and the maintenance and evolution of the software for the end user or 
prosumer, since these are challenges that are not in their hands to solve, but in the other agents that are providing the software and hardware facilities.

\subsubsection{Viewpoint names}

Although the main objective of including the IEEE/IEC/ISO 42010 standard is describing <Common Middleware Architecture> according to its principles, there are some other characteristics of ancillary concepts that should be included as well, such as the idea of architecture viewpoint. According to the standard, an Architecture Description may contain several different views, which adhere to the features of what is referred to as architecture viewpoint. An architecture viewpoint can be defined as a collection of conventions introduced in views, which at the same time address specific concerns of interest for the stakeholders [163]. In the previous reference, it is stated how decision detail, relationship, chronology and stakeholder involvement viewpoints are provided for forces in architecture decisions. According to the template that is expected to be used to describe an architecture viewpoint [164], there are several features that define the latter that are also present in the description of an architecture, such as an overview, concerns and stakeholders or model kinds. As far as $<$ Common Middleware Architecture $>$ is concerned, there are two different viewpoints that have been added, according to the two domains where the $<$ Smart Grid $>$ is more heavily involved:

1. One has been named <Electricity>. This viewpoint includes all the features related to the generation, usage and consumption of electricity in the power grid.

2. The second viewpoint is $\langle\mid \mathrm{ICT}\rangle$. This one deals with all the features that are related to Information and Communication Technologies.

The viewpoints that have been included provide a more detailed explanation of the architecture description, as they allow expanding the knowledge of the two areas of knowledge that have more features composing the $<$ Smart Grid $>$.

\subsubsection{Overview for viewpoints}

The viewpoint <Electricity> considers the system of interest of the <Smart Grid> from the perspective of electricity. Therefore, it is its main interest how the <Smart Grid> impacts in every aspect of <Electricity>, such as the patterns of its consumption, where it can be generated, how it impacts on the production and need of regular power plants, etc. Other aspect relevant to this point of view is trading: if there are newly added small energy producers, this energy can be traded in the markets so that revenues can be obtained by the prosumers. However, legislation may have to be modified, or prosumers may have to conform to it if they want to participate. It can be said the same about other actors that may have prominent changes under the $<$ Smart Grid $>$, like aggregators or DSOs.

On the other hand, $\langle\mathrm{ICT}\rangle$ convers all the aspects related to Information and Communication Technologies. These are related mostly to the aspects involving software for the $<$ Smart Grid $>$ 
devices and appliances present in a deployment. Therefore, this viewpoint comprehends all the facilities related to the services that can be provided for end users/prosumers, such as device and service registration, security, context awareness, and any other service that will be included in the middleware architecture. Data that is transferred from one device to another will usually fall within the domain of this viewpoint.

\subsubsection{Stakeholders, concerns and "anti-concerns" for viewpoints}

Each of these features reflects the description made before for the architecture. For example, regarding the concerns and the stakeholders related to the $\langle$ Electricity $\rangle$ and $\langle\mid \mathrm{CT}\rangle$ viewpoints, concerns will name areas of interest in a system [159]. The ones that can be mentioned are as follows:

1. Concerns for <Electricity> involve mostly the reliability of the power grid when it has to deliver the electricity required. In addition to that, the required changes of voltage throughout the system must also be considered (from a very high voltage in the facilities of the DSO to a high one in the TSO transmission, a medium one when it is delivered to the aggregator or the aggregator-like company that is selling it to the end users and low voltage for end users).

2. Concerns for $\langle\mid \mathrm{C} T\rangle$ are wider due to the plethora of services expected to be included in the middleware solution. The main concerns involve, as it happened with the previous viewpoint, the reliability of the architecture in terms of data delivery and service availability. Other areas of interest that may be cited are the ones dealing with each of the services the architecture is expected to provide, such as a) device registration, b) security of information transfers, c) semantic capabilities and d) application access to the middleware via Middleware Access Points.

Stakeholders and "anti-concerns" have also to be taken into account. On the one hand, the stakeholders that are relevant for each of the viewpoints are the same that the ones that were mentioned in the architecture description. However, they are part of the area of influence of each of the viewpoints that have been settled.

1. Stakeholders for the <Electricity> viewpoint involve many of the ones that were mentioned in the architecture description: users (the parties that use <Electricity>, either as a commodity -consumers- or as a good to make a profit with), operators (parties involved in the transmission or distribution of <Electricity>, like the DSO or the TSO), acquirers (parties that buy <Electricity>), owners (parties that own the facilities required to produce <Electricity>), suppliers (agents whose main role is supplying $<$ Electricity>), builders (parties interested in building the infrastructures linked to $<$ Electricity>; the DSO and the TSO appear as the ones more evident) and maintainers (parties involved in the maintenance of <Electricity>-related goods and services; DSO and TSO appear again as the most interested in this kind of maters). No developers 
have been considered for this viewpoint, as the development of software is regarded as an activity more related to the $\langle\mathrm{ICT}\rangle$ viewpoint.

2. Stakeholders from the $\langle\mid \mathrm{ICT}\rangle$ point of view are quite similar to the ones that have been described before, since there is usually a party interested in the same kind of activities, even though they are more focused on the software side in the system of interest represented by the $\langle$ Smart Grid $\rangle$. Therefore, users (users of the software deployed in the <Smart Grid>), operators (staff monitoring the software), owners (parties that have purchased the software, which are likely to be either the DSO, TSO or the aggregator, if present), suppliers (staff providing the services included in each of the software components), developers (the staff that implemented the software that has been included in the middleware architecture) and maintainers (staff responsible for the stable performance of the software and its update, if necessary) are cited as the stakeholders with a prominent role in this viewpoint. Builders are left outside this viewpoint because the term feels more related to hardware than Information and Communication Technologies. Staff that takes part in the $<\mid \mathrm{ICT}>$ are usually part of the companies that either generate, distribute or transmit the electricity in the $<$ Smart Grid>; end users will take a lesser participation in this case, unless they have been turned into prosumers.

"Anti-concerns", on the other hand, summarize the challenges not useful to be tackled by a point of view. Regarding the two viewpoints that have been established for the system of interest called the <Smart Grid>, and considering that they cover by themselves the overwhelming majority of features and behaviour that can be found in the system of interest, it can be said that the issues that they both deal with are mutually exclusive, so <Electricity> will not result useful to understand the challenges associated to software services, device registration or data retrieval, whereas $\langle\mid C T\rangle$ cannot be used for issues related to power flow or electricity transmission and distribution.

\subsubsection{Operations on views}

If operations are defined as the methods to be applied to views and their models [159], there are several actions that can be regarded as operations in the <Common Middleware Architecture>. According to the previous reference, there are at least four different kinds of operations to bear in mind: construction methods (used to construct views according to a certain viewpoint; process guidance or work product guidance are cited as examples of operations), interpretation methods (used to guide readers when interpreting and learning about the architecture views and their models), analysis methods (utilized to perform several actions, such as reasoning about any topic, transformations, predictions, evaluation of architectural results or checking a feature) and implementation methods (means to design and build systems using a specific view). These elements have already been included as part of the $\mathrm{UML}$ diagrams that were described in the previous section, so that information has already been provided. Nevertheless, a summarization of the criteria that has been followed to include them is offered here as a summary of those actions: 
1. Construction methods are roughly equivalent the constructor methods that are used in each of the classes of the class diagrams of each of the components that have been included previously for each of the software components described in the <Common Middleware Architecture>, as they are used to create the instances of the objects required to execute the software when required.

2. Interpretation methods as defined in this context can be referred to all the UML diagrams that have been created for the description of the middleware architecture, as they provide those diagrams as a way to know what the proposal of this thesis is made of and how it behaves when running in a system.

3. Analysis methods can be referred to the requirement analysis that has been performed before, so that the required components of the <Common Middleware Architecture $>$ could be determined. The evaluation of the results provided by the usage of this model of architecture will be offered in the next section of the thesis.

4. Implementation methods have been heavily described both in section 4 and in the Platform Specific Models, where information is offered regarding how the architecture was developed. Lastly, the methods present in the class diagrams that have been included are mostly about how operations made by each of the instances are done.

\subsubsection{Views and views name}

Another major feature of this standard is the existence of Views, that is to say, the conventions that have been chosen from the different governing viewpoints. It is stated in the template used to describe architecture views that an architecture view for each viewpoint must be included, along with all its characteristics. Consequently, the following views and their names have been included:

1. <Electricity Deployment> has been chosen as the view for the <Electricity> viewpoint, as it best includes the stakeholders, concerns and "anti-concerns" that were formulated previously. It deals with the deployment of the entire electric infrastructure required for power generation, distribution, transmission and commercialization. In the end, it represents all the elements present in a conventional grid that makes no use of <Smart Grid>-like ICT elements.

2. <ICT architecture>, on the other hand, includes all the Information and Communication Technology-based features of the <Smart Grid>. The term "architecture" makes reference to the kind of middleware solution that $<$ Common Middleware Architecture > can be best described for. It also establishes a more specific perspective for this view, as $\langle\mathrm{ICT}>$ as a viewpoint does not hint what kind of software development is being done.

\subsubsection{Models and model name}

As it happened with the previous features, each of the models that have been included must also be given a name. It is stated in the template used for the description of architecture 
models that each of the architecture views must have at least one model, since the former is composed by the latter. In addition to that, it is expected from each of the models that will address each of the concerns that were highlighted by the viewpoints, as well as covering the whole system from that particular viewpoint. Thus, the following aspects have been added to the description of the model:

1. From the viewpoint of < Electricity $>$ that is addressed by the <Electricity Deployment $>$ view, an architecture model has been created; it is called <Electricity Deployment Model>. This model deals with all the previously described concerns by taking the following actions:

1. Reliability of the power grid is guaranteed by several actions that have been taken since the power grid was firstly made possible: forecasting the energy that is going to be demanded from the <Smart Grid> is one of the most useful ones (for example, forecasting wind speed as a way to know how much electricity will be injected [165]), as it prevents unforeseen overloads that may result in blackouts. Redundancy may also be used as a way to have a power grid functional despite occasional failures [166].

2. Required changes of voltage are also guaranteed by well-known regular power infrastructure: power converters can be used for conversion of frequencies in electrical energy [167] or voltage [168]. They are a proven solution that successfully solves these challenges.

2. As far as the $<I C T$ Architecture $>$ view is concerned, a model called $<I C T$ Architecture Model $>$ has been created. The following actions are undertaken by it so as to tackle the concerns of this viewpoint:

1. Data delivery: it is made sure that data will arrive to their destination due to the interoperability that the middleware solution installed in the $\langle$ Smart Grid $>$ is capable of offering. Services in the upper layer will collect the information about the queries done by the users of the system and will be sent to the hardware connected to the distributed software architecture via network and lower levels.

2. Service availability: this is ensured by the context awareness component that is present in the middleware solution: if there is any service that is not available, context awareness will look an equivalent one that can perform a similar functionality, or will communicate its availability if no replacement can be done.

3. Each of the present services: a collection of services will be installed so that end user will have a wide range of software facilities: device registration, security of information transfers, semantic capabilities or applications used to access the middleware architecture will be ready for the end users.

All these aspects have been summarized in Table 13: 
Table 13. Viewpoints and their associated views and architecture models

\begin{tabular}{|l|l|l|l|l|}
\hline Viewpoint & Associated view & $\begin{array}{l}\text { Associated } \\
\text { architecture } \\
\text { model }\end{array}$ & Concerns & $\begin{array}{l}\text { Actions to deal } \\
\text { with the } \\
\text { concerns }\end{array}$ \\
\hline$<$ Electricity & $\begin{array}{l}<\text { Electricity } \\
\text { Deployment }>\end{array}$ & $\begin{array}{l}<\text { Electricity } \\
\text { Deployment } \\
\text { Model }\end{array}$ & $\begin{array}{l}\text { Reliability of the } \\
\text { power grid; } \\
\text { voltage changes. }\end{array}$ & $\begin{array}{l}\text { Forecasting and } \\
\text { redundancy; } \\
\text { power } \\
\text { converters. }\end{array}$ \\
\hline$<\mathrm{ICT}>$ & $\begin{array}{l}\text { ACT } \\
\text { Architecture }>\end{array}$ & $\begin{array}{l}<\mathrm{ICT} \\
\text { Architecture } \\
\text { Model }>\end{array}$ & $\begin{array}{l}\text { Data delivery; } \\
\text { service } \\
\text { availability; } \\
\text { present services. }\end{array}$ & $\begin{array}{l}\text { Middleware } \\
\text { interoperability } \\
\text { capabilities; } \\
\text { context } \\
\text { awareness; } \\
\text { middleware } \\
\text { encased services. }\end{array}$ \\
\hline
\end{tabular}

\subsubsection{Known issues with views}

According to the documentation provided for the standard, any open issue with the views that have been included must be described. The challenges that can be pointed out are closely linked to the open issues that were mentioned at the end of section 2 (imprecision in service availability, no common solutions, ambiguity in middleware design and solutions, no specific methodology). All these issues are primarily focused on the <ICT Architecture $>$ view rather than the power grid, since the former is the main interest of this thesis. In any case, <Common Middleware Architecture $>$ attempts to partially solve them by means of the portrayed solutions that have been described.

\subsubsection{Consistency and correspondences}

The consistency requirements that have been chosen for this architecture can be divided in two different levels. On the one hand, they deal mostly with the ones expected with a middleware architecture in the application domain of the $\langle$ Smart Grid $\rangle$. On the other hand, they result from the idea of using <Common Middleware Architecture> as a standard, so it will be required to observe the services and methodologies described here to have an implementation compliant with the proposal.

1. The critical consistency requirement for any architecture that is described according to the services, components and interfaces that have been described is that it will abstract hardware heterogeneity for the upper applications, so that it will provide the illusion of requesting locally any service middleware is expected to provide. As a way of access those services, access via Middleware Access Points will have to be enabled as well.

2. The major consistency requirement for any system implementing this solution is that the a) services, b) protocols, c) API and d) procedures to implement the middleware 
solution based on <Common Middleware Architecture>. Under occasional circumstances, though, some services may not have to be implemented, provided that there is a good reason for it (if, for example, middleware is going to be used in a system that offers security via certifications, the security component may not be necessary).

\subsubsection{Known inconsistencies}

By inconsistencies, it is meant the differences between the objectives put forward by the architecture models and their actual views. So far, there are not significant inconsistencies to be detected, due to the fact that the implementations that have been carried out according to the ideas that have been put forward in this thesis have been done so with a) very tight requirements obtained from the analysis, which makes it hard to have deviations from the original expectations and b) the implementations that have been carried out are still not widespread and the performance of the studied systems does not vary greatly from one implementation to another.

\subsubsection{Correspondences in the Architecture Description}

The correspondences existing in the architecture description are closely linked to the distributed nature of the two systems of interest within the application domain of the <Smart Grid>: both the power grid and the ICT infrastructure are located in several devices scattered in a certain environment and transfer a commodity -either electricity or data- from one device to another in order to provide services to end users. However, there are still more prominent differences than similarities between the two viewpoints represented by <Electricity> and $<\mid C T>$ : energy storage is way more complex (and more expensive) to perform than data storage, and there are no losses to be taken into account if data is transferred or kept for a prolonged amount of time. Additionally, trading activities that are done with energy are harder to do with the data that has been transferred, and it may be even possible due to privacy concerns.

\subsubsection{Correspondence rules}

Correspondence rules that have been fixed in the proposal are primarily focused on the software tools and standards that are used to represent the information and the data interchanges that are performed as part of the $\langle\mathrm{ICT}>$ viewpoint, since the scope of this thesis is mainly related to providing a software architecture for the power grid. Therefore, the following rules can be used for correspondence between this viewpoint and the ones that may be present in other architectures:

1. Protocol Data Units must be used with the same fields and purposes that the ones shown in section 3.5. While its actual implementation may vary from one architecture or another depending on the needs of the deployment, using high-level data representation formats 
(JSON) or metadata (XML) can be considered as the most useful options, due to the fact that they are an already accepted way to depict data and can easily be formatted in almost any imaginable solution. This flexibility and the usage of a set of parameters widely known (sensor information, electricity data, network addresses for sources and data destinations, etc.) make up for the verbosity of these solutions.

2. Enterprise Service Bus as a way to encase the services present in the middleware architecture. There are three different kinds of services (device registration, context awareness and securitization) that must always be present in middleware for the <Smart Grid> in order to provide the bare minimum of required functionalities.

3. Application Programming Interfaces must be offered to the application layer in the way that will be explained in the following section. This is done so to guarantee that the applications (and the end users) will be able to access the middleware solution. Again, even though technologies to be used may differ depending on the requirements of a particular project (REST is used in the Platform Specific models that have been included in this thesis), the API provided must implement the services offered in the Appendix B of this manuscript.

\subsubsection{Architecture decisions and rationale}

Lastly, the rationale behind the decisions that have been made have been listed here in a summarized way, as suggested in the template used for the middleware description. This decisions mirror the key ones that were described previously:

1. The middleware architecture to be developed must provide interoperability, high-level interfaces and encase services for registration, securitization and context awareness.

2. It must be a distributed solution in order to include all the devices available in one deployment with heterogeneous hardware.

3. Adding the middleware solution to the deployment must not hinder the performance of the system that is put to run in a particular location.

\subsection{Protocol Data Units}

The Common Middleware Architecture that is presented here includes in its description the PDUs that have been used for data transmission in the implementation works of the Smart Grid-related research projects where they were required. The PDUs were conceived to solve the typical duties than can be expected from middleware solutions in the Smart Grid:

1. Device registration: if the hardware elements present in a deployment are to be accessed to retrieve data from them, they must be registered in the system before so that they will be acknowledged.

2. Service request: once the devices have been registered, they can be requested about the information that they have obtained.

3. Service reply: the devices will encapsulate the significant information that is provided in return for the request that was previously formulated. 
4. Service listing of services: it is a useful functionality when tasks related to service discovery are being carried out.

The PDUs to be shown here contain the information that is transferred both at the high and the low levels of CMA, since communications will have different features at both levels of the middleware architecture. Next sections describe the appearance of the PDUs that have been used in each of the levels.

\subsubsection{Generic PDU format}

The PDUs have been defined will have the same format overall: a header will have the most important elements required for the information exchange to take place (the type of request that is being done, the identifier of the destination, etc.), whereas the payload will contain all the information required in the data exchange (the name of the operation, its parameters, the output obtained from that result, etc.). Consequently, the PDUs used for information requests to the middleware have the format portrayed in Figure 79:

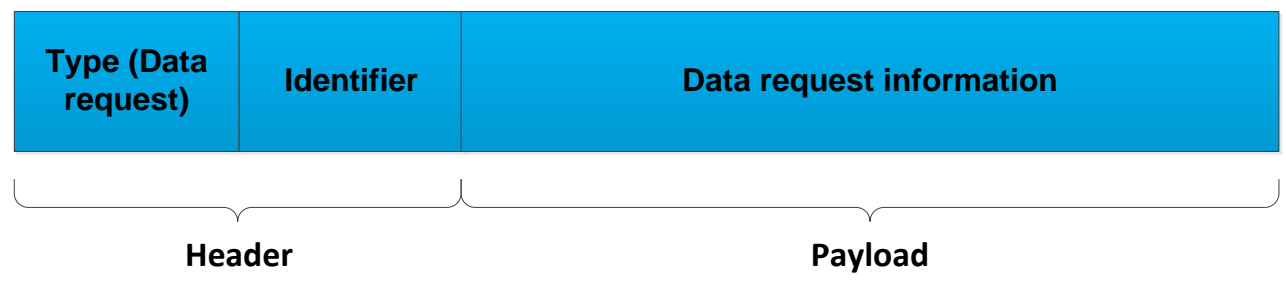

Figure 79. PDU format for a high level data request

The three elements present in the header and the payload can be further described as having the following features:

1. The type defines what kind of PDU is being used. While all the PDUs that are launched from the high level are service requests, the same field has been used for the low level ones, so it was decided to leave it the same way for any kind of PDU type.

2. The identifier that has been located in the header is required to identify the service that is going to be requested and differentiate it from the other, so that it can be directed to the hardware device (or middleware component) that can receive it.

3. The payload is also present in all the PDUs. In this case, it will contain information about the data requests that are transmitted from the application layer.

It is important noting that the PDUs that are described in this section are the ones used for data requests, so the formats of how data are transferred from the lower levels are defined according to the low level PDU formats. The REST interfaces will be used to transfer these PDUs to the high levels of the middleware solution. In order to do so, they will be invoked via URI, so each of the URIs used will correspond to a specific kind of data request (and therefore will contain different information from that request in the payload). 
It could happen that, due to the checking done by the Context Awareness component, there will be no way to obtain the information that has been requested (most likely due to the fact that a device is being requested about information but it is unable to provide it because it is not present in the system at that specific moment). In this case, a PDU will be sent from the Middleware Access point component that received it stating that there was an error. The appearance of this PDU, as shown in Figure 80 , would be very simple, as only the message is required.

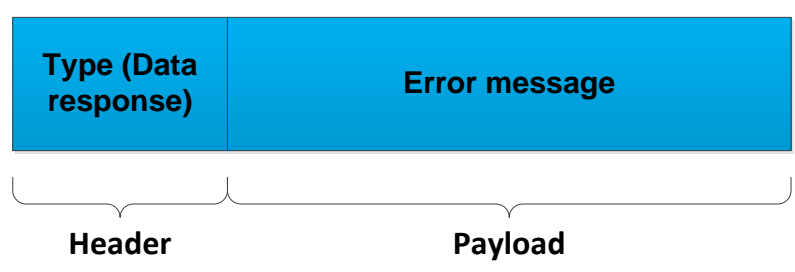

Figure 80. PDU for registration failure

\subsubsection{Low level PDU formats}

There are several kinds of PDUs that have to be used at the lower level, due all the operations that can be done (device or service registration, data response, etc.). The header is used in this case to contain the information required for the registration, that is to say: the type of PDU that is being transferred (a registration request), an identifier for the device that will be implied in the data transmission and the kind of heartbeat message that will be present (if any). On the other hand, the payload will contain information about the device and service (or services, in case the device to be registered contains more than one) that are going to be included in the system. All these data have been included in Figure 81.

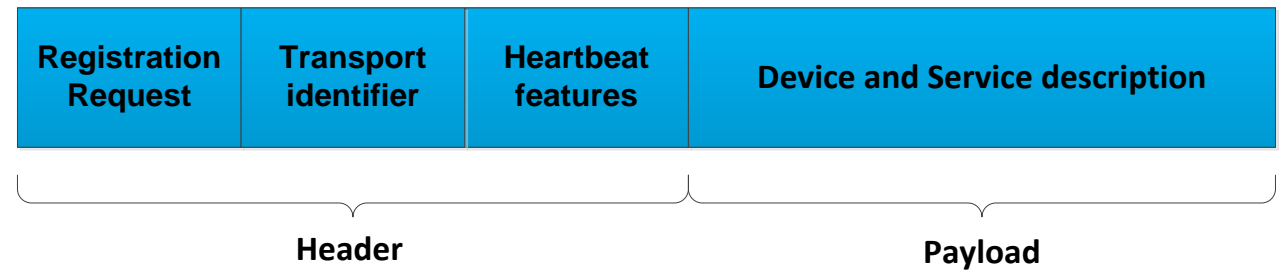

Figure 801. PDU for registration requests

Depending on the information detected by the middleware architecture (mainly, the previous presence of a device with identical features or not), a registration answer will be sent back to the device that triggered it in the first place. The header will contain just information about the kind of PDU that is transferred back to the device (since it could also be a data request generated in the middleware) that became registered and either a confirmation of the registration having taken place or a refusal if there was any kind of problem. As it happened with the error PDU, this one will only require an identifier to establish what kind of PDU it is, along with the information about the success or failure of the registration, as depicted in Figure 82. 


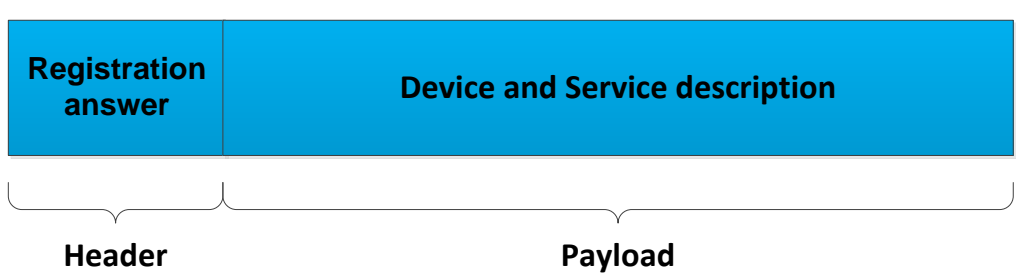

Figure 812. PDU for registration answer

Last but not least, a PDU has to be designed for data response in order to format the information that is going to be sent back to the application layer. While the complexity of the information that is sent back could more significant than in other cases, the overall structure of the PDU transferred will be rather simple, as the information identifying destination or kinds of data will already be included in the payload. The data that have been added to this PDU will be usually related to sensor information, so it has been chosen to place sensor reading information as the information that is collected by the device. Furthermore, an identifier for the service that was requested will also be included so as to let the middleware components be aware of where the data should be sent back. Figure 83 represents all the concepts described for this PDU.

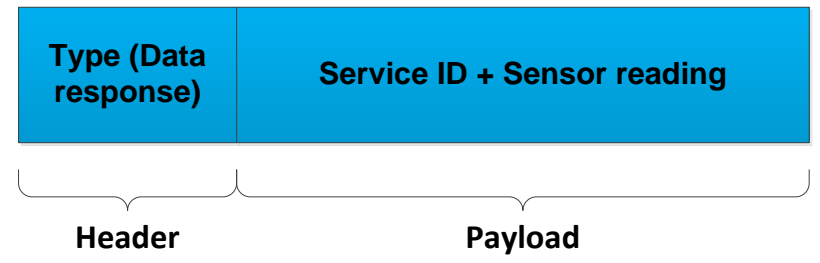

Figure 823. PDU for data response

\subsection{Business models and middleware exploitation}

Another topic of major importance for this thesis is the usability of middleware as a part of the necessary infrastructure to make the Smart Grid profitable, especially for the prosumers that are willing to take part of it. As it has been previously mentioned, the integration of prosumers is a critical objective for the Smart Grid, since they represent small scale energy producers (and consumers) that can assist the overall power grid not only with their own supply of electricity, but also with their changed patterns of behaviour when consuming energy. In this case, middleware will be used as the entity giving access to the economic part of electricity generation, trading and consumption, since it will make possible for the prosumers to trade their produced energy at will. This is what is referred to in the thesis as middleware exploitation: the creation of business models that will suppose an opportunity for prosumers and regular end users of the Smart Grid to make a profit, with the mandatory cooperation of a middleware solution that will integrate their facilities in the whole power grid infrastructure.

As represented in [34], there are several business models that can be used with end users and the power grid, depending on the level of intelligence of both the power grid where the models can be included and the infrastructure of the end user. Note that although middleware 
is rare to be explicitly mentioned, it is the underlying software solution that is making possible the business models that have been represented, along with their mathematical models. Also, models that rely on fewer software elements might not be using the Smart Grid infrastructures by default. In these cases, middleware will still be useful, but as a way to interchange information between the elements present in the TSO and DSO. As described in [34], the models that have been conceived as a way to provide middleware exploitation are as follows:

\subsubsection{Offline model without the Smart Grid}

This scenario has been designed for the use case where end users will not necessarily be prosumers, and the Smart Grid will not necessarily be present (middleware is still able to exist, though, as software components in the TSO and DSO parts of the power distribution). The end user will employ three different elements to obtain a profit in this scenario: an energy storage solution (typically, a home battery), a trading algorithm with access to energy markets and a Home Load Controller (HLC) used to monitor the energy consumed in the facilities where it is installed. Trading operations done with the algorithm use the differences between prices in energy during peak and valley (or off-peak) hours to its advantage: since energy is more expensive during peak hours and cheaper during the valley ones, it can be bought at a certain buying price for a number of kilowatts per hour (Bpk) and sold to others member of the power grid (such as the DSO) at a certain selling price for the kilowatt per hour (Spk), according to the actual needs and availability of the end user, who may choose to use some of that energy for their own personal consumption if they need it for themselves. These price variations will result in a profit for the user, which has been called Upk in (23). In this scenario, this profit has to be understood as the cost reduction that results after applying the installed infrastructure at the end user's facilities. As it will be explained in the next lines, this profit will be an actual one when the Smart grid becomes fully enabled.

$$
U p k=S p k-B p k
$$

Another way to describe this profit is taking into account the energy storage solution is located in the premises used by the end user. If the energy can be measured as charge cycles, then an amount of energy $E c$ will be stored in the battery each time it is charged within its warranty period. In this way, end user profit can be modeled as the profit for each of the battery charges done to the battery, thus obtaining a user profit called Upc, represented by the profit for each kilowatt per hour (Upk) and the kilowatts per hour that an energy storage solution can hold (EC). These ideas have been expressed in (24).

$$
U p c=U p k \cdot E c
$$

Bearing this in mind, it is possible to model the user profit as something that will be repeated during a certain amount of time. This idea can referred to as user profit during a certain amount of time (Upt) ranging from 0 to $n$ for every $i$, as expressed in (25).

$$
U p t=\sum_{i=0}^{n} U p c i
$$


The consequence of all this will be that the end users adopting this model with their available infrastructure (energy storage, trading algorithm, Home Load Controller) will be able to pay less for the electricity they produce. As depicted in (26), the cost of the energy used during a period of time (Int) will result from the regular expenditures (Ext) of the end user during the same period time described in (26) and the subtraction of the user profit during that same period (Upt). In the most drastic scenario, it might even be possible that the profit obtained is larger than the costs of electricity used by them, so they will have a net profit from the available pieces of equipment and software.

$$
\operatorname{Int}=E x t-U p t
$$

In the end, the end user will be able to fully pay the investment done in the energy storage solution and the other elements that allow them to engage into trading activities. When this happens, the Break Event Point or BEP will be reached and the costs of the investment (Inv) is equal to the profit (or in this scenario, cost reduction) obtained by the end user during a certain amount of time (Upt), as represented in (27). The amount of time that the investment is fully returned, though, will depend on the extent of modernization of the power grid and how the end user is involved in trading activities.

$$
B E P \rightarrow U p t=\operatorname{Inv}
$$

Finally, the number of charge cycles required to obtain the user profit that was previously mentioned will be defined depending on the Investment (Inv) done in order to purchase all the required infrastructure and the user profit obtained from the battery charges $(U p c)$. This number of charges has been defined as $N c$ in (28), which represents the idea of calculating the number of battery charges required by an end user to obtain a profit considering the amount of capital that has been invested.

$$
N c=\frac{I n v}{U p c}
$$

\subsubsection{Offline model within the Smart Grid}

As far as this model is concerned, not only it will make use of the equations of the mathematical model previously introduced, but also some new are introduced regarding the location of the Trading Algorithm used, which will be located in the facilities of an entity (aggregator, DSO, TSO, etc.) that is not the end user. In addition to that, since the power grid has been enhanced (or at least, enhanced enough) to be regarded as smart, the user profit obtained from a Smart Grid-like environment (Upsg) can be modelled as the profit obtained from the regular amount as the profit per kilowatt hour (Upk) and the amount of time they are connected to the facilities of the Smart Grid $(t)$. This profit will result as represented in (29):

$$
U p s g=U p k \cdot t
$$


The usage of the infrastructure owned by the Smart Grid will guarantee than an additional profit is obtained, due to the fact that it will be used to enable further services that were not previously available. Equation (30) represents this fact by adding the profits obtained from the usage of the Smart Grid infrastructures to the ones obtained from the battery charges (Upc). This latter gained has been referred to as Upcsg.

$$
U p c s g=U p c+U p s g
$$

\subsubsection{Online model without the Smart Grid}

The main addition that this model brings when compared to the previous ones is that it includes online services for the end user. The idea that is put forward by this model is that there will be a company selling the required hardware and software components (energy reservoir, Trading Algorithm and the Home Load Controller). Consequently, benefits will be shared between the end user and the entity that is providing the necessary infrastructure to keep the former online. In addition to that, a different quantity of profits will be obtained from the end user, which will be defined as the user profit during a specific amount of time (Upt). The components will be sold to the end user at a lower cost than the one they would have to afford if they were bought by themselves, but this end user will be charged with an additional fee for using them. In this way, the selling price established for a specific period of time (Spt) will be the one for the kilowatts per hour that was established in (23) multiplied by the number of time units used to generate the electricity to be sold $(t)$, as depicted in (31):

$$
S p t=S p k \cdot t
$$

The same kind of procedure will be carried out for the buying price, which will result in defining a new term called Buying price for a specific amount of time (Bpt) during an amount of time $t$, as shown in (32):

$$
B p t=B p k \cdot t
$$

The final user profit per amount of time in this use case, or Upt, will be obtained by taking into account the buying price of electricity during a specific amount of time Bpt, its selling price or Spt, and the cost assigned to the platform that is used for the online activities, which as has been modelled as a percentage obtained from the profit made by enabling trading activities (Pch). Overall, the benefits obtained for the end user, and represented by (33) are most suitable for an end user that prefer not doing a first significant investment; since a percentage from the benefits is charge by time unit, all the infrastructure ends up being effectively paid during that amount of time.

$$
U p t=(1-P c h) \cdot(S p t-B p t)
$$


In the same way, the infrastructure will end up being paid to the third party providing support to the end user during an amount of time $t$ when, as described in (32), the investment that has been made by the third party will be returned if the percentage charged to the end user during $t$ makes up for the total cost of the infrastructure sold to the end user (represented as $\operatorname{In} v_{\%}$ ). These concepts have been included as in (34).

$$
\operatorname{Inv} v_{\%}=P c h \cdot t
$$

At the same time, the business made by this third party company can also be modelled as the business profit of a period of time (Bspt) resulting from the difference between the selling and buying prices of electricity (Spt and Bpt) and the percentage that is charged to the end user that is using their platform, as represented in (35).

$$
B s p t=P c h \cdot(S p t-B p t)
$$

\subsubsection{Online model within the Smart Grid}

While all the other equations are still valid for this use case, a new one will be included that reflects the new environment where the Smart Grid is fully functional. Therefore, if the Smart Grid is going to be accessed during a fixed amount of time, similar trading operations will be carried out. In the end, and as reflected in (36), all the benefits that are obtained both by any third party implied in the trading activities and the end user will be obtained from trading with the electricity that has been generated and injected in the power grid. This concept has been included in (36), where the profits made by both entities (Upt for the end user and Bspt for the Spt and Bpt) are the same as the ones that are obtained from the overall trading activities that are carried out in the electricity markets.

$$
U p t+B s p t=S p t-B p t
$$

\subsection{Section summary}

A collection of concepts essential to understand this thesis has been presented in this chapter. To begin with, the open issues found during the analysis of the state of the art have been born in mind so that the proposal that is done here is capable of improving the existing developments. Since the most prominent issues found were about the lack of clear definition of middleware, which services to include or how to create a standard, the proposal presented attempts to tackle all of them. This has been done so by means of a requirement analysis that self-contains all the challenges that were found in the previous section. From those open issues, a list of components has been elaborated so that they can be included in a proposal for 
a Common Middleware Architecture. From an UML perspective, the typical problems that will have to be tackled were modelled into use cases. These ones, in turn, have been further analysed via sequence diagrams, resulting in a collection of classes that have been kept as part of the components that were identified as necessary during the requirement analysis stage. Furthermore, a deeper analysis of the proposal that shows its behaviour and different transitioning states has also been provided with the usage of UML state and activity diagrams. They have shown that the aforementioned challenges can be solved with the principles described in this section. In section for it will be explained where those components were located for the implementation works, as defined in Figure 84.

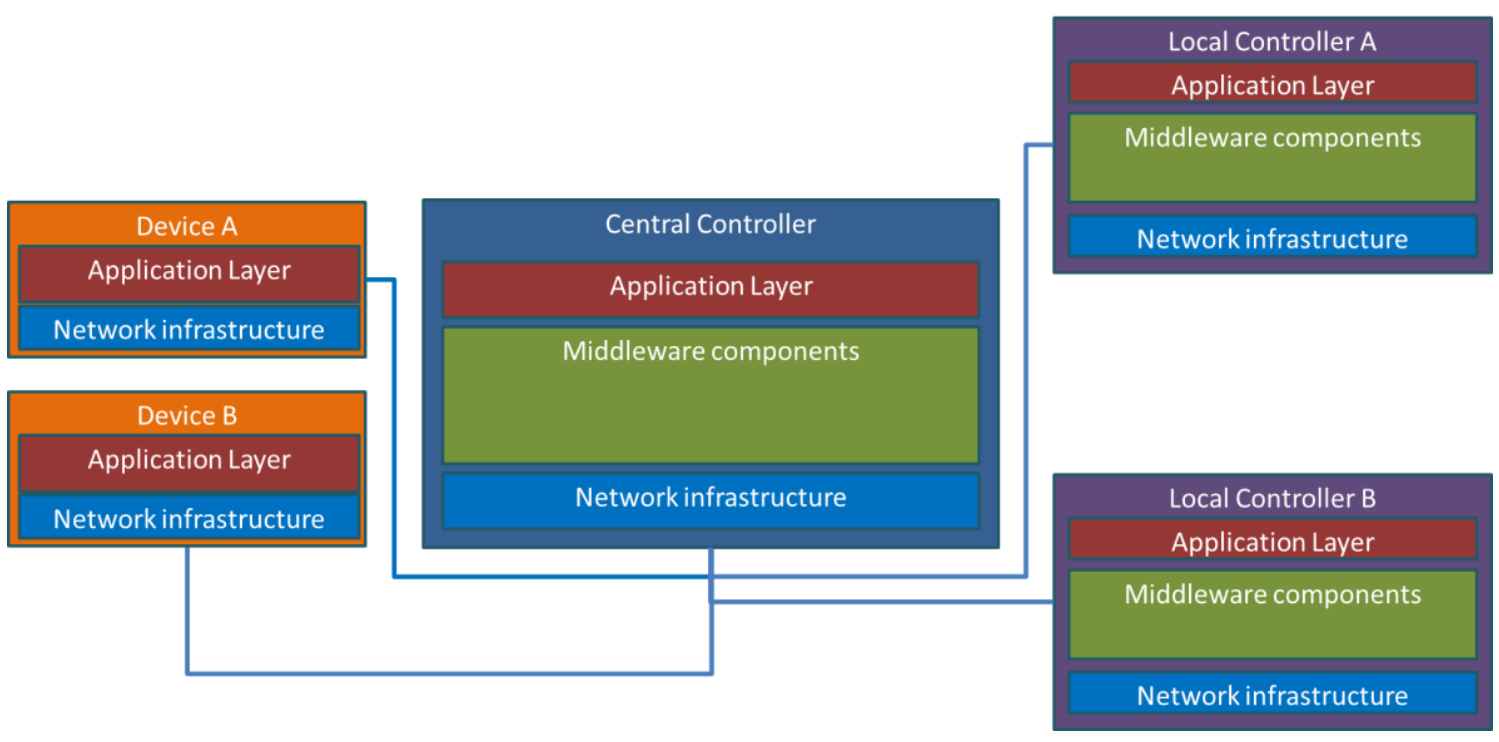

Figure 834. High-level view of component deployments

Data representation formats are also to be considered in the description of the proposal, as they will change depending on the environment where they are used. Furthermore, how middleware is located in the business models where new agents (aggregators and more specifically, prosumers) are included in the system, and the business models that become possible due to the inclusion of middleware in the Smart Grid, have also been represented. They have been subdivided in different scenarios where they can be applied, taking into account the level of development of the Smart Grid and the hardware devices available for the end users. It has been explicitly described how the integration of the different RESs within a distributed, Cyber-Physical System that is offered by semantic middleware offers economic benefits to prosumers, who can choose to buy, sell or store power either among them or to other parties participating in energy markets. While the legislative framework changes that would be required to include prosumers in them is beyond the scope of this thesis, it is stated that it is possible to do so from a purely technological point of view.

Besides, Common Middleware Architecture offers a framework where the design, structure and location of the services are already provided. In addition to that, the standardization perspective is tackled by describing the middleware architecture according to the information details provided by the proposal ISO/IEC/IEEE 42010, where ideas as viewpoints or 
stakeholders are also considered. Lastly, a protocol for data transmission, referred to as Low Level CMA Protocol, has also been provided as a way to offer a template on how information should be transferred to the upper layers of the middleware. This low-level protocol has its counterpart in a high level service protocol, called High Level CMA Protocol, which also offers a way to request information from the application layer. Some of the most important concepts of this latter standardization process have been contained in Table 14.

Table 14. Main concepts for ISO/IEC/IEEE 42010 standardization

\begin{tabular}{|l|l|l|l|l|}
\hline Viewpoint & Associated view & $\begin{array}{l}\text { Associated } \\
\text { architecture } \\
\text { model }\end{array}$ & Concerns & $\begin{array}{l}\text { Actions to deal } \\
\text { with the } \\
\text { concerns }\end{array}$ \\
\hline$<$ Electricity $>$ & $\begin{array}{l}<\text { Electricity } \\
\text { Deployment }>\end{array}$ & $\begin{array}{l}<\text { Electricity } \\
\text { Deployment } \\
\text { Model }>\end{array}$ & $\begin{array}{l}\text { Reliability of the } \\
\text { power grid; } \\
\text { voltage changes }\end{array}$ & $\begin{array}{l}\text { Forecasting and } \\
\text { redundancy; } \\
\text { power converters }\end{array}$ \\
\hline$<\mid C T>$ & $\begin{array}{l}<\mathrm{ICT} \\
\text { Architecture }>\end{array}$ & $\begin{array}{l}<\mathrm{ICT} \\
\text { Architecture } \\
\text { Model }>\end{array}$ & $\begin{array}{l}\text { Data delivery; } \\
\text { service } \\
\text { availability; } \\
\text { present services }\end{array}$ & $\begin{array}{l}\text { Middleware } \\
\text { interoperability } \\
\text { capabilities; } \\
\text { context } \\
\text { awareness; } \\
\text { middleware } \\
\text { encased services }\end{array}$ \\
\hline
\end{tabular}

Another aspect that must be taken into account is the scope that CMA intends to cover. The idea that is transmitted in this thesis is that rather than having yet another standard that attempts to cover similar areas and issues where other are still struggling to do so, the concepts, developments and ideas of CMA can be integrated in the most fitting already existing standardization works. As a way to prove this point, a description of CMA according the rules of architectures descriptions put forward in the ISO/IEC/IEEE 42010 standard has been added to the thesis as an Appendix. While other options were considered, this was the easiest to take, due to the fact that standardization groups integrated in the IEEE are easier to take part in, considering the background acquired and the economic costs that the author of the thesis could afford.

Therefore, standardization groups related to software architectures and the Smart Grid were searched, in order to obtain knowledge about the criteria that were used in these groups for standardization, as well as providing ideas and feedback during the standardization activities that are carried out in these areas of knowledge. Currently, the following standardization groups have been joined:

1. P42020 - Standard for Systems and Software Engineering - Architecture Processes [183].

2. P20000-1: Information Technology - Service Management - Part 1: Service Management System Requirements [184]. 
4. Validation of the proposal and results 
This chapter is focused on the validation of the principles described previously in the thesis. In order to do so, there are two main actions that have been taken in this section of the document: the implementation works that were carried out during the e-GOTHAM and I3RES projects have been described here, highlighting the most critical parts of them. Additionally, the deployment that was done in two different demonstrators related to the projects has also been described, so that the functionalities and innovations introduced by the middleware solutions inspired by this Common Middleware Architecture proposal can be seen when dealing with actual needs for services and communications at the data level. There are two scenarios that have been chosen for validation purposes: the first one was the residential scenario of the e-GOTHAM project, where middleware was deployed, among other components, in the Finnish town of Ylivieska. In the second one, middleware was deployed for the I3RES project in the Norwegian town of Steinkjer.

\subsection{Introduction}

The theoretical principles that have been described in the previous sections become part of an actual implementation in this one. For example, each of the levels that has been previously described (application, middleware, network, etc.) have been turned into actual software elements that are part of components and solutions that have been tested and installed in demonstrators. As it can be seen in Figure 85, each of the levels of the layered architecture has become part of a specific implementation work.

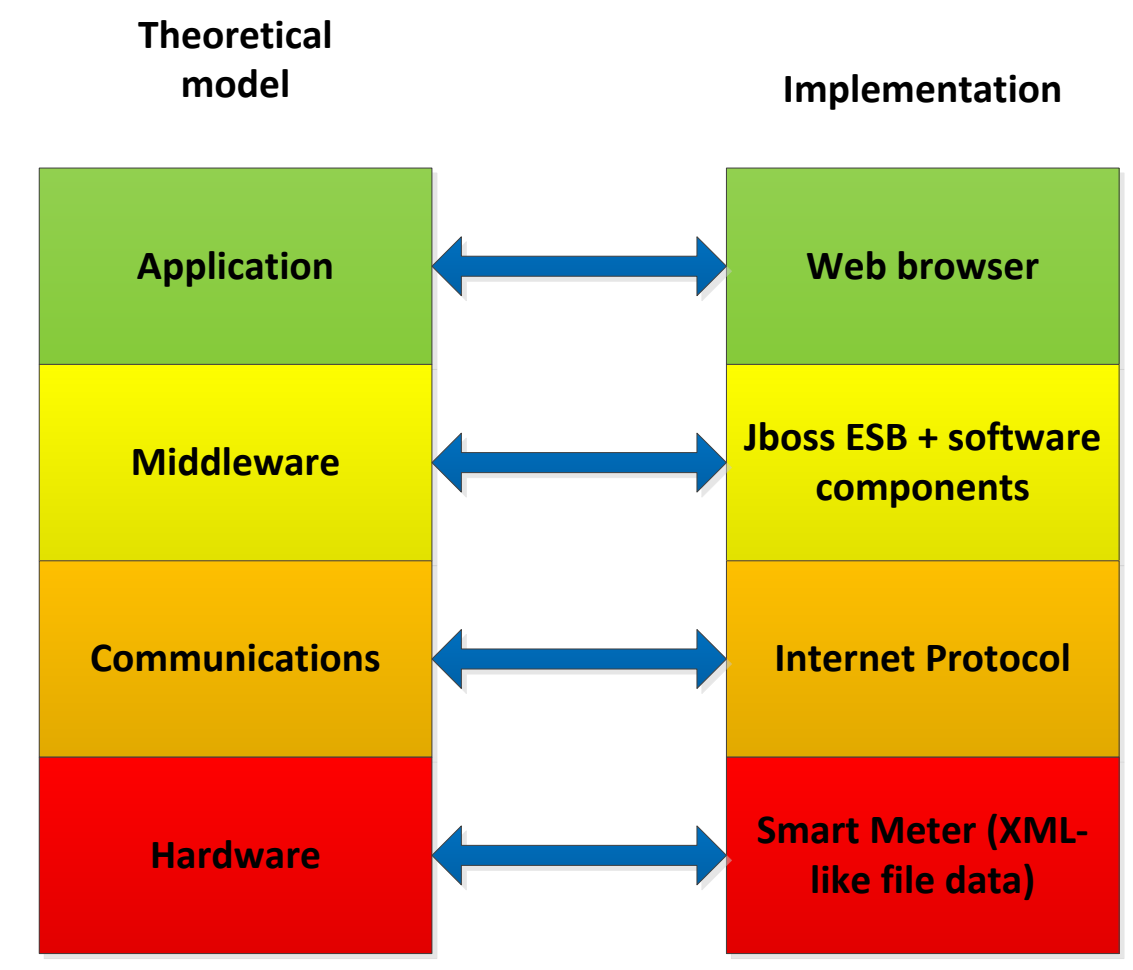

Figure 845. Theoretical and actual implementations of the middleware and its adjacent layers

This chapter has been divided in several sections that show the importance of the implementation works that were carried out in both the developments done during the e- 
GOTHAM and I3RES research projects, as well as when comparing the ideas that have been put into practice in the other solutions aiming to create a middleware for the Smart Grid. Firstly, the framework that has been used to contain all the services and components that have been included is described. "Framework" in this context means the collection of tools, devices and services that have been used for the implementation of the middleware iterations used in the aforementioned research projects. This idea is of major importance, due to the fact that it summarizes two of the four pillars Common Middleware Architecture is coming from. In addition to that, the implementation works carried out during the period of time comprehended in the research projects will be displayed in this chapter. When those implementation works were carried out they were tested in two different kinds of environments at three different locations (one set of tests took place in a laboratory, whereas the other tests were carried out in the demonstrators used for components deployment). The section summary that has been introduced in this chapter will highlight the main achievements depicted.

Another characteristic of this section is featuring the methodology used for the development of the middleware solution. Basically, a merge between incremental prototyping and the waterfall model for software development has been used in the research projects where middleware for the Smart Grid was featured. Under this methodology, analysis, design, implementation and testing activities are made carried out every time a development iteration is completed. Therefore, prototypes of increasing complexity can be deployed under the assumption that the previous version has worked as expected. This methodology -which combines the traditional waterfall model with feedback and incremental prototyping- ensures that unforeseen events are kept at a minimal. While there might be a small delay regarding the achievement of lesser, partial goals rather than pointing at the final ones, reliability of the obtained results, which is sometimes jeopardized in complex, long-term projects, makes up for it. Overall, this procedure has been depicted in Figure 86. Aside from some minor differences in the activities that have to be made in each stage, such as the setting of the framework at the very first stage, or the maintenance of the final software product created, the steps to be taken are essentially the same in every iteration of the incremental prototype. 


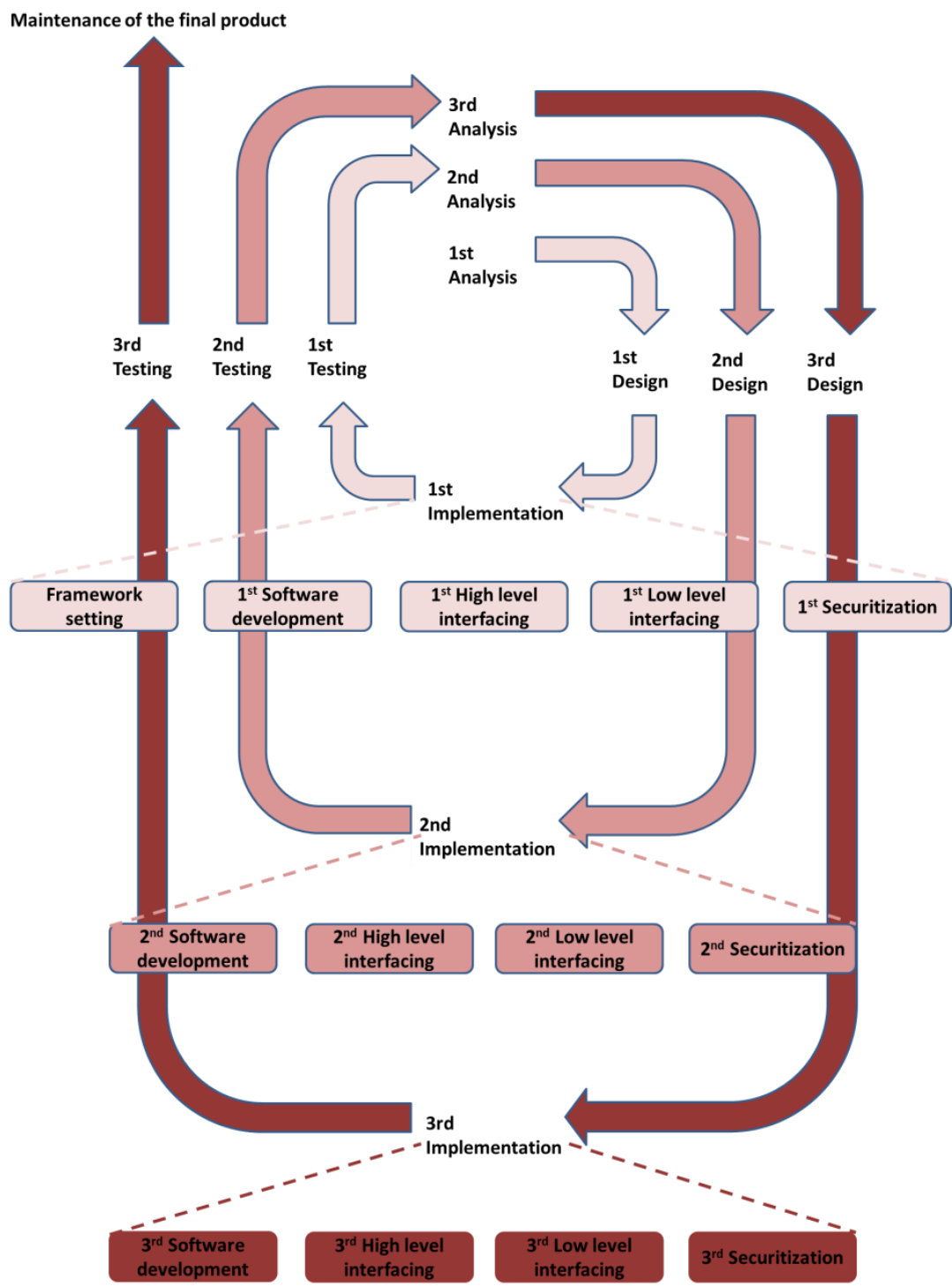

Figure 856. Waterfall model (enhanced with feedback and incremental prototyping) for middleware development

As far as this section is concerned, the main procedures that have been included deal with the steps that have been used to develop the middleware solutions that have been used as a way to infer the Common Middleware Architecture that was used before. Purely implementation details have been included in Annex A, whereas part of the API that resulted is in Annex B.

\subsection{Framework for the Common Middleware Architecture}

When describing the elements that are used for describing the main features that have been added to the framework of the implementation works, there are five of them that must be highlighted:

1. The Enterprise Service Bus implementation that has been chosen. As it was described in Chapter 2, an Enterprise Service Bus is used as the most suitable way to install services as 
part of a middleware architecture that, according to the needs of the Smart Grid, will have them contained.

2. The programming language used for the services. It has been considered that, due to the experience gathered during the developments done and its sheer popularity, Java should be used as the programming language for the services.

3. High level interfaces. Representational State Transfer (REST) facilities have been chosen as the way to implement how communications are done when interfacing the application layer with the highest layer of the CMA.

4. Low level interfaces. They are required to collect information from the devices. There have been two different strategies that have been followed: on the one hand, the XML format that was referred to before has been defined for data transfers. On the other hand, middleware has used interfaces with AMQP in order to collect the information from the lower layers. AMQP was chosen by a partner in the research project that was responsible for the communication layer falling just below middleware. The purpose for using AMQP was decoupling communications between middleware and lower layers, since it offered Publish/Subscribe facilities (and the middleware could subscribe to the hardware devices that would publish information, so that the data would be already available in the middleware once it was requested from the application layer). Therefore, interfaces were specifically tailored for the interconnectivity between the AMQP-based and all the other components.

5. Interconnectivity among software components. The software elements that have been defined as part of the middleware have to transfer information among them. Therefore, they must have interfaces that are utilized for inner middleware information transmissions.

Considering all these aspects, the next sections will provide a description of the framework tools that have been included in this thesis.

\subsubsection{Enterprise Service Bus}

Among the possible ESBs that could be used, the final decision was to implement middleware solutions with Fuse ESB, which has been recently transformed into JBoss Fuse [105]. In this case, the software components that are developed have been included in the ESB as bundles, that is to say, executable software packages that are run when the main ESB is launched. There were several reasons to choose this ESB solution over other similar:

1. It was offered for free to be downloaded from the Internet [169]. Taking into account that a proprietary or costly solution would have imposed strain on the budget, and the fact that there were satisfactory solutions that were offered for free, JBoss Fuse could be used without restrictions.

2. The community that is behind it is significant enough in case there is any issue that requires being solved fast. JBoss Fuse is a red hat development, so there is a great availability amount of documentation that can be checked. Also, researchers and developers that have already been implied in the development of solutions with JBoss Fuse can provide some feedback. 
3. From a more technical point of view, the software bundles that are deployed in the middleware can be done so without having to stop the running ESB and having to start it again once the bundle has been. This is a great advantage whenever middleware has to be updated because already functional services can be kept working while at the same time new services are added, according to the needs that interoperability and scalability may impose.

Furthermore, all the advantages previously explained in Chapter 2 are still valid for this ESB. As it has been explained in [154], and summarized in Figure 87, an ESB will ensure that a) the developed services can be stored in a distributed manner, b) applications written in different languages and accessed in different ways can be interacted and c) the bus will improve the efficiency of data interchanges.

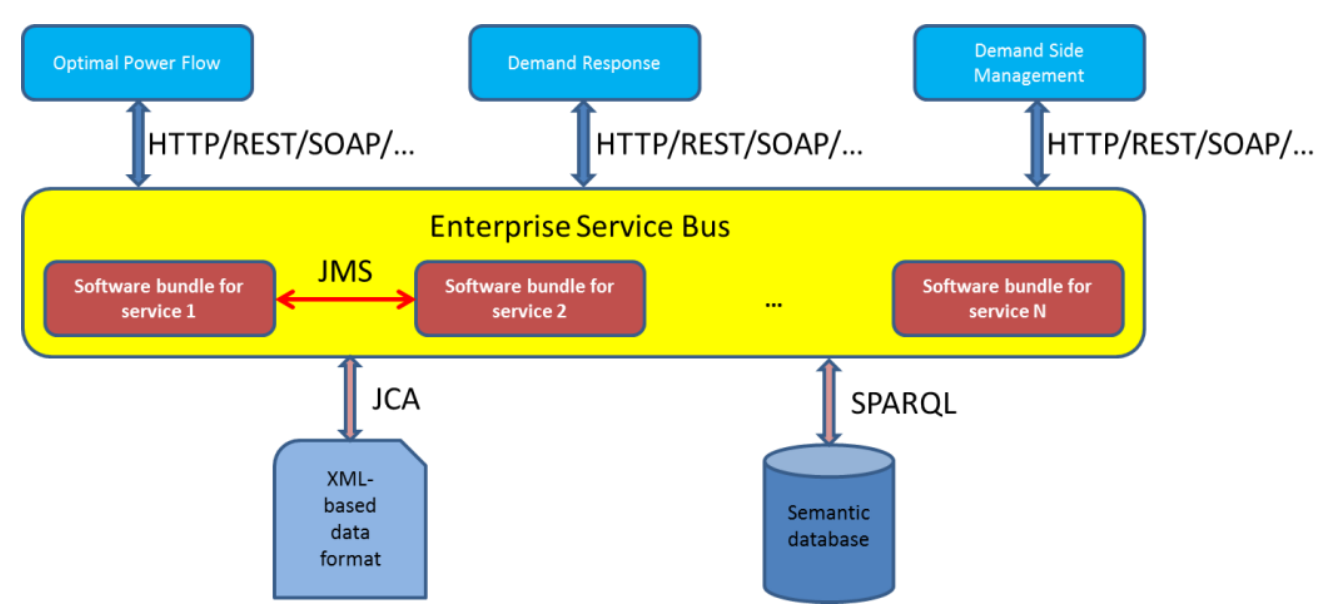

Figure 867. Connectivity among elements of an ESB, as depicted in [154].

Among the possible ways of communicating bundles with each other, the one that has been chosen is Open Services Gateway Initiative (OSGi), as it was included in other similar developments described in the previous chapter. Other option that considered for inner communications was using Java Message Service (JMS, [170]) for message transmission between the software components but it was deemed that OSGi offered a way simpler framework to operate among bundles. Nevertheless, this latter one was used during the development and testing activities that are portrayed in [141] and, since JMS could be used in a useful manner among distributed hardware components. How the bundles interact with each other by means of these OSGi interfaces is described in section 4.2.5.

\subsubsection{Programming language and Integrated Development Environment}

Among the possible options for programming, Java was regarded as the most suitable one due to its sheer popularity [171]. The used version of Java was Java 2 Enterprise Edition (J2EE), which will be referred to as Java hereinafter. In addition to that, it was also one of the programming languages that could be used to implement software bundles for JBoss Fuse. The implementation works that were carried out had to take into account, though, when bundles 
had to be developed, since they are required to be compliant with the Apache Maven build system. In order to do that there were several procedures that must be taken into account when codifying these in an Integrated Development Environment (IDE). Apache Maven [172] is used as a way to have runnable software bundles located in one repository that will execute them when it is commanded to do so. In a way they are executable compressed files that will be triggered as soon as the directory where they have been located is involved in a program execution.

\subsubsection{High level interfaces}

When high level interfaces had to be implemented, there were three ideas that had to be born in mind: a) how to enable a way for applications to interact with the high level services of the middleware architecture, $b$ ) what kind of information should be sent from the applications and c) how it should be formatted in order to make it understandable by the middleware solution supposed to interact with them. These two issues were solved by using REST as they way to establish interfaces with the application layer (which was represented firstly by a web browser and afterwards as an Android GUI) and a specific XML message format for application data exchange. REST interfaces were implemented by making three additions to the Java Maven projects that had been created and codified previously. On the one hand, the Java libraries that are supporting REST functionalities have to be imported. That was done by typing the following lines of code in the classes that, as was shown in the previous section, include the methods required to invoke the REST operations. Those imported libraries are:

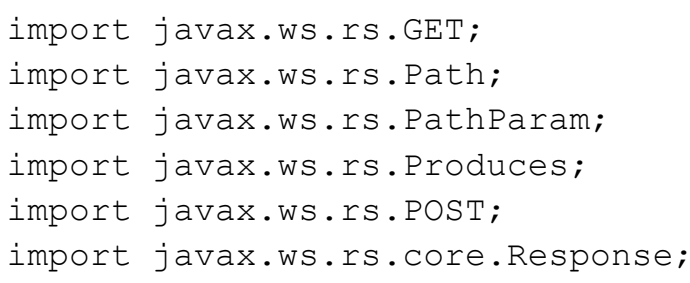

Another additional edition that has to be introduced is related to the nature of a Maven project. Whenever it is created, there are several adjacent files that are made available. These ancillary files reflect, among other features, the dependencies that the project has with the maven software facilities. For example, the blueprint.xml file contains the most prominent features that are part of the project. Another one of the files is the one referred to Project Object Model (POM), which makes use of XML to describe a representation of a Maven project. This file, though, must specify how the specifications of the Maven project are made compatible with the usage of JBoss Fuse and the Web service-based that are being used. Therefore, the following lines of code have to be included:

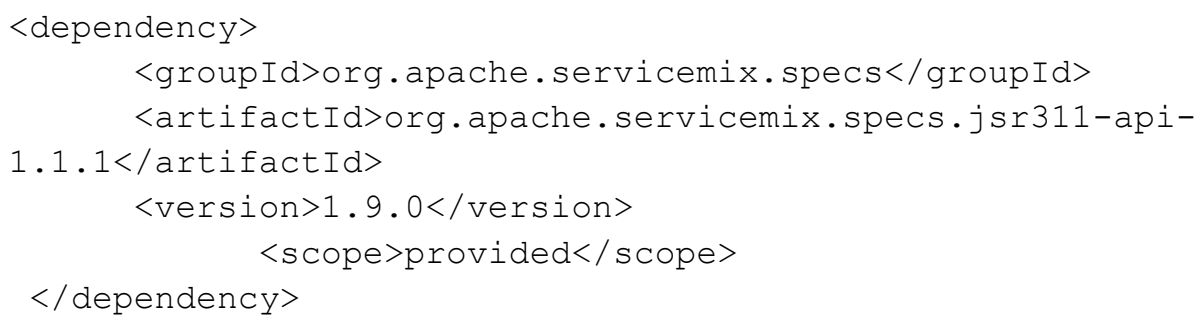


If the overall POM.xml file is taken into account, they will be located within the dependencies section of the file, as represented in Figure 88.

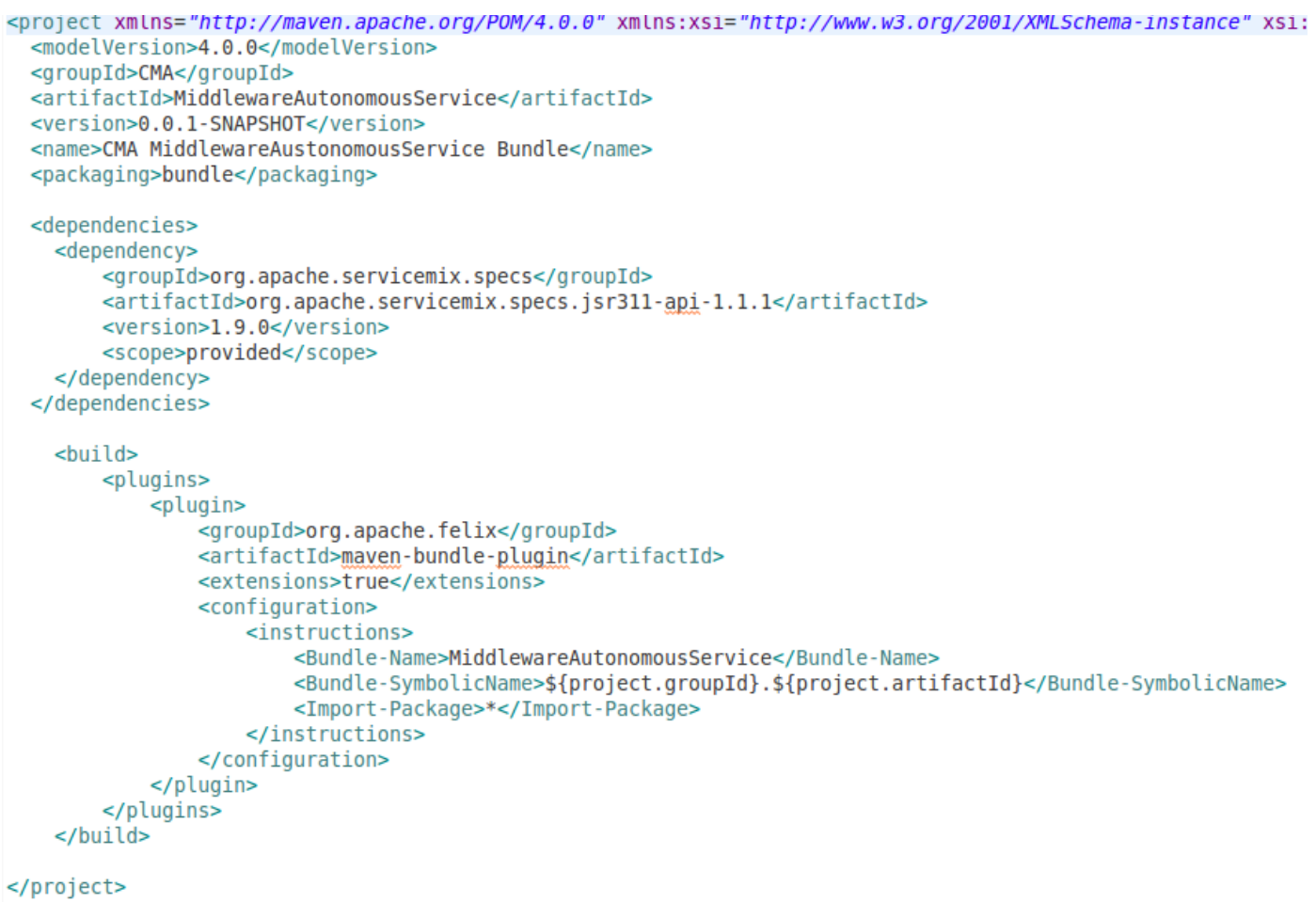

Figure 878. Web service dependencies location

The last edition that has to be carried out is all the sections of the URI that will be used in order to invoke the services. This is done so by introducing parameters immediately before the header of the methods that are used as the operations to be invoked in the code; they will be codified by adding "@” before the kind of operation ("GET" will be the most frequent due to the fact that information is retrieved from the application, rather than provided to it when a request is done via URI), the part of the URI that is invoked (or the "path", as it is added to the code) and the "result" that is produced (xml document, plain text, etc.). An example of what the code looks like has been included in the next lines.

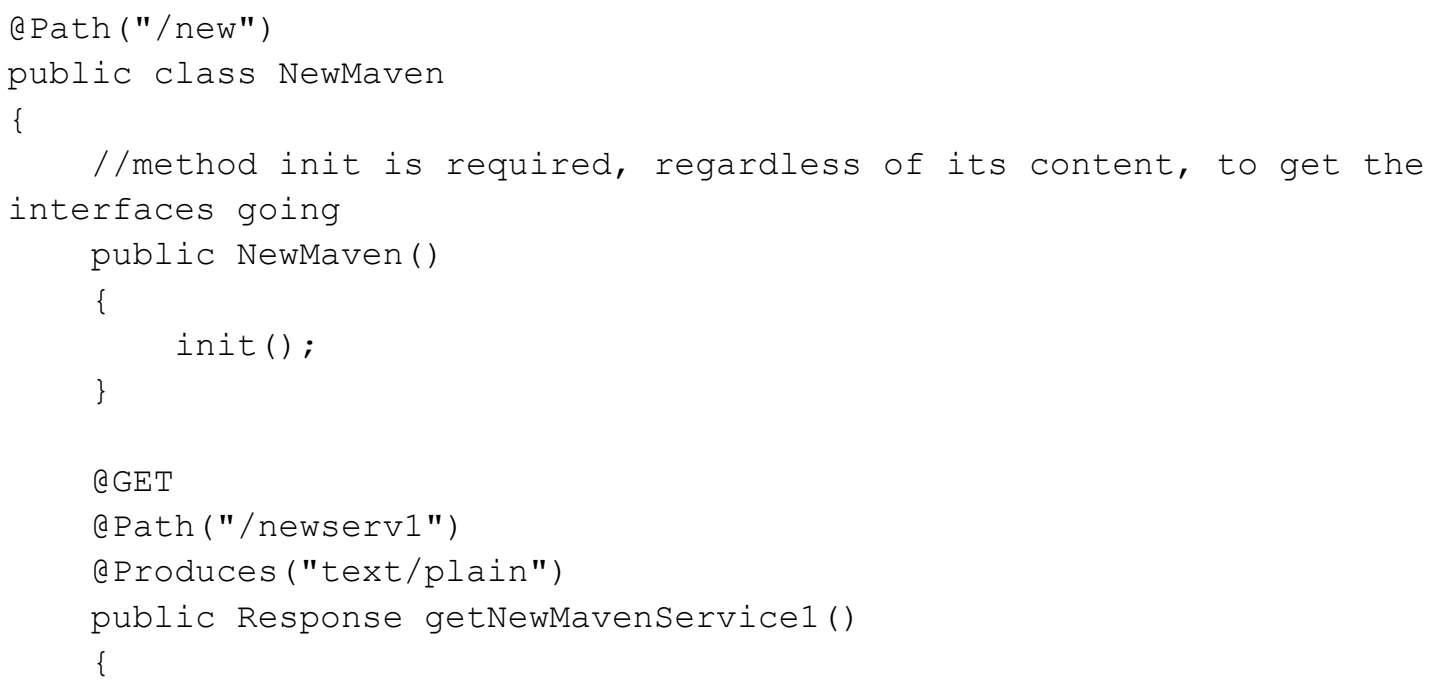




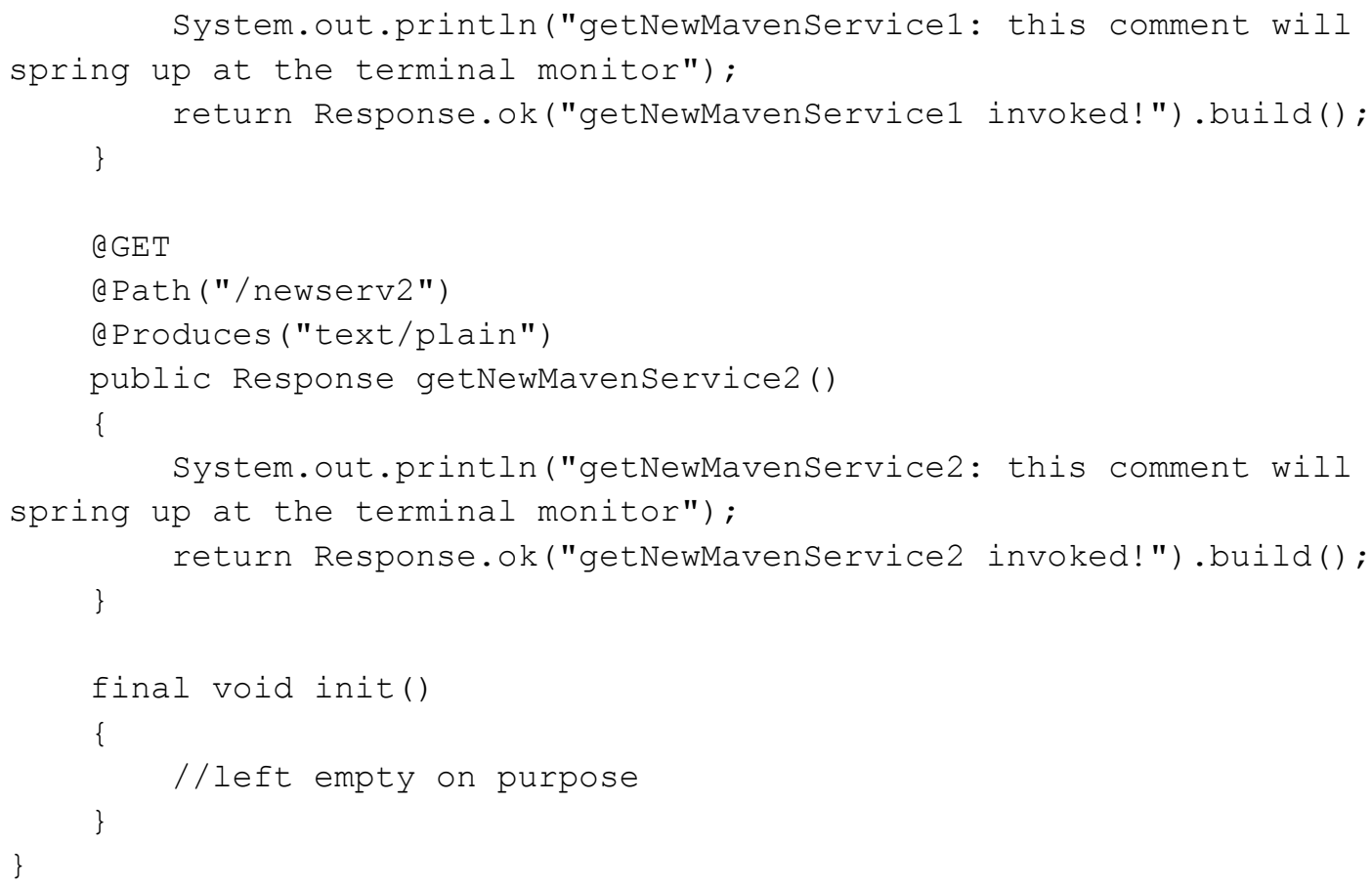

With all these aspects taken into account, it was decided to incorporate URIs to all the hardware devices that could be added to the middleware solution that was planned to be installed, all according to their own capabilities. As far as the e-GOTHAM project was concerned, there were ten possible devices to be accessed (as well as other URIs for data storage and other services). Depending on the services that could be provided, the URIs associated with each of them would be different. The structure that has been followed for the Uniform Resource Identifiers could be described as follows:

\section{http://[IP address]:8181/cxf/support/support/\{service name\}/\{serviceID\}/\{input\}}

The first part of the URL remains fixed for all the URIs that can be invoked. The only parameter that changes is the IP address of the machine that is running the most significant parts of the middleware (that is, the Central Controller; for the sake of readability it is referred to as [IP address] in the URIs that have been presented). There are two support tags in the URIs that have been used because the initial tests for implementation activities were made in an eGOTHAM component (which was equivalent to what a Middleware Access Point is in Common Middleware Architecture) used for support where all of the tasks were supposed to be support related. The original idea was to move those interfaces to other components that would have received a different name, but due to the fact that the functionality was already been offered in that component, they were left as URIs accessible from the support component. The finished middleware architecture conceived from e-GOTHAM has been summarized in [141]. After those tags, the variable part of the URI is added: the service name represents the service that is requested, the service identifier is the unique number that the device it belongs to was given during the registration process and the input is a parameter that may be required for the service data delivery; this last parameter is optional, as it may not be needed by the hardware device that will receive the request (for example, if real-time current is requested to a 
particular device with an identifier there is no need to specify other parameters, but if a device is requested to take an action, such as turning on or off a load, it might need so).

It has to be noted that a URI is not the same as a Uniform Resource Location (URL), due to the fact that the former points to the name of the very resource that is accessed, whereas a URL points at the location of the resource, instead of to the resource itself. With this in mind, invoking services from one device or the middleware itself became a matter of using the suitable URI containing the service invocation and the required parameters. As far as the eGOTHAM project was concerned, there were several devices to obtain services from:

1. Local Controller: this is a device that was tailored for the e-GOTHAM project which contains several services aimed to control several features of a dwell, such as power consumption or temperature.

2. Energy meter at the Ylivieska demonstrator: device used to measure energy consumed. The demonstrator that was deployed in Ylivieska relied on these devices to collect information about consumed energy.

3. Pressure meter at the Ylivieska demonstrator: device used to collect pressure measurements from sensors monitoring a District Heating Network present in the Ylivieska demonstrator.

4. Remote Terminal Unit: these are pieces of equipment to manage transmission and distribution automation. They had several electricity-related services that could be invoked by using the URIs that have been previously described.

5. Combined temperature and humidity measurement device: used to measure that kind of parameters in the demonstrator of Ylivieska

6. Phasor Measurement Unit: PMUs are used for measuring and synchronizing electric waves transmitted through the power grid. Starting and stopping a specific service number that could be provided by them was listed as the only potential facility that could be managed from the middleware architecture.

7. Pressure device at Herrfors facilities: it is used for the same purpose that the pressure meter was used for, but it is installed in Herrfors, which is a local power company that was involved in the e-GOTHAM project as one of the utilities that would use the results of the project to their advantage [173].

8. Data device at Herrfors facilities: this URI was added in case a device used to obtain generic information was added to the project. Even though no new devices were used, it was kept as part of the development works done.

9. Enfo ESS Box: an appliance used to gather information about energy consumed in a certain amount of time. Enfo is a Norwegian company heavily involved in facilities for the Smart Grid, such as business models, Demand Side Management and energy trading [174].

10. E-GOTHAM Box: device tailored for the e-GOTHAM project used for several actions: turning off loads, obtaining information about power consumption, etc.

The features that were invoked by the URIs used by the services have been described according to the URIs that the high level services of the middleware got programmed with: 
1. Local Controller prototype: this device is capable of turning on and off electricity loads, as well as providing information about the consumed power by the appliance and the temperature for a user that is expected to be at home.

http://[IP address]:8181/cxf/support/support/contro|Appliance1/\{servicelD\}/\{input\}

http://[IP address]:8181/cxf/support/support/controlAppliance2/\{servicelD\}/\{input\}

http://[IP address]:8181/cxf/support/support/getPowerAppliance1/\{servicelD\}

http://[IP address]:8181/cxf/support/support/getPowerAppliance2/\{serviceID\}

http://[IP address]:8181/cxf/support/support/getTemperatureatHome/\{serviceID\}

2. Ylivieska energy measurement device: this device is capable of obtaining the latest results of the consumed energy that is measured.

http://[IP address]:8181/cxf/support/support/getEnergyLastResults/\{serviceID\}

3. Ylivieska pressure measurement device: this device is capable of obtaining the latest results of measured pressure.

http://[IP address]:8181/cxf/support/support/getPressureLastResults/\{servicelD\}

4. iRTU: this Real-Time Unit is used in the Norwegian pilot to provide information about binary inputs and errors, as well as information about temperature and real-time voltage.

http://[IP address]:8181/cxf/support/support/digital in/\{digital input\}/\{serviceID\}

http://[IP address]:8181/cxf/support/support/error/\{digital input\}/\{serviceID\}

http://[IP address]:8181/cxf/support/support/ground voltage/\{servicelD\}

http://[IP address]:8181/cxf/support/support/line voltage/\{digital input\}/\{servicelD\}

http://[IP address]:8181/cxf/support/support/temperature/\{servicelD\}

http://[IP address]:8181/cxf/support/support/current/\{digital input\}/\{serviceID\}

5. Ylivieska temperature and humidity measurement device: this device is capable obtaining the latest results of temperature and humidity that are measured.

http://[IP address]:8181/cxf/support/support/getTempHumLastResults/\{servicelD\}

6. Phasor Measurement Unit: this device has an interface in case it is started and stopped from an application.

http://[IP address]:8181/cxf/support/support/start/\{serviceID\}

http://[IP address]:8181/cxf/support/support/stop/\{servicelD\} 
Chapter 4. Validation of the proposal and results

7. Herrfors pressure device: as in previous devices, this one is capable of obtaining the latest results of the pressure that is measured.

http://[IP address]:8181/cxf/support/support/getHerrforsPressureLastResults/\{servicelD\}

8. Herrfors data device: this device is capable obtaining the latest results of the data that is being gathered from the deployment.

http://[IP address]:8181/cxf/support/support/getHerrforsDataLastResults/\{servicelD\}

9. Enfo ESS Box: application interfaces have been developed for energy-centred operations: energy measurements, accumulated energy or average energy values are taken into account.

http://[IP address]:8181/cxf/support/support/energy/\{servicelD\}

http://[IP address]:8181/cxf/support/support/get accumulated energy/\{serviceID\}

http://[IP address]:8181/cxf/support/support/get accumulated energy interval/\{serviceID\}

http://[IP address]:8181/cxf/support/support/get average power/\{servicelD\}

http://[IP address]:8181/cxf/support/support/get average power interval/\{servicelD\}

http://[IP address]:8181/cxf/support/support/get energy/\{servicelD\}

http://[IP address]:8181/cxf/support/support/get energy interval/\{servicelD\}

10. e-GOTHAM Box: this device is used in the Norwegian pilot to deal with energy data, such as temperature, power consumption, turning off one energy load, etc.

http://[IP address]:8181/cxf/support/support/getFlexibleLoad/\{servicelD\}

http://[IP address]:8181/cxf/support/support/getTemperature/\{serviceID\}

http://[IP address]:8181/cxf/support/support/getTotalPowerConsuption/\{servicelD\}

http://[IP address]:8181/cxf/support/support/turnOffLoad/\{power off\}/\{serviceID\}

In addition to the former URIs, there were some other that were used during testing activities with the purpose of assessing how the software and interfaces that had been developed were behaving. All those testing interfaces can be described as follows:

1. URIs used as an example of how to use applications that already have a request message to be sent, rather than relying on the middleware for that task:

http://[IP address]:8181/cxf/support/support/performRequest.

http://[IP address]:8181/cxf/support/support/performRequest/\{servicelD\}

2. URIs used to test the early Logical Device that was developed by UPM, regarding how current could be requested: 
Chapter 4. Validation of the proposal and results

http://[IP address]:8181/cxf/support/support/getCurrent

http://[IP address]:8181/cxf/support/support/getCurrent/\{servicelD\}

3. URIs used as an example of how service list could be generated:

http://[IP address]:8181/cxf/support/support/ejemploList

http://[IP address]:8181/cxf/support/support/ejemploListNoCab

http://[IP address]:8181/cxf/support/support/ejemploListText

4. URIs used as an example of how Monitoring and testing and Discovery components could be accessed.

http://[IP address]:8181/cxf/monitoringTesting/monitoringTesting/getMonitoringTesting

http://[IP address]:8181/cxf/discovery/discovery/getDiscovery

Finally, there are some other URIs used by the Discovery component with the purpose of listing these services, the active services (according to how they are defined as active by the Context Awareness component) and listing devices and the operations each of them is capable of providing.

http://[IP address]:8181/cxf/discovery/discovery/listServices

http://[IP address]:8181/cxf/discovery/discovery/listActiveServices

http://[IP address]:8181/cxf/discovery/discovery/listDevices

http://[IP address]:8181/cxf/discovery/discovery/listServiceOperations/\{servicelD\}

An example of how URIs can be used to get a grasp on the available middleware components at the highest level by just requesting the available ones is executing a URI request in the controller that is supposed to contain all the services. This can be done by simply invoking the first fixed part of the URIs that have been used as a way to request data, that is, introducing http://[IP address]:8181/cxf/ in a web browser or any software that has the full TCP/IP or OSI stack installed. An example of what will be obtained as a result has been portrayed in Figure 89. Note that despite using REST interfaces, the services that are listed have been regarded as SOAP-based ones by the JBoss Fuse, thus hinting that the Simple Object Access Protocol is used in a subtle way. 
Available SOAP services:

Available RESTful services:

Endpoint address: http://localhost:8181/cxf/devreg

WADL : http://localhost:8181/cxf/devreg? wadl

Endpoint address: http://localhost:8181/cxf/monitoringTesting

WADL : http://localhost:8181/cxf/monitoringTesting? wadl

Endpoint address: http://localhost:8181/cxf/support

WADL : http://localhost:8181/cxf/support? wadl

Endpoint address: http://localhost:8181/cxf/discovery

WADL : http://localhost:8181/cxf/discovery? wadl

Figure 889. URIs for available middleware high level components

\subsubsection{Low level interfaces}

Rather than just being focused on how information is recovered from devices, low level interfaces implement a specific low level format that was designed as a way to establish a convention on how data was expected to be retrieved. That convention has already been described in the second-to-last subsection of the previous chapter (the PDUs to be used for data transfers and delivery), but the implementation details have been included here. Since middleware works at a data level, it was decided that XML messages were the most suitable way to represent the Processing Data Units that were included in the system. The specification and the simple protocol that has been developed so as to undertake all these procedures are referred to in [154] as Smart Grid Data Interchange Protocol (SGDIP). The procedures contain all the required information to manage the facilities mentioned before: device registration and deregistration, heartbeats or devices leaving the system where they have been deployed.

As far as registration is concerned, the procedure to have a service registered differs greatly from the way used to register a device. In addition to that, differences between legacy and tailored devices may have to be taken into account as well. Services that were registered in the middleware by making use of XML messages should have the following appearance:

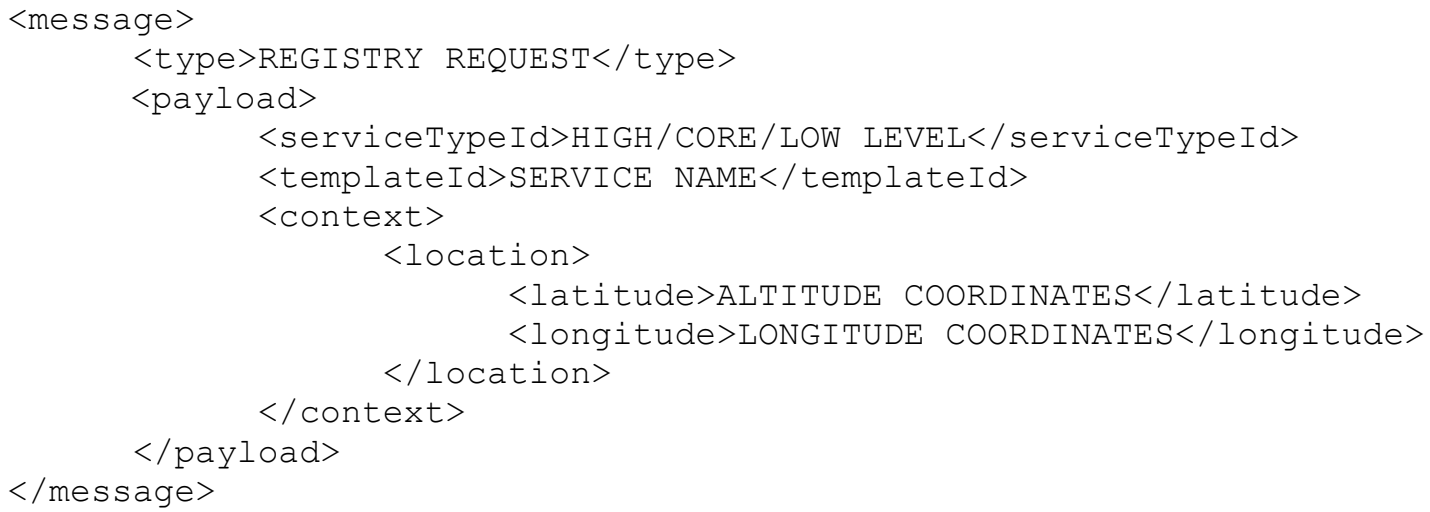


As it can be seen, they have the summarized content of all the features that can be contained by them and do not add some other concepts mandatory for other registration procedures, such as the features of the hardware to be included or the services it is capable of providing. It has to be noted that once these services have been registered, no other message will be sent back to confirm the registration, as the general consensus was avoiding the transfer of XML messages within the semantic middleware unless it was strictly necessary.

As for the nature of the devices integrated in the middleware, there are two kinds that have been considered: on the one hand, devices that have been developed from scratch for the projects where the content of this thesis takes place were able to have all the features that were required for them, not only in terms of middleware or software, but also for network connectivity, sensing, etc. These are referred to as Tailored Devices. On the other hand, there are devices that cannot have any middleware installed in them and may be additional interfacing work to be integrated in a deployment. These latter are referred to as "Legacy Devices" and are found far more frequently due to several reasons (they precede the development activities carried out in e-GOTHAM and I3RES projects, they represent the interests that manufacturers and companies had in the projects, etc.). While the existence of two different kinds of devices was troublesome for other parts of the project (it effectively made not possible to install middleware components in them for management of data requests and deliveries), the registration procedure was barely affected at all. A Tailored Device would send a registration message with the following appearance:

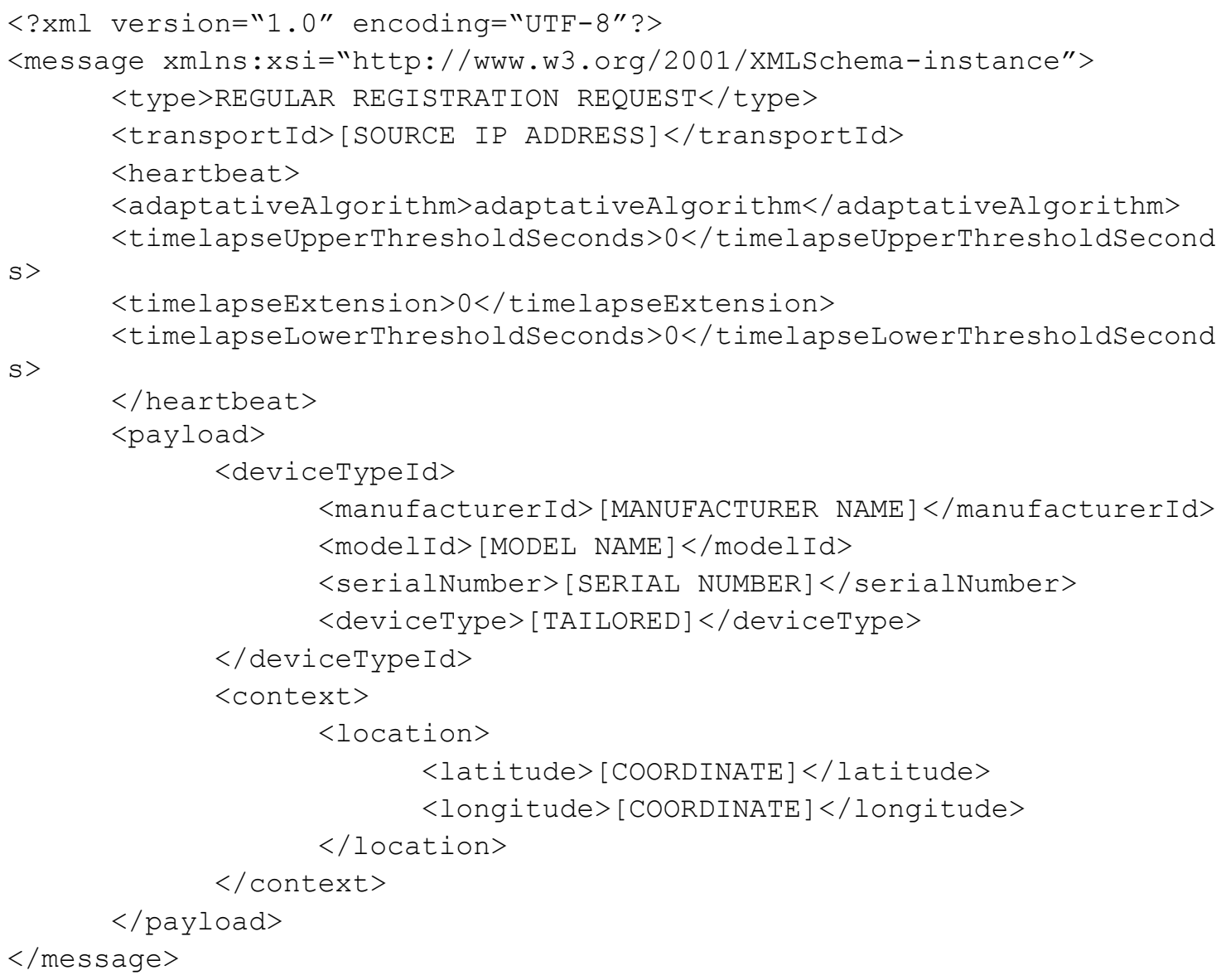


The textfields that have been added have been done so according to the criteria introduced in the previous section. As previously mentioned, the only impact that having two different kinds of devices make is the fact that the type of registration is changed to what was defined in eGOTHAM as "physical".

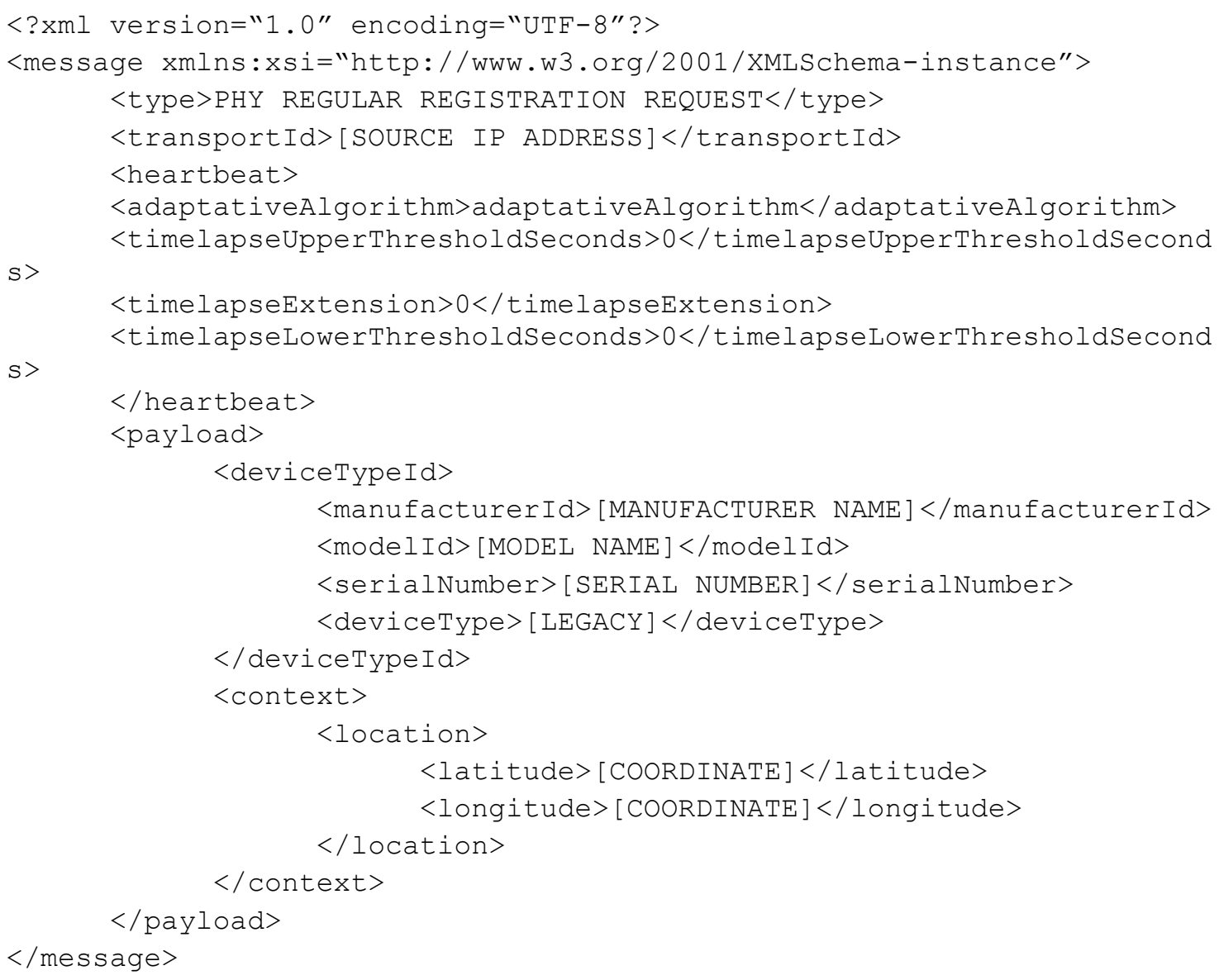

Both messages have the transport id field added as part of the content transmitted to the network because such an identification is the one needed to distinguish the device sending the request and must therefore receive the answer; it is also a way to contain an identifier used to send later requests and responses whenever data that can be provided by the device is needed at the application layer. The only features that remain to be included is all the services and parameters that the devices can provide. One of the decisions that had to be made was regarding how to transfer information from one side to the other one of the development, as $\mathrm{XML}$ messages can be very verbose.

A decision was taken in order to include the information of the available hardware devices immediately before the registration was completed. This was made possible by composing a series of templates that included all the features of the devices to be registered, according to the information that had been previously provided by the partners involved in their manufacture. Each of the templates was assigned a number, and so were the devices to be included in the middleware. A total of 13 templates were created for device registration that included tailored devices, RTU units or PMUs. For example, the template of a tailored device 
used as a way to check all the content included in the registration procedure has been included in the database of the middleware solution.

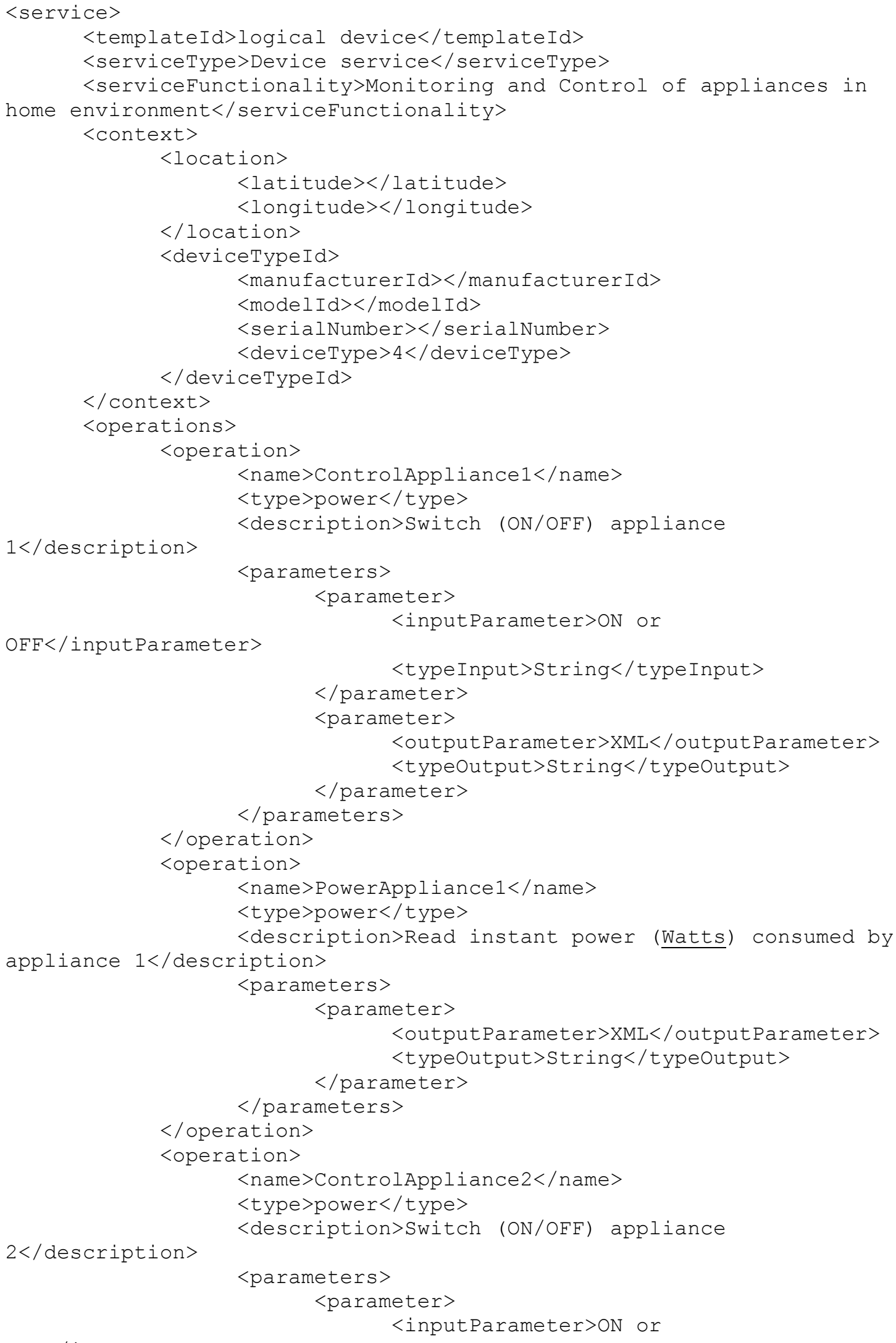

$\mathrm{OFF}</$ inputParameter $>$ 


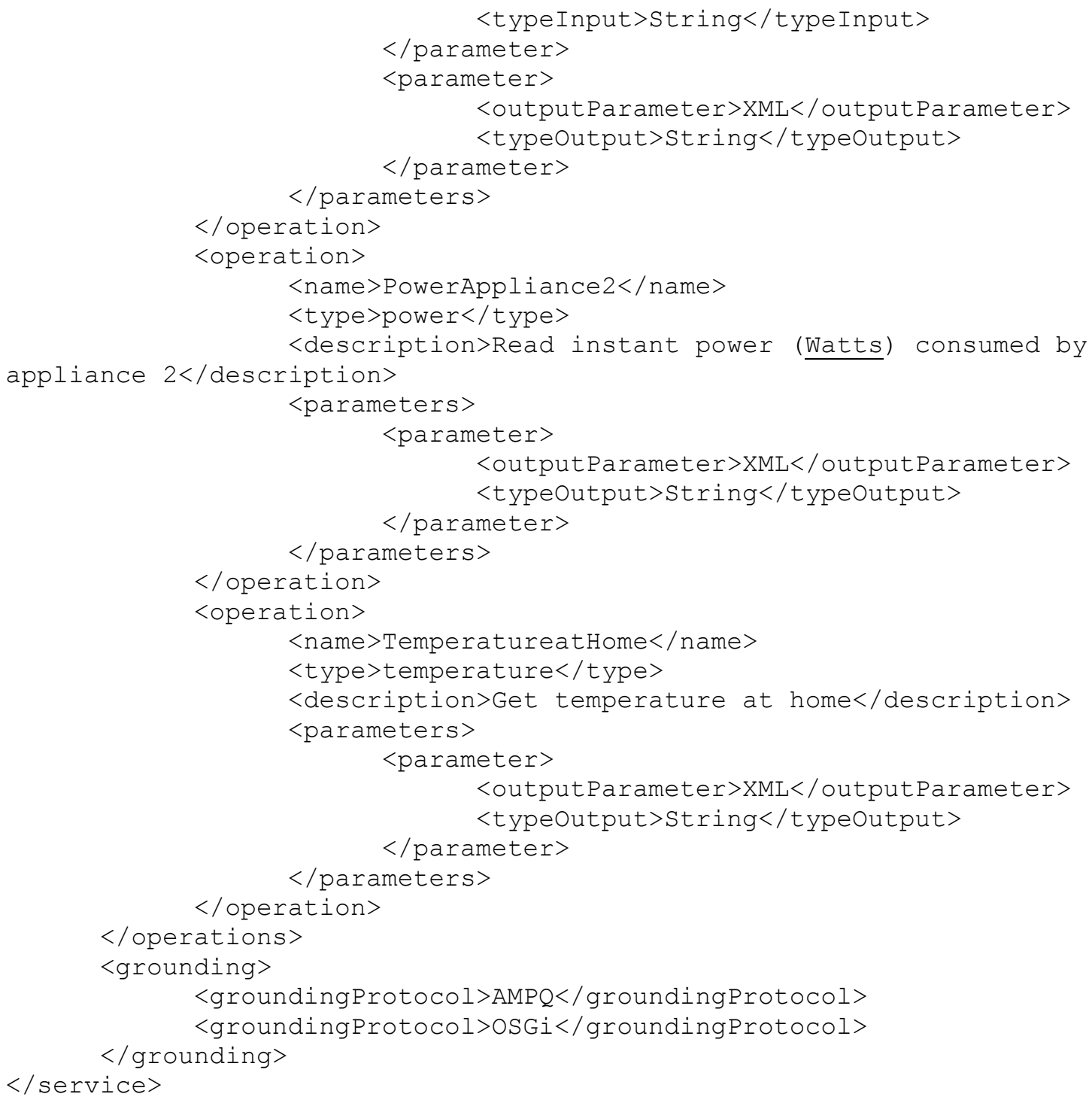

As it can be seen in the previous template, the amount of information regarding the services that the device can provide is way larger than the one sent in the registration request message. This was made possible because partners of the e-GOTHAM project were requested about the information to be included in the demonstrators. The procedure to do so was filling up a table with all the data regarding operations, features and input or output parameters required for the services. This table looked like Table 15.

Table 15. Device information table filled by e-GOTHAM partners

\begin{tabular}{|l|l|}
\hline Field name & Content \\
\hline Service functionality & Describes the usefulness of the service [String] \\
\hline Latitude location & $\begin{array}{l}\text { Indicates the latitude, in degrees, where the } \\
\text { service is located [double] }\end{array}$ \\
\hline Longitude location & $\begin{array}{l}\text { Indicates the longitude, in degrees, where the } \\
\text { service is located [double] }\end{array}$ \\
\hline
\end{tabular}


Chapter 4. Validation of the proposal and results

\begin{tabular}{|c|c|}
\hline Device manufacturer & $\begin{array}{l}\text { Describes the manufacturer of the physical } \\
\text { device, for example of the smart meter [String] }\end{array}$ \\
\hline Device model & Model name of the device [String] \\
\hline Serial number & identifies the device uniquely [String] \\
\hline Operation name 1 & $\begin{array}{l}\text { Name of the operation associated to that } \\
\text { service [String]. The service can have one or } \\
\text { more operations depending on its available } \\
\text { functionalities }\end{array}$ \\
\hline Operation description 1 & $\begin{array}{l}\text { Describes the functionality of the operation } \\
\text { [String] }\end{array}$ \\
\hline Input parameter operation 1 & $\begin{array}{l}\text { Input parameter employed for the execution of } \\
\text { the operation. It follows the e-GOTHAM data } \\
\text { model information, that is, CIM + SSN. None, } \\
\text { one or more input parameters can appear }\end{array}$ \\
\hline Input parameter operation $N$ & Input parameter for operation number $\mathrm{N}$ \\
\hline Output parameter operation 1 & $\begin{array}{l}\text { It is the output parameter that is obtained such } \\
\text { as result of the operation. It follows the e- } \\
\text { GOTHAM data model information, that is, CIM } \\
+ \text { SSN. None, one or more output parameters } \\
\text { can appear }\end{array}$ \\
\hline Output parameter operation $N$ & Output parameter number $\mathrm{N}$ \\
\hline Precondition parameter operation 1 & $\begin{array}{l}\text { If the operation needs some precondition } \\
\text { before its execution, this field indicates it. It } \\
\text { follows the e-GOTHAM data model } \\
\text { information, that is, CIM + SSN. None, one or } \\
\text { more input parameters can appear. }\end{array}$ \\
\hline Precondition parameter operation $N$ & Precondition $\mathrm{N}$ \\
\hline Operation name $\mathrm{N}$ & Name of Operation $\mathrm{N}$ \\
\hline Operation description $N$ & Description of Operation N \\
\hline
\end{tabular}

Once the device with its ancillary services became registered, the registration responses that are sent from the middleware solution to the devices are simple messages that will reflect the success or failure of the operation that they were trying in the first place. The appearance of the XML message that will be sent has been included below this paragraph. As it was mentioned in the previous chapter, this will largely depend on whether a device has already been registered in the system or not. A service id and the acknowledgement of the registration procedure (marked with a " $Y$ " in the registered field) are included in the message.

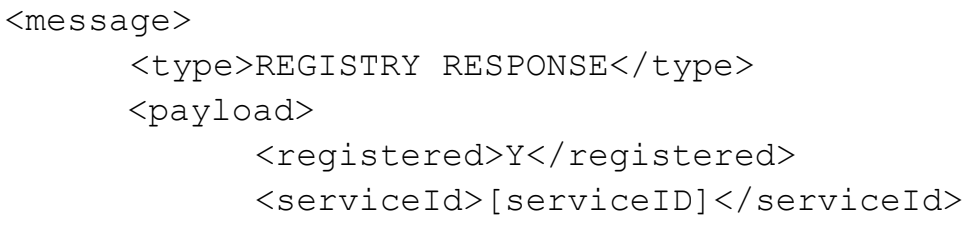




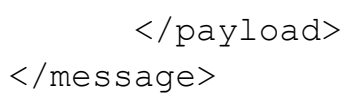

The message that is obtained from the middleware solution in case the registration did not take place due to the reasons mentioned in the previous chapter is essentially the same as the one shown before, with the exception that a capital " $\mathrm{N}$ " will be used instead of a " $\mathrm{Y}$ ". An error code and description are also offered so as to provide more information about the potential failure.

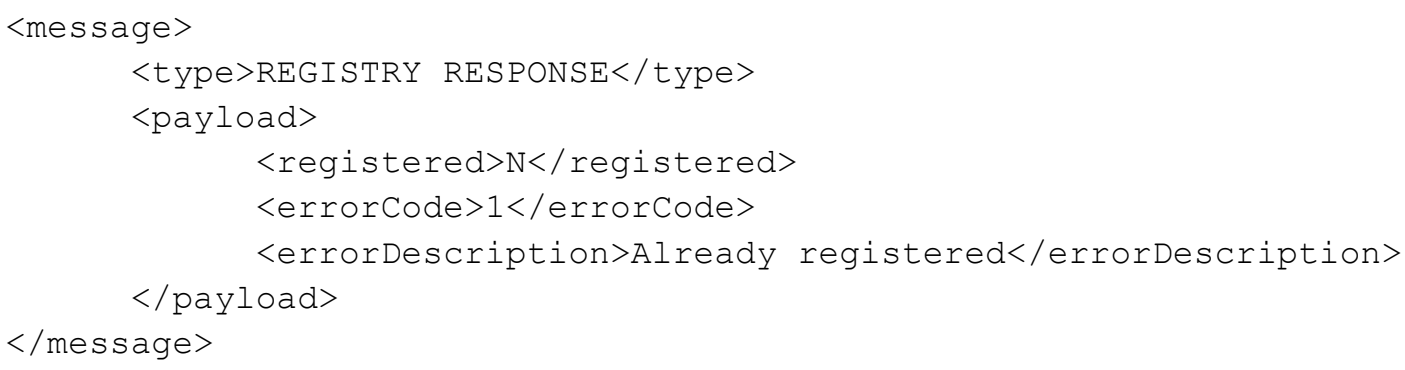

Another message that has also been conceived for the protocol to be used with the low level interfaces includes the voluntary removal of a device from the deployment, due to any reason (device replacement, testing, etc.). For this kind of situations, a "bye" message has been conceived. Its appearance is as included in the XML message described right below this paragraph.

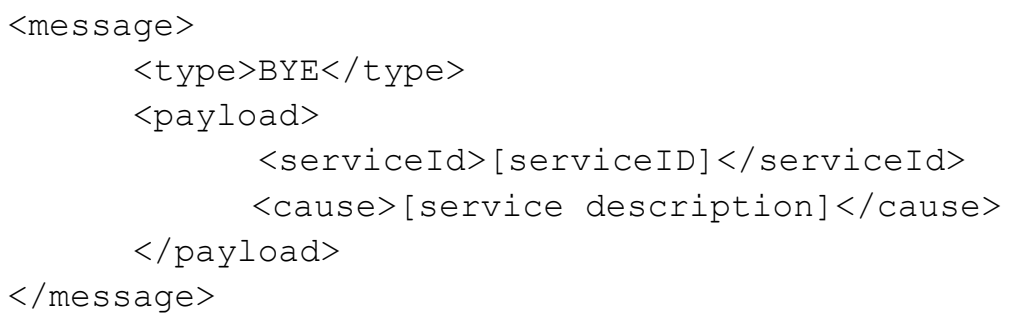

Other message that comes in handy for discovery and context-related services is the heartbeat messages that are supposed to be sent by devices that have already been registered and included in the system. The structure of this message will follow the textfields introduced in the PDUs of section 3. No extra content is required to be included, as it will be only required for the message to contain the code of the service that has been registered. Additionally, the amount of keep alive/heartbeat messages can be regulated depending on the information contained in the fields related to heartbeats in the registration message.

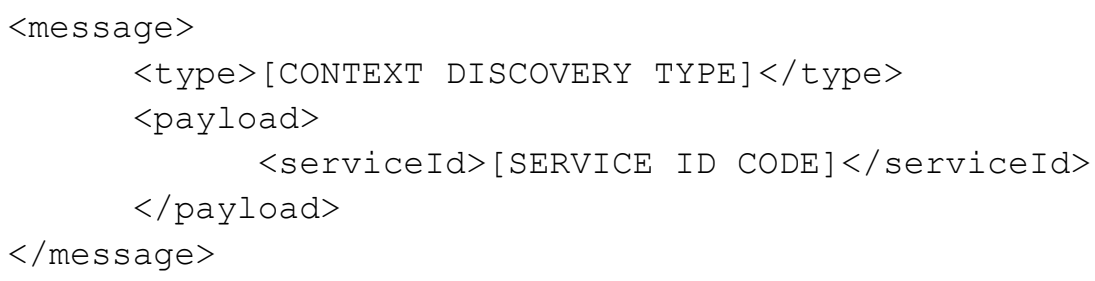


Lastly, deregistration can also be started from the middleware if it is required. The procedure that has to be followed implies sending a message to the device that is going to become unattached from the system.

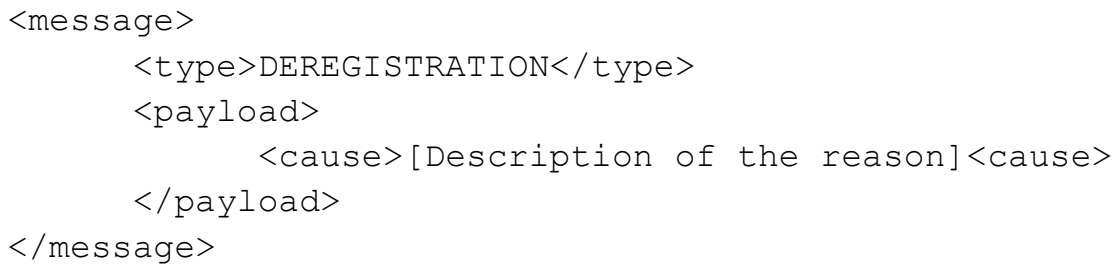

These are all the messages that can be sent from the lower interfaces to the higher level middleware services. Plus, since there is an action needed between the data that were sent to the middleware solution and how they were stored in it, an ontology-based component must be included as a support for the registration functionalities. The data format that was sent to the ontology connector component, where they are supposed to be reformatted according to the elements defined by the semantic capabilities, will be used for registration. Figure 90 shows how data are transferred among the different elements of the middleware, in the way they were designed for the e-GOTHAM project.

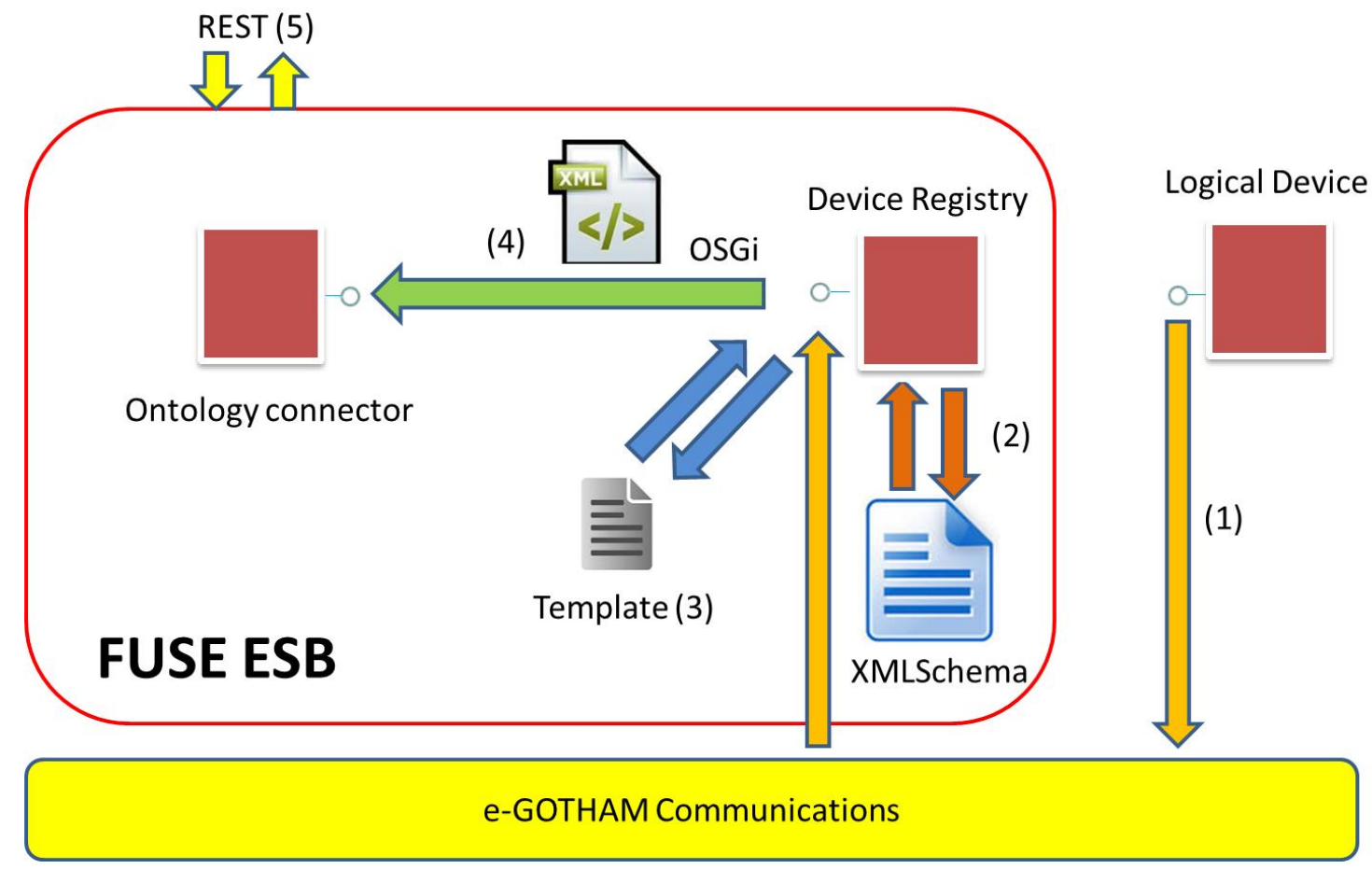

Figure 90. Device registration with the intervention of the ontology of the middleware, as developed in the eGOTHAM project

As it can be seen, when the information reaches the software component used for device registration (1). The request that is obtained is compared to an XMLSchema to verify that is correctly built (2). It is in that software component where it has been decided that the template used for service and device registration (3), which will be filled according to the information regarding the kind of device that is planned to be registered in the system. Finally, the XML template filled with the required information will be sent to the software component 
called Ontology connector, which was used to encase the ontology used in the deployment. The important concept to have clear is that the format used by the devices to be registered will be as the one shown for registration of physical and logical devices previously, whereas the data format that is used to store the information about the devices and the services is the one shown in the template of the device type number 4.

\subsubsection{Interconnectivity of software components}

The software components that have been implemented are capable of performing operations by themselves (especially when they become Middleware Autonomous Services), interact with high or lower levels of the architecture by means of either REST interfaces and URIs (for Middleware Access Points) or lower level identifiers and information transmission conventions. (hardware abstraction capabilities). However, if data has to be sent from one component to another one, other software technologies that do not require leaving the middleware will have to be used. It was explained previously in this manuscript that OSGi interfaces can be used as a way to interface software components. Considering that they can also be used as part of the JBoss Fuse components, it was decided to incorporate them to the software components that were being developed as part of the middleware in e-GOTHAM and I3RES projects. In order to do so, though, there are several modifications that must be done to the implementation works that are being carried out.

It has been explained that while creating the maven project there are several ancillary files that become part of the project, such as the blueprint.xml or POM.xml. These latter two files have to be modified to reflect the dependencies on OSGi and OSGi as a technology to offer interfaces among software components. For example, if there were two JBoss Fuse bundles to communicate with each other in a Client/Server, it should be reflected that a) OSGi interfaces are being used and b) those interfaces connect the client bundle with the server bundle. It can be appreciated in the next lines of code how the technology used is reflected: an OSGi reference has been included mentioning both the identifier that has been given to the service (helloWorldService) and the codified interface. These text lines were not present in the blueprint.xml file that was firstly obtained from the generation of the Maven project. The example that has been used here has as its only purpose to clarify how interconnectivity is done among software bundles, as other communication paradigms can be used as well (for example, a Publish/Subscribe paradigm is possible to be used in case it is needed to have data sent and received following the same fashion).

$<$ ?xml version="1.0" encoding="UTF-8"?>

$<$ blueprint xmlns="http://www.osgi.org/xm/ns/blueprint/v1.0.0" xmlns:xsi="http://www.w3.org/2001/XMLSchema-instance" xsi:schemaLocation $="$ http://www.osgi.org/xmlns/blueprint/v1.0.0

http://www.osgi.org/xmlns/blueprint/v1.0.0/blueprint.xsd">

$<$ !-- OSGi reference -->

$<$ reference id="helloWorldService"

interface = "org. mycompany. server.osgi. IHelloWorldSvc" />

$<$ bean id="clientBean" class="org.mycompany. client. osgi. Client"> 


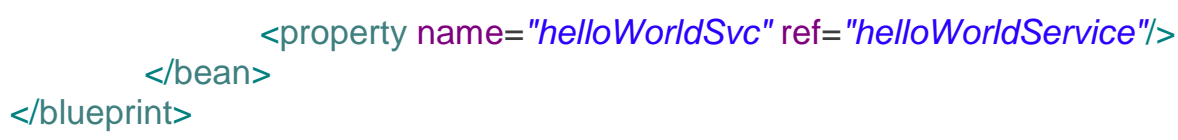

In the same fashion, extra lines are added to the server part of the communications that reflect both the interfaces that have been defined (OSGi Interface) and the implementation main class used (OSGi implementation).

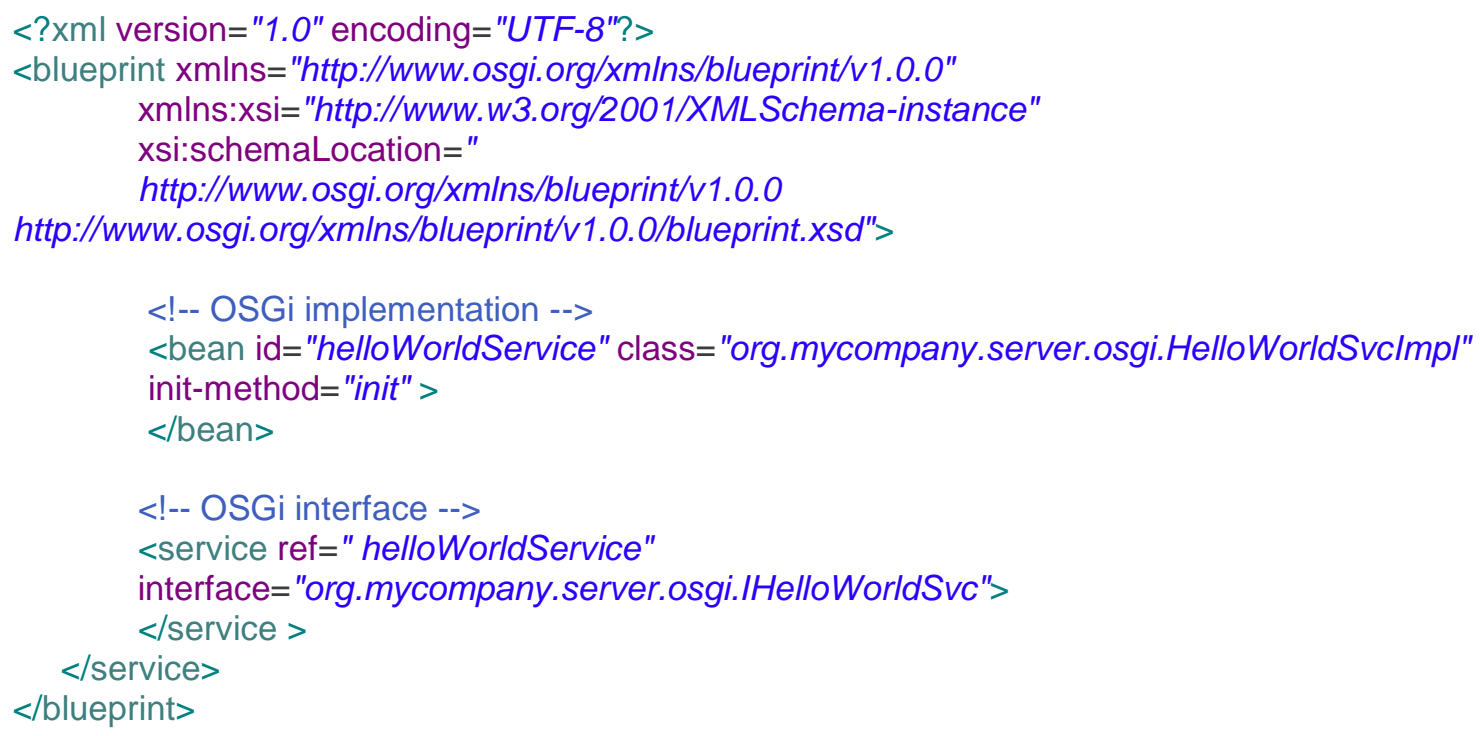

Lastly, the POM.xml files that are located in both ends of the communication must also be edited. The editions that must be done involve two tags: dependencies and plugin. The dependencies tag will have to incorporate the other bundles used to send and receive information (for example, the bundle with the server part of the communications). In addition to that, the plugin referred to the artefact id will have to be included, as depicted in the code lines included below.

Overall, the procedures that have been described here are based on the experience and knowhow acquired by the author of the thesis while performing implementation works for the eGOTHAM and I3RES projects. They may differ depending on other variables that are much closer to an implementation, lower level; for example, the creation of a Maven project might be different if instead of using Eclipse in Ubuntu as the IDE and operating system, NetBeans and Windows are used instead. In any case, the underlying ideas will be still valid, as it is made clear how it is needed to have an IDE running in a machine to perform the codification of the software services.

<project xmIns="http://maven.apache.org/POM/4.0.0"

xmlns:xsi="http://www.w3.org/2001/XMLSchema-instance"

xsi:schemaLocation="http://maven. apache.org/POM/4.0.0 http://maven.apache.org/xsd/maven-

4.0.0.xsd">

$<$ modelVersion $>4.0 .0<$ /modelVersion>

$<$ groupld $>$ org.mycompany $<$ /groupld $>$

$<$ artifactld $>$ helloWorld-client $</$ artifactld $>$

$<$ version>0.0.1-SNAPSHOT</version>

$<$ packaging $>$ bundle $<$ /packaging $>$

$<$ dependencies> 


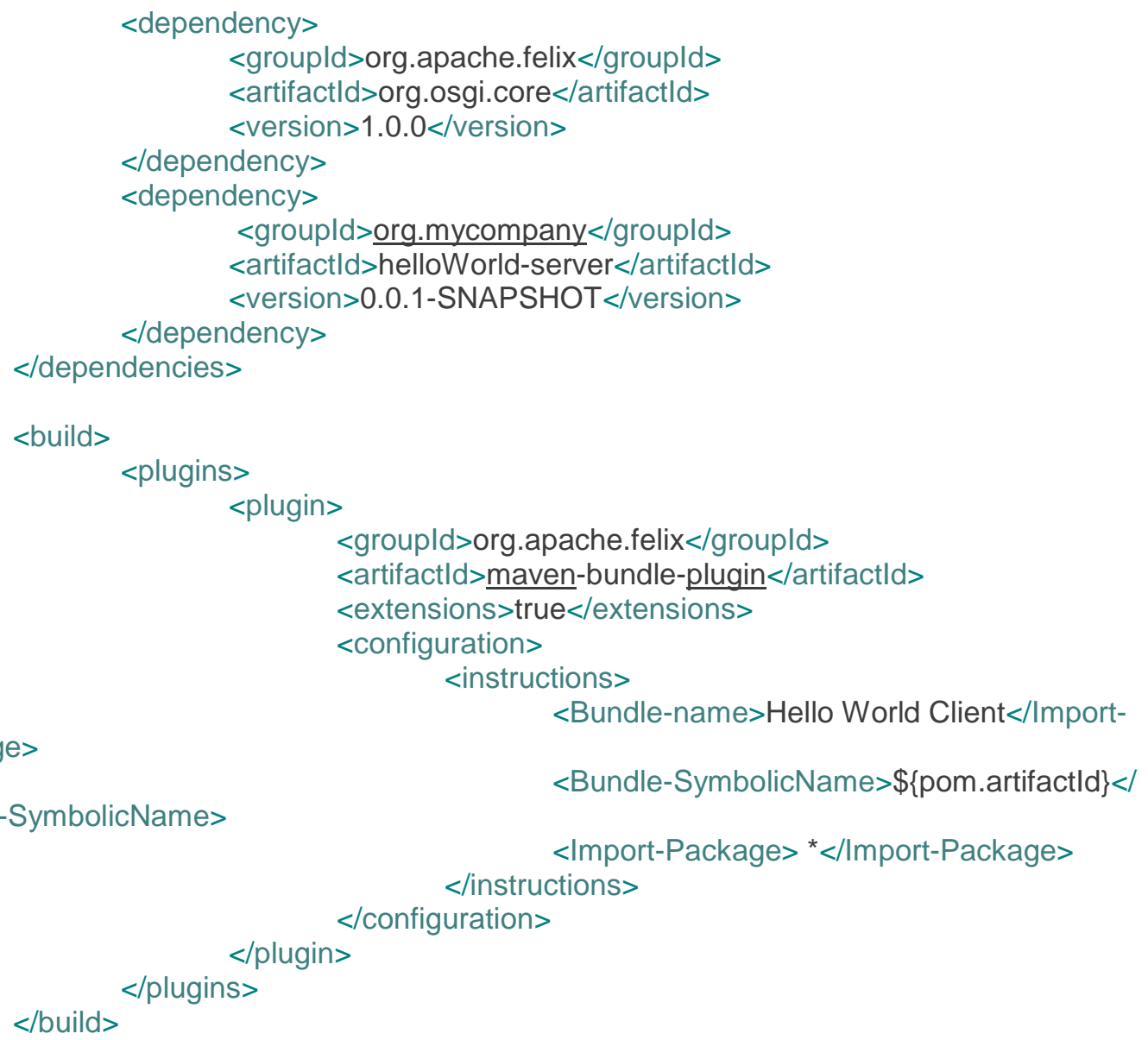
<Import-Package> * </lmport-Package> $<$ /instructions > $</$ configuration $>$

Bundle-SymbolicName>

The same operations are carried out for the server part of the communication. Dependencies regarding OSGi must be included as well for the server.

<project xmlns="http://maven.apache.org/POM/4.0.0"

xmlns:xsi="http://www.w3.org/2001/XMLSchema-instance"

xsi:schemaLocation="http://maven.apache.org/POM/4.0.0 http://maven.apache.org/xsd/maven-

4.0.0.xsd">

$<$ modelVersion $>4.0 .0<$ /modelVersion $>$

$<$ groupld $>$ org.mycompany $<$ groupld $>$

$<$ artifactld $>$ helloWorld-client $<$ /artifactld $>$

$<$ version>0.0.1-SNAPSHOT</version>

$<$ packaging $>$ bundle $<$ /packaging $>$

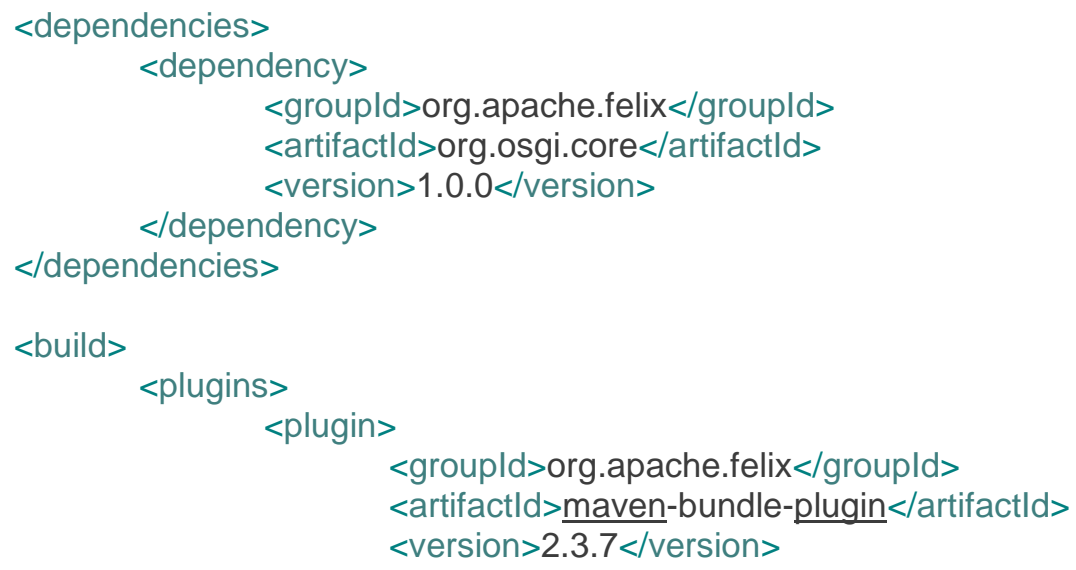


$<$ extensions $>$ true $<$ /extensions $>$

$<$ configuration>

$<$ instructions $>$

Package>

$<$ Bundle-name>Hello World Client</Import-

Bundle-SymbolicName>

$<$ Bundle-SymbolicName $>\$\{$ pom.artifactld $\}<1$

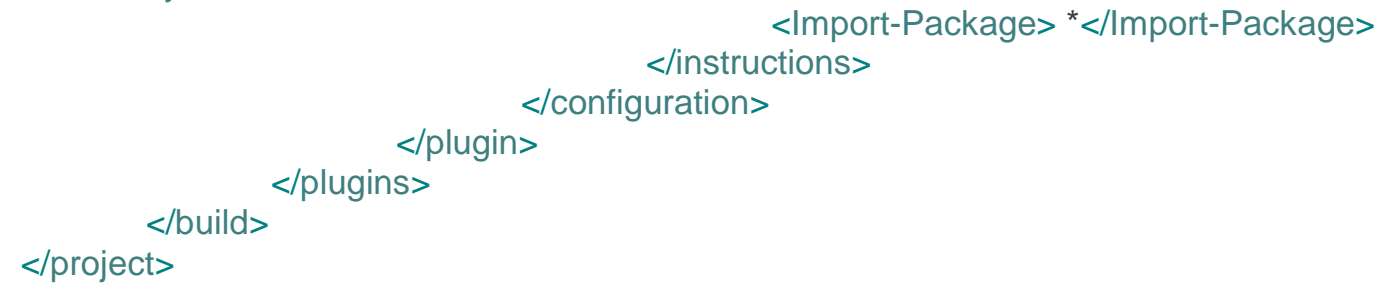

Once all the operations regarding interfaces have been described, implementation works can be further explained in the next section.

\subsubsection{CMA ontology implementation}

As previously listed, a software component has been created to control the registration of devices and services in any deployment where the middleware is involved. In order to make that process possible, though, an ontology must be created in order to contain all the information related to the services and devices present in the system. In this way, the most frequent elements of a deployment where the Smart Grid and middleware are involved can be modelled as a collection of interrelations that are tightly interweaved with each other. These elements have been considered to be:

1. Features related to devices: concepts such as the manufacturer, the series number or the kind of functionality that a device can provide have been included in the ontology.

2. Features related to services: in the same way, characteristics related to the services present in the middleware, like their functionalities, have been added to the ontology as well.

3. Features related to the existing environment: physical, network and the application layer compose the grounding elements used for the correct performance of the middleware and its functionalities.

These concepts have been included in the ontology as the following elements:

1. Classes: these are used to include the elements from the Smart Grid that be roughly regarded as the ones. CMA includes the ones related to grounding (network, physical layer and application), hardware (device services) and middleware (the software components).

2. Object properties: the properties describe the relationships between two instances or individuals. By "instances" is meant individuals, which are elements linked to the classes that were previously defined. 
3. Data properties: the properties that have been included in the ontology relationships are used to establish between instances and individuals and data values. In this case, their value is a data literal, as described in [175].

4. Individuals: these are characteristics that have been included in the classes, which express a feature about a specific feature of the device. They are instances of the objects that are used at the most basic level.

The methodology that has been used to include the ontology as part of the Common Middleware Architecture components is referred to as Methontology. It makes use of the following steps in order to make sure that a realistic result can be obtained:

1. Building the glossary of terms: this stage consists of listing all the prominent elements of the application domain where the ontology is located. In this case, characteristics related to devices, middleware services and the required infrastructure for them to work flawlessly were included.

2. Building taxonomy concepts: it consists of creating associations (or even a hierarchy) between the listed elements depending on their position in the environment. For example, a concept can be regarded as a subclass from another one,

3. Building diagrams of ad hoc binary relationships: they are based on the relationships that elements from the ontology may have among them, usually focused on an entity $A$ having $B$ and, at the same time, $B$ being part of $A$.

4. Building the dictionary of concepts: in this step, the characteristics of each of the concepts that have been included in the ontology are explained. Typically, concepts will be linked to several attributes (this is especially evident for devices and the services of the middleware architecture) that will establish a set of relationships among them.

5. Describing the binary, ad hoc relationships: in this step, the name of each of the relations ships that were created, as well as their domain (the class it is associated to), cardinality (number of potential iterations of the relationship), range (the kind of attribute related to the relationship) and inverse relationship (which is essentially the relationship name from the opposite perspective).

6. Describing the attributes: in this stage the attributes are described according to the concept they are linked to and are given a kind of value that will be used for their implementation (it will usually match the primitive types provided by any high-level programming language). Possible range of values and the cardinality between the attribute name and the concept is usually given as well.

Once all these steps have been carried out, the ontology can be normalized, as it has been described in [175]. The ontology that has been created to include such a functionality has been displayed in Figure 91. As it can be seen, all the previous elements have been integrated in it, along with the relationships that they keep with each other. The ontology that is portrayed in this thesis can be regarded as a starting ground to describe how the different elements of a semantic middleware for the Smart Grid can be included in a deployment of this nature. It can be expanded in either future iterations of the CMA or can be included as a branch in other standards where CMA makes its own contributions for their improvement. 


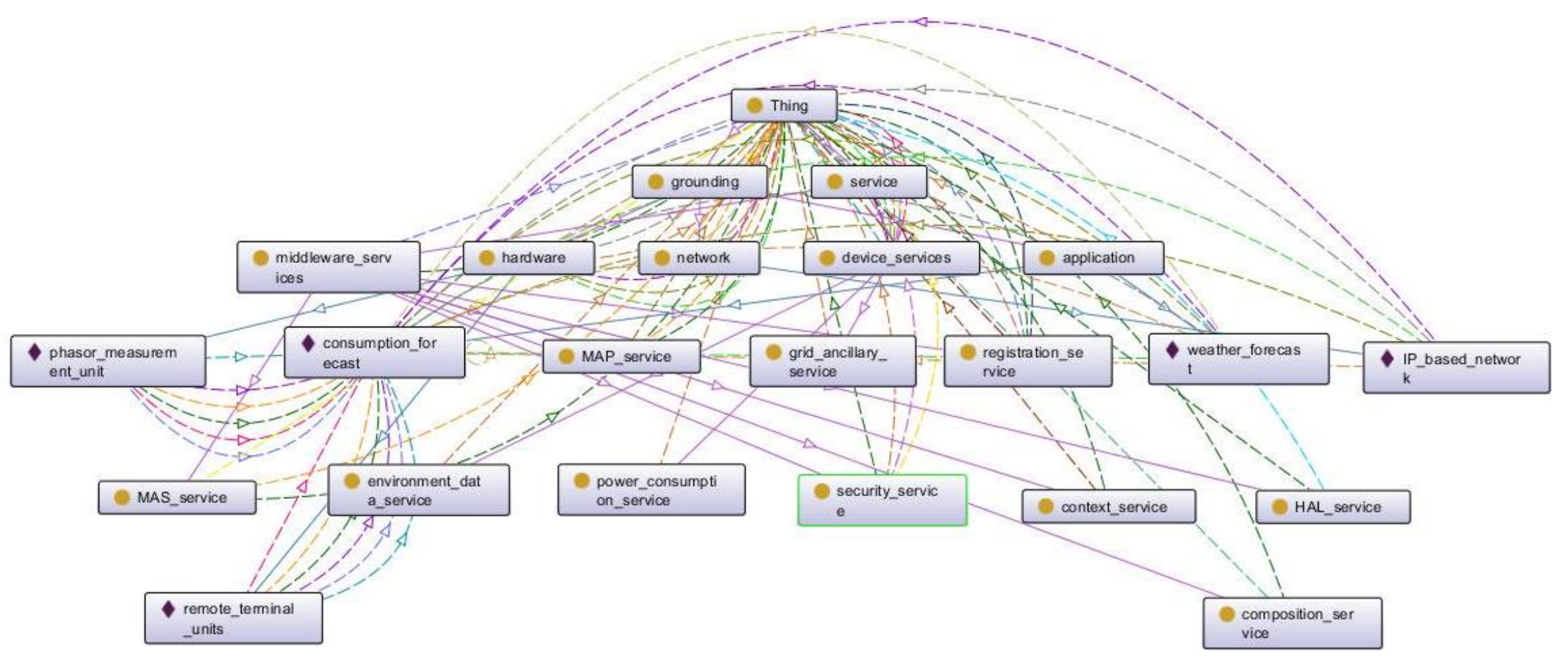

Figure 91. Conceived CMA ontology 
The ontology that is represented in is also represented as an OWL file that describes what elements for a Smart Grid deployment with the middleware are and how they are related to each other. Part of this OWL file has been included in the Appendix B regarding middleware implementation activities.

\subsection{Implementation works}

Most of the implementation works have been carried out with the central objective of having bundles (software components of the middleware) included in the JBoss Fuse (the middleware framework) performing their expected functionalities. After the codification activities have been carried out and the projects have been compiled with all their classes, they will have to be installed and executed. The place to perform such actions in JBoss Fuse is the directory called "deploy", where all the developed components will be stored for their usage. When JBoss Fuse is run, all the bundles (which in the end are executable jar files) that have been deployed to the deploy directory start running, as it has been depicted in Figure 92 .

During implementation works, the components will be deployed repeatedly and it is only natural that the services will not work as planned the first time. Due to this fact, it would be surprising that sometimes the status of those components is defined as "Failure" rather than "Active". Some other times interfaces may malfunction; in that case, bundles will be characterized as "Installed", but not active. Therefore, bugs must be fixed before the component is deployed again, as any minor issue might make the bundle completely useless. These aspects are part of the troubleshooting activities that were carried out during the implementation works. 


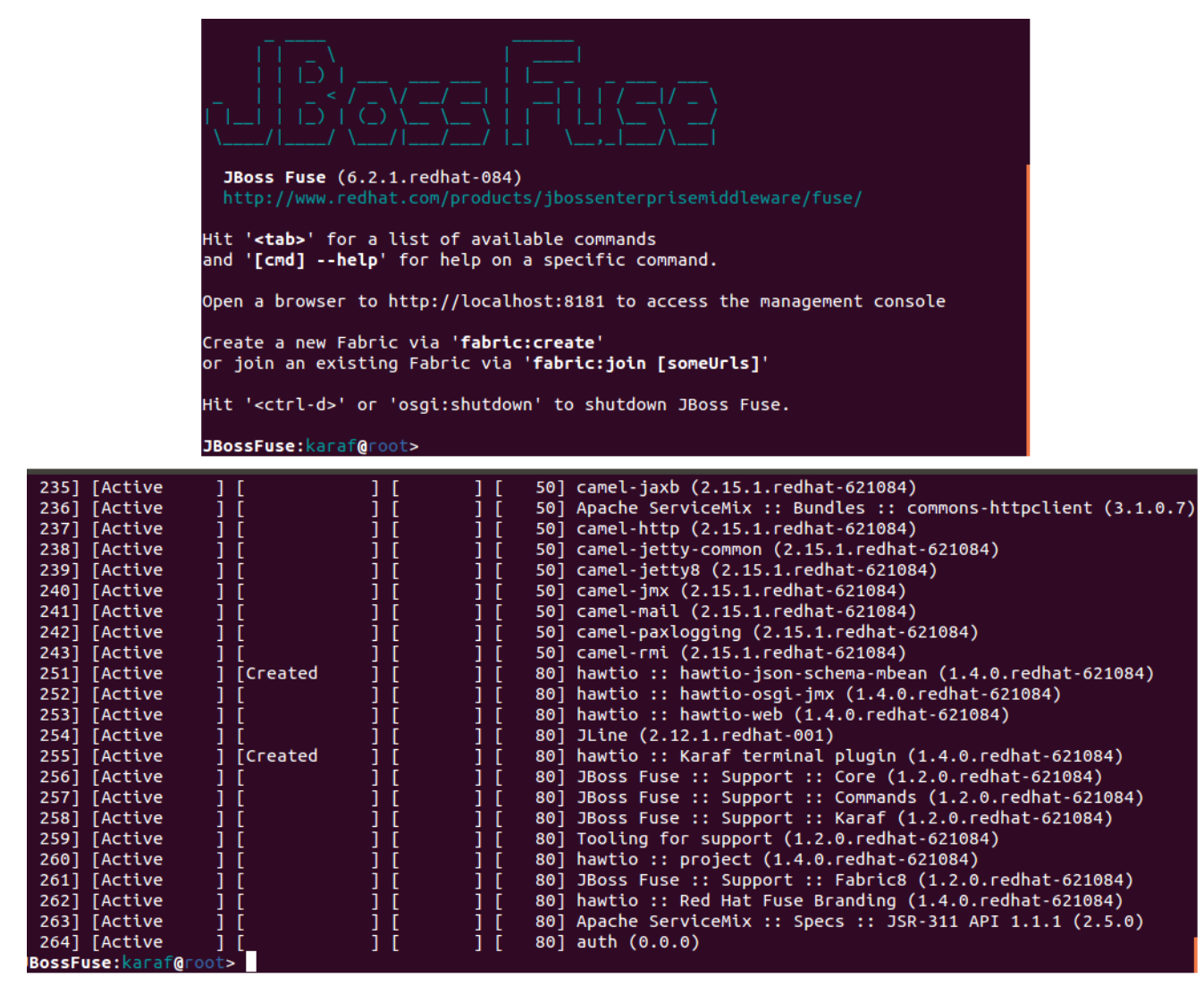

Figure 89. Running JBoss Fuse with the list of active bundles

1. The JBoss Fuse and the communication network will be considered as already pre-existing, even though there might be no devices registered. This is easy to know by just executing an URI on a web browser requesting that available services and devices are listed. The URI to be executed would be: http://[IP address]:8181/cxf/discovery/discovery/listServices. When there are no services registered, the list will appear empty (even though it will still have all the labels required to define the components that will have once there is content in it), as shown in Figure 93.

This XML file does not appear to have any style information associated with it. The document tree is shown below.

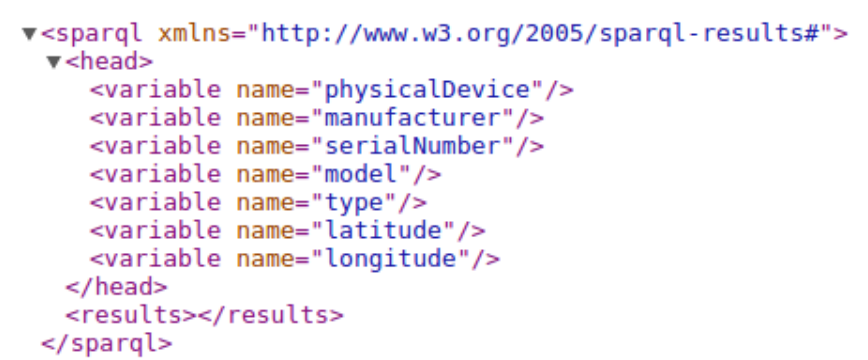

Figure 903. Empty services list

2. As explained, registration of a device can be done by either having the device sending the request or sending the request from the application side. The procedure that is shown here is the one that was been carried out while implementation works happened and 
there were no finished devices available, so the REST interfaces had to be used for that purpose. Registration messages would be sent by invoking the correct service to the device registration component that behaved as a Middleware Access Point via URI, which implies selecting:

\section{http://[IP address]:8181/cxf/devreg/deviceregistry/registerDevice/rpi mbus/6}

A message showing the identifier given to the device would be shown, as depicted in Figure 94. This identifier would be used later as a way to request services to the device.

\section{Apache Karaf Web C $\times$ http://localhost:818 $\times$ localhost:8181/cxf/c $\times$} localhost:8181/cxf/devreg/deviceregistry/registerDevice/rpi_mbus/6

ModelID from physical device has been registered with id 1331

Figure 914: registration message with the identifier.

3. After the registration process there will be several major changes taking place. One of them will be the generation of a bundle representing the registered device, as shown in Figure 95. It is important to take into account, though, that the creation of software bundles whenever there is a new device registered was a decision that a) only applied to the e-GOTHAM project (other iterations of middleware may use other software resources, such as executable programs) and b) was done once because the main objective of including new bundles is getting track of the already existing devices, rather than overloading the middleware with too many bundles in the middleware.

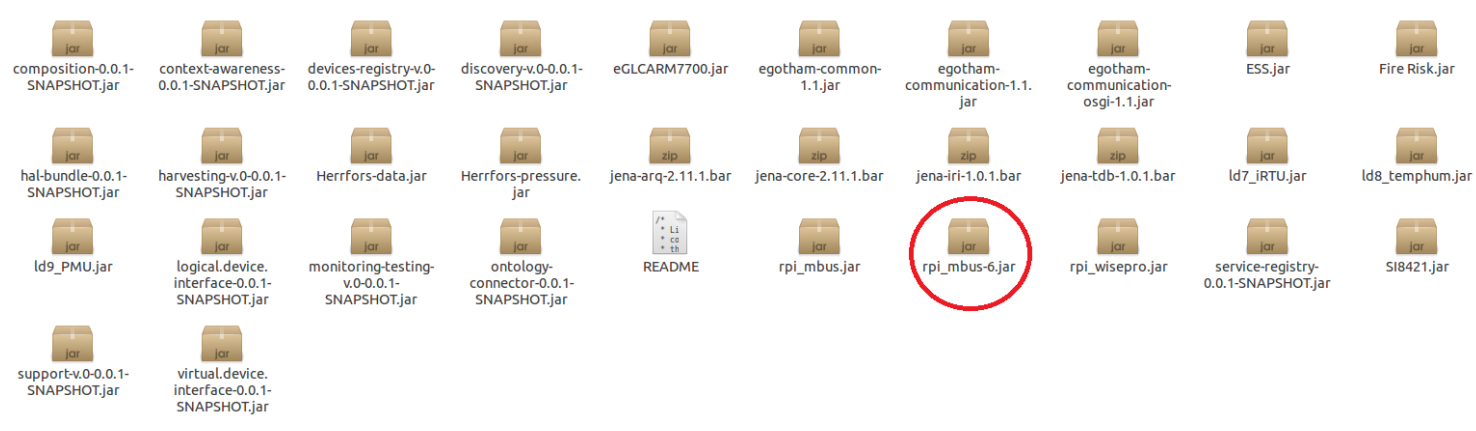

Figure 925. Middleware bundles and the new one created during registration

4. The features that were included in the list of registered devices and services will change to reflect the new devices that have been included. Once a device becomes registered, all the information that is provided about it becomes integrated in the system. In Figure 96, the new list that has been created includes the device that has become registered and its features. In addition to that, any service that can be provided by it will become available as well. 


\section{localhost:8181/cxf/discovery/discovery/listDevices}

This XML file does not appear to have any style information associated with it. The document tree i

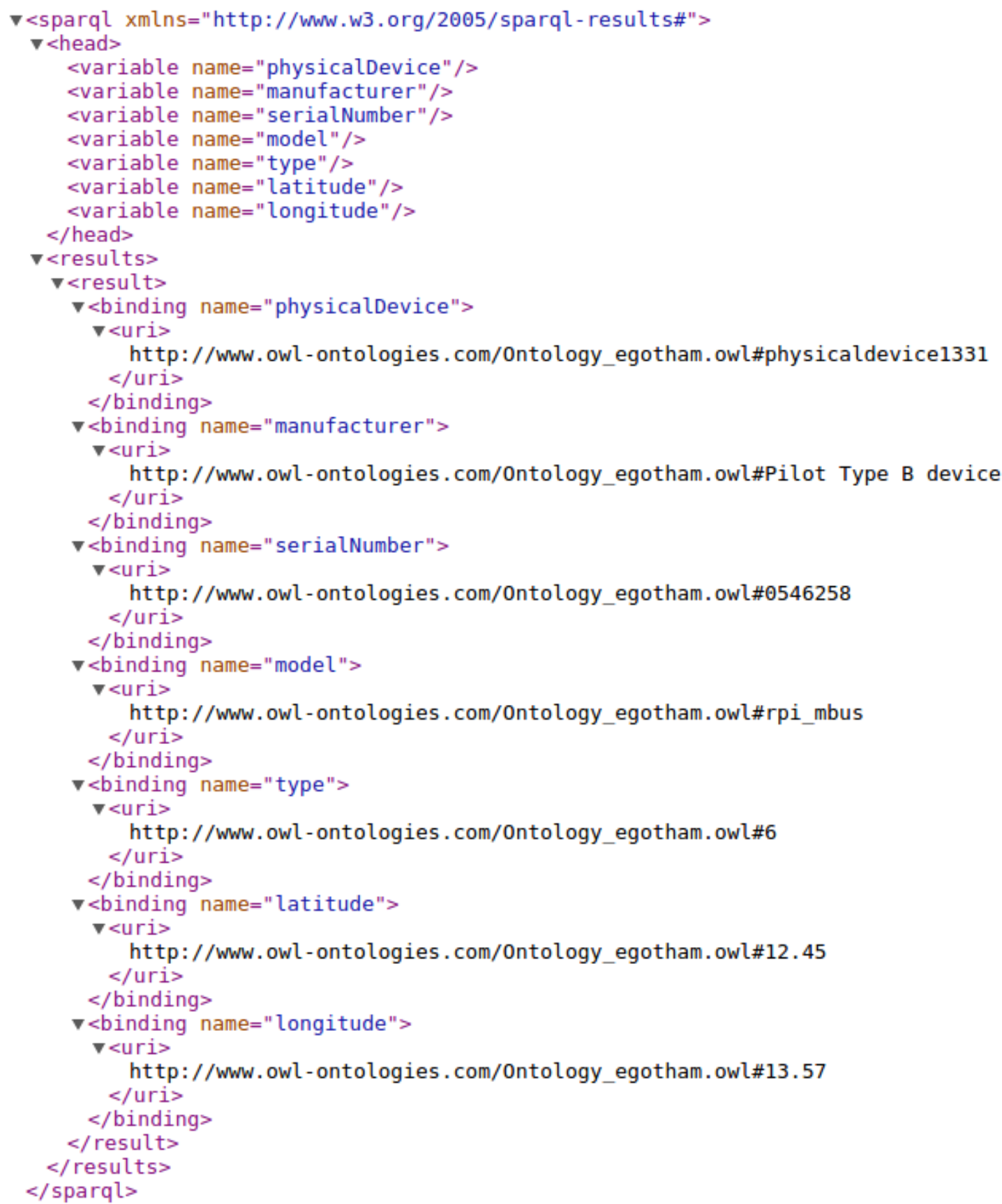

\section{Figure 936. Features of the device that has become registered}

5. After the registration, and provided that there were no issues during it, the services that can be offered by the device can be invoked from the application layer. Data requests will be carried out in the same fashion that was explained previously, invoking the service that wants to be used for data gathering. For example, if real-time electric current is requested, it will be done so via an XML message sent from the Middleware Access Point service connected to the application that started the request via URI by accessing the REST 
interface. The following XML is an example of how it would be used to send the request for electric current at a specific time.

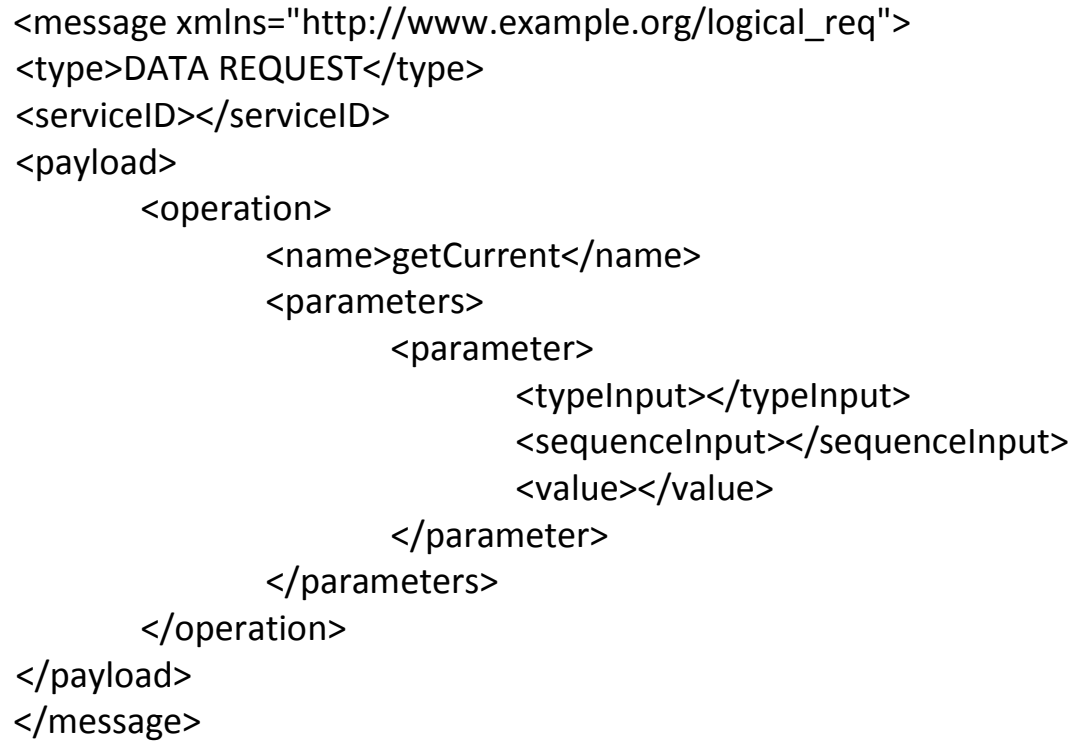

6. The request will be transferred through the distributed system until it reaches the device that is expected to have the information needed to answer it. Once the information is provided, it will be returned to the application that asked for it and the result will be shown by means of any resource that the application has, such as a Graphical User Interface or alike.

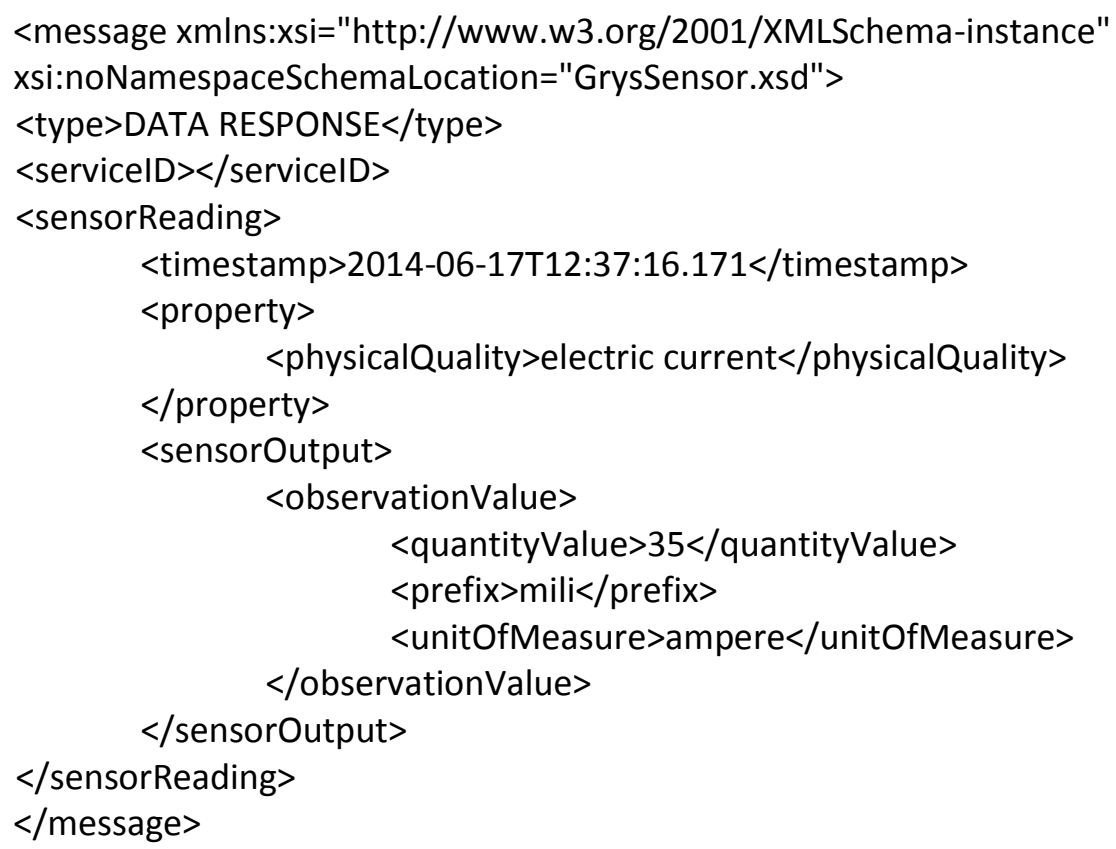

7. Lastly, this procedure has been carried out considering that the securitization of the interfaces has already been completed. It has been considered that the detailed procedure to make possible the securitization of the interfaces should rely in certifications rather 
than software components. Therefore, the procedure that has been included in this thesis reflects the one that was described in [154] and depicted in Figure 97:

1. In order to have secure transactions between elements using access from the layer where applications are located, some security -related infrastructure must be added to the system. An HTTP Apache server can be used to provide that infrastructure, as long as there is a machine using it [171]. Once the Apache Server has been created, a new SSL will be integrated in the system.

2. Whenever there is a communication, data will have to be re-directed through the securitized site. To make this possible, a new directive in the non-secure VirtualHost will have to be created at the default configuration file, so that the process will become automated anytime. The default fine is stored at /apache2/sitesavailable/000-default.conf in UNIX-based machines, and the sentence that has to be added to the file will be Redirect permanent / [URL used for securitized access]/. DocumentRoot resources will also need to be disabled and swapped by the former order.

3. Certificates will be created for the user willing to participate in data interchanges. Having the certificate as something provided by them as a self-signed Certificate Authority (CA) might be required. Additionally, entities participating in the communication require a user key and user certificate request; these latter must be signed to enable the Certificate Authority to create the certificate.

4. Consequently, the Certificate Signing Request (CSR) will be sent for its verification at the CA. If everything goes as planned and there are no issues found, the CSR will be signed with the key owned by the CA.

5. Finally, the signed Server/Client certificate will be delivered the Server/Client side of the communications link (in case the CA is not involved in the communication about to take place) and be used for information exchange.

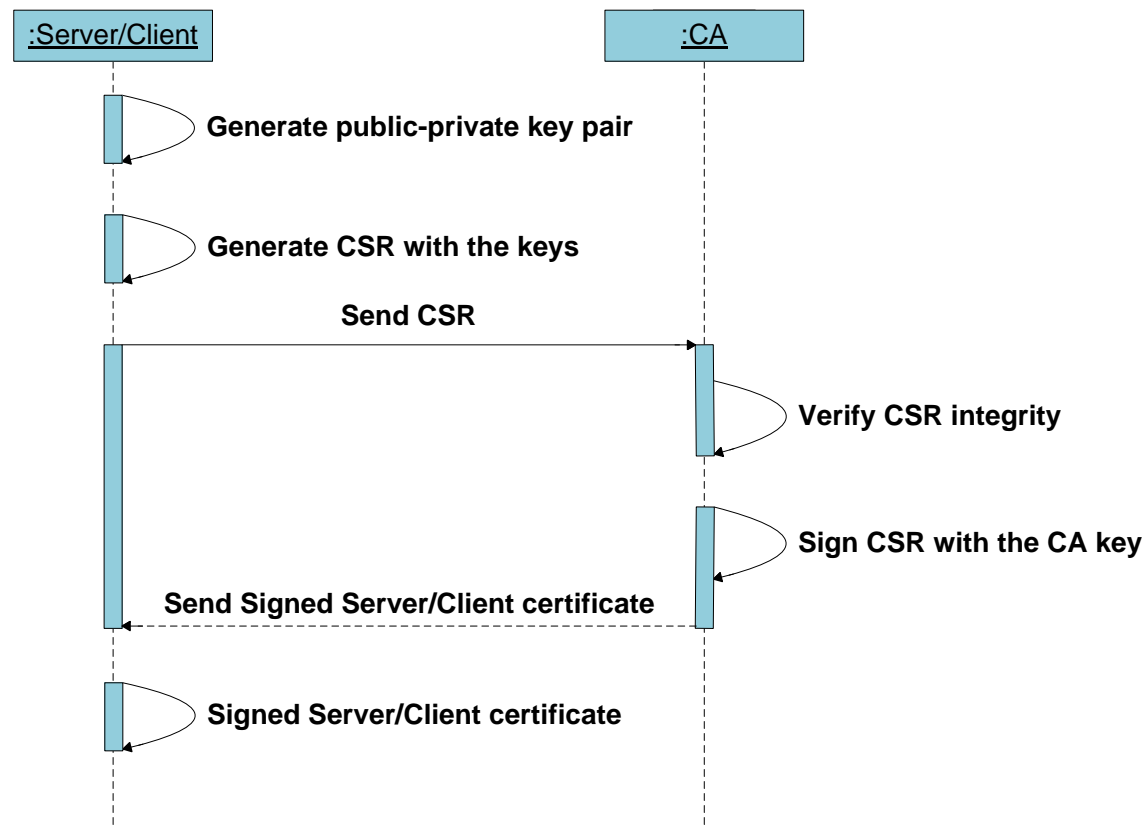

Figure 947. Security sequence of steps, as shown in [154] 
As far as security is concerned, while the option that was decided to be used was the one described here, there is also the possibility of having the securitization features as another separated software component in the middleware architecture. This is the option that has been applied in [176], where bundles for a JBoss Fuse have been equipped with the security capabilities provided by the library Bouncy Castle [155]. The component that used those libraries was tested to figure out its performance when either symmetric or asymmetric cyphering were used; it can be regarded as satisfactory (even though asymmetric ciphering may take even some minutes when 4092 bits long public and private keys are used, it is still acceptable because asymmetric cyphering takes always a longer time to be performed when compared to its symmetric counterpart, especially with long keys), so it can be concluded that the addition of components focused on providing security does not worsen the output of the overall system that has been used.

\subsection{Demonstrator tests}

The developed middleware solutions where deployed in two different demonstrators where the principles of what would become the Common Middleware Architecture would be used. It has to be remembered that the conception of the middleware architectural model that has been put forward in this document is a result of the development works made for the eGOTHAM and I3RES research projects, as well as the weaknesses found in the existing middleware architectures for the Smart Grid and the challenges associated to its standardization activities.

\subsubsection{Preliminary tests}

The main principles that guide semantic middleware for the Smart Grid were firstly introduced when preliminary versions of the middleware implementation works that were carried out in the LifeWear project. The middleware architecture that was present relied on software components installed in the nodes of a Wireless Sensor Network. The main challenge that had to be faced with this implementation was related to the capabilities of the motes of the WSN, which had a computational capability lower than usual pieces of equipment, such as a PC, or other pieces of equipment present in the Smart Grid, such as smart meters, PMUs or RTUs. Testing activities were carried out to assess the performance of the system, as it has been described in [177]. As it can be seen in Figure 98, several sets of simple service requests were carried out in order to know how it would take for data to be transferred in the wireless environment. The average time used to satisfy those requests after installing the middleware components responsible for simple and composed service requests was slightly larger than 700 milliseconds; it was deemed as acceptable considering the computational capabilities of the motes and the verbosity of the messages that was used. 


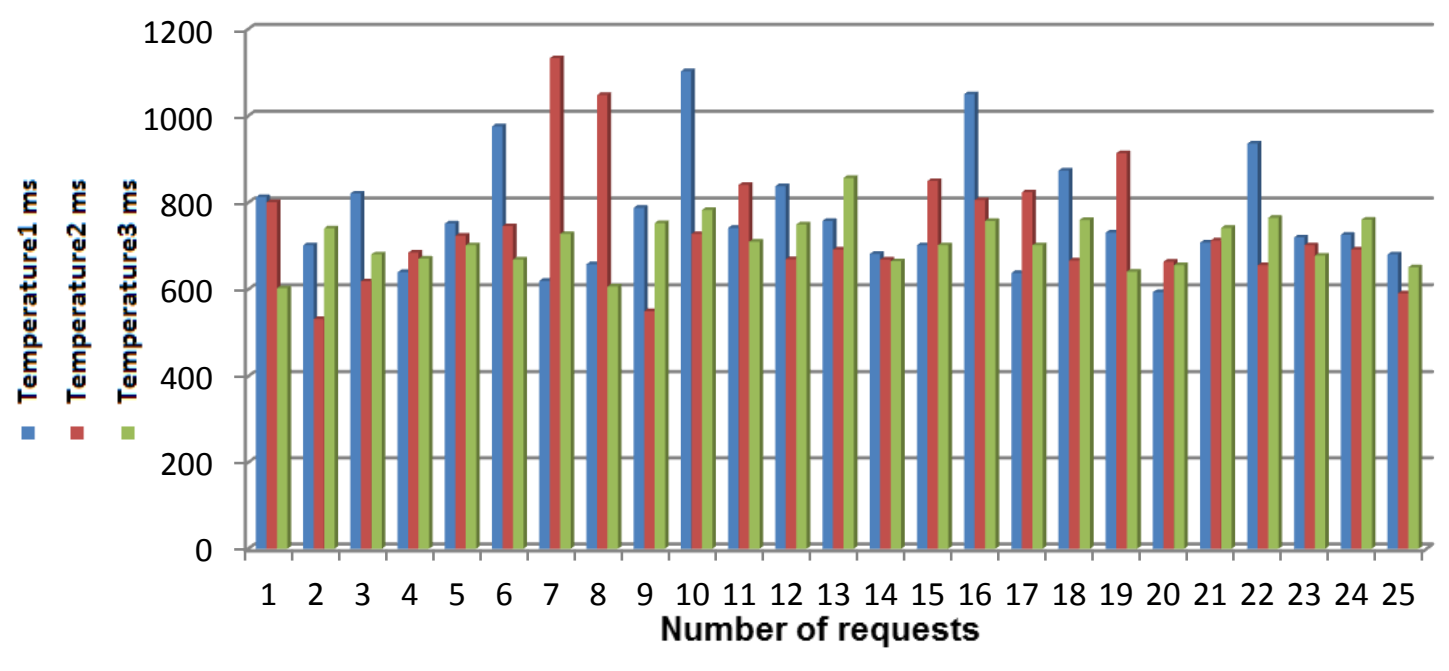

Figure 958. Time used to answer temperature requests done to nodes, as described in [177]

More tests were done for composed services: in the case that is portrayed in Figure 99, the time used in each of the requests that were performed has been included in the chart. The composed service was conceived as using three different kinds of data (body temperature, environment temperature and heartbeat rate) collected from different nodes in the network. The output would result in a high, medium or low risk of getting injuries while performing an exercise. Considering that three different pieces of information were required, as well as composing the service and sending the information back to the application layer, performance results were worse than the ones that had been obtained previously.

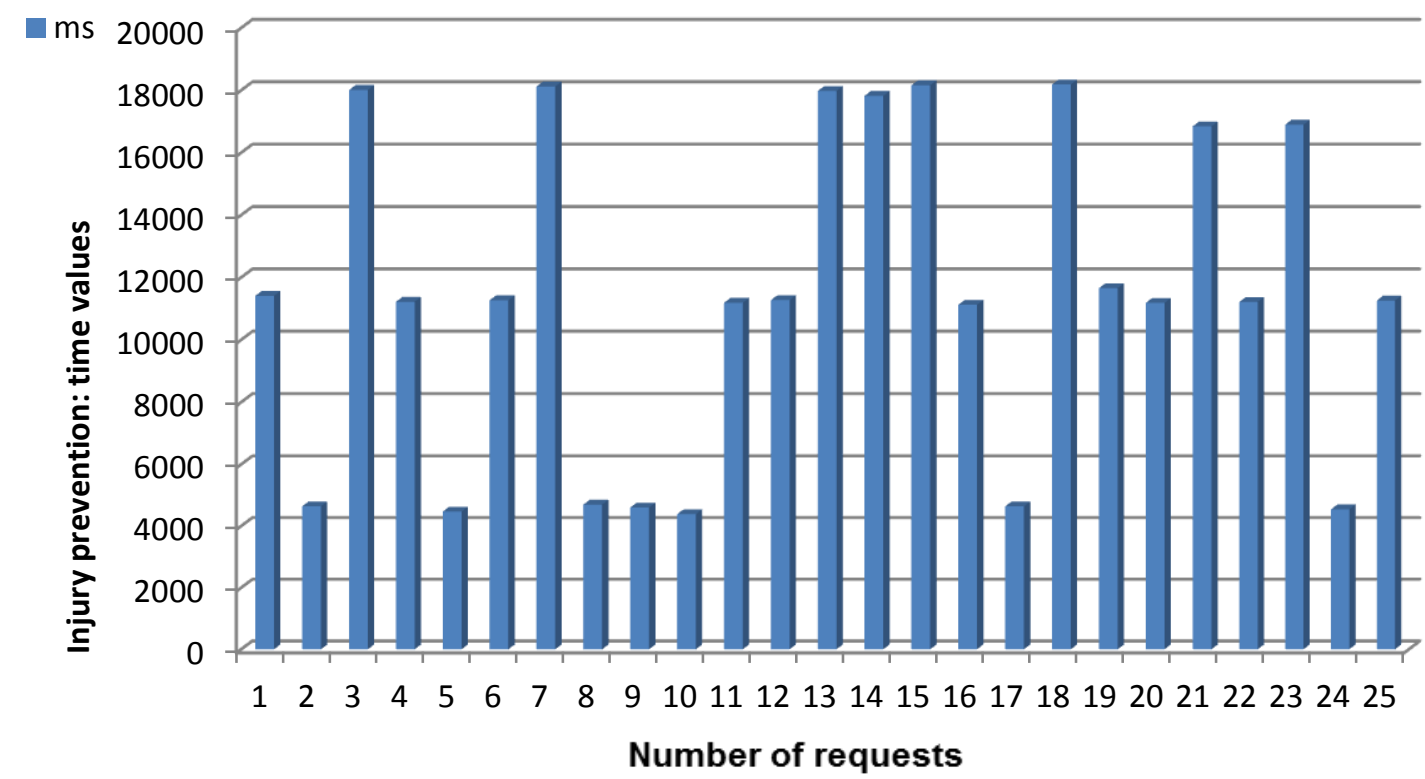

Figure 969. Time used to answer to obtain data from a composed service, as described in [177] 
After this first iteration of the middleware, a more advanced one that was expected to be used in the I3RES project was implemented in another WSN in order to check its future feasibility in a future Smart Grid environment. Although motes from a WSN are devices that cannot be easily compared to the ones present in the Smart Grid, the middleware that was developed this time had many features in common with what would become the middleware developments done for I3RES and e-GOTHAM. For example, as depicted in [178], simple and composed service requests were still carried out, but there were other elements that took part in the development works that had been carried out, such a semantic component or facilities for device and service registration. Furthermore, a first UML design of the elements that a middleware architecture for the Smart Grid should have were made as well. Some results regarding performance have been included in Figure 100. In this case, compose services were obtained in way lower time that before, but it was mostly due to the fact that only two simple services where used for composition, and the WSN that was built for testing purposes did not have as many nodes as the previous one.
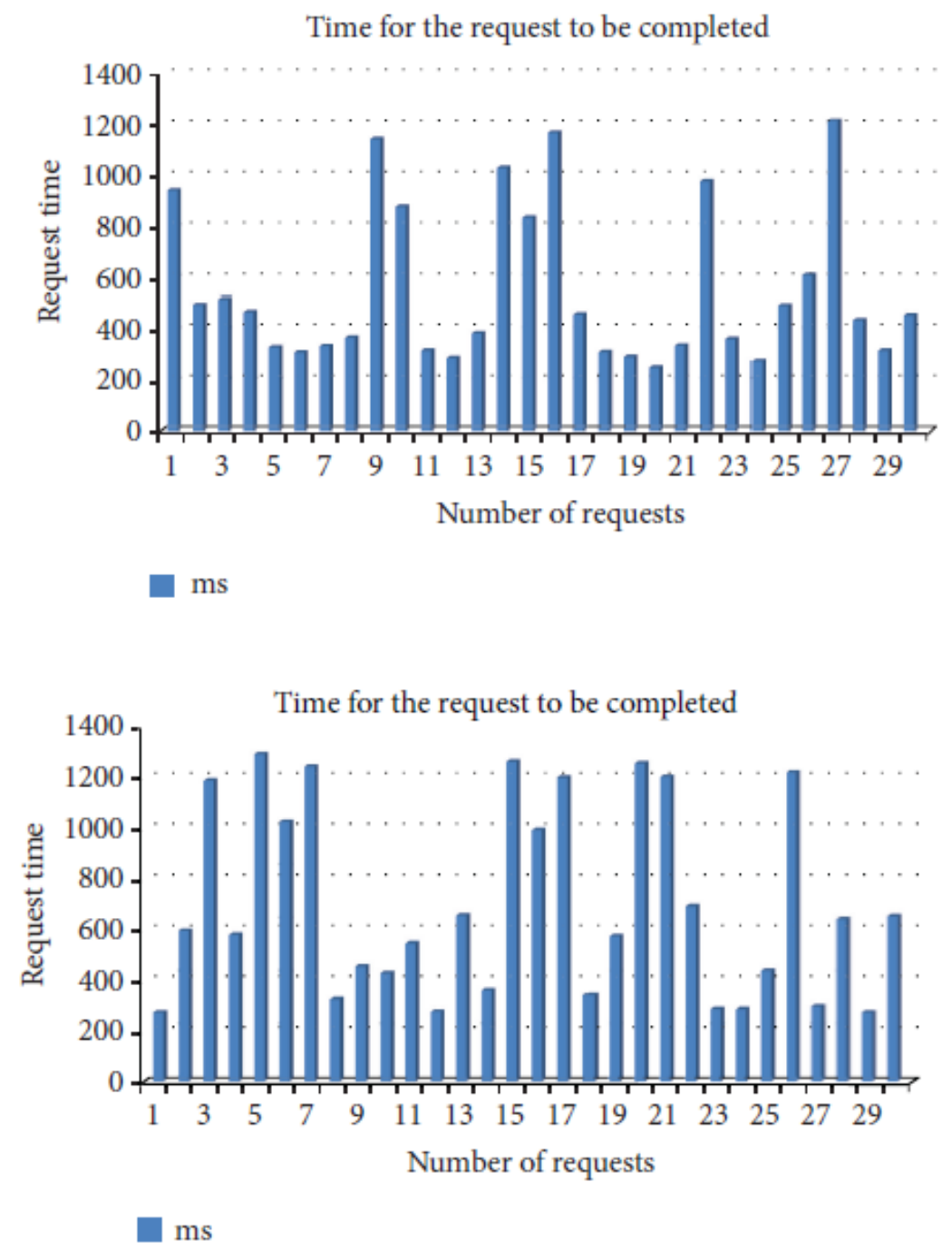

Figure 100. Performance results for simple (high graph) and composed (low graph) service requests, as described in [178] 
Lastly, while the implementation works in the e-GOTHAM project were being carried out, further testing activities were made as middleware components were codified. The main novelty of these testing activities was that an actual Local Controller was used. It was a Raspberry Pi-based project that made use of Arduino platforms and a sensor used to measure real-time current usage. The Local Controller features and performance are the main topic of the Bachelor thesis made by Luis Moreno [179].

\subsubsection{Laboratory scenario}

The first batch of tests was made in laboratories of partners that offered their equipment in order to find out how the developed software components would behave in other environment out of the local tests done in the equipment used for implementation. Although the pieces of hardware that were used for those tests were quite similar to the ones that had been used locally (regarding CPU and hardware capabilities), there were some extra actions that had to be carried out, which were related to the remote infrastructure required to access to the facilities of the partners and being able to execute commands remotely.

During the activities done with regards to testing in the e-GOTHAM project, more thorough and deep batches were developed in order to ensure that the middleware would behave as expected in the specifications that had been put forward. The two most prominent components that were checked have been included in the following tables. For example, the support component was tested on all the facilities that had been programmed to access the devices via Uniform Resource Identifier. The results can be seen in Table 16. 
Table 16. Support middleware component testing

\begin{tabular}{|c|c|c|c|c|c|c|}
\hline \multicolumn{7}{|c|}{ Test information for the Support component (e-GOTHAM) } \\
\hline Test Number & Test & Test Data & Expected result & Actual result & Status & Notes \\
\hline \multirow{4}{*}{1} & \multirow{4}{*}{ /contro|Appliance1/\{serviceID $\} /\{$ input $\}$} & $\begin{array}{l}\text { servicelD= } 4347 \\
\text { Input=on }\end{array}$ & ok & ok & $\begin{array}{l}\bigotimes \text { Pass } \\
\square \text { Fail }\end{array}$ & \\
\hline & & $\begin{array}{l}\text { servicelD= } 4347 \\
\text { Input=test }\end{array}$ & $\begin{array}{l}\text { Error - } \\
\text { parameters error }\end{array}$ & $\begin{array}{l}\text { Error - } \\
\text { parameters error }\end{array}$ & $\begin{array}{l}\bigotimes \text { Pass } \\
\square \text { Fail }\end{array}$ & \\
\hline & & $\begin{array}{l}\text { servicelD= test } \\
\text { Input=on }\end{array}$ & $\begin{array}{l}\text { Error - } \\
\text { Parameters error }\end{array}$ & Error 500 & $\begin{array}{l}\bigotimes \text { Pass } \\
\square \text { Fail }\end{array}$ & \\
\hline & & $\begin{array}{l}\text { servicelD= } 3423 \\
\text { Input=on }\end{array}$ & $\begin{array}{l}\text { Error - } \\
\text { parameters error }\end{array}$ & $\begin{array}{l}\text { Error - } \\
\text { parameters error }\end{array}$ & $\begin{array}{l}\bigotimes \text { Pass } \\
\square \text { Fail }\end{array}$ & $\begin{array}{l}3423 \text { was a } \\
\text { random number }\end{array}$ \\
\hline \multirow{4}{*}{2} & \multirow{4}{*}{ /controlAppliance1/\{serviceID\}/\{input\} } & $\begin{array}{l}\text { servicelD= } 4347 \\
\text { Input=on }\end{array}$ & ok & ok & $\begin{array}{l}\bigotimes \text { Pass } \\
\square \text { Fail }\end{array}$ & \\
\hline & & $\begin{array}{l}\text { servicelD= } 4347 \\
\text { Input=test }\end{array}$ & $\begin{array}{l}\text { Error - } \\
\text { parameters error }\end{array}$ & $\begin{array}{l}\text { Error - } \\
\text { parameters error }\end{array}$ & $\begin{array}{l}\bigotimes \text { Pass } \\
\square \text { Fail }\end{array}$ & \\
\hline & & $\begin{array}{l}\text { servicelD= test } \\
\text { Input=on }\end{array}$ & $\begin{array}{l}\text { Error - } \\
\text { Parameters error }\end{array}$ & Error 500 & $\begin{array}{l}\bigotimes \text { Pass } \\
\square \text { Fail }\end{array}$ & \\
\hline & & $\begin{array}{l}\text { servicelD= } 3423 \\
\text { Input=on }\end{array}$ & $\begin{array}{l}\text { Error - } \\
\text { parameters error }\end{array}$ & $\begin{array}{l}\text { Error - } \\
\text { parameters error }\end{array}$ & $\begin{array}{l}\bigotimes \text { Pass } \\
\square \text { Fail }\end{array}$ & $\begin{array}{l}3423 \text { was a } \\
\text { random number }\end{array}$ \\
\hline \multirow{3}{*}{3} & \multirow{3}{*}{ /controlAppliance1/\{serviceID\}/\{input\} } & $\begin{array}{l}\text { Digital input= on } \\
\text { servicelD=4347 }\end{array}$ & ok & ok & $\begin{array}{l}\bigotimes \text { Pass } \\
\square \text { Fail }\end{array}$ & \\
\hline & & $I D=4347$ & ok & ok & $\begin{array}{l}\bigotimes \text { Pass } \\
\square \text { Fail }\end{array}$ & \\
\hline & & $I D=3423$ & $\begin{array}{l}\text { Error - } \\
\text { parameters error }\end{array}$ & $\begin{array}{l}\text { Error - } \\
\text { parameters error }\end{array}$ & $\begin{array}{l}\bigotimes \text { Pass } \\
\square \text { Fail }\end{array}$ & $\begin{array}{l}3423 \text { was a } \\
\text { random number }\end{array}$ \\
\hline \multirow[t]{2}{*}{4} & \multirow[t]{2}{*}{ /controlAppliance1/\{serviceID $\} /\{$ input $\}$} & ID=test & $\begin{array}{l}\text { Error - } \\
\text { parameters error }\end{array}$ & $\begin{array}{l}\text { Error - } \\
\text { parameters error }\end{array}$ & $\begin{array}{l}\triangle \text { Pass } \\
\square \text { Fail }\end{array}$ & \\
\hline & & Digital input $=$ on & ok & ok & $\bigotimes$ Pass & \\
\hline
\end{tabular}


Chapter 4. Validation of the proposal and results

\begin{tabular}{|c|c|c|c|c|c|c|}
\hline & & servicelD=4347 & & & Fail & \\
\hline & & $\mathrm{ID}=4347$ & ok & $\begin{array}{l}\text { ok, but no } \\
\text { content returned } \\
(204)\end{array}$ & $\begin{array}{l}\bigotimes \text { Pass } \\
\square \text { Fail }\end{array}$ & \\
\hline & & $I D=3423$ & $\begin{array}{l}\text { Error - } \\
\text { parameters error }\end{array}$ & $\begin{array}{l}\text { Error - } \\
\text { parameters error }\end{array}$ & $\begin{array}{l}\triangle \text { Pass } \\
\square \text { Fail }\end{array}$ & $\begin{array}{l}3423 \text { was a } \\
\text { random number }\end{array}$ \\
\hline \multirow{4}{*}{5} & \multirow{4}{*}{ /controlAppliance $1 /\{$ servicelD $\} /\{$ input $\}$} & $I D=$ test & $\begin{array}{l}\text { Error- } \\
\text { parameters error }\end{array}$ & $\begin{array}{l}\text { Error - } \\
\text { parameters error }\end{array}$ & $\begin{array}{l}\bigotimes \text { Pass } \\
\square \text { Fail }\end{array}$ & \\
\hline & & $\begin{array}{l}\text { Get request with } \\
\text { no parameters }\end{array}$ & ok & Error 500 & $\begin{array}{l}\triangle \text { Pass } \\
\square \text { Fail }\end{array}$ & \\
\hline & & $\mathrm{Id}=4347$ & ok & ok & $\begin{array}{l}\bigotimes \text { Pass } \\
\square \text { Fail }\end{array}$ & \\
\hline & & $\mathrm{Id}=3423$ & $\begin{array}{l}\text { Error - } \\
\text { parameters error }\end{array}$ & $\begin{array}{l}\text { Error - } \\
\text { parameters error }\end{array}$ & $\begin{array}{l}\bigotimes \text { Pass } \\
\square \text { Fail }\end{array}$ & $\begin{array}{l}3423 \text { was a } \\
\text { random number }\end{array}$ \\
\hline \multirow{4}{*}{6} & \multirow{4}{*}{ /controlAppliance1/\{serviceID\}/\{input\} } & Id=test & $\begin{array}{l}\text { Error - } \\
\text { parameters error }\end{array}$ & $\begin{array}{l}\text { Error - } \\
\text { parameters error }\end{array}$ & $\begin{array}{l}\bigotimes \text { Pass } \\
\square \text { Fail }\end{array}$ & \\
\hline & & $\mathrm{Id}=4347$ & ok & ok & $\begin{array}{l}\bigotimes \text { Pass } \\
\square \text { Fail }\end{array}$ & \\
\hline & & $\mathrm{Id}=3423$ & $\begin{array}{l}\text { Error - } \\
\text { parameters error }\end{array}$ & $\begin{array}{l}\text { Error - } \\
\text { parameters error }\end{array}$ & $\begin{array}{l}\bigotimes \text { Pass } \\
\square \text { Fail }\end{array}$ & $\begin{array}{l}3423 \text { was a } \\
\text { random number }\end{array}$ \\
\hline & & Id=test & $\begin{array}{l}\text { Error - } \\
\text { parameters error }\end{array}$ & $\begin{array}{l}\text { Error - } \\
\text { parameters error }\end{array}$ & $\begin{array}{l}\bigotimes \text { Pass } \\
\square \text { Fail }\end{array}$ & \\
\hline \multirow{4}{*}{7} & \multirow{4}{*}{ /controlAppliance1/\{serviceID\}/\{input\} } & $\mathrm{Id}=4347$ & ok & ok & $\begin{array}{l}\bigotimes \text { Pass } \\
\square \text { Fail }\end{array}$ & \\
\hline & & $\mathrm{Id}=3423$ & $\begin{array}{l}\text { Error - } \\
\text { parameters error }\end{array}$ & $\begin{array}{l}\text { Error - } \\
\text { parameters error }\end{array}$ & $\begin{array}{l}\bigotimes \text { Pass } \\
\square \text { Fail }\end{array}$ & $\begin{array}{l}3423 \text { was a } \\
\text { random number }\end{array}$ \\
\hline & & Id=test & $\begin{array}{l}\text { Error - } \\
\text { parameters error }\end{array}$ & $\begin{array}{l}\text { Error - } \\
\text { parameters error }\end{array}$ & $\begin{array}{l}\bigotimes \text { Pass } \\
\square \text { Fail }\end{array}$ & \\
\hline & & $\mathrm{Id}=4347$ & ok & ok & $\bigotimes$ Pass & \\
\hline
\end{tabular}


Chapter 4. Validation of the proposal and results

\begin{tabular}{|c|c|c|c|c|c|c|}
\hline & & & & & Fail & \\
\hline \multirow{4}{*}{8} & \multirow{4}{*}{ /controlAppliance $1 /\{$ serviceID $\} /\{$ input $\}$} & $\mathrm{Id}=3423$ & $\begin{array}{l}\text { Error - } \\
\text { parameters error }\end{array}$ & $\begin{array}{l}\text { Error - } \\
\text { parameters error }\end{array}$ & $\begin{array}{l}\bigotimes \text { Pass } \\
\square \text { Fail }\end{array}$ & $\begin{array}{l}3423 \text { was a } \\
\text { random number }\end{array}$ \\
\hline & & $I d=$ test & $\begin{array}{l}\text { Error - } \\
\text { parameters error }\end{array}$ & $\begin{array}{l}\text { Error - } \\
\text { parameters error }\end{array}$ & $\begin{array}{l}\bigotimes \text { Pass } \\
\square \text { Fail }\end{array}$ & \\
\hline & & $\mathrm{Id}=4347$ & ok & ok & $\begin{array}{l}\bigotimes \text { Pass } \\
\square \text { Fail }\end{array}$ & \\
\hline & & $\mathrm{ld}=3423$ & $\begin{array}{l}\text { Error - } \\
\text { parameters error }\end{array}$ & $\begin{array}{l}\text { Error - } \\
\text { parameters error }\end{array}$ & $\begin{array}{l}\bigotimes \text { Pass } \\
\square \text { Fail }\end{array}$ & $\begin{array}{l}3423 \text { was a } \\
\text { random number }\end{array}$ \\
\hline \multirow{4}{*}{9} & \multirow{4}{*}{ /controlAppliance1/\{serviceID\}/\{input $\}$} & Id=test & $\begin{array}{l}\text { Error - } \\
\text { parameters error }\end{array}$ & $\begin{array}{l}\text { Error - } \\
\text { parameters error }\end{array}$ & $\begin{array}{l}\bigotimes \text { Pass } \\
\square \text { Fail }\end{array}$ & \\
\hline & & $I d=4347$ & ok & ok & $\begin{array}{l}\bigotimes \text { Pass } \\
\square \text { Fail }\end{array}$ & \\
\hline & & $\mathrm{Id}=3423$ & $\begin{array}{l}\text { Response with } \\
\text { empty results }\end{array}$ & $\begin{array}{l}\text { Response with } \\
\text { empty results }\end{array}$ & $\begin{array}{l}\bigotimes \text { Pass } \\
\square \text { Fail }\end{array}$ & \\
\hline & & Id=test & $\begin{array}{l}\text { Response with } \\
\text { empty results }\end{array}$ & $\begin{array}{l}\text { Response with } \\
\text { empty results }\end{array}$ & $\begin{array}{l}\triangle \text { Pass } \\
\square \text { Fail }\end{array}$ & \\
\hline \multirow{4}{*}{10} & \multirow{4}{*}{ /controlAppliance1/\{servicelD\}/\{input $\}$} & $\mathrm{Id}=4347$ & $\begin{array}{l}\text { Response with } \\
\text { empty results }\end{array}$ & $\begin{array}{l}\text { Response with } \\
\text { empty results }\end{array}$ & $\begin{array}{l}\triangle \text { Pass } \\
\square \text { Fail }\end{array}$ & \\
\hline & & $\mathrm{Id}=3423$ & $\begin{array}{l}\text { Response with } \\
\text { empty results }\end{array}$ & $\begin{array}{l}\text { Response with } \\
\text { empty results }\end{array}$ & $\begin{array}{l}\bigotimes \text { Pass } \\
\square \text { Fail }\end{array}$ & \\
\hline & & $I d=$ test & $\begin{array}{l}\text { Response with } \\
\text { empty results }\end{array}$ & $\begin{array}{l}\text { Response with } \\
\text { empty results }\end{array}$ & $\begin{array}{l}\bigotimes \text { Pass } \\
\square \text { Fail }\end{array}$ & \\
\hline & & $\mathrm{Id}=4347$ & ok & ok & $\begin{array}{l}\bigotimes \text { Pass } \\
\square \text { Fail }\end{array}$ & \\
\hline \multirow[t]{2}{*}{11} & \multirow[t]{2}{*}{ /controlAppliance1/\{servicelD\}/\{input\} } & Id=test & $\begin{array}{l}\text { Error - } \\
\text { parameters error }\end{array}$ & $\begin{array}{l}\text { Error - } \\
\text { parameters error }\end{array}$ & $\begin{array}{l}\bigotimes \text { Pass } \\
\square \text { Fail }\end{array}$ & \\
\hline & & $\mathrm{Id}=4347$ & ok & ok & $\bigotimes$ Pass & \\
\hline
\end{tabular}


Chapter 4. Validation of the proposal and results

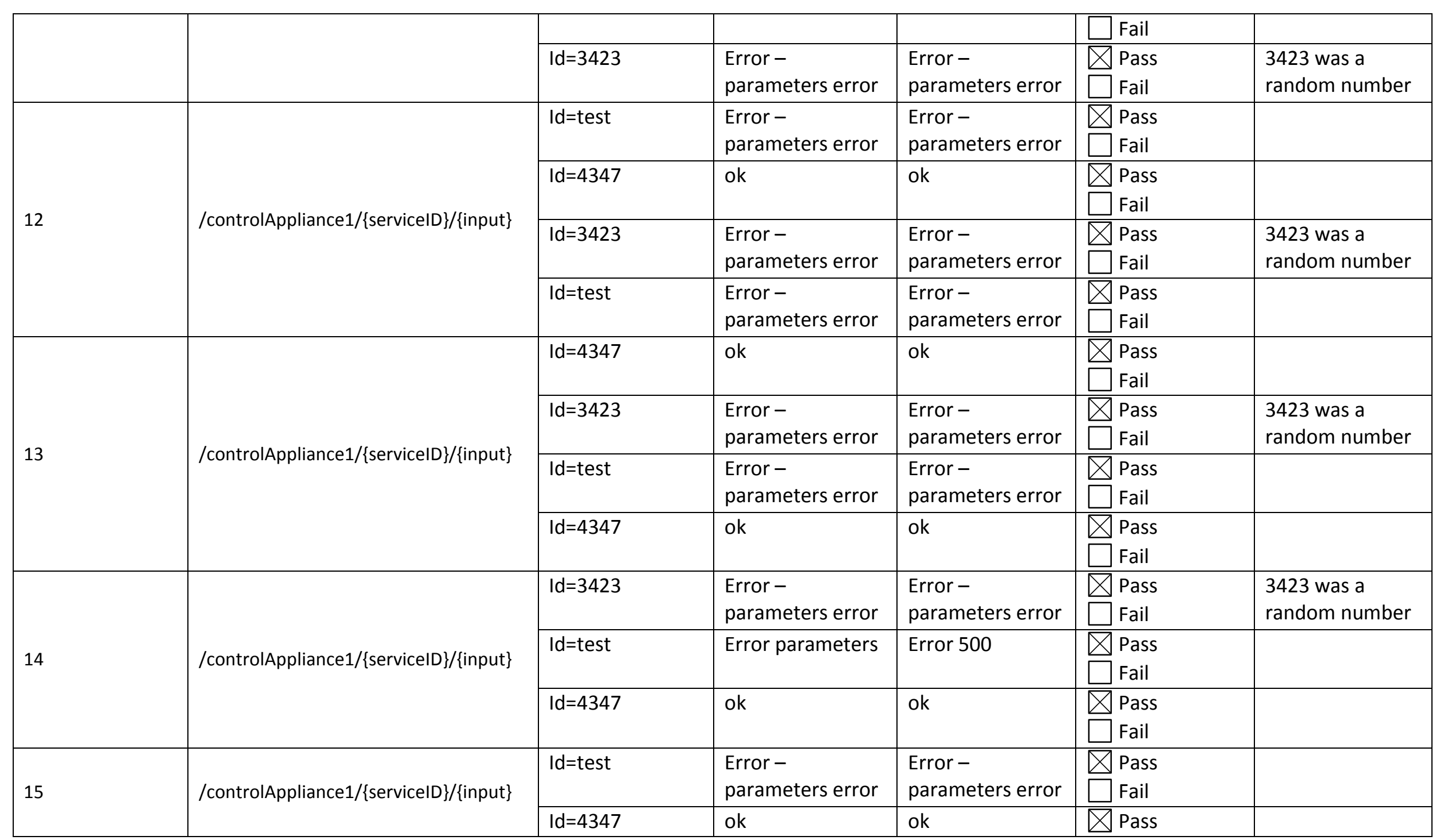


Chapter 4. Validation of the proposal and results

\begin{tabular}{|c|c|c|c|c|c|c|c|}
\hline & & & & & & Fail & \\
\hline & & $I d=3423$ & Error parameters & $\begin{array}{l}\text { Server does not } \\
\text { respond. }\end{array}$ & 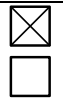 & $\begin{array}{l}\text { Pass } \\
\text { Fail }\end{array}$ & $\begin{array}{l}3423 \text { was a } \\
\text { random number }\end{array}$ \\
\hline & & Id=test & $\begin{array}{l}\text { Error - } \\
\text { parameters error }\end{array}$ & $\begin{array}{l}\text { Error - } \\
\text { parameters error }\end{array}$ & $\triangle$ & $\begin{array}{l}\text { Pass } \\
\text { Fail }\end{array}$ & \\
\hline \multirow{4}{*}{16} & \multirow{4}{*}{ /controlAppliance1/\{serviceID\}/\{input\} } & $\mathrm{Id}=4347$ & ok & ok & $\triangle$ & $\begin{array}{l}\text { Pass } \\
\text { Fail }\end{array}$ & \\
\hline & & $\mathrm{Id}=3423$ & $\begin{array}{l}\text { Error - } \\
\text { parameters error }\end{array}$ & $\begin{array}{l}\text { Error - } \\
\text { parameters error }\end{array}$ & 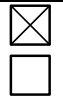 & $\begin{array}{l}\text { Pass } \\
\text { Fail }\end{array}$ & $\begin{array}{l}3423 \text { was a } \\
\text { random number }\end{array}$ \\
\hline & & Id=test & $\begin{array}{l}\text { Error - } \\
\text { parameters error }\end{array}$ & $\begin{array}{l}\text { Error - } \\
\text { parameters error }\end{array}$ & 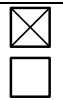 & $\begin{array}{l}\text { Pass } \\
\text { Fail }\end{array}$ & \\
\hline & & $\mathrm{Id}=4347$ & ok & ok & 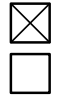 & $\begin{array}{l}\text { Pass } \\
\text { Fail }\end{array}$ & \\
\hline \multirow{4}{*}{17} & \multirow{4}{*}{ /controlAppliance1/\{serviceID\}/\{input\} } & $I d=3423$ & $\begin{array}{l}\text { Error - } \\
\text { parameters error }\end{array}$ & $\begin{array}{l}\text { Error - } \\
\text { parameters error }\end{array}$ & 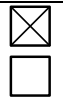 & $\begin{array}{l}\text { Pass } \\
\text { Fail }\end{array}$ & $\begin{array}{l}3423 \text { was a } \\
\text { random number }\end{array}$ \\
\hline & & Id=test & $\begin{array}{l}\text { Error - } \\
\text { parameters error }\end{array}$ & $\begin{array}{l}\text { Error - } \\
\text { parameters error }\end{array}$ & 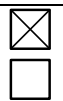 & $\begin{array}{l}\text { Pass } \\
\text { Fail }\end{array}$ & \\
\hline & & $I d=4347$ & ok & ok & 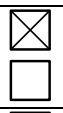 & $\begin{array}{l}\text { Pass } \\
\text { Fail }\end{array}$ & \\
\hline & & $I d=3423$ & $\begin{array}{l}\text { Error - } \\
\text { parameters error }\end{array}$ & $\begin{array}{l}\text { Error - } \\
\text { parameters error }\end{array}$ & 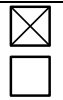 & $\begin{array}{l}\text { Pass } \\
\text { Fail }\end{array}$ & $\begin{array}{l}3423 \text { was a } \\
\text { random number }\end{array}$ \\
\hline \multirow{4}{*}{18} & \multirow{4}{*}{ /controlAppliance $1 /\{$ servicelD $\} /\{$ input $\}$} & Id=test & $\begin{array}{l}\text { Error - } \\
\text { parameters error }\end{array}$ & $\begin{array}{l}\text { Error - } \\
\text { parameters error }\end{array}$ & & $\begin{array}{l}\text { Pass } \\
\text { Fail }\end{array}$ & \\
\hline & & $\mathrm{Id}=4347$ & ok & ok & 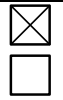 & $\begin{array}{l}\text { Pass } \\
\text { Fail }\end{array}$ & \\
\hline & & $I d=3423$ & $\begin{array}{l}\text { Error - } \\
\text { parameters error }\end{array}$ & $\begin{array}{l}\text { Error - } \\
\text { parameters error }\end{array}$ & & $\begin{array}{l}\text { Pass } \\
\text { Fail }\end{array}$ & $\begin{array}{l}3423 \text { was a } \\
\text { random number }\end{array}$ \\
\hline & & $I d=$ test & Error - & Error - & $\bigotimes$ & Pass & \\
\hline
\end{tabular}


Chapter 4. Validation of the proposal and results

\begin{tabular}{|c|c|c|c|c|c|c|}
\hline & & & parameters error & parameters error & $\square$ Fail & \\
\hline \multirow{4}{*}{19} & \multirow{4}{*}{ /controlAppliance1/\{servicelD\}/\{input\} } & $\mathrm{Id}=4347$ & ok & ok & $\begin{array}{l}\bigotimes \text { Pass } \\
\square \text { Fail }\end{array}$ & \\
\hline & & $\mathrm{Id}=3423$ & $\begin{array}{l}\text { Error - } \\
\text { parameters error }\end{array}$ & $\begin{array}{l}\text { Error - } \\
\text { parameters error }\end{array}$ & $\begin{array}{l}\bigotimes \text { Pass } \\
\square \text { Fail }\end{array}$ & $\begin{array}{l}3423 \text { was a } \\
\text { random number }\end{array}$ \\
\hline & & Id=test & $\begin{array}{l}\text { Error - } \\
\text { parameters error }\end{array}$ & $\begin{array}{l}\text { Error - } \\
\text { parameters error }\end{array}$ & $\begin{array}{l}\triangle \text { Pass } \\
\square \text { Fail }\end{array}$ & \\
\hline & & $I d=4347$ & ok & ok & $\begin{array}{l}\bigotimes \text { Pass } \\
\square \text { Fail }\end{array}$ & \\
\hline \multirow{4}{*}{20} & \multirow{4}{*}{ /controlAppliance1/\{servicelD\}/\{input $\}$} & $I d=3423$ & $\begin{array}{l}\text { Error - } \\
\text { parameters error }\end{array}$ & $\begin{array}{l}\text { Error - } \\
\text { parameters error }\end{array}$ & $\begin{array}{l}\bigotimes \text { Pass } \\
\square \text { Fail }\end{array}$ & $\begin{array}{l}3423 \text { was a } \\
\text { random number }\end{array}$ \\
\hline & & Id=test & $\begin{array}{l}\text { Error - } \\
\text { parameters error }\end{array}$ & $\begin{array}{l}\text { Error - } \\
\text { parameters error }\end{array}$ & $\begin{array}{l}\bigotimes \text { Pass } \\
\square \text { Fail }\end{array}$ & \\
\hline & & $\mathrm{Id}=4347$ & ok & ok & $\begin{array}{l}\bigotimes \text { Pass } \\
\square \text { Fail }\end{array}$ & \\
\hline & & $I d=3423$ & $\begin{array}{l}\text { Error - } \\
\text { parameters error }\end{array}$ & $\begin{array}{l}\text { Error - } \\
\text { parameters error }\end{array}$ & $\begin{array}{l}\triangle \text { Pass } \\
\square \text { Fail }\end{array}$ & $\begin{array}{l}3423 \text { was a } \\
\text { random number }\end{array}$ \\
\hline \multirow{4}{*}{21} & \multirow{4}{*}{ /controlAppliance1/\{servicelD\}/\{input $\}$} & Id=test & $\begin{array}{l}\text { Error - } \\
\text { parameters error }\end{array}$ & $\begin{array}{l}\text { Error - } \\
\text { parameters error }\end{array}$ & $\begin{array}{l}\bigotimes \text { Pass } \\
\square \text { Fail }\end{array}$ & \\
\hline & & $\mathrm{Id}=4347$ & ok & ok & $\begin{array}{l}\bigotimes \text { Pass } \\
\square \text { Fail }\end{array}$ & \\
\hline & & $\mathrm{Id}=3423$ & $\begin{array}{l}\text { Error- } \\
\text { parameters error }\end{array}$ & $\begin{array}{l}\text { Error - } \\
\text { parameters error }\end{array}$ & $\begin{array}{l}\bigotimes \text { Pass } \\
\square \text { Fail }\end{array}$ & $\begin{array}{l}3423 \text { was a } \\
\text { random number }\end{array}$ \\
\hline & & Id=test & $\begin{array}{l}\text { Error - } \\
\text { parameters error }\end{array}$ & $\begin{array}{l}\text { Error - } \\
\text { parameters error }\end{array}$ & $\begin{array}{l}\triangle \text { Pass } \\
\square \text { Fail }\end{array}$ & \\
\hline \multirow[t]{2}{*}{22} & \multirow[t]{2}{*}{ /controlAppliance1/\{servicelD\}/\{input $\}$} & $\begin{array}{l}\text { Digital input= on } \\
\text { servicelD=4347 }\end{array}$ & ok & ok & $\begin{array}{l}\triangle \text { Pass } \\
\square \text { Fail }\end{array}$ & \\
\hline & & $\mathrm{Id}=4347$ & ok & ok & $\triangle$ Pass & \\
\hline
\end{tabular}


Chapter 4. Validation of the proposal and results

\begin{tabular}{|c|c|c|c|c|c|c|}
\hline & & & & & Fail & \\
\hline & & Id=test & $\begin{array}{l}\text { Error - } \\
\text { parameters error }\end{array}$ & $\begin{array}{l}\text { Error - } \\
\text { parameters error }\end{array}$ & $\begin{array}{l}\bigotimes \text { Pass } \\
\square \text { Fail }\end{array}$ & \\
\hline & & $\begin{array}{l}\text { Get request with } \\
\text { no parameters }\end{array}$ & ok & ok & $\begin{array}{l}\bigotimes \text { Pass } \\
\square \text { Fail }\end{array}$ & \\
\hline \multirow{4}{*}{23} & \multirow{4}{*}{ /controlAppliance1/\{serviceID\}/\{input\} } & $\mathrm{Id}=4347$ & ok & ok & $\begin{array}{l}\triangle \text { Pass } \\
\square \text { Fail }\end{array}$ & \\
\hline & & $\mathrm{Id}=3423$ & $\begin{array}{l}\text { Error - } \\
\text { parameters error }\end{array}$ & $\begin{array}{l}\text { Error - } \\
\text { parameters error }\end{array}$ & $\begin{array}{l}\bigotimes \text { Pass } \\
\square \text { Fail }\end{array}$ & $\begin{array}{l}3423 \text { was a } \\
\text { random number }\end{array}$ \\
\hline & & Id=test & $\begin{array}{l}\text { Error - } \\
\text { parameters error }\end{array}$ & $\begin{array}{l}\text { Error - } \\
\text { parameters error }\end{array}$ & $\begin{array}{l}\triangle \text { Pass } \\
\square \text { Fail }\end{array}$ & \\
\hline & & $\mathrm{Id}=4347$ & ok & ok & $\begin{array}{l}\bigotimes \text { Pass } \\
\square \text { Fail }\end{array}$ & \\
\hline \multirow{4}{*}{24} & \multirow{4}{*}{ /controlAppliance $1 /\{$ serviceID $\} /\{$ input $\}$} & $\mathrm{Id}=3423$ & $\begin{array}{l}\text { Error - } \\
\text { parameters error }\end{array}$ & $\begin{array}{l}\text { Error - } \\
\text { parameters error }\end{array}$ & $\begin{array}{l}\bigotimes \text { Pass } \\
\square \text { Fail }\end{array}$ & $\begin{array}{l}3423 \text { was a } \\
\text { random number }\end{array}$ \\
\hline & & Id=test & $\begin{array}{l}\text { Error - } \\
\text { parameters error }\end{array}$ & $\begin{array}{l}\text { Error - } \\
\text { parameters error }\end{array}$ & $\begin{array}{l}\triangle \text { Pass } \\
\square \text { Fail }\end{array}$ & \\
\hline & & $I d=4347$ & ok & ok & $\begin{array}{l}\bigotimes \text { Pass } \\
\square \text { Fail }\end{array}$ & \\
\hline & & $I d=3423$ & $\begin{array}{l}\text { Error - } \\
\text { parameters error }\end{array}$ & $\begin{array}{l}\text { Error - } \\
\text { parameters error }\end{array}$ & $\begin{array}{l}\bigotimes \text { Pass } \\
\square \text { Fail }\end{array}$ & $\begin{array}{l}3423 \text { was a } \\
\text { random number }\end{array}$ \\
\hline \multirow{4}{*}{25} & \multirow{4}{*}{ /controlAppliance1/\{servicelD $\} /\{$ input $\}$} & Id=test & $\begin{array}{l}\text { Error - } \\
\text { parameters error }\end{array}$ & $\begin{array}{l}\text { Error - } \\
\text { parameters error }\end{array}$ & $\begin{array}{l}\bigotimes \text { Pass } \\
\square \text { Fail }\end{array}$ & \\
\hline & & $\begin{array}{l}\text { Power_off=off } \\
\text { Id=4347 }\end{array}$ & ok & ok & $\begin{array}{l}\bigotimes \text { Pass } \\
\square \text { Fail }\end{array}$ & \\
\hline & & $\mathrm{Id}=3423$ & $\begin{array}{l}\text { Error - } \\
\text { parameters error }\end{array}$ & $\begin{array}{l}\text { Error - } \\
\text { parameters error }\end{array}$ & $\begin{array}{l}\bigotimes \text { Pass } \\
\square \text { Fail }\end{array}$ & $\begin{array}{l}3423 \text { was a } \\
\text { random number }\end{array}$ \\
\hline & & $I d=$ test & Error - & Error - & $\triangle$ Pass & \\
\hline
\end{tabular}


Chapter 4. Validation of the proposal and results

\begin{tabular}{|c|c|c|c|c|c|c|c|}
\hline & & & parameters error & parameters error & & Fail & \\
\hline \multirow{4}{*}{26} & \multirow{4}{*}{ /controlAppliance $1 /\{$ servicelD $\} /\{$ input $\}$} & $\begin{array}{l}\text { servicelD= } 4347 \\
\text { Input=on }\end{array}$ & ok & ok & 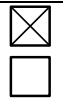 & $\begin{array}{l}\text { Pass } \\
\text { Fail }\end{array}$ & \\
\hline & & $\begin{array}{l}\text { servicelD= } 4347 \\
\text { Input=test }\end{array}$ & $\begin{array}{l}\text { Error - } \\
\text { parameters error }\end{array}$ & $\begin{array}{l}\text { Error - } \\
\text { parameters error }\end{array}$ & 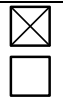 & $\begin{array}{l}\text { Pass } \\
\text { Fail }\end{array}$ & \\
\hline & & $\begin{array}{l}\text { servicelD= test } \\
\text { Input=on }\end{array}$ & $\begin{array}{l}\text { Error - } \\
\text { Parameters error }\end{array}$ & Error 500 & $\triangle$ & $\begin{array}{l}\text { Pass } \\
\text { Fail }\end{array}$ & \\
\hline & & $\begin{array}{l}\text { servicelD= } 3423 \\
\text { Input=on }\end{array}$ & $\begin{array}{l}\text { Error - } \\
\text { parameters error }\end{array}$ & $\begin{array}{l}\text { Error - } \\
\text { parameters error }\end{array}$ & 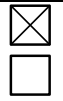 & $\begin{array}{l}\text { Pass } \\
\text { Fail }\end{array}$ & $\begin{array}{l}3423 \text { was a } \\
\text { random number }\end{array}$ \\
\hline \multirow{3}{*}{27} & \multirow{3}{*}{ /controlAppliance1/\{servicelD\}/\{input $\}$} & $\begin{array}{l}\text { servicelD= } 4347 \\
\text { Input=on }\end{array}$ & ok & ok & $\begin{array}{l}\square \\
\square\end{array}$ & $\begin{array}{l}\text { Pass } \\
\text { Fail }\end{array}$ & \\
\hline & & $\begin{array}{l}\text { servicelD= } 4347 \\
\text { Input=test }\end{array}$ & $\begin{array}{l}\text { Error - } \\
\text { parameters error }\end{array}$ & $\begin{array}{l}\text { Error - } \\
\text { parameters error }\end{array}$ & $\bar{\square}$ & $\begin{array}{l}\text { Pass } \\
\text { Fail }\end{array}$ & \\
\hline & & $\begin{array}{l}\text { servicelD= } 3423 \\
\text { Input=on }\end{array}$ & $\begin{array}{l}\text { Error - } \\
\text { parameters error }\end{array}$ & $\begin{array}{l}\text { Error - } \\
\text { parameters error }\end{array}$ & $\begin{array}{l}\square \\
\square\end{array}$ & $\begin{array}{l}\text { Pass } \\
\text { Fail }\end{array}$ & $\begin{array}{l}3423 \text { was a } \\
\text { random number }\end{array}$ \\
\hline \multirow{4}{*}{28} & \multirow{4}{*}{ /contro|Appliance1/\{serviceID\}/\{input\} } & $\begin{array}{l}\text { Digital input= on } \\
\text { servicelD=4347 }\end{array}$ & ok & $\begin{array}{l}\text { Error } 400 \text { (not } \\
\text { found) }\end{array}$ & $\square$ & $\begin{array}{l}\text { Pass } \\
\text { Fail }\end{array}$ & \\
\hline & & $\begin{array}{l}\text { Digital input= on } \\
\text { servicelD=4347 }\end{array}$ & ok & ok & $\begin{array}{l}\square \\
\square\end{array}$ & $\begin{array}{l}\text { Pass } \\
\text { Fail }\end{array}$ & \\
\hline & & $I D=4347$ & ok & ok & & $\begin{array}{l}\text { Pass } \\
\text { Fail }\end{array}$ & \\
\hline & & $I D=3423$ & $\begin{array}{l}\text { Error - } \\
\text { parameters error }\end{array}$ & $\begin{array}{l}\text { Error - } \\
\text { parameters error }\end{array}$ & & $\begin{array}{l}\text { Pass } \\
\text { Fail }\end{array}$ & $\begin{array}{l}3423 \text { was a } \\
\text { random number }\end{array}$ \\
\hline \multirow{3}{*}{29} & \multirow{3}{*}{ /contro|Appliance1/\{serviceID\}/\{input\} } & $I D=$ test & $\begin{array}{l}\text { Error - } \\
\text { parameters error }\end{array}$ & $\begin{array}{l}\text { Error - } \\
\text { parameters error }\end{array}$ & & $\begin{array}{l}\text { Pass } \\
\text { Fail }\end{array}$ & \\
\hline & & $\begin{array}{l}\text { Digital input= on } \\
\text { servicelD=4347 }\end{array}$ & ok & ok & & $\begin{array}{l}\text { Pass } \\
\text { Fail }\end{array}$ & \\
\hline & & $I D=4347$ & ok & ok & $\bigotimes$ & Pass & \\
\hline
\end{tabular}


Chapter 4. Validation of the proposal and results

\begin{tabular}{|c|c|c|c|c|c|c|c|}
\hline & & & & & & Fail & \\
\hline & & $\mathrm{ID}=3423$ & $\begin{array}{l}\text { Error - } \\
\text { parameters error }\end{array}$ & $\begin{array}{l}\text { Error - } \\
\text { parameters error }\end{array}$ & 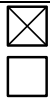 & $\begin{array}{l}\text { Pass } \\
\text { Fail }\end{array}$ & $\begin{array}{l}3423 \text { was a } \\
\text { random number }\end{array}$ \\
\hline \multirow{4}{*}{30} & \multirow{4}{*}{ /controlAppliance $1 /\{$ servicelD $\} /\{$ input $\}$} & $I D=$ test & $\begin{array}{l}\text { Error - } \\
\text { parameters error }\end{array}$ & $\begin{array}{l}\text { Error - } \\
\text { parameters error }\end{array}$ & $\triangle$ & $\begin{array}{l}\text { Pass } \\
\text { Fail }\end{array}$ & \\
\hline & & $\begin{array}{l}\text { Get request with } \\
\text { no parameters }\end{array}$ & ok & Error 500 & $\bigotimes$ & $\begin{array}{l}\text { Pass } \\
\text { Fail }\end{array}$ & \\
\hline & & $\mathrm{Id}=4347$ & ok & ok & $\bigotimes$ & $\begin{array}{l}\text { Pass } \\
\text { Fail }\end{array}$ & \\
\hline & & $I d=3423$ & $\begin{array}{l}\text { Error - } \\
\text { parameters error }\end{array}$ & $\begin{array}{l}\text { Error - } \\
\text { parameters error }\end{array}$ & \begin{tabular}{|l}
$\square$ \\
$\square$
\end{tabular} & $\begin{array}{l}\text { Pass } \\
\text { Fail }\end{array}$ & $\begin{array}{l}3423 \text { was a } \\
\text { random number }\end{array}$ \\
\hline \multirow{4}{*}{31} & \multirow{4}{*}{ /controlAppliance $1 /\{$ serviceID $\} /\{$ input $\}$} & Id=test & $\begin{array}{l}\text { Error - } \\
\text { parameters error }\end{array}$ & $\begin{array}{l}\text { Error- } \\
\text { parameters error }\end{array}$ & $\bar{\square}$ & $\begin{array}{l}\text { Pass } \\
\text { Fail }\end{array}$ & \\
\hline & & $\mathrm{Id}=4347$ & ok & ok & $\begin{array}{l}\bigotimes \\
\square\end{array}$ & $\begin{array}{l}\text { Pass } \\
\text { Fail }\end{array}$ & \\
\hline & & $I d=3423$ & $\begin{array}{l}\text { Error - } \\
\text { parameters error }\end{array}$ & $\begin{array}{l}\text { Error - } \\
\text { parameters error }\end{array}$ & 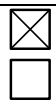 & $\begin{array}{l}\text { Pass } \\
\text { Fail }\end{array}$ & $\begin{array}{l}3423 \text { was a } \\
\text { random number }\end{array}$ \\
\hline & & Id=test & $\begin{array}{l}\text { Error - } \\
\text { parameters error }\end{array}$ & $\begin{array}{l}\text { Error - } \\
\text { parameters error }\end{array}$ & $\bar{\bigotimes}$ & $\begin{array}{l}\text { Pass } \\
\text { Fail }\end{array}$ & \\
\hline \multirow{4}{*}{32} & \multirow{4}{*}{ /controlAppliance1/\{servicelD\}/\{input\} } & $\mathrm{Id}=4347$ & ok & ok & $\frac{\bigotimes}{\square}$ & $\begin{array}{l}\text { Pass } \\
\text { Fail }\end{array}$ & \\
\hline & & $\mathrm{Id}=3423$ & $\begin{array}{l}\text { Error - } \\
\text { parameters error }\end{array}$ & $\begin{array}{l}\text { Error - } \\
\text { parameters error }\end{array}$ & 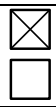 & $\begin{array}{l}\text { Pass } \\
\text { Fail }\end{array}$ & $\begin{array}{l}3423 \text { was a } \\
\text { random number }\end{array}$ \\
\hline & & $\mathrm{Id}=$ test & $\begin{array}{l}\text { Error - } \\
\text { parameters error }\end{array}$ & $\begin{array}{l}\text { Error - } \\
\text { parameters error }\end{array}$ & $\bar{\square}$ & $\begin{array}{l}\text { Pass } \\
\text { Fail }\end{array}$ & \\
\hline & & $\mathrm{Id}=4347$ & ok & ok & 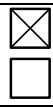 & $\begin{array}{l}\text { Pass } \\
\text { Fail }\end{array}$ & \\
\hline 33 & /controlAppliance1/\{serviceID\}/\{input\} & $I d=3423$ & Error - & Error - & $\bigotimes$ & Pass & 3423 was a \\
\hline
\end{tabular}


Chapter 4. Validation of the proposal and results

\begin{tabular}{|c|c|c|c|c|c|c|}
\hline & & & parameters error & parameters error & Fail & random number \\
\hline & & Id=test & $\begin{array}{l}\text { Error- } \\
\text { parameters error }\end{array}$ & $\begin{array}{l}\text { Error- } \\
\text { parameters error }\end{array}$ & $\begin{array}{l}\square \text { Pass } \\
\square \text { Fail }\end{array}$ & \\
\hline & & $\mathrm{Id}=4347$ & ok & ok & $\begin{array}{l}\square \text { Pass } \\
\square \text { Fail }\end{array}$ & \\
\hline & & $\mathrm{Id}=3423$ & $\begin{array}{l}\text { Error- } \\
\text { parameters error }\end{array}$ & $\begin{array}{l}\text { Error- } \\
\text { parameters error }\end{array}$ & $\begin{array}{l}\square \text { Pass } \\
\square \text { Fail }\end{array}$ & $\begin{array}{l}3423 \text { was a } \\
\text { random number }\end{array}$ \\
\hline \multirow{3}{*}{34} & \multirow{3}{*}{ /controlAppliance $1 /\{$ servicelD $\} /\{$ input $\}$} & Id=test & $\begin{array}{l}\text { Error - } \\
\text { parameters error }\end{array}$ & $\begin{array}{l}\text { Error - } \\
\text { parameters error }\end{array}$ & $\begin{array}{l}\bar{\square} \text { Pass } \\
\square \text { Fail }\end{array}$ & \\
\hline & & $I d=3423$ & $\begin{array}{l}\text { Response with } \\
\text { empty results }\end{array}$ & $\begin{array}{l}\text { Response with } \\
\text { empty results }\end{array}$ & $\begin{array}{l}\square \text { Pass } \\
\square \text { Fail }\end{array}$ & \\
\hline & & Id=test & $\begin{array}{l}\text { Response with } \\
\text { empty results }\end{array}$ & $\begin{array}{l}\text { Response with } \\
\text { empty results }\end{array}$ & $\begin{array}{l}\square \text { Pass } \\
\square \text { Fail }\end{array}$ & \\
\hline \multirow{2}{*}{35} & \multirow{2}{*}{ /controlAppliance1/\{servicelD $\} /\{$ input $\}$} & $\mathrm{Id}=4347$ & $\begin{array}{l}\text { Response with } \\
\text { empty results }\end{array}$ & $\begin{array}{l}\text { Response with } \\
\text { empty results }\end{array}$ & $\begin{array}{l}\bar{\square} \text { Pass } \\
\square \text { Fail }\end{array}$ & \\
\hline & & $\mathrm{Id}=4347$ & ok & ok & $\begin{array}{l}\square \text { Pass } \\
\square \text { Fail }\end{array}$ & \\
\hline \multirow{3}{*}{36} & \multirow{3}{*}{ /controlAppliance1/\{servicelD $\} /\{$ input $\}$} & Id=test & $\begin{array}{l}\text { Error- } \\
\text { parameters error }\end{array}$ & $\begin{array}{l}\text { Error- } \\
\text { parameters error }\end{array}$ & $\begin{array}{l}\square \text { Pass } \\
\square \text { Fail }\end{array}$ & \\
\hline & & $I d=4347$ & ok & ok & $\begin{array}{l}\bar{\square} \text { Pass } \\
\square \text { Fail }\end{array}$ & \\
\hline & & $\mathrm{Id}=3423$ & Error- & Error- & $\triangle$ Pass & 3423 was a \\
\hline
\end{tabular}


Chapter 4. Validation of the proposal and results

\begin{tabular}{|c|c|c|c|c|c|c|}
\hline & & & parameters error & parameters error & Fail & random number \\
\hline \multirow{4}{*}{37} & \multirow{4}{*}{ /controlAppliance $1 /\{$ servicelD $\} /\{$ input\} } & Id=test & $\begin{array}{l}\text { Error - } \\
\text { parameters error }\end{array}$ & $\begin{array}{l}\text { Error - } \\
\text { parameters error }\end{array}$ & $\begin{array}{l}\square \text { Pass } \\
\square \text { Fail }\end{array}$ & \\
\hline & & $I d=4347$ & ok & ok & $\begin{array}{l}\square \text { Pass } \\
\square \text { Fail }\end{array}$ & \\
\hline & & $I d=3423$ & $\begin{array}{l}\text { Error- } \\
\text { parameters error }\end{array}$ & $\begin{array}{l}\text { Error- } \\
\text { parameters error }\end{array}$ & $\begin{array}{l}\square \text { Pass } \\
\square \text { Fail }\end{array}$ & $\begin{array}{l}3423 \text { was a } \\
\text { random number }\end{array}$ \\
\hline & & Id=test & $\begin{array}{l}\text { Error- } \\
\text { parameters error }\end{array}$ & $\begin{array}{l}\text { Error- } \\
\text { parameters error }\end{array}$ & $\begin{array}{l}\square \text { Pass } \\
\square \text { Fail }\end{array}$ & \\
\hline
\end{tabular}

An equivalent set of tests was done with the component used for device registration. Several attempts and refinements of the software were carried out until all the procedures were finally successful. Results have been shown in Table 17.

Table 17. Device registration testing activities

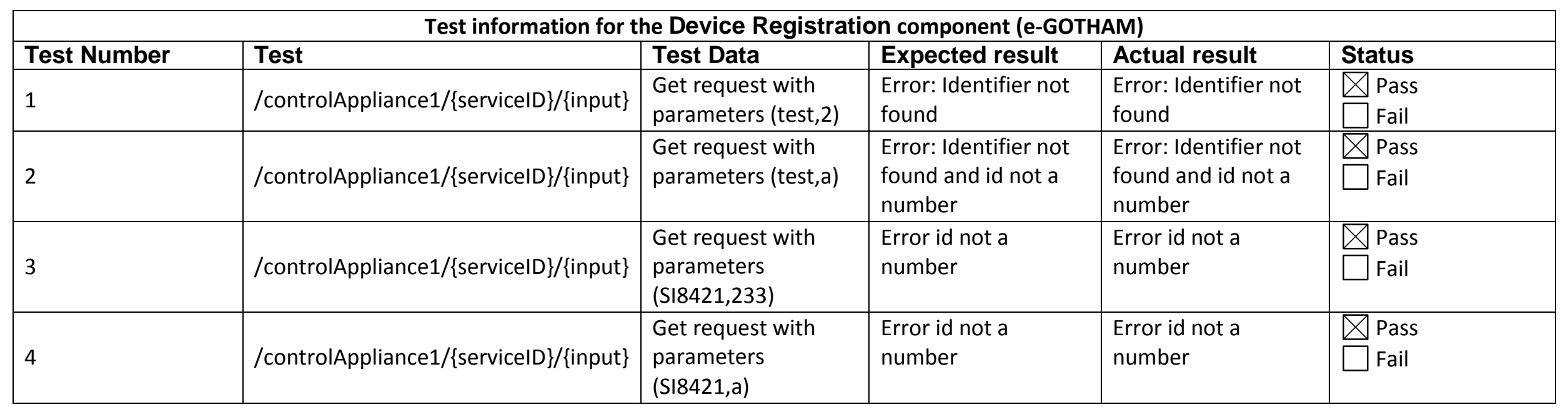


Chapter 4. Validation of the proposal and results

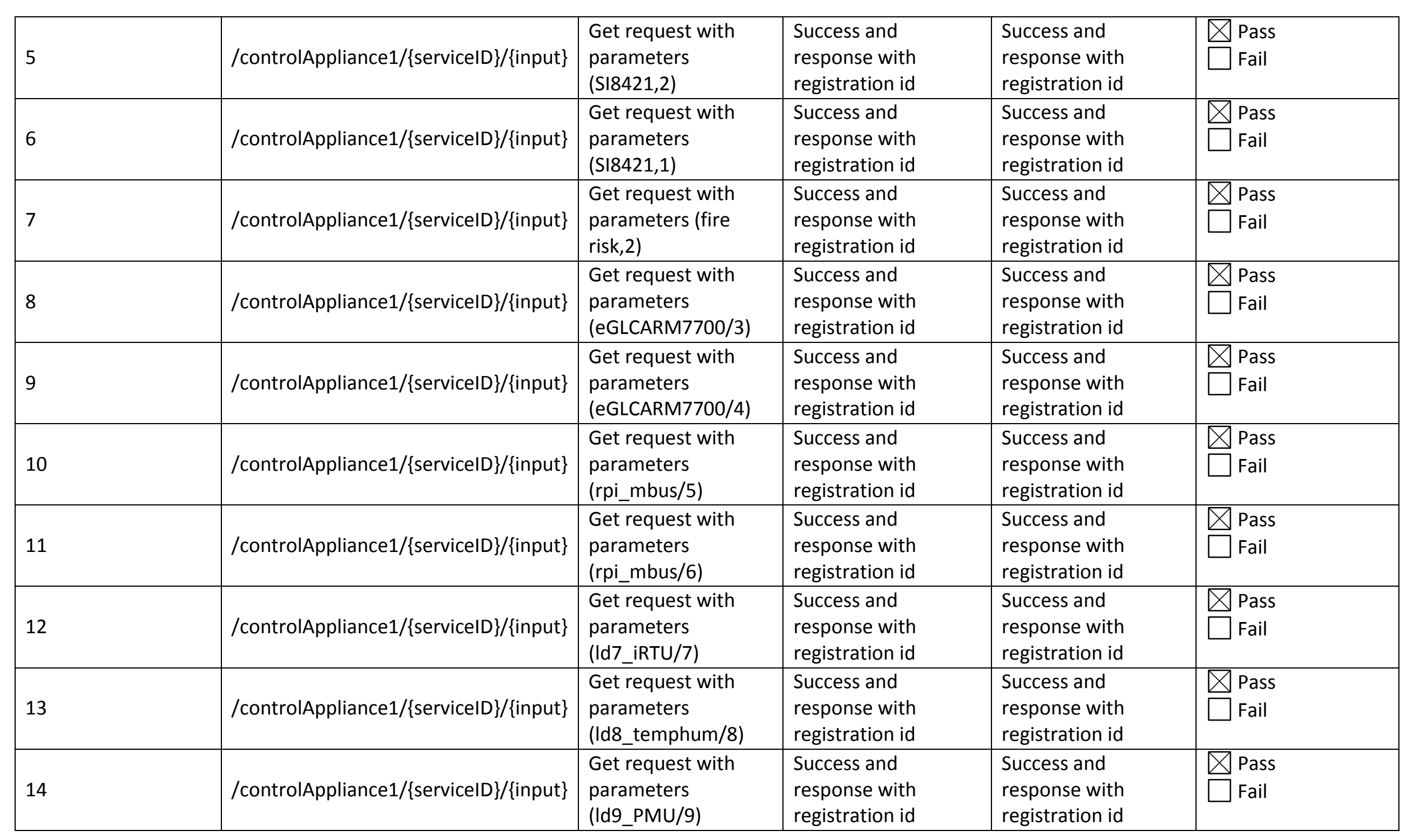


Chapter 4. Validation of the proposal and results

\begin{tabular}{|l|l|l|l|l|}
\hline 15 & /controlAppliance1/\{serviceID\}/\{input\} & $\begin{array}{l}\text { Get request with } \\
\text { parameters } \\
\text { (Herrfors- } \\
\text { pressure/10) }\end{array}$ & $\begin{array}{l}\text { Success and } \\
\text { response with } \\
\text { registration id }\end{array}$ & $\begin{array}{l}\text { Success and } \\
\text { response with } \\
\text { registration id }\end{array}$ \\
\hline 16 & /controlAppliance1/\{serviceID\}/\{input\} & $\begin{array}{l}\text { Get request with } \\
\text { parameters } \\
\text { (Herrfors-data/11) }\end{array}$ & $\begin{array}{l}\text { Success and } \\
\text { response with } \\
\text { registration id }\end{array}$ & $\begin{array}{l}\text { Success and } \\
\text { response with } \\
\text { registration id }\end{array}$ \\
\hline 17 & /controlAppliance1/\{serviceID\}/\{input\} & $\begin{array}{l}\text { Get request with } \\
\text { parameters (ESS/12) }\end{array}$ & $\begin{array}{l}\text { Success and } \\
\text { response with } \\
\text { registration id }\end{array}$ & $\begin{array}{l}\text { Success and } \\
\text { response with } \\
\text { registration id }\end{array}$ \\
\hline
\end{tabular}




\subsubsection{Ylivieska scenario}

When the testing activities proved that the middleware components had reached a suitable level of maturity, middleware was deployed in the two demonstrators that had been arranged for its usage. The first one was used in the Finnish city of Ylivieska, where middleware was used to contain several communication facilities and software components [180]. The Enterprise Service Bus that was used during testing facilities was employed again when the actual deployment took place. Additionally, the hardware capabilities of the unit running the central Controller had no special requirements (as stated in [154], middleware was installed in a Ubuntu-based virtual machine running the 14.04 Long Time Support version of the operating system. The hardware had a $3 \mathrm{GHz}$ CPU, 4 GB of RAM memory, $160 \mathrm{~GB}$ of Hard Disk and Gigabit Ethernet communications). As portrayed in Figure 101, the deployment was done in line to what had been conceived during the design and implementation stages for middleware: a somewhat centralized element contained most of the elements in the middleware architecture, which was chosen to have the most complex services to be installed. Some of them had functionalities comparable to the ones defined as Middleware Access Points, where software components were used to access other parts of the middleware. Others performed functionalities typical of a Smart Grid (energy consumption forecasting, information, management, etc.) and did not require any other middleware service (and therefore behaved as Middleware Autonomous Services).

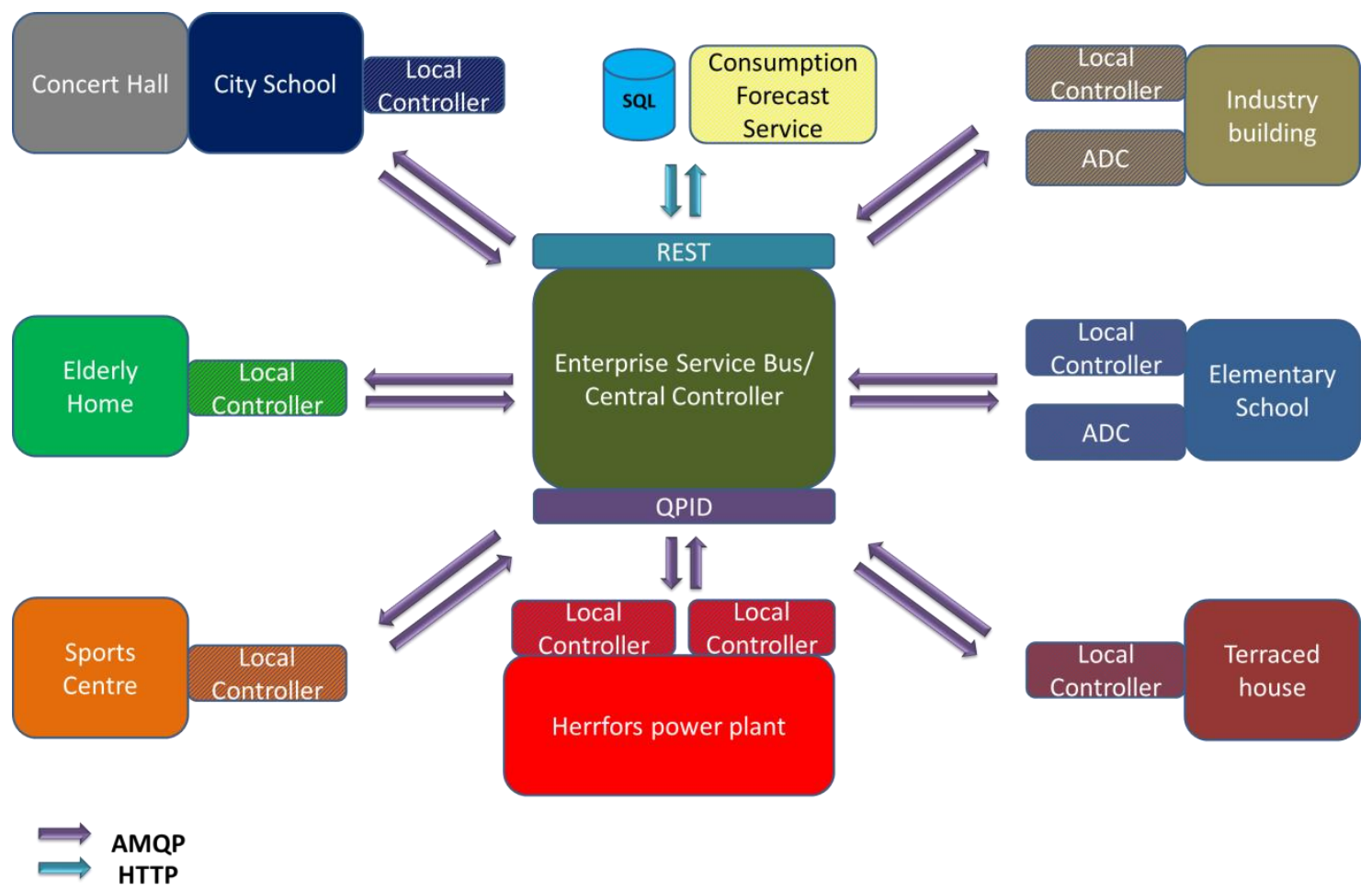

Figure 101. Deployment made in Ylivieska. Middleware is at the centre of the deployment

According to what was used in the Ylivieska scenario, the following pieces of equipment were installed in different facilities of the town: Local Controllers were installed in the school of the 
city, elderly home, sports centre, an industrial facility, an elementary school and a rowhouse. Furthermore, two of them were installed in a power plant owned by a DSO called Herrfors, due to the higher complexity of its installations. The Local Controllers that were communicating with the middleware were based on raspberry Pi model B hardware, which had enough computational power to receive and transfer information from their location to the one where they were deployed [91]. Data was gathered by means of shields that were manufactured and sold by Open Energy Monitor [181]. All in all, the deployment guaranteed that data could be sent from the Local Controllers to the central one and have requests done throughout the interfaces, both on top of the middleware (via HTTP) and below it (by means of AMQP).

\subsubsection{Steinkjer scenario}

This was the demonstrator that was chosen to be used in the I3RES project [182]. While the middleware components that were deployed had several major differences from the ones that had been created for the e-GOTHAM project (both in their implemented code and purpose), the computer where they were deployed was still of regular characteristics, so the solution could be deployed in a fashion that resembled the one carried out previously. The hardware that was to be interacted reflected the higher stress in power transmission made by I3RES, in the sense that were units such as weather stations or tertiary buildings expected to send their information about their status and performance. The final appearance of the deployment, along with its components, has been depicted in Figure 102.

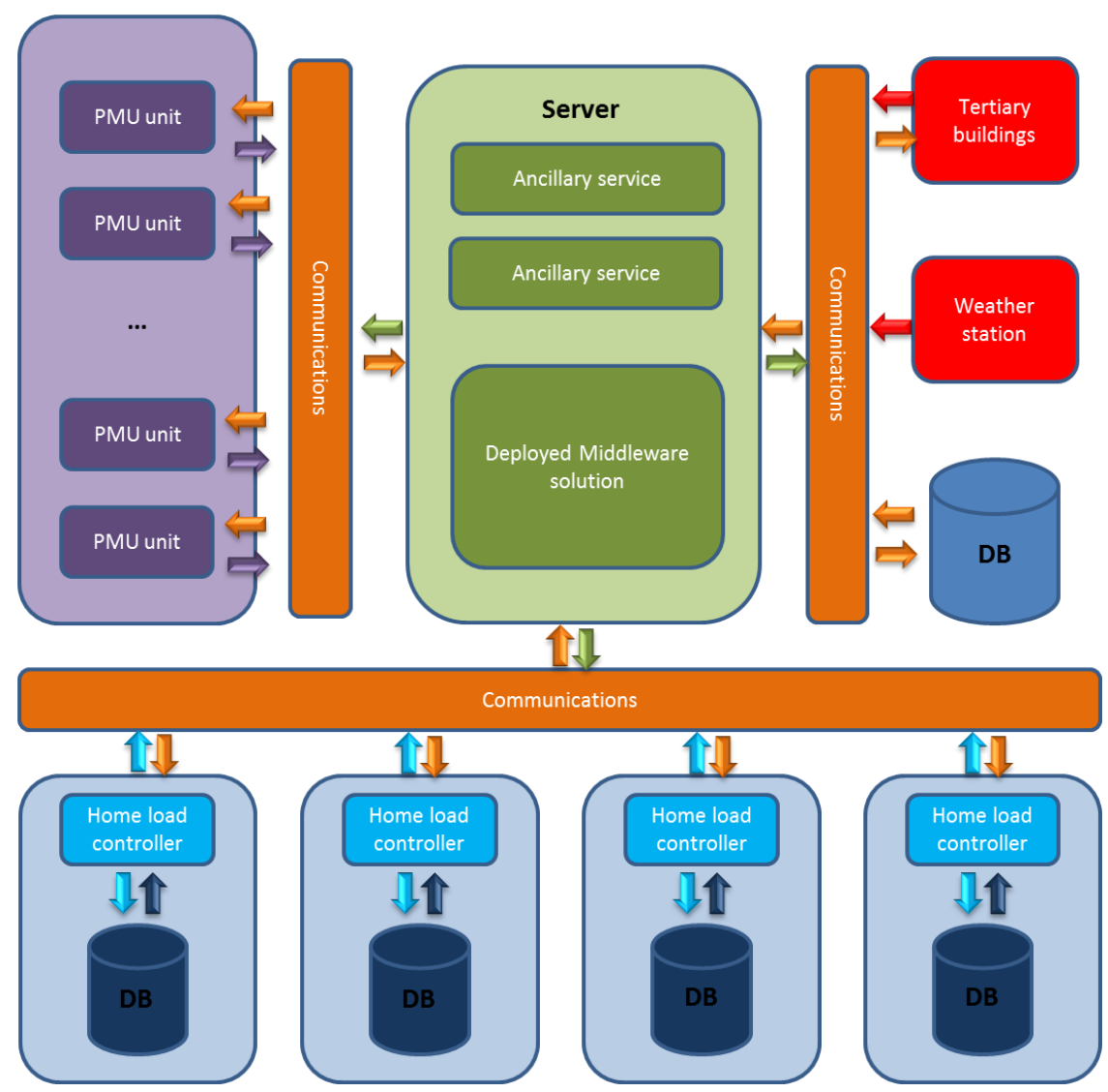

Figure 102. Steinjker deployment with the middleware solution, as depicted in [34] 
Part of the testing activities that were carried out before the deployment of the middleware solution created for the i3RES project consisted of checking whether the performance of the middleware solution that had been developed resulted good enough considering the operations of data transmission that to be done. Consequently, tests were run with other partners working together in order to ensure that, when the deployment was done, it should not suppose a problem to have the middleware architecture installed in the pieces of equipment used. One thousand requests were done onto a fully operational middleware solution to assess its performance; graphical results are shown in Figure 103.

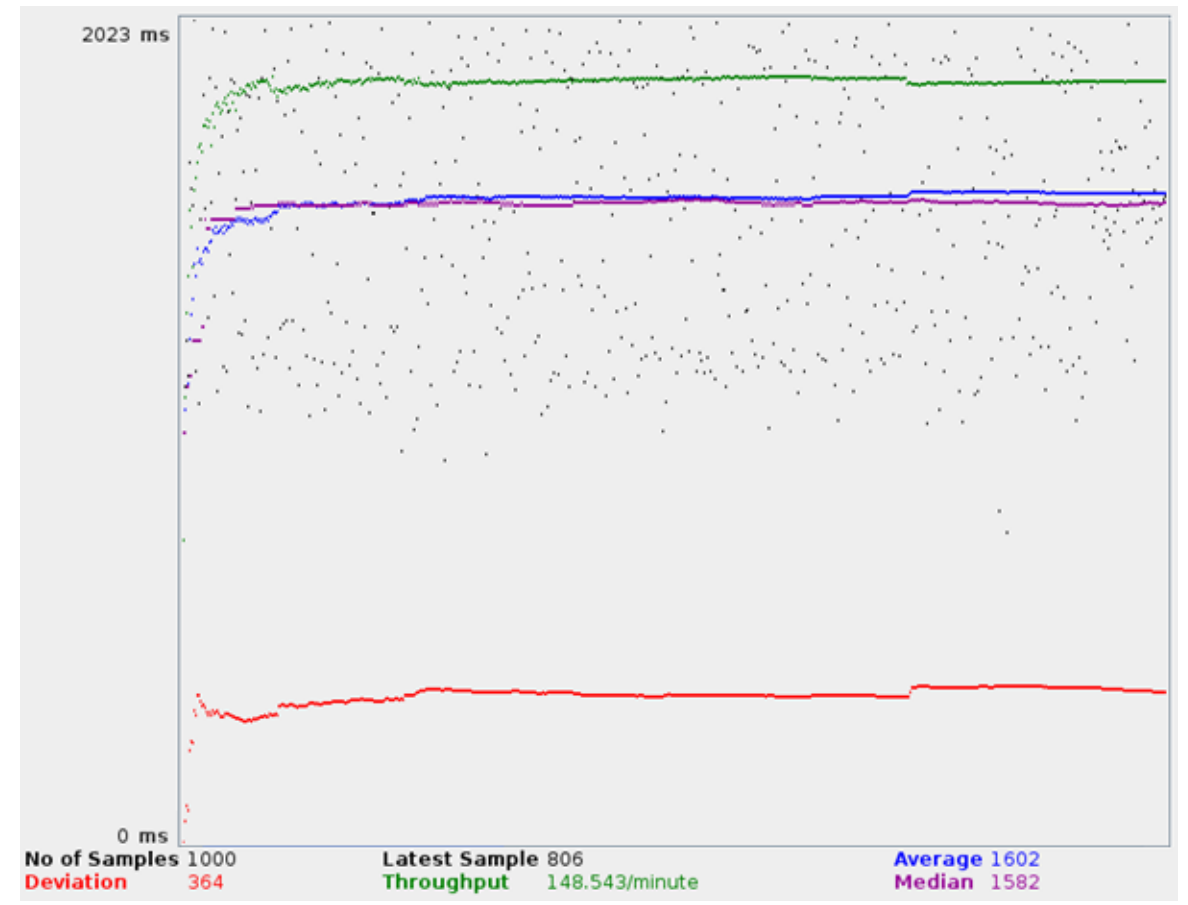

Figure 97. Middleware tests results, as shown in [34]

The performance results obtained can be described with the most important parameters that were assessed [34]:

1. Average value to attend a request was measured at 1602 milliseconds. This can be regarded as a satisfactory result for end users and clients, due to the fact that they can have their information in a relatively insignificant period of time. More importantly, when the number of requests increases, the figure obtained did not substantially increase, showing that the system was able to offer a high degree of robustness.

2. Median value to attend a request was 1582 milliseconds. While this is also a satisfactory value, the most important conclusion that can be extracted from it is that it is a very close value to the one that was obtained as the average, which shows that the system behaved with a great degree of regularity and without the influence of any outliers obtained in the results.

3. Deviation value was 364 milliseconds. As it was given away from the previous conclusions, the value obtained for deviation was comparatively low due to the regular 
performance of the middleware solution. The dispersion level obtained from the 100 samples that were registered is also acceptable, as it does not suppose a significant time value for an end user, a consumer or even a prosumer.

4. Throughput value was 148.543 queries/minute. Considering the scale of the system that was implemented and were it was deployed, it can be considered as an acceptable figure, as there was not a chance that there would be such a number of requests per minute done to the middleware solution once it was deployed to the demonstrator.

\subsubsection{Smart Grid scenario test results}

The results that have been presented in this section provide all the testing activities that were carried out in order to assess the feasibility of the solution put forward in this thesis. Aside from the testing activities that were carried out in a controlled environment (such as laboratories and the facilities of Vitrociset), software services and implementation works that have been depicted in this section, along with all the activities required to prove them until they are reliable enough to be deployed in an actual scenario. The performance obtained in the laboratory tests done for the I3RES project confirmed that its behaviour is good enough for the purposes of the middleware. In addition to that, the middleware deployment done in the cities of Ylivieska and Steinkjer proves that it can be used to receive and transfer information at the data level. The software components that were included in those latter two scenarios also show how middleware can be used to provide services for the Smart Grid.

When comparing the results that have been obtained with this middleware architecture against the results that the first versions of the middleware implemented in the Wireless Sensor Networks, it can be seen that the capacity and the improvement that have been carried out are remarkable: not only there are more services that can be used (secure access to the middleware architecture, semantic capabilities, device registration in a database, etc.) but also the amount of requests that can be handled by the middleware is far greater than the one that was used before. In addition to that, the performance of the semantic middleware architecture is able to guarantee that service requests will be satisfied at an acceptable rate, so it can be claimed that including a middleware architecture in a system related to the Smart Grid does not impact negatively its performance, while at the same time there are more services available for end users or prosumers.

\subsection{Section summary}

This section has offered a thorough description about how the proposal for a common middleware architecture for the Smart Grid has been implemented in order to test its performance and feasibility in a realistic scenario. The implementation activities that have been offered describe the software tools that have been used for such an implementation (Java as the programming language, JBoss Fuse as the Enterprise Service Bus), along with the hardware devices that were used during the testing and deployment of the solutions in two 
different demonstrators, as arranged in the e-GOTHAM and I3RES projects. The results that have been provided show that services have been added in a way that had not been conceived before, while at the same time keeping an acceptable performance for the overall system.

The inference process that resulted in conceiving the Common Middleware Architecture must also be included in this part of the chapter. During the implementation works that were taking place in e-GOTHAM and I3RES research projects, there were several aspects that were found out to be common in both projects:

1. Existing solutions for middleware in the Smart Grid were not completely satisfactory. There were several services like semantics, security or context awareness that were not included in previous solutions, or they were so in a way that could not be used to the overall system's advantage (only partial solutions, laboratory testing in simulators as opposed to real world deployments, etc.).

2. A way to encase services had to be figured out. Since what was deemed as most useful was the design and implementation of a middleware architecture, a way to contain all the software services that had to be included had to be thought. In order to do so, Enterprise Service Bus solutions seemed like the best ones, as they were able to contain those services, establish a way to have them communicating with each other and enabling its capability to interact with other elements of a distributed or Cyber-Physical System.

3. Software technologies used for communications between elements alien to the middleware architecture had to be included. The importance of interfacing other software developments was stressed from the very first stages of the projects, where the requirements were defined for each of the elements present in what would become the deployed system.

4. The solutions had to be tested in actual deployments rather than only in controlled environments or laboratories. The nature of the European research projects defined by ARTEMIS or FP7 initiatives made almost mandatory having the development works tested in environments where they could provide real usefulness to a system that contained the other elements development by other partners in the consortiums that had cooperated in the projects.

5. Similarities and differences among projects could be defined and given boundaries. While the objectives in the projects that were participated were different from each other (the eGOTHAM project was mostly focused on device integration and interoperability whereas I3RES was focused on Demand Response and Demand Side Management for the Smart Grid), there were similarities in requirements (distributed system with software components interacting with a plethora of devices) among them, so a common ground could be found that complemented the works done in a) previous middleware instalments and b) standards that, to a greater or a lesser extent, were involved, in hardware interoperability for the Smart Grid (or at least, the power grid). Those are the four different areas of knowledge that have been heavily considered for the analysis, design, implementation and testing works that have resulted in the conception of CMA, which can be located at the intersection of all of those initiatives, as depicted in Figure 104. 


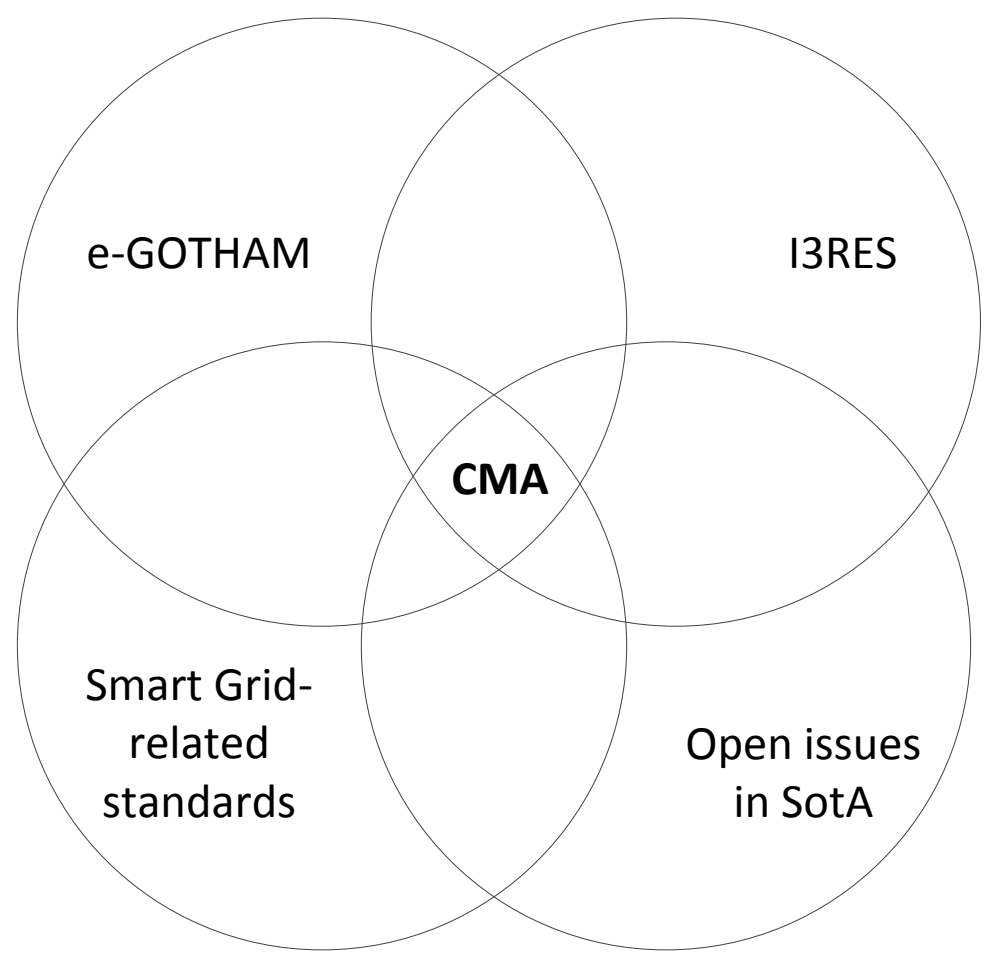

Figure 98. Different areas of knowledge and projects used for the conception of CMA

It can be inferred from the previous figure that the combination of different sources of demands form research projects and weaknesses detected in previous pieces of work have been the main motivation for the development of Common Middleware Architecture.

6. The inclusion of a middleware architecture (as part of the implementation works required to integrate it with all the other parts of the system) does not pose a threat in terms of performance. Even though the results obtained for middleware in the scenario where WSNs were used as the hardware where the architecture was installed show that nodes may struggle to get a near real-time result for composed services, this issue was basically non-existent in the other deployments where more capable hardware was used to deliver data readings and service-related information. 


\section{Conclusions and future}

works 
This chapter of the thesis has been used to include the main conclusions that can be extracted from the work that has led to its completion. It highlights all the lessons that have been learned about the topics that were introduced (middleware for the Smart Grid, integration of renewable energies, etc.) and pinpoints where future works can be aimed at.

\subsection{Original contributions of the thesis}

This thesis has described several significant original contributions that have been listed in this section as a way to provide a starting ground for future research activities.

1. Viability of middleware solutions for the Smart Grid. The usefulness of having a middleware solution for the Smart Grid has been proven in this thesis. Middleware can be used for functionalities of major importance for the Smart Grid, such as hardware abstraction, device integration and addition of software services, as long as it is configured as a software architecture where new functionalities can be encased. Its inclusion in a deployment improves the overall status of the Smart Grid, due to the fact that it enables the use of advanced ICT functionalities (service registration, security, semantics, etc.) that would be harder or impossible to count on without middleware.

2. Standardization for middleware architectures in the Smart Grid. Services that are available with a middleware solution for the Smart Grid, as well as how to access them with an Application Programming Interface, have been depicted in this thesis. These activities had not been done before for a middleware architecture for the Smart Grid, so they are most useful for any development that has to be done in this application domain, since they are providing a starting ground that will ease developments and will further aid its future refinement. In addition to that, it has been proved why a software architecture is the most suitable option for middleware in a Smart Grid. Among the different ways to include middleware, there are options such as using just hardware abstraction or MessageOriented Middleware. The study on the state of the art that has been carried out proves that the most useful existing options, and the ones capable of providing a greater array of services, are the ones based on software architectures that encase software components within them.

3. Exploitation of middleware via business models for prosumers and small-scale facilities. Semantic middleware has been proven to be a critical component to make possible the integration, from a more economics-related point of view, of the prosumers that are expected to play a significant role in the Smart Grid (due to the fact that they provide their own electricity to buy and sell in a distributed, small-sized way). Business models have been provided for prosumers that, by making use of an intermediation architecture, will be able to trade the electricity they produce in the markets. While the legislative part required to be modified is beyond the scope of this thesis, it is proven from the technical point of view that it is possible to perform operations of this nature for their benefit, thus making the grid more reliable and democratizing access to energy. 


\subsection{Conclusions}

This thesis has been structured in a way that the contributions have been described in the introduction, as a way to make a first mention of the objectives that were set for it. That is why a study of the current State of the Art regarding middleware architectures for the Smart Grid was made. This study resulted in the inference of several open issues and challenges that were deemed as necessary to be tackled in any future solution that was going to be developed in this application domain. In addition to that, since it was mentioned during the study that a collection of services would have to interchange information between the application layer and the hardware, another study of open source Enterprise Service Bus implementations was added.

Several requirements were set so that the highlighted open issues and challenges could be dealt with in the most efficient way possible. With this list of requirements, a collection of components was established and implemented. The requirement analysis that was performed for each of the research projects differ in results, due to the fact that they had different orientations (e-GOTHAM was mostly focused on the integration of the ICT part of the Smart Grid within the power grid, whereas I3RES was more oriented to the enhancement of energy consumption behaviour by means of services such as Demand Side Management or Demand Response). Nevertheless, the obtained complementary results, combined with the features that the existing State of the Art offered, as well as the different aspects that were contemplated in the existing standards within this area of knowledge resulted in the conception of Common Middleware Architecture, a proposal for a semantic middleware architecture in the context of the Smart Grid that supersedes many of the issues that were found before. When all is said and done, the following conclusions can be inferred from the presented work:

1. Challenges in existing solutions of middleware for the Smart Grid are profuse. When all the studied solutions where researched, it was found that none of them would completely address the issues found regarding interoperability, service availability, distribution or usability. Therefore, there was a solution that had to be achieved based on the challenges found in the ones preceding the development works done during the e-GOTHAM and I3RES research projects, and the requirements of these projects.

2. The bulk of the issues that were found has been addressed. CMA provides a framework were services can be included in an ordered fashion, with each of their functionalities and boundaries clearly defined. In addition to that, the procedures that must be followed in order to have an equivalent implementation have been described as well.

3. Feasibility of middleware for the Smart Grid. Rather than having just a theoretical work of design, implementation activities were carried out so that developments could be used in an actual scenario. It was due to those successful implementation activities that common features could be inferred and combined with the weaknesses found in the existing standards, thus making possible the conception of CMA. In addition to that, performance results measured and deployments done in two different pilots prove that the development of semantic middleware for the Smart Grid is feasible and desirable. Indeed, 
the validation results obtained in the two previously described scenarios show that middleware and its encased services can be implemented in a realistic manner, where characteristics like context awareness or semantics can be offered without significant impact in the rates they are offered at.

4. Benefits of standardization have been offered. Since one of the main goals of middleware is guarantee interoperability among heterogeneous distributed devices, a standard that defines the services to be used and how information is supposed to be mapped and integrated becomes an appealing objective. The establishment of a standard for interoperability software architectures used as something consensual in the Smart Grid would greatly improve any development done in this application domain, as it will provide a blueprint of the services to be included and how to interface them.

5. Implementation via open source resources. An important feature to consider here is that the development works described have been done with open source tools (JBoss Fuse being the most prominent of them all), which is of major importance to lower the overall cost of the project and facilitate the implementation with examples and troubleshooting procedures that can be found with next to no problem. Also, it is easier to improve the state of the art in semantic middleware for the Smart Grid using this kind of software resources, since any component detail or the overall appearance of the architecture can be described.

6. A commercial point of view has also been provided in order to justify how the business models that make use of middleware as part of the required technology to integrate prosumers (or even regular end users) in the Smart Grid. By being able to guarantee information interchanges between several distributed entities, middleware enhances the capacities of the power grid and includes new actors that are likely to contribute to the power flow of the overall grid, as well as demand and offer new services according to their needs and capabilities.

\subsection{Future works}

The following future works have been programmed for future research:

1. Portability to other Cyber-Physical Systems is already been made. Many of the principles of CMA have been included in the SWARMs project [20], even though the application domain of maritime robotics has many major differences if it is compared to the Smart Grid (despite sharing several underlying features) such as being a Cyber-Physical System with distributed hardware heterogeneous elements in a constrained environment from the computational and communications point of view.

2. Further integration with other similarly themed standards. As it has already been mentioned, the objective of CMA is providing new ideas that other proposals and standards may lack in order to provide the most holistic and complete point of view to the standardization processes that are being carried out for now. The promotion of the concepts of CMA (layered middleware architecture, core services, access services, procedure for middleware implementation and testing, interfaces within and without 
middleware) and their inclusion in other more developed proposals for standardization preceding the ones made here for years will be emphasized during the next period of time.

3. Extension to other software tools and languages. It has already been proven how CMA can be implemented by means of an open source JBoss Fuse as an ESB and Java as the programming language. Other developers, though, may prefer other kinds of tools due to their background or the requirements of their own projects. Offering consulting to other possible implementations or Platform Specific Models will be done as soon as an interest is spotted both within and without the research group where these implementation works have been carried out.

4. Increase of the intelligence of the services included in the middleware. As described in this document and supported by research papers, services make extensive use of semantic capabilities. Its further enhancement of their performance by means of already familiar or new tools (deep learning, machine learning, etc.) is a likely development to take place.

\subsection{Publications and projects}

The following section includes all the scientific publications that have been made that can be considered as part of this thesis. They comprehend a timespan of more than four years from the first one to the last one. Currently there are other publications that are under consideration for some other journals, but they have yet to be accepted and published.

\subsubsection{SCl-indexed journals}

The following scientific papers have been published as a result of the research works done. At the end of each of the references, a description has been added to clarify what they provide as a contribution to the thesis, as well as a way to refer to them in Figure 106, which portrays in a graphical manner a timeline with all the outputs aimed to satisfy the objectives of the thesis.

1. Rodríguez-Molina, Jesús; Martínez, José-Fernán; Castillejo, Pedro; López, Lourdes, "Combining Wireless Sensor Networks and Semantic Middleware for an Internet of ThingsBased Sportsman/Woman Monitoring Application," Sensors, vol. 13, p. 1787, 2013.

This paper summarizes the work that has been done for the LifeWear project regarding the extension of the previously existing middleware works, as well as other new services that were added both to the project and the content of this thesis, such as interfaces to the application layer (based on REST). This is the first paper co-authored by the author of this manuscript where service composition was used: there were two services (injury prevention, assessing the data obtained from body temperature, environmental temperature and heartbeat rate, and temperature control, making use of environmental and body temperature). This paper is referred to as Sensors 1 in Figure 106.

2. Rodríguez-Molina, Jesús; Martínez, José-Fernán; Castillejo, Pedro; de Diego, Rubén; "SMArc: A Proposal for a Smart, Semantic Middleware Architecture Focused on Smart City 
Energy Management," International Journal of Distributed Sensor Networks, vol. 2013, p. 17, 2013.

This was a first approach to what middleware should be for distributed and Cyber-Physical Systems. Ideas like having a middleware layer with several components providing services, as well as abstracting hardware heterogeneity, semantic capabilities and offering access points to the applications. The design principles and some of the implementation works were included as part of the I3RES project. It is referred to as IJDSN in Figure 106.

3. Rodríguez-Molina, Jesús; Martínez-Núñez, Margarita; Martínez, José-Fernán; PérezAguiar, Waldo. "Business Models in the Smart Grid: Challenges, Opportunities and Proposals for Prosumer Profitability". Energies, vol. 7, no. 9, p. 6142, 2014.

This is the first approach that was made with the idea of integrating prosumers into a business model that makes use of middleware as the underlying software. A study of the possibilities that were offered for prosumers was included, as well as how they could impact the Smart Grid with their activities. This manuscript is referred to as Energies 3 in Figure 106.

4. Rodríguez-Molina, Jesús; Martínez, José-Fernán; Castillejo, Pedro. "A Study on Applicability of Distributed Energy Generation, Storage and Consumption within Small Scale Facilities". Energies, vol. 9, no. 9, p. 745, 2016.

This manuscript improves the ideas put forward in the former one by providing a mathematical model used to prove how either payment reduction regarding electricity or even profits can be obtained. This model is the foundation of the Section 2.4 present in this thesis. Performance details of the middleware tested for the I3RES project have been included in this manuscript. It is referred to as Energies 4 in Figure 106.

5. Rodríguez-Molina, Jesús; Martínez, José-Fernán; Castillejo, Pedro; Rubio, Gregorio. "Development of middleware applied to microgrids by means of an open source Enterprise Service Bus" [154]. Energies, vol. 10, no. 2, p. 172, 2017.

This research paper focuses on how an ESB is used to gather all the services present in a middleware solution, as well as offering information about the data that is to be included in the messages that are interchanged among the different parties where middleware has been installed. It is referred to as Energies 5 in Figure 106.

6. Martínez, José-Fernán; Rodríguez-Molina, Jesús; Castillejo, Pedro; de Diego, Rubén. "Middleware Architectures for the Smart Grid: Survey and Challenges in the Foreseeable Future". Energies, vol. 6, no. 9, p. 3593, 2013.

This paper offers a first study of the state of the art regarding middleware architectures for the Smart Grid, in order to have an exact idea of what is used as such and their features, strong points and weaknesses. While the studied proposals of the paper have been 
included in this thesis, there are many others that have been included as well in order to have an overall more complete study. This manuscript is referred to as Energies 1 in Figure 106.

7. Castillejo, Pedro; Martinez, José-Fernán; Rodriguez-Molina, Jesús; Cuerva, Alexandra. "Integration of wearable devices in a wireless sensor network for an E-health application," in IEEE Wireless Communications, vol. 20, no. 4, p. 38, 2013 [28].

This paper describes how a middleware architecture can be used as part of a distributed system where both wearable and non-wearable elements are combined together in the same deployment due to its hardware abstraction properties. It is referred to as IEEE WC in Figure 106.

8. de Diego, Rubén; Martínez, José-Fernán; Rodríguez-Molina, Jesús.; Cuerva, Alexandra. "A Semantic Middleware Architecture Focused on Data and Heterogeneity Management within the Smart Grid". Energies vol. 7, no. 9, p. 5953, 2014.

This paper offers a first approach to how to build middleware for a Smart Grid in a realistic manner that will integrate devices to be found in a deployment. It also includes information about how semantics are built as part of the system and how components can be interfaced among them and from outside the middleware. It is referred to as Energies 2 in Figure 106.

9. Li, Xin; Martínez, José-Fernán; Rodríguez-Molina, Jesús; Lucas Martínez, Néstor. "A Survey on Intermediation Architectures for Underwater Robotics." Sensors, vol. 16, no. 2, p. 190 [185].

This manuscript provides information about how middleware architectures can be used and are present in other CPSs that, even though are different to the Smart Grid, contain a significant number of similarities (hardware heterogeneity, need for interoperability, devices scattered in an area sharing information collected from their environment, etc.). It is referred to as Sensors 2 in Figure 106.

\subsubsection{Conferences}

The following contributions have been made to conferences regarding the scope of this thesis:

1. Rodríguez-Molina, Jesús; Martínez, José-Fernán; Rubio, Gregorio.; Hernández, Vicente. "A Proposal for an Internet of Things-based Monitoring System Composed by Low Capability, Open Source and Open Hardware Devices". 3rd International Conference on Sensor Networks (SENSORNETS), 2014 [186].

This conference was used to show how middleware can be used to unify under a same system several devices reliant on the IoT to collect and transfer information. Aside 
from middleware there were several levels that were studied to do such integration. In this case, middleware was oriented to messages rather than offering a whole architecture with services. It is referred to as SENSORN in Figure 106.

2. Rodríguez-Molina, Jesús; Martínez-Núñez, Margarita; Pérez-Aguiar, Waldo; de Diego, Rubén. "A study on challenges and opportunities for energy business developments in the Smart City". 8th International Conference on Industrial Engineering and Industrial Management (CIO-ICIEOM-IIIE), 2014 [187].

The piece of work that was shown in this conference dealt with the possibility of having different business strategies for Smart Cities where the Smart Grid played a prominent role. Prosumer activities were included as a way to describe that prosumers could result in a new agent with a critical importance in this kind of environment. It is referred to as $\mathrm{ClO}$ in Figure 106.

3. Martín-Wanton, T.; Muelas, E.; Santos de la Cámara, R.; Rodríguez-Molina, J., Li, X., Martinez-Ortega, J-F. "Modelado de vehículos autónomos y la incertidumbre de su entorno para la seguridad de operaciones marítimas". IV Congreso Nacional de i+d en Defensa y Seguridad (DESEi+d), 2016 [188].

The collaboration is this conference was focused on showing how the middleware could be used in other systems resembling the Smart Grid, despite being included in other different CPSs. It is referred to as DESEi+d in Figure 106.

\subsubsection{Book chapters}

This thesis has been involved in the following book chapters:

1. Rodríguez-Molina, J. "The Role of Middleware in Distributed Energy Systems Integrated in the Smart Grid". This is Chapter 5 of "Energy Management of Distributed Generation Systems" [189].

This book chapter describes how the development of middleware can be eased with a collection of steps and resources that are usually the same during its development. Furthermore, it offers a perspective of how middleware can be standardized as a collection of services in a middleware architecture and the services it could contain. It is referred to as Chapter 2 in Figure 106.

2. Hernández, V.; Martínez, J-F.; Cuerva, A.; Rodríguez-Molina, J.; Rubio, G.; Jara, A. "Semantic as an Interoperability Enabler in Internet of Things". This is Chapter 9 of "Internet of Things: Converging Technologies for Smart Environments and Integrated Ecosystems" [190]. 
This book chapter had as its main idea how semantic middleware is a critical concept in the IOT. It describes how middleware is used as a way to withhold hardware differences in a distributed deployment and how homogeneity is provided with the aid of semantics. Its content can also be easily ported to the Smart Grid, due to its resemblance to some major features in loT-based deployments. It is referred in Figure 106 as Chapter 1.

\subsubsection{Research projects}

This thesis has been done in the context of the following European research projects:

1. LifeWear: Mobilized Lifestyle with Wearables (ITEA2) [23] was focused on providing simple and composed services for wearables and distributed systems. Among other aspects, a Wireless Sensor Network was used to transfer data from a Body Area Network (BAN) to a mobile phone application by means of middleware, an open source Enterprise Service Bus, and REST-based interfaces. The foundations used to develop semantic middleware for the Smart Grid were firstly introduced during this project, such as hardware abstraction or URIs to access interfaces.

2. WoO: Web of Objects (ITEA2). The goals of this project were described as "turn up the Internet of Things promises by simplifying object and application deployment, commissioning, maintenance, operation and service composition inside building infrastructures" [24]. Middleware was also involved as a way to abstract hardware heterogeneity from the different devices that could be present in the network.

3. e-GOTHAM: Sustainable-Smart Grid Open System for the Aggregated Control, Monitoring and Management of Energy (ARTEMIS) [21]. As it has been mentioned before, this project dealt with the Integration of Information and Communication Technologies at the microgrid level by creating a new aggregated energy demand model, so that different appliances related to the latter could be monitored and requested for services. The requirement analysis, design procedures and implementation works done in this project are of critical importance to the thesis.

4. I3RES: ICT-based intelligent integration of Renewable Energy Sources (FP7) aimed to "integrate renewable energy sources in the distribution grid by incorporating intelligence at three different levels: in the integration of Renewable Energy Sources (RES) and the development of control and management mechanisms that reduce the impact of its intermittency; in the facilitation of the participation of all actors in the electricity market; and in the overall operation of the network" [53]. Even though activities in this project were dealing mostly with the services that could be obtained from the demand side, the requirement analysis carried out and the middleware solution that was specified for this project were also pivotal for the conception of CMA as a general semantic middleware architecture for the Smart Grid.

5. SWARMs: Smart and Networking UnderWAter Robots in Cooperation Meshes (ECSEL) [20]. This project has set as main objective spreading the usage of autonomous maritime vehicles with the purpose of easing the creation, planning and performance of tasks and 
missions in water-based environments. Although it is not explicitly related to the Smart Grid, it is an example of how Cyber-Physical Systems with similar challenges (heterogeneous hardware, set of services to be defined, interfaces with the application layer of a distributed system, etc.) can use middleware solutions that resemble the basic principles of other CPSs.

\subsubsection{Other produced results}

Aside from the scientific results that have been previously shown regarding publications and projects where this thesis is embedded, there are some other achievements that must be mentioned, as they are closely related to the activities that have been carried out in relation with this manuscript.

The software components that were developed for e-GOTHAM have been registered as original contributions made by the researches involved in the project. The documentation required to be delivered to the Technology Transfer Office (OTT) of the Technical University of Madrid clearly states that the author of the thesis has been an active co-operator in the design, implementation and testing of the software solutions that have been created as the middleware solutions in the e-GOTHAM research project. Among other features, procedures showing how the semantic middleware components were conceived, designed, implemented and tested have been included in the thesis.

The ideas that have been included in this thesis were also the cornerstone for a business models. Exploitation of the results that have been obtained in the projects are a major area or interest in the projects funded by the European Commission, so aside of including the development activities carried out regarding middleware in several demonstrators (with the most prominent ones shown in the previous section) an idea for a business based on integration of Renewable Energy Sources based on semantic middleware was put forward for the $13^{\text {th }}$ competition for start-up ideas made during 2016 called Actúa UPM [191]. The startup idea was applied under the name "Middlegrid". Among the 506 business ideas that were presented, the one co-authored by the author of thesis was considered among the top 10 ones and received a cash prize of $1000 €$ [192] and a certificate that has been included as Figure 105. 


\section{ZCTÚZZuPm}

El Programa de Creación de Empresas UPM otorga al proyecto:

\section{Middlegrid}

\section{DIPLOMA A LA MEJOR IDEA DE NEGOCIO}

Por el que se reconoce el potencial de desarrollo de la idea de negocio presentada a la XIII Competición de Creación de Empresas UPM, actúaupm, por D. Pedro Castillejo, Dña. Esther Moreno, D. José Fernán Martínez y D. Jesús Rodríguez con un premio dotado con $1.000 €$.

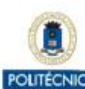

CAMPUS
DE EXCELENCIA INTERNACIONA

CA:T

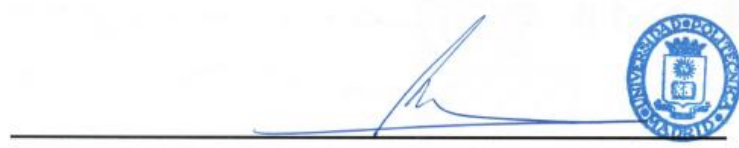

D. Gonzalo León Serrano Director del Centro de Apoyo a la Innovación Tecnológica (CAIT) En Madrid, a 7 de abril de 2016

Figure 99. Cash prize certificate for Middlegrid (Spanish)

The main achievements that have been produced as a result of the implementation works that have been carried out for this thesis have been summarized in Figure 106 in a chronological order. The notation that has been used is the same as the one that was referred to those achievements in the previous pages. Note that the activities related to commercial aspects where the middleware is involved have been referred to as business model (for the preparation of the Middlegrid proposal) and E-G/I3 Reg. for the registration of the middleware produced in e-GOTHAM and I3RES projects. 


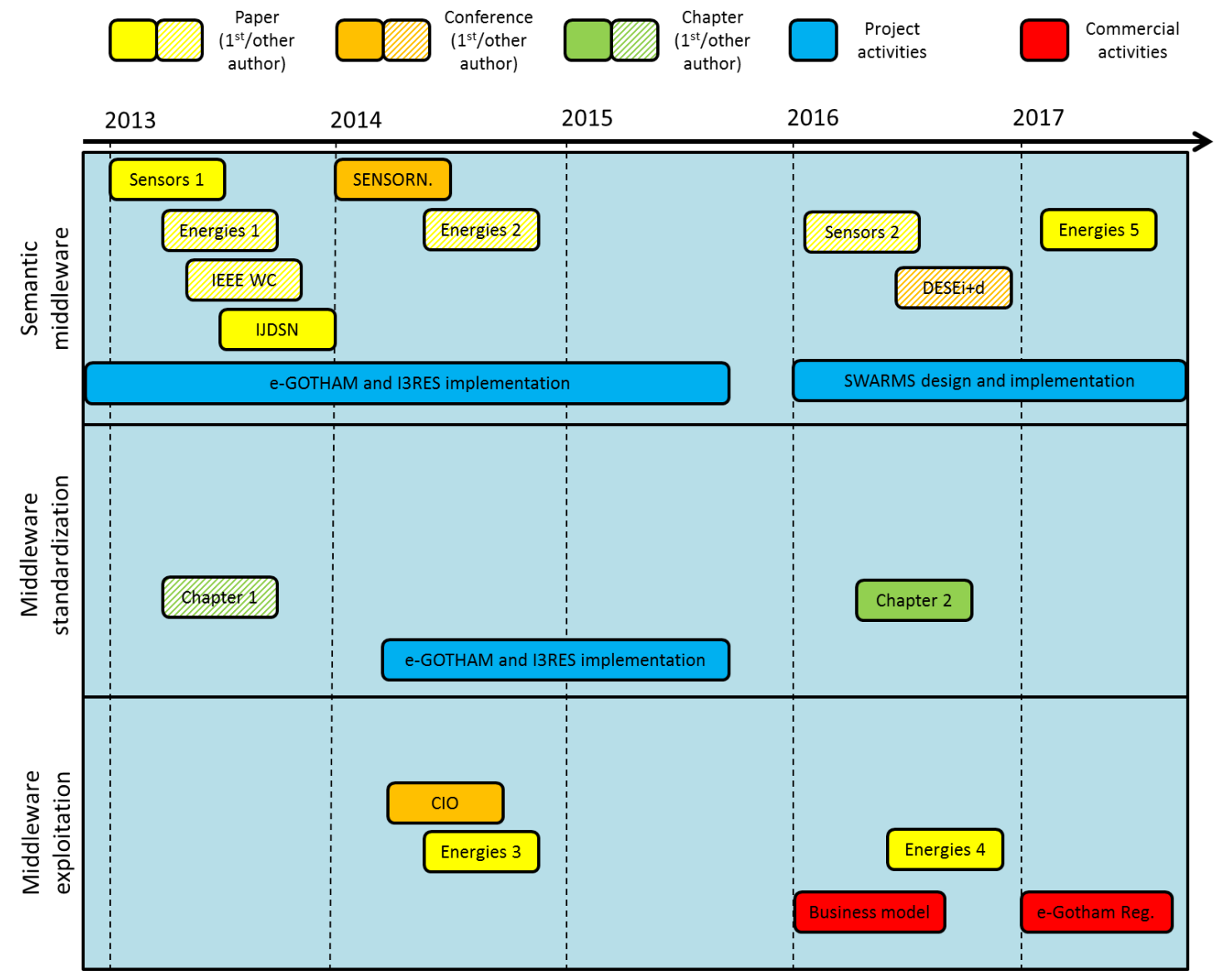

Figure 100. Chronological summarization of all the outputs resulting in the thesis 
Appendix A: development procedures 
This Appendix is used to include some of the implementation works that, although do not strictly fall within research activities (rather, implementation took place as an output of the research activities done) are useful to have as some extra content, in case it can be useful as a guide on semantic middleware architectures for the Smart Grid.

For starters, it must be remembered that there are two actions that must be carried out In order to have them working as an Apache Maven piece of work:

1. The option to create an Apache Maven project must be available from the IDE: depending on the version of the latter, it may be accessible right away or a plug-in will have to be downloaded. For example, in Figure 107, it can be seen how it is accessed by following the File $\rightarrow$ New $\rightarrow$ Maven Project options.

\section{\& New Maven Project}

\section{New Maven project}

Select project name and location

Create a simple project (skip archetype selection)

Use default Workspace location

Location:

Browse...

Add project(s) to working set

Working set:

- Advanced

Figure 101. Maven project access in Eclipse IDE for Ubuntu

2. The group and artifact identifiers must be set so that bundles will be able to communicate with each other via OSGi interfaces. This will be done by setting the name of the Group ID as the one that will be used in order to univocally identify the project among the other ones. The Artifact ID, on the other hand, will be used to identify the future .jar file to be executed that contains the developed service. The configuration is included in Figure 108.

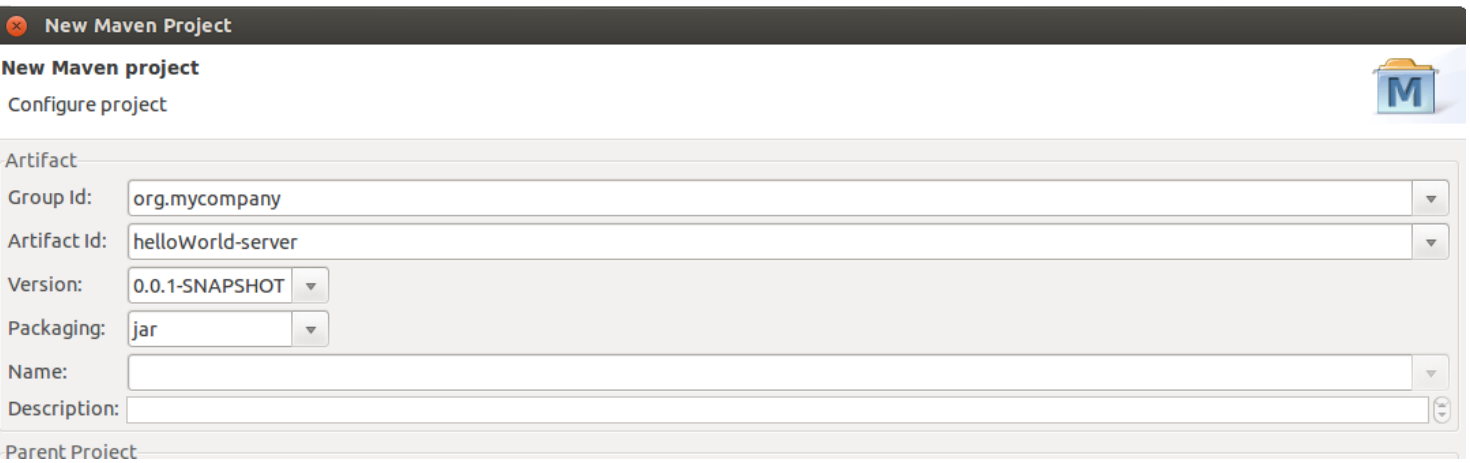

Figure 102. Creation of a maven project for Eclipse in Ubuntu 
There are several other software components that are part of JBoss Fuse and play different roles in it, and even some others that can be installed in order to enhance the qualities of the services that are provided. For example, in Figure 109 it can be seen where the software components were supposed to be included, as well as the broker that was used in order to establish a layer of AMQP communications right below the middleware architecture. There are some features from the JBoss Fuse that can be seen, but they are common to other ESB implementations and systems (dependencies, uninstalling executable program, etc.).

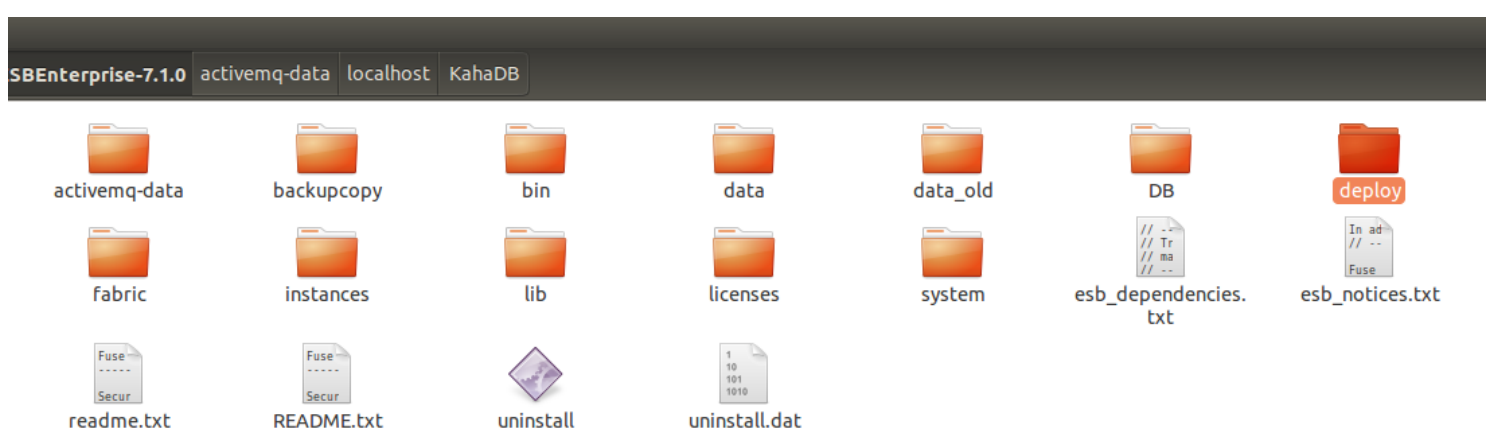

Figure 103. Location of the activemq broker and deploy directories

JBoss Fuse also makes available a web interface with several capabilities that can be accessed for a better understanding of the software components that have been deployed. It is also a very useful tool for debugging any failure that may have happened when deploying a bundle to the JBoss Fuse, as it will show a brief message that tells any issue found. This feature has been depicted in Figure 110.

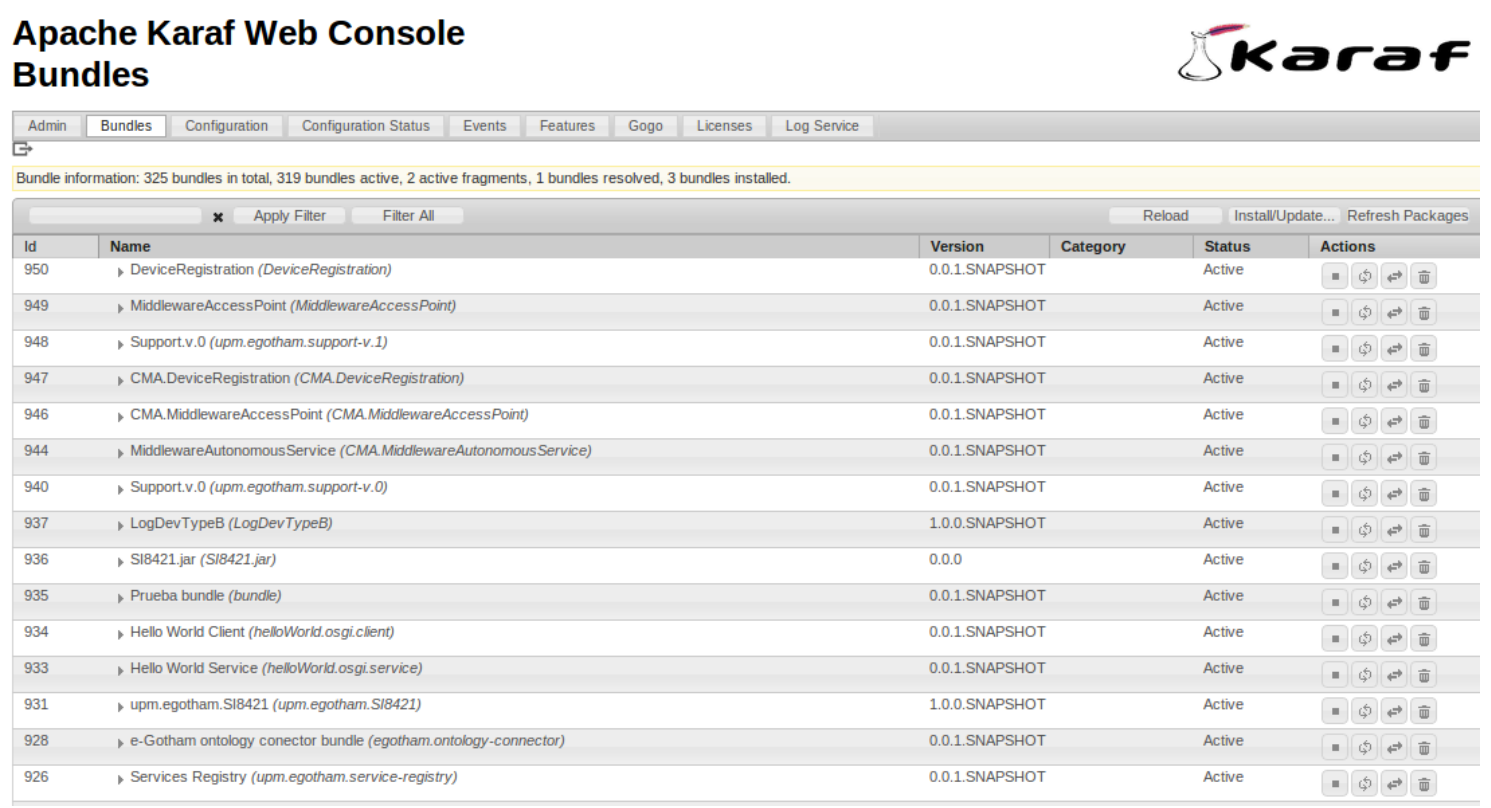

Figure 104. Web interface of the JBoss Fuse

The last aspect to take into account when using the ESB is that there are several permissions that must be enabled in order to have full access to the testing and deployment of the presented solutions. These permissions are properties that must be addressed by editing the user.properties file that usually stored in the /etc directory of JBoss Fuse (several minor 
changes may happen depending on the version of the ESB, though). As displayed in Figure 111, commonly there will be two categories; one is referred to the end users and the other to the administrator. Logins and passwords will be set to the end users in order to have them use the system in a more extensive fashion or not, depending on the privileges that they have been given.

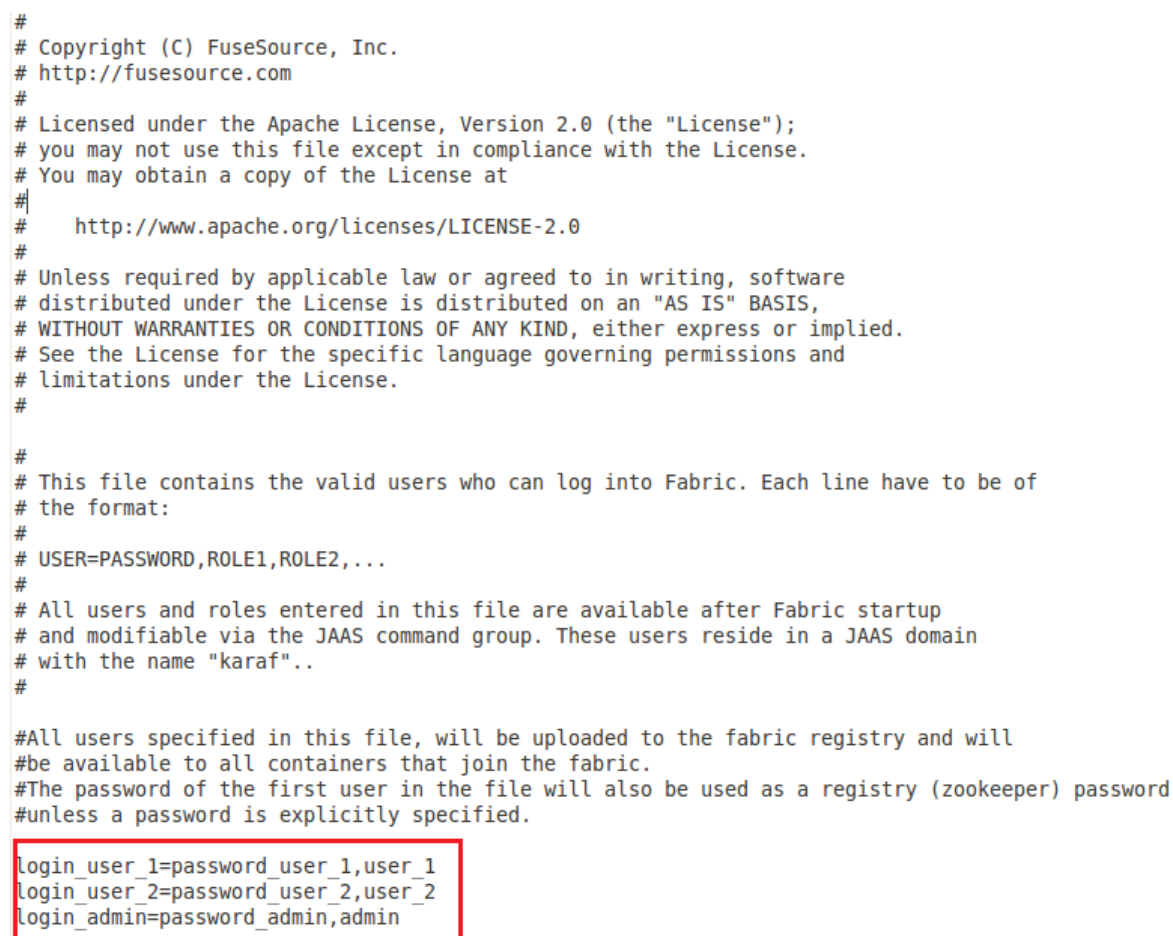

Figure 105. User.properties file in JBoss Fuse

A final glimpse of the appearance of the Java bundles developed as the software components of the middleware architecture is offered in Figure 112. This is the result stored in the /deploy directory of JBoss Fuse. They can be divided in different kinds, depending on what they are related to:

1. Middleware services related to different levels: these are the software bundles that have been obtained regarding the features that have been described before (high level, core and low level services).

2. Registered devices: these are the software bundles used to contain all the features and services that are provided for a registered device. This decision was taken due to the specific developments in the e-GOTHAM, so bundles containing descriptions of the services may or may not be present in other research projects.

3. Ancillary software components. These latter ones are shown as a way to prove that external works can also be included in the JBoss Fuse, as long as they are converted in functional bundles. 


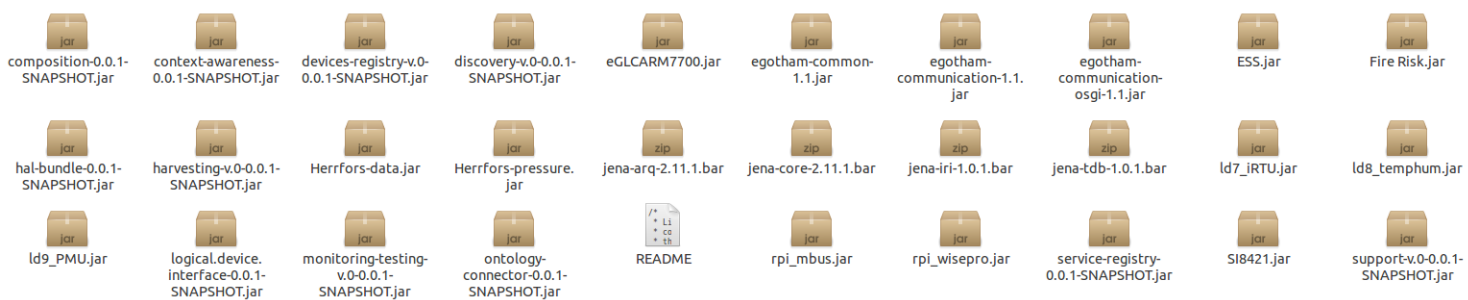

Figure 106. Software bundles from the middleware

One of the most important actions to be undertaken during the development works of the eGOTHAM project was connecting to those facilities by means of a Virtual Private Network (VPN) which was offered to us as a way to perform remote testing, while at the same time having a securitized environment that can offer confidentiality with data authentication. The parameters required to access this VPN were as shown in Figure 113.

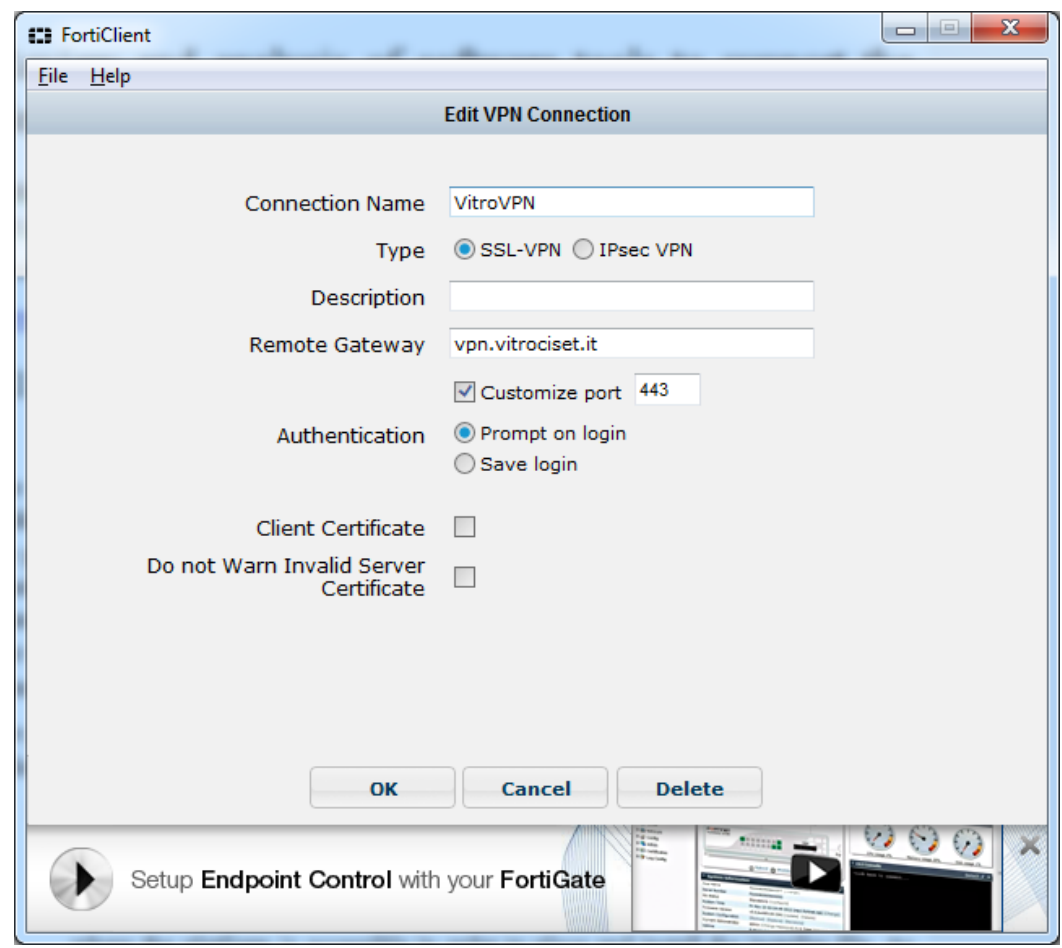

Figure 107. Virtual Private Network settings for remote connections to the Vitrociset laboratory

Once the parameters, login and password were correctly set, there was no problem in accessing remotely to the hardware used for service testing. The software components of the middleware would be loaded to the remote piece of equipment by means of a Secure File Transfer Protocol (SFTP) called Filezilla, and the operation with them via Command Line Interface would be done in the same way as if local equipment was being handled. The version of JBoss Fuse that was installed in those facilities was Fuse ESB, a slightly older version of the ESB that was commonly used locally (which switched from Fuse ESB to JBoss Fuse as it was made available), as it had been agreed with other partners to use this one over making any change that would consume time. The components had the appearance in the Command Line Interface as portrayed in Figure 114. 

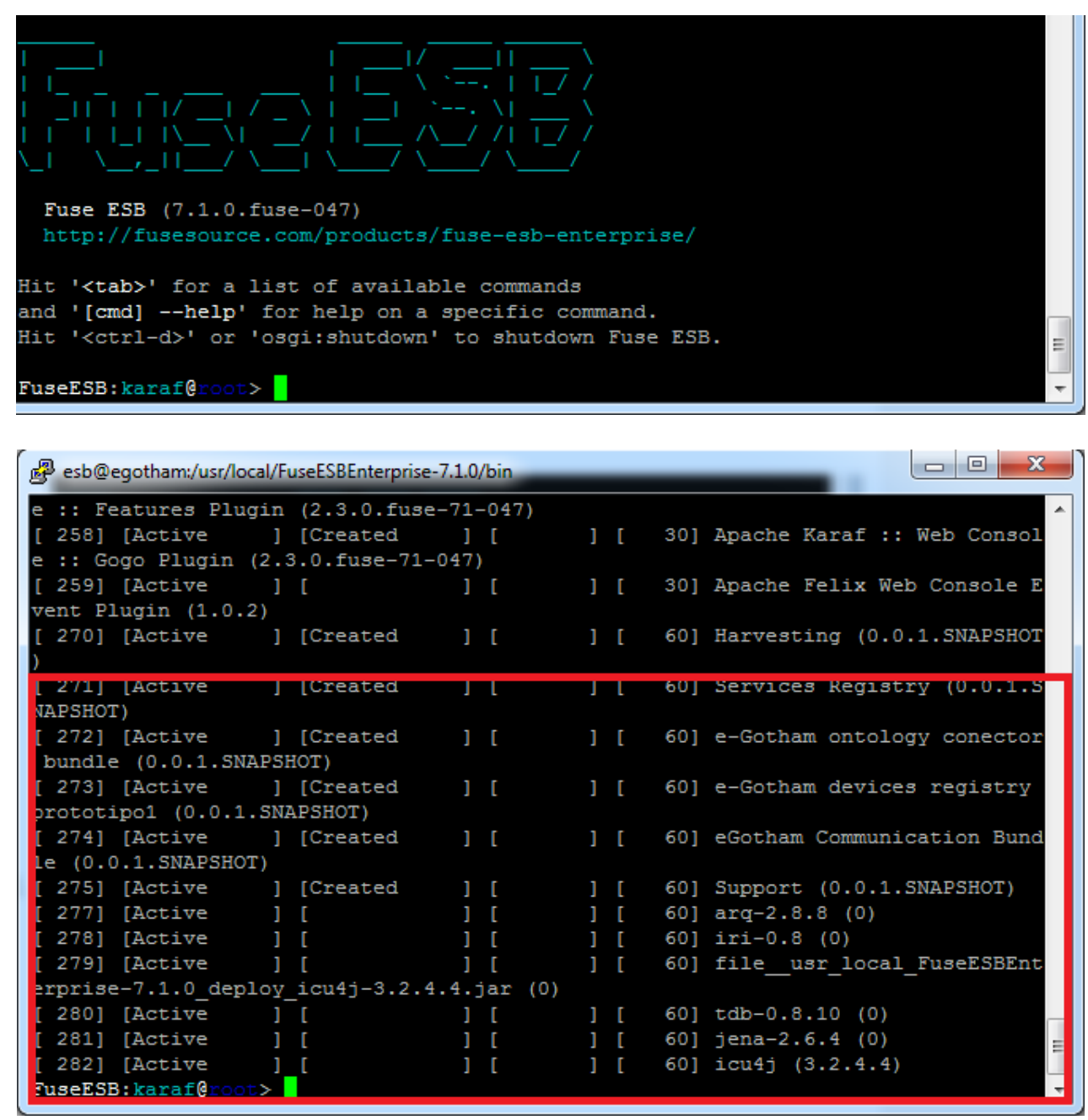

Figure 108. Example of ESB and components deployed in Vitrociset facilities

Lastly, while implementation works were carried out for the ontology used as the starting ground of the different features represented by the CMA, an OWL file was obtained after adding all the elements that were described in Section 4. Some of the content of this file has been included in the next pages. Both the ontology and the OWL file were developed by using Protegé as the tool for this implementation works.

?xml version="1.0"?>

$<$ !DOCTYPE rdf:RDF [

<!ENTITY owl "http://www.w3.org/2002/07/owl\#" >

<!ENTITY xsd "http://www.w3.org/2001/XMLSchema\#" >

<!ENTITY rdfs "http://www.w3.org/2000/01/rdf-schema\#" >

<!ENTITY rdf "http://www.w3.org/1999/02/22-rdf-syntax-ns\#" > 
]>

<rdf:RDF xmlns="http://www.semanticweb.org/jesús/ontologies/2017/3/untitledontology-12\#"

xml:base="http://www.semanticweb.org/jesús/ontologies/2017/3/untitled-ontology$12 "$

xmlns:rdfs="http://www.w3.org/2000/01/rdf-schema\#"

xmlns:owl="http://www.w3.org/2002/07/owl\#"

xmlns:xsd="http://www.w3.org/2001/XMLSchema\#"

xmlns:rdf="http://www.w3.org/1999/02/22-rdf-syntax-ns\#">

<owl:Ontology

rdf:about="http://www.semanticweb.org/jesús/ontologies/2017/3/untitled-ontology-12"/>

$<!-$

|||||||||||||||||||||||||||||||||||||||||||||||||||||||||||||||||||||||||||||||||||

/l

// Object Properties

//

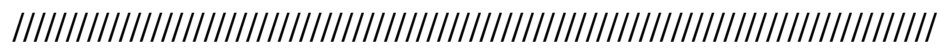

$-->$

$<!--$ http://www.semanticweb.org/jesús/ontologies/2017/3/untitled-ontology-

12\#hasAManufacturer -->

$<$ owl:ObjectProperty

rdf:about="http://www.semanticweb.org/jesús/ontologies/2017/3/untitled-ontology-

12\#hasAManufacturer">

$<$ rdfs:domain

rdf:resource="http://www.semanticweb.org/jesús/ontologies/2017/3/untitled-ontology12\#hardware"/>

$</ o w l: O b j e c t P r o p e r t y>$

$<!--$ http://www.semanticweb.org/jesús/ontologies/2017/3/untitled-ontology-

12\#hasAName -->

$<$ owl:ObjectProperty

rdf:about="http://www.semanticweb.org/jesús/ontologies/2017/3/untitled-ontology-

12\#hasAName"> 
$<$ rdfs:domain

rdf:resource="http://www.semanticweb.org/jesús/ontologies/2017/3/untitled-ontology12\#hardware"/>

$</$ owl:ObjectProperty $>$

$<!--$ http://www.semanticweb.org/jesús/ontologies/2017/3/untitled-ontology-

12\#hasASimpleService -->

$<$ owl:ObjectProperty

rdf:about="http://www.semanticweb.org/jesús/ontologies/2017/3/untitled-ontology-

12\#hasASimpleService">

$<$ rdfs:domain

rdf:resource="http://www.semanticweb.org/jesús/ontologies/2017/3/untitled-ontology12\#device_services"/>

$<$ rdfs:domain

rdf:resource="http://www.semanticweb.org/jesús/ontologies/2017/3/untitled-ontology12\#hardware"/>

</owl:ObjectProperty>

$<!--$ http://www.semanticweb.org/jesús/ontologies/2017/3/untitled-ontology12\#hasAStatus -->

<owl:ObjectProperty

rdf:about="http://www.semanticweb.org/jesús/ontologies/2017/3/untitled-ontology-

12\#hasAStatus">

$<$ rdfs:domain

rdf:resource="http://www.semanticweb.org/jesús/ontologies/2017/3/untitled-ontology12\#hardware"/>

$<$ owl:ObjectProperty>

$<$ !-- http://www.semanticweb.org/jesús/ontologies/2017/3/untitled-ontology12\#hasAnApplication -->

<owl:ObjectProperty

rdf:about="http://www.semanticweb.org/jesús/ontologies/2017/3/untitled-ontology-

12\#hasAnApplication">

$<$ rdfs:domain

rdf:resource="http://www.semanticweb.org/jesús/ontologies/2017/3/untitled-ontology12\#grounding"/>

</owl:ObjectProperty> 
<!-- http://www.semanticweb.org/jesús/ontologies/2017/3/untitled-ontology12\#hasAnInnerInterface -->

$<$ owl:ObjectProperty

rdf:about="http://www.semanticweb.org/jesús/ontologies/2017/3/untitled-ontology-

12\#hasAnInnerInterface">

$<$ rdfs:domain

rdf:resource="http://www.semanticweb.org/jesús/ontologies/2017/3/untitled-ontology12\#HAL_service"/>

$<$ rdfs:domain

rdf:resource="http://www.semanticweb.org/jesús/ontologies/2017/3/untitled-ontology12\#MAP_service"/>

$<$ rdfs:domain

rdf:resource="http://www.semanticweb.org/jesús/ontologies/2017/3/untitled-ontology12\#MAS_service"/>

$<$ rdfs:domain

rdf:resource="http://www.semanticweb.org/jesús/ontologies/2017/3/untitled-ontology12\#composition_service"/>

$<$ rdfs:domain

rdf:resource="http://www.semanticweb.org/jesús/ontologies/2017/3/untitled-ontology12\#context_service"/>

$<$ rdfs:domain

rdf:resource="http://www.semanticweb.org/jesús/ontologies/2017/3/untitled-ontology12\#middleware_services"/>

$<$ rdfs:domain

rdf:resource="http://www.semanticweb.org/jesús/ontologies/2017/3/untitled-ontology12\#registration_service"/>

$<$ rdfs:domain

rdf:resource="http://www.semanticweb.org/jesús/ontologies/2017/3/untitled-ontology12\#security_service"/>

$</$ owl:ObjectProperty $>$

<!-- http://www.semanticweb.org/jesús/ontologies/2017/3/untitled-ontology12\#hasAnOuterInterface -->

<owl:ObjectProperty

rdf:about="http://www.semanticweb.org/jesús/ontologies/2017/3/untitled-ontology12\#hasAnOuterInterface"> 
$<$ rdfs:domain

rdf:resource="http://www.semanticweb.org/jesús/ontologies/2017/3/untitled-ontology12\#MAP_service"/>

$<$ rdfs:domain

rdf:resource="http://www.semanticweb.org/jesús/ontologies/2017/3/untitled-ontology12\#MAS_service"/>

$<$ rdfs:domain

rdf:resource="http://www.semanticweb.org/jesús/ontologies/2017/3/untitled-ontology12\#network"/>

$</$ owl:ObjectProperty $>$

<!-- http://www.semanticweb.org/jesús/ontologies/2017/3/untitled-ontology12\#hasAsymmetricCyphering -->

$<$ owl:ObjectProperty

rdf:about="http://www.semanticweb.org/jesús/ontologies/2017/3/untitled-ontology-

12\#hasAsymmetricCyphering">

$<$ rdfs:domain

rdf:resource="http://www.semanticweb.org/jesús/ontologies/2017/3/untitled-ontology12\#security_service"/>

$</$ owl:ObjectProperty $>$

$<!--$ http://www.semanticweb.org/jesús/ontologies/2017/3/untitled-ontology12\#hasCompositionFunctionality -->

<owl:ObjectProperty

rdf:about="http://www.semanticweb.org/jesús/ontologies/2017/3/untitled-ontology-

12\#hasCompositionFunctionality">

$<$ rdfs:domain

rdf:resource="http://www.semanticweb.org/jesús/ontologies/2017/3/untitled-ontology12\#composition_service"/>

$</$ owl:ObjectProperty $>$

$<!--$ http://www.semanticweb.org/jesús/ontologies/2017/3/untitled-ontology12\#hasContextAwarenessFunctionality -->

$<$ owl:ObjectProperty

rdf:about="http://www.semanticweb.org/jesús/ontologies/2017/3/untitled-ontology12\#hasContextAwarenessFunctionality"> 
$<$ rdfs:domain

rdf:resource="http://www.semanticweb.org/jesús/ontologies/2017/3/untitled-ontology12\#context_service"/>

$</$ owl:ObjectProperty $>$

$<!--$ http://www.semanticweb.org/jesús/ontologies/2017/3/untitled-ontology12\#hasDeviceList -->

$<$ owl:ObjectProperty

rdf:about="http://www.semanticweb.org/jesús/ontologies/2017/3/untitled-ontology12\#hasDeviceList">

$<$ rdfs:domain

rdf:resource="http://www.semanticweb.org/jesús/ontologies/2017/3/untitled-ontology12\#registration_service"/>

$</ o w l: O b j e c t P r o p e r t y>$

$<!--$ http://www.semanticweb.org/jesús/ontologies/2017/3/untitled-ontology12\#hasFeatures -->

<owl:ObjectProperty

rdf:about="http://www.semanticweb.org/jesús/ontologies/2017/3/untitled-ontology12\#hasFeatures">

$<$ rdfs:domain

rdf:resource="http://www.semanticweb.org/jesús/ontologies/2017/3/untitled-ontology12\#hardware"/>

$</ o w l: O b j e c t P r o p e r t y>$

$<!--$ http://www.semanticweb.org/jesús/ontologies/2017/3/untitled-ontology12\#hasGpsCoordinates -->

<owl:ObjectProperty

rdf:about="http://www.semanticweb.org/jesús/ontologies/2017/3/untitled-ontology12\#hasGpsCoordinates">

$<$ rdfs:domain

rdf:resource="http://www.semanticweb.org/jesús/ontologies/2017/3/untitled-ontology12\#hardware"/>

$</ o w l: O b j e c t P r o p e r t y>$

$<!--$ http://www.semanticweb.org/jesús/ontologies/2017/3/untitled-ontology-

12\#hasHALFunctionality --> 
<owl:ObjectProperty

rdf:about="http://www.semanticweb.org/jesús/ontologies/2017/3/untitled-ontology-

12\#hasHALFunctionality">

$<$ rdfs:domain

rdf:resource="http://www.semanticweb.org/jesús/ontologies/2017/3/untitled-ontology12\#HAL_service"/>

$</$ owl:ObjectProperty $>$

$<!--$ http://www.semanticweb.org/jesús/ontologies/2017/3/untitled-ontology12\#hasMAPFunctionality -->

$<$ owl:ObjectProperty

rdf:about="http://www.semanticweb.org/jesús/ontologies/2017/3/untitled-ontology-

12\#hasMAPFunctionality">

$<$ rdfs:domain

rdf:resource="http://www.semanticweb.org/jesús/ontologies/2017/3/untitled-ontology12\#MAP_service"/>

$</$ owl:ObjectProperty>

$<!--$ http://www.semanticweb.org/jesús/ontologies/2017/3/untitled-ontology12\#hasMASFunctionality -->

$<$ owl:ObjectProperty

rdf:about="http://www.semanticweb.org/jesús/ontologies/2017/3/untitled-ontology-

12\#hasMASFunctionality">

$<$ rdfs:domain

rdf:resource="http://www.semanticweb.org/jesús/ontologies/2017/3/untitled-ontology12\#MAS_service"/>

$</$ owl:ObjectProperty $>$ 
Appendix B: Application
Programming Interface 
Considering the work that was carried out during the implementation of the middleware solution, as well as the information that is presented in other software standards of widespread use in distributed systems (COAP, AMQP, MQTT, energy interoperability solutions, etc.), it has been considered as mandatory to provide and Application Programming Interface that can be used as a way to provide a starting ground for middleware developments in the Smart Grid. It has to be noted that the thesis is focused on providing an API for the middleware rather than the implementation of specific services such as security or semantic capabilities. Consequently, these latter two services can be further expanded with the usage of further methods that will make possible the usage of more functionalities provided by the systems where they are deployed.

The API that has been included in this section is the one that belongs to several classes that were developed for the e-GOTHAM project. The reason for choosing just some of them is due to the fact that they have a huge amount of methods, and in fact there are a total of 230 classes that were developed during the semantic middleware implementation works. Having the API of all the classes and methods would result in a very long document, where the majority of the content would be the API itself, which is unrelated to the main objectives of this thesis.

To begin with, the API from the SupportConnectorREST class has been included. As it can be inferred from the name, it is used to guarantee connectivity between the application layer and all the other components from the middleware. Its appearance has been included in Figure 115.

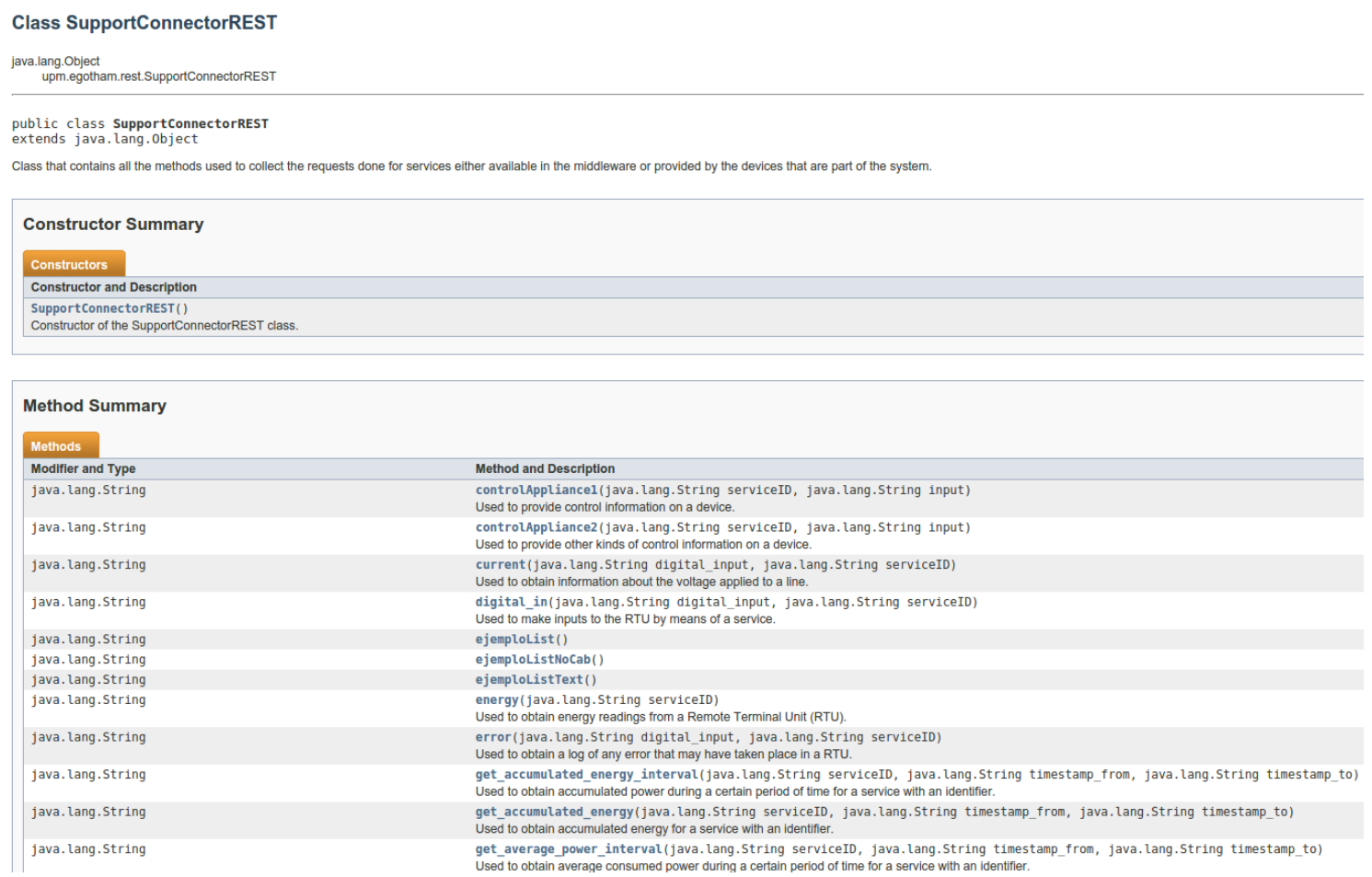




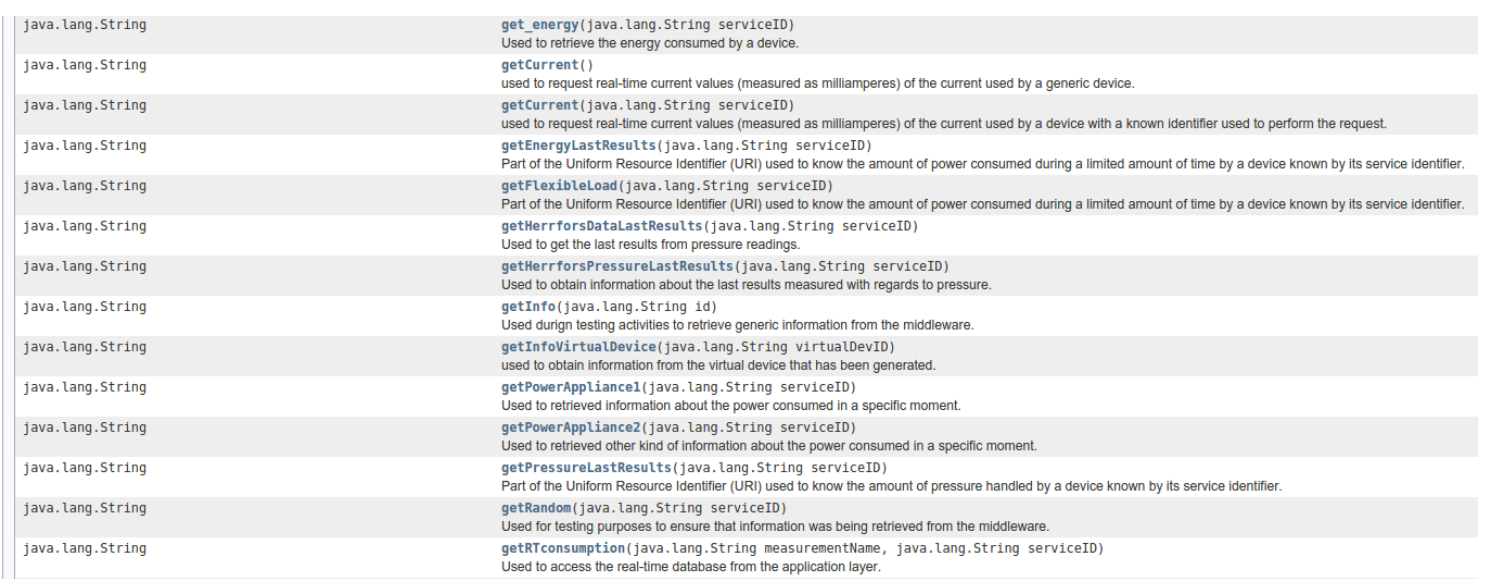

Figure 109. API from the SupportREST class

The Support class, on the other hand, makes sure that the core functionalities of the support software component will be carried out. Compared to what has been described in the CMA, this component can be regarded as a Middleware Access Point, so it will provide access to other functionalities from the semantic middleware architecture that has been installed. The methods that have been codified are in Figure 116.

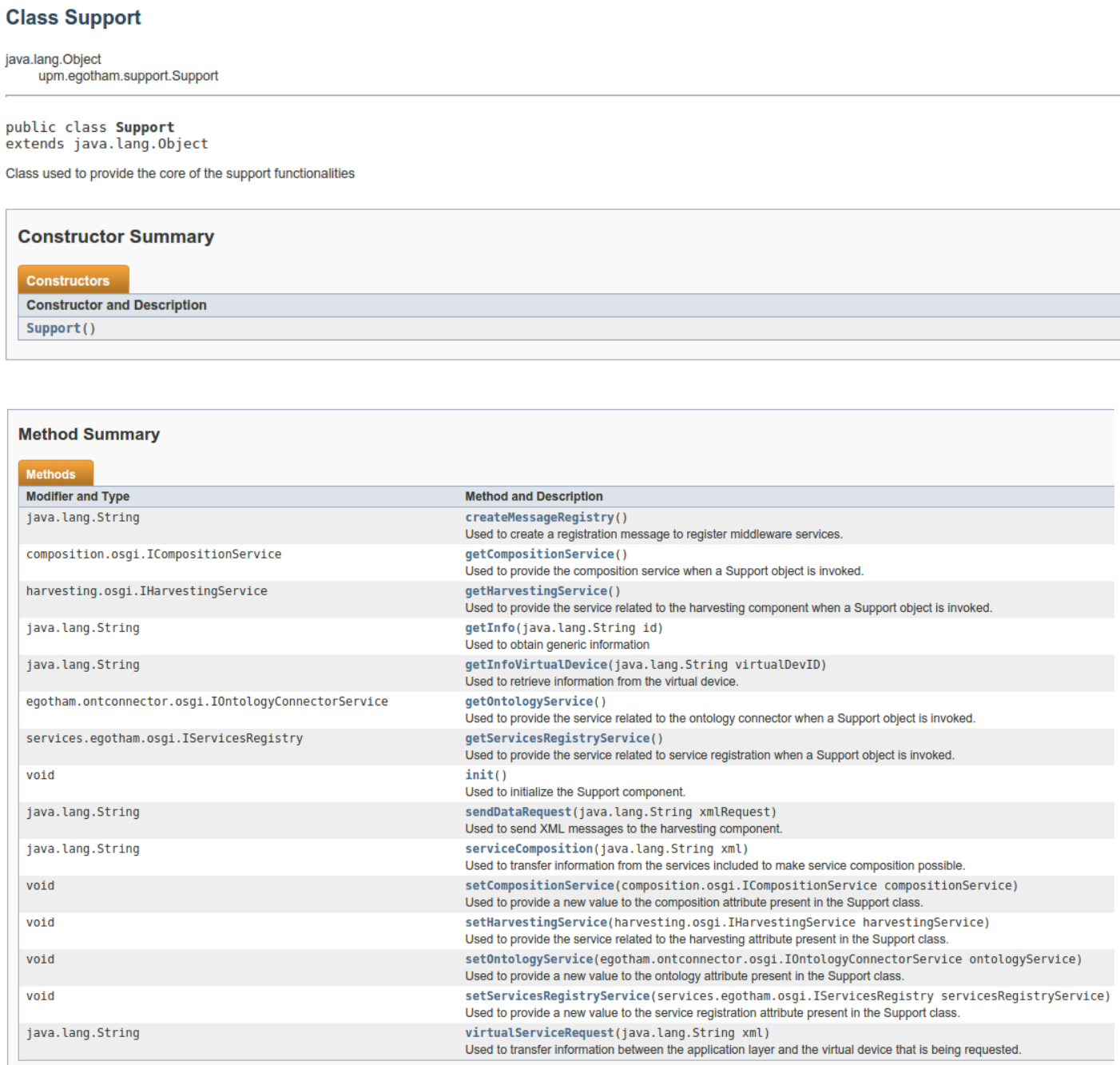


In the same way, the ContextAwarenessController class is used in order to manage the functionalities used for context awareness functionalities. In case of this class, it becomes important to include functionalities that will be capable of interacting with all the other components required for the context awareness functionalities. Therefore, the ontology connector and the methods related to device registration have been included as part of the API. The methods that were implemented have been included in Figure 117.

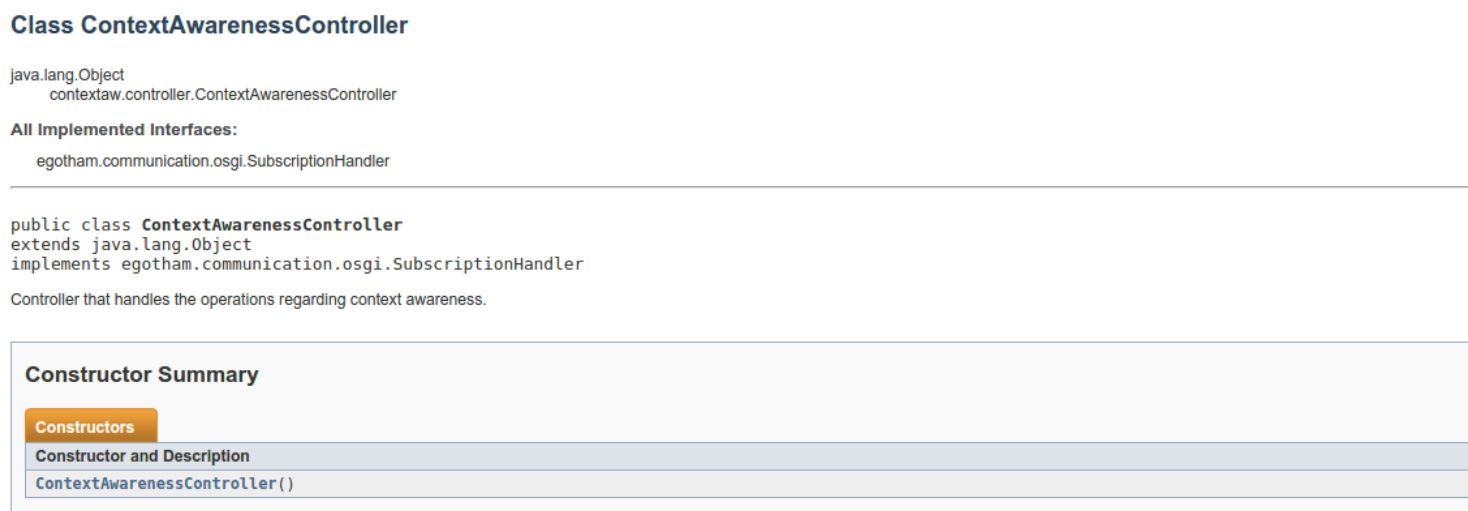

\begin{tabular}{|c|c|}
\hline \multicolumn{2}{|l|}{ Method Summary } \\
\hline \multicolumn{2}{|l|}{ Methods } \\
\hline Modifier and Type & Method and Description \\
\hline java.lang.String & $\begin{array}{l}\text { createMlessageRegistry () } \\
\text { CUsed to create the XML message that will be used to register the context awareness service registration. }\end{array}$ \\
\hline egotham. communication. osgi. ICommunication & $\begin{array}{l}\text { getCommunicationService() } \\
\text { Used to get the communication facilities used by the context awareness component. }\end{array}$ \\
\hline java.util. Map<java. lang.String, java. lang.String> & $\begin{array}{l}\text { getDevicesMap () } \\
\text { Used to provide the map of the registered services. }\end{array}$ \\
\hline java. lang.String & $\begin{array}{l}\text { getEf fect iveT ransportID ( } j \text { ava. lang. String serviceID) } \\
\text { Used to look for a transport identifier that will be used to substitute services by means of a service identifier. }\end{array}$ \\
\hline services. egotham. osgi. IServicesRegistry & $\begin{array}{l}\text { getServicesRegistryService() } \\
\text { Used to obtain the services registration parameter. }\end{array}$ \\
\hline java. lang.String & $\begin{array}{l}\text { getTypeByServiceId ( java. lang. Long serviceId) } \\
\text { Used to provide the deviceType for a given service identifier. }\end{array}$ \\
\hline void & $\begin{array}{l}\text { handleMessage ( } \mathrm{java} \text {. lang. String mes sage) } \\
\text { Used to process an XML message retrieved from the communication component. }\end{array}$ \\
\hline void & $\begin{array}{l}\text { init () } \\
\text { Used to initialize the instance of the object created from the class. }\end{array}$ \\
\hline boolean & $\begin{array}{l}\text { isDeviceTypeDeployed ( } j \text { ava. lang. String deviceType) } \\
\text { Used to know whether there is a type b bundle for a specific type of device }\end{array}$ \\
\hline void & $\begin{array}{l}\text { onDeviceFailure ( } \text { java. lang.String serviceID) } \\
\text { Used to replace the malfunctioning service with the one expected to substitute it. }\end{array}$ \\
\hline void & $\begin{array}{l}\text { onDeviceRestore ( } \text { java. lang.String serviceID) } \\
\text { used to manage the process of making the service again usable. }\end{array}$ \\
\hline void & $\begin{array}{l}\text { process XML ( java. lang. String message) } \\
\text { Used to process the XML message received by the component. }\end{array}$ \\
\hline void & $\begin{array}{l}\text { registerTypeBDevice ( } \mathrm{j} \text { ava. lang. String deviceType, java.lang.Long serviceId) } \\
\text { Used to match a new type b device with its deviceType }\end{array}$ \\
\hline void & $\begin{array}{l}\text { setCommunicationservice (egotham. communication. osgi. I Communication communicationservice) } \\
\text { Used to establish the communication facilities used by the context awareness component. }\end{array}$ \\
\hline void & $\begin{array}{l}\text { setontologyconnector (egotham. ont connector. osgi. IOntologyConnectorService ontologyconnector) } \\
\text { Used to establish the variable used to invoke facilities from the ontology connector. }\end{array}$ \\
\hline void & $\begin{array}{l}\text { setServicesRegistryService (services. egotham. osgi. IServicesRegistry servicesRegistryService) } \\
\text { Used to establish the service registration parameter. }\end{array}$ \\
\hline void & $\begin{array}{l}\text { stop () } \\
\text { Used to stop actions in the context awareness component. }\end{array}$ \\
\hline
\end{tabular}

Figure 111. API of the ContextAwarenessController class

The Composition Controller class is mostly used to manage the composition operations used to merge different simple services into a single one capable of providing different functionalities that are still related with the original simple services, as it has been previously described. As it happens with other components, there are also methods used for the interaction of this service with other ones present in the middleware (hence the set and get methods related to the message registration or the ontology component). A screenshot of the API from this class has been included in Figure 118. 


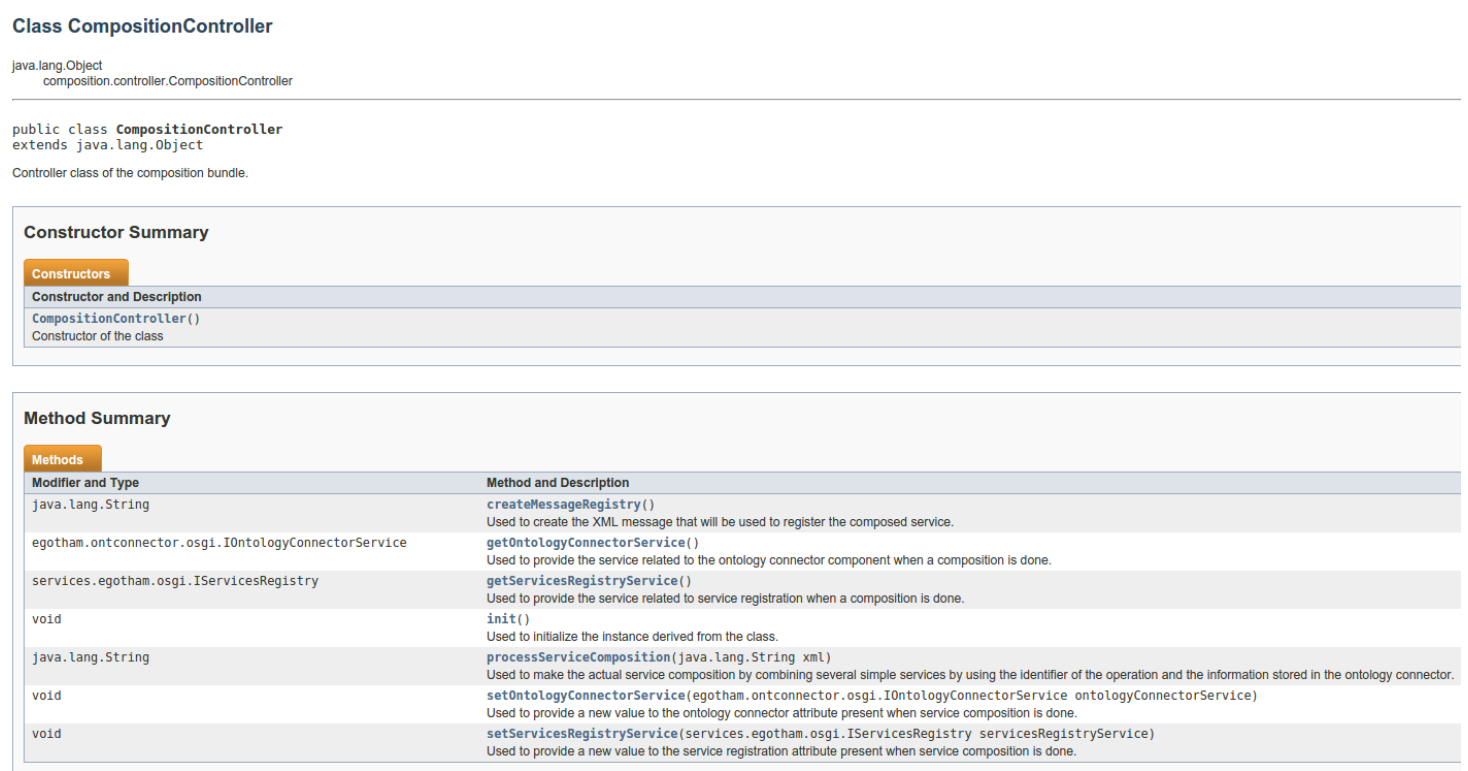

Figure 112. API of the CompositionController class

Lastly, the class used as the controller for the Hardware Abstraction Layer component has been included as well. Its functionalities are well reflected in the methods that have been included, as they are used to collect messages that come from middleware components located at a higher level and deal with lower level concepts, such as transport identifiers and producers. The API has been represented in Figure 119.

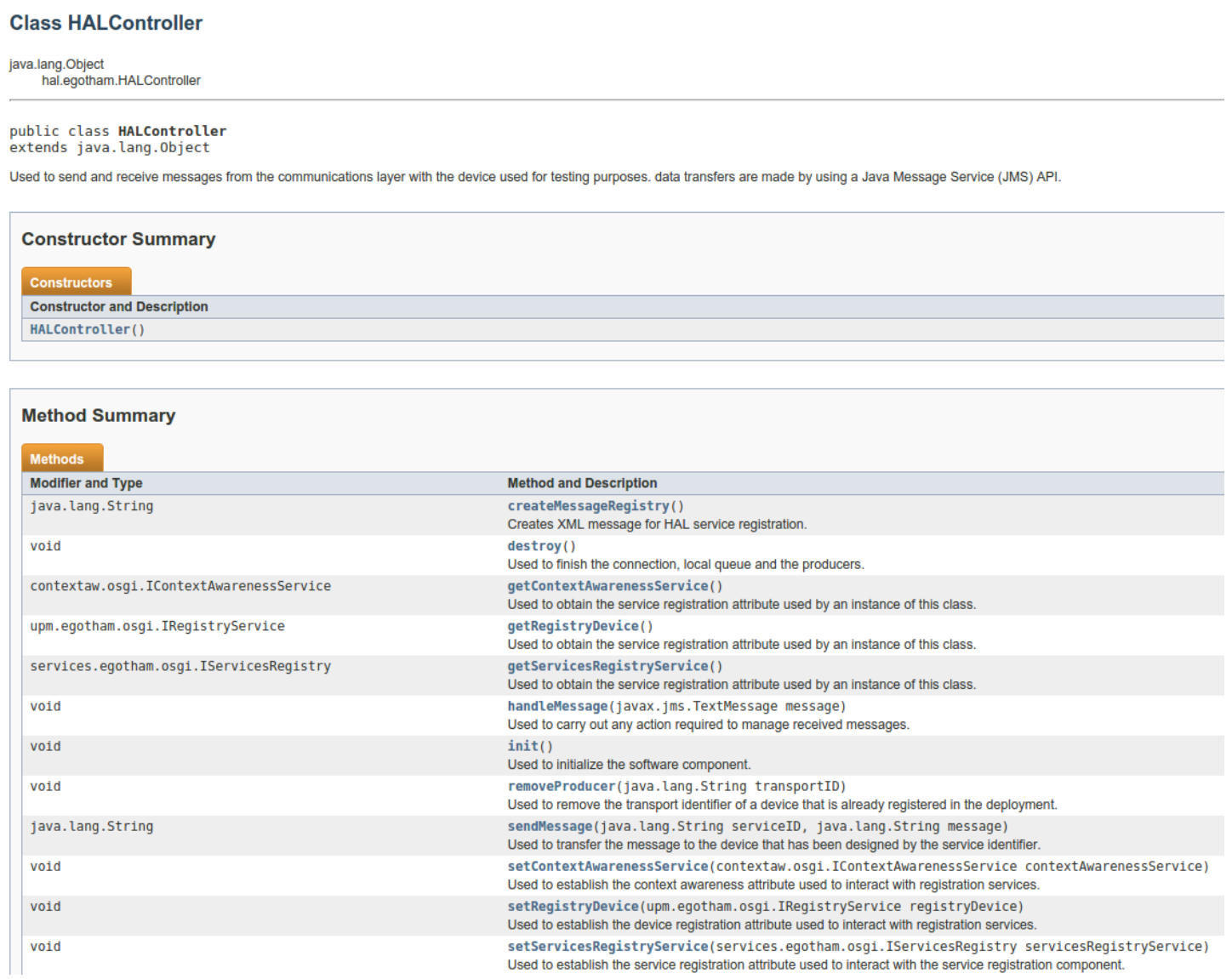

Figure 113. API of the HALController class 
6. Bibliography 


\subsection{References}

The following format has been used for the references that have been included:

1. Scientific papers: first author surname, first character of first author's name; second author surname, first character of second author's name, $\mathrm{N}$ author surname, first character of $\mathrm{N}$ author's name; name of the scientific paper [in italics]. Name of the journal, date details, volume, number of issue, pages [if applicable].

2. Conference papers: first author surname, first character of first author's name; second author surname, first character of second author's name, $\mathrm{N}$ author surname, first character of $\mathrm{N}$ author's name; name of the scientific paper [in italics]. Name of the conference (abbreviation of the conference), year, volume, number of issue, pages [if applicable].

3. Electronic books: name of the book. Available from: URL of the book. Date details. Last time it was accessed.

4. Web sites: name of the website. Available from: URL of the website. Date details. Last time it was accessed.

5. Other pieces of work (Bachelor theses, Master theses, etc.): name of the website. URL of the website. Last time it was accessed.

[1] Atkeson, A.; Kehoe, P. The Transition to a New Economy After the Second Industrial Revolution. Proceedings, Federal Reserve Bank of San Francisco. November 2002.

[2] Liu, Z.; Yu, J.; Guo, X.; Sun, T.; Zhang, J. Survey of technologies of line commutated converter based high voltage direct current transmission in China. CSEE Journal of Power and Energy Systems, 2015. vol. 1 no. 2. pp. 1-8.

[3] Brittain, J.E., Electrical Engineering Hall of Fame: Nikola Tesla. Proceedings of the IEEE, 2005. vol. 93, no.5. pp. 1057-1059.

[4] Pourbeik, P.; Kundur, P.S.; Taylor, C.W. The anatomy of a power grid blackout - Root causes and dynamics of recent major blackouts. IEEE Power and Energy Magazine, 2006. vol. 4, no. 5. pp. 22-29.

[5] Sardella, M. Stuck In the Sixties: Power Grid Modernization Remains Elusive. Available from:

http://static1.1.sqspcdn.com/static/f/205172/26761467/1451508058660/2015 1230 Stuck+in+the+60\%27s.pdf?token=qkFxflR3vL3p6\%2FWMibvzA4e8gmo\%3D. 2015. Last access $16^{\text {th }}$ May 2017.

[6] Tverberg, G. The U. S. electric grid: will it be our undoing? Available from: http://www.theoildrum.com/node/3934. 2008. Last access $16^{\text {th }}$ May 2017.

[7] Bu, S.; Yu, F. R.; Liu, P. X.; Zhang, P. Distributed Scheduling in Smart Grid Communications with Dynamic Power Demands and Intermittent Renewable Energy 
Resources. IEEE International Conference on Communications Workshops (ICC). 2011. pp. 1-5.

[8] Torres Sibille, A. del C.; Cloquell-Ballester Víctor-Andrés; Cloquell-Ballester VicenteAgustín; Darton, R. Development and validation of a multicriteria indicator for the assessment of objective aesthetic impact of wind farms. Renewable and Sustainable Energy Reviews. 2009. vol 13, no. 1. pp. 40-66.

[9] Rybach, L., Geothermal Power Growth 1995-2013-A Comparison with Other Renewables. Energies. 2014. vol 7 no. 8. pp. 4802-4812.

[10] Singhal, A.; Saxena, R.P. Software models for Smart Grid. International Workshop on Software Engineering for the Smart Grid (SE4SG). 2012.

[11] Rodríguez-Molina, J.; Martínez-Núñez, M.; Martínez, J.-F.; Pérez-Aguiar, W. Business Models in the Smart Grid: Challenges, Opportunities and Proposals for Prosumer Profitability. Energies. 2014. vol. 7 no. 9. pp. 6142-6171.

[12] European Energy Exchange AG, E.E.E. Available from: https://www.eex.com/en/. 2017. Last access $16^{\text {th }}$ May 2017.

[13] Bauer, F.L.; Bolliet, L.; Helms H. J. Software Engineering: Report on a conference sponsored by the NATO science committee, Peter Naur and Brian Randell, Editors. 1968. Last access $16^{\text {th }}$ May 2017.

[14] Uribe, O.H.; Martin J.P.; García-Alegre, J.P.; Santos, M.; Guinea, D. Smart Building: Decision Making Architecture for Thermal Energy Management. Sensors. 2015. vol 15 no. 11. pp. 27543-27568.

[15] Hussain, S.; Bang, J.H.; Han, M.; Ahmed, M.I.; Amin, M.B.; Lee, S.; Nugent, C.; McClean, S.; Scotney, B.; Parr, G. Behavior Life Style Analysis for Mobile Sensory Data in Cloud Computing through MapReduce. Sensors. 2014. vol. 14 no. 11. pp. 22001-22020.

[16] Razzaque, M.A.; Milojevic-Jevric, M.; Palade, A. ; S. Clarke. Middleware for Internet of Things: A Survey. IEEE Internet of Things Journal. 2016. vol 3, no. 1. pp. 70-95.

[17] Yurur, O.; C.H. Liu; Moreno, W. A survey of context-aware middleware designs for human activity recognition. IEEE Communications Magazine. 2014. vol 52 no 6. pp. 2431.

[18] Aguilar, J.; Jerez, M.; Exposito, E.; Villemur, T.. CARMiCLOC: Context Awareness Middleware in Cloud Computing. Latin American Computing Conference (CLEI). 2015.

[19] Yang, J., L. Zhang, and X.A. Wang. On Cloud Computing Middleware Architecture. in 2015 10th International Conference on P2P, Parallel, Grid, Cloud and Internet Computing (3PGCIC). 2015.

[20] SWARMs consortium. SWARMs: Smart and Networking UnderWAter Robots in Cooperation Meshes. Available from: http://www.swarms.eu/. 2017. Last access $17^{\text {th }}$ May 2017. 
[21] e-GOTHAM consortium. e-GOTHAM: Sustainable-Smart Grid Open System for the Aggregated Control, Monitoring and Management of Energy. Available from: http://ses.jrc.ec.europa.eu/e-gotham. 2012. Last access $17^{\text {th }}$ May 2017.

[22] CORDIS (Community Research and Development Information Service). ICT-based Intelligent management of Integrated RES for the smart grid optimal operation. Available from: http://cordis.europa.eu/project/rcn/106338 es.html. 2016. Last access $17^{\text {th }}$ May 2017.

[23] ITEA 3 Initiative. LifeWear: Mobilized Lifestyle With Wearables. Available from: https://itea3.org/project/lifewear.html. 2013. Last access $17^{\text {th }}$ May 2017.

[24] ITEA 3 Initiative. WoO: Web of Objects. Available from: https://itea3.org/project/webof-objects.html. 2013. Last access $17^{\text {th }}$ May 2017.

[25] Che, L.; Shahidehpour, M.; Alabdulwahab, A.; Al-Turki, Y. Hierarchical Coordination of a Community Microgrid With AC and DC Microgrids. IEEE Transactions on Smart Grid. 2015. vol. 6 no. 6. pp. 3042-3051.

[26] Ding, K.; Deng, B.; Zhang, X.; Ge, L. Optimization of service selection algorithm for complex event processing in Enterprise Service Bus platform. 4th International Conference on Computer Science \& Education. ICCSE '09. 2009. pp. 582-586.

[27] Jieming, W.; Xiaoli, T. Research of enterprise application integration based-on ESB. 2nd International Conference on Advanced Computer Control (ICACC). 2010. vol. 5. pp. 9093.

[28] Castillejo, P.; Martinez, J.-F.; Rodriguez-Molina, J.; Cuerva, A.. Integration of wearable devices in a wireless sensor network for an E-health application. IEEE Wireless Communications. vol 20, no. 4. pp. 38-49.

[29] Alghamdi, A.; Nasir, M.; Ahmad, I.; Nafjan, K.A. An interoperability study of ESB for C4I systems. International Symposium on Information Technology. 2010.

[30] Eurich, M. and R. Boutellier. Middleware integration platforms: A new challenge to business models of ICT companies: Unleashing the business potential of horizontalization. in e-Business (ICE-B), Proceedings of the 2010 International Conference on. 2010. vol 2. pp. 733-738.

[31] Chongnan, W.; Zongtao, W.; Hongwei, X. Design of message-oriented middleware with publish/subscribe model on Telemetry and Command computer. 2nd International Conference on Systems and Informatics (ICSAI). 2014.

[32] Bahsoon, R., W. Emmerich, and J. Macke, Using real options to select stable middleware-induced software architectures. IEE Proceedings - Software, 2005. vol 152. no 4. pp. 167-186.

[33] Ramakrishnan, R.; Gaur, L. Smart electricity distribution in residential areas: Internet of Things (IOT) based advanced metering infrastructure and cloud analytics. International Conference on Internet of Things and Applications (IOTA). 2016. pp. 46-51. 
[34] Rodríguez-Molina, J.; Martínez, J.-F.; Castillejo, P. A Study on Applicability of Distributed Energy Generation, Storage and Consumption within Small Scale Facilities. Energies. 2016. vol 9. no. 9. pp. 745.

[35] Wu, D.; Aliprantis, D.C.; Ying, L. Load Scheduling and Dispatch for Aggregators of PlugIn Electric Vehicles. IEEE Transactions on Smart Grid. 2012. vol 3. no 1. pp. 368-376.

[36] Sattarpour, T.; Daryoosh, N.; Golshannavaz, S.; Siano, P. A multi-objective hybrid GA and TOPSIS approach for sizing and siting of DG and RTU in smart distribution grids. Journal of Ambient Intelligence and Humanized Computing. 2016. pp. 1-18.

[37] Barella, R.; Nguyen, D.; Winter, R.; Lu, K.-T.; Wallace, S.; Zhao, X.; Cotilla-Sanchez, E. A Sensitivity Based Approach for Efficient PMU Deployment on Smart Grid. Smart Cities, Green Technologies, and Intelligent Transport Systems: 4th International Conference (SMARTGREENS). 2015. 1st International Conference (VEHITS). 2015. Lisbon, Portugal. May 20-22, 2015. Revised Selected Papers. pp. 199-215.

[38] Wu, X.; Zhu, X.; Wu, G. Q.; Ding, W. Data mining with big data. IEEE Transactions on Knowledge and Data Engineering, 2014. vol 26. no. 1. p. 97-107.

[39] Capra, L. MaLM: Machine Learning Middleware to Tackle Ontology Heterogeneity. Fifth Annual IEEE International Conference on Pervasive Computing and Communications Workshops (PerCom Workshops '07). 2007. pp. 449-454.

[40] Valls, M.G.; Lopez, I.R.; Villar, L.F. iLAND: An Enhanced Middleware for Real-Time Reconfiguration of Service Oriented Distributed Real-Time Systems. IEEE Transactions on Industrial Informatics. 2013. vol 9. no 1. pp. 228-236.

[41] Nishida, H.; Nguyen, T. Optimal Client/Server Assignment for Internet Distributed Systems. IEEE Transactions on Parallel and Distributed Systems. 2013. vol. 24. no. 3. pp. 565-575.

[42] Heard, N.; Rubin-Delanchy, P.; Lawson, D.J. Filtering Automated Polling Traffic in Computer Network Flow Data. Intelligence and Security Informatics Conference (JISIC), IEEE Joint. 2014. pp. 268-271.

[43] Croucher, D.A.; Engelbrecht, H.A. A peer-to-peer middleware plugin for an existing MMOG Client/Server architecture. 2012 IEEE International Workshop on Haptic Audio Visual Environments and Games (HAVE). 2012. pp. 140-141.

[44] Gjermundrod, H.; Bakken, D. E.; Hauser, C. H.; Bose, A. GridStat: A Flexible QoSManaged Data Dissemination Framework for the Power Grid. IEEE Transactions on Power Delivery. 2009. vol 24. no 1. pp. 136-143.

[45] Johnston, R. A.; Hauser, C. H.; Gjermundrod, K. H.; Bakken, D. E. Distributing TimeSynchronous Phasor Measurement Data Using the GridStat Communication Infrastructure. Proceedings of the 39th Annual Hawaii International Conference on System Sciences (HICSS'06). 2006. vol. 10. pp 245. 
[46] Viddal, E. S.; Bakken, D. E.; Gjermundr, H.; Hauser, C. H. Wide-Area Actuator RPC over GridStat with Timeliness, Redundancy, and Safety. International Conference on Complex, Intelligent and Software Intensive Systems (CISIS). 2010. pp. 17-24.

[47] Zhou, L.; Rodrigues, J.J.P.C. Service-oriented middleware for smart grid: Principle, infrastructure, and application. IEEE Communications Magazine. 2013. vol 51. no 1. pp. 84-89.

[48] Zaballos, A.; Vallejo, A.; Selga, J. M. Heterogeneous communication architecture for the smart grid. IEEE Network. 2011. vol 25. no 5. pp. 30-37.

[49] Sajjad, S.M.; Yousaf, M. Security analysis of IEEE 802.15.4 MAC in the context of Internet of Things (IOT). Conference on Information Assurance and Cyber Security (CIACS). 2014. pp. 9-14.

[50] Park, S.O.; Kim, J. S.; Kim, S. J. An object-based middleware supporting efficient interoperability on a smart home network. Multimedia Tools and Applications. 2013. vol. 63. no. 1. pp. 227-246.

[51] Li, Z.; Wang, Z.; Tournier, J. C.; Peterson, W.; Li, W.; Wang, Y. A Unified Solution for Advanced Metering Infrastructure Integration with a Distribution Management System. First IEEE International Conference on Smart Grid Communications (SmartGridComm). 2010. pp. 566-571.

[52] OASIS. Devices Profile for Web Services Version 1.1. Available from: http://docs.oasisopen.org/ws-dd/dpws/1.1/os/wsdd-dpws-1.1-spec-os.pdf. 2009. Last access $17^{\text {th }}$ May 2017.

[53] Mark Adamiak, D.B.; Mackiewicz, R. IEC 61850 Communication Networks and Systems In Substations: An Overview for Users. Available from: http://citeseerx.ist.psu.edu/viewdoc/download?doi=10.1.1.698.1497\&rep=rep1\&type =pdf. 2009. Last access $17^{\text {th }}$ May 2017.

[54] Sucic, S.; Bony, B.; Guise, L. Standards-compliant event-driven SOA for semanticenabled smart grid automation: Evaluating IEC 61850 and DPWS integration. IEEE International Conference on Industrial Technology (ICIT). 2012. pp. 403-408.

[55] Dewen, W.; Yongli, Z.; Peipei, L.; Jiancai, H.; Wenqing, Z. Research on distributed transmission of power telecontrol information based on ACSI/MMS. 3rd IEEE Conference on Industrial Electronics and Applications. 2008. pp. 670-674.

[56] Garc, A. P.; Oliver, J.; Gosch, D. An intelligent agent-based distributed architecture for Smart-Grid integrated network management. IEEE 35th Conference on Local Computer Networks (LCN). 2010. pp. 1013-1018.

[57] Awad, A.; German, R. Self-Organizing Smart Grid Services. Sixth International Conference on Next Generation Mobile Applications, Services and Technologies. 2012. pp. 205-210. 
[58] Kim, Y. J.; Thottan, M.; Kolesnikov, V.; Lee, W. A secure decentralized data-centric information infrastructure for smart grid. IEEE Communications Magazine. 2010. vol 48. no. 11. pp. 58-65.

[59] Fang, X.; Yang, D.; Xue, G. Evolving Smart Grid Information Management Cloudward: A Cloud Optimization Perspective. IEEE Transactions on Smart Grid. 2013. vol 4. no. 1. pp. 111-119.

[60] Moghe, U.; Lakkadwala, P.; Mishra, D. K. Cloud computing: Survey of different utilization techniques. CSI Sixth International Conference on Software Engineering (CONSEG). 2012. pp. 1-4.

[61] Liao, W.H.; Kuai, S. C. Leau, Y. R. Auto-scaling Strategy for Amazon Web Services in Cloud Computing. IEEE International Conference on Smart City/SocialCom/SustainCom (SmartCity). 2015. pp. 1-6.

[62] Souidi, S.; Boccio, D.; Mierzwa, S.; Aguilar, J. The feasibility of using Microsoft Azure infrastructure for a monitoring and evaluation system solution in sub-Saharan Africa. IEEE Global Humanitarian Technology Conference (GHTC). 2015. pp. 226-232.

[63] Subhashini, P.; Nalla, S. Data retrieval mechanism using Amazon simple storage service and Windows Azure. 3rd International Conference on Computing for Sustainable Global Development (INDIACom). 2016. pp. 412-414.

[64] Lee, J.; Kim, Y.; Hahn, J.; Hoseok S. Customer energy management platform in the Smart Grid. 14th Asia-Pacific Network Operations and Management Symposium (APNOMS). 2012. pp. 1-4.

[65] Shi, K.; Bi, Y.; Jiang, L. Middleware-based implementation of smart micro-grid monitoring using data distribution service over IP networks. 49th International Universities Power Engineering Conference (UPEC). 2014. pp. 1-5.

[66] Object Management Group. What's in the DDS Standard? Available from: http://portals.omg.org/dds/omg-dds-standard/. 2016. Last access $17^{\text {th }}$ May 2017.

[67] Object Management group, I. Documents Associated With Data Distribution Service ${ }^{T M}$, V1.4. 2015 [cited 2016 4th November 2016]; Available from: http://www.omg.org/spec/DDS/1.4/.

[68] Object Management Group. Documents Associated With The Real-Time PublishSubscribe Wire Protocol DDS Interoperability ${ }^{T M}$ Wire Protocol Specification, V2.2. Available from: http://www.omg.org/spec/DDSI-RTPS/2.2/. 2014. $17^{\text {th }}$ May 2017.

[69] RTI. Data Distribution Service (DDS) Community RTI Connext Users: Domain. Available from: https://community.rti.com/glossary/domain. 2016. $17^{\text {th }}$ May 2017.

[70] RTI community. Fundamentals of DDS Domains and DomainParticipants. Available from:

https://community.rti.com/static/documentation/connext-

$\mathrm{dds} / 5.2 .0 / \mathrm{doc} / \mathrm{manuals} / \mathrm{connext} \mathrm{dds} / \mathrm{html}$ files/RTI ConnextDDS CoreLibraries User sManual/Content/UsersManual/Fundamentals of DDS Domains and DomainPa.htm . 2015. Last access $17^{\text {th }}$ May 2017. 
[71] OpenDDS. OpenDDS. Available from: http://opendds.org/. 2016. Last access $17^{\text {th }}$ May 2017.

[72] PrismTech. DDS community. Available from: http://www.prismtech.com/ddscommunity. 2017. Last access $17^{\text {th }}$ May 2017.

[73] eProsima. Introduction to DDS. Available from:

http://www.eprosima.com/index.php/resources-all/dds-all. 2015. Last access $17^{\text {th }}$ May 2017.

[74] Twin Oaks Computing, Inc. CoreDX DDS Data Distribution Service Middleware. Available from: http://www.twinoakscomputing.com/coredx. 2017. Last access $17^{\text {th }}$ May 2017.

[75] GNU Operating System initiative. GNU Lesser General Public License. Available from: https://www.gnu.org/licenses/lgpl-3.0.en.html. 2007. Last access $17^{\text {th }}$ May 2017.

[76] Guang L.; Seed, D.; Starsinic, M.; Wang, C.; Russell, P. Enabling Smart Grid with ETSI M2M standards. IEEE Wireless Communications and Networking Conference Workshops (WCNCW). 2012. pp. 148-153.

[77] European Telecommunications Standards Institute. Available from: http://www.etsi.org/about. 2017. Last access $17^{\text {th }}$ May 2017.

[78] Oliveira, J. P. C. M.; Rodrigues, A. W. d. O.; Sá, R. C.; Araújo, P.; Araújo, A. L. C. Smart Middleware Device for Smart Grid integration. IEEE 24th International Symposium on Industrial Electronics (ISIE). 2015. pp. 779-781.

[79] Ashok, A.; Hahn, A.; Govindarasu, M. Cyber-physical security of Wide-Area Monitoring, Protection and Control in a smart grid environment. Journal of Advanced Research, 2014. vol 5. no. 4. p. 481-489.

[80] Hu, Y.; Donnelly, M.; Helmer, T.; Tram, H.; Martin, K.; Govindarasu, M.; Uluski, R.; Cioni, M. NASPInet Specification - An Important Step toward Its Implementation. 43rd Hawaii International Conference on System Sciences (HICSS). 2010. pp. 1-9.

[81] Hoefling, M.; Heimgaertner, F.; Menth, M.; Katsaros, K. V.; Romano, P.; Zanni, L.; Kamel, G. Enabling resilient smart grid communication over the information-centric $C$ DAX middleware. International Conference and Workshops on Networked Systems (NetSys). 2015. pp. 1-8.

[82] C-DAX Consortium. Cyber-secure Data and Control Cloud for power grids. Available from: http://www.cdax.eu/. 2012. Last access $17^{\text {th }}$ May 2017.

[83] Martin, S.; Hernandez, J.; Valmaseda, C. A novel middleware for smart grid data exchange towards the energy efficiency in buildings. International Conference and Workshops on Networked Systems (NetSys). 2015. pp. 1-8.

[84] OSGi Alliance Initiative. OSGi Alliance - About us.

Available from: https://www.osgi.org/about-us/. 2017. Last access $17^{\text {th }}$ May 2017. 
[85] Villa, D.; Martín, C.; Villanueva, F. J.; Moya, F.; López J. C.. Middleware-based management for smart grids. IEEE International Conference on Consumer Electronics (ICCE). 2011. pp. 545-546.

[86] Object Management Group. CORBA - Home. Available from: http://www.corba.org/. 2017. Last access 17th May 2017.

[87] Henning, M.; Spruiell, M. Distributed programming with Ice. Available from: http://www.triodia.com/staff/michi/lce-3.4.1.pdf. 2010. Last access $17^{\text {th }}$ May 2017.

[88] Lemenager, F.; Joannic, C.; Soriano, R.; Bachiller Prieto, R.; Alberto Monferrer, M.; Espejo Portero, N.; Mosshammer, R. Assessment and outlook of the OpenNode Smart Grid architecture. 22nd International Conference and Exhibition on Electricity Distribution (CIRED). 2013. pp. 1-4.

[89] OpenNode consortium. Available from: http://opennode.atosresearch.eu/. 2012. Last access 17th May 2017.

[90] Wilcox, J.; Kaleshi, D.; Sooriyabandara, M. DIRECTOR: A distributed communication transport manager for the Smart Grid. IEEE International Conference on Communications (ICC). 2014. pp. 4227-4232.

[91] RS Components. Raspberry Pi Model B. Available from:

http://docs-europe.electrocomponents.com/webdocs/127d/0900766b8127da4b.pdf.

2017. Last access 17th May 2017.

[92] Youssef, T.A.; Elsayed, A. T.; Mohammed, O. A. DDS based interoperability framework for Smart Grid Testbed infrastructure. IEEE 15th International Conference on Environment and Electrical Engineering (EEEIC). 2015. pp. 219-224.

[93] OpenESB community. OpenESB: the Open Enterprise Service Bus. Available from: http://www.open-esb.net/. 2017. Last access 17th May 2017.

[94] OpenESB V2.3 team. OpenESB V2.3 Data Sheet. Available from: http://www.openesb.net/files/OpenESB\%20Documents/Community/v2.3\%20datasheet/ProductSheetOpenESB\%20V2.3\%20.pdf. 2017. Last access 17th May 2017.

[95] Oracle Corp. JSR 208: JavaTM Business Integration (JBI). Available from: https://jcp.org/en/jsr/detail?id=208. 2010. Last access 17th May 2017.

[96] OpenESB community. Available from: https://en.wikipedia.org/wiki/Open ESB. 2017. Last access 17th May 2017.

[97] Oracle Corp. Oracle GlassFish Server 3.1 Quick Start Guide. Available from: http://docs.oracle.com/cd/E18930 01/html/821-2432/index.html. 2011. Last access 17th May 2017.

[98] Red Hat Inc.. Red Hat JBoss Enterprise Application Platform. Available from: http://www.jboss.org/products/eap/download/. 2016. Last access 17th May 2017. 
[99] Pymma Services. OpenESB SE Enterprise Edition V3.0 Installation guide. Available from:

http://www.open-

esb.net/files/OpenESB\%20Documents/Pymma/rt@!\&\%60\%60WERTO/770-

001\%20Startup\%20with\%200penESB\%20SE.pdf. 2014. Last access 17th May 2017.

[100] Open Source Initiative. Common Development and Distribution License (CDDL-1.0). Available from: https://opensource.org/licenses/CDDL-1.0. 2017. Last access 17th May 2017.

[101] WSO2 consortium. WSO2: Comprehensive and open platform for your connected business. Available from: http://wso2.com/products/. 2017. Last access 17th May 2017.

[102] WSO2 ESB consortium. WSO2 ESB architecture.

Available from: https://docs.wso2.com/display/ESB490/Architecture. 2016. Last access 17th May 2017.

[103] WSO2 ESB documentation. ESB Transports. Available from:

https://docs.wso2.com/display/ESB490/ESB+Transports. 2016. Last access 17th May 2017.

[104] Pietrzak, M. Scalability of WSO2 integration bus. Available from: https://www.bluesoft.net.pl/2012/04/scalability-of-wso2-integration-bus/. 2015. Last access 17th May 2017.

[105] Red hat Inc. Introducing Red Hat JBoss Fuse. Available from:

https://access.redhat.com/documentation/en-

US/Red Hat JBoss Fuse/6.0/html/Product Introduction/files/ESBProdIntroOverview. html. 2015. Last access 17th May 2017.

[106] Open Source Initiative. GNU General Public License, version 2 (GPL-2.0). Available from: https://opensource.org/licenses/GPL-2.0. 2017. Last access 17th May 2017.

[107] JBoss Community. Getting Started Guide: Your guide to starting out with the JBoss ESB. Available from:

http://docs.jboss.org/ibossesb/docs/4.12/manuals/html/Getting Started Guide/index .html\#gpl. 2010. Last access 17th May 2017.

[108] OSGi Alliance. OSGi: Architecrture.

Available from: https://www.osgi.org/developer/architecture/. 2017. Last access 17th May 2017.

[109] Red Hat Inc. Architectural Levels. Available from: https://access.redhat.com/documentation/enUS/Red Hat JBoss Fuse/6.0/html/Product Introduction/files/ESBProdIntroAnatomy. html. 2017. Last access 17th May 2017.

[110] Red Hat Inc. How Red Hat JBoss Fuse Activates a Deployed Bundle. Available from: https://access.redhat.com/documentation/en- 
US/Red Hat JBoss Fuse/6.0/html/Product Introduction/files/ESBexecution.html. 2017. Last access 17th May 2017.

[111] Rintanen, J.; Suhonen, J.; Hännikäinen, M.; Hämäläinen, T. D. International Workshop on Embedded Computer Systems. 2008. pp. 248-257.

[112] Apache Software Foundation. Service Mix. Available from: http://servicemix.apache.org/. 2011. Last access 17th May 2017.

[113] Rouse, M. business process management (BPM). Available from: http://searchcio.techtarget.com/definition/business-process-management. 2017. Last access 17th May 2017.

[114] Vogel, L. The OSGi declarative services functionality. Available from: http://www.vogella.com/tutorials/OSGiServices/article.html\#declarativeservices over view. 2016. Last access 17th May 2017.

[115] Spring Framework consortium. Chapter 1. Why Spring Dynamic Modules? Available from:

http://docs.spring.io/osgi/docs/current/reference/html/why-Spring\%20DM.html. 2017. Last access 17th May 2017.

[116] Apache Software Foundation. ActiveMQ. Available from: http://activemq.apache.org/. 2011. Last access 17th May 2017.

[117] Oracle Corp. Java SE Technologies - Database.

Available from: http://www.oracle.com/technetwork/java/javase/jdbc/index.html. 2017. Last access 17th May 2017.

[118] Apache Software Foundation. Apache Camel. Available from: http://camel.apache.org/. 2017. Last access 17th May 2017.

[119] Apache Software Foundation. Apache CXF: An Open-Source Services Framework. Available from: http://cxf.apache.org/. 2017. Last access 17th May 2017.

[120] Apache Software Foundation. Apache Karaf. Available from: http://karaf.apache.org/. 2016. Last access 17th May 2017.

[121] Red Hat Inc. Scalable Deployment with Fuse Fabric. Available from: https://access.redhat.com/documentation/enUS/Red Hat JBoss Fuse/6.0/html/Getting Started/files/Deploy-Scalable.html. 2017. Last access 17th May 2017.

[122] Petals ESB Consortium. Available from: https://doc.petalslink.com/display/petalsesb/Petals+ESB. 2014. Last access 17th May 2017.

[123] OW2 consortium. Introducing the OW2 consortium. Available from: https://www.ow2.org/bin/view/About/OW2 Consortium. 2017. Last access 17th May 2017. 
[124] Petals ESB community. Monitoring Petals ESB with Cacti. Available from: https://doc.petalslink.com/display/petalsesbsnapshot/Monitoring+Petals+ESB+with+C acti. 2017. Last access 17th May 2017.

[125] Nagios Enterprises. Nagios: The industry standard inlT infrastructure monitoring. Available from: https://www.nagios.com/. 2017. Last access 17th May 2017.

[126] Petals ESB community. Petals Technical Overview. Available from: https://doc.petalslink.com/display/petalsesb/Petals+Technical+Overview. 2017. Last access 17th May 2017.

[127] Mulesoft Inc. Mule User Guide. Available from: https://docs.mulesoft.com/mule-user-guide/v/3.6/. 2017. Last access 17th May 2017.

[128] MuleSoft Inc. What is Mule ESB? Available from: https://www.mulesoft.com/resources/esb/what-mule-esb. 2009. Last access 17th May 2017.

[129] MuleSoft Inc. Mule ESB Enterprise. Available from: https://www.mulesoft.com/platform/soa/mule-esb-enterprise. 2009. Last access 17th May 2017.

[130] MuleSoft Inc., Mule ESB Enterprise Performance. Available from: https://www.mulesoft.com/lp/whitepaper/soa/esb-enterprise-performance 2014. (pp. 5). Last access 17th May 2017.

[131] Talend community. Talend Open Studio Documentation. Available from: https://help.talend.com/\#/home. 2017. Last access 17th May 2017.

[132] Talend Inc. Talend Open Source ESB. Available from: https://www.talend.com/resource/open-source-esb.html. 2017. Last access 17th May 2017.

[133] Talend ESB community. Enterprise Service Bus. Available from: https://www.talend.com/resource/enterprise-service-bus/. 2017. Last access 17th May 2017.

[134] Talend ESB community. Technical Overview - Talend ESB. Available from: https://info.talend.com/esbtechoverview.html?type=seo\& ga=1.131380428.2118450 593.1479400308. 2017. Last access 17th May 2017.

[135] Zato community. Zato 2.0.7 documentation. Available from: https://zato.io/docs/index.html. 2017. Last access 17th May 2017.

[136] Zato community. Open Source ESB, SOA, REST, APIs and Cloud Integrations in Python. Available from: https://zato.io/blog/. 2017. Last access 17th May 2017.

[137] Zato community. What ESB and SOA are anyway. Available from: https://zato.io/docs/intro/esb-soa.html. 2017. Last access 17th May 2017.

[138] Zato community. Securing APIs with Zato and Vault, part 1. Available from: 
https://zato.io/blog/posts/securing-apis-zato-vault.html. 2016. Last access 17th May 2017.

[139] Zato. Zato downloads. Available from: https://zato.io/downloads.html. 2017. Last access 17th May 2017.

[140] Zachar, M.; Daoutidis, P. Microgrid/Macrogrid Energy Exchange: A Novel Market Structure and Stochastic Scheduling. IEEE Transactions on Smart Grid. 2016. vol. 8. no 1. pp. 178-189.

[141] de Diego, R.; Martínez, J.-F.; Rodríguez-Molina, J.; Cuerva, A. A Semantic Middleware Architecture Focused on Data and Heterogeneity Management within the Smart Grid. Energies. 2014. vol. 7 no. 9. pp. 5953-5994.

[142] Grady Booch, J.R.; Jacobson, I. The Unified Modeling Language User Guide, 2nd Edition, ed. Addison-Wesley. 2005.

[143] IEEE/IEC/ISO 42010 group,. ISO/IEC/IEEE 42010 Website. Available from: http://www.iso-architecture.org/ieee-1471/. 2017. Last access 17th May 2017.

[144] Object Management Group. The Architecture of Choice for a Changing World (Executive Overview). Available from:

http://www.omg.org/mda/executive overview.htm. 2016. Last access 17th May 2017.

[145] Ciancarini, P. Model transformations. Available from:

http://www.slideshare.net/kronat/6-architetture-software-model-transformation.

2011. Last access 17th May 2017.

[146] Garrido, J. L.; Noguera, M.; González, M.; Hurtado, M. V.; Rodríguez, M. L. Definition and use of Computation Independent Models in an MDA-based groupware development process. Science of Computer Programming. 2007. vol 66. no 1. pp. 2543.

[147] Yashwant S.; Sood, M. The Impact of the Computational Independent Model for Enterprise Information System Development. Available from: http://citeseerx.ist.psu.edu/viewdoc/download?doi=10.1.1.206.5131\&rep=rep1\&type =pdf. 2010. Last access 17th May 2017.

[148] Object Management Group. Model Driven Architecture (MDA) FAQ. Available from: http://www.omg.org/mda/faq mda.htm. 2017. Last access 17th May 2017.

[149] MQTT community. MQTT Version 3.1.1. Available from: http://docs.oasisopen.org/mqtt/mqtt/v3.1.1/os/mqtt-v3.1.1-os.pdf. 2014. Last access 17th May 2017.

[150] AMQP community. AMQP - Advanced Message Queuing Protocol. Available from: https://www.amqp.org/. 2014. Last access 17th May 2017.

[151] European Committee for Standardization. CEN, the European Committee for Standardization: who we are. Available from:

https://www.cen.eu/about/Pages/default.aspx. 2017. Last access 17th May 2017. 
[152] European Committee for Electrotechnical Standardization consortium (CENELEC). CENELEC: Who we are. Available from: https://www.cenelec.eu/aboutcenelec/whoweare/. 2017. Last access 17th May 2017.

[153] CEN-CENELEC-ETSI Smart Grid Coordination Group. Smart Grid Reference Architecture. Available from: https://ec.europa.eu/energy/sites/ener/files/documents/xpert group1 reference arc hitecture.pdf. 2012. Last access 17th May 2017.

[154] Rodríguez-Molina, J.; Martínez, J.-F.; Castillejo, P.; Rubio, G. Development of Middleware Applied to Microgrids by Means of an Open Source Enterprise Service Bus. Energies. 2017. vol. 10. no. 2. pp. 172-221.

[155] The Legion of the Bouncy Castle. Bouncy Castle Crypto APIs. Available from: https://www.bouncycastle.org/. 2013. Last access 17th May 2017.

[156] Apache Software Foundation. Apache Jena: A free and open source Java framework for building Semantic Web and Linked Data applications. Available from: http://jena.apache.org/index.html. 2017. Last access 17th May 2017.

[157] World Wide Web Consortium. Resource Description Framework (RDF). Available from: http://www.w3.org/RDF/. 2014. Last access 17th May 2017.

[158] World Wide Web Consortium. SPARQL 1.1 Query Language. Available from: https://www.w3.org/TR/sparql11-query/. 2013. Last access 17th May 2017.

[159] Hilliard, R. Architecture description template for use with ISO/IEC/IEEE 42010:2011. Available from: http://www.iso-architecture.org/42010/templates/42010-adtemplate.pdf. 2011. Last access 17th May 2017.

[160] International Standard Organization, International Electrotechnical Commission, Institute of Electrical and Electronics Engineers. Systems and software engineering Architecture description (International Standard ISO/IEC/IEEE 42010). Available from: http://ieeexplore.ieee.org/stamp/stamp.jsp?arnumber=6129467\&tag=1. 2011. Last access 17th May 2017.

[161] Kim, K.D.; Kumar, P. R. Cyber-Physical Systems: A Perspective at the Centennial. Proceedings of the IEEE. 2012. vol 100 (Special Centennial Issue). pp. 1287-1308.

[162] Sakurama, K. Control of Large-Scale Cyber-Physical Systems with Agents via a Representative Model. IEEE 4th International Conference on Cyber-Physical Systems, Networks, and Applications (CPSNA). 2016. pp. 40-43.

[163] Heesch, U.v.; Avgeriou, P.; Hilliard, R. Forces on Architecture Decisions - A Viewpoint. Joint Working IEEE/IFIP Conference on Software Architecture and European Conference on Software Architecture. 2012. pp. 101-110.

[164] IEEE/IEC/ISO, Architecture Viewpoint Template for ISO/IEC/IEEE 42010. Available from: http://www.iso-architecture.org/ieee-1471/templates/42010-vp-template.pdf. 2011. 2011. Last access 17th May 2017. 
[165] Sharma, D.; Lie, T.T.; Nair, N. K. C., Vallès, B. Wind speed forecasting using ANN, ARMA and AIC hybrid to ensure power grid reliability. IEEE Innovative Smart Grid Technologies - Asia (ISGT ASIA). 2015. pp. 1-5.

[166] Al-Khannak, R.; Bitzer, B. Grid Computing as an innovative solution for power system's reliability and redundancy. International Conference on Clean Electrical Power. 2009. pp. 790-797.

[167] Noshahr, J.B. Emission phenomenon of supra-harmonics caused by switching of fullpower frequency converter of wind turbines generator (PMSG) in smart grid. IEEE 16th International Conference on Environment and Electrical Engineering (EEEIC). 2016. pp. $1-6$.

[168] Ivry, P. M.; Rawa, M. J.; Thomas, D. W. P.; Sumner, M. Power quality of a voltage source converter in a smart grid. IEEE Grenoble Conference. 2013. pp. 1-6.

[169] Red Hat Inc. Download JBoss Fuse for Development Use. Available from: https://developers.redhat.com/products/fuse/download/. 2016. Last access 17th May 2017.

[170] Oracle Corp. The JMS API Programming Model. Available from: http://docs.oracle.com/iavaee/6/tutorial/doc/bnceh.html. 2013. Last access 17th May 2017.

[171] Redmonk Community,. The RedMonk Programming Language Rankings: January 2015. Available from: http://redmonk.com/sogrady/2015/01/14/language-rankings-1-15/. 2015. Last access 17th May 2017.

[172] Apache Maven Project. Welcome to Apache Maven. Available from: https://maven.apache.org/. 2015. Last access 17th May 2017.

[173] Herrfors katerno Group. Herrfors Ltd., Your local power company (Finnish). Available from: https://www.herrfors.fi/. 2017. Last access 17th May 2017.

[174] Enfo Ltd. About Enfo. Available from: http://www.enfo.no/about-enfo/. 2017. Last access 17th May 2017.

[175] Cuerva, A. Modelo de información común de un middleware semántico para Internet de la Energía (Spanish). Available from: http://oa.upm.es/43690/. 2016. Last access 17th May 2017.

[176] Martos, M.d.I.C.. Servicio de seguridad combinable mediante técnicas de orquestación de servicios (Spanish). Available from: http://oa.upm.es/38799/. 2015. Last access 17th May 2017.

[177] Rodríguez-Molina, J.; Martínez, J.-F.; Castillejo, P.; López, L. Combining Wireless Sensor Networks and Semantic Middleware for an Internet of Things-Based Sportsman/Woman Monitoring Application. Sensors. 2013. vol 13. no 2. p. 1787. 
[178] Rodríguez-Molina, J.; Martínez, J.-F.; Castillejo, P.; de Diego, R. SMArc: A Proposal for a Smart, Semantic Middleware Architecture Focused on Smart City Energy Management. International Journal of Distributed Sensor Networks. 2013. vol 9. no 12. pp. 560418.

[179] Rodríguez, L.M.M. Evaluación del dispositivo Raspberry Pi como elemento de despliegue de servicios en el marco de las Smart Grids (Spanish). Available from: http://oa.upm.es/32612/. 2014. Last access 17th May 2017.

[180] e-GOTHAM consortium. e-GOTHAM in short. Available from: http://ylivieska.centria.fi/egotham/eGenglish.html. 2015. Last access $22^{\text {nd }}$ March 2017.

[181] OpenEnergyMonitor Project. About OpenEnergyMonitor Project. Available from: https://learn.openenergymonitor.org/sustainable-energy/energy/introduction. 2017. Last access 17th May 2017.

[182] NTE - Demo Steinkjer (Norwegian). Available from:

http://smartgridnorway.no/?dt portfolio=nte-demo-steinkjer. 2017. Last access 17th May 2017.

[183] International Standard Association. P42020 - Standard for Systems and Software Engineering -- Architecture Processes.

Available from: https://standards.ieee.org/develop/project/42020.html. 2017. Last access 17th May 2017.

[184] Society, I.S. P20000-1 - 20000-1, Information Technology -- Service Management -- Part 1: Service Management System Requirements.

Available from: https://standards.ieee.org/develop/project/20000-1.html. 2017. Last access 17th May 2017.

[185] Li, X.; Martínez, J.-F.; Rodríguez-Molina, J.; Martínez, N.L. A Survey on Intermediation Architectures for Underwater Robotics. Sensors. 2016. vol 16 no. 2. pp. 190.

[186] Rodríguez-Molina, J.; Martínez, J.-F.; Rubio, G.; Hernández, V. A Proposal for an Internet of Things-based Monitoring System Composed by Low Capability, Open Source and Open Hardware Devices. SENSORNETS 2014 - 3rd International Conference on Sensor Networks. 2014. vol. 3. pp. 87-94.

[187] Rodríguez-Molina, J.M.-N., M.; Pérez-Aguiar, W.; de Diego, R, A study on challenges and opportunities for energy business developments in the Smart City. 8th International Conference on Industrial Engineering and Industrial Management. 2014. pp. 254-261.

[188] Martín-Wanton, T.; Muelas, E.; Santos de la Cámara, R.; Rodríguez-Molina, J.; Li, X.; Martinez-Ortega, J-F. Modelado de vehículos autónomos y la incertidumbre de su entorno para la seguridad de operaciones marítimas (Spanish). IV Congreso Nacional de $i+d$ en Defensa y Seguridad (DESEi+d 2016). 2016. pp. 599-606.

[189] Rodríguez-Molina, J., The Role of Middleware in Distributed Energy Systems Integrated in the Smart Grid. Energy Management of Distributed Generation Systems.

Available from: 
https://www.intechopen.com/books/energy-management-of-distributed-generationsystems/the-role-of-middleware-in-distributed-energy-systems-integrated-in-thesmart-grid. 2016. pp. 133-154.

[190] Hernández, V.; Martínez J.-F.; Cuerva, A.; Rodríguez-Molina, J.; Rubio, G.; Jara, A.; Semantic as an Interoperability Enabler in Internet of Things. Vol. 1. 2013. pp. 315-342.

[191] Actúa UPM blogspot. iLa XIII Competición actúaupm alcanza sus mejores cifras! (Spanish). Available from: http://actuaupm.blogspot.com.es/2016/04/la-xiiicompeticion-actuaupm-alcanza.html. 2016. Last access 17th May 2017.

[192] Universidad Politécnica de Madrid web manager. XIII Competición de creación de empresas UPM. Premios de mil euros a las mejores ideas de negocio. (Spanish). Available from:

http://www.upm.es/sfs/Rectorado/Gabinete\%20del\%20Rector/Notas\%20de\%20Prens a/2016/04/documentos/premiadoscompeticionactua.pdf. 2016. Last access 17th May 2017. 


\subsection{List of acronyms}

\begin{tabular}{|c|c|}
\hline Acronym & Extended name \\
\hline ACSI & Abstract Communication Service Interface \\
\hline ADN & Active Distribution Networks \\
\hline AMI & Advanced Metering Infrastructure \\
\hline AMQP & Advanced Message Queuing Protocol \\
\hline API & Application Programming Interface \\
\hline ARM & Advanced RISC Machines \\
\hline ARTEMIS & Advanced Research and Technology for Embedded Intelligence and Systems \\
\hline AUV & Autonomous Underwater Vehicle \\
\hline AWS & Amazon Web Services \\
\hline BAN & Body Area Network \\
\hline BIM & Building Information Model \\
\hline BMS & Building Management System \\
\hline BPEL & Business Process Execution Language \\
\hline BSD & Berkeley Software Distribution \\
\hline BSS & Business Supporting System \\
\hline $\mathrm{CC}$ & Central Controller \\
\hline C-DAX & Cyber-secure Data and Control Cloud for power grids \\
\hline CDDL & Common Development and Distribution License \\
\hline CEMS & Customer Energy Management Systems \\
\hline $\mathrm{CLI}$ & Command Line Interface \\
\hline CLL & Communication Logic Layer \\
\hline CMA & Common Middleware Architecture \\
\hline CORBA & Common Object Request Broker Architecture \\
\hline CPS & Cyber-Physical System \\
\hline DACM & Data Acquisition and Control Manager \\
\hline DC & Domain Controller \\
\hline DCPS & Data-Centric Publish/Subscribe \\
\hline DDS & Data Distributed Service \\
\hline DER & Distributed Energy Resource \\
\hline DMS & Distributed Management System \\
\hline DNP3 & Distributed Network Protocol, version 3 \\
\hline DN & Designated Node \\
\hline DPWS & Device Profile for Web Services \\
\hline DR & Demand Response \\
\hline DRMS & Demand Response Management System \\
\hline DSM & Demand Side Management \\
\hline DSO & Distribution System Operator \\
\hline ECSEL & Electronic Components and Systems for European Leadership \\
\hline EDA & Event Driven Architecture \\
\hline
\end{tabular}




\begin{tabular}{|c|c|}
\hline e-GOTHAM & $\begin{array}{l}\text { Sustainable-Smart Grid Open System for the Aggregated Control, Monitoring } \\
\text { and Management of Energy }\end{array}$ \\
\hline EMD & Embedded Metering Devices \\
\hline EMS & Energy Management System \\
\hline ESB & Enterprise Service Bus \\
\hline ETSI & European Telecommunications Standards Institute \\
\hline FP7 & Framework Programme 7 \\
\hline GNU & GNU's Not Unix! \\
\hline GPRS & General Packet Radio Service \\
\hline GUI & Graphical User Interface \\
\hline HEMS & Home Energy Management System \\
\hline HTTP & Hypertext Transfer Protocol \\
\hline HVDC & High Voltage Direct Current \\
\hline I3RES & $\begin{array}{l}\text { ICT-based Intelligent management of Integrated RES for the smart grid optimal } \\
\text { operation }\end{array}$ \\
\hline IAP & Intelligent Agents Platform \\
\hline IBM & International Business Machines \\
\hline ICT & Information and Communication Technology \\
\hline IDL & Interface Description Language \\
\hline IED & Intelligent Electronic Device \\
\hline INMS & Integrated Network Management \\
\hline IoT & Internet of Things \\
\hline IP & Internet Protocol \\
\hline IPRs & Intellectual Property Rights \\
\hline ITEA & Information Technology for European Advancement \\
\hline $\mathrm{JBI}$ & Java Business Integration \\
\hline JCR & Journal Citation Report \\
\hline JDBC & Java Database Connectivity \\
\hline JSON & JavaScript Object Notation \\
\hline JVM & Java Virtual Machine \\
\hline LC & Local Controller \\
\hline LCE & Loosely Couple Event \\
\hline LGPLv3 & GNU Lesser General Public License \\
\hline $\mathrm{M} 2 \mathrm{M}$ & Machine-to-Machine \\
\hline MD & Mediation Device \\
\hline MDI & Meter Data Integration \\
\hline MDMS & Meter Data Management System \\
\hline MMS & Manufacturing Message Specification \\
\hline MOM & Message-Oriented Middleware \\
\hline MOS & Mean Option Score \\
\hline NASPI & North American Synchro-Phasor Initiative \\
\hline NATO & North Atlantic Treaty Organization \\
\hline NGB & Next Generation Network \\
\hline
\end{tabular}




\begin{tabular}{|c|c|}
\hline OEM & Original Equipment Manufacturer \\
\hline OMG & Object Management Group \\
\hline OPF & Optimal Power Flow \\
\hline OSGi & Open Services Gateway initiative \\
\hline OSHNet & Object-Based Middleware for Smart Home Network \\
\hline PC & Personal Computer \\
\hline PDC & Phasor Data Concentrators \\
\hline PIM & Platform Independent Model \\
\hline PLC & Power Line Communication \\
\hline PMU & Phasor Measurement Unit \\
\hline POM & Project Object Model \\
\hline PSM & Platform Specific Model \\
\hline QoE & Quality of Experience \\
\hline QoS & Quality of Service \\
\hline RAM & Random Access Memory \\
\hline REMS & Renewable Energy Management System \\
\hline RES & Renewable Energy Source \\
\hline REST & Representational State Transfer \\
\hline $\mathrm{RPC}$ & Remote Procedure Call \\
\hline RTP & Real Time Protocol \\
\hline RTPS & Real Time Publish Subscriber \\
\hline RTSE & Real-Time State Estimation \\
\hline RTU & Remote Terminal Unit \\
\hline SC & Service Consumers \\
\hline SCADA & Supervisory Control And Data Acquisition system \\
\hline SEDA & Stage Event-Driven Architecture \\
\hline SMTP & Simple Mail Transfer Protocol \\
\hline SOA & Service Oriented Architecture \\
\hline SOAP & Simple Object Access Protocol \\
\hline SP & Service Providers \\
\hline SQL & Structured Query Language \\
\hline SSN & Secondary Substation Node \\
\hline SWARMs & Smart and Networking UnderWAter Robots in Cooperation Meshes \\
\hline TCP & Transmission Control Protocol \\
\hline TSO & Transmission System Operator \\
\hline UDP & User Datagram Protocol \\
\hline USN & Ubiquitous Sensor Network Middleware \\
\hline VNA & Virtual Network Adapter \\
\hline VPN & Virtual Private Network \\
\hline VPP & Virtual Power Plant \\
\hline WAC & Wide-Area Control \\
\hline WAMPAC & Wide Area Monitoring, Protection, and Control Systems \\
\hline
\end{tabular}


Chapter 6. Bibliography

\begin{tabular}{|l|l|}
\hline WAMS & Wide-Area Monitoring Systems \\
\hline Wi-Fi & Wireless Fidelity \\
\hline WiMAX & Worldwide Interoperability for Microwave Access \\
\hline WSDL & Web Services Description Language \\
\hline WSN & Wireless Sensor Network \\
\hline XML & eXtensible Markup Language \\
\hline XSD & XML Schema Definition \\
\hline
\end{tabular}

